Symbolic Hierarchical Interface-based Supervisory Control 



\title{
Symbolic Synthesis and Verification of Hierarchical Interface-BASEd Supervisory CONTROL
}

\author{
By \\ RaOguang Song, B.Eng.
}

\begin{abstract}
A Thesis
Submitted to the School of Graduate Studies in Partial Fulfillment of the Requirements

for the Degree

Master of Applied Science

McMaster University

(C)Copyright by Raoguang Song, Mar. 2006
\end{abstract}


Master of Applied Science(2006)

Computing And Software
McMaster University

Hamilton, Ontario

TITLE: Symbolic Synthesis and Verification of Hierarchical Interface-based Supervisory Control

AUTHOR: Raoguang Song, B.Eng.

SUPERVISOR: Dr. Ryan J. Leduc

NUMBER OF PAGES: xvii, 249 


\section{Abstract}

Hierarchical Interface-based Supervisory Control (HISC) is a method to alleviate the state-explosion problem when verifying the controllable and nonblocking properties of a large discrete event system. By decomposing a system as a number of subsystems according to the HISC method, we can verify the subsystems separately and the global system controllability and nonblocking property are guaranteed, so that potentially great computation effort is saved.

In this thesis, we first present a predicate-based synthesis algorithm for each type of subsystem and then prove the correctness of the algorithms. Then a predicatebased verification algorithm for each type of subsystem is provided. Based on the predicate-based algorithms, a symbolic implementation is proposed by using Binary Decision Diagrams (BDD) and the fact that a subsystem is usually composed of a number of components. With the symbolic implementation, we can handle a much larger subsystem of each type.

Two large and complicated examples (with estimated worst-case state space on the order of $10^{30}$ ) extended from the AIP example are provided for demonstrating the capabilities of the algorithms and the implementation. A software tool for the synthesis and verification using our approach is also developed. 


\section{Acknowledgements}

First I would like to sincerely thank my supervisor, Dr. Ryan Leduc, for his insightful guidance, great patience and generous support; for his careful review and corrections to the draft of this thesis.

A special thanks to Dr. Chuan Ma for his help on the BDD implementation techniques and STS. I must acknowledge preceding research works on HISC synthesis method done by Pengcheng Dai and many useful discussions with him.

At last, I am very much indebted to my wife Ning Zhou and my daughter Karen. To them, I dedicate this thesis. 


\section{Contents}

List of Figures xiii

List of Tables $\quad$ xvii

1 Introduction 1

1.1 Research Review . . . . . . . . . . . . . . . . . . . 3

1.2 Thesis Overview . . . . . . . . . . . . . . . . . 6

1.3 Thesis Structure . . . . . . . . . . . . . . . . . . . . . 7

2 Preliminaries $\quad 9$

2.1 Algebra .............................. 9

2.1.1 Posets and Lattices . . . . . . . . . . . . . . . . . . . 9

2.1.2 Equivalence Relations . . . . . . . . . . . . . . . . 11

2.1.3 Monotone Functions and Fixpoints . . . . . . . . . . . . . . . 11

2.2 Languages . . . . . . . . . . . . . . . . . . . . . . 14

2.3 DES . . . . . . . . . . . . . . . . . . . 15

2.4 Operations on DES . . . . . . . . . . . . . . . . . . 18

2.4 .1 Product and Meet . . . . . . . . . . . . . 18

2.4.2 Synchronous Product . . . . . . . . . . . . . . . . 18

2.5 Predicates and Predicate Transformers . . . . . . . . . . . . . 20 
2.5 .1 Predicates . . . . . . . . . . . . . . . . 20

2.5.2 Predicate Transformers . . . . . . . . . . . . . . . 22

2.5.3 Languages Induced by Predicates . . . . . . . . . . . 27

2.6 Supervisory Control . . . . . . . . . . . . . . . . . . . . . . . . . . . 32

2.6 .1 Controllable Languages . . . . . . . . . . . . . . . . . . 32

2.6.2 Supervisory Control _. . . . . . . . . . . . . . . . 33

2.6.3 Implementation of MNSC $V$ for $\mathbf{G} \ldots \ldots \ldots \ldots$

2.6 .4 Supervisor Synthesis . . . . . . . . . . . . . . 38

3 HISC Overview $\quad 39$

3.1 System Structure . . . . . . . . . . . . . . . . . . . . . . . . . . 39

3.1 .1 Command-pair Interfaces . . . . . . . . . . . . . . . . 42

3.1 .2 Flat System . . . . . . . . . . . . . . . . . 43

3.2 Local Conditions . . . . . . . . . . . . . . . . . . . . 44

3.2 .1 Interface Consistent . . . . . . . . . . . . . 45

3.2.2 Local Conditions for Global Nonblocking . . . . . . . . . 54

3.2.3 Local Conditions for Global Controllability . . . . . . . . . 54

3.3 Level-wise Interface Controllable Supervisor . . . . . . . . . . . 55

4 Synthesis of HISC $\quad 59$

4.1 Introduction . . . . . . . . . . . . . . . . . . . . . . 59

4.2 High-level Supervisor Synthesis _. . . . . . . . . . . . 62

4.2.1 High-level Interface Controllable Language . . . . . . . . . . 62

4.2.2 $\sup \mathcal{C}_{H}\left(L_{m}\left(\mathcal{G}_{H}\right)\right)$ and the Greatest Fixpoint of $\Omega_{H} \ldots \ldots 66$

4.2.3 Computing the greatest fixpoint of $\Omega_{H} \ldots \ldots \ldots \ldots$

4.2.4 Computing $\sup \mathcal{C}_{H}\left(L\left(\mathcal{G}_{H}, \mathcal{P}_{h a}\right)\right) \ldots \ldots \ldots \ldots \ldots$

4.2 .5 Computing $\Omega_{H N B}\left(L\left(\mathcal{G}_{H}, P\right)\right) \ldots \ldots \ldots 2$ 
4.2.6 The Algorithm to Compute $\sup \mathcal{C}_{H}\left(L_{m}\left(\mathcal{G}_{H}\right)\right) \ldots \ldots \ldots$

4.3 Low-level Supervisor Synthesis . . . . . . . . . . . . . . . . . . . 101

4.3.1 The $j^{\text {th }}$ Low-level P4 Interface Controllable Language . . . . . 101

4.3.2 The $j^{\text {th }}$ Low-level Interface Controllable Language . . . . . . . 110

4.3.3 $\sup \mathcal{C}_{L_{j}}\left(L_{m}\left(\mathcal{G}_{L_{j}}\right)\right)$ and the Greatest Fixpoint of $\Omega_{L_{j}} \ldots \ldots 113$

4.3.4 Computing the Greatest Fixpoint of $\Omega_{L_{j}} \ldots \ldots \ldots \ldots \ldots$

4.3.5 The Algorithm to Compute $\sup \mathcal{C}_{L_{j}}\left(L_{m}\left(\mathcal{G}_{L_{j}}\right) \ldots \ldots \ldots\right.$

5 Verification of HISC 143

6 Symbolic Computation for HISC Synthesis and Verification 145

6.1 Symbolic Representation of State Subsets and Transitions . . . . . 146

6.1.1 Symbolic Representation of State Subsets . . . . . . . . 147

6.1.2 Symbolic Representation of Transitions . . . . . . . . . . . 151

6.2 Symbolic Computation of Predicate Transformers . . . . . . . . . 154

6.2.1 Computation of Transition and Inverse Transition . . . . . . 155

$6.2 .2 \quad$ Computation of $R \ldots \ldots \ldots \ldots \ldots \ldots$

6.2 .3 Computation of $T R \ldots \ldots \ldots \ldots \ldots \ldots$

6.2.4 Computation of $\mathcal{C} \mathcal{R} \ldots \ldots \ldots \ldots \ldots$

6.3 Miscellaneous Computation for HISC Synthesis and Verification . . 168

6.3.1 Computation of $\operatorname{pr}\left(\operatorname{Bad}_{H}\right) \ldots \ldots \ldots \ldots$

6.3.2 Computation of $\operatorname{pr}\left(\operatorname{Bad}_{L_{j}}\right) \ldots \ldots \ldots \ldots \ldots \ldots$

6.3.3 Miscellaneous Computation in Algorithm 4.2 . . . . . . . 172

6.3.4 Verifying Event Partition . . . . . . . . . . . . . . 173

6.3.5 Verifying Command-pair Interfaces _ . . . . . . . . . . 174

6.4 BDD Implementations of Algorithms . . . . . . . . . . . 177

6.4.1 State Variable Ordering . . . . . . . . . . . . . . 178 
6.5 Controller Implementation . . . . . . . . . . . . . . . . . 180

6.5.1 Simplifying Control Predicates . . . . . . . . . . . . . 188

6.6 A Small Example . . . . . . . . . . . . . . . . . . . 193

7 The AIP Example $\quad 199$

7.1 Introduction of the AIP . . . . . . . . . . . . . . . 200

7.2 Control Specifications . . . . . . . . . . . . . . . . . . 203

7.3 System Structure . . . . . . . . . . . . . . . . . . 205

7.4 The Interface DES . . . . . . . . . . . . . . . . . . 207

7.5 Low-level Subsystems . . . . . . . . . . . . . . . . . . . . 208

7.6 The High-level Subsystem . . . . . . . . . . . . . . . . . . . . 209

7.6.1 Plant Components . . . . . . . . . . . . . . . 210

7.6.2 Supervisor Components . . . . . . . . . . . . . 214

7.7 Verifying Properties . . . . . . . . . . . . . . . . 222

7.8 Synthesizing Supervisors . . . . . . . . . . . . . . 226

7.9 Results For the AIP Example . . . . . . . . . . . . . . . . . 228

8 Multiple AIP Example $\quad 229$

8.1 System Model . . . . . . . . . . . . . . . . . . 230

8.2 Verifying Properties . . . . . . . . . . . . . . . . 234

8.3 Synthesizing Supervisors . . . . . . . . . . . . . . . . 236

9 Conclusions and Future Work 239

9.1 Conclusions . . . . . . . . . . . . . . . . . . . 239

9.2 Future Work . . . . . . . . . . . . . . . . . . . . . 241

9.2.1 Intermediate BDD Size Problem . . . . . . . . . . . . . 241

9.2.2 Complexity Analysis of the Algorithms . . . . . . . . . . . . 242 
9.2.3 Symbolic Computation for Timed HISC System . . . . . . . . 242

A HISC Software Program $\quad 251$

A.1 Introduction of HISC Software Program . . . . . . . . . . . . . . 251

A.2 Source Code . . . . . . . . . . . . . . . . . . . 253 


\section{List of Figures}

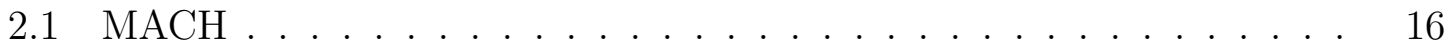

2.2 Example for predicate transformers $\ldots \ldots \ldots \ldots$

3.1 HISC block diagram . . . . . . . . . . . . . . . . . 40

3.2 HISC system structure . . . . . . . . . . . . . . . . . . . . 42

3.3 Example interface . . . . . . . . . . . . . . . . . . . . . . . . 43

6.1 Small factory example . . . . . . . . . . . . . . . . . 148

6.2 Example DES $\mathbf{G}_{E} \ldots \ldots \ldots \ldots \ldots \ldots \ldots \ldots$

6.3 Example system $\mathbf{M} \ldots \ldots \ldots$

6.4 Control diagram . . . . . . . . . . . . . . . . . . . . . . 183

6.5 New control diagram . . . . . . . . . . . . . . . . . . . . 187

6.6 System diagram for small example . . . . . . . . . . . . . . . . 193

6.7 The small example . . . . . . . . . . . . . . . . . . . . . . . . . . . 194

6.8 Synthesized high-level proper supervisor _ . . . . . . . . . . . . . . 195

6.9 Synthesized low-level proper supervisor for low-level $m 1$. . . . . . . 195

6.10 Synthesized low-level proper supervisor for low-level $m 2$. . . . . . . 195

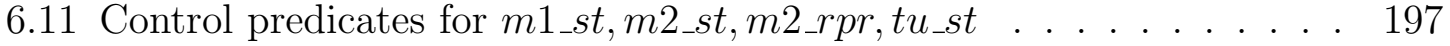

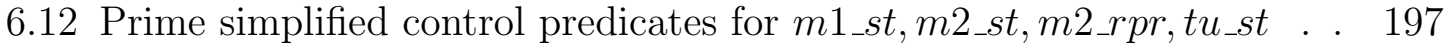

6.13 Triple-prime simplified control predicates for $m 1 \_s t, m 2 \_s t, m 2 \_r p r, t u \_s t 197$ 


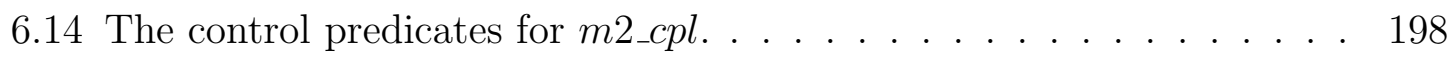

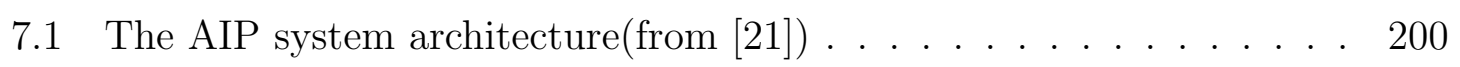

7.2 Assembly station of external loop $\mathrm{X}=1,2,3$ (from [21]) . . . . . . 201

7.3 Transport unit for external loop $\mathrm{X}=1,2,3,4$ (from [21]) . . . . . . 202

7.4 The AIP system structure . . . . . . . . . . . . . . 205

7.5 Interface to low-level $w=1,2$ (from [23]) . . . . . . . . . . . 207

7.6 Interface to low-level 3 (from [23]) . . . . . . . . . . . . . . . . 207

7.7 Interface to low-level $v=4,5,7$ (from [23]) . . . . . . . . . . 207

7.8 Interface to low-level 6 (from [23]) . . . . . . . . . . . . . . . 207

7.9 Interface to low-level $8 \ldots \ldots \ldots$. . . . . . . . . . . 208

7.10 CapGateEL_2.AS3 . . . . . . . . . . . . . . . . . . 208

7.11 OperateGateEL_2.AS3 . . . . . . . . . . . . . . . . 209

7.12 PalletArvGateSenEL_2.AS3 . . . . . . . . . . . . . . . 209

7.13 Low-level 8 Subsystem . . . . . . . . . . . . . . . . . 209

7.14 Component DES in the AIP high-level . . . . . . . . . . . . . 210

7.15 ASStoreUpState. $k=$ AS1, AS2 (from [23]) . . . . . . . . . 211

7.16 QueryPalletAtTU.i, $i=\mathrm{TU} 1, \mathrm{TU} 2, \mathrm{TU} 3, \mathrm{TU} 4 \ldots \ldots . . \ldots 211$

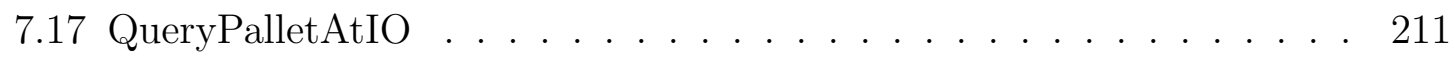

7.18 CapEL12, CapEL22, CapEL32, CapEL42 . . . . . . . . . . . . . 212

7.19 CapC1, CapC2, CapC3, CapC4 . . . . . . . . . . . 213

7.20 CapEL11, CapEL21, CapEL31, CapEL41 . . . . . . . . . . . 214

7.21 ManageTU1 . . . . . . . . . . . . . . . . 216

7.22 ManageTU2 . . . . . . . . . . . . . . . . 216

7.23 ManageTU3 . . . . . . . . . . . . . . . . 217

7.24 ManageTU4 . . . . . . . . . . . . . . . . 217 
7.25 DetWhichStnUp (from [21]) . . . . . . . . . . . . . . . . 218

7.26 HndlComEventsAS(from [21]) . . . . . . . . . . . . . . 218

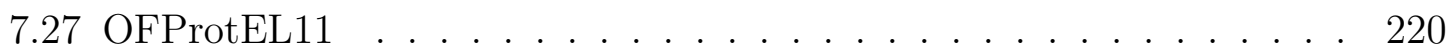

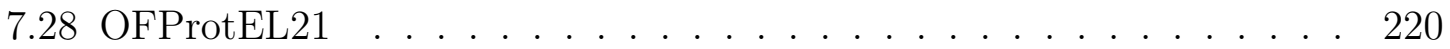

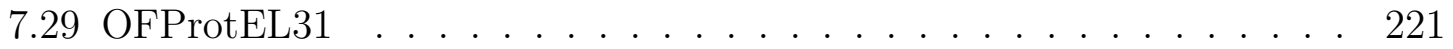

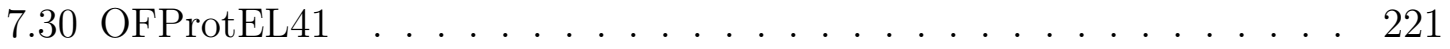

7.31 OFProtEL12, OFProtEL22, OFProtEL32, OFProtEL42 … . . 222

7.32 OFProtC1, OFProtC2, OFProtC3, OFProtC4 . . . . . . . . 223

7.33 AltMvInTypes . . . . . . . . . . . . . . . . . . . . 223

7.34 ManageIO . . . . . . . . . . . . . . . . . . . . . 223

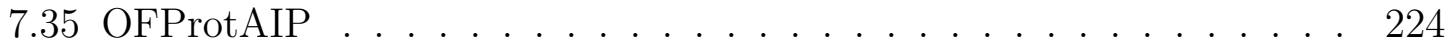

8.1 Block diagram of multiple AIPs . . . . . . . . . . . . . . 230

$8.2 \operatorname{IntfAIP-j}(\mathrm{j}=1,2,3) \ldots \ldots \ldots \ldots \ldots \ldots \ldots \ldots \ldots$

8.3 New low-level plants in 3-2 MAIP system(verfication) . . . . . . . . . 231

8.4 Components in low-level subsystem MAIP- $j,(j=1,2,3) \ldots \ldots$

8.5 High-level plant components . . . . . . . . . . . . . . . . . 233

8.6 High-level supervisor components . . . . . . . . . . . . . . . . 234

8.7 New low-level plants for the 5-4 MAIP system(verification) . . . . . 236

8.8 New low-level plants for the 3-2 MAIP system(synthesis) $\ldots \ldots$. . . 237

9.1 Number of BDD nodes and time for $C R$ operation at each iteration . 242 


\section{List of Tables}

7.1 The AIP example data (verification) $\ldots \ldots \ldots \ldots \ldots$

7.2 The AIP example data (synthesis) . . . . . . . . . . . . 227

8.1 The multiple AIP example data(verification) . . . . . . . . 235

8.2 The multiple AIP example data(synthesis) $\ldots \ldots \ldots \ldots$

A.1 Source code files in the software library . . . . . . . . . . . 252

A.2 Source code files for the library usage example . . . . . . . . . 252 
Master Thesis - R. Song - McMaster - Computing and Software 


\section{Chapter 1}

\section{Introduction}

A Discrete Event System (DES) is a dynamic system that is discrete in state space and is event-driven. Ramadge and Wonham (RW) [36] proposed the supervisory control theory framework for a class of general DES, in which the DES is represented as an automaton over an event alphabet, and its behavior is described by the language generated by the automaton. The events in the alphabet are partitioned as controllable events which can be disabled and uncontrollable events which can not be disabled. By disabling and enabling controllable events, a system controller can restrict the behavior of a plant. Such a controller is also called a supervisor. Two properties, controllability and nonblocking, are usually desirable for the behavior of the controlled system.

Although the supervisory control theory does not require the language to be regular, in practice the language should be, so that the automaton representing the language has a finite state space. The control action in the theory is based on the strings generated by the plant. However, the number of the strings could be infinite. In order to implement such a controller, a supervisor DES can be designed so that the synchronous product of the plant DES and the supervisor DES can generate the 


\section{Master Thesis - R. Song - McMaster - Computing and Software}

desired behavior. We can design the supervisor DES by hand, and then verify the controllable and nonblocking properties.

In [48], RW provided an algorithm to synthesize an optimal supervisor according to an arbitrary specification language. An optimal supervisor means that it is a minimally restrictive controller that satisfies the specification and is nonblocking and controllable. The complexity of this algorithm is polynomial on the number of the DES states.

The most intuitive but inefficient method to store a DES in a computer is to save the state space and transitions in look-up tables directly. To check the controllable and nonblocking properties, we have to traverse the DES which means we have to go through and search the look-up tables. However, most practical systems have a very large state space since they are usually modeled as the synchronous product of a group of component DES, so the state space size is increased exponentially on the number of the components. Such a problem is known as the state explosion problem.

The software TCT [47] is a tool using the above method to store a DES. It provides a synthesizing routine (supcon) to compute the optimal supervisor and other routines to help model a system and check properties. Due to the state explosion problem, TCT can only handle some simple systems under the memory limitations (With 1GB RAM, TCT can handle a system on the order of $10^{7}$ states). Therefore, it is necessary to find other efficient approaches to model and represent DES such that for a large system a supervisor can be synthesized and nonblocking and controllable properties can be verified. 


\section{Master Thesis - R. Song-McMaster-Computing and Software}

\section{$1.1 \quad$ Research Review}

In order to overcome the state explosion problem, usually two categories of approaches can be applied: to explore control architectures and to explore internal structures of DES.

Modular control $[13,34,49]$ is an early and widely used method, which implements control action by designing multiple supervisors. Each supervisor is responsible to implement part of the control specifications. In $[3,30,39,45,50]$, this approach is extended as decentralized control by allowing each supervisor access only to partial observations of the plant. These architectures successfully solved the controllability verification but not for the nonblocking verification.

Another architectural approach is the model aggregation methods or the bottom up methods $[8,18,33,41,46,52]$. In these methods, the aggregation model(high-level) is obtained from low-level models by language aggregation or state aggregation. The "hierarchical consistency" concept is introduced to make sure that a high-level supervisor can be implemented in low-levels. However, as aggregate models are constructed from low-levels sequentially, a given level can not be constructed until all the levels below it have been constructed. Furthermore, the conditions for this type of approaches are sufficient and necessary, which means that a change on one level may affect all the levels above it.

In [35], RW introduced a static state feedback supervisory control framework by using predicates to store the state space and define the specifications. In [47], a dynamic state feedback control is also introduced by using memory components. However, they did not mention how to represent a predicate.

In the VDES $[9,10,28,29]$ or Petri Net $[32,42,43]$ framework, a system state is represented by a vector with integer components, and state transitions by integer 


\section{Master Thesis - R. Song - McMaster - Computing and Software}

vector addition. The specifications are defined in the form of linear inequalities. The supervisor is synthesized by using linear programming algorithms. These approaches are applied well on the systems with high degree of regularity. Moreover, to verify the nonblocking property or synthesize a nonblocking controller, a reachability graph may need to be constructed first.

STCT [51] utilized the fact that a system is usually modeled as a group of DES components, so that each state of the system can be represented as a vector. It then does the synthesis process based on the synchronous product of the plant and the specification components but does not actually build the synchronous product. By using IDD (Integer Decision Diagram, an extension of Binary Decision Diagram (BDD) [6]) to encode the state space and transitions of the system, STCT can synthesize an optimal controllable and nonblocking supervisor for a system with closed-loop state space size on the order of $10^{23}$, but that system is quite artificial, and there is no rigorous theoretical foundation for the algorithms.

Ma [31] presented a top-down multi-level design model called State Tree Structures (STS) which was initiated in [44] by using the idea of state charts [17]. A STS includes a State Tree to store states and holons to store local dynamics. A sub-state-tree is used to store a state set and a basic sub-state-tree is used to represent one state. Set operations such as union could be done by the join operation of two sub-statetrees, and global dynamics could be constructed from the local holons, but there is no easy sub-state-tree operation corresponding to the intersection of two state sets. Furthermore, not all of the state sets can be represented as one sub-state-tree. However, by using STS, one could model a system from the top level and treat each module as a superstate, and later on the module can be further designed in detail. These modeling steps reflect the hierarchical nature of most complex systems, so modeling a system as a STS will be straightforward. 


\section{Master Thesis - R. Song-McMaster-Computing and Software}

To solve the above problems in STS, Ma used BDDs to represent state sets. Due to the rich structure of STS, the state sets can be represented by BDDs compactly. By using the optimized recursive algorithms, he modeled and synthesized a state-based supervisor for a system with an estimated state space on the order of $10^{24}$. However, in order to construct the global transitions from local transitions, STS requires the local coupling condition: no shared events between two holons matched to the OR superstates that are not AND-adjacent to the same AND superstates. This restriction could force a modeler to flatten the STS, so that it makes the STS less structural and some optimization techniques (e.g. coreachable inference) work less efficiently. We will talk more about this in the AIP example chapter. Furthermore, in STS, the synthesis process is still working on the global state space, which grows exponentially on the number of components.

Leduc et al. [21-27] proposed the Hierarchical Interface-based Supervisory Control (HISC) by bringing the information hiding theory from software engineering into supervisory control. By using this method, a system is decomposed into one high-level subsystem and multiple low-level subsystems. Between the high-level subsystem and each low-level subsystem, there is a well-defined interface which restricts the interaction of the subsystems. All the communications between the high-level subsystem and low-level subsystems must be done through a specific interface. Therefore, after modeling the interfaces, we can model those subsystems simultaneously. The really valuable thing in HISC is that as long as each subsystem and its interface satisfies certain local conditions, then the global controllable and nonblocking properties can be guaranteed, which means that we only need to work on the states in each subsystem instead of the states of the synchronous product of the whole system. With automata based algorithms, Leduc successfully verified a system with an estimated worst-case

closed-loop state space on the order of $10^{21}$. Richter et al. [37] also modeled and 


\section{Master Thesis - R. Song - McMaster - Computing and Software}

verified a bottling plant by using the HISC method with estimated state space on the order of $10^{47}$ (They obtained this number by multiplying the number of states in each component DES).

\subsection{Thesis Overview}

Because the algorithms in [21] (Leduc) explicitly list the states and transitions, each subsystem could not be very large. With 1GB of RAM, he could handle individual components only as large as $10^{6}-10^{7}$ states. This might put limitations on modeling a real system especially for the high-level. Dai [14] is developing synthesis algorithms to compute the supervisors for each subsystem. However, his algorithms are still based on the explicit state and transition listing, thus do not save much computation.

As each subsystem in HISC is usually modeled as a group of plant DES and a group of specification/supervisor DES, each subsystem-wide state can be represented as a vector, where each member of the vector is the state of a component DES. Therefore, we can use BDD to represent the state space and transitions for each subsystem, and develop algorithms based on BDD representations to verify or synthesize supervisors for it. By using the BDD representation and algorithms, our program is able to handle much larger subsystem, so the power of HISC is greatly improved.

In this thesis, we need to verify not only the controllable and nonblocking properties but also the interface consistent conditions in [21] for the high-level and low-level subsystems. The conditions we used are from $[23,24]$, an equivalent version of the conditions in [21]. A proof for the equivalence between the two can be found in [14,24]. We first present the synthesis algorithms based on specifications for each type of subsystem, and then give the verification algorithms based on modular supervisors for 


\section{Master Thesis - R. Song-McMaster-Computing and Software}

each type of subsystem. The correctness of the algorithms is proved. A software tool to implement these algorithms is also developed.

Two examples of industrial size are provided to show the capability of our algorithms in this thesis. The first one is extended from the AIP example in [21], which synthesizes a high-level supervisor with state space on the order of $10^{15}$. In the second example, we build a feedback system by treating each high-level subsystem in the first example as a low-level system, and then synthesize a low-level supervisor.

As the structure of each subsystem could be thought of as a special STS with three levels (Level 0 is the root AND superstate, and each state in Level 1 is an OR superstate corresponding to each component DES, and each state in Level 2 is a simple state corresponding to each state in the component DES), we borrowed many ideas from [31], especially with respect to BDD implementation.

\subsection{Thesis Structure}

The remaining chapters in this thesis are organized as follows.

In Chapter 2, we present the necessary algebra and language foundations for proofs in this thesis. Basic supervisory control theory of DES and some fundamental operations of DES and propositions are also presented.

In Chapter 3, we give an overview for the HISC method, and then give an equivalent definition of the interface consistent condition for the purpose of proving our synthesis algorithms.

In Chapter 4 and 5, the synthesis and verification algorithms based on predicate operations for an HISC system are presented and proved.

In Chapter 6, we give a BDD-based implementation of the algorithms in Chapter 4 and 5. The encoding techniques and optimization techniques for the BDD imple- 


\section{Master Thesis - R. Song - McMaster - Computing and Software}

mentations are the main focus of this chapter.

In Chapter 7 and 8, examples to show the capabilities of our algorithms and BDD implementations are presented.

Finally, in Chapter 9, we discuss the future work and conclusions. 


\section{Chapter 2}

\section{Preliminaries}

In this chapter, we first discuss some basic algebraic and linguistic concepts that will be used in the later chapters. Then we introduce the predicate and predicate transformers which are important for our later algorithms. After that, we give an overview of DES and the RW supervisory control theory. We also state and prove several fundamental propositions. This chapter is largely based on [47]. Proposition 2.1 is an extension of Theorem 3.18 from [19] by Huth and Ryan.

\subsection{Algebra}

\subsubsection{Posets and Lattices}

Let $X$ be a set, and $\leq$ be a binary relation on $X$. $\leq$ is a partial order if it has the following three properties:

- reflexive: $(\forall x \in X) x \leq x$

- transitive: $(\forall x, y, z \in X) x \leq y \& y \leq z \Rightarrow x \leq z$

- antisymmetric: $(\forall x, y \in X) x \leq y \& y \leq x \Rightarrow x=y$ 


\section{Master Thesis - R. Song - McMaster - Computing and Software}

If $\leq$ is a partial order on $X$, then the pair $(X, \leq)$ is called a poset. If $\leq$ is understood, we could call $X$ a poset.

Let $(X, \leq)$ be a poset, $x, y \in X$. An element $l \in X$ is a meet of $x$ and $y$ (denote it as $\left.x \sqcap y=l^{1}\right)$, if

$$
l \leq x \& l \leq y \&((\forall a \in X) a \leq x \& a \leq y \Rightarrow a \leq l)
$$

Dually, an element $u \in X$ is a join of $x$ and $y($ denote it as $x \sqcup y=u$ ), if

$$
x \leq u \& y \leq u \&((\forall b \in X) x \leq b \& y \leq b \Rightarrow u \leq b)
$$

Let $L$ be a set. A poset $(L, \leq)$ is a lattice if the meet and join of any two elements in $L$ always exist.

Let $S$ be a nonempty subset of $L$ and $l \in L$. Element $l$ is the infimum of $S(\inf S)$ if

$$
(\forall y \in S) l \leq y \quad \& \quad(\forall z \in L)((\forall y \in S) z \leq y) \Rightarrow z \leq l
$$

Dually, $u \in L$ is the supremum of $(\sup (S))$ if

$$
(\forall y \in S) y \leq u \quad \& \quad(\forall z \in L)((\forall y \in S) y \leq z) \Rightarrow u \leq z
$$

A lattice $(L, \leq)$ is complete if, for any nonempty subset $S$ of $L$, both $\inf S$ and $\sup S$ always exist. Every finite lattice is complete.

Let $X$ be a set. Define $\operatorname{Pwr}(X)$ as the set consisting of all the subsets of $X$. $(\operatorname{Pwr}(X), \subseteq)$ is a complete lattice $[15]$. This fact is fundamental for the correctness of our algorithms.

\footnotetext{
${ }^{1}$ In [47], the meet of elements $x$ and $y$ is denoted as $x \wedge y$, and the join of them is denoted as $x \vee y$, but we will use the notation $\wedge$ for predicate operation and and $\vee$ for predicate operation or, because the predicate operations are heavily used in this thesis.
} 


\section{Master Thesis - R. Song-McMaster-Computing and Software}

\subsubsection{Equivalence Relations}

Let $X$ be a nonempty set, and $E$ a binary relation on $X$. E is an equivalence relation if

- $(\forall x \in X) x E x \quad$ (reflexive)

- $\left(\forall x, x^{\prime} \in X\right) x E x^{\prime} \Rightarrow x^{\prime} E x \quad$ (symmetric)

- $\left(\forall x, x^{\prime}, x^{\prime \prime} \in X\right) x E x^{\prime} \& x^{\prime} E x^{\prime \prime} \Rightarrow x E x^{\prime \prime} \quad$ (transitive)

Let $x \in X$, define $[x] \subseteq X$ as $[x]:=\left\{x^{\prime} \in X \mid x^{\prime} E x\right\}$. $[x]$ is called the coset or equivalent class of $x$ with respect to the equivalence relation $E$.

By $x \in[x],[x]$ is nonempty.

Let $x, y \in X$, then it can be shown that either $[x]=[y]$ or $[x] \cap[y]=\emptyset$. Namely, $[x]$ and $[y]$ are either equal or disjoint.

\subsubsection{Monotone Functions and Fixpoints}

Let $(X, \leq)$ be a complete lattice. A function $f: X \rightarrow X$ is monotone if

$$
(\forall x, y \in X) x \leq y \Rightarrow f(x) \leq f(y) .
$$

Let $X$ be a set, and $f: X \rightarrow X$ be a function. An element $x \in X$ is a fixpoint of $f$ if $x=f(x)$. Let $(X, \leq)$ be a complete lattice. The element $x$ is the greatest fixpoint of $f$ if

$$
\left(\forall x^{\prime} \in X\right) x^{\prime}=f\left(x^{\prime}\right) \Rightarrow x^{\prime} \leq x .
$$

Let $X$ be a set, and $f: X \rightarrow X$ be a function. For $x \in X$, denote $f^{0}(x)$ for $x$, $f^{1}(x)$ for $f(x), f^{2}(x)$ for $f(f(x)), \ldots$, and $f^{i}(x)$ for 
Master Thesis - R. Song - McMaster - Computing and Software

$$
\underbrace{f(f(\ldots f}_{i}(x))), i \in\{0,1,2, \ldots\} .
$$

We now state Theorem 2.1 which is the well known Knaster-Tarski Theorem [40].

Theorem 2.1 (Knaster-Tarski Theorem). Let $(X, \leq)$ be a complete lattice, and let $f: X \rightarrow X$ be a monotone function. Then the greatest fixpoint of $f$ exists and is equal to $\sup \{x \mid x \leq f(x)\}$.

Note that the set $X$ can be uncountable infinite, and that this theorem does not say anything about how to compute the greatest fixpoint. It only states the existence.

For our usage, the complete lattice is $\left(\operatorname{Pwr}\left(\Sigma^{*}\right), \subseteq\right) . \Sigma^{*}$ is countable infinite, while $\operatorname{Pwr}\left(\Sigma^{*}\right)$ is uncountable infinite.

Proposition 2.1 is a special case of the Knaster-Tarski theorem, and it also gives a method to compute the greatest fixpoint for the special case.

Proposition 2.1. Let $X$ be a finite set with $n$ elements, $n \in\{0,1, \ldots\}$. If a function $f: \operatorname{Pwr}(X) \rightarrow \operatorname{Pwr}(X)$ is a monotone function with respect to $\subseteq$, then there exists $k \in\{0,1,2, \ldots\}$ such that $k \leq n$ and $f^{k}(X)$ is the greatest fixed point of $f$ with respect to $(P w r(X), \subseteq)$, and $(\forall i \in\{1,2, \ldots\}) i>k \Rightarrow f^{i}(X)=f^{k}(X)$.

\section{Proof:}

1. Show that there exists $k \leq n, f^{k}(X)$ is a fixpoint of $f$, i.e. $f^{k}(X)=f^{k+1}(X)$.

We know that $f(X) \subseteq X$,

$$
\begin{aligned}
& \Rightarrow f^{2}(X) \subseteq f(X), \quad \text { as } f \text { is monotone. } \\
& \Rightarrow f^{3}(X) \subseteq f^{2}(X) \\
& \ldots \\
& \Rightarrow f^{n+1}(X) \subseteq f^{n}(X)
\end{aligned}
$$

So, we have 


$$
\begin{gathered}
\text { Master Thesis - R. Song - McMaster - Computing and Software } \\
\qquad f^{n+1}(X) \subseteq f^{n}(X) \subseteq \cdots \subseteq f^{1}(X) \subseteq X
\end{gathered}
$$

Assume $\nexists k \leq n, f^{k}(X)=f^{k+1}(X)$, then

$$
f^{n+1}(X) \subset f^{n}(X) \subset \cdots \subset f^{1}(X) \subset X
$$

This is impossible, because $X$ only contains $n$ elements, and $f^{k+1}(X) \subset f^{k}(X)$ $(k \in\{0,1, \ldots\})$ means that $f^{k+1}(X)$ contains at least one less element than $f^{k}(X)$ does.

2. Show that $f^{k}(X)$ is the greatest fixpoint of $f$, i.e. $(\forall S \in P w r(X)) S=f(S) \Rightarrow$ $S \subseteq f^{k}(X)$.

Let $S \in \operatorname{Pwr}(X)$, and assume $S=f(S)$.

Must show this implies $S \subseteq f^{k}(X)$.

We know that $S \subseteq X$

$\Rightarrow f(S) \subseteq f(X), \quad$ as $f$ is monotone

$\Rightarrow S \subseteq f(X), \quad$ as $S=f(S)$

$\Rightarrow f(S) \subseteq f^{2}(X), \quad$ as $f$ is monotone

$\Rightarrow S \subseteq f^{2}(X), \quad$ as $S=f(S)$

$\cdots$

$\Rightarrow S \subseteq f^{k}(X)$

3. Show that $(\forall i \in\{1,2, \ldots\}) i>k \Rightarrow f^{i}(X)=f^{k}(X)$.

This follows immediately since $f^{k}(X)$ is a fixpoint of $f$, i.e. $f^{k}(X)=f^{k+1}(X)$. 
Master Thesis - R. Song - McMaster - Computing and Software

\section{$2.2 \quad$ Languages}

Let $\Sigma$ be a finite set of symbols, and we refer to $\Sigma$ as an alphabet. A string over $\Sigma$ is a finite sequence of symbols in $\Sigma$. Denote $\Sigma^{+}$as the set of all such strings over $\Sigma$.

Let $\epsilon(\notin \Sigma)$ be the empty string (no symbols), we write $\Sigma^{*}=\Sigma^{+} \cup\{\epsilon\}$.

We define the operation of concatenation of strings: cat $: \Sigma^{*} \times \Sigma^{*} \rightarrow \Sigma^{*}$ according to

$$
\begin{gathered}
\operatorname{cat}(\epsilon, s)=\operatorname{cat}(s, \epsilon)=s, \quad s \in \Sigma^{*}, \\
\operatorname{cat}(s, t)=s t, \quad s, t \in \Sigma^{+} .
\end{gathered}
$$

A language $L$ is a subset of $\Sigma^{*}$, and thus an element of $\operatorname{Pwr}\left(\Sigma^{*}\right)$.

Let $s, t \in \Sigma^{*} . t$ is said to be a prefix of $s(t \leq s)$ if $\left(\exists u \in \Sigma^{*}\right) t u=s$.

Let $L \subseteq \Sigma^{*}$. The (prefix) closure of $L$ is defined as:

$$
\bar{L}:=\left\{t \in \Sigma^{*} \mid(\exists s \in L) t \leq s\right\}
$$

A language $L$ is closed if $L=\bar{L}$.

The following proposition will be used in following chapters.

Proposition 2.2. Let $L_{1}, L_{2} \in \operatorname{Pwr}\left(\Sigma^{*}\right)$. Then the following holds:

$$
\overline{L_{1}} \cup \overline{L_{2}}=\overline{L_{1} \cup L_{2}}
$$

\section{proof:}

$\overline{L_{1}} \cup \overline{L_{2}} \subseteq \overline{L_{1} \cup L_{2}}$ follows immediately since $\overline{L_{1}} \subseteq \overline{L_{1} \cup L_{2}}$ and $\overline{L_{2}} \subseteq \overline{L_{1} \cup L_{2}}$.

We now show $\overline{L_{1} \cup L_{2}} \subseteq \overline{L_{1}} \cup \overline{L_{2}}$.

Let $s \in \overline{L_{1} \cup L_{2}}$

Must show this implies $s \in \overline{L_{1}} \cup \overline{L_{2}}$

By (1), we know $\left(\exists s^{\prime} \in \Sigma^{*}\right) s s^{\prime} \in L_{1} \cup L_{2}$

$\Rightarrow s s^{\prime} \in L_{1}$ or $s s^{\prime} \in L_{2}$ 


$$
\text { Master Thesis - R. Song - McMaster - Computing and Software }
$$

If $s s^{\prime} \in L_{1}$, then $s \in \overline{L_{1}}$. We thus have $s \in \overline{L_{1}} \cup \overline{L_{2}}$.

Similarly, if $s s^{\prime} \in L_{2}$, then $s \in \overline{L_{2}}$. We thus also have $s \in \overline{L_{1}} \cup \overline{L_{2}}$.

By (2), (3) and (4), we can conclude $s \in \overline{L_{1}} \cup \overline{L_{2}}$.

Let $L \subseteq \Sigma^{*}$ and $s \in \Sigma^{*}$. The operator $\operatorname{Elig}_{L}: \Sigma^{*} \rightarrow \operatorname{Pwr}(\Sigma)$ is used to get the set of eligible events for $s$ with respect to $L$.

$$
\operatorname{Elig}_{L}(s):=\{\sigma \in \Sigma \mid s \sigma \in L\}
$$

Let $L \subseteq \Sigma^{*}$. The Nerode equivalence relation on $\Sigma^{*}$ with respect to $L$ is defined as

$$
\left(\forall s, t \in \Sigma^{*}\right) s \equiv_{L} t \text { iff }\left(\forall u \in \Sigma^{*}\right) s u \in L \Leftrightarrow t u \in L .
$$

Write $\|L\|$ as the cardinality of the set of the equivalence class of $\equiv_{L}$. If $\|L\|$ is finite, we say $L$ is regular. All the languages in this thesis are regular unless otherwise stated.

For a regular language $L$, if we treat each Nerode equivalence class as a state, then we can build a recognizer with finite number of states to tell if a string $s \in \Sigma^{*}$ is in $L$ or not. Such a recognizer is said to be canonical.

\section{$2.3 \quad$ DES}

A DES $\mathbf{G}$ is a generator, and formally defined as a five tuple

$$
\mathbf{G}:=\left(Q, \Sigma, \delta, q_{0}, Q_{m}\right)
$$

where $Q$ is the state set; $\Sigma=\Sigma_{c} \dot{\cup} \Sigma_{u}$, where $\Sigma_{c}$ is the set of controllable events, and $\Sigma_{u}$ is the set of uncontrollable events; $\delta: Q \times \Sigma \rightarrow Q$ is the (partial) transition function; $q_{0}$ is the initial state, and $Q_{m} \subseteq Q$ is the set of marker states. We always assume $Q$ and $\Sigma$ are finite. 
Master Thesis - R. Song - McMaster - Computing and Software

Let $q \in Q, \sigma \in \Sigma$. We use $\delta(q, \sigma)$ ! to mean that $\delta(q, \sigma)$ is defined.

The transition function $\delta$ can be extended to $\delta: Q \times \Sigma^{*} \rightarrow Q$ according to

$$
\begin{gathered}
\delta(q, \epsilon)=q \\
\delta(q, s \sigma)=\delta(\delta(q, s), \sigma)
\end{gathered}
$$

provided $q^{\prime}:=\delta(q, s)$ ! and $\delta\left(q^{\prime}, \sigma\right)$ !. Note that $\delta(q, \epsilon)$ is always defined.

A DES over $\Sigma$ is the empty DES if $Q=\emptyset$, and we denote it as EMPTY.

A DES G can be represented as a transition graph. The nodes(circles) in the graph represent states in $Q$. The edges represent transitions. The initial state is labeled with an entering arrow $(\rightarrow \infty)$, and the marker states are labels with an exiting $\operatorname{arrow}(\bullet \rightarrow)$. If the initial state is also a marker state, it will be labeled with a double side $\operatorname{arrow}(\circ \leftrightarrow)$. The controllable transitions are labeled with a slash on the edge. Figure 2.1 is the transition graph representation for the well known MACH DES. The DES MACH includes three states $s 0, s 1$ and $s 2$. State $s 0$ is the initial state and

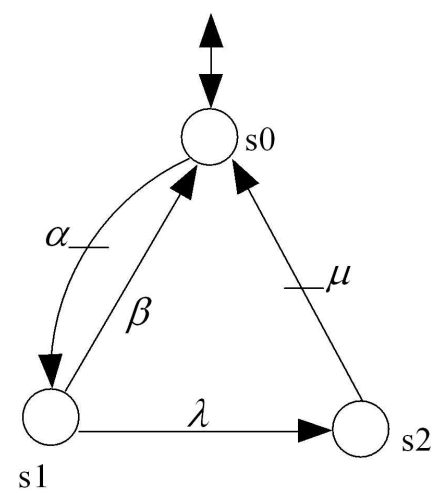

Figure 2.1: $\mathrm{MACH}$

the only marker state. There are four transitions: $(s 0, \alpha, s 1),(s 1, \beta, s 0),(s 1, \lambda, s 2)$, $(s 2, \mu, s 0)$. Events $\alpha, \mu$ are controllable events and events $\beta, \lambda$ are uncontrollable events. Note that the event set $\Sigma$ may include events other than the four events shown in the graph. 


\section{Master Thesis - R. Song - McMaster-Computing and Software}

A DES G generates two languages: $L(\mathbf{G})$ and $L_{m}(\mathbf{G})$, which are called the closed behavior of $\mathbf{G}$ and the marked behavior of $\mathbf{G}$ respectively, and are defined as follows.

$$
\begin{gathered}
L(\mathbf{G}):=\left\{s \in \Sigma^{*} \mid \delta\left(q_{0}, s\right) !\right\} \\
L_{m}(\mathbf{G}):=\left\{s \in \Sigma^{*} \mid \delta\left(q_{0}, s\right) \in Q_{m}\right\}
\end{gathered}
$$

The language $L(\mathbf{G})$ is closed, and $L_{m}(\mathbf{G}) \subseteq L(\mathbf{G})$.

Let $q \in Q$. State $q$ is reachable if $\exists s \in \Sigma^{*}$ such that $\delta\left(q_{0}, s\right)=q$. State $q$ is coreachable if $\exists s \in \Sigma^{*}$ such that $\delta(q, s) \in Q_{m}$.

A DES $\mathbf{G}$ is reachable, if every state in $Q$ is reachable.

A DES $\mathbf{G}$ is coreachable, if every state in $Q$ is coreachable.

A DES $\mathbf{G}$ is trim, if every state in $Q$ is reachable and coreachable.

A DES $\mathbf{G}$ is nonblocking, if every reachable state in $Q$ is coreachable. i.e. $\overline{L_{m}(\mathbf{G})}=$ $L(\mathbf{G})$.

We say that DES $\mathbf{G}$ represents a language $K \subseteq \Sigma^{*}$ if $\mathbf{G}$ is nonblocking and $L_{m}(\mathbf{G})=K$. We thus have $L(\mathbf{G})=\bar{K}$.

In the tuple definition of $\mathbf{G}$, we define that $\delta: Q \times \Sigma \rightarrow Q$ is a partial function. We say such a DES $\mathbf{G}$ is deterministic. Instead, one can define a DES $\mathbf{T}:=$ $\left(Q, \Sigma, \tau, q_{0}, Q_{m}\right)$, where $Q, \Sigma, q_{0}$ and $Q_{m}$ are defined as in $\mathbf{G}$, but $\tau: Q \times \Sigma \rightarrow \operatorname{Pwr}(Q)$ is a total function. We say such a DES $\mathbf{T}$ is nondeterministic.

As every nondeterministic DES can be converted to a deterministic DES, we assume that all the DES in this thesis are deterministic. 
Master Thesis - R. Song - McMaster - Computing and Software

\subsection{Operations on DES}

\subsubsection{Product and Meet}

Let $\mathbf{G}_{\mathbf{1}}=\left(Q_{1}, \Sigma, \delta_{1}, q_{1_{0}}, Q_{1_{m}}\right)$ and $\mathbf{G}_{\mathbf{2}}=\left(Q_{2}, \Sigma, \delta_{2}, q_{2_{0}}, Q_{2_{m}}\right)$ be two DES defined over the same alphabet $\Sigma$. The product of two DES is defined as:

$$
\mathbf{G}_{\mathbf{1}} \times \mathbf{G}_{\mathbf{2}}:=\left(Q_{1} \times Q_{2}, \Sigma, \delta_{1} \times \delta_{2},\left(q_{1_{0}}, q_{2_{0}}\right), Q_{1_{m}} \times Q_{2_{m}}\right),
$$

where $\delta_{1} \times \delta_{2}: Q_{1} \times Q_{2} \times \Sigma \rightarrow Q_{1} \times Q_{2}$ is given by

$$
\left(\delta_{1} \times \delta_{2}\right)\left(\left(q_{1}, q_{2}\right), \sigma\right):=\left(\delta_{1}\left(q_{1}, \sigma\right), \delta_{2}\left(q_{2}, \sigma\right)\right)
$$

whenever $\delta_{1}\left(q_{1}, \sigma\right) !$ and $\delta_{2}\left(q_{2}, \sigma\right) !$.

From the definition, we have $L\left(\mathbf{G}_{\mathbf{1}} \times \mathbf{G}_{\mathbf{2}}\right)=L\left(\mathbf{G}_{\mathbf{1}}\right) \cap L\left(\mathbf{G}_{\mathbf{2}}\right)$, and $L_{m}\left(\mathbf{G}_{\mathbf{1}} \times \mathbf{G}_{\mathbf{2}}\right)=$ $L_{m}\left(\mathbf{G}_{\mathbf{1}}\right) \cap L_{m}\left(\mathbf{G}_{\mathbf{2}}\right)$.

The meet of DES $\mathbf{G}_{\mathbf{1}}$ and $\mathbf{G}_{\mathbf{2}}$ is defined as $\mathbf{G}_{\mathbf{1}} \times \mathbf{G}_{\mathbf{2}}$ but only including the reachable states, and we denote it as $\operatorname{meet}\left(\mathbf{G}_{\mathbf{1}}, \mathbf{G}_{\mathbf{2}}\right)$.

Obviously, $L\left(\operatorname{meet}\left(\mathbf{G}_{\mathbf{1}}, \mathbf{G}_{\mathbf{2}}\right)\right)=L\left(\mathbf{G}_{\mathbf{1}} \times \mathbf{G}_{\mathbf{2}}\right)$ and $L_{m}\left(\operatorname{meet}\left(\mathbf{G}_{\mathbf{1}}, \mathbf{G}_{\mathbf{2}}\right)\right)=L_{m}\left(\mathbf{G}_{\mathbf{1}} \times\right.$ $\left.\mathrm{G}_{2}\right)$.

\subsubsection{Synchronous Product}

\section{Synchronous Product on Languages}

Let $\Sigma_{1}, \Sigma_{2}$ be two alphabets, $\Sigma=\Sigma_{1} \cup \Sigma_{2}$. 


\section{Master Thesis - R. Song - McMaster - Computing and Software}

Define the natural projection $P_{i}: \Sigma^{*} \rightarrow \Sigma_{i}^{*}(i=1,2)$ according to

$$
\begin{gathered}
P_{i}(\epsilon)=\epsilon \\
P_{i}(\sigma)= \begin{cases}\epsilon & \text { if } \sigma \notin \Sigma_{i} \\
\sigma & \text { if } \sigma \in \Sigma_{i}\end{cases} \\
P_{i}(s \epsilon)=P_{i}(s) P_{i}(\sigma) \quad s \in \Sigma^{*}, \sigma \in \Sigma
\end{gathered}
$$

Clearly, $\left(\forall s, t \in \Sigma^{*}\right) P_{i}(s t)=P_{i}(s) P_{i}(t)$. In other words, $P_{i}$ is catenative.

Let $P_{i}^{-1}: \operatorname{Pwr}\left(\Sigma_{i}^{*}\right) \rightarrow \operatorname{Pwr}\left(\Sigma^{*}\right)$ be the inverse image function of $P_{i}$, namely for $H \subseteq \Sigma_{i}^{*}$,

$$
P_{i}^{-1}(H):=\left\{s \in \Sigma^{*} \mid P_{i}(s) \in H\right\} .
$$

Let $L_{1} \subseteq \Sigma_{1}^{*}, L_{2} \subseteq \Sigma_{2}^{*}$. The synchronous product $L_{1} \| L_{2} \subseteq \Sigma^{*}$ is defined as

$$
L_{1}|| L_{2}:=P_{1}^{-1}\left(L_{1}\right) \cap P_{2}^{-1}\left(L_{2}\right) .
$$

\section{Selfloop}

Let $\mathbf{G}_{\mathbf{1}}=\left(Q_{1}, \Sigma_{1}, \delta_{1}, q_{1_{0}}, Q_{1_{m}}\right)$ be a DES defined on alphabet $\Sigma_{1}$, and $\Sigma_{2}$ be another alphabet with $\Sigma_{1} \cap \Sigma_{2}=\emptyset$. The selfloop operation on $\mathbf{G}_{\mathbf{1}}$ is used to generate a new DES $\mathbf{G}$ by selflooping each event in $\Sigma_{2}$ on each state of $\mathbf{G}_{\mathbf{1}}$. Formally,

$$
\mathbf{G}=\operatorname{selfloop}\left(\mathbf{G}_{1}, \Sigma_{2}\right)=\left(Q_{1}, \Sigma_{1} \cup \Sigma_{2}, \delta_{2}, q_{1_{0}}, Q_{1_{m}}\right),
$$

where $\delta_{2}: Q_{1} \times\left(\Sigma_{1} \cup \Sigma_{2}\right) \rightarrow Q_{1}$ is a partial function and defined as

$$
\delta_{2}(q, \sigma):= \begin{cases}\delta_{1}(q, \sigma), & \sigma \in \Sigma_{1}, \delta_{1}(q, \sigma) ! \\ q, & \sigma \in \Sigma_{2} \\ \text { undefined, } & \text { otherwise }\end{cases}
$$


Master Thesis - R. Song - McMaster - Computing and Software

Let $P_{1}:\left(\Sigma_{1} \cup \Sigma_{2}\right)^{*} \rightarrow \Sigma_{1}^{*}$ be a natural projection, then we have

$$
\begin{aligned}
L\left(\operatorname{selfloop}\left(\mathbf{G}_{\mathbf{1}}, \Sigma_{2}\right)\right) & =P_{1}^{-1}\left(L\left(\mathbf{G}_{\mathbf{1}}\right)\right) \\
L_{m}\left(\operatorname{selfloop}\left(\mathbf{G}_{\mathbf{1}}, \Sigma_{2}\right)\right) & =P_{1}^{-1}\left(L_{m}\left(\mathbf{G}_{\mathbf{1}}\right)\right)
\end{aligned}
$$

\section{Synchronous Product on DES}

Let $\mathbf{G}_{\mathbf{1}}=\left(Q_{1}, \Sigma_{1}, \delta_{1}, q_{1_{0}}, Q_{1_{m}}\right), \mathbf{G}_{\mathbf{2}}=\left(Q_{2}, \Sigma_{2}, \delta_{2}, q_{2_{0}}, Q_{2_{m}}\right)$ be two DES. The synchronous product of $\mathbf{G}_{\mathbf{1}}$ and $\mathbf{G}_{\mathbf{2}}$ is defined as ${ }^{2}$

$$
\mathbf{G}_{\mathbf{1}} \| \mathbf{G}_{\mathbf{2}}:=\operatorname{selfloop}\left(\mathbf{G}_{\mathbf{1}}, \Sigma_{2}-\Sigma_{1}\right) \times \operatorname{selfloop}\left(\mathbf{G}_{\mathbf{2}}, \Sigma_{1}-\Sigma_{2}\right) .
$$

Note that the alphabet set of $\mathbf{G}_{\mathbf{1}} \| \mathbf{G}_{\mathbf{2}}$ is $\Sigma_{1} \cup \Sigma_{2}$. The product of two DES $\mathbf{G}_{\mathbf{1}}, \mathbf{G}_{\mathbf{2}}$ is actually a special case of the synchronous product where $\Sigma_{1}=\Sigma_{2}$.

We define $\operatorname{sync}\left(\mathbf{G}_{\mathbf{1}}, \mathbf{G}_{\mathbf{2}}\right)$ as the DES $\mathbf{G}_{\mathbf{1}} \| \mathbf{G}_{\mathbf{2}}$ but only with reachable states. Then, we have

$$
\begin{gathered}
L\left(\mathbf{G}_{\mathbf{1}} \| \mathbf{G}_{\mathbf{2}}\right)=L\left(\operatorname{sync}\left(\mathbf{G}_{\mathbf{1}}, \mathbf{G}_{\mathbf{2}}\right)\right)=L\left(\mathbf{G}_{\mathbf{1}}\right) \| L\left(\mathbf{G}_{\mathbf{2}}\right)=P_{1}^{-1}\left(L\left(\mathbf{G}_{\mathbf{1}}\right)\right) \cap P_{1}^{-1}\left(L\left(\mathbf{G}_{\mathbf{2}}\right)\right) \\
L_{m}\left(\mathbf{G}_{\mathbf{1}} \| \mathbf{G}_{\mathbf{2}}\right)=L_{m}\left(\operatorname{sync}\left(\mathbf{G}_{\mathbf{1}}, \mathbf{G}_{\mathbf{2}}\right)\right)=L_{m}\left(\mathbf{G}_{\mathbf{1}}\right) \| L_{m}\left(\mathbf{G}_{\mathbf{2}}\right)=P_{1}^{-1}\left(L_{m}\left(\mathbf{G}_{\mathbf{1}}\right)\right) \cap P_{1}^{-1}\left(L_{m}\left(\mathbf{G}_{\mathbf{2}}\right)\right)
\end{gathered}
$$

Note that our synchronous product operation on DES is associative. Namely, for DES $\mathbf{G}_{\mathbf{1}}, \mathbf{G}_{\mathbf{2}}, \mathbf{G}_{\mathbf{3}}$, we have

$$
\begin{aligned}
\left(\left(\mathbf{G}_{\mathbf{1}} \| \mathrm{G}_{\mathbf{2}}\right) \| \mathrm{G}_{\mathbf{3}}\right) & =\left(\mathrm{G}_{\mathbf{1}} \|\left(\mathrm{G}_{\mathbf{2}} \| \mathrm{G}_{\mathbf{3}}\right)\right) \\
\operatorname{sync}\left(\operatorname{sync}\left(\mathrm{G}_{\mathbf{1}}, \mathbf{G}_{\mathbf{2}}\right), \mathbf{G}_{\mathbf{3}}\right) & =\operatorname{sync}\left(\mathbf{G}_{\mathbf{1}}, \operatorname{sync}\left(\mathbf{G}_{\mathbf{2}}, \mathbf{G}_{\mathbf{3}}\right)\right)
\end{aligned}
$$

\subsection{Predicates and Predicate Transformers}

\subsubsection{Predicates}

Let $\mathbf{G}=\left(Q, \Sigma, \delta, q_{0}, Q_{m}\right)$ be a DES. A predicate $P$ defined on $Q$ is a function $P: Q \rightarrow\{1,0\} . P$ is identified by a corresponding state subset

\footnotetext{
${ }^{2}$ The operator is same as the synchronous product operator on languages, but they can be easily distinguished by context.
} 


$$
\begin{gathered}
\text { Master Thesis - R. Song - McMaster - Computing and Software } \\
Q_{P}:=\{q \in Q \mid P(q)=1\} \subseteq Q .
\end{gathered}
$$

If $q \in Q_{P}$, we write $q \models P$ and say $q$ satisfies $P$ or $P$ includes $q$. So, $q \models P$ if and only if $P(q)=1$. We write $\operatorname{Pred}(Q)$ for the set of all predicates defined on $Q$, so $\operatorname{Pred}(Q)$ can be identified by $\operatorname{Pwr}(Q)$. With the predicates, we can build boolean expression using the following operations:

$$
\begin{array}{lll}
(\neg P)(q)=1 & \text { iff } & P(q)=0 \\
\left(P_{1} \wedge P_{2}\right)(q)=1 & \text { iff } & P_{1}(q)=1 \text { and } P_{2}(q)=1 \\
\left(P_{1} \vee P_{2}\right)(q)=1 & \text { iff } & P_{1}(q)=1 \text { or } P_{2}(q)=1 \\
\left(P_{1}-P_{2}\right)(q)=1 & \text { iff } & P_{1}(q)=1 \text { and } P_{2}(q)=0
\end{array}
$$

where $P, P_{1}, P_{2} \in \operatorname{Pred}(Q)$ and $q \in Q$. Clearly, $P_{1}-P_{2}=P_{1} \wedge \neg P_{2}$.

The two special predicates true and false are identified by $Q$ and $\emptyset$ respectively. The predicate $P_{m}$ is identified by $Q_{m}$.

For convenience, if $Q$ is understood, for $Q_{1} \subseteq Q$, denote the predicate identified by $Q_{1}$ as $\operatorname{pr}\left(Q_{1}\right)$. For a predicate $P_{1} \in \operatorname{Pred}(Q)$, denote the corresponding state subset $Q_{P_{1}}$ as $s t\left(P_{1}\right)$.

The relation $\preceq$ on $\operatorname{Pred}(Q)$ is defined as

$$
\left(\forall P_{1}, P_{2} \in \operatorname{Pred}(Q)\right) P_{1} \preceq P_{2} \text { iff } P_{1} \wedge P_{2}=P_{1}
$$

It can easily be shown that $\preceq$ is a partial order on $\operatorname{Pred}(Q)$. Clearly, $P_{1} \preceq P_{2}$ iff $Q_{P_{1}} \subseteq Q_{P_{2}}$, so $(\forall q \in Q) q \models P_{1} \Rightarrow q \models P_{2}$.

If $P_{1} \preceq P_{2}$, we say that $P_{1}$ is stronger than $P_{2}$, and we also say that $P_{1}$ is a subpredicate of $P_{2}$.

Under the identification of $\operatorname{Pred}(Q)$ with $\operatorname{Pwr}(Q)$ and $\preceq$ with $\subseteq$, it is clear that $(\operatorname{Pred}(Q), \preceq)$ is a complete lattice. The top element is true, and the bottom element 


\section{Master Thesis - R. Song - McMaster - Computing and Software}

is false.

For a predicate $P \in \operatorname{Pred}(Q)$, we define $S u b(P)$ to be the set of all the subpredicates of $P$. Clearly, $S u b(P)$ is identified by $P w r\left(Q_{P}\right)$, and $(S u b(P), \preceq)$ is also a complete lattice with top element $P$ and bottom element false.

Proposition 2.3. Let $Q$ be the state set of a DES $\mathbf{G}$ with $n$ states, $n \in\{0,1, \ldots\}$, and let $P$ be a predicate on $Q$. If a function $f: S u b(P) \rightarrow S u b(P)$ is a monotone function with respect to $\preceq$, then there exists $k \in\{0,1, \ldots\}$ such that $k \leq n$ and $f^{k}(P)$ is the greatest fixed point of $f$ with respect to $(S u b(P), \preceq)$, and $(\forall i \in\{1,2, \ldots\}) i>$ $k \Rightarrow f^{i}(P)=f^{k}(P)$.

\section{proof:}

Immediately from Proposition 2.1 and the identifying relationship of $S u b(P)$ with $\operatorname{Pwr}\left(Q_{P}\right), \preceq$ with $\subseteq, P$ with $Q_{P}$, and false with $\emptyset$.

\subsubsection{Predicate Transformers}

Let $\mathbf{G}=\left(Q, \Sigma, \delta, q_{0}, Q_{m}\right)$ be a fixed DES. A predicate transformer is a function $f: \operatorname{Pred}(Q) \rightarrow \operatorname{Pred}(Q)$. We now introduce several predicate transformers which will be used later on.

\section{- $R(\mathbf{G},$.}

Let $P \in \operatorname{Pred}(Q)$. The reachability predicate $R(\mathbf{G}, P)$ is defined to hold precisely on those states that can be reached in $\mathbf{G}$ from $q_{0}$ via states satisfying $P$. Formally,

1. $q_{0} \models P \Rightarrow q_{0} \models R(\mathbf{G}, P)$

2. $q \models R(\mathbf{G}, P) \& \sigma \in \Sigma \& \delta(q, \sigma) ! \& \delta(q, \sigma) \models P \Rightarrow \delta(q, \sigma) \models R(\mathbf{G}, P)$ 


\section{Master Thesis - R. Song-McMaster-Computing and Software}

3. No other states satisfy $R(\mathbf{G}, P)$.

In other words, $q \models R(\mathbf{G}, P)$ if and only if there exists a path in $\mathbf{G}$ from $q_{0}$ to $q$ and each state on the path satisfies $P$. Therefore, $q_{0} \not \models P \Rightarrow R(\mathbf{G}, P)=$ false.

Clearly, $R(\mathbf{G}, P) \preceq P$ and $R(\mathbf{G},$.$) is monotone with respect to \preceq$. Note that $R(\mathbf{G}$, true $)$ exactly includes all the reachable states in $Q$.

\section{- $C R(\mathbf{G},$.}

Let $P \in \operatorname{Pred}(Q)$. The coreachability predicate $C R(\mathbf{G}, P)$ is defined to hold precisely on those states that can reach a marker state in $\mathbf{G}$ via states satisfying $P$. Formally,

1. $P_{m} \wedge P=$ false $\Rightarrow C R(\mathbf{G}, P)=$ false

2. $q \models P_{m} \wedge P \Rightarrow q \models C R(\mathbf{G}, P)$

3. $q \models C R(\mathbf{G}, P) \& q^{\prime} \models P \& \sigma \in \Sigma \& \delta\left(q^{\prime}, \sigma\right)$ ! \& $\delta\left(q^{\prime}, \sigma\right)=q \Rightarrow q^{\prime}=$ $C R(\mathbf{G}, P)$

4. No other states satisfy $C R(\mathbf{G}, P)$.

In other words, $q \models C R(\mathbf{G}, P)$ if and only if there exists a path in $\mathbf{G}$ from $q$ to a marker state satisfying $P$ and each state on the path satisfies $P$.

Clearly, $C R(\mathbf{G}, P) \preceq P$ and $C R(\mathbf{G},$.$) is monotone with respect to \preceq$. Note that $C R(\mathbf{G}$, true $)$ exactly includes all the coreachable states in $Q$.

- $\operatorname{TR}\left(\mathbf{G}, ., \Sigma^{\prime}\right)$

Let $\Sigma^{\prime} \subseteq \Sigma$. With $\mathbf{G}$ and $\Sigma^{\prime}$ fixed, $T R\left(\mathbf{G}, ., \Sigma^{\prime}\right)$ is a predicate transformer. Let $P \in \operatorname{Pred}(Q) . T R\left(\mathbf{G}, P, \Sigma^{\prime}\right)$ is defined to hold precisely on those states that can reach a state satisfying $P$ in $\mathbf{G}$ only via transitions with events in $\Sigma^{\prime}$. Formally, 
Master Thesis - R. Song - McMaster - Computing and Software

1. $P=$ false $\Rightarrow \operatorname{TR}\left(\mathbf{G}, P, \Sigma^{\prime}\right)=$ false

2. $q \models P \Rightarrow q \models T R\left(\mathbf{G}, P, \Sigma^{\prime}\right)$

3. $q \models T R\left(\mathbf{G}, P, \Sigma^{\prime}\right) \& q^{\prime} \in Q \& \sigma \in \Sigma^{\prime} \& \delta\left(q^{\prime}, \sigma\right)$ ! \& $\delta\left(q^{\prime}, \sigma\right)=q \Rightarrow q^{\prime} \models$ $T R\left(\mathbf{G}, P, \Sigma^{\prime}\right)$

4. No other states satisfy $T R\left(\mathbf{G}, P, \Sigma^{\prime}\right)$.

In other words, $q \models T R\left(\mathbf{G}, P, \Sigma^{\prime}\right)$ if and only if there exists a path in $\mathbf{G}$ from $q$ to a state satisfying $P$ and each transition event in the path is in $\Sigma^{\prime}$. Note that the states on the path (excluding the end point state) do not need to satisfy $P$. Clearly, $P \preceq T R\left(\mathbf{G}, P, \Sigma^{\prime}\right)$, and $T R\left(\mathbf{G}, P, \Sigma^{\prime}\right)$ is monotone with respect to $\preceq$.

- $\mathcal{C} \mathcal{R}\left(\mathbf{G}, P^{\prime}, \Sigma^{\prime},.\right)$

Let $P^{\prime} \in \operatorname{Pred}(Q)$ and $\Sigma^{\prime} \subseteq \Sigma$. With $\mathbf{G}, P^{\prime}$ and $\Sigma^{\prime}$ fixed, $\mathcal{C} \mathcal{R}\left(\mathbf{G}, P^{\prime}, \Sigma^{\prime},.\right)$ is also a predicate transformer. Let $P \in \operatorname{Pred}(Q) \cdot \mathcal{C R}\left(\mathbf{G}, P^{\prime}, \Sigma^{\prime}, P\right)$ is defined to hold precisely on those states that can reach a state satisfying $P^{\prime}$ in $\mathbf{G}$ via states satisfying $P$ and transitions with events in $\Sigma^{\prime}$. Formally,

1. $P^{\prime} \wedge P=$ false $\Rightarrow \mathcal{C} \mathcal{R}\left(\mathbf{G}, P^{\prime}, \Sigma^{\prime}, P\right)=$ false

2. $q \models P^{\prime} \wedge P \Rightarrow q \models \mathcal{C} \mathcal{R}\left(\mathbf{G}, P^{\prime}, \Sigma^{\prime}, P\right)$

3. $q \models \mathcal{C R}\left(\mathbf{G}, P^{\prime}, \Sigma^{\prime}, P\right) \& q^{\prime} \models P \& \sigma \in \Sigma^{\prime} \& \delta\left(q^{\prime}, \sigma\right) ! \& \delta\left(q^{\prime}, \sigma\right)=q \Rightarrow q^{\prime} \models$ $\mathcal{C R}\left(\mathbf{G}, P^{\prime}, \Sigma^{\prime}, P\right)$

4. No other states satisfy $\mathcal{C} \mathcal{R}\left(\mathbf{G}, P^{\prime}, \Sigma^{\prime}, P\right)$.

In other words, $q \models \mathcal{C} \mathcal{R}\left(\mathbf{G}, P^{\prime}, \Sigma^{\prime}, P\right)$ if and only if there exists a path in $\mathbf{G}$ from $q$ to a state which satisfies $P^{\prime}$ and each state on the path satisfies $P$ and each transition event is in $\Sigma^{\prime}$. 


\section{Master Thesis - R. Song - McMaster - Computing and Software}

Clearly, $\mathcal{C R}\left(\mathbf{G}, P^{\prime}, \Sigma^{\prime}, P\right) \preceq P$ and $P^{\prime} \wedge P \preceq \mathcal{C R}\left(\mathbf{G}, P^{\prime}, \Sigma^{\prime}, P\right)$. Note that $C R(\mathbf{G},$.$) is actually a special case of \mathcal{C R}\left(\mathbf{G}, P^{\prime}, \Sigma^{\prime},.\right)$ with $P^{\prime}=P_{m}$ and $\Sigma^{\prime}=\Sigma$.

Proposition 2.4. For a fixed $\mathbf{G}$, fixed predicate $P^{\prime} \in \operatorname{Pred}(Q)$, and $\Sigma^{\prime} \subseteq \Sigma$, the predicate transformer $\mathcal{C R}\left(\mathbf{G}, P^{\prime}, \Sigma^{\prime},.\right)$ is monotone with respect to $\preceq$, i.e.

$$
\left(\forall P_{1}, P_{2} \in \operatorname{Pred}(Q)\right) P_{1} \preceq P_{2} \Rightarrow \mathcal{C R}\left(\mathbf{G}, P^{\prime}, \Sigma^{\prime}, P_{1}\right) \preceq \mathcal{C R}\left(\mathbf{G}, P^{\prime}, \Sigma^{\prime}, P_{2}\right)
$$

\section{proof:}

Let $P_{1}, P_{2} \in \operatorname{Pred}(Q)$. Assume $P_{1} \preceq P_{2}$. Must show this implies

$$
\mathcal{C R}\left(\mathbf{G}, P^{\prime}, \Sigma^{\prime}, P_{1}\right) \preceq \mathcal{C R}\left(\mathbf{G}, P^{\prime}, \Sigma^{\prime}, P_{2}\right) .
$$

Let $q \models \mathcal{C R}\left(\mathbf{G}, P^{\prime}, \Sigma^{\prime}, P_{1}\right)$.

We now show implies $q \models \mathcal{C R}\left(\mathbf{G}, P^{\prime}, \Sigma^{\prime}, P_{2}\right)$.

From (1), we know $(\exists k \in\{1,2, \ldots\})\left(\exists q_{1}, q_{2}, \ldots, q_{k} \in Q\right)\left(\exists \sigma_{1}, \sigma_{2}, \ldots, \sigma_{k-1} \in \Sigma^{\prime}\right)$,

$$
\begin{gathered}
q_{1}=q, q_{k} \models P^{\prime} \\
q_{i+1}=\delta\left(q_{i}, \sigma_{i}\right), \quad i=1,2, \ldots, k-1 \\
q_{i} \models P_{1}, \quad i=1,2, \ldots, k
\end{gathered}
$$

By $P_{1} \preceq P_{2}$, we have

$$
\begin{gathered}
(\exists k \in\{1,2, \ldots\})\left(\exists q_{1}, q_{2}, \ldots, q_{k} \in Q\right)\left(\exists \sigma_{1}, \sigma_{2}, \ldots, \sigma_{k-1} \in \Sigma^{\prime}\right) \\
q_{1}=q, q_{k}=P^{\prime} \\
q_{i+1}=\delta\left(q_{i}, \sigma_{i}\right), \quad i=1,2, \ldots, k-1 \\
q_{i} \models P_{2}, \quad i=1,2, \ldots, k
\end{gathered}
$$

As these predicate transformers play important roles in our algorithms, we give an example for them. Let $\mathbf{G}$ be the DES shown in Figure 2.2(a). A state labeled 
with a predicate $\left(P\right.$ or $\left.P^{\prime}\right)$ means that it satisfies that predicate. For example, state $s 3$ satisfies $P$ but not $P^{\prime}$, state $s 1$ satisfies neither $P$ nor $P^{\prime}$, and state $s 2$ satisfies both $P$ and $P^{\prime}$. The other figures in Figure 2.2 show the result of the above predicate transformers. The states filled with gray color satisfy the corresponding predicate transformer result.

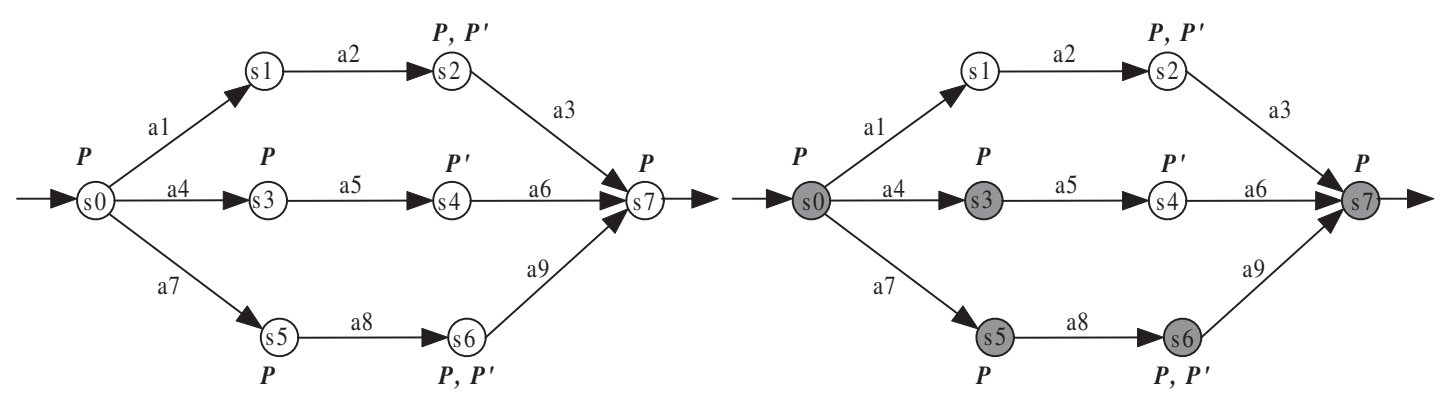

(a) Example $\mathbf{G}$

(b) $R(\mathbf{G}, P)=\operatorname{pr}(\{s 0, s 3, s 5, s 6, s 7\})$

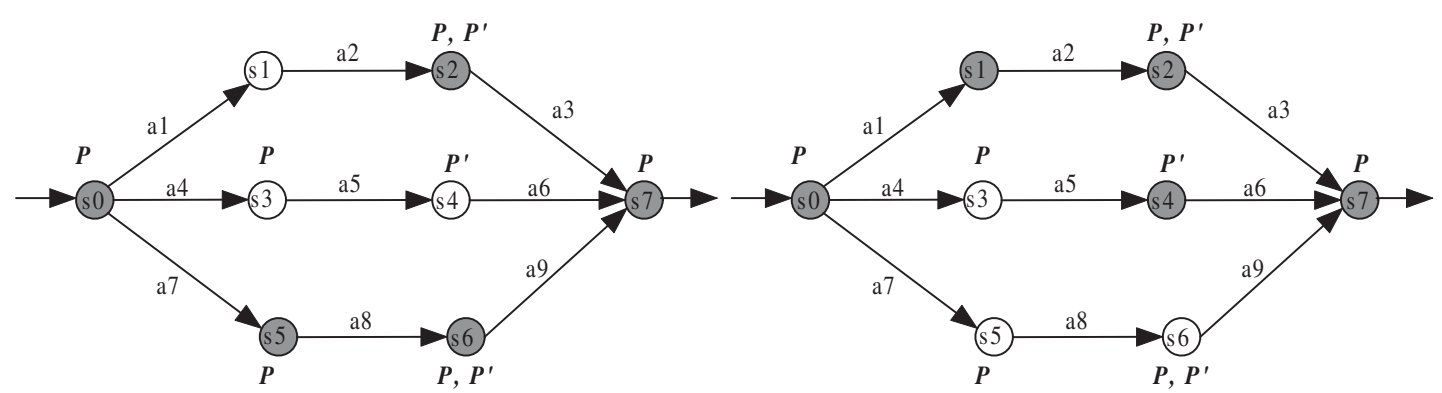

(c) $C R(\mathbf{G}, P)=\operatorname{pr}(\{s 0, s 2, s 5, s 6, s 7\})$

(d) $\operatorname{TR}(\mathbf{G}, \operatorname{pr}(\{s 7\}),\{a 1, a 2, a 3, a 4, a 6\})=\operatorname{pr}(\{$ $s 0, s 1, s 2, s 4, s 7\})$

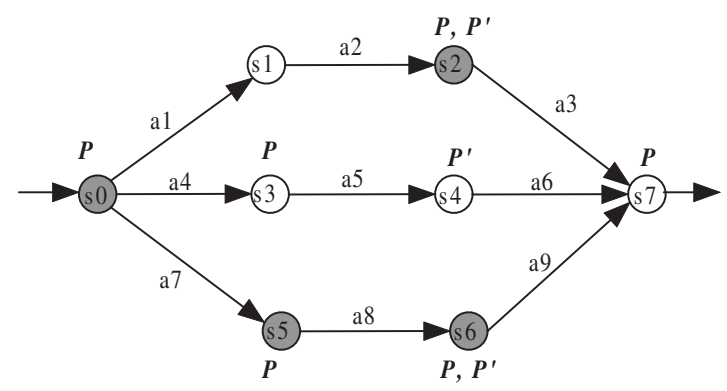

(e) $\mathcal{C R}\left(\mathbf{G}, P^{\prime},\{a 1, a 2, a 4, a 5, a 7, a 8\}, P\right)=\operatorname{pr}(\{$ $s 0, s 2, s 5, s 6\})$

Figure 2.2: Example for predicate transformers 


\section{Master Thesis - R. Song-McMaster-Computing and Software}

\subsubsection{Languages Induced by Predicates}

Let $\mathbf{G}=\left(Q, \Sigma, \delta, q_{0}, Q_{m}\right)$ be a DES, and $P \in \operatorname{Pred}(Q)$ be a predicate on $Q$. The language $L(\mathbf{G}, P)$ is the closed language induced by $P$. Informally, $L(\mathbf{G}, P)$ is the closed language generated by the DES $\mathbf{G}$ with only those states satisfying $P$. Formally,

$$
L(\mathbf{G}, P):=\left\{w \in \Sigma^{*} \mid(\forall v \leq w) \delta\left(q_{0}, v\right) \models P\right\} .
$$

Similarly, we define $L_{m}(\mathbf{G}, P)$ as the marked language induced by $P$.

$$
L_{m}(\mathbf{G}, P):=\left\{w \in L(\mathbf{G}, P) \mid \delta\left(q_{0}, w\right) \in Q_{m}\right\}
$$

We can define a DES $\mathbf{G}^{\prime}$ based on DES $\mathbf{G}$ and $P$ as follows. If $q_{0} \not \models P$, then $\mathbf{G}^{\prime}$ is defined as the EMPTY DES. Otherwise let $\mathbf{G}^{\prime}:=\left(Q^{\prime}, \Sigma, \delta^{\prime}, q_{0}, Q_{m}^{\prime}\right)$, where $Q^{\prime}:=Q_{P}, Q_{m}^{\prime}:=Q_{P} \cap Q_{m}$, and the partial function $\delta^{\prime}: Q^{\prime} \times \Sigma \rightarrow Q^{\prime}$ is defined according to

$$
\left(\forall q^{\prime} \in Q^{\prime}\right)(\forall \sigma \in \Sigma) \delta^{\prime}\left(q^{\prime}, \sigma\right):= \begin{cases}\delta\left(q^{\prime}, \sigma\right), & \text { if } \delta\left(q^{\prime}, \sigma\right) ! \& \delta\left(q^{\prime}, \sigma\right) \models P \\ \text { undefined, } & \text { otherwise. }\end{cases}
$$

Essentially, the DES $\mathbf{G}^{\prime}$ is the DES $\mathbf{G}$ after removing all the states not satisfying $P$ and all the transitions with source states or target states not satisfying $P$. Now one may think of $L(\mathbf{G}, P)$ and $L_{m}(\mathbf{G}, P)$ as $L\left(\mathbf{G}^{\prime}\right)$ and $L_{m}\left(\mathbf{G}^{\prime}\right)$ respectively.

The following two propositions will be used in following chapters.

Proposition 2.5. Let $\mathbf{G}=\left(Q, \Sigma, \delta, q_{0}, Q_{m}\right)$ be a DES, then the following holds:

$$
(\forall P \in \operatorname{Pred}(Q))\left(\forall s \in \Sigma^{*}\right) s \in L(\mathbf{G}, P) \Leftrightarrow \delta\left(q_{0}, s\right) \models R(\mathbf{G}, P) .
$$

\section{proof:}

Let $P \in \operatorname{Pred}(Q)$ and $s \in \Sigma^{*}$. Must show implies $s \in L(\mathbf{G}, P) \Leftrightarrow \delta\left(q_{0}, s\right) \models$ $R(\mathbf{G}, P)$. 
Master Thesis - R. Song - McMaster - Computing and Software

$$
\begin{aligned}
& s \in L(\mathbf{G}, P) \\
& \Leftrightarrow(\forall t \leq s) \delta\left(q_{0}, t\right) \models P, \quad \text { by definition of } L(\mathbf{G}, P) \\
& \Leftrightarrow(\exists n \in\{0,1,2, \ldots\})\left(\exists \sigma_{0}, \sigma_{1}, \ldots, \sigma_{n-1} \in \Sigma\right)\left(\exists q_{1}, q_{2}, \ldots, q_{n} \in Q\right) \\
& s=\sigma_{0} \sigma_{1} \cdots \sigma_{n-1} \\
& q_{n}=\delta\left(q_{0}, s\right) \\
& q_{i} \models P, \quad i=0,1, \ldots, n \\
& q_{i+1}=\delta\left(q_{i}, \sigma_{i}\right), \quad i=0,1, \ldots, n-1
\end{aligned}
$$

From this proposition, it is clear that the language $L(\mathbf{G}, P)$ only depends on those states satisfying $R(\mathbf{G}, P)$.

Proposition 2.6. Let $\mathbf{G}=\left(Q, \Sigma, \delta, q_{0}, Q_{m}\right)$ be a DES, then the following holds:

$$
(\forall P \in \operatorname{Pred}(Q))(\forall s, t \in L(\mathbf{G}, P)) \delta\left(q_{0}, s\right)=\delta\left(q_{0}, t\right) \Rightarrow s \equiv_{L(\mathbf{G}, P)} t \& s \equiv_{L_{m}(\mathbf{G}, P)} t
$$

where $\equiv_{L(\mathbf{G}, P)}$ and $\equiv_{L_{m}(\mathbf{G}, P)}$ are the Nerode equivalence relations on $\Sigma^{*}$ with respect to $L(\mathbf{G}, P)$ and $L_{m}(\mathbf{G}, P)$ respectively.

\section{proof:}

Let $P \in \operatorname{Pred}(Q), s \in L(\mathbf{G}, P)$ and $t \in L(\mathbf{G}, P)$.

Assume $\delta\left(q_{0}, s\right)=\delta\left(q_{0}, t\right)$.

1. Show $s \equiv_{L(\mathbf{G}, P)} t$.

Sufficient to show that $\left(\forall u \in \Sigma^{*}\right) s u \in L(\mathbf{G}, P) \Leftrightarrow t u \in L(\mathbf{G}, P)$.

Let $u \in \Sigma^{*}$. Must show the following two points. 
Master Thesis - R. Song-McMaster-Computing and Software

(a) $s u \in L(\mathbf{G}, P) \Rightarrow t u \in L(\mathbf{G}, P)$

Assume $s u \in L(\mathbf{G}, P)$.

We now show that $t u \in L(\mathbf{G}, P)$.

By (3) and Proposition 2.5, we know $\delta\left(q_{0}, s u\right) \models R(\mathbf{G}, P)$.

If $u=\epsilon$, then $t u=t \in L(\mathbf{G}, P)$ from (1). So, we assume $u \neq \epsilon$.

Let $u:=\sigma_{1} \sigma_{2} \cdots \sigma_{n}$, where $n \in\{1,2, \ldots\}$ and $\sigma_{1}, \sigma_{2}, \ldots, \sigma_{n} \in \Sigma$.

By (4) and the definition of $R(\mathbf{G}, P)$, we have

$$
\delta\left(q_{0}, s \sigma_{1}\right) \models P, \delta\left(q_{0}, s \sigma_{1} \sigma_{2}\right) \models P, \ldots, \delta\left(q_{0}, s \sigma_{1} \sigma_{2} \cdots \sigma_{n}\right) \models P .
$$

By (2) and $\mathbf{G}$ is deterministic, we have

$$
\begin{gathered}
\delta\left(q_{0}, s \sigma_{1}\right)=\delta\left(q_{0}, t \sigma_{1}\right), \delta\left(q_{0}, s \sigma_{1} \sigma_{2}\right)=\delta\left(q_{0}, t \sigma_{1} \sigma_{2}\right), \cdots, \\
\delta\left(q_{0}, s \sigma_{1} \sigma_{2} \cdots \sigma_{n}\right)=\delta\left(q_{0}, t \sigma_{1} \sigma_{2} \cdots \sigma_{n}\right) .
\end{gathered}
$$

By (5)(6), we have

$$
\delta\left(q_{0}, t \sigma_{1}\right) \models P, \delta\left(q_{0}, t \sigma_{1} \sigma_{2}\right) \models P, \ldots, \delta\left(q_{0}, t \sigma_{1} \sigma_{2} \cdots \sigma_{n}\right) \models P .
$$

From (1), we know $t \in L(\mathbf{G}, P)$. From this and Proposition 2.5, we have

$$
\delta\left(q_{0}, t\right) \models R(\mathbf{G}, P)
$$

By $(7),(8)$ and the definition of $R(\mathbf{G}, P)$, we have $\delta\left(q_{0}, t u\right) \models R(\mathbf{G}, P)$.

$\Rightarrow t u \in L(\mathbf{G}, P), \quad$ by Proposition 2.5

(b) $t u \in L(\mathbf{G}, P) \Rightarrow s u \in L(\mathbf{G}, P)$

Identical to Part 1(a) by exchanging $s$ and $t$.

2. Show $s \equiv_{L_{m}(\mathbf{G}, P)} t$.

Sufficient to show that $\left(\forall u \in \Sigma^{*}\right) s u \in L_{m}(\mathbf{G}, P) \Leftrightarrow t u \in L_{m}(\mathbf{G}, P)$.

Let $u \in \Sigma^{*}$. Must show the following two points. 
Master Thesis - R. Song - McMaster - Computing and Software

(a) $s u \in L_{m}(\mathbf{G}, P) \Rightarrow t u \in L_{m}(\mathbf{G}, P)$

Assume $s u \in L_{m}(\mathbf{G}, P)$.

We now show that $t u \in L_{m}(\mathbf{G}, P)$.

By (9) and the definition of $L_{m}(\mathbf{G}, P)$, we have

$s u \in L(\mathbf{G}, P) \& \delta\left(q_{0}, s u\right) \in Q_{m}$

$\Rightarrow \delta\left(q_{0}, s u\right) \models R(\mathbf{G}, P) \& \delta\left(q_{0}, s u\right) \in Q_{m}, \quad$ by Proposition 2.5

If $u=\epsilon$, then $t u=t \in L_{m}(\mathbf{G}, P)$ from (1) and (2). So, we assume $u \neq \epsilon$.

Let $u:=\sigma_{1} \sigma_{2} \cdots \sigma_{n}$, where $n \in\{1,2, \ldots\}$ and $\sigma_{1}, \sigma_{2}, \ldots, \sigma_{n} \in \Sigma$.

By (10) and the definition of $R(\mathbf{G}, P)$, we have

$$
\begin{gathered}
\delta\left(q_{0}, s \sigma_{1}\right) \models P, \delta\left(q_{0}, s \sigma_{1} \sigma_{2}\right) \models P, \ldots, \delta\left(q_{0}, s \sigma_{1} \sigma_{2} \cdots \sigma_{n}\right) \models P \\
\delta\left(q_{0}, s \sigma_{1} \sigma_{2} \cdots \sigma_{n}\right) \in Q_{m}
\end{gathered}
$$

By (2) and $\mathbf{G}$ is deterministic, we have

$$
\begin{gathered}
\delta\left(q_{0}, s \sigma_{1}\right)=\delta\left(q_{0}, t \sigma_{1}\right), \delta\left(q_{0}, s \sigma_{1} \sigma_{2}\right)=\delta\left(q_{0}, t \sigma_{1} \sigma_{2}\right), \cdots \\
\delta\left(q_{0}, s \sigma_{1} \sigma_{2} \cdots \sigma_{n}\right)=\delta\left(q_{0}, t \sigma_{1} \sigma_{2} \cdots \sigma_{n}\right) .
\end{gathered}
$$

By (11)(12), we have

$$
\begin{gathered}
\delta\left(q_{0}, t \sigma_{1}\right) \models P, \delta\left(q_{0}, t \sigma_{1} \sigma_{2}\right) \models P, \ldots, \delta\left(q_{0}, t \sigma_{1} \sigma_{2} \cdots \sigma_{n}\right) \models P . \\
\delta\left(q_{0}, t \sigma_{1} \sigma_{2} \cdots \sigma_{n}\right) \in Q_{m}
\end{gathered}
$$

From (1), we know $t \in L(\mathbf{G}, P)$. From this and Proposition 2.5, we have

$$
\delta\left(q_{0}, t\right) \models R(\mathbf{G}, P)
$$

By (13),(14) and the definition of $R(\mathbf{G}, P)$, we have $\delta\left(q_{0}, t u\right) \models R(\mathbf{G}, P) \& \delta\left(q_{0}, t u\right) \in Q_{m}$ 


$$
\begin{aligned}
& \text { Master Thesis }-R \text {. Song - McMaster - Computing and Software } \\
& \Rightarrow t u \in L(\mathbf{G}, P) \& \delta\left(q_{0}, t u\right) \in Q_{m}, \quad \text { by Proposition } 2.5 \\
& \Rightarrow t u \in L_{m}(\mathbf{G}, P), \quad \text { by definition of } L_{m}(\mathbf{G}, P)
\end{aligned}
$$

(b) $t u \in L_{m}(\mathbf{G}, P) \Rightarrow s u \in L_{m}(\mathbf{G}, P)$

Identical to Part 2(a) by exchanging $s$ and $t$.

Let $s \in L(\mathbf{G}, P)$. We define the following set of strings

$$
L^{s}(\mathbf{G}, P):=\left\{t \in L(\mathbf{G}, P) \mid(\exists u \leq t) \delta\left(q_{0}, u\right)=\delta\left(q_{0}, s\right)\right\}
$$

We see that $L^{s}(\mathbf{G}, P)$ exactly includes all the strings in $L(\mathbf{G}, P)$ reaching or passing through the state $\delta\left(q_{0}, s\right)$.

From the definition of $L^{s}(\mathbf{G}, P)$, we have

$$
L(\mathbf{G}, P)-L^{s}(\mathbf{G}, P)=\left\{t \in L(\mathbf{G}, P) \mid(\forall u \leq t) \delta\left(q_{0}, u\right) \neq \delta\left(q_{0}, s\right)\right\}
$$

Let $K \subseteq L(\mathbf{G}, P)$. The following definition will be useful later on.

$$
L^{K}(\mathbf{G}, P)=\bigcup_{s \in K} L^{s}(\mathbf{G}, P)
$$

Proposition 2.7. Let $\mathbf{G}=\left(Q, \Sigma, \delta, q_{0}, Q_{m}\right)$ be a DES, $P, P^{\prime} \in \operatorname{Pred}(Q), s \in L(\mathbf{G}, P)$ and $q=\delta\left(q_{0}, s\right)$. Let $P^{\prime}:=P-\operatorname{pr}(\{q\})$, then the following holds

$$
L\left(\mathbf{G}, P^{\prime}\right)=L(\mathbf{G}, P)-L^{s}(\mathbf{G}, P)
$$

\section{proof:}

1. Show that $L\left(\mathbf{G}, P^{\prime}\right) \subseteq L(\mathbf{G}, P)-L^{s}(\mathbf{G}, P)$.

Let $t \in L\left(\mathbf{G}, P^{\prime}\right)$. 
Master Thesis - R. Song - McMaster - Computing and Software

We now show this implies $t \in L(\mathbf{G}, P)-L^{s}(\mathbf{G}, P)$.

By (1) and the fact $P^{\prime} \preceq P$, we have $t \in L(\mathbf{G}, P)$.

By (1) and the definition of $L\left(\mathbf{G}, P^{\prime}\right)$, we also have

$$
\begin{aligned}
& (\forall v \leq t) \delta\left(q_{0}, v\right) \models P^{\prime} \\
& \Rightarrow(\forall v \leq t) \delta\left(q_{0}, v\right) \neq q, \quad \text { by definition of } P^{\prime} \\
& \Rightarrow(\forall v \leq t) \delta\left(q_{0}, v\right) \neq \delta\left(q_{0}, s\right) \\
& \Rightarrow t \in L(\mathbf{G}, P)-L^{s}(\mathbf{G}, P), \quad \text { by Equation } 2.1 \text { and }(2)
\end{aligned}
$$

2. Show that $L(\mathbf{G}, P)-L^{s}(\mathbf{G}, P) \subseteq L\left(\mathbf{G}, P^{\prime}\right)$.

Let $t \in L(\mathbf{G}, P)-L^{s}(\mathbf{G}, P)$.

We now show this implies $t \in L\left(\mathbf{G}, P^{\prime}\right)$.

By (3) and (2.1), we know $(\forall v \leq t) \delta\left(q_{0}, v\right) \neq \delta\left(q_{0}, s\right)$

$$
\begin{aligned}
& \Rightarrow(\forall v \leq t) \delta\left(q_{0}, v\right) \neq q \\
& \Rightarrow(\forall v \leq t) \delta\left(q_{0}, v\right) \models P^{\prime}, \quad \text { as } t \in L(\mathbf{G}, P) \\
& \Rightarrow t \in L\left(\mathbf{G}, P^{\prime}\right)
\end{aligned}
$$

This proposition states that if we remove all the strings in $L^{s}(\mathbf{G}, P)$, it is equivalent to removing the state $\delta\left(q_{0}, s\right)$ from $P$.

\subsection{Supervisory Control}

\subsubsection{Controllable Languages}

Let $\mathbf{G}=\left(Q, \Sigma, \delta, q_{0}, Q_{m}\right)$ be a DES with $\Sigma=\Sigma_{c} \dot{\cup} \Sigma_{u}$.

Let $K \subseteq \Sigma^{*} . K$ is controllable with respect to $\mathbf{G}$ if 


$$
\begin{gathered}
\text { Master Thesis - R. Song - McMaster - Computing and Software } \\
(\forall s \in \bar{K})\left(\forall \sigma \in \Sigma_{u}\right) s \sigma \in L(\mathbf{G}) \Rightarrow s \sigma \in \bar{K}
\end{gathered}
$$

Let $S \subseteq \Sigma^{*}$, and $\Sigma_{0} \subseteq \Sigma$. Denote $S \Sigma_{0}$ as the set $\left\{s \sigma \mid s \in S, \sigma \in \Sigma_{0}\right\}$. The condition for language $K$ being controllable with respect to $\mathbf{G}$ can be rewritten as

$$
\bar{K} \Sigma_{u} \cap L(\mathbf{G}) \subseteq \bar{K}
$$

We can also use the Elig operator to express that $K$ is controllable with respect to $\mathbf{G}$ as

$$
(\forall s \in \bar{K} \cap L(\mathbf{G})) \operatorname{Elig}_{L(\mathbf{G})}(s) \cap \Sigma_{u} \subseteq \operatorname{Elig}_{\bar{K}}(s)
$$

The above three representations for controllable language will be used in this thesis interchangeably.

Clearly, $\emptyset, L(\mathbf{G})$ and $\Sigma^{*}$ are always controllable with respect to $\mathbf{G}$.

Let $E \subseteq \Sigma^{*}$ be an arbitrary language. Define the set of all sublanguages of $E$ that are controllable with respect to $\mathbf{G}$ as

$$
\mathcal{C}(E):=\{K \subseteq E \mid K \text { is controllable with respect to } \mathbf{G}\}
$$

Proposition 2.8 (From [47]). $\mathcal{C}(E)$ is nonempty and is closed under arbitrary unions. In particular, the supremal element $\sup \mathcal{C}(E) \in \mathcal{C}(E)$.

This proposition says that $\sup \mathcal{C}(E) \subseteq E$. Therefore, we can compute $\sup \mathcal{C}(E)$ by removing strings from $E$.

\subsubsection{Supervisory Control}

Let $\mathbf{G}=\left(Q, \Sigma, \delta, q_{0}, Q_{m}\right)$ be a nonempty DES with $\Sigma=\Sigma_{c} \dot{\cup} \Sigma_{u}$. A control pattern is the union of $\Sigma_{u}$ and a subset of $\Sigma_{c}$. Then, the set all of control patterns is 


$$
\begin{gathered}
\text { Master Thesis }- \text { R. Song - McMaster - Computing and Software } \\
\Gamma:=\left\{\gamma \in P w r(\Sigma) \mid \gamma \supseteq \Sigma_{u}\right\}
\end{gathered}
$$

where $\gamma$ is a control pattern.

A supervisory control for $\mathbf{G}$ is any map $V: L(\mathbf{G}) \rightarrow \Gamma$. We write the pair $(\mathbf{G}, V)$ as $V / \mathbf{G}$. We also refer to $\mathbf{G}$ as the plant (DES).

The closed behavior of $V / \mathbf{G}$ is defined to be the language $L(V / \mathbf{G}) \subseteq L(\mathbf{G})$, which is described as follows.

1. $\epsilon \in L(V / \mathbf{G})$

2. If $s \in L(V / \mathbf{G}), \sigma \in V(s)$, and $s \sigma \in L(\mathbf{G})$ then $s \sigma \in L(V / \mathbf{G})$

3. No other strings belong to $L(V / \mathbf{G})$.

Clearly, $\epsilon \in L(V / \mathbf{G})$ and $L(V / \mathbf{G})$ is closed and $\{\epsilon\} \subseteq L(V / \mathbf{G}) \subseteq L(\mathbf{G})$.

The marked behavior of $V / \mathbf{G}$ is defined as $L_{m}(V / \mathbf{G})=L(V / \mathbf{G}) \cap L_{m}(\mathbf{G})$. We always have $\emptyset \subseteq L_{m}(V / \mathbf{G}) \subseteq L(\mathbf{G})$.

$V$ is a nonblocking supervisory control(NSC) for $\mathbf{G}$ if $\overline{L_{m}(V / \mathbf{G})}=L(V / \mathbf{G})$.

Usually, given a plant DES $\mathbf{G}$, we want the plant $\mathbf{G}$ to behave in a desired way. That means that we want a sublanguage of $L_{m}(\mathbf{G})$ which represents a set of desired tasks that the plant is supposed to complete. It is also desired that the controlled system be nonblocking. However, it is not always true that we can find a nonblocking supervisory control for a sublanguage of $L_{m}(\mathbf{G})$.

Let $K \subseteq L \subseteq \Sigma^{*}$. We say the language $K$ is $L$-closed if $K=\bar{K} \cap L$.

Theorem 2.2 (From [47]). Let $K \subseteq L_{m}(\mathbf{G}), K \neq \emptyset$. There exists a $N S C V$ for $\mathbf{G}$ such that $L_{m}(V / \mathbf{G})=K$ if and only if $K$ is controllable with respect to $\mathbf{G}$ and $K$ is $L_{m}(\mathbf{G})$-closed 


\section{Master Thesis - R. Song-McMaster-Computing and Software}

The above supervisory control $V$ does not play a role in marking a string, so the desired sublanguage $K$ is required to be $L_{m}(\mathbf{G})$-closed. If we also allow $V$ to include marking, then we can implement a more flexible supervisory control.

Let $K \subseteq L_{m}(\mathbf{G})$. We redefine the marked behavior of $V / \mathbf{G}$ as $L_{m}(V / \mathbf{G})=$ $L(V / \mathbf{G}) \cap K$. The map $V: L(\mathbf{G}) \rightarrow \Gamma$ is called a marking nonblocking supervisory control (MNSC) for the pair $(K, \mathbf{G})$ if $\overline{L_{m}(V / \mathbf{G})}=L(V / \mathbf{G})$.

Theorem 2.3 (From [47]). Let $K \subseteq L_{m}(\mathbf{G}), K \neq \emptyset$. There exists a $M N S C V$ for the pair $(K, \mathbf{G})$ such that $L_{m}(V / \mathbf{G})=K$ if and only if $K$ is controllable with respect to G.

In this thesis, we will focus on MNSC.

\subsubsection{Implementation of MNSC $V$ for G}

The supervisory control $V: L(\mathbf{G}) \rightarrow \Gamma$ is mainly used for theoretical purpose. In reality, it is not convenient to build such a map.

Let $K \subseteq L_{m}(\mathbf{G})$ be a desired marked sublanguage for a nonempty DES $\mathbf{G}=$ $\left(Q, \Sigma, \delta, q_{0}, Q_{m}\right)$. Assume $K$ is controllable. By Theorem 2.3, there exists a MNSC $V$ such that $K=L_{m}(V / \mathbf{G}), \bar{K}=L(V / \mathbf{G})$.

In order to implement the language $K$, we construct a DES over $\Sigma=\Sigma_{c} \dot{\cup} \Sigma_{u}$ which we will refer to as a supervisor, say $\mathbf{S}$, such that $K=L_{m}(\mathbf{S} \times \mathbf{G}) \subseteq L_{m}(\mathbf{G})$ and $L(\mathbf{S} \times \mathbf{G})=\bar{K}$. Then we say that $\mathbf{S}$ implements $V$. In order to ensure that $S$ implements V, by Theorem 2.3 and the MNSC definition, the following verification conditions are required.

1. $L_{m}(\mathbf{S} \times \mathbf{G})$ is controllable with respect to $\mathbf{G}$.

2. $\overline{L_{m}(\mathbf{S} \times \mathbf{G})}=L(\mathbf{S} \times \mathbf{G})$. 
Master Thesis - R. Song - McMaster - Computing and Software

The first condition can be written as

$$
\overline{L_{m}(\mathbf{S} \times \mathbf{G})} \Sigma_{u} \cap L(\mathbf{G}) \subseteq \overline{L_{m}(\mathbf{S} \times \mathbf{G})}
$$

As we also require the second condition to hold, the first condition is equivalent to

$$
L(\mathbf{S} \times \mathbf{G}) \Sigma_{u} \cap L(\mathbf{G}) \subseteq L(\mathbf{S} \times \mathbf{G})
$$

A supervisor $\mathbf{S}$ is controllable with respect to $\mathbf{G}$ if it satisfies the first condition. A supervisor $\mathbf{S}$ is nonblocking for $\mathbf{G}$ if it satisfies the second condition. The second condition also states that $\mathbf{S} \times \mathbf{G}$ is nonblocking.

In [21], the first condition is defined as $L(\mathbf{S}) \Sigma_{u} \cap L(\mathbf{G}) \subseteq L(\mathbf{S})$, we now show that our condition is equivalent to it.

Proposition 2.9. Let $\mathbf{G}$ be a plant DES, $\mathbf{S}$ be a supervisor for $\mathbf{G}$, and both $\mathbf{G}$ and $\mathbf{S}$ are defined over event set $\Sigma=\Sigma_{c} \dot{\cup} \Sigma_{u}$. Then we have

$$
L(\mathbf{S} \times \mathbf{G}) \Sigma_{u} \cap L(\mathbf{G}) \subseteq L(\mathbf{S} \times \mathbf{G}) \text { iff } L(\mathbf{S}) \Sigma_{u} \cap L(\mathbf{G}) \subseteq L(\mathbf{S})
$$

proof:

1. (if) Assume $L(\mathbf{S}) \Sigma_{u} \cap L(\mathbf{G}) \subseteq L(\mathbf{S})$.

Must show this implies $L(\mathbf{S} \times \mathbf{G}) \Sigma_{u} \cap L(\mathbf{G}) \subseteq L(\mathbf{S} \times \mathbf{G})$.

Let $s \in L(\mathbf{S} \times \mathbf{G}) \Sigma_{u} \cap L(\mathbf{G})$.

We now show implies $s \in L(\mathbf{S} \times \mathbf{G})$.

By (2) we know $s \in L(\mathbf{S}) \Sigma_{u} \cap L(\mathbf{G}) \& s \in L(\mathbf{G})$

$\Rightarrow s \in L(\mathbf{S}) \& s \in L(\mathbf{G}), \quad$ by $(1)$

$\Rightarrow s \in L(\mathbf{S} \times \mathbf{G}), \quad$ by definition of $L(\mathbf{S} \times \mathbf{G})$ 
2. (only if) Assume $L(\mathbf{S} \times \mathbf{G}) \Sigma_{u} \cap L(\mathbf{G}) \subseteq L(\mathbf{S} \times \mathbf{G})$.

Must show this implies $L(\mathbf{S}) \Sigma_{u} \cap L(\mathbf{G}) \subseteq L(\mathbf{S})$

Let $s \in L(\mathbf{S}) \Sigma_{u} \cap L(\mathbf{G})$.

We now show implies $s \in L(\mathbf{S})$.

By (4), we know $\left(\exists s^{\prime} \in L(\mathbf{S})\right)\left(\exists \sigma \in \Sigma_{u}\right) s=s^{\prime} \sigma \& s \in L(\mathbf{G})$

$\Rightarrow s^{\prime} \in L(\mathbf{S}) \& s^{\prime} \sigma \in L(\mathbf{G})$

$\Rightarrow s^{\prime} \in L(\mathbf{S}) \& s^{\prime} \in L(\mathbf{G}) \& s^{\prime} \sigma \in L(\mathbf{G}), \quad$ as $L(\mathbf{G})$ is closed

$\Rightarrow s^{\prime} \in L(\mathbf{S} \times \mathbf{G}) \& s^{\prime} \sigma \in L(\mathbf{G})$

$\Rightarrow s^{\prime} \sigma \in L(\mathbf{S} \times \mathbf{G}) \Sigma_{u} \& s^{\prime} \sigma \in L(\mathbf{G})$

$\Rightarrow s^{\prime} \sigma \in L(\mathbf{S} \times \mathbf{G}), \quad$ by $(3)$

$\Rightarrow s^{\prime} \sigma \in L(\mathbf{S}), \quad$ by definition of $L(\mathbf{S} \times \mathbf{G})$

$\Rightarrow s \in L(\mathbf{S}), \quad$ by $(5)$

Except for some trivial systems, usually both the plant $\mathbf{G}$ and the supervisor $\mathbf{S}$ are modeled as a group of modular(component) DES. The final $\mathbf{G}$ and $\mathbf{S}$ are the synchronous product of their modular components. For convenience, we sometimes add some artificial events to supervisor DES. The events must be selflooped at each state of the plant G. Similarly, the supervisors may not care about some events in the plant DES, so those events may not appear in any of the supervisor DES. Then, we have to selfloop all the "don't care events" at each state of $\mathbf{S}$.

Let $\mathbf{S}:=\mathbf{S}_{1}\|\cdots\| \mathbf{S}_{m}, \mathbf{G}:=\mathbf{G}_{1}\|\cdots\| \mathbf{G}_{n}, m, n \in\{1,2, \ldots\}$. Let $S^{\prime}:=$ $\operatorname{selfloop}\left(\mathbf{S}, \Sigma_{G}-\Sigma_{S}\right)$ and $\mathbf{G}^{\prime}:=\operatorname{selfloop}\left(\mathbf{G}, \Sigma_{S}-\Sigma_{G}\right)$, where $\Sigma_{S}$ and $\Sigma_{G}$ are the event set for $\mathbf{S}$ and $\mathbf{G}$ respectively. The verification conditions (Equation 2.2) become 
Master Thesis - R. Song - McMaster - Computing and Software

1. $L\left(\mathbf{S}^{\prime} \times \mathbf{G}^{\prime}\right) \Sigma_{u} \cap L\left(\mathbf{G}^{\prime}\right) \subseteq L\left(\mathbf{S}^{\prime} \times \mathbf{G}^{\prime}\right)$,

2. $\left.\overline{L_{m}\left(\mathbf{S}^{\prime} \times \mathbf{G}^{\prime}\right)}=L\left(\mathbf{S}^{\prime} \times \mathbf{G}^{\prime}\right)\right)$.

\subsubsection{Supervisor Synthesis}

Sometimes, it is difficult to design a controllable and nonblocking supervisor $\mathbf{S}$ for a plant $\mathbf{G}$, especially for a complicated system.

To this end, we need a synthesis method to build a controllable and nonblocking supervisor $\mathbf{S}$ from a specification DES $\mathbf{E}$, which only cares about the system requirements. However, $\mathbf{E}$ is usually not controllable with respect to $\mathbf{G}$ and $\mathbf{E} \times \mathbf{G}$ may block, so we would like to find the supremal sublanguage $\sup \mathcal{C}\left(L_{m}(\mathbf{E} \times \mathbf{G})\right)$. Then according to Theorem 2.3, a MNSC $V$ can be built. If we construct a nonblocking DES KDES representing $\sup \mathcal{C}\left(L_{m}(\mathbf{E} \times \mathbf{G})\right)$, then KDES implements $V$. 


\section{Chapter 3}

\section{HISC Overview}

The Hierarchical Interface-based Supervisory Control (HISC) framework was proposed by Leduc et al. in [21-27] to alleviate the state explosion problem. In this chapter, we first give an overview of the HISC system structure and then give the definitions of high-level and low-level proper supervisors.

\subsection{System Structure}

An HISC system currently is a two-level system which includes one high-level subsystem and $n$ low-level subsystems $(n \geq 1)$. The high-level subsystem communicates with each low-level subsystem through a separate interface. Figure 3.1 shows the system block diagram and the conceptual flow of information. In [21], if $n=1$, the system is called a serial interface system, otherwise the system is called a $n^{\text {th }} d e$ gree parallel interface system. Because the serial interface system is a special case of a parallel interface system, here we only discuss the parallel interface system and

call the $n^{\text {th }}$ degree parallel interface system as the $n^{\text {th }}$ degree interface system. All the subsystems and interfaces are modeled as DES automata. In order to restrict 
Master Thesis - R. Song - McMaster - Computing and Software

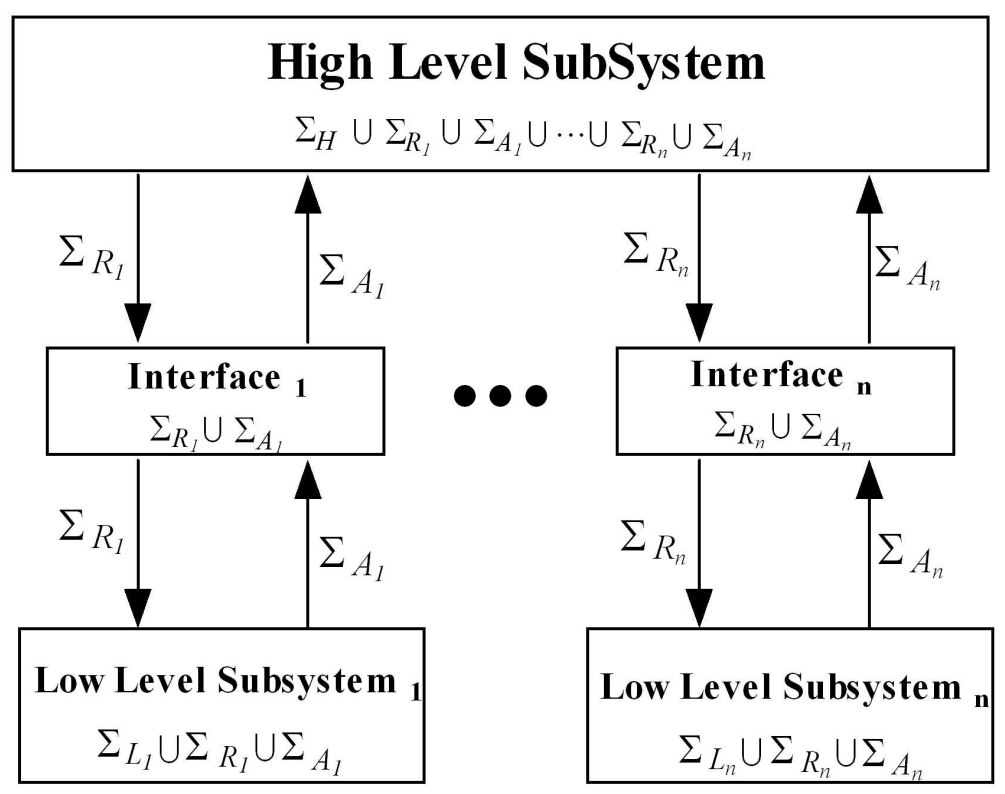

Figure 3.1: HISC block diagram

the information flow at the interface, for an $n^{\text {th }}$ degree interface system, the system alphabet is partitioned into pairwise disjoint alphabets:

$$
\Sigma:=\Sigma_{H} \dot{\cup} \bigcup_{k \in\{1, \ldots, n\}}\left[\Sigma_{L_{k}} \dot{\cup} \Sigma_{R_{k}} \dot{\cup} \Sigma_{A_{k}}\right]
$$

In the remainder of this chapter, $j$ is always an index with range $\{1, \ldots, n\}$. The high-level subsystem is modeled by DES $\mathbf{G}_{H}$, which is the product of the highlevel plant $\mathbf{G}_{H}^{p}$ and the high-level supervisor $\mathbf{S}_{H}$ (both are defined over event set $\left.\Sigma_{H} \dot{\cup}\left(\dot{\cup}_{k \in\{1, \ldots, n\}}\left[\Sigma_{R_{k}} \dot{\cup} \Sigma_{A_{k}}\right]\right)\right)$. The $j^{t h}$ low-level subsystem is modeled by DES $\mathbf{G}_{L_{j}}$, which is the product of the $j^{\text {th }}$ low-level plant $\mathbf{G}_{L_{j}}^{p}$ and the $j^{\text {th }}$ low-level supervisor $\mathbf{S}_{L_{j}}$ (both are defined over event set $\Sigma_{L_{j}} \dot{\cup} \Sigma_{R_{j}} \dot{\cup} \Sigma_{A_{j}}$ ), and the $j^{\text {th }}$ interface is modeled by $\mathbf{G}_{I_{j}}$ (defined over event set $\Sigma_{R_{j}} \dot{\cup} \Sigma_{A_{j}}$ ). The description for each event partition is as follows:

$\Sigma_{H}$ : The set of high-level events, exist only in high-level subsystem. 


\section{Master Thesis - R. Song - McMaster - Computing and Software}

$\Sigma_{R_{j}}$ : The set of request events for the $j^{\text {th }}$ interface.

$\Sigma_{A_{j}}$ : The set of answer events for the $j^{\text {th }}$ interface.

$\Sigma_{L_{j}}$ : The set of $j^{\text {th }}$ low-level events, exist only in the $j^{\text {th }}$ low-level subsystem.

For controllability, the event set $\Sigma$ is also partitioned as $\Sigma=\Sigma_{c} \cup \dot{\cup} \Sigma_{u}$, where $\Sigma_{c}$ is the controllable event set and $\Sigma_{u}$ is the uncontrollable event set.

We refer to DES $\mathcal{G}_{H}:=\mathbf{G}_{H}\left\|\mathbf{G}_{I_{1}}\right\| \ldots \| \mathbf{G}_{I_{n}}$ as the high-level and DES $\mathcal{G}_{L_{j}}:=\mathbf{G}_{I_{j}} \| \mathbf{G}_{L_{j}}$ as the $j^{\text {th }}$ low-level. For convenience, the following event sets are also defined:

$$
\begin{array}{ll}
\Sigma_{I_{j}}:=\Sigma_{R_{j}} \dot{\cup} \Sigma_{A_{j}} & \text { The set of interface events for the } j^{\text {th }} \text { interface } \\
\Sigma_{A}:=\dot{\cup}_{k \in\{1, \ldots, n\}} \Sigma_{A_{k}} & \text { The set of all the answer events } \\
\Sigma_{I H}:=\left(\dot{\cup}_{k \in\{1, \ldots, n\}} \Sigma_{I_{k}}\right) \cup \dot{ } \Sigma_{H} & \text { The set of interface and high-level events } \\
\Sigma_{I L_{j}}:=\Sigma_{L_{j}} \cup \dot{U} \Sigma_{I_{j}} & \text { The set of } j^{\text {th }} \text { interface and low-level events } \\
\Sigma_{I L}:=\dot{\cup}_{k \in\{1, \ldots, n\}} \Sigma_{I L_{k}} & \text { The set of all interface and low-level events } \\
\Sigma_{h u}:=\Sigma_{I H} \cap \Sigma_{u} & \text { The set of the high-level uncontrollable events } \\
\Sigma_{h c}:=\Sigma_{I H} \cap \Sigma_{c} & \text { The set of the high-level controllable events } \\
\Sigma_{l u_{j}}:=\Sigma_{I L_{j}} \cap \Sigma_{u} & \text { The set of the } j^{\text {th }} \text { low-level uncontrollable events } \\
\Sigma_{l c_{j}}:=\Sigma_{I L_{j}} \cap \Sigma_{c} & \text { The set of the } j^{\text {th }} \text { low-level controllable events }
\end{array}
$$

Then the high-level subsystem and the high-level are defined over event set $\Sigma_{I H}$. The $j^{t h}$ interface is defined over event set $\Sigma_{I_{j}}$, and the $j^{\text {th }}$ low-level subsystem and the $j^{\text {th }}$ low-level are defined over event set $\Sigma_{I L_{j}}$. We also have $\Sigma_{I H}=\Sigma_{h u} \dot{\cup} \Sigma_{h c}$ and $\Sigma_{I L_{j}}=\Sigma_{l u_{j}} \dot{\cup} \Sigma_{l c_{j}}$. The overall system structure is shown in Figure 3.2. 
Master Thesis - R. Song - McMaster - Computing and Software

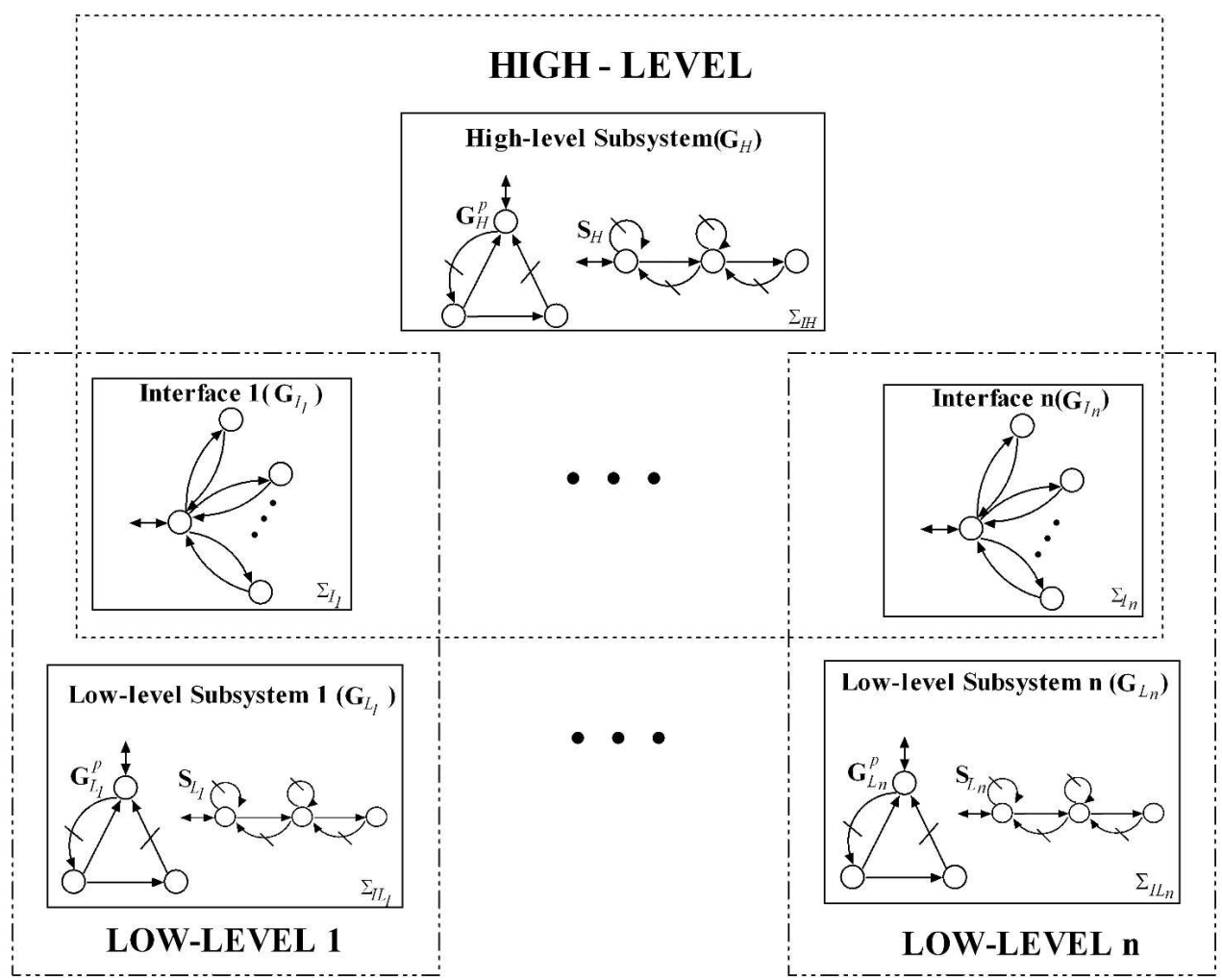

Figure 3.2: HISC system structure

\subsubsection{Command-pair Interfaces}

The interface DES play a very important role in the HISC structure. The highlevel subsystem $\mathbf{G}_{H}$ send requests through an interface to a low-level subsystem. Once the low-level subsystem completes the requested job, it sends back an answer through the interface to the high-level subsystem. However, until the low-level subsystem completes the requested job, it can not accept another request from the high-level. To enforce such a mechanism, the interface DES is required to be a command-pair interface as defined below.

Definition 3.1. For the $n^{\text {th }}$ degree interface system composed of plant components $\mathbf{G}_{H}^{p}, \mathbf{G}_{L_{1}}^{p}, \ldots, \mathbf{G}_{L_{n}}^{p}$, supervisors $\mathbf{S}_{H}, \mathbf{S}_{L_{1}}, \ldots, \mathbf{S}_{L_{n}}$, and interfaces $\mathbf{G}_{I_{1}}, \ldots, \mathbf{G}_{I_{n}}$, the $j^{\text {th }}$ 
interface DES $\mathbf{G}_{I_{j}}=\left(X_{j}, \Sigma_{R_{j}} \dot{\cup} \Sigma_{A_{j}}, \xi_{j}, x_{j_{0}}, X_{j_{m}}\right)$ is a command-pair interface if ${ }^{1}$ :

(A) $L\left(\mathbf{G}_{I_{j}}\right) \subseteq \overline{\left(\Sigma_{R_{j}} \cdot \Sigma_{A_{j}}\right)^{*}}$

(B) $L_{m}\left(\mathbf{G}_{I_{j}}\right)=\left(\Sigma_{R_{j}} \cdot \Sigma_{A_{j}}\right)^{*} \cap L\left(\mathbf{G}_{I_{j}}\right)$

An example command-pair interface from [23], with $\Sigma_{R_{j}}:=\left\{\rho_{i} \mid i=1,2,3\right\}$ and $\Sigma_{A_{j}}:=\left\{\alpha_{i} \mid i=1, \ldots, 7\right\}$, is shown in Figure 3.3.

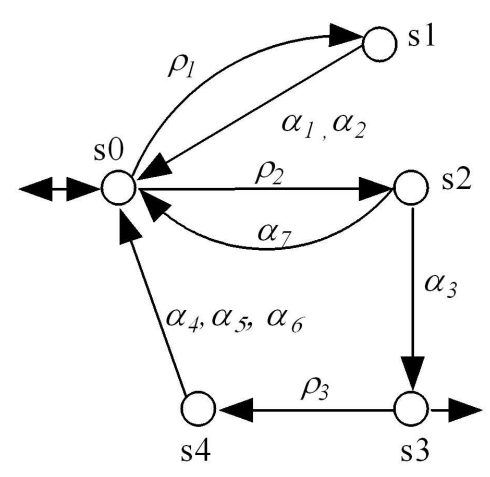

Figure 3.3: Example interface

\subsubsection{Flat System}

An HISC system is actually a structured flat system. The flat plant is defined as

$$
\operatorname{PLANT}:=\mathbf{G}_{H}^{p}\left\|\mathbf{G}_{L_{1}}^{p}\right\| \cdots \| \mathbf{G}_{L_{n}}^{p}
$$

and the flat supervisor is defined as

$$
\mathbf{S U P}:=\mathbf{S}_{H}\left\|\mathbf{S}_{L_{1}}\right\| \cdots\left\|\mathbf{S}_{L_{n}}\right\| \mathbf{G}_{I_{1}}\|\cdots\| \mathbf{G}_{I_{n}} .
$$

Therefore, both PLANT and SUP are defined over event set $\Sigma$. The whole flat system is the product of the flat supervisor and the flat plant.

\footnotetext{
${ }^{1}$ As we require $\mathbf{G}_{I_{j}}$ to be expressible as a tuple including initial state $x_{j_{0}}$, it thus can not be an empty DES. It follows that the empty string belongs to $L\left(\mathbf{G}_{I_{j}}\right)$, and thus to $L_{m}\left(\mathbf{G}_{I_{j}}\right)$ by point B.
} 
Master Thesis - R. Song - McMaster - Computing and Software

\section{SYSTEM $:=\mathrm{SUP} \times$ PLANT}

As stated in Chapter 2, we want to ensure SYSTEM satisfies the following two properties.

1. $L(\mathbf{S Y S T E M}) \Sigma_{u} \cap L(\mathbf{P L A N T}) \subseteq L(\mathbf{S Y S T E M})$

2. $\overline{L_{m}(\text { SYSTEM })}=L($ SYSTEM $)$

The first property is called global controllability, and the second property is called global nonblocking.

\subsection{Local Conditions}

One of the most important benefits of modeling a system as an HISC system is that we can guarantee global controllability and nonblocking by only checking local conditions on the high-level and each low-level separately. The conditions here are based on [23] and [27]. Please refer to them for a more detailed discussion. All our conditions here are based on either the high-level languages or the low-level languages. We do not directly use the conditions in [23] because our conditions are more convenient to prove the correctness of the predicate algorithms in the next chapters. All we do here mainly is to remove low-level event selfloops from the languages in high-level conditions in [23], and to remove high-level event selfloops from the languages in low-level conditions in [23].

Definition 3.2. For the $n^{\text {th }}$ degree interface system that respects the alphabet partition given by 3.1 and is composed of plant components $\mathbf{G}_{H}^{p}, \mathbf{G}_{L_{1}}^{p}, \ldots, \mathbf{G}_{L_{n}}^{p}$, supervisors $\mathbf{S}_{H}, \mathbf{S}_{L_{1}}, \ldots, \mathbf{S}_{L_{n}}$, and interfaces $\mathbf{G}_{I_{1}}, \ldots, \mathbf{G}_{I_{n}}$, for all $j \in\{1, \ldots, n\}$, define

$$
\mathbf{G}_{I_{j}}^{h}:=\operatorname{selfloop}\left(\mathbf{G}_{I_{j}}, \Sigma_{I H}-\Sigma_{I_{j}}\right)
$$




$$
\begin{gathered}
\text { Master Thesis }- \text { R. Song - McMaster - Computing and Software } \\
\qquad \mathbf{G}_{I_{j}}^{l}:=\operatorname{selfloop}\left(\mathbf{G}_{I_{j}}, \Sigma_{L_{j}}\right)
\end{gathered}
$$

From the definition, $\mathbf{G}_{I_{j}}^{h}$ is defined over $\Sigma_{I H}$, and $\mathbf{G}_{I_{j}}^{l}$ is defined over $\Sigma_{I L_{j}}$. Now we define

$$
\mathbf{G}_{I}^{h}:=\mathbf{G}_{I_{1}}^{h} \times \cdots \times \mathbf{G}_{I_{n}}^{h}
$$

Therefore, the high-level $\mathcal{G}_{H}$ and $j^{\text {th }}$ low-level $\mathcal{G}_{L_{j}}$ can be defined as

$$
\mathcal{G}_{H}=\mathbf{S}_{H} \times \mathbf{G}_{H}^{p} \times \mathrm{G}_{I}^{h} \quad \mathcal{G}_{L_{j}}=\mathbf{S}_{L_{j}} \times \mathrm{G}_{L_{j}}^{p} \times \mathbf{G}_{I_{j}}^{l}
$$

\subsubsection{Interface Consistent}

Definition 3.3. The $n^{\text {th }}$ degree interface system composed of plant components $\mathbf{G}_{H}^{p}, \mathbf{G}_{L_{1}}^{p}, \ldots, \mathbf{G}_{L_{n}}^{p}$, supervisors $\mathbf{S}_{H}, \mathbf{S}_{L_{1}}, \ldots, \mathbf{S}_{L_{n}}$, and interfaces $\mathbf{G}_{I_{1}}, \ldots, \mathbf{G}_{I_{n}}$ is interface consistent with respect to the alphabet partition given by 3.1, if for all $j \in\{1, \ldots, n\}$, the following conditions are satisfied:

- Multi-level Properties

1. The event set of $\mathbf{G}_{H}$ is $\Sigma_{I H}$, and the event set of $\mathbf{G}_{L_{j}}$ is $\Sigma_{I L_{j}}$.

2. $\mathbf{G}_{I_{j}}$ is a command-pair interface.

- High-level Properties

3. $L\left(\mathbf{G}_{H} \times \prod_{\substack{k=1, \ldots, n \\ k \neq j}} \mathbf{G}_{I_{k}}^{h}\right) \Sigma_{A_{j}} \cap P_{I_{j} \mid \Sigma_{I H}}^{-1}\left(L\left(\mathbf{G}_{I_{j}}\right)\right) \subseteq L\left(\mathbf{G}_{H} \times \prod_{\substack{k=1, \ldots, n \\ k \neq j}} \mathbf{G}_{I_{k}}^{h}\right)$, where $\prod_{\substack{k=1, \ldots, n \\ k \neq j}} \mathbf{G}_{I_{k}}^{h}:=\mathbf{G}_{I_{1}}^{h} \times \cdots \times \mathbf{G}_{I_{j-1}}^{h} \times \mathbf{G}_{I_{j+1}}^{h} \times \cdots \times \mathbf{G}_{I_{n}}^{h}$; $P_{I_{j} \mid \Sigma_{I H}}: \Sigma_{I H}^{*} \rightarrow \Sigma_{I_{j}}^{*}$ is a natural projection. 
Master Thesis - R. Song - McMaster - Computing and Software

- Low-level Properties

4. $L\left(\mathbf{G}_{L_{j}}\right) \Sigma_{R_{j}} \cap P_{I_{j} \mid \Sigma_{I L_{j}}}^{-1}\left(L\left(\mathbf{G}_{I_{j}}\right)\right) \subseteq L\left(\mathbf{G}_{L_{j}}\right)$,

where $P_{I_{j} \mid \Sigma_{I L_{j}}}: \Sigma_{I L_{j}}^{*} \rightarrow \Sigma_{I_{j}}^{*}$ is a natural projection.

5. $\left(\forall s \in \Sigma_{I L_{j}}^{*} \cdot \Sigma_{R_{j}} \cap L\left(\mathcal{G}_{L_{j}}\right)\right) \operatorname{Elig}_{L\left(\mathcal{G}_{L_{j}}\right)}\left(s \Sigma_{L_{j}}^{*}\right) \cap \Sigma_{A_{j}}=\operatorname{Elig}_{L\left(\mathbf{G}_{I_{j}}\right)}\left(P_{I_{j}}(s)\right) \cap \Sigma_{A_{j}}$, where $\operatorname{Elig}_{L\left(\mathcal{G}_{L_{j}}\right)}\left(s \Sigma_{L_{j}}^{*}\right):=\cup_{l \in \Sigma_{L_{j}}^{*}} \operatorname{Elig}_{L\left(\mathcal{G}_{L_{j}}\right)}(s l)$

$$
P_{I_{j}}: \Sigma^{*} \rightarrow \Sigma_{I_{j}}^{*} \text { is a natural projection. }
$$

6. $\left(\forall s \in L\left(\mathcal{G}_{L_{j}}\right)\right) \quad P_{I_{j}}(s) \in L_{m}\left(\mathbf{G}_{I_{j}}\right) \Rightarrow\left(\exists l \in \Sigma_{L_{j}}^{*}\right) s l \in L_{m}\left(\mathcal{G}_{L_{j}}\right)$

For the purpose of later proofs, we will give an equivalent interface consistent definition in Definition 3.5. For convenience to prove the equivalence between Definition 3.3 and Definition 3.5, we first present an intermediate version of the interface consistent definition.

Definition 3.4. The $n^{\text {th }}$ degree interface system composed of plant components $\mathbf{G}_{H}^{p}, \mathbf{G}_{L_{1}}^{p}, \ldots, \mathbf{G}_{L_{n}}^{p}$, supervisors $\mathbf{S}_{H}, \mathbf{S}_{L_{1}}, \ldots, \mathbf{S}_{L_{n}}$, and interfaces $\mathbf{G}_{I_{1}}, \ldots, \mathbf{G}_{I_{n}}$ is interface consistent with respect to the alphabet partition given by 3.1, if for all $j \in\{1, \ldots, n\}$, the following conditions are satisfied:

- Multi-level Properties

1. The event set of $\mathbf{G}_{H}^{p}$ and $\mathbf{S}_{H}$ is $\Sigma_{I H}$, and the event set of $\mathbf{G}_{L_{j}}^{p}$ and $\mathbf{S}_{L_{j}}$ is $\Sigma_{I L_{j}}$.

2. $\mathbf{G}_{I_{j}}$ is a command-pair interface. 
Master Thesis - R. Song - McMaster - Computing and Software

- High-level Properties

3. $L\left(\mathcal{G}_{H}\right) \Sigma_{A_{j}} \cap L\left(\mathbf{G}_{I_{j}}^{h}\right) \subseteq L\left(\mathcal{G}_{H}\right)$

- Low-level Properties

4. $L\left(\mathcal{G}_{L_{j}}\right) \Sigma_{R_{j}} \cap L\left(\mathbf{G}_{I_{j}}^{l}\right) \subseteq L\left(\mathcal{G}_{L_{j}}\right)$

5. $\left(\forall s \in \Sigma_{I L_{j}}^{*} . \Sigma_{R_{j}} \cap L\left(\mathcal{G}_{L_{j}}\right)\right) \quad \operatorname{Elig}_{L\left(\mathcal{G}_{L_{j}}\right)}\left(s \Sigma_{L_{j}}^{*}\right) \cap \Sigma_{A_{j}}=\operatorname{Elig}_{L\left(\mathbf{G}_{I_{j}}^{l}\right)}(s) \cap \Sigma_{A_{j}}$, where $\operatorname{Elig}_{L\left(\mathcal{G}_{L_{j}}\right)}\left(s \Sigma_{L_{j}}^{*}\right):=\cup_{l \in \Sigma_{L_{j}}^{*}} \operatorname{Elig}_{L\left(\mathcal{G}_{L_{j}}\right)}(s l)$

6. $\left(\forall s \in L\left(\mathcal{G}_{L_{j}}\right)\right) s \in L_{m}\left(\mathbf{G}_{I_{j}}^{l}\right) \Rightarrow\left(\exists l \in \Sigma_{L_{j}}^{*}\right) s l \in L_{m}\left(\mathcal{G}_{L_{j}}\right)$

Proposition 3.1. Definition 3.3 is equivalent to Definition 3.4.

\section{proof:}

For the $n^{\text {th }}$ degree interface system that respects the alphabet partition given by 3.1 and is composed of plant components $\mathbf{G}_{H}^{p}, \mathbf{G}_{L_{1}}^{p}, \ldots, \mathbf{G}_{L_{n}}^{p}$, supervisors $\mathbf{S}_{H}, \mathbf{S}_{L_{1}}, \ldots, \mathbf{S}_{L_{n}}$, and interfaces $\mathbf{G}_{I_{1}}, \ldots, \mathbf{G}_{I_{n}}$, we prove the equivalence between each point in Definition 3.3 and Definition 3.4.

1. Point 1 in both definitions are equivalent.

As we defined $\mathbf{G}_{H}:=\mathbf{S}_{H} \times \mathbf{G}_{H}^{p}$ and $\mathbf{G}_{L_{j}}:=\mathbf{S}_{L_{j}} \times \mathbf{G}_{L_{j}}^{p}$ in Section 3.1, Point 1 in both definitions are clearly equivalent.

2. Point 2 in both definitions are equivalent.

Point 2 in both definitions are exactly the same. 
Master Thesis - R. Song - McMaster - Computing and Software

3. Point 3 in both definitions are equivalent.

By the definitions of $P_{I_{j} \mid \Sigma_{I H}}$ and $\mathbf{G}_{I_{j}}^{h}$, we have $P_{I_{j} \mid \Sigma_{I H}}^{-1}\left(L\left(\mathbf{G}_{I_{j}}\right)\right)=L\left(\mathbf{G}_{I_{j}}^{h}\right)$. Thus we know Point 3 in Definition 3.3 is equivalent to

$$
L\left(\mathbf{G}_{H} \times \prod_{\substack{k=1, \ldots, n \\ k \neq j}} \mathbf{G}_{I_{k}}^{h}\right) \Sigma_{A_{j}} \cap L\left(\mathbf{G}_{I_{j}}^{h}\right) \subseteq L\left(\mathbf{G}_{H} \times \prod_{\substack{k=1, \ldots, n \\ k \neq j}} \mathbf{G}_{I_{k}}^{h}\right)
$$

We now show that (1) is equivalent to Point 3 in Definition 3.4.

By the definition of $\mathcal{G}_{H}$, we have $\mathcal{G}_{H}=\mathbf{G}_{H} \times \mathbf{G}_{I_{1}}^{h} \times \cdots \times \mathbf{G}_{I_{n}}^{h}$.

The rest proof of this point is identical to the proof of Proposition 2.9 by substituting $\Sigma$ with $\Sigma_{I H}, \Sigma_{u}$ with $\Sigma_{A_{j}}, \Sigma_{c}$ with $\Sigma_{I H}-\Sigma_{A_{j}}, \mathbf{S}$ with $\mathbf{G}_{H} \times$ $\prod_{\substack{k=1, \ldots, n \\ k \neq j}} \mathbf{G}_{I_{k}}^{h}, \mathbf{G}$ with $\mathbf{G}_{I_{j}}^{h}$, and $\mathbf{S} \times \mathbf{G}$ with $\mathcal{G}_{H}$.

4. Point 4 in both definitions are equivalent.

By the definitions of $P_{I_{j} \mid \Sigma_{I L_{j}}}$ and $\mathbf{G}_{I_{j}}^{l}$, we have $P_{I_{j} \mid \Sigma_{I L_{j}}}^{-1}\left(L\left(\mathbf{G}_{I_{j}}\right)\right)=L\left(\mathbf{G}_{I_{j}}^{l}\right)$. Thus we know Point 4 in Definition 3.3 is equivalent to

$$
L\left(\mathbf{G}_{L_{j}}\right) \Sigma_{A_{j}} \cap L\left(\mathbf{G}_{I_{j}}^{l}\right) \subseteq L\left(\mathbf{G}_{L_{j}}\right)
$$

We now show that (2) is equivalent to Point 4 in Definition 3.4.

By the definition of $\mathcal{G}_{L_{j}}$, we have $\mathcal{G}_{L_{j}}=\mathbf{G}_{L_{j}} \times \mathbf{G}_{I_{j}}$.

The rest proof of this point is identical to the proof of Proposition 2.9 by substituting $\Sigma$ with $\Sigma_{I L_{j}}, \Sigma_{u}$ with $\Sigma_{R_{j}}, \Sigma_{c}$ with $\Sigma_{I L_{j}}-\Sigma_{R_{j}}$, S with $\mathbf{G}_{L_{j}}$, G with $\mathbf{G}_{I_{j}}^{l}$, and $\mathbf{S} \times \mathbf{G}$ with $\mathcal{G}_{L_{j}}$.

5. Point 5 in both definitions are equivalent.

By comparing Point 5 in Definition 3.3 and Point 5 in Definition 3.4, it is sufficient to show 
Master Thesis - R. Song - McMaster - Computing and Software

$\left(\forall s \in \Sigma_{I_{j}}^{*} . \Sigma_{R_{j}} \cap L\left(\mathcal{G}_{L_{j}}\right)\right) \operatorname{Elig}_{L\left(\mathbf{G}_{I_{j}}^{l}\right)}(s) \cap \Sigma_{A_{j}}=\operatorname{Elig}_{L\left(\mathbf{G}_{I_{j}}\right)}\left(P_{I_{j}}(s)\right) \cap \Sigma_{A_{j}}$.

Let $s \in \Sigma_{I L_{j}}^{*} . \Sigma_{R_{j}} \cap L\left(\mathcal{G}_{L_{j}}\right)$.

(a) Show that $\operatorname{Elig}_{L\left(\mathbf{G}_{I_{j}}^{l}\right)}(s) \cap \Sigma_{A_{j}} \subseteq \operatorname{Elig}_{L\left(\mathbf{G}_{I_{j}}\right)}\left(P_{I_{j}}(s)\right) \cap \Sigma_{A_{j}}$

Let $\alpha \in \operatorname{Elig}_{L\left(\mathbf{G}_{I_{j}}^{l}\right)}(s) \cap \Sigma_{A_{j}}$.

We now show that $\alpha \in \operatorname{Elig}_{L\left(\mathbf{G}_{I_{j}}\right)}\left(P_{I_{j}}(s)\right) \cap \Sigma_{A_{j}}$.

By (4), we know $s \alpha \in L\left(\mathbf{G}_{I_{j}}^{l}\right)$

$\Rightarrow s \alpha \in P_{I_{j} \mid \Sigma_{I L_{j}}}^{-1}\left(L\left(\mathbf{G}_{I_{j}}\right)\right), \quad$ by the definitions of $\mathbf{G}_{I_{j}}^{l}$ and $P_{I_{j} \mid \Sigma_{I L_{j}}}$

$\Rightarrow P_{I_{j} \mid \Sigma_{I L_{j}}}(s \alpha) \in L\left(\mathbf{G}_{I_{j}}\right)$

$\Rightarrow P_{I_{j} \mid \Sigma_{I L_{j}}}(s) \alpha \in L\left(\mathbf{G}_{I_{j}}\right), \quad$ as $\alpha \in \Sigma_{A_{j}}$ by (4)

From (3), we know $s \in \Sigma_{I L_{j}}^{*}$. By the definitions of $P_{I_{j} \mid \Sigma_{I L_{j}}}$ and $P_{I_{j}}$, we thus have $P_{I_{j}}(s) \alpha \in L\left(\mathbf{G}_{I_{j}}\right)$

$\Rightarrow \alpha \in \operatorname{Elig}_{L\left(\mathbf{G}_{I_{j}}\right)}\left(P_{I_{j}}(s)\right) \cap \Sigma_{A_{j}}$.

(b) Show that $\operatorname{Elig}_{L\left(\mathbf{G}_{I_{j}}\right)}\left(P_{I_{j}}(s)\right) \cap \Sigma_{A_{j}} \subseteq \operatorname{Elig}_{L\left(\mathbf{G}_{I_{j}}^{l}\right)}(s) \cap \Sigma_{A_{j}}$

Let $\alpha \in \operatorname{Elig}_{L\left(\mathbf{G}_{I_{j}}\right)}\left(P_{I_{j}}(s)\right) \cap \Sigma_{A_{j}}$.

We now show that $\alpha \in \operatorname{Elig}_{L\left(\mathbf{G}_{I_{j}}^{l}\right)}(s) \cap \Sigma_{A_{j}}$.

By (5), we know $P_{I_{j}}(s) \alpha \in L\left(\mathbf{G}_{I_{j}}\right)$

From (3), we know $s \in \Sigma_{I L_{j}}^{*}$. By the definitions of $P_{I_{j} \mid \Sigma_{I L_{j}}}$ and $P_{I_{j}}$, we thus have $P_{I_{j} \mid \Sigma_{I L_{j}}}(s) \alpha \in L\left(\mathbf{G}_{I_{j}}\right)$

$\Rightarrow P_{I_{j} \mid \Sigma_{I L_{j}}}(s \alpha) \in L\left(\mathbf{G}_{I_{j}}\right), \quad$ as $\alpha \in \Sigma_{A_{j}}$ by (5)

$\Rightarrow s \alpha \in P_{I_{j} \mid \Sigma_{I L_{j}}}^{-1}\left(L\left(\mathbf{G}_{I_{j}}\right)\right)$

$\Rightarrow s \alpha \in L\left(\mathbf{G}_{I_{j}}^{l}\right)$, by the definitions of $\mathbf{G}_{I_{j}}^{l}$ and $P_{I_{j} \mid \Sigma_{I L_{j}}}$

$\Rightarrow \alpha \in \operatorname{Elig}_{L\left(\mathbf{G}_{I_{j}}^{l}\right)}(s) \cap \Sigma_{A_{j}}$. 
Master Thesis - R. Song - McMaster - Computing and Software

6. Point 6 in both definitions are equivalent.

By comparing Point 6 in Definition 3.3 and Point 6 in Definition 3.4, it is sufficient to show

$$
\left(\forall s \in L\left(\mathcal{G}_{L_{j}}\right)\right) s \in L_{m}\left(\mathbf{G}_{I_{j}}^{l}\right) \text { iff } P_{I_{j}}(s) \in L_{m}\left(\mathbf{G}_{I_{j}}\right)
$$

Let $s \in L\left(\mathcal{G}_{L_{j}}\right)$.

$\Rightarrow s \in \Sigma_{I L_{j}}^{*}, \quad$ by the fact that $\mathcal{G}_{L_{j}}$ is defined over $\Sigma_{I L_{j}}$.

Let function $P_{I_{j} \mid \Sigma_{I L_{j}}}: \Sigma_{I L_{j}}^{*} \rightarrow \Sigma_{I_{j}}^{*}$ be a natural projection.

(a) Show that $s \in L_{m}\left(\mathbf{G}_{I_{j}}^{l}\right) \Rightarrow P_{I_{j}}(s) \in L_{m}\left(\mathbf{G}_{I_{j}}\right)$.

Assume $s \in L_{m}\left(\mathbf{G}_{I_{j}}^{l}\right)$. Must show this implies $P_{I_{j}}(s) \in L_{m}\left(\mathbf{G}_{I_{j}}\right)$.

From assumption $s \in L_{m}\left(\mathbf{G}_{I_{j}}^{l}\right)$, by the definitions of $P_{I_{j} \mid \Sigma_{I L_{j}}}$ and $\mathbf{G}_{I_{j}}^{l}$, we have $s \in P_{I_{j} \mid \Sigma_{I L_{j}}}^{-1}\left(L_{m}\left(\mathbf{G}_{I_{j}}\right)\right)$.

$\Rightarrow P_{I_{j} \mid \Sigma_{I L_{j}}}(s) \in L_{m}\left(\mathbf{G}_{I_{j}}\right)$

$\Rightarrow P_{I_{j}}(s) \in L_{m}\left(\mathbf{G}_{I_{j}}\right), \quad$ by $(6)$ and the definitions of $P_{I_{j}}$ and $P_{I_{j} \mid \Sigma_{I L_{j}}}$.

(b) Show that $P_{I_{j}}(s) \in L_{m}\left(\mathbf{G}_{I_{j}}\right) \Rightarrow s \in L_{m}\left(\mathbf{G}_{I_{j}}^{l}\right)$

Assume $P_{I_{j}}(s) \in L_{m}\left(\mathbf{G}_{I_{j}}\right)$. Must show this implies $s \in L_{m}\left(\mathbf{G}_{I_{j}}^{l}\right)$.

From assumption $P_{I_{j}}(s) \in L_{m}\left(\mathbf{G}_{I_{j}}\right)$, by (6) and the definitions of $P_{I_{j}}$ and $P_{I_{j} \mid \Sigma_{I L_{j}}}$, we have $P_{I_{j} \mid \Sigma_{I L_{j}}}(s) \in L_{m}\left(\mathbf{G}_{I_{j}}\right)$.

$\Rightarrow s \in P_{I_{j} \mid \Sigma_{I L_{j}}}^{-1}\left(L_{m}\left(\mathbf{G}_{I_{j}}\right)\right)$

$\Rightarrow s \in L_{m}\left(\mathbf{G}_{I_{j}}^{l}\right), \quad$ by the definitions of $P_{I_{j} \mid \Sigma_{I L_{j}}}$ and $\mathbf{G}_{I_{j}}^{l}$.

We now give our final interface consistent definition. We will always use this definition in the rest of this thesis. 
Master Thesis - R. Song - McMaster - Computing and Software

Definition 3.5. The $n^{\text {th }}$ degree interface system composed of plant components $\mathbf{G}_{H}^{p}, \mathbf{G}_{L_{1}}^{p}, \ldots, \mathbf{G}_{L_{n}}^{p}$, supervisors $\mathbf{S}_{H}, \mathbf{S}_{L_{1}}, \ldots, \mathbf{S}_{L_{n}}$, and interfaces $\mathbf{G}_{I_{1}}, \ldots, \mathbf{G}_{I_{n}}$ is interface consistent with respect to the alphabet partition given by 3.1, if for all $j \in\{1, \ldots, n\}$, the following conditions are satisfied ${ }^{2}$ :

- Multi-level Properties

1. The event set of $\mathbf{G}_{H}^{p}$ and $\mathbf{S}_{H}$ is $\Sigma_{I H}$, and the event set of $\mathbf{G}_{L_{j}}^{p}$ and $\mathbf{S}_{L_{j}}$ is $\Sigma_{I L_{j}}$

2. $\mathbf{G}_{I_{j}}$ is a command-pair interface.

- High-level Properties

3. $L\left(\mathcal{G}_{H}\right) \Sigma_{A_{j}} \cap L\left(\mathbf{G}_{I_{j}}^{h}\right) \subseteq L\left(\mathcal{G}_{H}\right)$

- Low-level Properties

4. $L\left(\mathcal{G}_{L_{j}}\right) \Sigma_{R_{j}} \cap L\left(\mathbf{G}_{I_{j}}^{l}\right) \subseteq L\left(\mathcal{G}_{L_{j}}\right)$

5. $\left(\forall s \in L\left(\mathcal{G}_{L_{j}}\right)\right)\left(\forall \rho \in \Sigma_{R_{j}}\right)\left(\forall \alpha \in \Sigma_{A_{j}}\right)$

$s \rho \alpha \in L\left(\mathbf{G}_{I_{j}}^{l}\right) \Rightarrow\left(\exists l \in \Sigma_{L_{j}}^{*}\right) s \rho l \alpha \in L\left(\mathcal{G}_{L_{j}}\right)$

6. $\left(\forall s \in L\left(\mathcal{G}_{L_{j}}\right)\right) s \in L_{m}\left(\mathbf{G}_{I_{j}}^{l}\right) \Rightarrow\left(\exists l \in \Sigma_{L_{j}}^{*}\right) s l \in L_{m}\left(\mathcal{G}_{L_{j}}\right)$

Proposition 3.2. Definition 3.5 is equivalent to Definition 3.4.

\section{proof:}

For the $n^{\text {th }}$ degree interface system that respects to the alphabet partition given by 3.1 and is composed of plant components $\mathbf{G}_{H}^{p}, \mathbf{G}_{L_{1}}^{p}, \ldots, \mathbf{G}_{L_{n}}^{p}$, supervisors $\mathbf{S}_{H}, \mathbf{S}_{L_{1}}, \ldots, \mathbf{S}_{L_{n}}$, and interfaces $\mathbf{G}_{I_{1}}, \ldots, \mathbf{G}_{I_{n}}$,

\footnotetext{
${ }^{2}$ This definition is only for proving our synthesis algorithm. For understanding HISC, please see the interface consistent definition in [23] by Leduc.
} 
Master Thesis - R. Song - McMaster - Computing and Software

1. assume for all $j \in\{1, \ldots, n\}$, the system satisfies all the conditions in Definition 3.4. Must show this implies the system satisfies all the conditions in Definition 3.5 as well.

Point 1, 2, 3, 4 and 6 in both definitions are exactly same, so the system satisfies Point 1, 2, 3, 4 and 6 in Definition 3.5.

We now show that the system also satisfies Point 5 in Definition 3.5.

Let $j \in\{1, \ldots, n\}, s \in L\left(\mathcal{G}_{L_{j}}\right), \rho \in \Sigma_{R_{j}}, \alpha \in \Sigma_{A_{j}}$.

Assume $s \rho \alpha \in L\left(\mathbf{G}_{I_{j}}^{l}\right)$.

Must show implies $\left(\exists l \in \Sigma_{L_{j}}^{*}\right)$ s $\rho l \alpha \in L\left(\mathcal{G}_{L_{j}}\right)$.

By (1), we know $s \in L\left(\mathcal{G}_{L_{j}}\right), \rho \in \Sigma_{R_{j}}$

$\Rightarrow s \rho \in L\left(\mathcal{G}_{L_{j}}\right), \quad$ by Point 4 in Definition 3.4

$\Rightarrow s \rho \in \Sigma_{I L_{j}}^{*} \cdot \Sigma_{R_{j}} \cap L\left(\mathcal{G}_{L_{j}}\right)$

$\Rightarrow \operatorname{Elig}_{L\left(\mathcal{G}_{L_{j}}\right)}\left(s \rho \Sigma_{L_{j}}^{*}\right) \cap \Sigma_{A_{j}}=\operatorname{Elig}_{L\left(\mathbf{G}_{I_{j}}^{l}\right)}(s \rho) \cap \Sigma_{A_{j}}$,

by Point 5 in Definition 3.4

By (2), we have $s \rho \alpha \in L\left(\mathbf{G}_{I_{j}}^{l}\right)$

$\Rightarrow \alpha \in \operatorname{Elig}_{L\left(\mathbf{G}_{I_{j}}^{l}\right)}(s \rho) \cap \Sigma_{A_{j}}$.

$\Rightarrow \alpha \in \operatorname{Elig}_{L\left(\mathcal{G}_{L_{j}}\right)}\left(s \rho \Sigma_{L_{j}}^{*}\right) \cap \Sigma_{A_{j}}, \quad$ by $(3)$

$\Rightarrow\left(\exists l \in \Sigma_{L_{j}}^{*}\right) s \rho l \alpha \in L\left(\mathcal{G}_{L_{j}}\right)$

2. assume for all $j \in\{1, \ldots, n\}$, the system satisfies all the conditions in Definition 3.5, must show this implies the system satisfies all the conditions in Definition 3.4 as well.

Point 1, 2, 3, 4 and 6 in both definitions are exactly same, so the system satisfies Point 1, 2, 3, 4 and 6 in Definition 3.4. 


\section{Master Thesis - R. Song - McMaster - Computing and Software}

We now show that the system also satisfies Point 5 in Definition 3.4.

Let $j \in\{1, \ldots, n\}$ and $s \in \Sigma_{I L_{j}}^{*} . \Sigma_{R_{j}} \cap L\left(\mathcal{G}_{L_{j}}\right)$.

Must show this implies $\operatorname{Elig}_{L\left(\mathcal{G}_{L_{j}}\right)}\left(s \Sigma_{L_{j}}^{*}\right) \cap \Sigma_{A_{j}}=\operatorname{Elig}_{L\left(\mathbf{G}_{I_{j}}^{l}\right)}(s) \cap \Sigma_{A_{j}}$.

(a) Show that $\operatorname{Elig}_{L\left(\mathcal{G}_{L_{j}}\right)}\left(s \Sigma_{L_{j}}^{*}\right) \cap \Sigma_{A_{j}} \subseteq \operatorname{Elig}_{L\left(\mathbf{G}_{I_{j}}^{l}\right)}(s) \cap \Sigma_{A_{j}}$.

Sufficient to show that

$\left(\forall \alpha \in \Sigma_{A_{j}}\right)\left(\forall l \in \Sigma_{L_{j}}^{*}\right) \alpha \in \operatorname{Elig}_{L\left(\mathcal{G}_{L_{j}}\right)}(s l) \Rightarrow \alpha \in \operatorname{Elig}_{L\left(\mathbf{G}_{I_{j}}^{l}\right)}(s)$.

Let $\alpha \in \Sigma_{A_{j}}, l \in \Sigma_{L_{j}}^{*}$.

Assume $\alpha \in \operatorname{Elig}_{L\left(\mathcal{G}_{L_{j}}\right)}(s l)$.

Must show this implies $\alpha \in \operatorname{Elig}_{L\left(\mathbf{G}_{I_{j}}^{l}\right)}(s)$

By $\mathcal{G}_{L_{j}}=\mathbf{S}_{L_{j}} \times \mathbf{G}_{L_{j}}^{p} \times \mathbf{G}_{I_{j}}^{l}$, we have $L\left(\mathcal{G}_{L_{j}}\right) \subseteq L\left(\mathbf{G}_{I_{j}}^{l}\right)$

By (4) and (5), we have $\alpha \in \operatorname{Elig}_{L\left(\mathbf{G}_{I_{j}}^{l}\right)}(s l)$

As $\mathbf{G}_{I_{j}}^{l}:=\operatorname{selfloop}\left(\mathbf{G}_{I_{j}}, \Sigma_{L_{j}}\right)$, we have $\operatorname{Elig}_{L\left(\mathbf{G}_{I_{j}}^{l}\right)}(s)=\operatorname{Elig}_{L\left(\mathbf{G}_{I_{j}}^{l}\right)}(s l)$.

So, $\alpha \in \operatorname{Elig}_{L\left(\mathbf{G}_{I_{j}}^{l}\right)}(s)$.

(b) Show that $\operatorname{Elig}_{L\left(\mathbf{G}_{I_{j}}^{l}\right)}(s) \cap \Sigma_{A_{j}} \subseteq \operatorname{Elig}_{L\left(\mathcal{G}_{L_{j}}\right)}\left(s \Sigma_{L_{j}}^{*}\right) \cap \Sigma_{A_{j}}$.

Sufficient to show that $\left(\forall \alpha \in \Sigma_{A_{j}}\right) s \alpha \in L\left(\mathbf{G}_{I_{j}}^{l}\right) \Rightarrow\left(\exists l \in \Sigma_{L_{j}}^{*}\right)$ sl $\alpha \in$ $L\left(\mathcal{G}_{L_{j}}\right)$

From (3), we know $s \in \Sigma_{I L_{j}}^{*} \cdot \Sigma_{R_{j}} \cap L\left(\mathcal{G}_{L_{j}}\right)$

$\Rightarrow\left(\exists s^{\prime} \in L\left(\mathcal{G}_{L_{j}}\right)\right)\left(\exists \rho \in \Sigma_{R_{j}}\right) s^{\prime} \rho=s$

$\Rightarrow\left(\forall \alpha \in \Sigma_{A_{j}}\right) s^{\prime} \rho \alpha \in L\left(\mathbf{G}_{I_{j}}^{l}\right) \Rightarrow\left(\exists l \in \Sigma_{L_{j}}^{*}\right) s^{\prime} \rho l \alpha \in L\left(\mathcal{G}_{L_{j}}\right)$,

by Point 5 in Definition 3.5

$\Rightarrow\left(\forall \alpha \in \Sigma_{A_{j}}\right) s \alpha \in L\left(\mathbf{G}_{I_{j}}^{l}\right) \Rightarrow\left(\exists l \in \Sigma_{L_{j}}^{*}\right) s l \alpha \in L\left(\mathcal{G}_{L_{j}}\right)$. 


\section{Master Thesis - R. Song-McMaster - Computing and Software}

Note that although Point 5 in Definition 3.5 is stronger than Point 5 in Definition 3.4, the two definitions are equivalent, because Point 5 in Definition 3.5 only requires some extra conditions which are already present in Point 4 of both definitions.

By Proposition 3.1 and Proposition 3.2, we know that Definition 3.3 and Definition 3.5 are equivalent.

\subsubsection{Local Conditions for Global Nonblocking}

Definition 3.6. The $n^{\text {th }}$ degree interface system composed of plant components $\mathbf{G}_{H}^{p}, \mathbf{G}_{L_{1}}^{p}, \ldots, \mathbf{G}_{L_{n}}^{p}$, supervisors $\mathbf{S}_{H}, \mathbf{S}_{L_{1}}, \ldots, \mathbf{S}_{L_{n}}$, and interfaces $\mathbf{G}_{I_{1}}, \ldots, \mathbf{G}_{I_{n}}$ is said to be level-wise nonblocking with respect to the alphabet partition given by 3.1, if the following two conditions are satisfied:

1. Nonblocking at the high-level: $\overline{L_{m}\left(\mathcal{G}_{H}\right)}=L\left(\mathcal{G}_{H}\right)$

2. Nonblocking at the low-level: $(\forall j \in\{1, \ldots, n\}) \overline{L_{m}\left(\mathcal{G}_{L_{j}}\right)}=L\left(\mathcal{G}_{L_{j}}\right)$

Theorem 3.1 (From [24]). If the $n^{\text {th }}$ degree interface system composed of plant components $\mathbf{G}_{H}^{p}, \mathbf{G}_{L_{1}}^{p}, \ldots, \mathbf{G}_{L_{n}}^{p}$, supervisors $\mathbf{S}_{H}, \mathbf{S}_{L_{1}}, \ldots, \mathbf{S}_{L_{n}}$, and interfaces $\mathbf{G}_{I_{1}}, \ldots, \mathbf{G}_{I_{n}}$, is level-wise nonblocking and interface consistent with respect to the alphabet partition given by 3.1, then

$$
\overline{L_{m}(\text { SYSTEM })}=L(\text { SYSTEM })
$$

\subsubsection{Local Conditions for Global Controllability}

Definition 3.7. The $n^{\text {th }}$ degree interface system composed of plant components $\mathbf{G}_{H}^{p}, \mathbf{G}_{L_{1}}^{p}, \ldots, \mathbf{G}_{L_{n}}^{p}$, supervisors $\mathbf{S}_{H}, \mathbf{S}_{L_{1}}, \ldots, \mathbf{S}_{L_{n}}$, and interfaces $\mathbf{G}_{I_{1}}, \ldots, \mathbf{G}_{I_{n}}$ is said 


\section{Master Thesis - R. Song-McMaster-Computing and Software}

to be level-wise controllable with respect to the alphabet partition given by 3.1, if for all $j \in\{1, \ldots, n\}$ the following two conditions are satisfied:

1. The alphabet of $\mathbf{G}_{H}^{p}$ and $\mathbf{S}_{H}$ is $\Sigma_{I H}$, the alphabet of $\mathbf{G}_{L_{j}}^{p}$ and $\mathbf{S}_{L_{j}}$ is $\Sigma_{I L_{j}}$, and the alphabet of $\mathbf{G}_{I_{j}}$ is $\Sigma_{I_{j}}$

2. Controllable at the high-level: $L\left(\mathcal{G}_{H}\right) \Sigma_{h u} \cap L\left(\mathbf{G}_{H}^{p} \times \mathbf{G}_{I}^{h}\right) \subseteq L\left(\mathcal{G}_{H}\right)$

3. Controllable at the low-level: $L\left(\mathcal{G}_{L_{j}}\right) \Sigma_{l u_{j}} \cap L\left(\mathbf{G}_{L_{j}}^{p}\right) \subseteq L\left(\mathcal{G}_{L_{j}}\right)$

Theorem 3.2. If the $n^{\text {th }}$ degree interface system composed of plant components $\mathbf{G}_{H}^{p}, \mathbf{G}_{L_{1}}^{p}, \ldots, \mathbf{G}_{L_{n}}^{p}$, supervisors $\mathbf{S}_{H}, \mathbf{S}_{L_{1}}, \ldots, \mathbf{S}_{L_{n}}$, and interfaces $\mathbf{G}_{I_{1}}, \ldots, \mathbf{G}_{I_{n}}$, is levelwise controllable with respect to the alphabet partition given by 3.1, then

$$
L(\mathbf{S Y S T E M}) \Sigma_{u} \cap L(\mathbf{P L A N T}) \subseteq L(\text { SYSTEM })
$$

proof:

Follows immediately from Theorem 2 of [24] and Proposition 2.9.

\subsection{Level-wise Interface Controllable Supervisor}

From Section 3.2, we can see that all the conditions are either based on the highlevel or a single low-level. Therefore, we can verify the global controllability and global nonblocking by only verifying the local conditions. For convenience, we now group the conditions by levels and give several corresponding definitions.

Definition 3.8. For the $n^{\text {th }}$ degree interface system that respects the alphabet partition given by 3.1 and is composed of plant components $\mathbf{G}_{H}^{p}, \mathbf{G}_{L_{1}}^{p}, \ldots, \mathbf{G}_{L_{n}}^{p}$, supervisors $\mathbf{S}_{H}, \mathbf{S}_{L_{1}}, \ldots, \mathbf{S}_{L_{n}}$, and interfaces $\mathbf{G}_{I_{1}}, \ldots, \mathbf{G}_{I_{n}}$, define the following: 
Master Thesis - R. Song - McMaster - Computing and Software

- $\mathbf{S}_{H}$ is high-level interface controllable (HIC), if the following conditions are satisfied:

1. $L\left(\mathcal{G}_{H}\right) \Sigma_{h u} \cap L\left(\mathbf{G}_{H}^{p} \times \mathbf{G}_{I}^{h}\right) \subseteq L\left(\mathcal{G}_{H}\right)$

2. $(\forall j \in\{1, \ldots, n\}) L\left(\mathcal{G}_{H}\right) \Sigma_{A_{j}} \cap L\left(\mathbf{G}_{I_{j}}^{h} \subseteq L\left(\mathcal{G}_{H}\right)\right.$

- $\mathbf{S}_{H}$ is a high-level proper supervisor, if the following conditions are satisfied:

1. $\mathbf{S}_{H}$ is high-level interface controllable.

2. $\overline{L_{m}\left(\mathcal{G}_{H}\right)}=L\left(\mathcal{G}_{H}\right)$

- For all $j \in\{1, \ldots, n\}, \mathbf{S}_{L_{j}}$ is $j^{\text {th }}$ low-level interface controllable $\left(\mathrm{LIC}_{j}\right)$, if the following conditions are satisfied:

1. $L\left(\mathcal{G}_{L_{j}}\right) \Sigma_{l u_{j}} \cap L\left(\mathbf{G}_{L_{j}}^{p}\right) \subseteq L\left(\mathcal{G}_{L_{j}}\right)$

2. $L\left(\mathcal{G}_{L_{j}}\right) \Sigma_{R_{j}} \cap L\left(\mathbf{G}_{I_{j}}^{l}\right) \subseteq L\left(\mathcal{G}_{L_{j}}\right)$

3. $\left(\forall s \in L\left(\mathcal{G}_{L_{j}}\right)\right)\left(\forall \rho \in \Sigma_{R_{j}}\right)\left(\forall \alpha \in \Sigma_{A_{j}}\right)$ $s \rho \alpha \in L\left(\mathbf{G}_{I_{j}}^{l}\right) \Rightarrow\left(\exists l \in \Sigma_{L_{j}}^{*}\right)$ s $\rho l \alpha \in L\left(\mathcal{G}_{L_{j}}\right)$

4. $\left(\forall s \in L\left(\mathcal{G}_{L_{j}}\right)\right) s \in L_{m}\left(\mathbf{G}_{I_{j}}^{l}\right) \Rightarrow\left(\exists l \in \Sigma_{L_{j}}^{*}\right) s l \in L_{m}\left(\mathcal{G}_{L_{j}}\right)$

- For all $j \in\{1, \ldots, n\}, \mathbf{S}_{L_{j}}$ is a $j^{\text {th }}$ low-level proper supervisor, if the following conditions are satisfied:

1. $\mathbf{S}_{L_{j}}$ is $j^{\text {th }}$ low-level interface controllable.

2. $\overline{L_{m}\left(\mathcal{G}_{L_{j}}\right)}=L\left(\mathcal{G}_{L_{j}}\right)$

Proposition 3.3. For the $n^{\text {th }}$ degree interface system that respects the alphabet partition given by 3.1 and is composed of plant components $\mathbf{G}_{H}^{p}, \mathbf{G}_{L_{1}}^{p}, \ldots, \mathbf{G}_{L_{n}}^{p}$, supervisors 


$$
\text { Master Thesis - R. Song - McMaster - Computing and Software }
$$

$\mathbf{S}_{H}, \mathbf{S}_{L_{1}}, \ldots, \mathbf{S}_{L_{n}}$, and interfaces $\mathbf{G}_{I_{1}}, \ldots, \mathbf{G}_{I_{n}}$, the flat system is nonblocking and the flat supervisor is controllable for the flat plant if for all $j \in\{1, \ldots, n\}$, the following conditions are satisfied:

1. The alphabet of $\mathbf{G}_{H}^{p}$ and $\mathbf{S}_{H}$ is $\Sigma_{I H}$.

2. The alphabet of $\mathbf{G}_{L_{j}}^{p}$ and $\mathbf{S}_{L_{j}}$ is $\Sigma_{I L_{j}}$.

3. $\mathbf{G}_{I_{j}}$ is a command-pair interface.

4. $\mathbf{S}_{H}$ is a high-level proper supervisor.

5. $\mathbf{S}_{L_{j}}$ is a $j^{\text {th }}$ low-level proper supervisor.

\section{proof:}

If the system satisfies all the above five conditions, clearly, it is interface consistent, level-wise nonblocking and level-wise controllable, so the flat system is is nonblocking and the flat supervisor is controllable for the flat plant by Theorem 3.1 and 3.2 . 
Master Thesis - R. Song - McMaster - Computing and Software 


\section{Chapter 4}

\section{Synthesis of HISC}

In [21-27], the supervisors in an HISC system are designed by hand, and then the system is verified for the interface consistent, level-wise controllable and nonblock-

ing conditions. However, for a complicated system it is very desirable to synthesize the supervisors from some specifications which specify the desired system behavior but the system containing them does not necessarily satisfy all the required conditions. In this chapter, we first discuss how to compute the supremal high-level and low-level interface controllable sublanguages, and then give the predicate-based algorithms. These algorithms can easily be implemented by using Binary Decision Diagrams(BDD) as we will discuss in Chapter 6.

\subsection{Introduction}

In the previous chapter, we specified that a $n^{\text {th }}$ degree interface system is composed of plants, supervisors and interfaces. By supervisors, we expect that the system containing them not only have the desired behavior but also is interface consistent, level-wise controllable and level-wise nonblocking. As we discuss the synthesis process 


\section{Master Thesis - R. Song - McMaster - Computing and Software}

in this chapter, we will assume that a $n^{\text {th }}$ degree parallel interface system is composed of plants, specifications and interfaces. By specifications, we mean that they specify the desired behavior but the system containing them is likely not interface consistent, level-wise controllable and level-wise nonblocking.

For the $n^{\text {th }}$ degree interface system that respects the alphabet partition given by 3.1 and is composed of plant components $\mathbf{G}_{H}^{p}, \mathbf{G}_{L_{1}}^{p}, \ldots, \mathbf{G}_{L_{n}}^{p}$, supervisors $\mathbf{S}_{H}, \mathbf{S}_{L_{1}}$, $\ldots, \mathbf{S}_{L_{n}}$, and interfaces $\mathbf{G}_{I_{1}}, \ldots, \mathbf{G}_{I_{n}}$, if we replace the high-level supervisor $\mathbf{S}_{H}$ by a high-level specification DES $\mathbf{E}_{H}$ (defined over $\Sigma_{I H}$ ), and for all $j \in\{1, \ldots, n\}$, we replace the $j^{\text {th }}$ low-level supervisor $\mathbf{S}_{L_{j}}$ by a $j^{\text {th }}$ low-level specification DES $\mathbf{E}_{L_{j}}$ (defined over $\Sigma_{I L_{j}}$ ), then the resulting system is called a $n^{\text {th }}$ degree specification interface system. We refer to the original system with supervisors as a $n^{\text {th }}$ degree supervisor interface system. In case we do not care whether a system is composed of specifications or supervisors, we refer to it as a $n^{\text {th }}$ degree interface system.

We can apply all the concepts defined for a $n^{\text {th }}$ degree supervisor interface system to a $n^{\text {th }}$ degree specification interface system by replacing each supervisor DES by its corresponding specification DES. To make things clear, we give the following corresponding definitions for a $n^{\text {th }}$ degree specification system.

- The high-level subsystem: $\mathbf{G}_{H}:=\mathbf{E}_{H} \times \mathbf{G}_{H}^{p}$.

- The $j^{\text {th }}$ low-level subsystem: $\mathbf{G}_{L_{j}}:=\mathbf{E}_{L_{j}} \times \mathbf{G}_{L_{j}}^{p}, j \in\{1, \ldots, n\}$

- The high-level: $\mathcal{G}_{H}:=\mathbf{E}_{H} \times \mathbf{G}_{H}^{p} \times \mathbf{G}_{I}^{h}$.

- The $j^{\text {th }}$ low-level: $\mathcal{G}_{L_{j}}:=\mathbf{E}_{L_{j}} \times \mathbf{G}_{L_{j}}^{p} \times \mathbf{G}_{I_{j}}^{l}, j \in\{1, \ldots, n\}$

We now give the starting point for the synthesis process.

Definition 4.1. The $n^{\text {th }}$ degree parallel interface specification system composed of plant components $\mathbf{G}_{H}^{p}, \mathbf{G}_{L_{1}}^{p}, \ldots, \mathbf{G}_{L_{n}}^{p}$, specifications $\mathbf{E}_{H}, \mathbf{E}_{L_{1}}, \ldots, \mathbf{E}_{L_{n}}$, and interfaces 


\section{Master Thesis - R. Song-McMaster-Computing and Software}

$\mathbf{G}_{I_{1}}, \ldots, \mathbf{G}_{I_{n}}$, is HISC-valid with respects to the alphabet partition given by 3.1, if for all $j \in\{1, \ldots, n\}$, the following conditions are satisfied:

1. The alphabet of $\mathbf{G}_{H}^{p}$ and $\mathbf{E}_{H}$ is $\Sigma_{I H}$.

2. The alphabet of $\mathbf{G}_{L_{j}}^{p}$ and $\mathbf{E}_{L_{j}}$ is $\Sigma_{I L_{j}}$.

3. $\mathbf{G}_{I_{j}}$ is a command-pair interface.

Obviously, the three conditions are exactly same as the first three conditions in Propositions 3.3 except that we use the specification DES instead. Given a $n^{\text {th }}$ degree HISC-valid parallel interface specification system, our main objective in this chapter is to develop a method to synthesize a proper high-level supervisor and proper low-level supervisors such that the marked behavior of the constructed $n^{\text {th }}$ degree supervisor interface system is as large as possible. That is, we would like to compute the largest marked sublanguages of the high-level and each of the low-levels such that the DES representing each is either a proper high-level supervisor or a proper low-level supervisor, as appropriate. We say such a proper high-level supervisor or low-level supervisor is locally maximally permissive for its level.

Let $\boldsymbol{\Phi}$ be the $n^{\text {th }}$ degree HISC-valid specification interface system ${ }^{1}$ that respects the alphabet partition given by 3.1 and is composed of plant components $\mathbf{G}_{H}^{p}, \mathbf{G}_{L_{1}}^{p}$, $\ldots, \mathbf{G}_{L_{n}}^{p}$, specifications $\mathbf{E}_{H}, \mathbf{E}_{L_{1}}, \ldots, \mathbf{E}_{L_{n}}$, and interfaces $\mathbf{G}_{I_{1}}, \ldots, \mathbf{G}_{I_{n}}$. We assume that all the DES in system $\boldsymbol{\Phi}$ have a finite number of states. In this chapter, we always use the system $\boldsymbol{\Phi}$, and $j$ is always an index with range $\{1, \ldots, n\}$.

For later usage, we give the tuple definitions for the following DES:

$$
\mathcal{G}_{H}:=\left(Q_{H}, \Sigma_{I H}, \delta_{H}, q_{H_{0}}, Q_{H_{m}}\right), \mathbf{G}_{H}^{p}:=\left(Y_{H}, \Sigma_{I H}, \eta_{H}, y_{H_{0}}, Y_{H_{m}}\right),
$$

\footnotetext{
${ }^{1}$ We use the Greek letter to mean that $\boldsymbol{\Phi}$ is not a simple DES, but a structured system.
} 
Master Thesis - R. Song - McMaster - Computing and Software

$$
\begin{gathered}
\mathbf{E}_{H}:=\left(Z_{H}, \Sigma_{I H}, \zeta_{H}, z_{H_{0}}, Z_{H_{m}}\right), \mathbf{G}_{I_{j}}^{h}:=\left(X_{j}^{h}, \Sigma_{I H}, \xi_{j}^{h}, x_{j_{0}}^{h}, X_{j_{m}}^{h}\right), \\
\mathbf{G}_{I}^{h}:=\left(X^{h}, \Sigma_{I H}, \xi^{h}, x_{0}^{h}, X_{m}^{h}\right), \\
\mathcal{G}_{L_{j}}:=\left(Q_{L_{j}}, \Sigma_{I L_{j}}, \delta_{L_{j}}, q_{L j_{0}}, Q_{L j_{m}}\right), \quad \mathbf{G}_{L_{j}}^{p}:=\left(Y_{L_{j}}, \Sigma_{I L_{j}}, \eta_{L_{j}}, y_{L j_{0}}, Y_{L j_{m}}\right), \\
\mathbf{E}_{L_{j}}:=\left(Z_{L_{j}}, \Sigma_{I L_{j}}, \zeta_{L_{j}}, z_{L j_{0}}, Z_{L j_{m}}\right), \quad \mathbf{G}_{I_{j}}^{l}:=\left(X_{j}^{l}, \Sigma_{I L_{j}}, \xi_{j}^{l}, x_{j_{0}}^{l}, X_{j_{m}}^{l}\right) .
\end{gathered}
$$

So,

$$
\begin{gathered}
\mathcal{G}_{H}=\left(Z_{H} \times Y_{H} \times X^{h}, \Sigma_{I H}, \zeta_{H} \times \eta_{H} \times \xi^{h},\left(z_{H_{0}}, y_{H_{0}}, x_{0}^{h}\right), Z_{H_{m}} \times Y_{H_{m}} \times X_{m}^{h}\right) \\
\mathcal{G}_{L_{j}}=\left(Z_{L_{j}} \times Y_{L_{j}} \times X_{j}^{l}, \Sigma_{I L_{j}}, \zeta_{L_{j}} \times \eta_{L_{j}} \times \xi_{j}^{l},\left(z_{L j_{0}}, y_{L j_{0}}, x_{j_{0}}^{l}\right), Z_{L j_{m}} \times Y_{L j_{m}} \times X_{j_{m}}^{l}\right) \\
\mathbf{G}_{I}^{h}=\left(X_{1}^{h} \times \cdots \times X_{n}^{h}, \Sigma_{I H}, \xi_{1}^{h} \times \cdots \times \xi_{n}^{h},\left(x_{1_{0}}^{h}, \cdots, x_{n_{0}}^{h}\right), X_{1_{m}}^{h} \times \cdots \times X_{n_{m}}^{h}\right)
\end{gathered}
$$

\subsection{High-level Supervisor Synthesis}

In this section, we show how to synthesize a locally maximally permissive proper high-level supervisor for system $\boldsymbol{\Phi}$.

\subsubsection{High-level Interface Controllable Language}

Definition 4.2. For system $\Phi$, let $K \subseteq \Sigma_{I H}^{*}$ be a language. $K$ is high-level interface controllable(HIC) with respect to system $\mathbf{\Phi}$ if

1. $\bar{K} \Sigma_{h u} \cap L\left(\mathbf{G}_{H}^{p} \times \mathbf{G}_{I}^{h}\right) \subseteq \bar{K}$

2. $(\forall j \in\{1, \ldots, n\}) \bar{K} \Sigma_{A_{j}} \cap L\left(\mathbf{G}_{I_{j}}^{h}\right) \subseteq \bar{K}$

Obviously, the empty language $\emptyset$ is high-level interface controllable with respect to system $\boldsymbol{\Phi}$. Note that the definition is based on the language $\bar{K}$, so $K$ is high-level interface controllable with respect to system $\boldsymbol{\Phi}$ iff $\bar{K}$ is high-level interface controllable with respect to system $\boldsymbol{\Phi}$. 


\section{Master Thesis - R. Song-McMaster-Computing and Software}

For an arbitrary language $E \subseteq \Sigma_{I H}^{*}$, we define the set of all sublanguages of $E$ that are high-level interface controllable with respect to system $\boldsymbol{\Phi}$ as

$\mathcal{C}_{H}(E):=\{K \subseteq E \mid K$ is high-level interface controllable with respect to system $\mathbf{\Phi}\}$

Clearly, $\left(\mathcal{C}_{H}(E), \subseteq\right)$ is a poset. We now show that the supremum in this poset always exists in $\mathcal{C}_{H}(E)$.

Proposition 4.1. For system $\boldsymbol{\Phi}$, let $E \subseteq \Sigma_{I H}^{*}$. The set $\mathcal{C}_{H}(E)$ is nonempty and is closed under arbitrary unions. In particular, $\mathcal{C}_{H}(E)$ contains a (unique) supremal element, $\sup \mathcal{C}_{H}(E)=\cup\left\{K \mid K \in \mathcal{C}_{H}(E)\right\}$.

\section{proof:}

1. Show that $\mathcal{C}_{H}(E)$ is nonempty

The empty language $\emptyset$ is high-level interface controllable with respect to system $\Phi$, so $\emptyset \in \mathcal{C}_{H}(E)$.

2. Show that $\mathcal{C}_{H}(E)$ is closed under arbitrary unions.

Let $B$ be an index set. Assume that $(\forall \beta \in B) K_{\beta} \in \mathcal{C}_{H}(E)$. Sufficient to show that $\cup_{\beta \in B} K_{\beta} \in \mathcal{C}_{H}(E)$.

Let $K:=\cup_{\beta \in B} K_{\beta}$.

Sufficient to show that $K$ is high-level interface controllable with respect to system $\boldsymbol{\Phi}$. Then by Definition 4.2, we have to show the following:

(a) Show that $\bar{K} \Sigma_{h u} \cap L\left(\mathbf{G}_{H}^{p} \times \mathbf{G}_{I}^{h}\right) \subseteq \bar{K}$

Let $s \in \bar{K} \Sigma_{h u} \cap L\left(\mathbf{G}_{H}^{p} \times \mathbf{G}_{I}^{h}\right)$.

Must show this implies $s \in \bar{K}$.

From (2), we know $\left(\exists s^{\prime} \in \bar{K}\right)\left(\exists \sigma \in \Sigma_{h u}\right) s^{\prime} \sigma=s$ 
Master Thesis - R. Song - McMaster - Computing and Software

As $s^{\prime} \in \bar{K}$, we can conclude $\left(\exists u \in \Sigma_{I H}^{*}\right) s^{\prime} u \in K$

$\Rightarrow(\exists \beta \in B) s^{\prime} u \in K_{\beta}, \quad$ by $(1)$

$\Rightarrow s^{\prime} \in \overline{K_{\beta}}, \quad$ by $(1)$

$\Rightarrow s^{\prime} \sigma \in \overline{K_{\beta}} \Sigma_{h u}$

$\Rightarrow s^{\prime} \sigma \in \overline{K_{\beta}} \Sigma_{h u} \cap L\left(\mathbf{G}_{H}^{p} \times \mathbf{G}_{I}^{h}\right), \quad$ by (3) and (2)

$\Rightarrow s^{\prime} \sigma \in \overline{K_{\beta}}, \quad$ as $K_{\beta} \in \mathcal{C}_{H}(E)$

$\Rightarrow\left(\exists u^{\prime} \in \Sigma_{I H}^{*}\right) s^{\prime} \sigma u^{\prime} \in K_{\beta}$

$\Rightarrow s^{\prime} \sigma u^{\prime} \in K, \quad$ by $(1)$

$\Rightarrow s u^{\prime} \in K, \quad$ by $(3)$

$\Rightarrow s \in \bar{K}$

(b) Show that $(\forall j \in\{1, \ldots, n\}) \bar{K} \Sigma_{A_{j}} \cap L\left(\mathbf{G}_{I_{j}}^{h}\right) \subseteq \bar{K}$

Let $j \in\{1, \ldots, n\}$. Let $s \in \bar{K} \Sigma_{A_{j}} \cap L\left(\mathbf{G}_{I_{j}}^{h}\right)$. Must show this implies $s \in \bar{K}$. The rest is identical to Part 2(a) (from the line labeled with (3)) after substituting $\Sigma_{h u}$ with $\Sigma_{A_{j}}, \Sigma_{I H}$ with $\Sigma_{I L_{j}}$ and $L\left(\mathbf{G}_{H}^{p} \times \mathbf{G}_{I}^{h}\right)$ with $L\left(\mathbf{G}_{I_{j}}^{h}\right)$.

3. Show that $\sup \mathcal{C}_{H}(E)=\cup\left\{K \mid K \in \mathcal{C}_{H}(E)\right\}$.

Let $K_{\text {sup }}:=\cup\left\{K \mid K \in \mathcal{C}_{H}(E)\right\}$

Clearly, for all $K \in \mathcal{C}_{H}(E)$, we have $K \subseteq K_{\text {sup }}$

All that remains is to show:

$\left(\forall K^{\prime} \in P \operatorname{Pwr}\left(\Sigma_{I H}^{*}\right)\right)\left(\left(\left(\forall K \in \mathcal{C}_{H}(E)\right) K \subseteq K^{\prime}\right) \Rightarrow K_{\text {sup }} \subseteq K^{\prime}\right)$

Let $K^{\prime} \in \operatorname{Pwr}\left(\Sigma_{I H}^{*}\right)$

Assume $\left(\forall K \in \mathcal{C}_{H}(E)\right) K \subseteq K^{\prime}$

Must show this implies $K_{\text {sup }} \subseteq K^{\prime}$

Let $s \in K_{\text {sup }}$ 


\section{Master Thesis - R. Song - McMaster - Computing and Software}

Must show implies $s \in K^{\prime}$

By (4) and (6), we know $\left(\exists K \in \mathcal{C}_{H}(E)\right) s \in K$

$\Rightarrow s \in K^{\prime}, \quad$ by $(5)$

4. Show that $\sup \mathcal{C}_{H}(E) \in \mathcal{C}_{H}(E)$.

This immediately follows from Part 3: $\sup \mathcal{C}_{H}(E)=\cup\left\{K \mid K \in \mathcal{C}_{H}(E)\right\}$.

From Proposition 4.1, we have $\sup \mathcal{C}_{H}(E) \subseteq E$, which means that we can compute $\sup \mathcal{C}_{H}(E)$ by removing strings from $E$.

The following lemma will be used later.

Lemma 4.1. For system $\boldsymbol{\Phi}$, let $L \subseteq \Sigma_{I H}^{*}$. If $L$ is closed then $\sup \mathcal{C}_{H}(L)$ is also closed.

\section{proof:}

Assume $L=\bar{L}$. Must show this implies $\sup \mathcal{C}_{H}(L)=\overline{\sup \mathcal{C}_{H}(L)}$.

$\sup \mathcal{C}_{H}(L) \subseteq \overline{\sup \mathcal{C}_{H}(L)}$ is automatic. We now show $\overline{\sup \mathcal{C}_{H}(L)} \subseteq \sup \mathcal{C}_{H}(L)$.

Sufficient to show that $\overline{\sup \mathcal{C}_{H}(L)} \subseteq L$ and $\overline{\sup \mathcal{C}_{H}(L)}$ is high-level interface controllable with respect to system $\boldsymbol{\Phi}$.

$\overline{\sup \mathcal{C}_{H}(L)}$ is high-level interface controllable with respect to system $\boldsymbol{\Phi}$ automatically by the definition of high-level interface controllable language.

By Proposition 4.1, we know that $\sup \mathcal{C}_{H}(L) \subseteq L$.

$\Rightarrow \overline{\sup \mathcal{C}_{H}(L)} \subseteq \bar{L}$.

$\Rightarrow \overline{\sup \mathcal{C}_{H}(L)} \subseteq L, \quad$ as $L$ is closed. 
Master Thesis - R. Song - McMaster - Computing and Software

\subsection{2 $\sup \mathcal{C}_{H}\left(L_{m}\left(\mathcal{G}_{H}\right)\right)$ and the Greatest Fixpoint of $\Omega_{H}$}

For system $\boldsymbol{\Phi}$, if we compute the supremal high-level interface controllable sublanguage of $L_{m}\left(\mathcal{G}_{H}\right)$, then a DES representing this sublanguage is a maximally permissive proper high-level supervisor. Therefore, we have to find a method to compute $\sup \mathcal{C}_{H}\left(L_{m}\left(\mathcal{G}_{H}\right)\right)$. To this objective, we define the following function.

Definition 4.3. For system $\Phi$, define the function $\Omega_{H}: \operatorname{Pwr}\left(\Sigma_{I H}^{*}\right) \rightarrow \operatorname{Pwr}\left(\Sigma_{I H}^{*}\right)$ according to

$$
\left(\forall K \in \operatorname{Pwr}\left(\Sigma_{I H}^{*}\right)\right) \Omega_{H}(K)=\Omega_{H N B}(\mathrm{HIC}(K))
$$

where $\Omega_{H N B}$ and HC are functions for system $\boldsymbol{\Phi}$, which are defined as following:

- $\Omega_{H N B}: \operatorname{Pwr}\left(\Sigma_{I H}^{*}\right) \rightarrow \operatorname{Pwr}\left(\Sigma_{I H}^{*}\right)$

$$
\left(\forall K \in \operatorname{Pwr}\left(\Sigma_{I H}^{*}\right)\right) \Omega_{H N B}(K)=L_{m}\left(\mathcal{G}_{H}\right) \cap K
$$

- $\mathrm{HIC}: \operatorname{Pwr}\left(\Sigma_{I H}^{*}\right) \rightarrow \operatorname{Pwr}\left(\Sigma_{I H}^{*}\right)$

$$
\left(\forall K \in \operatorname{Pwr}\left(\Sigma_{I H}^{*}\right)\right) \quad \operatorname{HC}(K)=\sup \mathcal{C}_{H}(\bar{K}) .
$$

Since the supremal element of $\sup \mathcal{C}_{H}(\bar{K})$ is unique and must exist by Proposition 4.1, the function HIC is well-defined. The function $\Omega_{H N B}$ and HIC both are defined on $\operatorname{Pwr}\left(\sum_{I H}^{*}\right)$, so we can composite them and the function $\Omega_{H}$ is well-defined.

Clearly, function $\Omega_{H N B}$ is monotone with respect to $\subseteq$, and HIC is also monotone with respect to $\subseteq$ by Proposition 4.1, so the function $\Omega_{H}$ is monotone with respect to $\subseteq$ as well.

By the Knaster-Tarski Theorem(Theorem 2.1), the greatest fixpoint of the function $\Omega_{H}$ with respect to $\left(\operatorname{Pwr}\left(\Sigma_{I H}^{*}\right), \subseteq\right)$ must exist. 


\section{Master Thesis - R. Song-McMaster-Computing and Software}

Proposition 4.2. For system $\boldsymbol{\Phi}, \sup \mathcal{C}_{H}\left(L_{m}\left(\mathcal{G}_{H}\right)\right)$ is the greatest fixpoint of the function $\Omega_{H}$ with respect to $\left(\operatorname{Pwr}\left(\Sigma_{I H}^{*}\right), \subseteq\right)$.

\section{proof:}

By the definition of $\Omega_{H}$, we can rewrite it as

$$
\left(\forall K \in \operatorname{Pwr}\left(\Sigma_{I H}^{*}\right)\right) \Omega_{H}(K)=L_{m}\left(\mathcal{G}_{H}\right) \cap \sup \mathcal{C}_{H}(\bar{K})
$$

Let $S=\sup \mathcal{C}_{H}\left(L_{m}\left(\mathcal{G}_{H}\right)\right)$.

1. Show that $S$ is a fixpoint of $\Omega_{H}$, i.e. $S=\Omega_{H}(S)$.

Sufficient to show that $S \subseteq \Omega_{H}(S)$ and $\Omega_{H}(S) \subseteq S$.

$\Omega_{H}(S)=L_{m}\left(\mathcal{G}_{H}\right) \cap \sup \mathcal{C}_{H}(\bar{S})$

As $S$ is high-level interface controllable with respect to system $\boldsymbol{\Phi}, \bar{S}$ is high-level interface controllable as well. Then,

$\Omega_{H}(S)=L_{m}\left(\mathcal{G}_{H}\right) \cap \bar{S}$

By Proposition 4.1, $S \subseteq L_{m}\left(\mathcal{G}_{H}\right)$. Also $S \subseteq \bar{S}$, so we have $S \subseteq \Omega_{H}(S)$.

Now we show that $\Omega_{H}(S) \subseteq S$.

Let $t \in \Omega_{H}(S)$.

Must show this implies $t \in S$.

By (3), we have $t \in \Omega_{H}(S)$

$\Rightarrow t \in L_{m}\left(\mathcal{G}_{H}\right)$ and $t \in \bar{S}, \quad$ by (1)

$\Rightarrow\{t\} \subseteq L_{m}\left(\mathcal{G}_{H}\right)$ and $\{t\} \subseteq \bar{S}$

$\Rightarrow\{t\} \subseteq L_{m}\left(\mathcal{G}_{H}\right)$ and $\overline{\{t\}} \subseteq \bar{S}$

Let $S^{\prime}=S \cup\{t\}$. 
Master Thesis - R. Song - McMaster - Computing and Software

$\Rightarrow S^{\prime} \subseteq L_{m}\left(\mathcal{G}_{H}\right)$,

by (4) and $S \subseteq L_{m}\left(\mathcal{G}_{H}\right)$

We now show that $S^{\prime}$ is high-level interface controllable with respect to system $\Phi$.

It is enough to show that

$\overline{S^{\prime}} \Sigma_{h u} \cap L\left(\mathbf{G}_{H}^{p} \times \mathbf{G}_{I}^{h}\right) \subseteq \overline{S^{\prime}}$ and $(\forall j \in\{1, \ldots, n\}) \overline{S^{\prime}} \Sigma_{A_{j}} \cap L\left(\mathbf{G}_{I_{j}}^{h}\right) \subseteq \overline{S^{\prime}}$

- Show that $\overline{S^{\prime}} \Sigma_{h u} \cap L\left(\mathbf{G}_{H}^{p} \times \mathbf{G}_{I}^{h}\right) \subseteq \overline{S^{\prime}}$

$S$ is high-level interface controllable, so $\bar{S} \Sigma_{h u} \cap L\left(\mathbf{G}_{H}^{p} \times \mathbf{G}_{I}^{h}\right) \subseteq \bar{S}$.

By (4) and (6), we have $\overline{\{t\}} \Sigma_{h u} \cap L\left(\mathbf{G}_{H}^{p} \times \mathbf{G}_{I}^{h}\right) \subseteq \bar{S}$

From (6) and (7), we have $(\bar{S} \cup \overline{\{t\}}) \Sigma_{h u} \cap L\left(\mathbf{G}_{H}^{p} \times \mathbf{G}_{I}^{h}\right) \subseteq \bar{S}$

$\Rightarrow(\overline{S \cup\{t\}}) \Sigma_{h u} \cap L\left(\mathbf{G}_{H}^{p} \times \mathbf{G}_{I}^{h}\right) \subseteq \bar{S}, \quad$ by Proposition 2.2

$\Rightarrow \overline{S^{\prime}} \Sigma_{h u} \cap L\left(\mathbf{G}_{H}^{p} \times \mathbf{G}_{I}^{h}\right) \subseteq \bar{S}$

$\Rightarrow \overline{S^{\prime}} \Sigma_{h u} \cap L\left(\mathbf{G}_{H}^{p} \times \mathbf{G}_{I}^{h}\right) \subseteq \overline{S^{\prime}}$,

as $S \subseteq S^{\prime}$ and thus $\bar{S} \subseteq \overline{S^{\prime}}$

- Show that $(\forall j \in\{1, \ldots, n\}) \overline{S^{\prime}} \Sigma_{A_{j}} \cap L\left(\mathbf{G}_{I_{j}}^{h}\right) \subseteq \overline{S^{\prime}}$.

Let $j \in\{1, \ldots, n\}$. We show $\overline{S^{\prime}} \Sigma_{A_{j}} \cap L\left(\mathbf{G}_{I_{j}}^{h}\right) \subseteq \overline{S^{\prime}}$.

$S$ is high-level interface controllable, so $\bar{S} \Sigma_{A_{j}} \cap L\left(\mathbf{G}_{I_{j}}^{h}\right) \subseteq \bar{S}$.

By (4) and (9), we have $\overline{\{t\}} \Sigma_{A_{j}} \cap L\left(\mathbf{G}_{I_{j}}^{h}\right) \subseteq \bar{S}$

From (9) and (10), we have $(\bar{S} \cup \overline{\{t\}}) \Sigma_{A_{j}} \cap L\left(\mathbf{G}_{I_{j}}^{h}\right) \subseteq \bar{S}$

$\Rightarrow(\overline{S \cup\{t\}}) \Sigma_{A_{j}} \cap L\left(\mathbf{G}_{I_{j}}^{h}\right) \subseteq \bar{S}, \quad$ by Proposition 2.2

$\Rightarrow \overline{S^{\prime}} \Sigma_{A_{j}} \cap L\left(\mathbf{G}_{I_{j}}^{h}\right) \subseteq \bar{S}$

$\Rightarrow \overline{S^{\prime}} \Sigma_{A_{j}} \cap L\left(\mathbf{G}_{I_{j}}^{h}\right) \subseteq \overline{S^{\prime}}$, as $S \subseteq S^{\prime}$ and thus $\bar{S} \subseteq \overline{S^{\prime}}$ 


\section{Master Thesis - R. Song - McMaster - Computing and Software}

By (5), (8), (11), we have $S^{\prime} \in \mathcal{C}_{H}\left(L_{m}\left(\mathcal{G}_{H}\right)\right)$. As $S$ is the supremal high-level interface controllable sublanguage of $L_{m}\left(\mathcal{G}_{H}\right)$, we get $S^{\prime} \subseteq S$.

$\Rightarrow\{t\} \subseteq S$

$\Rightarrow t \in S$

We thus have $\Omega_{H}(S) \subseteq S$. Combining it with (2) gives $\Omega_{H}(S)=S$.

2. Show that $S$ is the greatest fixpoint of $\Omega_{H}$. i.e. $\left(\forall T \in P w r\left(\Sigma_{I H}^{*}\right)\right) T=\Omega_{H}(T) \Rightarrow$ $T \subseteq S$.

Let $T \in P w r\left(\Sigma_{I H}^{*}\right)$. Assume $T=\Omega_{H}(T)$. Must show this implies $T \subseteq S$.

Sufficient to show that $T$ is high-level controllable with respect to system $\boldsymbol{\Phi}$ and $T \subseteq L_{m}\left(\mathcal{G}_{H}\right)$.

$$
\begin{aligned}
T & =\Omega_{H}(T) \\
& =L_{m}\left(\mathcal{G}_{H}\right) \cap \sup \mathcal{C}_{H}(\bar{T}) \\
& \subseteq \sup \mathcal{C}_{H}(\bar{T})
\end{aligned}
$$

By Lemma 4.1, $\sup \mathcal{C}_{H}(\bar{T})$ is closed, so combining with (12), we have

$$
\bar{T} \subseteq \sup \mathcal{C}_{H}(\bar{T})
$$

By Proposition 4.1, we know that $\sup \mathcal{C}_{H}(\bar{T}) \subseteq \bar{T}$. Therefore, we have

$$
\bar{T}=\sup \mathcal{C}_{H}(\bar{T})
$$

So, $\bar{T}$ is high-level controllable with respect to system $\boldsymbol{\Phi}$, which means that $T$ is high-level controllable with respect to system $\boldsymbol{\Phi}$.

From (12), clearly, $T \subseteq L_{m}\left(\mathcal{G}_{H}\right)$. 
Master Thesis - R. Song - McMaster - Computing and Software

\subsubsection{Computing the greatest fixpoint of $\Omega_{H}$}

From the above section, we know that we can compute the $\sup \mathcal{C}_{H}\left(L_{m}\left(\mathcal{G}_{H}\right)\right)$ by computing the greatest fixpoint of $\Omega_{H}$. It is tempting to compute the greatest fixpoint by iteration of $\Omega_{H}$.

Proposition 4.3. For system $\boldsymbol{\Phi}$, the set theoretic limit $\lim _{i \rightarrow \infty} \Omega_{H}^{i}\left(L\left(\mathcal{G}_{H}\right)\right), i \in$ $\{1,2, \ldots\}$ exists, and $\sup \mathcal{C}_{H}\left(L_{m}\left(\mathcal{G}_{H}\right)\right) \subseteq \lim _{i \rightarrow \infty} \Omega_{H}^{i}\left(L\left(\mathcal{G}_{H}\right)\right)$.

proof:

Let $S:=\sup \mathcal{C}_{H}\left(L\left(\mathcal{G}_{H}\right)\right)$.

1. Show that $\lim _{i \rightarrow \infty} \Omega_{H}^{i}\left(L\left(\mathcal{G}_{H}\right)\right)$ exists.

From the definition of $\Omega_{H}$, we know $\Omega_{H}\left(L\left(\mathcal{G}_{H}\right)\right)=L_{m}\left(\mathcal{G}_{H}\right) \cap \sup \mathcal{C}_{H}\left(L\left(\mathcal{G}_{H}\right)\right)$, so by $L_{m}\left(\mathcal{G}_{H}\right) \subseteq L\left(\mathcal{G}_{H}\right)$ and $\sup \mathcal{C}_{H}\left(L\left(\mathcal{G}_{H}\right)\right) \subseteq L\left(\mathcal{G}_{H}\right)$ by Proposition 4.1, we have

$\Omega_{H}\left(L\left(\mathcal{G}_{H}\right)\right) \subseteq L\left(\mathcal{G}_{H}\right)$

$\Rightarrow \Omega_{H}^{2}\left(L\left(\mathcal{G}_{H}\right)\right) \subseteq \Omega_{H}\left(L\left(\mathcal{G}_{H}\right)\right), \quad$ as $\Omega_{H}$ is monotone.

$\Rightarrow \Omega_{H}^{3}\left(L\left(\mathcal{G}_{H}\right)\right) \subseteq \Omega_{H}^{2}\left(L\left(\mathcal{G}_{H}\right)\right), \quad$ as $\Omega_{H}$ is monotone.

Then, we have

$$
L\left(\mathcal{G}_{H}\right) \supseteq \Omega_{H}\left(L\left(\mathcal{G}_{H}\right)\right) \supseteq \Omega_{H}^{2}\left(L\left(\mathcal{G}_{H}\right)\right) \supseteq \cdots
$$

Therefore, $\lim _{i \rightarrow \infty} \Omega_{H}^{i}\left(L\left(\mathcal{G}_{H}\right)\right)$ exists and equals to $\bigcap_{i=0}^{\infty} \Omega_{H}^{i}\left(L\left(\mathcal{G}_{H}\right)\right)$.

2. Show that $S \subseteq \lim _{i \rightarrow \infty} \Omega_{H}^{i}\left(L\left(\mathcal{G}_{H}\right)\right)$.

By Proposition 4.1, we have $S \subseteq L\left(\mathcal{G}_{H}\right)$ 
Master Thesis - R. Song - McMaster - Computing and Software

$$
\begin{aligned}
& \Rightarrow \Omega_{H}(S) \subseteq \Omega_{H}\left(L\left(\mathcal{G}_{H}\right)\right), \quad \text { as } \Omega_{H} \text { is monotone. } \\
& \Rightarrow S \subseteq \Omega_{H}\left(L\left(\mathcal{G}_{H}\right)\right), \quad \text { as } S=\Omega_{H}(S) \text { by Proposition 4.2. } \\
& \Rightarrow \Omega_{H}(S) \subseteq \Omega_{H}^{2}\left(L\left(\mathcal{G}_{H}\right)\right), \quad \text { as } \Omega_{H} \text { is monotone. } \\
& \Rightarrow S \subseteq \Omega_{H}^{2}\left(L\left(\mathcal{G}_{H}\right)\right), \quad \text { as } S=\Omega_{H}(S) \text { by Proposition } 4.2 . \\
& \ldots \\
& \Rightarrow S \subseteq \lim _{i \rightarrow \infty} \Omega_{H}^{i}\left(L\left(\mathcal{G}_{H}\right)\right) .
\end{aligned}
$$

Note that we start the iteration from the closed language $L\left(\mathcal{G}_{H}\right)$. It remains to show the reverse $\lim _{i \rightarrow \infty} \Omega_{H}^{i}\left(L\left(\mathcal{G}_{H}\right)\right) \subseteq \sup \mathcal{C}_{H}\left(L_{m}\left(\mathcal{G}_{H}\right)\right)$. For practical computation, we also have to show that the limit will be reached after a finite number of iterations on $\Omega_{H}$. In general case, actually $\lim _{i \rightarrow \infty} \Omega_{H}^{i}\left(L\left(\mathcal{G}_{H}\right)\right) \nsubseteq \sup \mathcal{C}_{H}\left(L_{m}\left(\mathcal{G}_{H}\right)\right)$. Please see [48] for details. However, in the case that all the languages are regular, the reverse holds and the number of iterations on $\Omega_{H}$ is finite, as we will show in the following sections.

To iterate the computation on the function $\Omega_{H}$, we first must know how to compute the function HIC, i.e. to compute the supremal high-level interface controllable sublanguage of a closed language. Here, we only focus on the special class of closed languages. Let $\operatorname{Pred}\left(Q_{H}\right)$ be the set of all predicates on $Q_{H}$, the state set of $\mathcal{G}_{H}$. Let $\mathcal{P}_{h a} \in \operatorname{Pred}\left(Q_{H}\right)$ be a predicate ${ }^{2}$, we now show how to compute $\sup \mathcal{C}_{H}\left(L\left(\mathcal{G}_{H}, \mathcal{P}_{h a}\right)\right)$. Note that $L\left(\mathcal{G}_{H}\right)=L\left(\mathcal{G}_{H}\right.$, true $)$, so at the starting point, we let $\mathcal{P}_{h a}=$ true and then compute $\sup \mathcal{C}_{H}\left(L\left(\mathcal{G}_{H}, \mathcal{P}_{h a}\right)\right)$.

We compute $\sup \mathcal{C}_{H}\left(L\left(\mathcal{G}_{H}, \mathcal{P}_{h a}\right)\right)$ by creating another function, and we will prove that $\sup \mathcal{C}_{H}\left(L\left(\mathcal{G}_{H}, \mathcal{P}_{h a}\right)\right)$ is the greatest fixpoint of that function.

\footnotetext{
${ }^{2} \mathcal{P}_{h a}$ is just an arbitrary predicate. We use this notation as we need to distinguish a given predicate $\left(\mathcal{P}_{h a}\right)$ with an arbitrary predicate $P$ in the next subsection.
} 
Master Thesis - R. Song - McMaster - Computing and Software

\subsubsection{Computing $\sup \mathcal{C}_{H}\left(L\left(\mathcal{G}_{H}, \mathcal{P}_{h a}\right)\right)$}

Definition 4.4. For system $\boldsymbol{\Phi}$, let $\mathcal{P}_{h a} \in \operatorname{Pred}\left(Q_{H}\right)$ be a given predicate. Define the function $\Omega_{H I C}: \operatorname{Pwr}\left(L\left(\mathcal{G}_{H}, \mathcal{P}_{h a}\right)\right) \rightarrow \operatorname{Pwr}\left(L\left(\mathcal{G}_{H}, \mathcal{P}_{h a}\right)\right)$ according to

$$
\begin{aligned}
& \left(\forall K \in \operatorname{Pwr}\left(L\left(\mathcal{G}_{H}, \mathcal{P}_{h a}\right)\right)\right) \\
& \Omega_{H I C}(K):=\left\{s \in K \mid \overline{\{s\}} \Sigma_{h u} \cap L\left(\mathbf{G}_{H}^{p} \times \mathbf{G}_{I}^{h}\right) \subseteq \bar{K} \quad \&\right. \\
& \left.\quad\left((\forall j \in\{1, \ldots, n\}) \overline{\{s\}} \Sigma_{A_{j}} \cap L\left(\mathbf{G}_{I_{j}}^{h}\right) \subseteq \bar{K}\right)\right\}
\end{aligned}
$$

Clearly $\Omega_{H I C}$ is monotone. Note that $\Omega_{H I C}(K) \subseteq K \subseteq L\left(\mathcal{G}_{H}, \mathcal{P}_{h a}\right)$, so this function is well-defined, and it only removes strings from $K$.

To make it easier to understand and use this function, the definition can be rewritten as

$$
\begin{gathered}
\left(\forall K \in \operatorname{Pwr}\left(L\left(\mathcal{G}_{H}, \mathcal{P}_{h a}\right)\right)\right) \\
\Omega_{H I C}(K):=\{s \in K \mid(\forall t \leq s) \\
\left(\left(\forall \sigma_{u} \in \Sigma_{h u}\right) t \sigma_{u} \in L\left(\mathbf{G}_{H}^{p} \times \mathbf{G}_{I}^{h}\right) \Rightarrow t \sigma_{u} \in \bar{K}\right) \& \\
\left(\left(\forall \sigma_{a_{1}} \in \Sigma_{A_{1}}\right) t \sigma_{a_{1}} \in L\left(\mathbf{G}_{I_{1}}^{h}\right) \Rightarrow t \sigma_{a_{1}} \in \bar{K}\right) \& \\
\ldots \\
\left.\left(\left(\forall \sigma_{a_{n}} \in \Sigma_{A_{n}}\right) t \sigma_{a_{n}} \in L\left(\mathbf{G}_{I_{n}}^{h}\right) \Rightarrow t \sigma_{a_{n}} \in \bar{K}\right)\right\} .
\end{gathered}
$$

Proposition 4.4. For system $\boldsymbol{\Phi}$, let $\mathcal{P}_{h a} \in \operatorname{Pred}\left(Q_{H}\right)$ be the given predicate for function $\Omega_{H I C}$. Then $\sup \mathcal{C}_{H}\left(L\left(\mathcal{G}_{H}, \mathcal{P}_{h a}\right)\right)$ is the greatest fixpoint of $\Omega_{H I C}$ with respect to $\left(\operatorname{Pwr}\left(L\left(\boldsymbol{\mathcal { G }}_{H}, \mathcal{P}_{h a}\right)\right), \subseteq\right)$.

\section{proof:}

Let $S=\sup \mathcal{C}_{H}\left(L\left(\mathcal{G}_{H}, \mathcal{P}_{h a}\right)\right)$. By Proposition 4.1, $S \subseteq L\left(\mathcal{G}_{H}, \mathcal{P}_{h a}\right), S$ is a valid input for $\Omega_{H I C}$. 


\section{Master Thesis - R. Song - McMaster - Computing and Software}

1. Show that $S=\Omega_{H I C}(S)$.

$$
\begin{aligned}
\Omega_{H I C}(S)=\left\{s \in S \mid \overline{\{s\}} \Sigma_{h u} \cap L\left(\mathbf{G}_{H}^{p} \times \mathbf{G}_{I}^{h}\right) \subseteq \bar{S} \&\right. \\
\left.\left((\forall j \in\{1, \ldots, n\}) \overline{\{s\}} \Sigma_{A_{j}} \cap L\left(\mathbf{G}_{I_{j}}^{h}\right) \subseteq \bar{S}\right)\right\}
\end{aligned}
$$

By the definition of $S$, clearly, all the strings in $S$ must satisfy the condition, so $\Omega_{H I C}(S)=S$.

2. Show that $S$ is the greatest fixpoint of $\Omega_{H I C}$, i.e. $\left(\forall T \in \operatorname{Pwr}\left(L\left(\mathcal{G}_{H}, \mathcal{P}_{h a}\right)\right)\right) T=\Omega_{H I C}(T) \Rightarrow T \subseteq S$.

Let $T \in \operatorname{Pwr}\left(L\left(\mathcal{G}_{H}, \mathcal{P}_{h a}\right)\right)$. Assume $T=\Omega_{H I C}(T)$.

Must show this implies $T \subseteq S$.

Sufficient to show that $T$ is high-level interface controllable with respect to system $\boldsymbol{\Phi}$.

$$
\begin{aligned}
\Omega_{H I C}(T)=\left\{s \in T \mid \overline{\{s\}} \Sigma_{h u} \cap L\left(\mathbf{G}_{H}^{p} \times \mathbf{G}_{I}^{h}\right) \subseteq \bar{T} \&\right. \\
\left.\left((\forall j \in\{1, \ldots, n\}) \overline{\{s\}} \Sigma_{A_{j}} \cap L\left(\mathbf{G}_{I_{j}}^{h}\right) \subseteq \bar{T}\right)\right\}
\end{aligned}
$$

So,

$$
\begin{aligned}
T=\left\{s \in T \mid \overline{\{s\}} \Sigma_{h u} \cap L\left(\mathbf{G}_{H}^{p} \times \mathbf{G}_{I}^{h}\right) \subseteq \bar{T} \&\right. \\
\left.\left((\forall j \in\{1, \ldots, n\}) \overline{\{s\}} \Sigma_{A_{j}} \cap L\left(\mathbf{G}_{I_{j}}^{h}\right) \subseteq \bar{T}\right)\right\}
\end{aligned}
$$

Then, we have

$\bar{T} \Sigma_{h u} \cap L\left(\mathbf{G}_{H}^{p} \times \mathbf{G}_{I}^{h}\right) \subseteq \bar{T} \quad \& \quad\left((\forall j \in\{1, \ldots, n\}) \bar{T} \Sigma_{A_{j}} \cap L\left(\mathbf{G}_{I_{j}}^{h}\right) \subseteq \bar{T}\right)$,

which means $T$ is high-level interface controllable with respect to system $\mathbf{\Phi}$.

From Proposition 4.4, we know that we can compute the $\sup \mathcal{C}_{H}\left(L\left(\mathcal{G}_{H}, \mathcal{P}_{h a}\right)\right)$ by computing the greatest fixpoint of $\Omega_{H I C}$. It is also tempting to compute the greatest fixpoint by iteration of $\Omega_{H I C}$. 
Master Thesis - R. Song - McMaster - Computing and Software

Proposition 4.5. For system $\boldsymbol{\Phi}$, let $\mathcal{P}_{h a} \in \operatorname{Pred}\left(Q_{H}\right)$ be the given predicate for the function $\Omega_{H I C}$. The set theoretic limit $\lim _{i \rightarrow \infty} \Omega_{H I C}^{i}\left(L\left(\mathcal{G}_{H}, \mathcal{P}_{h a}\right)\right), i \in\{1,2, \ldots\}$ exists, and $\sup \mathcal{C}_{H}\left(L\left(\mathcal{G}_{H}, \mathcal{P}_{h a}\right)\right) \subseteq \lim _{i \rightarrow \infty} \Omega_{H I C}^{i}\left(L\left(\mathcal{G}_{H}, \mathcal{P}_{h a}\right)\right)$

\section{proof:}

Let $S:=\sup \mathcal{C}_{H}\left(L\left(\mathcal{G}_{H}, \mathcal{P}_{h a}\right)\right)$.

1. Show that $\lim _{i \rightarrow \infty} \Omega_{H I C}^{i}\left(L\left(\mathcal{G}_{H}, \mathcal{P}_{h a}\right)\right)$ exists.

From the definition of $\Omega_{H I C}$, we have $\Omega_{H I C}\left(L\left(\mathcal{G}_{H}, \mathcal{P}_{h a}\right)\right) \subseteq L\left(\mathcal{G}_{H}, \mathcal{P}_{h a}\right)$

$\Rightarrow \Omega_{H I C}^{2}\left(L\left(\mathcal{G}_{H}, \mathcal{P}_{h a}\right)\right) \subseteq \Omega_{H I C}\left(L\left(\mathcal{G}_{H}, \mathcal{P}_{h a}\right)\right), \quad$ as $\Omega_{H I C}$ is monotone.

$\Rightarrow \Omega_{H I C}^{3}\left(L\left(\mathcal{G}_{H}, \mathcal{P}_{h a}\right)\right) \subseteq \Omega_{H I C}^{2}\left(L\left(\mathcal{G}_{H}, \mathcal{P}_{h a}\right)\right), \quad$ as $\Omega_{H I C}$ is monotone.

$\cdots$

Then, we have

$$
L\left(\mathcal{G}_{H}, \mathcal{P}_{h a}\right) \supseteq \Omega_{H I C}\left(L\left(\mathcal{G}_{H}, \mathcal{P}_{h a}\right)\right) \supseteq \Omega_{H I C}^{2}\left(L\left(\mathcal{G}_{H}, \mathcal{P}_{h a}\right)\right) \supseteq \cdots
$$

So, $\lim _{i \rightarrow \infty} \Omega_{H I C}^{i}\left(L\left(\mathcal{G}_{H}, \mathcal{P}_{h a}\right)\right)$ exists and equals to $\bigcap_{i=0}^{\infty} \Omega_{H I C}^{i}\left(L\left(\mathcal{G}_{H}, \mathcal{P}_{h a}\right)\right)$.

2. Show that $S \subseteq \lim _{i \rightarrow \infty} \Omega_{H I C}^{i}\left(L\left(\mathcal{G}_{H}, \mathcal{P}_{h a}\right)\right)$.

By Proposition 4.1 , we have $S \subseteq L\left(\mathcal{G}_{H}, \mathcal{P}_{h a}\right)$

$\Rightarrow \Omega_{H I C}(S) \subseteq \Omega_{H I C}\left(L\left(\mathcal{G}_{H}, \mathcal{P}_{h a}\right)\right), \quad$ as $\Omega_{H I C}$ is monotone.

$\Rightarrow S \subseteq \Omega_{H I C}\left(L\left(\mathcal{G}_{H}, \mathcal{P}_{h a}\right)\right), \quad$ as $S=\Omega_{H I C}(S)$ by Proposition 4.4.

$\Rightarrow \Omega_{H I C}(S) \subseteq \Omega_{H I C}^{2}\left(L\left(\mathcal{G}_{H}, \mathcal{P}_{h a}\right)\right), \quad$ as $\Omega_{H I C}$ is monotone.

$\Rightarrow S \subseteq \Omega_{H I C}^{2}\left(L\left(\mathcal{G}_{H}, \mathcal{P}_{h a}\right)\right), \quad$ as $S=\Omega_{H I C}(S)$ by Proposition 4.4.

$\cdots$

$\Rightarrow S \subseteq \lim _{i \rightarrow \infty} \Omega_{H I C}^{i}\left(L\left(\mathcal{G}_{H}, \mathcal{P}_{h a}\right)\right)$. 


\section{Master Thesis - R. Song - McMaster - Computing and Software}

It remains to show that $\lim _{i \rightarrow \infty} \Omega_{H I C}^{i}\left(L\left(\mathcal{G}_{H}, \mathcal{P}_{h a}\right)\right) \subseteq \sup \mathcal{C}_{H}\left(L\left(\mathcal{G}_{H}, \mathcal{P}_{h a}\right)\right)$ and that we only need to iterate a finite number of iterations in the case that $\mathcal{G}_{H}$ only has finite number of states.

Definition 4.5. For system $\boldsymbol{\Phi}$, define the function $W_{H}: \operatorname{Pred}\left(Q_{H}\right) \rightarrow \operatorname{Pwr}\left(\Sigma_{I H}^{*}\right)$ according to

$$
\begin{aligned}
& \left(\forall P \in \operatorname{Pred}\left(Q_{H}\right)\right) \\
& W_{H}(P):=\left\{s \in L\left(\mathcal{G}_{H}, P\right) \mid\left(\left(\exists \sigma_{u} \in \Sigma_{h u}\right) s \sigma_{u} \in L\left(\mathbf{G}_{H}^{p} \times \mathbf{G}_{I}^{h}\right) \& s \sigma_{u} \notin L\left(\mathcal{G}_{H}, P\right)\right)\right. \text { or } \\
& \left.\quad\left((\exists j \in\{1, \ldots, n\})\left(\exists \sigma_{a_{j}} \in \Sigma_{A_{j}}\right) s \sigma_{a_{j}} \in L\left(\mathbf{G}_{I_{j}}^{h}\right) \& s \sigma_{a_{j}} \notin L\left(\mathcal{G}_{H}, P\right)\right)\right\}
\end{aligned}
$$

Note that $W_{H}(P) \subseteq L\left(\mathcal{G}_{H}, P\right)$. The following two lemmas will be used in the proof of the next proposition.

Lemma 4.2. For system $\boldsymbol{\Phi}$, the following holds:

$$
\begin{aligned}
& \left(\forall P \in \operatorname{Pred}\left(Q_{H}\right)\right)\left(\forall s, t \in L\left(\mathcal{G}_{H}, P\right)\right) \\
& \quad s \in W_{H}(P) \&\left(\delta_{H}\left(q_{H_{0}}, s\right)=\delta_{H}\left(q_{H_{0}}, t\right)\right) \Rightarrow t \in W_{H}(P),
\end{aligned}
$$

where $q_{H_{0}}$ is the initial state of $\mathcal{G}_{H}$ as defined in section 4.1.

\section{proof:}

Let $P \in \operatorname{Pred}\left(Q_{H}\right)$, and $s, t \in L\left(\mathcal{G}_{H}, P\right)$.

Assume $\delta_{H}\left(q_{H_{0}}, s\right)=\delta_{H}\left(q_{H_{0}}, t\right)$ and $s \in W_{H}(P)$.

Must show this implies $t \in W_{H}(P)$.

Let $\equiv_{L\left(\mathcal{G}_{H}, P\right)}, \equiv_{L\left(\mathbf{G}_{H}^{p} \times \mathbf{G}_{I}^{h}\right)}, \equiv_{L\left(\mathbf{G}_{I_{j}}^{h}\right)}(j \in\{1, \ldots, n\})$ be the Nerode equivalence relation on $\Sigma_{I H}^{*}$ with respect to $L\left(\mathcal{G}_{H}, P\right), L\left(\mathbf{G}_{H}^{p} \times \mathbf{G}_{I}^{h}\right)$, and $L\left(\mathbf{G}_{I_{j}}^{h}\right)$ respectively.

From (2), we have $\delta_{H}\left(q_{H_{0}}, s\right)=\delta_{H}\left(q_{H_{0}}, t\right)$

By Proposition 2.6, we thus have $s \equiv_{L\left(\mathcal{G}_{H}, P\right)} t$ 
Master Thesis - R. Song - McMaster - Computing and Software

As $\mathcal{G}_{H}=\mathbf{E}_{H} \times \mathbf{G}_{H}^{p} \times \mathbf{G}_{I_{1}} \times \cdots \times \mathbf{G}_{I_{n}}$, we can conclude $\eta_{H} \times \xi^{h}\left(\left(y_{H_{0}}, x_{0}^{h}\right), s\right)=$ $\eta_{H} \times \xi^{h}\left(\left(y_{H_{0}}, x_{0}^{h}\right), t\right)$ and $(\forall j \in\{1, \ldots, n\}) \xi_{j}^{h}\left(x_{j_{0}}^{h}, s\right)=\xi_{j}^{h}\left(x_{j_{0}}^{h}, t\right)$.

By Proposition 2.6 and the fact $L\left(\mathbf{G}_{H}^{p} \times \mathbf{G}_{I}^{h}, \operatorname{true}\right)=L\left(\mathbf{G}_{H}^{p} \times \mathbf{G}_{I}^{h}\right)$ and $L\left(\mathbf{G}_{I_{j}}^{h}, \operatorname{true}\right)=$ $L\left(\mathbf{G}_{I_{j}}^{h}\right)$, we thus have $s \equiv_{L\left(\mathbf{G}_{H}^{p} \times \mathbf{G}_{I}^{h}\right)} t$ and $(\forall j \in\{1, \ldots, n\}) s \equiv_{L\left(\mathbf{G}_{I_{j}}^{h}\right)} t$

From (1), we have $s \in W_{H}(P)$

$$
\begin{aligned}
\Rightarrow & \left(\left(\exists \sigma_{u} \in \Sigma_{h u}\right) s \sigma_{u} \in L\left(\mathbf{G}_{H}^{p} \times \mathbf{G}_{I}^{h}\right) \& s \sigma_{u} \notin L\left(\mathcal{G}_{H}, P\right)\right) \text { or } \\
& \left((\exists j \in\{1, \ldots, n\})\left(\exists \sigma_{a_{j}} \in \Sigma_{A_{j}}\right) s \sigma_{a_{j}} \in L\left(\mathbf{G}_{I_{j}}^{h}\right) \& s \sigma_{a_{j}} \notin L\left(\mathcal{G}_{H}, P\right)\right) \\
\Rightarrow & \left(\left(\exists \sigma_{u} \in \Sigma_{h u}\right) t \sigma_{u} \in L\left(\mathbf{G}_{H}^{p} \times \mathbf{G}_{I}^{h}\right) \& t \sigma_{u} \notin L\left(\mathcal{G}_{H}, P\right)\right) \text { or } \\
& \left((\exists j \in\{1, \ldots, n\})\left(\exists \sigma_{a_{j}} \in \Sigma_{A_{j}}\right) t \sigma_{a_{j}} \in L\left(\mathbf{G}_{I_{j}}^{h}\right) \& t \sigma_{a_{j}} \notin L\left(\mathcal{G}_{H}, P\right)\right), \quad \text { by }(3)(4) \\
\Rightarrow & t \in W_{H}(P)
\end{aligned}
$$

Lemma 4.3. For system $\boldsymbol{\Phi}$, let $\mathcal{P}_{h a} \in \operatorname{Pred}\left(Q_{H}\right)$ be the given predicate for $\Omega_{H I C}$. Then the following holds:

$\left(\forall P \in S u b\left(\mathcal{P}_{h a}\right)\right)\left(\forall s \in L\left(\mathcal{G}_{H}, P\right)\right)$

$$
\left((\exists t \leq s) t \in W_{H}(P)\right) \Leftrightarrow s \notin \Omega_{H I C}\left(L\left(\mathcal{G}_{H}, P\right)\right)
$$

\section{proof:}

Let $P \in \operatorname{Sub}\left(\mathcal{P}_{h a}\right)$ and $s \in L\left(\mathcal{G}_{H}, P\right)$.

1. Show that $\left((\exists t \leq s) t \in W_{H}(P)\right) \Rightarrow s \notin \Omega_{H I C}\left(L\left(\mathcal{G}_{H}, P\right)\right)$

Assume $(\exists t \leq s) t \in W_{H}(P)$.

Must show this implies $s \notin \Omega_{H I C}\left(L\left(\mathcal{G}_{H}, P\right)\right)$.

From (2), we have

$$
\begin{aligned}
& (\exists t \leq s)\left(\left(\exists \sigma_{u} \in \Sigma_{h u}\right) t \sigma_{u} \in L\left(\mathbf{G}_{H}^{p} \times \mathbf{G}_{I}^{h}\right) \& t \sigma_{u} \notin L\left(\mathcal{G}_{H}, P\right)\right) \text { or } \\
& \left((\exists j \in\{1, \ldots, n\})\left(\exists \sigma_{a_{j}} \in \Sigma_{A_{j}}\right) t \sigma_{a_{j}} \in L\left(\mathbf{G}_{I_{j}}^{h}\right) \& t \sigma_{a_{j}} \notin L\left(\mathcal{G}_{H}, P\right)\right)
\end{aligned}
$$




$$
\begin{gathered}
\text { Master Thesis }-R \text {. Song - McMaster - Computing and Software } \\
\Rightarrow \neg\left((\forall t \leq s)\left(\left(\forall \sigma_{u} \in \Sigma_{h u}\right) t \sigma_{u} \in L\left(\mathbf{G}_{H}^{p} \times \mathbf{G}_{I}^{h}\right) \Rightarrow t \sigma_{u} \in L\left(\mathcal{G}_{H}, P\right)\right) \&\right. \\
\left(\left(\forall \sigma_{a_{1}} \in \Sigma_{A_{1}}\right) t \sigma_{a_{1}} \in L\left(\mathbf{G}_{I_{1}}^{h}\right) \Rightarrow t \sigma_{a_{1}} \in L\left(\mathcal{G}_{H}, P\right)\right) \& \\
\ldots \\
\left.\left(\left(\forall \sigma_{a_{n}} \in \Sigma_{A_{n}}\right) t \sigma_{a_{n}} \in L\left(\mathbf{G}_{I_{n}}^{h}\right) \Rightarrow t \sigma_{a_{n}} \in L\left(\mathcal{G}_{H}, P\right)\right)\right) \\
\Rightarrow s \notin \Omega_{H I C}\left(L\left(\mathcal{G}_{H}, P\right)\right), \quad \text { by definition of } \Omega_{H I C}
\end{gathered}
$$

2. Show that $s \notin \Omega_{H I C}\left(L\left(\mathcal{G}_{H}, P\right)\right) \Rightarrow(\exists t \leq s) t \in W_{H}(P)$.

Assume $s \notin \Omega_{H I C}\left(L\left(\mathcal{G}_{H}, P\right)\right)$.

Must show this implies $(\exists t \leq s) t \in W_{H}(P)$.

From (1), we know $s \in L\left(\mathcal{G}_{H}, P\right)$. From (3), we thus have

$$
\begin{gathered}
\Rightarrow \neg\left((\forall t \leq s)\left(\left(\forall \sigma_{u} \in \Sigma_{h u}\right) t \sigma_{u} \in L\left(\mathbf{G}_{H}^{p} \times \mathbf{G}_{I}^{h}\right) \Rightarrow t \sigma_{u} \in L\left(\mathcal{G}_{H}, P\right)\right) \&\right. \\
\left(\left(\forall \sigma_{a_{1}} \in \Sigma_{A_{1}}\right) t \sigma_{a_{1}} \in L\left(\mathbf{G}_{I_{1}}^{h}\right) \Rightarrow t \sigma_{a_{1}} \in L\left(\mathcal{G}_{H}, P\right)\right) \& \\
\ldots \\
\left.\left(\left(\forall \sigma_{a_{n}} \in \Sigma_{A_{n}}\right) t \sigma_{a_{n}} \in L\left(\mathbf{G}_{I_{n}}^{h}\right) \Rightarrow t \sigma_{a_{n}} \in L\left(\mathcal{G}_{H}, P\right)\right)\right) \\
\Rightarrow(\exists t \leq s)\left(\left(\exists \sigma_{u} \in \Sigma_{h u}\right) t \sigma_{u} \in L\left(\mathbf{G}_{H}^{p} \times \mathbf{G}_{I}^{h}\right) \& t \sigma_{u} \notin L\left(\mathcal{G}_{H}, P\right)\right) \text { or } \\
\left((\exists j \in\{1, \ldots, n\})\left(\exists \sigma_{a_{j}} \in \Sigma_{A_{j}}\right) t \sigma_{a_{j}} \in L\left(\mathbf{G}_{I_{j}}^{h}\right) \& t \sigma_{a_{j}} \notin L\left(\mathcal{G}_{H}, P\right)\right)
\end{gathered}
$$

As $s \in L\left(\mathcal{G}_{H}, P\right)$ and $L\left(\mathcal{G}_{H}, P\right)$ is closed, we have $t \in L\left(\mathcal{G}_{H}, P\right)$

By (4) and (5), we have $(\exists t \leq s) t \in W_{H}(P)$.

The above two lemmas state a very important fact. If a string $s \in L\left(\mathcal{G}_{H}, P\right)$ is in $W_{H}(P)$ with $P \preceq \mathcal{P}_{h a}$, Lemma 4.2 says that all strings in $L\left(\mathcal{G}_{H}, P\right)$ that go from $q_{H_{0}}$ to the state $\delta_{H}\left(q_{H_{0}}, s\right)$ are also in $W_{H}(P)$, while Lemma 4.3 says that all strings in $L\left(\mathcal{G}_{H}, P\right)$ that are extended from a string in $W_{H}(P)$ are not in $\Omega_{H I C}\left(L\left(\mathcal{G}_{H}, P\right)\right)$. It means that if a string $s \in L\left(\mathcal{G}_{H}, P\right)$ is in $W_{H}(P)$, then all the strings in $\left.L\left(\mathcal{G}_{H}, P\right)\right)$ 
Master Thesis - R. Song - McMaster - Computing and Software

that lead from $q_{H_{0}}$ to or pass through the state $\delta_{H}\left(q_{H_{0}}, s\right)$ are not in $\Omega_{H I C}\left(L\left(\mathcal{G}_{H}, P\right)\right)$. The fact is formally proved as follows by using these two lemmas.

Proposition 4.6. For system $\boldsymbol{\Phi}$, let $\mathcal{P}_{h a} \in \operatorname{Pred}\left(Q_{H}\right)$ be the given predicate for $\Omega_{H I C}$, then

$$
\left(\forall P \in S u b\left(\mathcal{P}_{h a}\right)\right) L\left(\mathcal{G}_{H}, P\right)-\Omega_{H I C}\left(L\left(\mathcal{G}_{H}, P\right)\right)=L^{W_{H}(P)}\left(\mathcal{G}_{H}, P\right),
$$

where $L^{W_{H}(P)}\left(\mathcal{G}_{H}, P\right)=\cup_{s \in W_{H}(P)} L^{s}\left(\mathcal{G}_{H}, P\right)$ as defined in Section 2.5.3.

proof:

Let $P \in S u b\left(\mathcal{P}_{h a}\right)$.

1. Show that $L^{W_{H}(P)}\left(\mathcal{G}_{H}, P\right) \subseteq L\left(\mathcal{G}_{H}, P\right)-\Omega_{H I C}\left(L\left(\mathcal{G}_{H}, P\right)\right)$.

Let $s \in L^{W_{H}(P)}\left(\mathcal{G}_{H}, P\right)$.

Must show this implies $s \in L\left(\mathcal{G}_{H}, P\right)-\Omega_{H I C}\left(L\left(\mathcal{G}_{H}, P\right)\right)$.

From (1), we know $\left(\exists t \in W_{H}(P)\right) s \in L^{t}\left(\mathcal{G}_{H}, P\right)$

$\Rightarrow\left(\exists t \in W_{H}(P)\right) s \in\left\{u \in L\left(\mathcal{G}_{H}, P\right) \mid(\exists v \leq u) \delta_{H}\left(q_{H_{0}}, v\right)=\delta_{H}\left(q_{H_{0}}, t\right)\right\}$

$\Rightarrow s \in L\left(\mathcal{G}_{H}, P\right) \&(\exists v \leq s)\left(\exists t \in W_{H}(P)\right) \delta_{H}\left(q_{H_{0}}, v\right)=\delta_{H}\left(q_{H_{0}}, t\right)$

$\Rightarrow s \in L\left(\mathcal{G}_{H}, P\right) \&(\exists v \leq s) v \in W_{H}(P)$,

by Lemma 4.2 and the fact $L\left(\mathcal{G}_{H}, P\right)$ is closed.

$\Rightarrow s \in L\left(\mathcal{G}_{H}, P\right) \& s \notin \Omega_{H I C}\left(L\left(\mathcal{G}_{H}, P\right)\right), \quad$ by Lemma 4.3

$\Rightarrow s \in L\left(\mathcal{G}_{H}, P\right)-\Omega_{H I C}\left(L\left(\mathcal{G}_{H}, P\right)\right)$

2. Show that $L\left(\mathcal{G}_{H}, P\right)-\Omega_{H I C}\left(L\left(\mathcal{G}_{H}, P\right)\right) \subseteq L^{W_{H}(P)}\left(\mathcal{G}_{H}, P\right)$.

Let $s \in L\left(\mathcal{G}_{H}, P\right)-\Omega_{H I C}\left(L\left(\mathcal{G}_{H}, P\right)\right)$.

Must show this implies $s \in L^{W_{H}(P)}\left(\mathcal{G}_{H}, P\right)$. 


\section{Master Thesis - R. Song-McMaster-Computing and Software}

Sufficient to show that $\left(\exists t \in W_{H}(P)\right) s \in L^{t}\left(\mathcal{G}_{H}, P\right)$

From (2), we know $s \in L\left(\mathcal{G}_{H}, P\right) \& s \notin \Omega_{H I C}\left(L\left(\mathcal{G}_{H}, P\right)\right)$.

$\Rightarrow s \in L\left(\mathcal{G}_{H}, P\right) \&(\exists t \leq s) \& t \in W_{H}(P), \quad$ by Lemma 4.3

$\Rightarrow\left(\exists t \in W_{H}(P)\right) s \in L^{t}\left(\mathcal{G}_{H}, P\right)$, as $t$ is a prefix of $s$ and $L\left(\mathcal{G}_{H}, P\right)$ is closed.

Now, if we look at Proposition 2.7, it seems that we can compute $\Omega_{H I C}\left(L\left(\mathcal{G}_{H}, P\right)\right)$ by removing all the states in $\left\{q \in Q_{H} \mid\left(\exists s \in W_{H}(P)\right) \delta_{H}\left(q_{H_{0}}, s\right)=q\right\}$ from the state set $Q_{H}$ of $\mathcal{G}_{H}$.

For any $q \in Q_{H}$, as $\mathcal{G}_{H}=\mathbf{E}_{H} \times \mathbf{G}_{H}^{p} \times \mathbf{G}_{I}^{h}$, there must exist unique $z \in Z_{H}, y \in Y_{H}$ and $x \in X^{h}$ such that $q=(z, y, x)$. For the state $x \in X^{h}$, as $\mathbf{G}_{I}^{h}=\mathbf{G}_{I_{1}}^{h} \times \cdots \times \mathbf{G}_{I_{n}}^{h}$, there must also exist unique $x_{1} \in X_{1}^{h}, \ldots, x_{n} \in X_{n}^{h}$ such that $x=\left(x_{1}, \ldots, x_{n}\right)$, so

$$
q=(z, y, x)=\left(z, y, x_{1}, \ldots, x_{n}\right)
$$

Definition 4.6. For system $\boldsymbol{\Phi}$, let $\mathcal{P}_{h a} \in \operatorname{Pred}\left(Q_{H}\right)$ be a given predicate. Define the function $\Pi_{H I C}: \operatorname{Sub}\left(\mathcal{P}_{h a}\right) \rightarrow \operatorname{Sub}\left(\mathcal{P}_{h a}\right)$ according to

$$
\begin{aligned}
& \left(\forall P \in \operatorname{Sub}\left(\mathcal{P}_{h a}\right)\right) \\
& \quad \Pi_{H I C}(P)=P-\operatorname{pr}\left(\operatorname{Bad}_{R H} \cup\left\{q \models R\left(\mathcal{G}_{H}, P\right) \mid\left(\exists \sigma \in \Sigma_{h u} \cup \Sigma_{A}\right) \delta_{H}(q, \sigma) \models \neg P\right\}\right),
\end{aligned}
$$

where $\operatorname{Bad}_{R H}=\left\{q \models R\left(\mathcal{G}_{H}, P\right) \mid\right.$

$$
\begin{aligned}
& \left(\left(\exists \sigma_{u} \in \Sigma_{h u}\right)\left(\eta_{H} \times \xi^{h}\left((y, x), \sigma_{u}\right) ! \& \quad \zeta_{H}\left(z, \sigma_{u}\right) \not y\right)\right. \text { or } \\
& \left(( \exists j \in \{ 1 , \ldots , n \} ) ( \exists \sigma _ { a _ { j } } \in \Sigma _ { A _ { j } } ) \left(\xi_{j}^{h}\left(x_{j}, \sigma_{a_{j}}\right) ! \&\right.\right. \\
& \left.\left.\left.\quad \zeta_{H} \times \eta_{H} \times \xi_{1}^{h} \times \cdots \times \xi_{j-1}^{h} \times \xi_{j+1}^{h} \times \cdots \times \xi_{n}^{h}\left(\left(z, y, x_{1}, \ldots, x_{j-1}, x_{j+1}, \ldots, x_{n}\right), \sigma_{a_{j}}\right) . .\right)\right)\right\}
\end{aligned}
$$

and $q=(z, y, x)=\left(z, y, x_{1}, \ldots, x_{n}\right)$ as in equation (4.1). 
Master Thesis - R. Song - McMaster - Computing and Software

Note that $\Pi_{H I C}(P) \preceq P \preceq \mathcal{P}_{h a}$, so the function is well-defined.

Proposition 4.7. For system $\boldsymbol{\Phi}$, let $\mathcal{P}_{h a} \in \operatorname{Pred}\left(Q_{H}\right)$ be the given predicate for $\Omega_{H I C}$, then

1. $\left(\forall P \in \operatorname{Sub}\left(\mathcal{P}_{h a}\right)\right) \Omega_{H I C}\left(L\left(\mathcal{G}_{H}, P\right)\right)=L\left(\mathcal{G}_{H}, \Pi_{H I C}(P)\right)$

2. $(\forall i \in\{0,1, \ldots\}) \Omega_{H I C}^{i}\left(L\left(\mathcal{G}_{H}, \mathcal{P}_{h a}\right)\right)=L\left(\mathcal{G}_{H}, \Pi_{H I C}^{i}\left(\mathcal{P}_{h a}\right)\right)$

proof:

1. Let $P \in \operatorname{Sub}\left(\mathcal{P}_{h a}\right)$. Must show that $\Omega_{H I C}\left(L\left(\mathcal{G}_{H}, P\right)\right)=L\left(\mathcal{G}_{H}, \Pi_{H I C}(P)\right)$.

We prove this proposition by a series of transformation.

$\Omega_{H I C}\left(L\left(\mathcal{G}_{H}, P\right)\right)$

$=L\left(\mathcal{G}_{H}, P\right)-L^{W_{H}(P)}\left(\mathcal{G}_{H}, P\right), \quad$ by Proposition 4.6

$=L\left(\mathcal{G}_{H}, P\right)-\cup_{w \in W_{H}(P)} L^{w}\left(\mathcal{G}_{H}, P\right)$

$=L\left(\mathcal{G}_{H}, P-\operatorname{pr}\left(\cup_{w \in W_{H}(P)}\left\{\delta_{H}\left(q_{H_{0}}, w\right)\right\}\right)\right), \quad$ by Proposition 2.7

$=L\left(\mathcal{G}_{H}, P-\operatorname{pr}\left(\left\{q \in R\left(\mathcal{G}_{H}, P\right) \mid \delta_{H}\left(q_{H_{0}}, w\right)=q\right.\right.\right.$ for some $\left.\left.\left.w \in W_{H}(P)\right\}\right)\right)$,

as $W_{H}(P) \subseteq L\left(\mathcal{G}_{H}, P\right)$ and by Proposition 2.5

$=L\left(\mathcal{G}_{H}, P-\operatorname{pr}\left(\left\{q \in R\left(\mathcal{G}_{H}, P\right) \mid\left(\exists w \in L\left(\mathcal{G}_{H}, P\right)\right) \delta_{H}\left(q_{H_{0}}, w\right)=q \&\right.\right.\right.$

$\left(\left(\exists \sigma_{u} \in \Sigma_{h u}\right) w \sigma_{u} \in L\left(\mathbf{G}_{H}^{p} \times \mathbf{G}_{I}^{h}\right) \& w \sigma_{u} \notin L\left(\mathcal{G}_{H}, P\right)\right)$ or

$\left.\left.\left.\left((\exists j \in\{1, \ldots, n\})\left(\exists \sigma_{a_{j}} \in \Sigma_{A_{j}}\right) w \sigma_{a_{j}} \in L\left(\mathbf{G}_{I_{j}}^{h}\right) \& w \sigma_{a_{j}} \notin L\left(\mathcal{G}_{H}, P\right)\right)\right\}\right)\right)$,

by definition of $W_{H}(P)$

$=L\left(\mathcal{G}_{H}, P-\operatorname{pr}\left(\left\{q \models R\left(\mathcal{G}_{H}, P\right) \mid\right.\right.\right.$

$\left(\left(\exists \sigma_{u} \in \Sigma_{h u}\right) \eta_{H} \times \xi^{h}\left((y, x), \sigma_{u}\right) ! \&\right.$

$\left(\delta_{H}\left(q, \sigma_{u}\right) \not\right.$ or $\left.\left.\left(\delta_{H}\left(q, \sigma_{u}\right) ! \& \delta_{H}\left(q, \sigma_{u}\right) \not \models P\right)\right)\right)$ or

$\left((\exists j \in\{1, \ldots, n\})\left(\exists \sigma_{a_{j}} \in \Sigma_{A_{j}}\right) \xi_{j}^{h}\left(x_{j}, \sigma_{a_{j}}\right) ! \&\right.$ 
Master Thesis - R. Song-McMaster-Computing and Software

$$
\left.\left.\left.\left.\left(\delta_{H}\left(q, \sigma_{a_{j}}\right) \text { y or }\left(\delta_{H}\left(q, \sigma_{a_{j}}\right) ! \& \delta_{H}\left(q, \sigma_{a_{j}}\right) \not \models P\right)\right)\right)\right\}\right)\right),
$$

where $q=(z, y, x)=\left(z, y, x_{1}, \ldots, x_{n}\right)$ as in equation (4.1).

$=L\left(\mathcal{G}_{H}, P-\operatorname{pr}\left(\left\{q \models R\left(\mathcal{G}_{H}, P\right) \mid\right.\right.\right.$

$\left(\left(\exists \sigma_{u} \in \Sigma_{h u}\right)\left(\eta_{H} \times \xi^{h}\left((y, x), \sigma_{u}\right) ! \& \delta_{H}\left(q, \sigma_{u}\right) \ell.\right)\right.$ or

$\left.\left(\eta_{H} \times \xi^{h}\left((y, x), \sigma_{u}\right) ! \& \delta_{H}\left(q, \sigma_{u}\right) ! \& \delta_{H}\left(q, \sigma_{u}\right) \not \models P\right)\right)$ or

$\left((\exists j \in\{1, \ldots, n\})\left(\exists \sigma_{a_{j}} \in \Sigma_{A_{j}}\right)\left(\xi_{j}^{h}\left(x_{j}, \sigma_{a_{j}}\right) ! \& \delta_{H}\left(q, \sigma_{a_{j}}\right) \not\right)\right.$ or

$$
\left.\left.\left.\left.\left(\xi_{j}^{h}\left(x_{j}, \sigma_{a_{j}}\right) ! \& \delta_{H}\left(q, \sigma_{a_{j}}\right) ! \& \delta_{H}\left(q, \sigma_{a_{j}}\right) \not \models P\right)\right)\right\}\right)\right),
$$

by logical distribution law.

$$
\begin{aligned}
& =L\left(\mathcal{G}_{H}, P-\operatorname{pr}\left(\left\{q \models R\left(\mathcal{G}_{H}, P\right) \mid\right.\right.\right. \\
& \left(\left(\exists \sigma_{u} \in \Sigma_{h u}\right)\left(\eta_{H} \times \xi^{h}\left((y, x), \sigma_{u}\right) ! \& \delta_{H}\left(q, \sigma_{u}\right) \not\right)\right. \text { or } \\
& \left.\left(\delta_{H}\left(q, \sigma_{u}\right) ! \& \delta_{H}\left(q, \sigma_{u}\right) \not \models P\right)\right) \text { or } \\
& \left((\exists j \in\{1, \ldots, n\})\left(\exists \sigma_{a_{j}} \in \Sigma_{A_{j}}\right)\left(\xi_{j}^{h}\left(x_{j}, \sigma_{a_{j}}\right) ! \& \delta_{H}\left(q, \sigma_{a_{j}}\right) \not .\right)\right. \text { or }
\end{aligned}
$$

$$
\left.\left.\left.\left.\left(\delta_{H}\left(q, \sigma_{a_{j}}\right) ! \& \delta_{H}\left(q, \sigma_{a_{j}}\right) \not \models P\right)\right)\right\}\right)\right),
$$

as $\delta_{H}\left(q, \sigma_{u}\right) ! \Rightarrow \eta_{H} \times \xi^{h}\left((y, x), \sigma_{u}\right) !$ and $\delta_{H}\left(q, \sigma_{a_{j}}\right) ! \Rightarrow \xi_{j}^{h}\left(x_{j}, \sigma_{a_{j}}\right) !$

by definition of $\delta_{H}$.

$=L\left(\mathcal{G}_{H}, P-\operatorname{pr}\left(\left\{q \models R\left(\mathcal{G}_{H}, P\right) \mid\right.\right.\right.$

$\left(\left(\exists \sigma_{u} \in \Sigma_{h u}\right)\left(\eta_{H} \times \xi^{h}\left((y, x), \sigma_{u}\right) ! \& \delta_{H}\left(q, \sigma_{u}\right) \not \cdot\right)\right.$ or $\left.\delta_{H}\left(q, \sigma_{u}\right) \not \models P\right)$ or

$\left((\exists j \in\{1, \ldots, n\})\left(\exists \sigma_{a_{j}} \in \Sigma_{A_{j}}\right)\left(\xi_{j}^{h}\left(x_{j}, \sigma_{a_{j}}\right) ! \& \delta_{H}\left(q, \sigma_{a_{j}}\right) \not\right)\right.$ or

$$
\left.\left.\left.\left.\delta_{H}\left(q, \sigma_{a_{j}}\right) \not \models P\right)\right\}\right)\right),
$$

as $\delta_{H}\left(q, \sigma_{u}\right) \not \models P \Leftrightarrow \delta_{H}\left(q, \sigma_{u}\right) ! \& \delta_{H}\left(q, \sigma_{u}\right) \not \models P$ and

$$
\delta_{H}\left(q, \sigma_{a_{j}}\right) \not \models P \Leftrightarrow \delta_{H}\left(q, \sigma_{a_{j}}\right) ! \& \delta_{H}\left(q, \sigma_{a_{j}}\right) \not \models P .
$$

$=L\left(\mathcal{G}_{H}, P-\operatorname{pr}\left(\left\{q \models R\left(\mathcal{G}_{H}, P\right) \mid\right.\right.\right.$

$\left(\left(\exists \sigma_{u} \in \Sigma_{h u}\right)\left(\eta_{H} \times \xi^{h}\left((y, x), \sigma_{u}\right) ! \& \zeta_{H}\left(z, \sigma_{u}\right)\right.\right.$ y.) or $\left.\delta_{H}\left(q, \sigma_{u}\right) \not \models P\right)$ or

$\left((\exists j \in\{1, \ldots, n\})\left(\exists \sigma_{a_{j}} \in \Sigma_{A_{j}}\right)\left(\xi_{j}^{h}\left(x_{j}, \sigma_{a_{j}}\right) ! \&\right.\right.$

$\zeta_{H} \times \eta_{H} \times \xi_{1}^{h} \times \cdots \times \xi_{j-1}^{h} \times \xi_{j+1}^{h} \times \cdots \times \xi_{n}^{h}\left(\left(z, y, x_{1}, \ldots, x_{j-1}, x_{j+1}, \ldots, x_{n}\right), \sigma_{a_{j}}\right)$ y.) or 
Master Thesis - R. Song - McMaster - Computing and Software

$$
\begin{aligned}
& \left.\left.\left.\left.\delta_{H}\left(q, \sigma_{a_{j}}\right) \not \models P\right)\right\}\right)\right) \text {, by definition of } \delta_{H} \text {. } \\
& =L\left(\mathcal{G}_{H}, P-\operatorname{pr}\left(\left\{q \models R\left(\mathcal{G}_{H}, P\right) \mid\right.\right.\right. \\
& \left(\left(\exists \sigma_{u} \in \Sigma_{h u}\right)\left(\eta_{H} \times \xi^{h}\left((y, x), \sigma_{u}\right) ! \& \zeta_{H}\left(z, \sigma_{u}\right) \not y\right) \text { or } \delta_{H}\left(q, \sigma_{u}\right) \models \neg P\right) \text { or } \\
& \left(( \exists j \in \{ 1 , \ldots , n \} ) ( \exists \sigma _ { a _ { j } } \in \Sigma _ { A _ { j } } ) \left(\xi_{j}^{h}\left(x_{j}, \sigma_{a_{j}}\right) ! \&\right.\right. \\
& \left.\zeta_{H} \times \eta_{H} \times \xi_{1}^{h} \times \cdots \times \xi_{j-1}^{h} \times \xi_{j+1}^{h} \times \cdots \times \xi_{n}^{h}\left(\left(z, y, x_{1}, \ldots, x_{j-1}, x_{j+1}, \ldots, x_{n}\right), \sigma_{a_{j}}\right) \not .\right) \text { or } \\
& \left.\left.\left.\left.\delta_{H}\left(q, \sigma_{a_{j}}\right) \models \neg P\right)\right\}\right)\right), \quad \text { by } q^{\prime} \not \models P \Leftrightarrow q^{\prime} \models \neg P \\
& =L\left(\mathcal{G}_{H}, P-\operatorname{pr}\left(\left\{q \models R\left(\mathcal{G}_{H}, P\right) \mid\right.\right.\right. \\
& \left(\left(\exists \sigma_{u} \in \Sigma_{h u}\right) \eta_{H} \times \xi^{h}\left((y, x), \sigma_{u}\right) ! \& \zeta_{H}\left(z, \sigma_{u}\right) \not\right) \text { or } \\
& \left(\left(\exists \sigma_{u} \in \Sigma_{h u}\right) \delta_{H}\left(q, \sigma_{u}\right) \models \neg P\right) \text { or } \\
& \left((\exists j \in\{1, \ldots, n\})\left(\exists \sigma_{a_{j}} \in \Sigma_{A_{j}}\right) \xi_{j}^{h}\left(x_{j}, \sigma_{a_{j}}\right) ! \&\right. \\
& \left.\zeta_{H} \times \eta_{H} \times \xi_{1}^{h} \times \cdots \times \xi_{j-1}^{h} \times \xi_{j+1}^{h} \times \cdots \times \xi_{n}^{h}\left(\left(z, y, x_{1}, \ldots, x_{j-1}, x_{j+1}, \ldots, x_{n}\right), \sigma_{a_{j}}\right) \not .\right) \text { or } \\
& \left.\left.\left.\left((\exists j \in\{1, \ldots, n\})\left(\exists \sigma_{a_{j}} \in \Sigma_{A_{j}}\right) \delta_{H}\left(q, \sigma_{a_{j}}\right) \models \neg P\right)\right\}\right)\right) \text {, by regrouping conditions } \\
& =L\left(\mathcal{G}_{H}, P-\operatorname{pr}\left(\operatorname { B a d } _ { R H } \cup \left\{q \models R\left(\mathcal{G}_{H}, P\right) \mid\right.\right.\right. \\
& \left(\left(\exists \sigma_{u} \in \Sigma_{h u}\right) \delta_{H}\left(q, \sigma_{u}\right) \models \neg P\right) \text { or } \\
& \left.\left.\left.\left((\exists j \in\{1, \ldots, n\})\left(\exists \sigma_{a_{j}} \in \Sigma_{A_{j}}\right) \delta_{H}\left(q, \sigma_{a_{j}}\right) \models \neg P\right)\right\}\right)\right),
\end{aligned}
$$$$
\text { by definition of } \operatorname{Bad}_{R H}
$$$$
=L\left(\mathcal{G}_{H}, P-\operatorname{pr}\left(\operatorname { B a d } _ { R H } \cup \left\{q \models R\left(\mathcal{G}_{H}, P\right) \mid\right.\right.\right.
$$$$
\left(\left(\exists \sigma_{u} \in \Sigma_{h u}\right) \delta_{H}\left(q, \sigma_{u}\right) \models \neg P\right) \text { or }
$$$$
\left.\left.\left.\left(\left(\exists \sigma_{a} \in \Sigma_{A}\right) \delta_{H}\left(q, \sigma_{a}\right) \models \neg P\right)\right\}\right)\right)
$$$$
=L\left(\mathcal{G}_{H}, P-\operatorname{pr}\left(\operatorname{Bad}_{R H} \cup\left\{q \models R\left(\mathcal{G}_{H}, P\right) \mid\left(\exists \sigma \in \Sigma_{h u} \cup \Sigma_{A}\right) \delta_{H}(q, \sigma) \models \neg P\right\}\right)\right)
$$$$
=L\left(\mathcal{G}_{H}, \Pi_{H I C}(P)\right)
$$

2. Show that $(\forall i \in\{0,1, \ldots\}) \Omega_{H I C}^{i}\left(L\left(\mathcal{G}_{H}, \mathcal{P}_{h a}\right)\right)=L\left(\mathcal{G}_{H}, \Pi_{H I C}^{i}\left(\mathcal{P}_{h a}\right)\right)$

We prove this by induction. 
Master Thesis - R. Song-McMaster-Computing and Software

(a) Base case: $(i=0)$

By the definitions of $\Omega_{H I C}^{0}\left(L\left(\mathcal{G}_{H}, \mathcal{P}_{h a}\right)\right)$ and $\Pi_{H I C}^{0}\left(\mathcal{P}_{h a}\right)$ (see section 2.1.3), we have

$\Omega_{H I C}^{0}\left(L\left(\mathcal{G}_{H}, \mathcal{P}_{h a}\right)\right)=L\left(\mathcal{G}_{H}, \mathcal{P}_{h a}\right)$

$L\left(\mathcal{G}_{H}, \Pi_{H I C}^{0}\left(\mathcal{P}_{h a}\right)\right)=L\left(\mathcal{G}_{H}, \mathcal{P}_{h a}\right)$

So, $\Omega_{H I C}^{0}\left(L\left(\mathcal{G}_{H}, \mathcal{P}_{h a}\right)\right)=L\left(\mathcal{G}_{H}, \Pi_{H I C}^{0}\left(\mathcal{P}_{h a}\right)\right)$

(b) Inductive step:

Let $k \in\{0,1, \ldots\}$. Assume $\Omega_{H I C}^{k}\left(L\left(\mathcal{G}_{H}, \mathcal{P}_{h a}\right)\right)=L\left(\mathcal{G}_{H}, \Pi_{H I C}^{k}\left(\mathcal{P}_{h a}\right)\right)$. Must show this implies $\Omega_{H I C}^{k+1}\left(L\left(\mathcal{G}_{H}, \mathcal{P}_{h a}\right)\right)=L\left(\mathcal{G}_{H}, \Pi_{H I C}^{k+1}\left(\mathcal{P}_{h a}\right)\right)$.

$\Omega_{H I C}^{k+1}\left(L\left(\mathcal{G}_{H}, \mathcal{P}_{h a}\right)\right)=\Omega_{H I C}\left(\Omega_{H I C}^{k}\left(L\left(\mathcal{G}_{H}, \mathcal{P}_{h a}\right)\right)\right)$

$=\Omega_{H I C}\left(L\left(\mathcal{G}_{H}, \Pi_{H I C}^{k}\left(\mathcal{P}_{h a}\right)\right)\right), \quad$ by assumption

$=L\left(\mathcal{G}_{H}, \Pi_{H I C}^{k+1}\left(\mathcal{P}_{h a}\right)\right)$, by taking $\Pi_{H I C}^{k}\left(\mathcal{P}_{h a}\right)$ as our

predicate $P$ in Point 1 of this proposition.

Now, if we take a look at the definition of the function $\Pi_{H I C}$, we can see that for any $P \preceq \mathcal{P}_{h a}$, the function $\Pi_{H I C}$ only removes states in $R\left(\mathcal{G}_{H}, P\right)$ from $P$. Precisely, $\Pi_{H I C}$ only removes all the states in $\operatorname{pr}\left(\operatorname{Bad}_{R H} \cup\left\{q \models R\left(\mathcal{G}_{H}, P\right) \mid\left(\exists \sigma \in \Sigma_{h u} \cup\right.\right.\right.$ $\left.\left.\left.\Sigma_{A}\right) \delta_{H}(q, \sigma) \models \neg P\right\}\right)$, which is a subpredicate of $R\left(\mathcal{G}_{H}, P\right)$. If we also remove any state that is not in $R\left(\mathcal{G}_{H}, P\right)$, then the language $L\left(\mathcal{G}_{H}, P\right)$ is not affected at all, because the $L\left(\mathcal{G}_{H}, P\right)$ is only dependent on $R\left(\mathcal{G}_{H}, P\right)$. Similarly, removing any state that is not in $R\left(\mathcal{G}_{H}, P\right)$ does not affect the language $L\left(\mathcal{G}_{H}, \Pi_{H I C}(P)\right)$ either, because it is only dependent on a subpredicate of $R\left(\mathcal{G}_{H}, P\right)$. The following function $\Gamma_{H I C}$ is a replacement of the function $\Pi_{H I C}$. 
Master Thesis - R. Song - McMaster - Computing and Software

Definition 4.7. For system $\boldsymbol{\Phi}$, let $\mathcal{P}_{h a} \in \operatorname{Pred}\left(Q_{H}\right)$ be a given predicate. Define the function $\Gamma_{H I C}: \operatorname{Sub}\left(\mathcal{P}_{h a}\right) \rightarrow \operatorname{Sub}\left(\mathcal{P}_{h a}\right)$ according to

$$
\begin{aligned}
& \left(\forall P \in \operatorname{Sub}\left(\mathcal{P}_{h a}\right)\right) \\
& \quad \Gamma_{H I C}(P):=P-\operatorname{pr}\left(\operatorname{Bad}_{H} \cup\left\{q \in Q_{H} \mid\left(\exists \sigma \in \Sigma_{h u} \cup \Sigma_{A}\right) \delta_{H}(q, \sigma) \models \neg P\right\}\right),
\end{aligned}
$$

where $\operatorname{Bad}_{H}:=\left\{q \in Q_{H} \mid\right.$

$$
\begin{aligned}
& \left(\left(\exists \sigma_{u} \in \Sigma_{h u}\right)\left(\eta_{H} \times \xi^{h}\left((y, x), \sigma_{u}\right) ! \& \zeta_{H}\left(z, \sigma_{u}\right) \not y\right)\right. \text { or } \\
& \left(( \exists j \in \{ 1 , \ldots , n \} ) ( \exists \sigma _ { a _ { j } } \in \Sigma _ { A _ { j } } ) \left(\xi_{j}^{h}\left(x_{j}, \sigma_{a_{j}}\right) ! \&\right.\right. \\
& \left.\left.\left.\quad \zeta_{H} \times \eta_{H} \times \xi_{1}^{h} \times \cdots \times \xi_{j-1}^{h} \times \xi_{j+1}^{h} \times \cdots \times \xi_{n}^{h}\left(\left(z, y, x_{1}, \ldots, x_{j-1}, x_{j+1}, \ldots, x_{n}\right), \sigma_{a_{j}}\right) . .\right)\right)\right\}
\end{aligned}
$$

and $q=(z, y, x)=\left(z, y, x_{1}, \ldots, x_{n}\right)$ as in equation (4.1).

Note that for system $\boldsymbol{\Phi}, \operatorname{Bad}_{H}$ is constant.

Corollary 4.1. For system $\boldsymbol{\Phi}$, let $\mathcal{P}_{h a} \in \operatorname{Pred}\left(Q_{H}\right)$ be the given predicate for the function $\Omega_{H I C}, \Pi_{H I C}$ and $\Gamma_{H I C}$. Then the following holds:

1. $\left(\forall P \in \operatorname{Sub}\left(\mathcal{P}_{h a}\right)\right) \Omega_{H I C}\left(L\left(\mathcal{G}_{H}, P\right)\right)=L\left(\mathcal{G}_{H}, \Gamma_{H I C}(P)\right)$

2. $(\forall i \in\{0,1, \ldots\}) \Omega_{H I C}^{i}\left(L\left(\mathcal{G}_{H}, \mathcal{P}_{h a}\right)\right)=L\left(\mathcal{G}_{H}, \Gamma_{H I C}^{i}\left(\mathcal{P}_{h a}\right)\right)$

\section{proof:}

1. Let $P \in \operatorname{Sub}\left(\mathcal{P}_{h a}\right)$, show that $\Omega_{H I C}\left(L\left(\mathcal{G}_{H}, P\right)\right)=L\left(\mathcal{G}_{H}, \Gamma_{H I C}(P)\right)$

By the definitions of $\Pi_{H I C}$ and $\Gamma_{H I C}$, and from the above description, we know that removing states that are not in $R\left(\mathcal{G}_{H}, P\right)$ does not affect the language $L\left(\mathcal{G}_{H}, \Pi_{H I C}(P)\right)$. We thus have

$$
L\left(\mathcal{G}_{H}, \Pi_{H I C}(P)\right)=L\left(\mathcal{G}_{H}, \Gamma_{H I C}(P)\right) .
$$

By Point 1 of Proposition 4.7, we know 
Master Thesis - R. Song - McMaster - Computing and Software

$$
\Omega_{H I C}\left(L\left(\mathcal{G}_{H}, P\right)\right)=L\left(\mathcal{G}_{H}, \Pi_{H I C}(P)\right) .
$$

By (1) and (2), we have $\Omega_{H I C}\left(L\left(\mathcal{G}_{H}, P\right)\right)=L\left(\mathcal{G}_{H}, \Gamma_{H I C}(P)\right)$.

2. Show that $(\forall i \in\{0,1, \ldots\}) \Omega_{H I C}^{i}\left(L\left(\mathcal{G}_{H}, \mathcal{P}_{h a}\right)\right)=L\left(\mathcal{G}_{H}, \Pi_{H I C}^{i}\left(\mathcal{P}_{h a}\right)\right)$

Identical to the proof of Part 2 of Proposition 4.7 after substituting $\Pi_{H I C}$ with $\Gamma_{H I C}$.

The following lemma will be used in the next proposition.

Lemma 4.4. For system $\boldsymbol{\Phi}$, let $\mathcal{P}_{h a} \in \operatorname{Pred}\left(Q_{H}\right)$ be the given predicate for the function $\Gamma_{H I C}$. The function $\Gamma_{H I C}$ is monotone with respect to $\preceq$, i.e.

$$
\left(\forall P_{1}, P_{2} \in \operatorname{Sub}\left(\mathcal{P}_{h a}\right)\right) P_{1} \preceq P_{2} \Rightarrow \Gamma_{H I C}\left(P_{1}\right) \preceq \Gamma_{H I C}\left(P_{2}\right)
$$

\section{proof:}

Let $P_{1}, P_{2} \in \operatorname{Sub}\left(\mathcal{P}_{h a}\right)$. Assume $P_{1} \preceq P_{2}$. Must show implies $\Gamma_{H I C}\left(P_{1}\right) \preceq \Gamma_{H I C}\left(P_{2}\right)$.

$$
\begin{aligned}
\Gamma_{H I C}\left(P_{1}\right) & =P_{1}-\operatorname{pr}\left(\operatorname{Bad}_{H} \cup\left\{q \in Q_{H} \mid\left(\exists \sigma \in \Sigma_{h u} \cup \Sigma_{A}\right) \delta_{H}(q, \sigma) \models \neg P_{1}\right\}\right) \\
& =P_{1} \wedge \neg \operatorname{pr}\left(\operatorname{Bad}_{H} \cup\left\{q \in Q_{H} \mid\left(\exists \sigma \in \Sigma_{h u} \cup \Sigma_{A}\right) \delta_{H}(q, \sigma) \models \neg P_{1}\right\}\right) \\
& =P_{1} \wedge\left(\neg \operatorname{pr}\left(\operatorname{Bad}_{H}\right)\right) \wedge \neg\left(\operatorname{pr}\left(\left\{q \in Q_{H} \mid\left(\left(\exists \sigma \in \Sigma_{h u} \cup \Sigma_{A}\right) \delta_{H}(q, \sigma) \models \neg P_{1}\right)\right\}\right)\right) \\
& =P_{1} \wedge\left(\neg \operatorname{pr}\left(\operatorname{Bad}_{H}\right)\right) \wedge \operatorname{pr}\left(\left\{q \in Q_{H} \mid\left(\left(\forall \sigma \in \Sigma_{h u} \cup \Sigma_{A}\right) \delta_{H}(q, \sigma) ! \Rightarrow \delta_{H}(q, \sigma) \models P_{1}\right)\right\}\right) \\
& \preceq P_{2} \wedge\left(\neg \operatorname{pr}\left(\operatorname{Bad}_{H}\right)\right) \wedge p r\left(\left\{q \in Q_{H} \mid\left(\left(\forall \sigma \in \Sigma_{h u} \cup \Sigma_{A}\right) \delta_{H}(q, \sigma) ! \Rightarrow \delta_{H}(q, \sigma) \models P_{2}\right)\right\}\right),
\end{aligned}
$$

by assumption $P_{1} \preceq P_{2}$.

$$
\begin{aligned}
& =P_{2} \wedge\left(\neg \operatorname{pr}\left(\operatorname{Bad}_{H}\right)\right) \wedge \neg\left(p r\left(\left\{q \in Q_{H} \mid\left(\left(\exists \sigma \in \Sigma_{h u} \cup \Sigma_{A}\right) \delta_{H}(q, \sigma) \models \neg P_{2}\right)\right\}\right)\right) \\
& =P_{2} \wedge \neg \operatorname{pr}\left(\operatorname{Bad}_{H} \cup\left\{q \in Q_{H} \mid\left(\exists \sigma \in \Sigma_{h u} \cup \Sigma_{A}\right) \delta_{H}(q, \sigma) \models \neg P_{2}\right\}\right) \\
& =P_{2}-\operatorname{pr}\left(\operatorname{Bad}_{H} \cup\left\{q \in Q_{H} \mid\left(\exists \sigma \in \Sigma_{h u} \cup \Sigma_{A}\right) \delta_{H}(q, \sigma) \models \neg P_{2}\right\}\right) \\
& =\Gamma_{H I C}\left(P_{2}\right)
\end{aligned}
$$


Master Thesis - R. Song - McMaster - Computing and Software

Proposition 4.8. For system $\boldsymbol{\Phi}$, let $\mathcal{P}_{h a} \in \operatorname{Pred}\left(Q_{H}\right)$ be the given predicate for the function $\Omega_{H I C}$ and $\Gamma_{H I C}$, then,

1. There exists $k \in\{0,1,2, \ldots\}$ with $k \leq\left|s t\left(\mathcal{P}_{h a}\right)\right|$ such that $\Gamma_{H I C}^{k}\left(\mathcal{P}_{h a}\right)$ is the greatest fixpoint of the function $\Gamma_{H I C}$ with respect to $\left(\operatorname{Sub}\left(\mathcal{P}_{h a}\right), \preceq\right)$, and where $\operatorname{st}\left(\mathcal{P}_{h a}\right)$ is the identifying state subset of $Q_{H}$ for $\mathcal{P}_{h a}$.

2. $\sup \mathcal{C}_{H}\left(L\left(\mathcal{G}_{H}, \mathcal{P}_{h a}\right)\right)=L\left(\mathcal{G}_{H}, \Gamma_{H I C}^{k}\left(\mathcal{P}_{h a}\right)\right)$

\section{proof:}

1. Show that there exists $k \leq\left|s t\left(\mathcal{P}_{h a}\right)\right|$ such that the greatest fixpoint of the function $\Gamma_{H I C}$ is equal to $\Gamma_{H I C}^{k}\left(\mathcal{P}_{h a}\right)$.

As we assume the number of states in $\mathcal{G}_{H}$ is finite (Section 4.1), the number of states in $\operatorname{st}\left(\mathcal{P}_{h a}\right)$ is also finite. By Lemma 4.4, we know that $\Gamma_{H I C}$ is monotone with respect to $\preceq$, so by Proposition 2.3, there exists $k \leq\left|\operatorname{st}\left(\mathcal{P}_{h a}\right)\right|$ such that the greatest fixpoint of the function $\Gamma_{H I C}$ is equal to $\Gamma_{H I C}^{k}\left(\mathcal{P}_{h a}\right)$.

2. Show that $\sup \mathcal{C}_{H}\left(L\left(\mathcal{G}_{H}, \mathcal{P}_{h a}\right)\right)=L\left(\mathcal{G}_{H}, \Gamma_{H I C}^{k}\left(\mathcal{P}_{h a}\right)\right)$

(a) Show that $\sup \mathcal{C}_{H}\left(L\left(\mathcal{G}_{H}, \mathcal{P}_{h a}\right)\right) \subseteq L\left(\mathcal{G}_{H}, \Gamma_{H I C}^{k}\left(\mathcal{P}_{h a}\right)\right)$

By Proposition 2.3, we know that

$$
\begin{aligned}
& (\forall i \in\{0,1, \ldots\}) i \geq k \Rightarrow L\left(\mathcal{G}_{H}, \Gamma_{H I C}^{i}\left(\mathcal{P}_{h a}\right)\right)=L\left(\mathcal{G}_{H}, \Gamma_{H I C}^{k}\left(\mathcal{P}_{h a}\right)\right) \\
& \Rightarrow \lim _{i \rightarrow \infty} L\left(\mathcal{G}_{H}, \Gamma_{H I C}^{i}\left(\mathcal{P}_{h a}\right)\right)=L\left(\mathcal{G}_{H}, \Gamma_{H I C}^{k}\left(\mathcal{P}_{h a}\right)\right) \\
& \Rightarrow \lim _{i \rightarrow \infty} \Omega_{H I C}^{i}\left(L\left(\mathcal{G}_{H}, \mathcal{P}_{h a}\right)\right)=L\left(\mathcal{G}_{H}, \Gamma_{H I C}^{k}\left(\mathcal{P}_{h a}\right)\right), \quad \text { by Corollary } 4.1 \\
& \Rightarrow \sup \mathcal{C}_{H}\left(L\left(\mathcal{G}_{H}, \mathcal{P}_{h a}\right)\right) \subseteq L\left(\mathcal{G}_{H}, \Gamma_{H I C}^{k}\left(\mathcal{P}_{h a}\right)\right), \quad \text { by Proposition } 4.5
\end{aligned}
$$

(b) Show that $L\left(\mathcal{G}_{H}, \Gamma_{H I C}^{k}\left(\mathcal{P}_{h a}\right)\right) \subseteq \sup \mathcal{C}_{H}\left(L\left(\mathcal{G}_{H}, \mathcal{P}_{h a}\right)\right)$

From Point 1 of this proposition and Proposition 2.3, we have 
Master Thesis - R. Song - McMaster - Computing and Software

$$
\begin{aligned}
& \Gamma_{H I C}^{k}\left(\mathcal{P}_{h a}\right)=\Gamma_{H I C}^{k+1}\left(\mathcal{P}_{h a}\right) \\
& \Rightarrow L\left(\mathcal{G}_{H}, \Gamma_{H I C}^{k}\left(\mathcal{P}_{h a}\right)\right)=L\left(\mathcal{G}_{H}, \Gamma_{H I C}^{k+1}\left(\mathcal{P}_{h a}\right)\right) \\
& \Rightarrow L\left(\mathcal{G}_{H}, \Gamma_{H I C}^{k}\left(\mathcal{P}_{h a}\right)\right)=\Omega_{H I C}^{k+1}\left(L\left(\mathcal{G}_{H}, \mathcal{P}_{h a}\right)\right), \quad \text { by Corollary } 4.1 \\
& \Rightarrow L\left(\mathcal{G}_{H}, \Gamma_{H I C}^{k}\left(\mathcal{P}_{h a}\right)\right)=\Omega_{H I C}\left(\Omega_{H I C}^{k}\left(L\left(\mathcal{G}_{H}, \mathcal{P}_{h a}\right)\right)\right) \\
& \Rightarrow L\left(\mathcal{G}_{H}, \Gamma_{H I C}^{k}\left(\mathcal{P}_{h a}\right)\right)=\Omega_{H I C}\left(L\left(\mathcal{G}_{H}, \Gamma_{H I C}^{k}\left(\mathcal{P}_{h a}\right)\right)\right), \quad \text { by Corollary } 4.1
\end{aligned}
$$

So, $L\left(\mathcal{G}_{H}, \Gamma_{H I C}^{k}\left(\mathcal{P}_{h a}\right)\right)$ is a fixpoint of the function $\Omega_{H I C}$. By Proposition 4.4, $\sup \mathcal{C}_{H}\left(L\left(\mathcal{G}_{H}, \mathcal{P}_{h a}\right)\right)$ is the greatest fixpoint of $\Omega_{H I C}$, thus,

$$
L\left(\mathcal{G}_{H}, \Gamma_{H I C}^{k}\left(\mathcal{P}_{h a}\right)\right) \subseteq \sup \mathcal{C}_{H}\left(L\left(\mathcal{G}_{H}, \mathcal{P}_{h a}\right)\right)
$$

We are now able to write a computer program to compute $\sup \mathcal{C}_{H}\left(L\left(\mathcal{G}_{H}, \mathcal{P}_{h a}\right)\right)$ by computing $\Gamma_{H I C}^{k}\left(\mathcal{P}_{h a}\right)(k \in\{0,1,2, \ldots\})$, and we know that after at most $\left|\operatorname{st}\left(\mathcal{P}_{h a}\right)\right|$ number of iterations, a fixpoint of $\Gamma_{H I C}$ will be reached, which is the greatest fixpoint of $\Gamma_{H I C}$ by Proposition 2.3. However, such a program based on function $\Gamma_{H I C}$ is not efficient enough. For instance, each time when we implement the $\Gamma_{H I C}$ function, we have to do the predicate subtracting operation. The following alternative method can avoid this.

Definition 4.8. For system $\boldsymbol{\Phi}$, define the function PHIC $: \operatorname{Pred}\left(Q_{H}\right) \rightarrow \operatorname{Pred}\left(Q_{H}\right)$ according to

$$
\left(\forall P \in \operatorname{Pred}\left(Q_{H}\right)\right) \operatorname{PHC}(P):=\neg T R\left(\mathcal{G}_{H}, \neg P \vee \operatorname{pr}\left(\operatorname{Bad}_{H}\right), \Sigma_{h u} \cup \Sigma_{A}\right) .
$$

The following lemma will be used in the next proposition.

Lemma 4.5. For system $\boldsymbol{\Phi}$, the following two points hold:

1. The function PHC is monotone with respect to $\preceq$, i.e.

$$
\left(\forall P_{1}, P_{2} \in \operatorname{Pred}\left(Q_{H}\right)\right) P_{1} \preceq P_{2} \Rightarrow \operatorname{PHC}\left(P_{1}\right) \preceq \operatorname{PHC}\left(P_{2}\right) .
$$


Master Thesis - R. Song-McMaster - Computing and Software

2. $\left(\forall P \in \operatorname{Pred}\left(Q_{H}\right)\right) \operatorname{PHC}(P) \preceq P$.

proof:

1. Show that PHIC is monotone.

Let $P_{1}, P_{2} \in \operatorname{Pred}\left(Q_{H}\right)$.

Assume $P_{1} \preceq P_{2}$. Must show this implies $\operatorname{PHC}\left(P_{1}\right) \preceq \operatorname{PHIC}\left(P_{2}\right)$.

By assumption $P_{1} \preceq P_{2}$, we have $\neg P_{2} \preceq \neg P_{1}$.

$\Rightarrow T R\left(\mathcal{G}_{H}, \neg P_{2} \vee \operatorname{pr}\left(\operatorname{Bad}_{H}\right), \Sigma_{h u} \cup \Sigma_{A}\right) \preceq T R\left(\mathcal{G}_{H}, \neg P_{1} \vee \operatorname{pr}\left(\operatorname{Bad}_{H}\right), \Sigma_{h u} \cup \Sigma_{A}\right)$,

as $T R$ is monotone

$\Rightarrow \neg \mathrm{PHC}\left(P_{2}\right) \preceq \neg \mathrm{PHC}\left(P_{1}\right)$

$\Rightarrow \operatorname{PHC}\left(P_{1}\right) \preceq \operatorname{PHC}\left(P_{2}\right)$

2. Show that $\left(\forall P \in \operatorname{Pred}\left(Q_{H}\right)\right) \operatorname{PHC}(P) \preceq P$.

Let $P \in \operatorname{Pred}\left(Q_{H}\right)$. We will now show that $\operatorname{PHC}(P) \preceq P$.

$$
\begin{aligned}
& \operatorname{PHIC}(P)=\neg T R\left(\mathcal{G}_{H}, \neg P \vee \operatorname{pr}\left(\operatorname{Bad}_{H}\right), \Sigma_{h u} \cup \Sigma_{A}\right) \\
& \Rightarrow \neg \operatorname{PHIC}(P)=T R\left(\mathcal{G}_{H}, \neg P \vee \operatorname{pr}\left(\operatorname{Bad}_{H}\right), \Sigma_{h u} \cup \Sigma_{A}\right) \\
& \Rightarrow \neg P \preceq \neg \operatorname{PHIC}(P), \quad \text { by definition of } T R \text { and fact } \neg P \preceq \neg P \vee \operatorname{pr}\left(\operatorname{Bad}_{H}\right) \\
& \Rightarrow \operatorname{PHC}(P) \preceq P .
\end{aligned}
$$

Proposition 4.9. For system $\mathbf{\Phi}$, the following holds:

$$
\left(\forall \mathcal{P}_{h a} \in \operatorname{Pred}\left(Q_{H}\right)\right) \sup \mathcal{C}_{H}\left(L\left(\mathcal{G}_{H}, \mathcal{P}_{h a}\right)\right)=L\left(\mathcal{G}_{H}, \operatorname{PHIC}\left(\mathcal{P}_{h a}\right)\right)
$$




\section{Master Thesis - R. Song-McMaster-Computing and Software}

\section{proof:}

Let $\mathcal{P}_{h a} \in \operatorname{Pred}\left(Q_{H}\right)$ be the given predicate for the function $\Gamma_{H I C}$ (i.e. $\operatorname{Sub}\left(\mathcal{P}_{h a}\right)$ is the domain and codomain for $\left.\Gamma_{H I C}\right)$.

From Lemma 4.5, we know $\operatorname{PHC}\left(\mathcal{P}_{h a}\right) \preceq \mathcal{P}_{h a}$, so $\operatorname{PHC}\left(\mathcal{P}_{h a}\right) \in \operatorname{Sub}\left(\mathcal{P}_{h a}\right)$ and is thus a valid input of $\Gamma_{H I C}$.

From Proposition 4.8, sufficient to show $\operatorname{PHC}\left(\mathcal{P}_{h a}\right)$ is the greatest fixpoint of $\Gamma_{H I C}$.

1. Show that $\operatorname{PHC}\left(\mathcal{P}_{h a}\right)$ is a fixpoint of $\Gamma_{H I C}$, i.e. $\operatorname{PHC}\left(\mathcal{P}_{h a}\right)=\Gamma_{H I C}\left(\operatorname{PHC}\left(\mathcal{P}_{h a}\right)\right)$.

$$
\begin{aligned}
& \Gamma_{H I C}\left(\operatorname{PHC}\left(\mathcal{P}_{h a}\right)\right)=\operatorname{PHC}\left(\mathcal{P}_{h a}\right)- \\
& \operatorname{pr}\left(\operatorname{Bad}_{H} \cup\left\{q \in Q_{H} \mid\left(\exists \sigma \in \Sigma_{h u} \cup \Sigma_{A}\right) \delta_{H}(q, \sigma) \models \neg \operatorname{PHC}\left(\mathcal{P}_{h a}\right)\right\}\right)
\end{aligned}
$$

To show $\Gamma_{H I C}\left(\operatorname{PHC}\left(\mathcal{P}_{h a}\right)\right)=\operatorname{PHIC}\left(\mathcal{P}_{h a}\right)$, sufficient to show that

$$
\begin{aligned}
&\left(\forall q^{\prime} \in Q_{H}\right) q^{\prime} \models \operatorname{PHC}\left(\mathcal{P}_{h a}\right) \Rightarrow q^{\prime} \not \models p r\left(\operatorname{Bad}_{H}\right) \& \\
& q^{\prime} \notin\left\{q \in Q_{H} \mid\left(\exists \sigma \in \Sigma_{h u} \cup \Sigma_{A}\right) \delta_{H}(q, \sigma) \models \neg \operatorname{PHC}\left(\mathcal{P}_{h a}\right)\right\}
\end{aligned}
$$

Let $q^{\prime} \in Q_{H}$. Assume $q^{\prime}=\operatorname{PHC}\left(\mathcal{P}_{h a}\right)$.

Must show the following two points.

(a) Show $q^{\prime} \not \models \operatorname{pr}\left(\operatorname{Bad}_{H}\right)$.

By $(1)$, we have $q^{\prime} \models \operatorname{PHC}\left(\mathcal{P}_{h a}\right)$

$$
\begin{aligned}
& \Rightarrow q^{\prime} \not \models T R\left(\mathcal{G}_{H}, \neg \mathcal{P}_{h a} \vee \operatorname{pr}\left(\operatorname{Bad}_{H}\right), \Sigma_{h u} \cup \Sigma_{A}\right) \\
& \Rightarrow q^{\prime} \not \models \operatorname{pr}\left(\operatorname{Bad}_{H}\right), \quad \text { by definition of } T R
\end{aligned}
$$

(b) Show $q^{\prime} \notin\left\{q \in Q_{H} \mid\left(\exists \sigma \in \Sigma_{h u} \cup \Sigma_{A}\right) \delta_{H}(q, \sigma) \models \neg \operatorname{PHC}\left(\mathcal{P}_{h a}\right)\right\}$

We prove this by contradiction.

$$
\begin{aligned}
& \text { Assume } q^{\prime} \in\left\{q \in Q_{H} \mid\left(\exists \sigma \in \Sigma_{h u} \cup \Sigma_{A}\right) \delta_{H}(q, \sigma) \models \neg \operatorname{PHC}\left(\mathcal{P}_{h a}\right)\right\} . \\
& \begin{array}{l}
\Rightarrow q^{\prime} \in\left\{q \in Q_{H} \mid\right. \\
\left.\quad\left(\exists \sigma \in \Sigma_{h u} \cup \Sigma_{A}\right) \delta_{H}(q, \sigma) \models T R\left(\mathcal{G}_{H}, \neg \mathcal{P}_{h a} \vee \operatorname{pr}\left(\operatorname{Bad}_{H}\right), \Sigma_{h u} \cup \Sigma_{A}\right)\right\}
\end{array}
\end{aligned}
$$


Master Thesis - R. Song - McMaster - Computing and Software

$$
\begin{aligned}
& \Rightarrow\left(\exists \sigma \in \Sigma_{h u} \cup \Sigma_{A}\right) \delta_{H}\left(q^{\prime}, \sigma\right) \models T R\left(\mathcal{G}_{H}, \neg \mathcal{P}_{h a} \vee \operatorname{pr}\left(\operatorname{Bad}_{H}\right), \Sigma_{h u} \cup \Sigma_{A}\right) \\
& \Rightarrow q^{\prime} \models T R\left(\mathcal{G}_{H}, \neg \mathcal{P}_{h a} \vee \operatorname{pr}\left(\operatorname{Bad}_{H}\right), \Sigma_{h u} \cup \Sigma_{A}\right), \quad \text { by definition of } T R \\
& \Rightarrow q^{\prime} \models \neg \operatorname{PHC}\left(\mathcal{P}_{h a}\right),
\end{aligned}
$$

which contradicts with (1): the assumption $q^{\prime} \models \operatorname{PHC}\left(\mathcal{P}_{h a}\right)$, so $q^{\prime} \notin\left\{q \in Q_{H} \mid\left(\exists \sigma \in \Sigma_{h u} \cup \Sigma_{A}\right) \delta_{H}(q, \sigma) \models \neg \operatorname{PHC}\left(\mathcal{P}_{h a}\right)\right\}$.

2. Show that $\operatorname{PHC}\left(\mathcal{P}_{h a}\right)$ is the greatest fixpoint of $\Gamma_{H I C}$, i.e.

$$
\left(\forall P^{\prime} \in S u b\left(\mathcal{P}_{h a}\right)\right) P^{\prime}=\Gamma_{H I C}\left(P^{\prime}\right) \Rightarrow P^{\prime} \preceq \operatorname{PHC}\left(\mathcal{P}_{h a}\right) .
$$

Let $P^{\prime} \in \operatorname{Sub}\left(\mathcal{P}_{h a}\right)$. Assume $P^{\prime}=\Gamma_{H I C}\left(P^{\prime}\right)$.

Must show this implies $P^{\prime} \preceq \operatorname{PHC}\left(\mathcal{P}_{h a}\right)$.

We show this by contradiction.

$$
\begin{aligned}
& \text { Assume } P^{\prime} \npreceq \operatorname{PHC}\left(\mathcal{P}_{h a}\right) \\
& \Rightarrow\left(\exists q^{\prime} \models P^{\prime}\right) q^{\prime} \not \models \operatorname{PHC}\left(\mathcal{P}_{h a}\right) \\
& \Rightarrow\left(\exists q^{\prime} \models P^{\prime}\right) q^{\prime} \models T R\left(\mathcal{G}_{H}, \neg \mathcal{P}_{h a} \vee \operatorname{pr}\left(\operatorname{Bad}_{H}\right), \Sigma_{h u} \cup \Sigma_{A}\right)
\end{aligned}
$$

By the definition of $\Gamma_{H I C}$, we have

$$
\begin{aligned}
\Gamma_{H I C}\left(P^{\prime}\right) & =P^{\prime}-\operatorname{pr}\left(\operatorname{Bad}_{H} \cup\left\{q \in Q \mid\left(\exists \sigma \in \Sigma_{h u} \cup \Sigma_{A}\right) \delta_{H}(q, \sigma) \models \neg P^{\prime}\right\}\right) \\
& =P^{\prime} \wedge \neg \operatorname{pr}\left(\operatorname{Bad}_{H} \cup\left\{q \in Q \mid\left(\exists \sigma \in \Sigma_{h u} \cup \Sigma_{A}\right) \delta_{H}(q, \sigma) \models \neg P^{\prime}\right\}\right) \\
& =P^{\prime} \wedge \neg \operatorname{pr}\left(\operatorname{Bad}_{H}\right) \wedge \neg p r\left(\left\{q \in Q \mid\left(\exists \sigma \in \Sigma_{h u} \cup \Sigma_{A}\right) \delta_{H}(q, \sigma) \models \neg P^{\prime}\right\}\right) \\
& =P^{\prime}-\operatorname{pr}\left(\operatorname{Bad}_{H}\right)-\operatorname{pr}\left(\left\{q \in Q \mid\left(\exists \sigma \in \Sigma_{h u} \cup \Sigma_{A}\right) \delta_{H}(q, \sigma) \models \neg P^{\prime}\right\}\right)(4)
\end{aligned}
$$

From (3), by the definition of $T R$, one of the following two conditions must be satisfied.

(a) $q^{\prime}=\neg \mathcal{P}_{h a} \vee \operatorname{pr}\left(\operatorname{Bad}_{H}\right)$

By (3), we also have $q^{\prime} \models P^{\prime}$ 


$$
\begin{aligned}
& \text { Master Thesis }-R \text {. Song }- \text { McMaster }- \text { Computing and Software } \\
& \Rightarrow q^{\prime}=\mathcal{P}_{h a}, \quad \text { as } P^{\prime} \in \operatorname{Sub}\left(\mathcal{P}_{h a}\right) \\
& \Rightarrow q^{\prime}=\neg\left(\neg \mathcal{P}_{h a}\right) \\
& \Rightarrow q^{\prime} \not \models \neg \mathcal{P}_{h a} \\
& \Rightarrow q^{\prime} \models \operatorname{pr}\left(\operatorname{Bad}_{H}\right), \quad \text { as } q^{\prime} \models \neg \mathcal{P}_{h a} \vee \operatorname{pr}\left(\operatorname{Bad}_{H}\right) .
\end{aligned}
$$

As $q^{\prime} \models \operatorname{pr}\left(\operatorname{Bad}_{H}\right)$ and $\Gamma_{H I C}\left(P^{\prime}\right)=P^{\prime}$, by $(4)$, we thus have $q^{\prime} \not \models P^{\prime}$, which contradicts $q^{\prime} \models P^{\prime}$ in $(3)$.

(b) $q^{\prime} \not \models \neg \mathcal{P}_{h a} \vee \operatorname{pr}\left(\operatorname{Bad}_{H}\right)$

$$
\begin{gathered}
\Rightarrow\left(\exists s \in\left(\Sigma_{h u} \cup \Sigma_{A}\right)^{+}\right) \delta_{H}\left(q^{\prime}, s\right) \models \neg \mathcal{P}_{h a} \vee \operatorname{pr}\left(\operatorname{Bad}_{H}\right), \text { by definition of } T R \\
\Rightarrow(\exists k \in\{1,2, \ldots\})\left(\exists q_{1}, q_{2}, \ldots, q_{k+1} \in Q_{H}\right)\left(\exists \sigma_{1}, \sigma_{2}, \ldots, \sigma_{k} \in \Sigma_{h u} \cup \Sigma_{A}\right) \\
q_{1}:=q^{\prime} \\
q_{k+1} \models \neg \mathcal{P}_{h a} \vee \operatorname{pr}\left(\operatorname{Bad}_{H}\right) \\
\delta_{H}\left(q_{i}, \sigma_{i}\right)=q_{i+1}, i=1,2, \ldots, k \\
s=\sigma_{1} \sigma_{2} \ldots \sigma_{k}
\end{gathered}
$$

First,we show that $q_{k+1} \models \neg P^{\prime}$.

By (5), we have $q_{k+1} \models \neg \mathcal{P}_{h a} \vee \operatorname{pr}\left(\operatorname{Bad}_{H}\right)$.

If $q_{k+1} \models \neg \mathcal{P}_{h a}$, as $P^{\prime} \preceq \mathcal{P}_{h a}$, we have $\neg \mathcal{P}_{h a} \preceq \neg P^{\prime}$; thus $q_{k+1} \models \neg P^{\prime}$.

If $q_{k+1} \models \operatorname{pr}\left(\operatorname{Bad}_{H}\right)$, by (4) and assumption $\Gamma_{H I C}\left(P^{\prime}\right)=P^{\prime}$, we also have $q_{k+1}=\neg P^{\prime}$.

Thus, in both cases we have $q_{k+1} \models \neg P^{\prime}$.

Now we show that $q_{k} \models \neg P^{\prime}$.

By (5), we know $\delta_{H}\left(q_{k}, \sigma_{k}\right)=q_{k+1}$. By (6), we know $q_{k+1} \models \neg P^{\prime}$. By (4) and assumption $\Gamma_{H I C}\left(P^{\prime}\right)=P^{\prime}$, we thus have $q_{k} \models \neg P^{\prime}$. 
Master Thesis - R. Song - McMaster - Computing and Software

Similarly, we can prove that $q_{i} \models \neg P^{\prime}$ for $i=k-1, \ldots, 1$. As $q_{1}=q^{\prime}$ by (5), we have $q^{\prime} \models \neg P^{\prime}$, which contradicts $q^{\prime} \models P^{\prime}$ in (3).

As Part (a) and Part (b) both will cause contradictions, we have $P^{\prime} \preceq \operatorname{PHIC}\left(\mathcal{P}_{h a}\right)$.

\subsubsection{Computing $\Omega_{H N B}\left(L\left(\mathcal{G}_{H}, P\right)\right)$}

For a predicate $P \in \operatorname{Pred}\left(Q_{H}\right)$, in the previous section, we have found a way to compute $\sup \mathcal{C}_{H}\left(L\left(\mathcal{G}_{H}, P\right)\right)$. To complete the computation for the function $\Omega_{H}$, we now show the method to compute $\Omega_{H N B}\left(L\left(\mathcal{G}_{H}, P\right)\right)$, i.e. the method to compute $L_{m}\left(\mathcal{G}_{H}\right) \cap L\left(\mathcal{G}_{H}, P\right)$.

Proposition 4.10. For system $\boldsymbol{\Phi}$, the following holds:

$$
\left(\forall P \in \operatorname{Pred}\left(Q_{H}\right)\right) L_{m}\left(\mathcal{G}_{H}\right) \cap L\left(\mathcal{G}_{H}, P\right)=L_{m}\left(\mathcal{G}_{H}, C R\left(\mathcal{G}_{H}, P\right)\right)
$$

proof:

Let $P \in \operatorname{Pred}\left(Q_{H}\right)$.

1. Show that $L_{m}\left(\mathcal{G}_{H}, C R\left(\mathcal{G}_{H}, P\right)\right) \subseteq L_{m}\left(\mathcal{G}_{H}\right) \cap L\left(\mathcal{G}_{H}, P\right)$

Clearly, $L_{m}\left(\mathcal{G}_{H}, C R\left(\mathcal{G}_{H}, P\right)\right) \subseteq L_{m}\left(\mathcal{G}_{H}\right)$.

By the definition of $C R\left(\mathcal{G}_{H},.\right)$, we know $C R\left(\mathcal{G}_{H}, P\right) \preceq P$.

$$
\begin{aligned}
& \Rightarrow L_{m}\left(\mathcal{G}_{H}, C R\left(\mathcal{G}_{H}, P\right)\right) \subseteq L_{m}\left(\mathcal{G}_{H}, P\right) \\
& \Rightarrow L_{m}\left(\mathcal{G}_{H}, C R\left(\mathcal{G}_{H}, P\right)\right) \subseteq L\left(\mathcal{G}_{H}, P\right)
\end{aligned}
$$

By (1) and (2), $L_{m}\left(\mathcal{G}_{H}, C R\left(\mathcal{G}_{H}, P\right)\right) \subseteq L_{m}\left(\mathcal{G}_{H}\right) \cap L\left(\mathcal{G}_{H}, P\right)$.

2. Show that $L_{m}\left(\mathcal{G}_{H}\right) \cap L\left(\mathcal{G}_{H}, P\right) \subseteq L_{m}\left(\mathcal{G}_{H}, C R\left(\mathcal{G}_{H}, P\right)\right)$

Let $s \in L_{m}\left(\mathcal{G}_{H}\right) \cap L\left(\mathcal{G}_{H}, P\right)$. 


\section{Master Thesis - R. Song - McMaster-Computing and Software}

Must show implies $s \in L_{m}\left(\mathcal{G}_{H}, C R\left(\mathcal{G}_{H}, P\right)\right)$.

Sufficient to show $s \in L\left(\mathcal{G}_{H}, C R\left(\mathcal{G}_{H}, P\right)\right)$ and $\delta_{H}\left(q_{H_{0}}, s\right) \in Q_{H_{m}}$, where $Q_{H_{m}}$ is the marker state set of $\mathcal{G}_{H}$ as defined in Section 4.1.

From (3), we know $s \in L_{m}\left(\mathcal{G}_{H}\right)$

$\Rightarrow \delta_{H}\left(q_{H_{0}}, s\right) \in Q_{H_{m}}$

From (3), we also know $s \in L\left(\mathcal{G}_{H}, P\right)$

$$
\begin{gathered}
\Rightarrow(\exists n \in\{0,1, \ldots\})\left(\exists q_{0}, q_{1}, \ldots, q_{n} \in Q_{H}\right)\left(\exists \sigma_{0}, \sigma_{1}, \ldots, \sigma_{n-1} \in \Sigma_{I H}\right) \\
q_{0}=q_{H_{0}} \quad\left(q_{H_{0}} \text { is the initial state of } \mathcal{G}_{H}\right) \\
q_{i} \models P, \quad i=0,1, \ldots, n \\
\delta_{H}\left(q_{i}, \sigma_{i}\right)=q_{i+1}, \quad i=0,1, \ldots, n-1 \\
s=\sigma_{0} \sigma_{1} \cdots \sigma_{n-1} \\
q_{n}=\delta_{H}\left(q_{H_{0}}, s\right) .
\end{gathered}
$$

By (4) and (5), we know $(\forall i \in\{0,1, \ldots, n\}) q_{i} \models C R\left(\mathcal{G}_{H}, P\right)$

$\Rightarrow s \in L\left(\mathcal{G}_{H}, C R\left(\mathcal{G}_{H}, P\right)\right)$, as $q_{0}=q_{H_{0}}$ by $(5)$

\subsubsection{The Algorithm to Compute $\sup \mathcal{C}_{H}\left(L_{m}\left(\mathcal{G}_{H}\right)\right)$}

It is time to put everything for the high-level together and to obtain our algorithm.

Definition 4.9. For system $\boldsymbol{\Phi}$, define the function $\Gamma_{H}: \operatorname{Pred}\left(Q_{H}\right) \rightarrow \operatorname{Pred}\left(Q_{H}\right)$ according to

$$
\left(\forall P \in \operatorname{Pred}\left(Q_{H}\right)\right) \quad \Gamma_{H}(P):=C R\left(\mathcal{G}_{H}, \operatorname{PHC}(P)\right)
$$


Master Thesis - R. Song - McMaster - Computing and Software

As PHIC (by Lemma 4.5) and the predicate transformer $C R\left(\mathcal{G}_{H},.\right)$ are monotone, the function $\Gamma_{H}$ is monotone.

Lemma 4.6. For system $\boldsymbol{\Phi}$, the following holds:

$$
\left(\forall P \in \operatorname{Pred}\left(Q_{H}\right)\right) \overline{L_{m}\left(\mathcal{G}_{H}, C R\left(\mathcal{G}_{H}, P\right)\right)}=L\left(\mathcal{G}_{H}, C R\left(\mathcal{G}_{H}, P\right)\right)
$$

\section{proof:}

Let $P \in \operatorname{Pred}\left(Q_{H}\right)$.

$\overline{L_{m}\left(\mathcal{G}_{H}, C R\left(\mathcal{G}_{H}, P\right)\right)} \subseteq L\left(\mathcal{G}_{H}, C R\left(\mathcal{G}_{H}, P\right)\right)$ is automatic.

We now show $L\left(\mathcal{G}_{H}, C R\left(\mathcal{G}_{H}, P\right)\right) \subseteq \overline{L_{m}\left(\mathcal{G}_{H}, C R\left(\mathcal{G}_{H}, P\right)\right)}$.

Let $s \in L\left(\mathcal{G}_{H}, C R\left(\mathcal{G}_{H}, P\right)\right)$.

Must show this implies $s \in \overline{L_{m}\left(\mathcal{G}_{H}, C R\left(\mathcal{G}_{H}, P\right)\right)}$.

Sufficient to show $\left(\exists s^{\prime} \in \Sigma_{I H}^{*}\right) s s^{\prime} \in L_{m}\left(\mathcal{G}_{H}, C R\left(\mathcal{G}_{H}, P\right)\right)$.

By (1), we know $s \in L\left(\mathcal{G}_{H}, C R\left(\mathcal{G}_{H}, P\right)\right)$

$$
\begin{gathered}
\Rightarrow(\exists k \in\{0,1, \ldots\})\left(\exists q_{0}, q_{1}, \ldots, q_{k} \in Q_{H}\right)\left(\exists \sigma_{0}, \sigma_{1}, \ldots, \sigma_{k-1} \in \Sigma_{I H}\right) \\
q_{0}=q_{H_{0}} \\
q_{i}=C R\left(\mathcal{G}_{H}, P\right), \quad i=0,1, \ldots, k \\
\delta_{H}\left(q_{i}, \sigma_{i}\right)=q_{i+1}, \quad i=0,1, \ldots, k-1 \\
s=\sigma_{0} \sigma_{1} \cdots \sigma_{k-1} .
\end{gathered}
$$

As $q_{k} \in C R\left(\mathcal{G}_{H}, P\right)$, it follows that

$$
\begin{gathered}
((\exists n \in\{0,1, \ldots\}) n \geq k)\left(\exists q_{k+1}, \ldots, q_{n} \in Q_{H}\right)\left(\exists \sigma_{k}, \sigma_{k+1}, \ldots, \sigma_{n-1} \in \Sigma_{I H}\right) \\
q_{n} \in Q_{H_{m}} \\
q_{i} \models C R\left(\mathcal{G}_{H}, P\right), \quad i=k, k+1, \ldots, n \\
\delta_{H}\left(q_{i}, \sigma_{i}\right)=q_{i+1}, \quad i=k, k+1, \ldots, n-1
\end{gathered}
$$


Let $s^{\prime}=\sigma_{k} \sigma_{k+1} \ldots \sigma_{n-1}$. Combining (2) and (3), we thus have

$$
\begin{gathered}
(\exists n \in\{0,1, \ldots\})\left(\exists q_{0}, q_{1}, \ldots, q_{n} \in Q_{H}\right)\left(\exists \sigma_{0}, \sigma_{1}, \ldots, \sigma_{n-1} \in \Sigma_{I H}\right) \\
q_{0}=q_{H_{0}} \\
q_{n} \in Q_{H_{m}} \\
q_{i} \models C R\left(\mathcal{G}_{H}, P\right), \quad i=0,1, \ldots, n \\
\delta_{H}\left(q_{i}, \sigma_{i}\right)=q_{i+1}, \quad i=0,1, \ldots, n-1 \\
s s^{\prime}=\sigma_{0} \sigma_{1} \cdots \sigma_{n-1} \\
\Rightarrow s s^{\prime} \in L_{m}\left(\mathcal{G}_{H}, C R\left(\mathcal{G}_{H}, P\right)\right)
\end{gathered}
$$

Lemma 4.7. For system $\boldsymbol{\Phi}$, the following holds:

$$
\left(\forall P \in \operatorname{Pred}\left(Q_{H}\right)\right)(\forall i \in\{1,2, \ldots\}) \overline{L_{m}\left(\mathcal{G}_{H}, \Gamma_{H}^{i}(P)\right)}=L\left(\mathcal{G}_{H}, \Gamma_{H}^{i}(P)\right)
$$

\section{proof:}

Let $i \in\{1,2, \ldots\}$, and $P \in \operatorname{Pred}\left(Q_{H}\right)$. By definition of $\Gamma_{H}$, we have

$$
\begin{aligned}
\overline{L_{m}\left(\mathcal{G}_{H}, \Gamma_{H}^{i}(P)\right)} & =\overline{L_{m}\left(\mathcal{G}_{H}, \Gamma_{H}\left(\Gamma_{H}^{i-1}(P)\right)\right)} \\
& =\overline{L_{m}\left(\mathcal{G}_{H}, C R\left(\mathcal{G}_{H}, \operatorname{PHC}\left(\Gamma_{H}^{i-1}(P)\right)\right)\right.}, \quad \text { by definition of } \Gamma_{H} \\
& =\overline{L_{m}\left(\mathcal{G}_{H}, C R\left(\mathcal{G}_{H}, P^{\prime}\right)\right)} \quad \text { by letting } P^{\prime}=\operatorname{PHC}\left(\Gamma_{H}^{i-1}(P)\right) \\
& =L\left(\mathcal{G}_{H}, C R\left(\mathcal{G}_{H}, P^{\prime}\right)\right), \quad \text { by Lemma } 4.6 \\
& =L\left(\mathcal{G}_{H}, C R\left(\mathcal{G}_{H}, \operatorname{PHC}\left(\Gamma_{H}^{i-1}(P)\right)\right)\right) \\
& =L\left(\mathcal{G}_{H}, \Gamma_{H}^{i}(P)\right), \quad \text { by definition of } \Gamma_{H}
\end{aligned}
$$

Proposition 4.11. For system $\mathbf{\Phi}$, the following two points hold:

1. $\left(\forall P \in \operatorname{Pred}\left(Q_{H}\right)\right) \Omega_{H}\left(L\left(\mathcal{G}_{H}, P\right)\right)=L_{m}\left(\mathcal{G}_{H}, \Gamma_{H}(P)\right)$

2. $\left(\forall P \in \operatorname{Pred}\left(Q_{H}\right)\right)(\forall i \in\{1,2, \ldots\}) \Omega_{H}^{i}\left(L\left(\mathcal{G}_{H}, P\right)\right)=L_{m}\left(\mathcal{G}_{H}, \Gamma_{H}^{i}(P)\right)$ 
Master Thesis - R. Song-McMaster - Computing and Software

proof:

1. Show that $\left(\forall P \in \operatorname{Pred}\left(Q_{H}\right)\right) \Omega_{H}\left(L\left(\mathcal{G}_{H}, P\right)\right)=L_{m}\left(\mathcal{G}_{H}, \Gamma_{H}(P)\right)$

Let $P \in \operatorname{Pred}\left(Q_{H}\right)$.

$$
\begin{aligned}
\Omega_{H}\left(L\left(\mathcal{G}_{H}, P\right)\right) & =L_{m}\left(\mathcal{G}_{H}\right) \cap \sup \mathcal{C}_{H}\left(\overline{L\left(\mathcal{G}_{H}, P\right)}\right), \quad \text { by definition of } \Omega_{H} \\
& =L_{m}\left(\mathcal{G}_{H}\right) \cap \sup \mathcal{C}_{H}\left(L\left(\mathcal{G}_{H}, P\right)\right) \\
& =L_{m}\left(\mathcal{G}_{H}\right) \cap L\left(\mathcal{G}_{H}, \operatorname{PHC}(P)\right), \quad \text { by Proposition } 4.9 \\
& =L_{m}\left(\mathcal{G}_{H}, C R\left(\mathcal{G}_{H}, \operatorname{PHC}(P)\right)\right), \quad \text { by Proposition } 4.10 \\
& =L_{m}\left(\mathcal{G}_{H}, \Gamma_{H}(P)\right), \quad \text { by definition of } \Gamma_{H}
\end{aligned}
$$

2. Show that $\left(\forall P \in \operatorname{Pred}\left(Q_{H}\right)\right)(\forall i \in\{1,2, \ldots\}) \Omega_{H}^{i}\left(L\left(\mathcal{G}_{H}, P\right)\right)=L_{m}\left(\mathcal{G}_{H}, \Gamma_{H}^{i}(P)\right)$

Let $P \in \operatorname{Pred}\left(Q_{H}\right)$. We prove this by induction.

(a) Base Case: $i=1$

By Point 1 of this proposition, we know $\Omega_{H}\left(L\left(\mathcal{G}_{H}, P\right)\right)=L_{m}\left(\mathcal{G}_{H}, \Gamma_{H}(P)\right)$

$$
\Rightarrow \Omega_{H}^{1}\left(L\left(\mathcal{G}_{H}, P\right)\right)=L_{m}^{1}\left(\mathcal{G}_{H}, \Gamma_{H}(P)\right)
$$

(b) Inductive step.

Let $k \in\{1,2, \ldots\}$. Assume $\Omega_{H}^{k}\left(L\left(\mathcal{G}_{H}, P\right)\right)=L_{m}\left(\mathcal{G}_{H}, \Gamma_{H}^{k}(P)\right)$. Must show

$$
\begin{aligned}
\Omega_{H}^{k+1}\left(L\left(\mathcal{G}_{H}, P\right)\right) & =L_{m}\left(\mathcal{G}_{H}, \Gamma_{H}^{k+1}(P)\right) . \\
\Omega_{H}^{k+1}\left(L\left(\mathcal{G}_{H}, P\right)\right) & =\Omega_{H}\left(\Omega_{H}^{k}\left(L\left(\mathcal{G}_{H}, P\right)\right)\right) \\
& =\Omega_{H}\left(L_{m}\left(\mathcal{G}_{H}, \Gamma_{H}^{k}(P)\right)\right), \quad \text { by inductive assumption } \\
& =L_{m}\left(\mathcal{G}_{H}\right) \cap \sup \mathcal{C}_{H}\left(\overline{L_{m}\left(\mathcal{G}_{H}, \Gamma_{H}^{k}(P)\right)}\right), \quad \text { by definition of } \Omega_{H} \\
& =L_{m}\left(\mathcal{G}_{H}\right) \cap \sup \mathcal{C}_{H}\left(L\left(\mathcal{G}_{H}, \Gamma_{H}^{k}(P)\right)\right), \quad \text { by Lemma } 4.7 \\
& =\Omega_{H}\left(L\left(\mathcal{G}_{H}, \Gamma_{H}^{k}(P)\right)\right), \quad \text { by definition of } \Omega_{H} \\
& =L_{m}\left(\mathcal{G}_{H}, \Gamma_{H}^{k+1}(P)\right), \quad \text { by Point } 1 \text { of this proposition. }
\end{aligned}
$$




\section{Master Thesis - R. Song-McMaster-Computing and Software}

Theorem 4.1. For system $\boldsymbol{\Phi}$, the following two points hold:

1. There exists $k \in\{0,1, \ldots\}$ such that $k \leq\left|Q_{H}\right|$ and $\Gamma_{H}^{k}$ (true) is the greatest fixpoint of the function $\Gamma_{H}$ with respect to $\left(\operatorname{Pred}\left(Q_{H}\right), \preceq\right)$.

2. $\sup \mathcal{C}_{H}\left(L_{m}\left(\mathcal{G}_{H}\right)\right)=\sup \mathcal{C}_{H}\left(L_{m}\left(\mathcal{G}_{H}\right.\right.$, true $\left.)\right)=L_{m}\left(\mathcal{G}_{H}, \Gamma_{H}^{k}(\right.$ true $\left.)\right)$.

\section{proof:}

1. Show that there exists $k \in\{0,1, \ldots\}$ such that $k \leq\left|Q_{H}\right|$ and $\Gamma_{H}^{k}($ true $)$ is the greatest fixpoint of the function $\Gamma_{H}$ with respect to $\left(\operatorname{Pred}\left(Q_{H}\right), \preceq\right)$.

As $\Gamma_{H}$ is monotone and $\left|Q_{H}\right|$ is assumed to be finite (Section 4.1), we know immediately from Proposition 2.3 that there exists $k \in\{0,1, \ldots\}$ such that $k \leq\left|Q_{H}\right|$ and $\Gamma_{H}^{k}($ true $)$ is the greatest fixpoint of the function $\Gamma_{H}$.

2. Show that $\sup \mathcal{C}_{H}\left(L_{m}\left(\mathcal{G}_{H}\right)\right)=\sup \mathcal{C}_{H}\left(L_{m}\left(\mathcal{G}_{H}\right.\right.$, true $\left.)\right)=L_{m}\left(\mathcal{G}_{H}, \Gamma_{H}^{k}(\right.$ true $\left.)\right)$. $\sup \mathcal{C}_{H}\left(L_{m}\left(\mathcal{G}_{H}\right)\right)=\sup \mathcal{C}_{H}\left(L_{m}\left(\mathcal{G}_{H}\right.\right.$, true $\left.)\right)$ is automatic as $L_{m}\left(\mathcal{G}_{H}\right)=L_{m}\left(\mathcal{G}_{H}\right.$, true $)$.

(a) Show that $\sup \mathcal{C}_{H}\left(L_{m}\left(\mathcal{G}_{H}\right.\right.$, true $\left.)\right) \subseteq L_{m}\left(\mathcal{G}_{H}, \Gamma_{H}^{k}(\right.$ true $\left.)\right)$

By Proposition 2.3, we know that

$$
\begin{aligned}
& (\forall i \in\{1,2, \ldots\}) i>k \Rightarrow L_{m}\left(\mathcal{G}_{H}, \Gamma_{H}^{i}(\text { true })\right)=L_{m}\left(\mathcal{G}_{H}, \Gamma_{H}^{k}(\text { true })\right) \\
& \Rightarrow \lim _{i \rightarrow \infty} L_{m}\left(\mathcal{G}_{H}, \Gamma_{H}^{i}(\text { true })\right)=L_{m}\left(\mathcal{G}_{H}, \Gamma_{H}^{k}(\text { true })\right) \\
& \Rightarrow \lim _{i \rightarrow \infty} \Omega_{H}^{i}\left(L\left(\mathcal{G}_{H}, \text { true }\right)\right)=L_{m}\left(\mathcal{G}_{H}, \Gamma_{H}^{k}(\text { true })\right), \quad \text { by Proposition } 4.11 \\
& \Rightarrow \sup \mathcal{C}_{H}\left(L_{m}\left(\mathcal{G}_{H}, \text { true }\right)\right) \subseteq L_{m}\left(\mathcal{G}_{H}, \Gamma_{H}^{k}(\text { true })\right), \quad \text { by Proposition } 4.3
\end{aligned}
$$

(b) Show that $L_{m}\left(\mathcal{G}_{H}, \Gamma_{H}^{k}(\right.$ true $\left.)\right) \subseteq \sup \mathcal{C}_{H}\left(L_{m}\left(\mathcal{G}_{H}\right.\right.$, true $\left.)\right)$.

Based on the value of $k$, we show this in two cases: 
Master Thesis - R. Song - McMaster - Computing and Software

- Case 1: $k \in\{1,2, \ldots\}$

By Point 1 of this theorem and Proposition 2.3, we have

$$
\begin{aligned}
& \Gamma_{H}^{k}(\text { true })=\Gamma_{H}^{k+1}(\text { true }) \\
& \Rightarrow L_{m}\left(\mathcal{G}_{H}, \Gamma_{H}^{k}(\text { true })\right)=L_{m}\left(\mathcal{G}_{H}, \Gamma_{H}^{k+1}(\text { true })\right) \\
& \Rightarrow L_{m}\left(\mathcal{G}_{H}, \Gamma_{H}^{k}(\text { true })\right)=\Omega_{H}^{k+1}\left(L\left(\mathcal{G}_{H}, \text { true }\right)\right), \quad \text { by Proposition } 4.11 \\
& \Rightarrow L_{m}\left(\mathcal{G}_{H}, \Gamma_{H}^{k}(\text { true })\right)=\Omega_{H}\left(\Omega_{H}^{k}\left(L\left(\mathcal{G}_{H}, \text { true }\right)\right)\right) \\
& \Rightarrow L_{m}\left(\mathcal{G}_{H}, \Gamma_{H}^{k}(\text { true })\right)=\Omega_{H}\left(L_{m}\left(\mathcal{G}_{H}, \Gamma_{H}^{k}(\text { true })\right)\right),
\end{aligned}
$$

by Proposition 4.11 and $k \in\{1,2, \ldots\}$

- Case 2: $k=0$

If $\Gamma_{H}^{0}($ true $)$ is the greatest fixpoint of $\Gamma_{H}$, then by Proposition 2.3 we know that

$$
\Gamma_{H}^{1}(\text { true })=\Gamma_{H}^{0}(\text { true })
$$

so $\Gamma_{H}^{1}($ true $)$ is also the greatest fixpoint of $\Gamma_{H}$.

$\Rightarrow L_{m}\left(\mathcal{G}_{H}, \Gamma_{H}^{1}(\right.$ true $\left.)\right)=\Omega_{H}\left(L_{m}\left(\mathcal{G}_{H}, \Gamma_{H}^{1}(\right.\right.$ true $\left.\left.)\right)\right), \quad$ by Case 1

$\Rightarrow L_{m}\left(\mathcal{G}_{H}, \Gamma_{H}^{0}(\right.$ true $\left.)\right)=\Omega_{H}\left(L_{m}\left(\mathcal{G}_{H}, \Gamma_{H}^{0}(\right.\right.$ true $\left.\left.)\right)\right), \quad$ by $(1)$

From Case 1 and Case 2, we know that $L_{m}\left(\mathcal{G}_{H}, \Gamma_{H}^{k}(\right.$ true $\left.)\right)$ is always a fixpoint of the function $\Omega_{H}$.

By Proposition 4.2, $\sup \mathcal{C}_{H}\left(L_{m}\left(\mathcal{G}_{H}\right.\right.$, true $\left.)\right)$ is the greatest fixpoint of $\Omega_{H}$, so $L_{m}\left(\mathcal{G}_{H}, \Gamma_{H}^{k}(\right.$ true $\left.)\right) \subseteq \sup \mathcal{C}_{H}\left(L_{m}\left(\mathcal{G}_{H}\right.\right.$, true $\left.)\right)$.

Corollary 4.2. For system $\boldsymbol{\Phi}$, let $\mathbf{S}_{H}$ be a DES defined over event set $\Sigma_{I H}$ with $L\left(\mathbf{S}_{H}\right)=L\left(\mathcal{G}_{H}, \Gamma_{H}^{k}(\right.$ true $\left.)\right)$ and $L_{m}\left(\mathbf{S}_{H}\right)=L_{m}\left(\mathcal{G}_{H}, \Gamma_{H}^{k}(\right.$ true $\left.)\right)$, where $k \in\{0,1, \ldots\}$ and $\Gamma_{H}^{k}($ true $)$ is the greatest fixpoint of $\Gamma_{H}$ with respect to $\left(\operatorname{Pred}\left(Q_{H}\right), \preceq\right)$. Then for the $n^{\text {th }}$ degree parallel interface system composed of $\mathbf{G}_{H}^{p}, \mathbf{G}_{L_{1}}^{p}, \ldots, \mathbf{G}_{L_{n}}^{p}, \mathbf{G}_{I_{1}}, \ldots$, 


\section{Master Thesis - R. Song-McMaster-Computing and Software}

$\mathbf{G}_{I_{n}}, \mathbf{S}_{H}, \mathbf{E}_{L_{1}}, \ldots, \mathbf{E}_{L_{n}}$ with respect to the alphabet partition in Equation 3.1, $\mathbf{S}_{H}$ is a high-level proper supervisor.

\section{proof:}

We first note that by Theorem 4.2, an appropriate $k \in\{0,1, \ldots\}$ exists such that $\Gamma_{H}^{k}($ true $)$ is the greatest fixpoint of $\Gamma_{H}$ with respect to $\left(\operatorname{Pred}\left(Q_{H}\right), \preceq\right)$.

1. Show that $\overline{L_{m}\left(\mathbf{S}_{H} \times \mathbf{G}_{H}^{p} \times \mathbf{G}_{I}^{h}\right)}=L\left(\mathbf{S}_{H} \times \mathbf{G}_{H}^{p} \times \mathbf{G}_{I}^{h}\right)$.

$$
\begin{aligned}
& L_{m}\left(\mathbf{S}_{H}\right)=L_{m}\left(\mathcal{G}_{H}, \Gamma_{H}^{k}(\text { true })\right) \\
& \Rightarrow L_{m}\left(\mathbf{S}_{H}\right) \subseteq L_{m}\left(\mathcal{G}_{H}\right) \\
& \Rightarrow L_{m}\left(\mathbf{S}_{H}\right) \subseteq L_{m}\left(\mathbf{E}_{H} \times \mathbf{G}_{H}^{p} \times \mathbf{G}_{I}^{h}\right) \\
& \Rightarrow L_{m}\left(\mathbf{S}_{H}\right) \subseteq L_{m}\left(\mathbf{G}_{H}^{p} \times \mathbf{G}_{I}^{h}\right) \\
& \Rightarrow L_{m}\left(\mathbf{S}_{H}\right)=L_{m}\left(\mathbf{S}_{H} \times \mathbf{G}_{H}^{p} \times \mathbf{G}_{I}^{h}\right)
\end{aligned}
$$

Similarly, we can show that $L\left(\mathbf{S}_{H}\right)=L\left(\mathbf{S}_{H} \times \mathbf{G}_{H}^{p} \times \mathbf{G}_{I}^{h}\right)$

If $k \in\{1,2, \ldots\}$ and $\Gamma_{H}^{k}($ true $)$ is the greatest fixpoint of $\Gamma_{H}$, then by Lemma 4.7 we know that $\overline{L_{m}\left(\mathbf{S}_{H}\right)}=L\left(\mathbf{S}_{H}\right)$.

If $k=0$ and $\Gamma_{H}^{0}($ true $)$ is the greatest fixpoint of $\Gamma_{H}$, then as $\left|Q_{H}\right|$ is assumed to be finite (Section 4.1), by Proposition 2.3 we know that $\Gamma_{H}^{1}($ true $)=\Gamma_{H}^{0}($ true) .

$\Rightarrow L\left(\mathbf{S}_{H}\right)=L\left(\mathcal{G}_{H}, \Gamma_{H}^{1}(\right.$ true $\left.)\right)$ and $L_{m}\left(\mathbf{S}_{H}\right)=L_{m}\left(\mathcal{G}_{H}, \Gamma_{H}^{1}(\right.$ true $\left.)\right)$

$\Rightarrow \overline{L_{m}\left(\mathbf{S}_{H}\right)}=L\left(\mathbf{S}_{H}\right), \quad$ by Lemma 4.7 .

Therefore, we always have

$$
\overline{L_{m}\left(\mathbf{S}_{H}\right)}=L\left(\mathbf{S}_{H}\right),
$$

when $k \in\{0,1, \ldots\}$ and $\Gamma_{H}^{k}($ true $)$ is the greatest fixpoint of $\Gamma_{H}$.

Combining (1), (2) and (3), we can conclude $\overline{L_{m}\left(\mathbf{S}_{H} \times \mathbf{G}_{H}^{p} \times \mathbf{G}_{I}^{h}\right)}=L\left(\mathbf{S}_{H} \times\right.$ $\left.\mathbf{G}_{H}^{p} \times \mathbf{G}_{I}^{h}\right)$. 
Master Thesis - R. Song - McMaster - Computing and Software

2. Show that $\mathbf{S}_{H}$ is high-level interface controllable.

By Part 1, Definition 3.8 and Definition 4.2, it is sufficient to show that $L_{m}\left(\mathbf{S}_{H} \times\right.$ $\left.\mathbf{G}_{H}^{p} \times \mathbf{G}_{I}^{h}\right)$ is high-level interface controllable.

From Theorem 4.1, we know $L_{m}\left(\mathbf{S}_{H}\right)$ is high-level interface controllable.

By (1), we can conclude $L_{m}\left(\mathbf{S}_{H} \times \mathbf{G}_{H}^{p} \times \mathbf{G}_{I}^{h}\right)$ is also high-level interface controllable.

The supervisor $\mathbf{S}_{H}$ can be built by trimming off states from $\mathcal{G}_{H}$ that do not satisfy $\Gamma_{H}^{k}($ true $)$. A trim supervisor DES $\mathbf{S}_{H}$ can be built by trimming states from $\mathcal{G}_{H}$ that do not satisfy $R\left(\mathcal{G}_{H}, \Gamma_{H}^{k}(\right.$ true $\left.)\right)$.

Algorithm 4.1 shows how to compute $\Gamma_{H}^{k}($ true $)$, where $k \in\{0,1, \ldots\}$ and $\Gamma_{H}^{k}($ true $)$ is the greatest fixpoint of $\Gamma_{H}$ with respect to $\left(\operatorname{Pred}\left(Q_{H}\right), \preceq\right)$. By Point 1 of Theorem 4.1, we know that the greatest fixpoint will be reached after finite number of iterations.

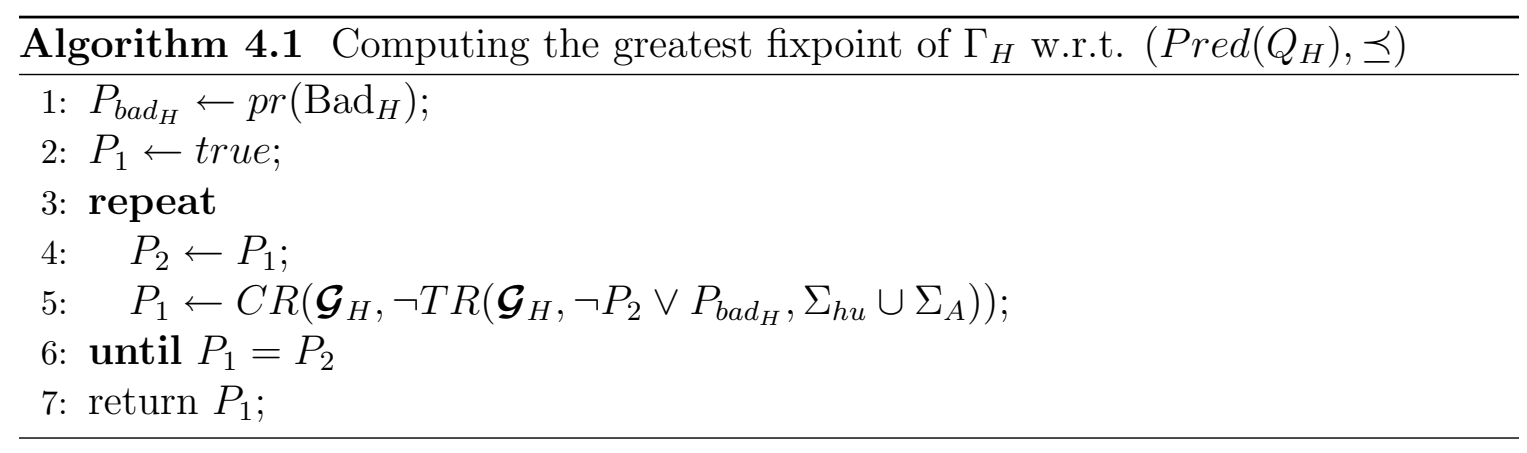

Line 5 computes $\Gamma_{H}\left(P_{2}\right)$, because $C R\left(\mathcal{G}_{H}, \neg T R\left(\mathcal{G}_{H}, \neg P_{2} \vee P_{\text {bad }}, \Sigma_{h u} \cup \Sigma_{A}\right)\right)=$ $C R\left(\mathcal{G}_{H}, \operatorname{PHC}\left(P_{2}\right)\right)=\Gamma_{H}\left(P_{2}\right)$. 


\section{Master Thesis - R. Song - McMaster - Computing and Software}

\subsection{Low-level Supervisor Synthesis}

In this section, we show how to synthesize a maximally permissible proper lowlevel supervisor for the $j^{\text {th }}$ low-level in system $\boldsymbol{\Phi}, j \in\{1, \ldots, n\}$. Unlike the high-level supervisor synthesis, the low-level supervisor synthesis is much more complicated. We first only discuss part of the conditions for the $j^{\text {th }}$ low-level.

\subsubsection{The $j^{\text {th }}$ Low-level P4 Interface Controllable Language}

Definition 4.10. Let $K \subseteq \Sigma_{I L_{j}}^{*}$ be a language. $K$ is $j^{\text {th }}$ low-level P4 interface controllable $\left(\mathrm{LPC}_{j}\right)$ with respect to system $\mathbf{\Phi}$ if the following conditions are satisfied:

1. $\bar{K} \Sigma_{l u_{j}} \cap L\left(\mathbf{G}_{L_{j}}^{p}\right) \subseteq \bar{K}$

2. $\bar{K} \Sigma_{R_{j}} \cap L\left(\mathbf{G}_{I_{j}}^{l}\right) \subseteq \bar{K}$

Clearly, the empty language $\emptyset$ is $j^{\text {th }}$ low-level P4 interface controllable and the definition is based on the language $\bar{K}$, so $K$ is $j^{\text {th }}$ low-level P4 interface controllable with respect to system $\boldsymbol{\Phi}$ iff $\bar{K}$ is $j^{\text {th }}$ low-level $\mathrm{P} 4$ interface controllable with respect to system $\boldsymbol{\Phi}$.

The low-level P4 interface controllable language definition is quite simple. Notice that the first condition here is identical to the first condition in the the high-level interface controllable language definition(Definition 4.2) by substituting $\Sigma_{I H}$ with $\Sigma_{I L_{j}}, \Sigma_{h u}$ with $\Sigma_{l u_{j}}, L\left(\mathbf{G}_{H}^{p} \times \mathbf{G}_{I}^{h}\right)$ with $L\left(\mathbf{G}_{L_{j}}^{p}\right)$. The second condition in Definition 4.2 is actually composed of $n$ sub-conditions. The second condition here is identical to the $j^{\text {th }}$ sub-conditions of the second condition in Definition 4.2 by substituting $\Sigma_{I H}$ with $\Sigma_{I L_{j}}, \Sigma_{A_{j}}$ with $\Sigma_{R_{j}}, L\left(\mathbf{G}_{I_{j}}^{h}\right)$ with $L\left(\mathbf{G}_{I_{j}}^{l}\right)$. Also notice that all the subconditions in the second condition of Definition 4.2 are independent. Therefore, the 


\section{Master Thesis - R. Song - McMaster - Computing and Software}

following propositions or lemmas are provided without detailed proofs, but with the corresponding propositions or lemmas in Section 4.2. The detailed proofs can be easily obtained by appropriate substitutions from the corresponding propositions or lemmas.

For an arbitrary language $E \subseteq \Sigma_{I L_{j}}^{*}$, we define the set of all sublanguages of $E$ that are $j^{\text {th }}$ low-level P4 interface controllable with respect to system $\boldsymbol{\Phi}$ as

$$
\begin{aligned}
\mathcal{L P C}_{j}(E):=\{K \subseteq E & \mid K \text { is } j^{\text {th }} \text { low-level } \\
& \mathrm{P} 4 \text { interface controllable with respect to system } \boldsymbol{\Phi} .\}
\end{aligned}
$$

Clearly, $\left(\mathcal{L P C}_{j}(E), \subseteq\right)$ is a poset. The following proposition shows that the supremum in this poset always exists in $\mathcal{L P C}_{j}(E)$.

Proposition 4.12. For system $\boldsymbol{\Phi}, \mathcal{L P C}_{j}(E)$ is nonempty and is closed under arbitrary unions. In particular, $\mathcal{L P C}_{j}(E)$ contains a (unique) supremal element, $\sup \mathcal{L P C}_{j}(E)=\cup\left\{K \mid K \in \mathcal{L P C}_{j}(E)\right\}$.

proof: Similar to Proposition 4.1.

From Proposition 4.12, we have $\sup \mathcal{L} \mathcal{P} \mathcal{C}_{j}(E) \subseteq E$, which means that we can compute $\sup \mathcal{L} \mathcal{P} \mathcal{C}_{j}(E)$ by removing strings from $E$.

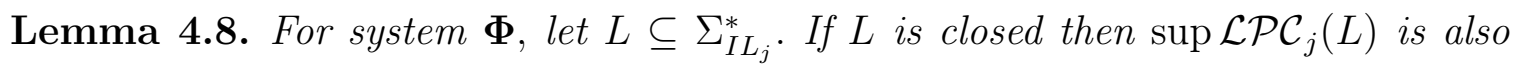
closed.

proof: Similar to Lemma 4.1.

Let $\mathcal{P}_{l a_{j}} \in \operatorname{Pred}\left(Q_{L_{j}}\right)$ be a given predicate, we now give our method to compute $\sup \mathcal{L} \mathcal{P} \mathcal{C}_{j}\left(L\left(\mathcal{G}_{L_{j}}, \mathcal{P}_{l a_{j}}\right)\right)$ 


\section{Master Thesis - R. Song-McMaster-Computing and Software}

Definition 4.11. For system $\boldsymbol{\Phi}$, let $\mathcal{P}_{l a_{j}} \in \operatorname{Pred}\left(Q_{L_{j}}\right)$ be a given predicate. Define the function $\Omega_{L P C_{j}}: \operatorname{Pwr}\left(L\left(\mathcal{G}_{L j}, \mathcal{P}_{l a_{j}}\right)\right) \rightarrow \operatorname{Pwr}\left(L\left(\mathcal{G}_{L_{j}}, \mathcal{P}_{l a_{j}}\right)\right)$ according to

$$
\begin{aligned}
& \left(\forall K \in \operatorname{Pwr}\left(L\left(\mathcal{G}_{L_{j}}, \mathcal{P}_{l a_{j}}\right)\right)\right) \\
& \quad \Omega_{L P C_{j}}(K):=\left\{s \in K \mid \overline{\{s\}} \Sigma_{l u_{j}} \cap L\left(\mathbf{G}_{L_{j}}^{p}\right) \subseteq \bar{K} \quad \&\left(\overline{\{s\}} \Sigma_{R_{j}} \cap L\left(\mathbf{G}_{I_{j}}^{l}\right) \subseteq \bar{K}\right)\right\}
\end{aligned}
$$

Clearly $\Omega_{L P C_{j}}$ is monotone. Note that $\Omega_{L P C_{j}}(K) \subseteq K \subseteq L\left(\mathcal{G}_{L_{j}}, \mathcal{P}_{l a_{j}}\right)$, so this function is well-defined, and it only removes strings from $K$.

To make it easier to understand and use this function, the definition can be rewritten as

$$
\begin{array}{r}
\left(\forall K \in \operatorname{Pwr}\left(L\left(\mathcal{G}_{L_{j}}, \mathcal{P}_{l a_{j}}\right)\right)\right) \\
\Omega_{L P C_{j}}(K):=\left\{s \in K \mid(\forall t \leq s)\left(\left(\forall \sigma_{u} \in \Sigma_{l u_{j}}\right) t \sigma_{u} \in L\left(\mathbf{G}_{L_{j}}^{p}\right) \Rightarrow t \sigma_{u} \in \bar{K}\right) \&\right. \\
\left.\left(\left(\forall \sigma_{r_{j}} \in \Sigma_{R_{j}}\right) t \sigma_{r_{j}} \in L\left(\mathbf{G}_{I_{j}}^{l}\right) \Rightarrow t \sigma_{r_{j}} \in \bar{K}\right)\right\} .
\end{array}
$$

Proposition 4.13. For system $\boldsymbol{\Phi}$, let $\mathcal{P}_{l a_{j}} \in \operatorname{Pred}\left(Q_{L_{j}}\right)$ be the given predicate for $\Omega_{L P C_{j}}$. Then $\sup \mathcal{L P C}_{j}\left(L\left(\mathcal{G}_{L_{j}}, \mathcal{P}_{l a_{j}}\right)\right)$ is the greatest fixpoint of $\Omega_{L P C_{j}}$ with respect to $\left(\operatorname{Pwr}\left(L\left(\mathcal{G}_{L_{j}}, \mathcal{P}_{l a_{j}}\right)\right), \subseteq\right)$.

proof: Similar to Proposition 4.4.

From Proposition 4.13, we know that we can compute the $\sup \mathcal{L} \mathcal{P} \mathcal{C}_{j}\left(L\left(\mathcal{G}_{L_{j}}, \mathcal{P}_{l a_{j}}\right)\right)$ by computing the greatest fixpoint of $\Omega_{L P C_{j}}$. It is also tempting to compute the greatest fixpoint by iteration of $\Omega_{L P C_{j}}$.

Proposition 4.14. For system $\boldsymbol{\Phi}$, let $\mathcal{P}_{l a_{j}} \in \operatorname{Pred}\left(Q_{L_{j}}\right)$ be the given predicate for the function $\Omega_{L P C_{j}}$. The set theoretic limit $\lim _{i \rightarrow \infty} \Omega_{L P C_{j}}^{i}\left(L\left(\mathcal{G}_{L j}, \mathcal{P}_{l a_{j}}\right)\right), i \in\{1,2, \ldots\}$ exists, and $\sup \mathcal{L P C}_{j}\left(L\left(\mathcal{G}_{L_{j}}, \mathcal{P}_{l a_{j}}\right)\right) \subseteq \lim _{i \rightarrow \infty} \Omega_{L P C_{j}}^{i}\left(L\left(\mathcal{G}_{L j}, \mathcal{P}_{l a_{j}}\right)\right)$. 
proof: Similar to Proposition 4.5.

It remains to show that $\lim _{i \rightarrow \infty} \Omega_{L P C_{j}}^{i}\left(L\left(\mathcal{G}_{L_{j}}, \mathcal{P}_{l a_{j}}\right)\right) \subseteq \sup \mathcal{L} \mathcal{P} \mathcal{C}_{j}\left(L\left(\mathcal{G}_{L_{j}}, \mathcal{P}_{l a_{j}}\right)\right)$ and that we only need to iterate a finite number of iterations in the case that $\mathcal{G}_{L_{j}}$ has a finite number of states.

Definition 4.12. For system $\Phi$, define the function $W_{L_{j}}: \operatorname{Pred}\left(Q_{L_{j}}\right) \rightarrow \operatorname{Pwr}\left(\Sigma_{I L_{j}}^{*}\right)$ according to

$$
\begin{aligned}
& \left(\forall P \in \operatorname{Pred}\left(Q_{L_{j}}\right)\right) \\
& \begin{aligned}
W_{L_{j}}(P):=\left\{s \in L\left(\mathcal{G}_{L_{j}}, P\right) \mid\right. & \left(\left(\exists \sigma_{u} \in \Sigma_{l u_{j}}\right) s \sigma_{u} \in L\left(\mathbf{G}_{L_{j}}^{p}\right) \& s \sigma_{u} \notin L\left(\mathcal{G}_{L_{j}}, P\right)\right) \text { or } \\
& \left.\left(\left(\exists \sigma_{r_{j}} \in \Sigma_{R_{j}}\right) s \sigma_{r_{j}} \in L\left(\mathbf{G}_{I_{j}}^{l}\right) \& s \sigma_{r_{j}} \notin L\left(\mathcal{G}_{L_{j}}, P\right)\right)\right\}
\end{aligned}
\end{aligned}
$$

Note that $W_{L_{j}}(P) \subseteq L\left(\mathcal{G}_{L_{j}}, P\right)$. The following two lemmas will be used in the proof of the next proposition.

Lemma 4.9. For system $\boldsymbol{\Phi}$, the following holds:

$\left(\forall P \in \operatorname{Pred}\left(Q_{L_{j}}\right)\right)\left(\forall s, t \in L\left(\mathcal{G}_{L_{j}}, P\right)\right)$

$$
s \in W_{L_{j}}(P) \&\left(\delta_{L_{j}}\left(q_{L j_{0}}, s\right)=\delta_{L_{j}}\left(q_{L j_{0}}, t\right)\right) \Rightarrow t \in W_{L_{j}}(P),
$$

where $q_{L j_{0}}$ is the initial state of $\mathcal{G}_{L_{j}}$ as defined in section 4.1 .

proof: Similar to Lemma 4.2.

Lemma 4.10. For system $\boldsymbol{\Phi}$, let $\mathcal{P}_{l a_{j}} \in \operatorname{Pred}\left(Q_{L_{j}}\right)$ be the given predicate for $\Omega_{L P C_{j}}$. Then the following holds:

$$
\begin{aligned}
&\left(\forall P \in S u b\left(\mathcal{P}_{l a_{j}}\right)\right)\left(\forall s \in L\left(\mathcal{G}_{L_{j}}, P\right)\right) \\
&\left((\exists t \leq s) t \in W_{L_{j}}(P)\right) \Leftrightarrow s \notin \Omega_{L P C_{j}}\left(L\left(\mathcal{G}_{L_{j}}, P\right)\right) .
\end{aligned}
$$




\section{Master Thesis - R. Song - McMaster - Computing and Software}

proof: Similar to Lemma 4.3.

The above two lemmas state a very important fact. If a string $s \in L\left(\mathcal{G}_{L_{j}}, P\right)$ is in $W_{L_{j}}(P)$ with $P \preceq \mathcal{P}_{l a_{j}}$, Lemma 4.9 says that all strings in $L\left(\mathcal{G}_{L_{j}}, P\right)$ that go from $q_{L j_{0}}$ to the state $\delta_{L_{j}}\left(q_{L j_{0}}, s\right)$ are also in $W_{L_{j}}(P)$, while Lemma 4.10 says that all strings in $L\left(\mathcal{G}_{L_{j}}, P\right)$ that are extended from a string in $W_{L_{j}}(P)$ are not in $\Omega_{L P C_{j}}\left(L\left(\mathcal{G}_{L_{j}}, P\right)\right)$. It means that if a string $s \in L\left(\mathcal{G}_{L_{j}}, P\right)$ is in $W_{L_{j}}(P)$, then all the strings in $\left.L\left(\mathcal{G}_{L_{j}}, P\right)\right)$ that lead from $q_{L j_{0}}$ to or pass through the state $\delta_{L_{j}}\left(q_{L j_{0}}, s\right)$ are not in $\Omega_{L P C_{j}}\left(L\left(\mathcal{G}_{L_{j}}, P\right)\right)$. The fact is formally proved as follows by using these two lemmas.

Proposition 4.15. For system $\boldsymbol{\Phi}$, let $\mathcal{P}_{l a_{j}} \in \operatorname{Pred}\left(Q_{L_{j}}\right)$ be the given predicate for $\Omega_{L P C_{j}}$, then

$$
\left(\forall P \in S u b\left(\mathcal{P}_{l a_{j}}\right)\right) L\left(\mathcal{G}_{L_{j}}, P\right)-\Omega_{L P C_{j}}\left(L\left(\mathcal{G}_{L_{j}}, P\right)\right)=L^{W_{L_{j}}(P)}\left(\mathcal{G}_{L_{j}}, P\right),
$$

where $L^{W_{L_{j}}(P)}\left(\mathcal{G}_{L_{j}}, P\right)=\cup_{s \in W_{L_{j}}(P)} L^{s}\left(\mathcal{G}_{L_{j}}, P\right)$ as defined in Section 2.5.3.

proof: Similar to Proposition 4.6.

Now, if we look at Proposition 2.7, it seems that we can compute $\Omega_{L P C_{j}}\left(L\left(\mathcal{G}_{L_{j}}, P\right)\right)$ by removing all the states in $\left\{q \in Q_{L_{j}} \mid\left(\exists s \in W_{L_{j}}(P)\right) \delta_{L_{j}}\left(q_{L j_{0}}, s\right)=q\right\}$ from the state set $Q_{L_{j}}$ of $\mathcal{G}_{L_{j}}$.

For any $q \in Q_{L_{j}}$, as $\mathcal{G}_{L_{j}}=\mathbf{E}_{L_{j}} \times \mathbf{G}_{L_{j}} \times \mathbf{G}_{I_{j}}^{l}$, there must exist $z \in Z_{L_{j}}, y \in Y_{L_{j}}$ and $x \in X_{j}^{l}$ such that

$$
q=(z, y, x)
$$

Definition 4.13. For system $\Phi$, let $\mathcal{P}_{l a_{j}} \in \operatorname{Pred}\left(Q_{L_{j}}\right)$ be a given predicate. Define the function $\Pi_{L P C_{j}}: S u b\left(\mathcal{P}_{l a_{j}}\right) \rightarrow S u b\left(\mathcal{P}_{l a_{j}}\right)$ according to 
Master Thesis - R. Song - McMaster - Computing and Software

$$
\begin{aligned}
& \left(\forall P \in \operatorname{Sub}\left(\mathcal{P}_{l a_{j}}\right)\right) \\
& \quad \Pi_{L P C_{j}}(P)=P-\operatorname{pr}\left(\operatorname{Bad}_{R L_{j}} \cup\right. \\
& \left.\quad\left\{q \models R\left(\mathcal{G}_{L_{j}}, P\right) \mid\left(\exists \sigma \in \Sigma_{l u_{j}} \cup \Sigma_{R_{j}}\right) \delta_{L_{j}}(q, \sigma) \models \neg P\right\}\right),
\end{aligned}
$$

where $\operatorname{Bad}_{R L_{j}}=\left\{q \models R\left(\mathcal{G}_{L_{j}}, P\right) \mid\right.$

$$
\begin{aligned}
& \left(\left(\exists \sigma_{u} \in \Sigma_{l u_{j}}\right) \eta_{L_{j}}\left(y, \sigma_{u}\right) ! \& \zeta_{L_{j}} \times \xi_{j}^{l}\left((z, x), \sigma_{u}\right) \not y\right) \text { or } \\
& \left.\left(\left(\exists \sigma_{r_{j}} \in \Sigma_{R_{j}}\right) \xi_{j}^{l}\left(x, \sigma_{r_{j}}\right) ! \& \zeta_{L_{j}} \times \eta_{L_{j}}\left((z, y), \sigma_{r_{j}}\right) \not y\right)\right\}
\end{aligned}
$$

and $q=(z, y, x)$ as in equation (4.2).

Note that $\Pi_{L P C_{j}}(P) \preceq P \preceq \mathcal{P}_{l a_{j}}$, so the function is well-defined. Let $P \in$ $\operatorname{Pred}\left(Q_{L_{j}}\right)$. Function $\Pi_{L P C_{j}}$ removes all the states in $\left\{q \in Q_{L_{j}} \mid\left(\exists s \in W_{L_{j}}(P)\right) \delta_{L_{j}}\left(q_{L j_{0}}, s\right)=\right.$ $q$ f from $P$. The following proposition shows this.

Proposition 4.16. For system $\boldsymbol{\Phi}$, let $\mathcal{P}_{l a_{j}} \in \operatorname{Pred}\left(Q_{L_{j}}\right)$ be the given predicate for $\Omega_{L P C_{j}}$, then

$$
\begin{aligned}
& \text { 1. }\left(\forall P \in S u b\left(\mathcal{P}_{l a_{j}}\right)\right) \Omega_{L P C_{j}}\left(L\left(\mathcal{G}_{L_{j}}, P\right)\right)=L\left(\mathcal{G}_{L_{j}}, \Pi_{L P C_{j}}(P)\right) \\
& \text { 2. }(\forall i \in\{0,1, \ldots\}) \Omega_{L P C_{j}}^{i}\left(L\left(\mathcal{G}_{L j}, \mathcal{P}_{l a_{j}}\right)\right)=L\left(\mathcal{G}_{L_{j}}, \Pi_{L P C_{j}}^{i}\left(\mathcal{P}_{l a_{j}}\right)\right)
\end{aligned}
$$

proof: Similar to Proposition 4.7.

Now, if we take a look at the definition of the function $\Pi_{L P C_{j}}$, we can see that for any $P \preceq \mathcal{P}_{l a_{j}}$, the function $\Pi_{L P C_{j}}$ only removes states in $R\left(\mathcal{G}_{L_{j}}, P\right)$ from $P$. Precisely, $\Pi_{L P C_{j}}$ only removes all the states in $\operatorname{pr}\left(\operatorname{Bad}_{R L_{j}} \cup\left\{q \models R\left(\mathcal{G}_{L_{j}}, P\right) \mid(\exists \sigma \in\right.\right.$ $\left.\left.\left.\Sigma_{l u_{j}} \cup \Sigma_{R_{j}}\right) \delta_{L_{j}}(q, \sigma) \models \neg P\right\}\right)$, which is a subpredicate of $R\left(\mathcal{G}_{L_{j}}, P\right)$. If we also remove any state that is not in $R\left(\mathcal{G}_{L_{j}}, P\right)$, then the language $L\left(\mathcal{G}_{L_{j}}, P\right)$ is not affected at all, because the $L\left(\mathcal{G}_{L_{j}}, P\right)$ is only dependent on $R\left(\mathcal{G}_{L_{j}}, P\right)$. Similarly, removing any state that is not in $R\left(\mathcal{G}_{L_{j}}, P\right)$ does not affect the language $L\left(\mathcal{G}_{L_{j}}, \Pi_{L P C_{j}}(P)\right)$ either, 


\section{Master Thesis - R. Song - McMaster - Computing and Software}

because it is only dependent on a subpredicate of $R\left(\mathcal{G}_{L_{j}}, P\right)$. The following function $\Gamma_{L P C_{j}}$ is a replacement of the function $\Pi_{L P C_{j}}$.

Definition 4.14. For system $\Phi$, let $\mathcal{P}_{l a_{j}} \in \operatorname{Pred}\left(Q_{L_{j}}\right)$ be a given predicate. Define the function $\Gamma_{L P C_{j}}: S u b\left(\mathcal{P}_{l a_{j}}\right) \rightarrow S u b\left(\mathcal{P}_{l a_{j}}\right)$ according to

$$
\begin{aligned}
& \left(\forall P \in \operatorname{Sub}\left(\mathcal{P}_{l a_{j}}\right)\right) \\
& \Gamma_{L P C_{j}}(P)=P-\operatorname{pr}\left(\operatorname{Bad}_{L_{j}} \cup\left\{q \models Q_{L_{j}} \mid\left(\exists \sigma \in \Sigma_{l u_{j}} \cup \Sigma_{R_{j}}\right) \delta_{L_{j}}(q, \sigma) \models \neg P\right\}\right), \\
& \text { where } \operatorname{Bad}_{L_{j}}=\left\{q \in Q_{L_{j}} \mid\right. \\
& \left(\left(\exists \sigma_{u} \in \Sigma_{l u_{j}}\right) \eta_{L_{j}}\left(y, \sigma_{u}\right) ! \& \zeta_{L_{j}} \times \xi_{j}^{l}\left((z, x), \sigma_{u}\right) \not\right) \text { or } \\
& \left.\left(\left(\exists \sigma_{r_{j}} \in \Sigma_{R_{j}}\right) \xi_{j}^{l}\left(x, \sigma_{r_{j}}\right) ! \& \zeta_{L_{j}} \times \eta_{L_{j}}\left((z, y), \sigma_{r_{j}}\right) \not y\right)\right\}
\end{aligned}
$$

and $q=(z, y, x)$ as in equation (4.2).

Note that for system $\boldsymbol{\Phi}, \operatorname{Bad}_{L_{j}}$ is constant.

Corollary 4.3. For system $\boldsymbol{\Phi}$, let $\mathcal{P}_{l a_{j}} \in \operatorname{Pred}\left(Q_{L_{j}}\right)$ be the given predicate for the function $\Omega_{L P C_{j}}, \Pi_{L P C_{j}}$ and $\Gamma_{L P C_{j}}$. Then the following holds:

1. $\left(\forall P \in S u b\left(\mathcal{P}_{l a_{j}}\right)\right) \Omega_{L P C_{j}}\left(L\left(\mathcal{G}_{L_{j}}, P\right)\right)=L\left(\mathcal{G}_{L_{j}}, \Gamma_{L P C_{j}}(P)\right)$

2. $(\forall i \in\{0,1, \ldots\}) \Omega_{L P C_{j}}^{i}\left(L\left(\mathcal{G}_{L_{j}}, \mathcal{P}_{l a_{j}}\right)\right)=L\left(\mathcal{G}_{L_{j}}, \Gamma_{L P C_{j}}^{i}\left(\mathcal{P}_{l a_{j}}\right)\right)$

proof: Similar to Corollary 4.1.

The following lemma will be used in the next proposition.

Lemma 4.11. For system $\boldsymbol{\Phi}$, let $\mathcal{P}_{l a_{j}} \in \operatorname{Pred}\left(Q_{L_{j}}\right)$ be the given predicate for the function $\Gamma_{L P C_{j}}$. The function $\Gamma_{L P C_{j}}$ is monotone with respect to $\preceq$, i.e.

$$
\left(\forall P_{1}, P_{2} \in S u b\left(\mathcal{P}_{l a_{j}}\right)\right) P_{1} \preceq P_{2} \Rightarrow \Gamma_{L P C_{j}}\left(P_{1}\right) \preceq \Gamma_{L P C_{j}}\left(P_{2}\right)
$$


Master Thesis - R. Song - McMaster - Computing and Software

proof: Similar to Lemma 4.4.

Proposition 4.17. For system $\boldsymbol{\Phi}$, let $\mathcal{P}_{l a_{j}} \in \operatorname{Pred}\left(Q_{L_{j}}\right)$ be the given predicate for the function $\Omega_{L P C_{j}}$ and $\Gamma_{L P C_{j}}$, then,

1. There exists $k \in\{0,1,2, \ldots\}$ with $k \leq\left|s t\left(\mathcal{P}_{l a_{j}}\right)\right|$ such that $\Gamma_{L P C_{j}}^{k}\left(\mathcal{P}_{l a_{j}}\right)$ is the greatest fixpoint of the function $\Gamma_{L P C_{j}}$ with respect to $\left(S u b\left(\mathcal{P}_{l a_{j}}\right), \preceq\right)$, and where $\operatorname{st}\left(\mathcal{P}_{l a_{j}}\right)$ is the identifying state subset of $Q_{L_{j}}$ for $\mathcal{P}_{l a_{j}}$.

2. $\sup \mathcal{L} \mathcal{P C}_{j}\left(L\left(\mathcal{G}_{L_{j}}, \mathcal{P}_{l a_{j}}\right)\right)=L\left(\mathcal{G}_{L_{j}}, \Gamma_{L P C_{j}}^{k}\left(\mathcal{P}_{l a_{j}}\right)\right)$

proof: Similar to Proposition 4.8.

Now, we are able to write a computer program to compute $\sup \mathcal{L} \mathcal{P} \mathcal{C}_{j}\left(L\left(\mathcal{G}_{L_{j}}, \mathcal{P}_{l a_{j}}\right)\right)$ by computing $\Gamma_{L P C_{j}}^{k}\left(\mathcal{P}_{l a_{j}}\right)(k \in\{0,1,2, \ldots\})$, and we know that after at most $\left|s t\left(\mathcal{P}_{l a_{j}}\right)\right|$ number of iterations, a fixpoint of $\Gamma_{L P C_{j}}$ will be reached, which is the greatest fixpoint of $\Gamma_{L P C_{j}}$ by Proposition 2.3. However, such a program based on function $\Gamma_{L P C_{j}}$ is not efficient enough. For instance, each time when we implement the $\Gamma_{L P C_{j}}$ function, we have to do the predicate subtracting operation. The following alternative method can avoid this.

Definition 4.15. For system $\boldsymbol{\Phi}$, define the function $\operatorname{PLPC}_{j}: \operatorname{Pred}\left(Q_{L_{j}}\right) \rightarrow \operatorname{Pred}\left(Q_{L_{j}}\right)$ according to

$$
\left(\forall P \in \operatorname{Pred}\left(Q_{L_{j}}\right)\right) \operatorname{PLPC}_{j}(P):=\neg T R\left(\mathcal{G}_{L_{j}}, \neg P \vee \operatorname{pr}\left(\operatorname{Bad}_{L_{j}}\right), \Sigma_{l u_{j}} \cup \Sigma_{R_{j}}\right) .
$$

Lemma 4.12. For system $\boldsymbol{\Phi}$, the following holds:

1. The function $\mathrm{PLPC}_{j}$ is monotone, i.e.

$$
\left(\forall P_{1}, P_{2} \in \operatorname{Pred}\left(Q_{L_{j}}\right)\right) P_{1} \preceq P_{2} \Rightarrow \operatorname{PLPC}_{j}\left(P_{1}\right) \preceq \operatorname{PLPC}_{j}\left(P_{2}\right) .
$$


2. $\left(\forall P \in \operatorname{Pred}\left(Q_{L_{j}}\right)\right) \operatorname{PLPC}_{j}(P) \preceq P$.

proof: Similar to Lemma 4.5.

Proposition 4.18. For system $\mathbf{\Phi}$, the following holds:

$$
\left(\forall \mathcal{P}_{l a_{j}} \in \operatorname{Pred}\left(Q_{L_{j}}\right)\right) \sup \mathcal{L P C}_{j}\left(L\left(\mathcal{G}_{L_{j}}, \mathcal{P}_{l a_{j}}\right)\right)=L\left(\mathcal{G}_{L_{j}}, \operatorname{PLPC}_{j}\left(\mathcal{P}_{l a_{j}}\right)\right)
$$

proof: Similar to Proposition 4.9.

The following lemma is obvious, but for later convenience, we give a proof here.

Lemma 4.13. For system $\boldsymbol{\Phi}$, the following holds:

$$
\left(\forall P \in \operatorname{Pred}\left(Q_{L_{j}}\right)\right)\left(\forall q \in Q_{L_{j}}\right) q \models \operatorname{PLPC}_{j}(P) \Rightarrow\left(\left(\forall \rho \in \Sigma_{R_{j}}\right) \xi_{j}^{l}(x, \rho) ! \Rightarrow \delta_{L_{j}}(q, \rho) !\right)
$$

\section{proof:}

Let $P \in \operatorname{Pred}\left(Q_{L_{j}}\right)$ and $q \in Q_{L_{j}}$.

Assume $q \models \operatorname{PLPC}_{j}(P)$.

Must show this implies $\left(\forall \rho \in \Sigma_{R_{j}}\right) \xi_{j}^{l}(x, \rho) ! \Rightarrow \delta_{L_{j}}(q, \rho) !$.

Let $\rho \in \Sigma_{R_{j}}$. Assume $\xi_{j}^{l}(x, \rho)$ !. We now show $\delta_{L_{j}}(q, \rho)$ !.

From (1), we have $q \models \operatorname{PLPC}_{j}(P)$

$$
\begin{aligned}
& \Rightarrow q \models \neg T R\left(\mathcal{G}_{L_{j}}, \neg P \vee \operatorname{pr}\left(\operatorname{Bad}_{L_{j}}\right), \Sigma_{l u_{j}} \cup \Sigma_{R_{j}}\right) \\
& \Rightarrow q \not \models T R\left(\mathcal{G}_{L_{j}}, \neg P \vee \operatorname{pr}\left(\operatorname{Bad}_{L_{j}}\right), \Sigma_{l u_{j}} \cup \Sigma_{R_{j}}\right) \\
& \Rightarrow q \not \models \operatorname{pr}\left(\operatorname{Bad}_{L_{j}}\right), \quad \text { by definition of } T R \\
& \Rightarrow \zeta_{L_{j}} \times \eta_{L_{j}}((z, y), \rho) !, \quad \text { by definition of } \operatorname{Bad}_{L_{j}} \text { and } q=(z, y, x) \text { and } \xi_{j}^{l}(x, \rho) ! \\
& \Rightarrow \delta_{L_{j}}(q, \rho) !, \quad \text { by } \delta_{L_{j}}=\zeta_{L_{j}} \times \eta_{L_{j}} \times \xi_{j}^{l} \text { and } \xi_{j}^{l}(x, \rho) ! .
\end{aligned}
$$


Master Thesis - R. Song - McMaster - Computing and Software

\subsubsection{The $j^{\text {th }}$ Low-level Interface Controllable Language}

Definition 4.16. Let $K \subseteq \Sigma_{I L_{j}}^{*}$. $K$ is $j^{\text {th }}$ low-level interface controllable $\left(\mathrm{LIC}_{j}\right)$ with respect to system $\boldsymbol{\Phi}$ if the following conditions are satisfied:

1. $K$ is $j^{\text {th }}$ low-level P4 interface controllable.

2. $(\forall s \in \bar{K})\left(\forall \rho \in \Sigma_{R_{j}}\right)\left(\forall \alpha \in \Sigma_{A_{j}}\right) s \rho \alpha \in L\left(\mathbf{G}_{I_{j}}^{l}\right) \Rightarrow\left(\exists l \in \Sigma_{L_{j}}^{*}\right) s \rho l \alpha \in \bar{K}$

3. $(\forall s \in \bar{K}) s \in L_{m}\left(\mathbf{G}_{I_{j}}^{l}\right) \Rightarrow\left(\exists l \in \Sigma_{L_{j}}^{*}\right) s l \in K$

Obviously, the empty language $\emptyset$ is $j^{\text {th }}$ low-level interface controllable with respect to system $\boldsymbol{\Phi}$. Note that the first and the second conditions are based on the closed language $\bar{K}$.

For a language $K \subseteq \Sigma_{I L_{j}}^{*}$, if $K$ satisfies the second condition of $\mathrm{LIC}_{j}$, we say that $K$ is $j^{\text {th }}$ low-level P5-satisfied, and if $K$ satisfies the third condition of $\mathrm{LIC}_{j}$, we say that $K$ is $j^{\text {th }}$ low-level P6-satisfied.

For an arbitrary language $E \subseteq \Sigma_{I L_{j}}^{*}$, we define the set of all sublanguages of $E$ that are $j^{\text {th }}$ low-level interface controllable with respect to system $\mathbf{\Phi}$ as

$\mathcal{C}_{L_{j}}(E):=\left\{K \subseteq E \mid K\right.$ is $j^{\text {th }}$ low-level interface controllable with respect to system $\left.\mathbf{\Phi}\right\}$

Clearly, $\left(\mathcal{C}_{L_{j}}(E), \subseteq\right)$ is a poset. We now show that the supremum always exists in $\mathcal{C}_{L_{j}}(E)$.

Proposition 4.19. Let $E \subseteq \Sigma_{I L_{j}}^{*}$. The set $\mathcal{C}_{L_{j}}(E)$ is nonempty and is closed under arbitrary unions. In particular, $\mathcal{C}_{L_{j}}(E)$ contains a (unique) supremal element, $\sup \mathcal{C}_{L_{j}}(E)=\cup\left\{K \mid K \in \mathcal{C}_{L_{j}}(E)\right\}$. 


\section{Master Thesis - R. Song-McMaster-Computing and Software}

\section{proof:}

1. Show that $\mathcal{C}_{L_{j}}(E)$ is nonempty

The empty language $\emptyset$ is $j^{\text {th }}$ low-level interface controllable with respect to system $\Phi$, so $\emptyset \in \mathcal{C}_{L_{j}}(E)$.

2. Show that $\mathcal{C}_{L_{j}}(E)$ is closed under arbitrary unions.

Let $B$ be an index set. Assume that $(\forall \beta \in B) K_{\beta} \in \mathcal{C}_{L_{j}}(E)$. Sufficient to show that $\cup_{\beta \in B} K_{\beta} \in \mathcal{C}_{L_{j}}(E)$.

Let $K:=\cup_{\beta \in B} K_{\beta} \in \mathcal{C}_{L_{j}}(E)$. It is sufficient to show that $K$ is $j^{\text {th }}$ low-level interface controllable with respect to system $\mathbf{\Phi}$. By Definition 4.16, we have to show the following:

(a) Show that $\bar{K} \Sigma_{l u_{j}} \cap L\left(\mathbf{G}_{L_{j}}^{p}\right) \subseteq \bar{K}$

Identical to the proof of Proposition 4.1(Part 2(a)) by substituting $\Sigma_{h u}$ with $\Sigma_{l u_{j}}, L\left(\mathbf{G}_{H}^{p} \times \mathbf{G}_{I}^{h}\right)$ with $L\left(\mathbf{G}_{L_{j}}^{p}\right)$ and $\mathcal{C}_{H}$ with $\mathcal{C}_{L_{j}}$.

(b) Show that $\bar{K} \Sigma_{R_{j}} \cap L\left(\mathbf{G}_{I_{j}}^{l}\right) \subseteq \bar{K}$

Identical to the proof of Proposition 4.1 (Part 2(a)) by substituting $\Sigma_{h u}$ with $\Sigma_{R_{j}}, L\left(\mathbf{G}_{H}^{p} \times \mathbf{G}_{I}^{h}\right)$ with $L\left(\mathbf{G}_{I_{j}}^{l}\right)$ and $\mathcal{C}_{H}$ with $\mathcal{C}_{L_{j}}$.

(c) Show that $(\forall s \in \bar{K})\left(\forall \rho \in \Sigma_{R_{j}}\right)\left(\forall \alpha \in \Sigma_{A_{j}}\right) s \rho \alpha \in L\left(\mathbf{G}_{I_{j}}^{l}\right) \Rightarrow(\exists l \in$ $\left.\Sigma_{L_{j}}^{*}\right) s \rho l \alpha \in \bar{K}$.

Let $s \in \bar{K}, \rho \in \Sigma_{R_{j}}, \alpha \in \Sigma_{A_{j}}$.

Assume $s \rho \alpha \in L\left(\mathbf{G}_{I_{j}}^{l}\right)$.

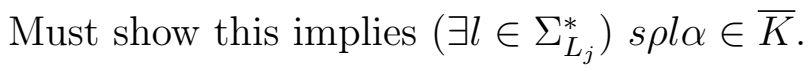

By (1), we have $s \in \bar{K}$

$\Rightarrow\left(\exists s^{\prime} \in \Sigma_{I L_{j}}^{*}\right) s s^{\prime} \in K$ 
Master Thesis - R. Song - McMaster - Computing and Software

$\Rightarrow(\exists \beta \in B) s s^{\prime} \in K_{\beta}$

$\Rightarrow s \in \overline{K_{\beta}}$

By (3), (2) and $K_{\beta} \in \mathcal{C}_{L_{j}}(E)$, we have $\left(\exists l \in \Sigma_{L_{j}}^{*}\right)$ s $\rho l \alpha \in \overline{K_{\beta}}$

$\Rightarrow\left(\exists l \in \Sigma_{L_{j}}^{*}\right) s \rho l \alpha \in \bar{K}, \quad$ as $K_{\beta} \subseteq K$

(d) Show that $(\forall s \in \bar{K}) s \in L_{m}\left(\mathbf{G}_{I_{j}}^{l}\right) \Rightarrow\left(\exists l \in \Sigma_{L_{j}}^{*}\right) s l \in K$

Let $s \in \bar{K}$.

Assume $s \in L_{m}\left(\mathbf{G}_{I_{j}}^{l}\right)$.

Must show this implies $\left(\exists l \in \Sigma_{L_{j}}^{*}\right) s l \in K$.

By (4), we have $s \in \bar{K}$

$\Rightarrow\left(\exists s^{\prime} \in \Sigma_{I L_{j}}^{*}\right) s s^{\prime} \in K$

$\Rightarrow(\exists \beta \in B) s s^{\prime} \in K_{\beta}$

$\Rightarrow s \in \overline{K_{\beta}}$

By (6), (5) and $K_{\beta} \in \mathcal{C}_{L_{j}}(E)$, we have $\left(\exists l \in \Sigma_{L_{j}}^{*}\right) s l \in K_{\beta}$

$\Rightarrow\left(\exists l \in \Sigma_{L_{j}}^{*}\right) s l \in K, \quad$ as $K_{\beta} \subseteq K$

3. Show that $\sup \mathcal{C}_{L_{j}}(E)=\cup\left\{K \mid K \in \mathcal{C}_{L_{j}}(E)\right\}$.

Identical to the proof of Proposition 4.1 (Part 3) by substituting $\mathcal{C}_{H}$ with $\mathcal{C}_{L_{j}}$ and $\Sigma_{I H}$ with $\Sigma_{I L_{j}}$.

4. Show that $\sup \mathcal{C}_{L_{j}}(E) \in \mathcal{C}_{L_{j}}(E)$.

This immediately follows from Part 2 and Part 3.

From Proposition 4.19, we have $\sup \mathcal{C}_{L_{j}}(E) \subseteq E$, which means that we can compute $\sup \mathcal{C}_{L_{j}}(E)$ by removing strings from $E$. 


\section{Master Thesis - R. Song-McMaster-Computing and Software}

\subsection{3 $\sup \mathcal{C}_{L_{j}}\left(L_{m}\left(\mathcal{G}_{L_{j}}\right)\right)$ and the Greatest Fixpoint of $\Omega_{L_{j}}$}

For system $\boldsymbol{\Phi}$, if we compute the supremal $j^{\text {th }}$ low-level interface controllable sublanguage of $L_{m}\left(\mathcal{G}_{L_{j}}\right)$, then a DES representing this sublanguage is a maximally permissible $j^{\text {th }}$ proper low-level supervisor. Therefore, we need to find a method to compute $\sup \mathcal{C}_{L_{j}}\left(L_{m}\left(\mathcal{G}_{L_{j}}\right)\right)$. For this purpose, we define the following function.

Definition 4.17. For system $\boldsymbol{\Phi}$, define the function $\Omega_{L_{j}}: \operatorname{Pwr}\left(\Sigma_{I L_{j}}^{*}\right) \rightarrow \operatorname{Pwr}\left(\Sigma_{I L_{j}}^{*}\right)$ according to

$$
\left(\forall K \in \operatorname{Pwr}\left(\Sigma_{I L_{j}}^{*}\right)\right) \Omega_{L_{j}}(K):=\Omega_{L N B_{j}}\left(\Omega_{p 6_{j}}\left(\Omega_{p 5_{j}}\left(\mathrm{LPC}_{j}(K)\right)\right)\right)
$$

where $\Omega_{L N B_{j}}, \Omega_{p 6_{j}}, \Omega_{p 5_{j}}, \mathrm{LPC}_{j}$ are functions for system $\boldsymbol{\Phi}$, which are defined as follows:

- $\mathrm{LPC}_{j}: \operatorname{Pwr}\left(\sum_{I L_{j}}^{*}\right) \rightarrow \operatorname{Pwr}\left(\Sigma_{I L_{j}}^{*}\right)$

$$
\left(\forall K \in \operatorname{Pwr}\left(\Sigma_{I L_{j}}^{*}\right)\right) \operatorname{LPC}_{j}(K):=\sup \mathcal{L P} \mathcal{C}_{j}(\bar{K})
$$

- $\Omega_{p 5_{j}}: \operatorname{Pwr}\left(\Sigma_{I L_{j}}^{*}\right) \rightarrow \operatorname{Pwr}\left(\Sigma_{I L_{j}}^{*}\right)$

$$
\begin{aligned}
& \left(\forall K \in \operatorname{Pwr}\left(\Sigma_{I L_{j}}^{*}\right)\right) \\
& \begin{aligned}
\Omega_{p 5_{j}}(K):=\{s \in K \mid & (\forall t \leq s)\left(\forall \rho \in \Sigma_{R_{j}}\right)\left(\forall \alpha \in \Sigma_{A_{j}}\right) \\
& \left.t \rho \alpha \in L\left(\mathbf{G}_{I_{j}}^{l}\right) \Rightarrow\left(\exists l \in \Sigma_{L_{j}}^{*}\right) t \rho l \alpha \in K\right\}
\end{aligned}
\end{aligned}
$$

- $\Omega_{p 6_{j}}: \operatorname{Pwr}\left(\Sigma_{I L_{j}}^{*}\right) \rightarrow \operatorname{Pwr}\left(\Sigma_{I L_{j}}^{*}\right)$

$\left(\forall K \in \operatorname{Pwr}\left(\Sigma_{I L_{j}}^{*}\right)\right)$

$$
\Omega_{p 6_{j}}(K):=\left\{s \in K \mid(\forall t \leq s) t \in L_{m}\left(\mathbf{G}_{I_{j}}^{l}\right) \Rightarrow\left(\exists l \in \Sigma_{L_{j}}^{*}\right) t l \in L_{m}\left(\mathcal{G}_{L_{j}}\right) \cap K\right\}
$$

- $\Omega_{L N B_{j}}: \operatorname{Pwr}\left(\Sigma_{I L_{j}}^{*}\right) \rightarrow \operatorname{Pwr}\left(\Sigma_{I L_{j}}^{*}\right)$

$$
\left(\forall K \in \operatorname{Pwr}\left(\Sigma_{I L_{j}}^{*}\right)\right) \Omega_{L N B_{j}}(K):=L_{m}\left(\mathcal{G}_{L_{j}}\right) \cap K
$$




\section{Master Thesis - R. Song - McMaster - Computing and Software}

As all the functions in $\Omega_{L_{j}}$ are defined on $\operatorname{Pwr}\left(\Sigma_{I L_{j}}^{*}\right)$, so we can compose them together.

Clearly, $\Omega_{p 5_{j}}, \Omega_{p 6_{j}}, \Omega_{L N B_{j}}$ are monotone with respect to $\subseteq$. By Proposition 4.12, it is also clear that $\mathrm{LPC}_{j}$ is monotone with respect to $\subseteq$. Therefore, the function $\Omega_{L_{j}}$ is monotone with respect to $\subseteq$ as well.

Note that $\left(\forall K \in \operatorname{Pwr}\left(\Sigma_{I L_{j}}^{*}\right)\right) \quad \operatorname{LPC}_{j}(K) \subseteq \bar{K}, \Omega_{p 5_{j}}(K) \subseteq K, \Omega_{p 6_{j}}(K) \subseteq K$ and $\Omega_{L N B_{j}}(K) \subseteq K$

By the Knaster-Tarski Theorem(Theorem 2.1), the greatest fixpoint of the function $\Omega_{L_{j}}$ with respect to $\left(\operatorname{Pwr}\left(\Sigma_{I L_{j}}^{*}\right), \subseteq\right)$ must exist. The following lemmas will be used later on.

Lemma 4.14. For system $\boldsymbol{\Phi}$, let $L \subseteq \Sigma_{I L_{j}}^{*}$ be a closed language, then $\Omega_{p 5_{j}}(L)$ and $\Omega_{p 6_{j}}(L)$ are also closed.

\section{proof:}

Assume $L=\bar{L}$. Must show implies $\overline{\Omega_{p 5_{j}}(L)}=\Omega_{p 5_{j}}(L)$ and $\overline{\Omega_{p 6_{j}}(L)}=\Omega_{p 6_{j}}(L)$.

$\Omega_{p 5_{j}}(L) \subseteq \overline{\Omega_{p 5_{j}}(L)}$ and $\Omega_{p 6_{j}}(L) \subseteq \overline{\Omega_{p 6_{j}}(L)}$ are automatic.

1. Show that $\overline{\Omega_{p 5_{j}}(L)} \subseteq \Omega_{p 5_{j}}(L)$

Let $s \in \overline{\Omega_{p 5_{j}}(L)}$.

Must show this implies $s \in \Omega_{p 5_{j}}(L)$.

From (1), we have $\left(\exists s^{\prime} \in \Sigma_{I L_{j}}^{*}\right) s s^{\prime} \in \Omega_{p 5_{j}}(L)$

$$
\begin{aligned}
& \Rightarrow s s^{\prime} \in L \&\left(\left(\forall t \leq s s^{\prime}\right)\left(\forall \rho \in \Sigma_{R_{j}}\right)\left(\forall \alpha \in \Sigma_{A_{j}}\right)\right. \\
& \left.\qquad t \rho \alpha \in L\left(\mathbf{G}_{I_{j}}^{l}\right) \Rightarrow\left(\exists l \in \Sigma_{L_{j}}^{*}\right) t \rho l \alpha \in L\right) \\
& \Rightarrow s \in L \&\left((\forall t \leq s)\left(\forall \rho \in \Sigma_{R_{j}}\right)\left(\forall \alpha \in \Sigma_{A_{j}}\right)\right. \\
& \left.\qquad t \rho \alpha \in L\left(\mathbf{G}_{I_{j}}^{l}\right) \Rightarrow\left(\exists l \in \Sigma_{L_{j}}^{*}\right) t \rho l \alpha \in L\right), \quad \text { as } L \text { is closed. } \\
& \Rightarrow s \in \Omega_{p 5_{j}}(L)
\end{aligned}
$$




\section{Master Thesis - R. Song - McMaster - Computing and Software}

2. Show that $\overline{\Omega_{p 6_{j}}(L)} \subseteq \Omega_{p 6_{j}}(L)$.

Let $s \in \overline{\Omega_{p 6_{j}}(L)}$.

Must show this implies $s \in \Omega_{p 6_{j}}(L)$.

From (2), we have $\left(\exists s^{\prime} \in \Sigma_{I L_{j}}^{*}\right) s s^{\prime} \in \Omega_{p 6_{j}}(L)$

$\Rightarrow s s^{\prime} \in L \&\left(\left(\forall t \leq s s^{\prime}\right) t \in L_{m}\left(\mathbf{G}_{I_{j}}^{l}\right) \Rightarrow\left(\exists l \in \Sigma_{L_{j}}^{*}\right) t l \in L_{m}\left(\mathcal{G}_{L_{j}}\right) \cap L\right)$

$\Rightarrow s \in L \&\left((\forall t \leq s) t \in L_{m}\left(\mathbf{G}_{I_{j}}^{l}\right) \Rightarrow\left(\exists l \in \Sigma_{L_{j}}^{*}\right) t l \in L_{m}\left(\mathcal{G}_{L_{j}}\right) \cap L\right)$,

as $L$ is closed.

$\Rightarrow s \in \Omega_{p 6_{j}}(L)$.

Lemma 4.15. For system $\Phi$ and for all $T \in P w r\left(\Sigma_{I L_{j}}^{*}\right)$,

1. $\bar{T}=\sup \mathcal{L P C}_{j}(\bar{T})$ iff $T$ is $j^{\text {th }}$ low-level P4 interface controllable.

2. $\bar{T}=\Omega_{p 5_{j}}(\bar{T})$ iff $T$ is $j^{\text {th }}$ low-level P5-satisfied.

\section{proof:}

Let $T \in \operatorname{Pwr}\left(\Sigma_{I L_{j}}^{*}\right)$.

1. Show that $\bar{T}=\sup \mathcal{L} \mathcal{P C}(\bar{T})$ iff $T$ is $j^{\text {th }}$ low-level $\mathrm{P} 4$ interface controllable.

We first show $\bar{T}=\sup \mathcal{L} \mathcal{P} \mathcal{C}_{j}(\bar{T})$ iff $\bar{T}$ is $j^{\text {th }}$ low-level P4 interface controllable.

(a) Show $\left(\bar{T}=\sup \mathcal{L P C}_{j}(\bar{T})\right) \Rightarrow\left(\bar{T}\right.$ is $j^{\text {th }}$ low-level P4 interface controllable $)$.

Assume $\bar{T}=\sup \mathcal{L P \mathcal { C }}(\bar{T})$.

Must show this implies $\bar{T}$ is $j^{\text {th }}$ low-level P4 interface controllable.

Sufficient to show that $\bar{T} \in \mathcal{L} \mathcal{P} \mathcal{C}_{j}(\bar{T})$.

By Proposition 4.12, we know $\sup \mathcal{L P \mathcal { C }}{ }_{j}(\bar{T})=\cup\left\{K \mid K \in \mathcal{L} \mathcal{P} \mathcal{C}_{j}(\bar{T})\right\}$. 
Master Thesis - R. Song - McMaster - Computing and Software

$\Rightarrow \bar{T}=\cup\left\{K \mid K \in \mathcal{L P C}_{j}(\bar{T})\right\}, \quad$ by $(1)$

$\Rightarrow \bar{T} \in \mathcal{L} \mathcal{P} \mathcal{C}_{j}(\bar{T}), \quad$ by Proposition 4.12

(b) Show $\left(\bar{T}\right.$ is $j^{\text {th }}$ low-level P4 interface controllable $) \Rightarrow\left(\bar{T}=\sup \mathcal{L P C}_{j}(\bar{T})\right)$.

Assume $\bar{T}$ is $j^{\text {th }}$ low-level P4 interface controllable.

Must show this implies $\bar{T}=\sup \mathcal{L P C}(\bar{T})$.

By (2), we know $\bar{T} \in \mathcal{L} \mathcal{P} \mathcal{C}_{j}(\bar{T})$

$\Rightarrow \bar{T} \subseteq \sup \mathcal{L P C}_{j}(\bar{T})$

By Proposition 4.12, we know $\sup \mathcal{L} \mathcal{P} \mathcal{C}_{j}(\bar{T}) \subseteq \bar{T}$

From (3) and (4), we have $\bar{T}=\sup \mathcal{L} \mathcal{P C} \mathcal{C}_{j}(\bar{T})$.

From Definition 4.10, we know that $T$ is $j^{\text {th }}$ low-level P4 interface controllable iff $\bar{T}$ is $j^{\text {th }}$ low-level P4 interface controllable.

So, we have $\bar{T}=\sup \mathcal{L} \mathcal{P C}(\bar{T})$ iff $T$ is $j^{\text {th }}$ low-level P4 interface controllable.

2. Show that $\bar{T}=\Omega_{p 5_{j}}(\bar{T})$ iff $T$ is $j^{\text {th }}$ low-level P5-satisfied.

$T$ is $j^{\text {th }}$ low-level P5-satisfied

$$
\begin{aligned}
& \Leftrightarrow(\forall s \in \bar{T})\left(\forall \rho \in \Sigma_{R_{j}}\right)\left(\forall \alpha \in \Sigma_{A_{j}}\right) s \rho \alpha \in L\left(\mathbf{G}_{I_{j}}^{l}\right) \Rightarrow\left(\exists l \in \Sigma_{L_{j}}^{*}\right) s \rho l \alpha \in \bar{T} \\
& \Leftrightarrow(\forall s \in \bar{T})(\forall t \leq s)\left(\forall \rho \in \Sigma_{R_{j}}\right)\left(\forall \alpha \in \Sigma_{A_{j}}\right) \\
& \qquad \quad t \rho \alpha \in L\left(\mathbf{G}_{I_{j}}^{l}\right) \Rightarrow\left(\exists l \in \Sigma_{L_{j}}^{*}\right) t \rho l \alpha \in \bar{T}, \quad \text { as } \bar{T} \text { is closed } \\
& \Leftrightarrow(\forall s \in \bar{T}) \quad s \in \Omega_{p 5_{j}}(\bar{T}) \\
& \Leftrightarrow \bar{T} \subseteq \Omega_{p 5_{j}}(\bar{T}) \\
& \Leftrightarrow \bar{T}=\Omega_{p 5_{j}}(\bar{T}), \quad \text { as } \Omega_{p 5_{j}}(\bar{T}) \subseteq \bar{T} \text { by definition of } \Omega_{p 5_{j}} .
\end{aligned}
$$




\section{Master Thesis - R. Song-McMaster-Computing and Software}

Proposition 4.20. For system $\boldsymbol{\Phi}, \sup \mathcal{C}_{L_{j}}\left(L_{m}\left(\mathcal{G}_{L_{j}}\right)\right)$ is the greatest fixpoint of the function $\Omega_{L_{j}}$ with respect to $\left(\operatorname{Pwr}\left(\Sigma_{I L_{j}}^{*}\right), \subseteq\right)$.

\section{proof:}

Let $S:=\sup \mathcal{C}_{L_{j}}\left(L_{m}\left(\mathcal{G}_{L_{j}}\right)\right)$.

1. Show that $S$ is a fixpoint of $\Omega_{L_{j}}$, i.e. $S=\Omega_{L_{j}}(S)$

(a) Show that $\bar{S}=\sup \mathcal{L P C} \mathcal{C}_{j}(\bar{S})$ and $\bar{S}=\Omega_{p 5_{j}}(\bar{S})$.

As $S$ is $j^{\text {th }}$ low-level interface controllable, $S$ is $j^{\text {th }}$ low-level P4 interface controllable and $j^{\text {th }}$ low-level P5-satisfied.

By Lemma 4.15, we have $\bar{S}=\sup \mathcal{L P \mathcal { C } _ { j }}(\bar{S})$ and $\bar{S}=\Omega_{p 5_{j}}(\bar{S})$.

(b) Show that $S=L_{m}\left(\mathcal{G}_{L_{j}}\right) \cap \bar{S}$

Sufficient to show that $S \subseteq L_{m}\left(\mathcal{G}_{L_{j}}\right) \cap \bar{S}$ and $L_{m}\left(\mathcal{G}_{L_{j}}\right) \cap \bar{S} \subseteq S$.

By Proposition 4.19, we have $S \subseteq L_{m}\left(\mathcal{G}_{L_{j}}\right)$.

$\Rightarrow S \subseteq L_{m}\left(\mathcal{G}_{L_{j}}\right) \cap \bar{S}, \quad$ as $S \subseteq \bar{S}$

Now we show $L_{m}\left(\mathcal{G}_{L_{j}}\right) \cap \bar{S} \subseteq S$.

Let $t \in L_{m}\left(\mathcal{G}_{L_{j}}\right) \cap \bar{S}$.

Must show this implies $t \in S$.

From (2), we have $t \in L_{m}\left(\mathcal{G}_{L_{j}}\right)$ and $t \in \bar{S}$

$\Rightarrow\{t\} \subseteq L_{m}\left(\mathcal{G}_{L_{j}}\right)$ and $\{t\} \subseteq \bar{S}$

$\Rightarrow\{t\} \subseteq L_{m}\left(\mathcal{G}_{L_{j}}\right)$ and $\overline{\{t\}} \subseteq \bar{S}$

Let $S^{\prime}:=S \cup\{t\}$.

$\Rightarrow S^{\prime} \subseteq L_{m}\left(\mathcal{G}_{L_{j}}\right), \quad$ by $(1)$ and $(3)$

We now show $S^{\prime}$ is $j^{\text {th }}$ low-level interface controllable with respect to system $\Phi$. 
Master Thesis - R. Song-McMaster - Computing and Software

i. Show that $\overline{S^{\prime}} \Sigma_{l u_{j}} \cap L\left(\mathbf{G}_{L_{j}}^{p}\right) \subseteq \overline{S^{\prime}}$

$S$ is $j^{\text {th }}$ low-level interface controllable, so $\bar{S} \Sigma_{l u_{j}} \cap L\left(\mathbf{G}_{L_{j}}^{p}\right) \subseteq \bar{S}$.

By (3) and (5), we have $\overline{\{t\}} \Sigma_{l_{u_{j}}} \cap L\left(\mathbf{G}_{L_{j}}^{p}\right) \subseteq \bar{S}$

From (5) and (6), we have $(\bar{S} \cup \overline{\{t\}}) \Sigma_{l u_{j}} \cap L\left(\mathbf{G}_{L_{j}}^{p}\right) \subseteq \bar{S}$

$\Rightarrow(\overline{S \cup\{t\}}) \Sigma_{l u_{j}} \cap L\left(\mathbf{G}_{L_{j}}^{p}\right) \subseteq \bar{S}, \quad$ by Proposition 2.2

$\Rightarrow \overline{S^{\prime}} \Sigma_{l u_{j}} \cap L\left(\mathbf{G}_{L_{j}}^{p}\right) \subseteq \bar{S}$

$\Rightarrow \overline{S^{\prime}} \Sigma_{l u_{j}} \cap L\left(\mathbf{G}_{L_{j}}^{p}\right) \subseteq \overline{S^{\prime}}$, as $S \subseteq S^{\prime}$ by definition of $S^{\prime}$

ii. Show that $\overline{S^{\prime}} \Sigma_{R_{j}} \cap L\left(\mathbf{G}_{I_{j}}^{l}\right) \subseteq \overline{S^{\prime}}$

$S$ is $j^{\text {th }}$ low-level interface controllable, so $\bar{S} \Sigma_{R_{j}} \cap L\left(\mathbf{G}_{I_{j}}^{l}\right) \subseteq \bar{S}$.

By (3) and (8), we have $\overline{\{t\}} \Sigma_{R_{j}} \cap L\left(\mathbf{G}_{I_{j}}^{l}\right) \subseteq \bar{S}$

From (8) and (9), we have $(\bar{S} \cup \overline{\{t\}}) \Sigma_{R_{j}} \cap L\left(\mathbf{G}_{I_{j}}^{l}\right) \subseteq \bar{S}$

$\Rightarrow(\overline{S \cup\{t\}}) \Sigma_{R_{j}} \cap L\left(\mathbf{G}_{I_{j}}^{l}\right) \subseteq \bar{S}, \quad$ by Proposition 2.2

$\Rightarrow \overline{S^{\prime}} \Sigma_{R_{j}} \cap L\left(\mathbf{G}_{I_{j}}^{l}\right) \subseteq \bar{S}$

$\Rightarrow \overline{S^{\prime}} \Sigma_{R_{j}} \cap L\left(\mathbf{G}_{I_{j}}^{l}\right) \subseteq \overline{S^{\prime}}$, as $S \subseteq S^{\prime}$ by definition of $S^{\prime}$

iii. Show that $\left(\forall s \in \overline{S^{\prime}}\right)\left(\forall \rho \in \Sigma_{R_{j}}\right)\left(\forall \alpha \in \Sigma_{A_{j}}\right) s \rho \alpha \in L\left(\mathbf{G}_{I_{j}}^{l}\right) \Rightarrow\left(\exists l \in \Sigma_{L_{j}}^{*}\right) s \rho l \alpha \in \overline{S^{\prime}}$

$S$ is $j^{\text {th }}$ low-level interface controllable, so

$(\forall s \in \bar{S})\left(\forall \rho \in \Sigma_{R_{j}}\right)\left(\forall \alpha \in \Sigma_{A_{j}}\right) s \rho \alpha \in L\left(\mathbf{G}_{I_{j}}^{l}\right) \Rightarrow\left(\exists l \in \Sigma_{L_{j}}^{*}\right) s \rho l \alpha \in \bar{S}$

By (3) and (11), $(\forall s \in \overline{\{t\}})\left(\forall \rho \in \Sigma_{R_{j}}\right)\left(\forall \alpha \in \Sigma_{A_{j}}\right)$

$$
s \rho \alpha \in L\left(\mathbf{G}_{I_{j}}^{l}\right) \Rightarrow\left(\exists l \in \Sigma_{L_{j}}^{*}\right) \text { s } \rho l \alpha \in \bar{S}
$$

By (11) and (12), $(\forall s \in \bar{S} \cup \overline{\{t\}})\left(\forall \rho \in \Sigma_{R_{j}}\right)\left(\forall \alpha \in \Sigma_{A_{j}}\right)$

$$
s \rho \alpha \in L\left(\mathbf{G}_{I_{j}}^{l}\right) \Rightarrow\left(\exists l \in \Sigma_{L_{j}}^{*}\right) \text { s } \rho l \alpha \in \bar{S}
$$

$\Rightarrow(\forall s \in \overline{S \cup\{t\}})\left(\forall \rho \in \Sigma_{R_{j}}\right)\left(\forall \alpha \in \Sigma_{A_{j}}\right)$

$s \rho \alpha \in L\left(\mathbf{G}_{I_{j}}^{l}\right) \Rightarrow\left(\exists l \in \Sigma_{L_{j}}^{*}\right)$ s $\rho l \alpha \in \bar{S}, \quad$ by Proposition 2.2

$\Rightarrow\left(\forall s \in \overline{S^{\prime}}\right)\left(\forall \rho \in \Sigma_{R_{j}}\right)\left(\forall \alpha \in \Sigma_{A_{j}}\right)$

$$
s \rho \alpha \in L\left(\mathbf{G}_{I_{j}}^{l}\right) \Rightarrow\left(\exists l \in \Sigma_{L_{j}}^{*}\right) s \rho l \alpha \in \overline{S^{\prime}} .
$$


Master Thesis - R. Song - McMaster - Computing and Software

iv. Show that $\left(\forall s \in \overline{S^{\prime}}\right) \quad s \in L_{m}\left(\mathbf{G}_{I_{j}}^{l}\right) \Rightarrow\left(\exists l \in \Sigma_{L_{j}}^{*}\right) s l \in S^{\prime}$

$S$ is $j^{\text {th }}$ low-level interface controllable, so

$(\forall s \in \bar{S}) s \in L_{m}\left(\mathbf{G}_{I_{j}}^{l}\right) \Rightarrow\left(\exists l \in \Sigma_{L_{j}}^{*}\right) s l \in S$.

By (3) and (14), we have

$(\forall s \in \overline{\{t\}}) s \in L_{m}\left(\mathbf{G}_{I_{j}}^{l}\right) \Rightarrow\left(\exists l \in \Sigma_{L_{j}}^{*}\right) s l \in S$.

By (14) and (15),we have

$(\forall s \in \bar{S} \cup \overline{\{t\}}) s \in L_{m}\left(\mathbf{G}_{I_{j}}^{l}\right) \Rightarrow\left(\exists l \in \Sigma_{L_{j}}^{*}\right) s l \in S$

$\Rightarrow(\forall s \in \overline{S \cup\{t\}}) s \in L_{m}\left(\mathbf{G}_{I_{j}}^{l}\right) \Rightarrow\left(\exists l \in \Sigma_{L_{j}}^{*}\right) s l \in S$, by Proposition 2.2

$\Rightarrow\left(\forall s \in \overline{S^{\prime}}\right) s \in L_{m}\left(\mathbf{G}_{I_{j}}^{l}\right) \Rightarrow\left(\exists l \in \Sigma_{L_{j}}^{*}\right) s l \in S^{\prime}$

By (4), (7), (10), (13) and (16), we have $S^{\prime} \in \mathcal{C}_{L_{j}}\left(L_{m}\left(\mathcal{G}_{L_{j}}\right)\right)$.

As $S=\sup \mathcal{C}_{L_{j}}\left(L_{m}\left(\mathcal{G}_{L_{j}}\right)\right)$, we then have $S^{\prime} \subseteq S$.

$\Rightarrow\{t\} \subseteq S$

$\Rightarrow t \in S$

(c) Show that $\bar{S}=\Omega_{p 6_{j}}(\bar{S})$

Sufficient to show that

$(\forall s \in \bar{S})(\forall t \leq s) t \in L_{m}\left(\mathbf{G}_{I_{j}}^{l}\right) \Rightarrow\left(\exists l \in \Sigma_{L_{j}}^{*}\right) t l \in L_{m}\left(\mathcal{G}_{L_{j}}\right) \cap \bar{S}$

Let $s \in \bar{S}$ and $t \leq s$.

Assume $t \in L_{m}\left(\mathbf{G}_{I_{j}}^{l}\right)$. Must show this implies $\left(\exists l \in \Sigma_{L_{j}}^{*}\right) t l \in L_{m}\left(\mathcal{G}_{L_{j}}\right) \cap \bar{S}$

From (17) and the assumption $t \in L_{m}\left(\mathbf{G}_{I_{j}}^{l}\right)$, by the definition of $S$, we have $\left(\exists l \in \Sigma_{L_{j}}^{*}\right) t l \in S$

$\Rightarrow\left(\exists l \in \Sigma_{L_{j}}^{*}\right) t l \in L_{m}\left(\mathcal{G}_{L_{j}}\right) \cap \bar{S}, \quad$ by Part $1(\mathrm{~b})$

By combining (a), (b), (c) and the definition of $\Omega_{L_{j}}$, we know $S=\Omega_{L_{j}}(S)$. 
Master Thesis - R. Song - McMaster - Computing and Software

2. Show that $S$ is the greatest fixpoint of $\Omega_{L_{j}}$. i.e.

$$
\left(\forall T \in \operatorname{Pwr}\left(\Sigma_{I L_{j}}^{*}\right)\right) T=\Omega_{L_{j}}(T) \Rightarrow T \subseteq S .
$$

Let $T \in \operatorname{Pwr}\left(\Sigma_{I L_{j}}^{*}\right)$. Assume $T=\Omega_{L_{j}}(T)$. Must show this implies $T \subseteq S$.

Sufficient to show that $T$ is $j^{\text {th }}$ low-level controllable with respect to system $\boldsymbol{\Phi}$ and $T \subseteq L_{m}\left(\mathcal{G}_{L_{j}}\right)$.

$$
\begin{aligned}
T & =\Omega_{L_{j}}(T) \\
& =\Omega_{L N B_{j}}\left(\Omega_{p 6_{j}}\left(\Omega_{p 5_{j}}\left(\operatorname{LPC}_{j}(T)\right)\right)\right) \\
& =L_{m}\left(\mathcal{G}_{L_{j}}\right) \cap \Omega_{p 6_{j}}\left(\Omega_{p 5_{j}}\left(\sup \mathcal{L P \mathcal { C } _ { j }}(\bar{T})\right)\right) \\
& \subseteq \Omega_{p 6_{j}}\left(\Omega_{p 5_{j}}\left(\sup \mathcal{L} \mathcal{P} \mathcal{C}_{j}(\bar{T})\right)\right)
\end{aligned}
$$

As $\bar{T}$ is closed, by Lemma 4.8, $\sup \mathcal{L P C}_{j}(\bar{T})$ is closed. Then by Lemma 4.14, $\Omega_{p 6_{j}}\left(\Omega_{p 5_{j}}\left(\sup \mathcal{L} \mathcal{P} \mathcal{C}_{j}(\bar{T})\right)\right)$ is also closed. So by (18), we have

$$
\bar{T} \subseteq \Omega_{p 6_{j}}\left(\Omega_{p 5_{j}}\left(\sup \mathcal{L P C}_{j}(\bar{T})\right)\right)
$$

For any $L \in \operatorname{Pwr}\left(\Sigma_{I L_{j}}^{*}\right)$, we have $\sup \mathcal{L P C}_{j}(L) \subseteq L$ by Proposition 4.12, $\Omega_{p 5_{j}}(L) \subseteq L$ by the definition of $\Omega_{p 5_{j}}$ and $\Omega_{p 6_{j}}(L) \subseteq L$ by the definition of $\Omega_{p 6_{j}}$, so we know

$$
\Omega_{p 6_{j}}\left(\Omega_{p 5_{j}}\left(\sup \mathcal{L} \mathcal{P} \mathcal{C}_{j}(\bar{T})\right)\right) \subseteq \bar{T}
$$

By (19) and (20), we have

$$
\bar{T}=\Omega_{p 6_{j}}\left(\Omega_{p 5_{j}}\left(\sup \mathcal{L P C}_{j}(\bar{T})\right)\right)
$$

Function $\Omega_{p 6_{j}}$ and $\Omega_{p 5_{j}}$ only remove strings from their inputs, and $\sup \mathcal{L P C _ { j }}(\bar{T}) \subseteq$ $\bar{T}$, so from (21) we have

$$
\bar{T}=\sup \mathcal{L P C}_{j}(\bar{T}), \bar{T}=\Omega_{p 5_{j}}(\bar{T}) \text { and } \bar{T}=\Omega_{p 6_{j}}(\bar{T})
$$




\section{Master Thesis - R. Song-McMaster-Computing and Software}

From $\bar{T}=\sup \mathcal{L P \mathcal { C } _ { j }}(\bar{T})$ and $\bar{T}=\Omega_{p 5_{j}}(\bar{T})$, by Lemma 4.15 , we know that $T$ is $j^{\text {th }}$ low-level P4 interface controllable and $j^{\text {th }}$ low-level P5-satisfied.

It remains to show that $T$ is $j^{\text {th }}$ low-level P6-satisfied.

Sufficient to show that $(\forall u \in \bar{T}) u \in L_{m}\left(\mathbf{G}_{I_{j}}^{l}\right) \Rightarrow\left(\exists l \in \Sigma_{L_{j}}^{*}\right) u l \in T$.

Let $u \in \bar{T}$. Assume $u \in L_{m}\left(\mathbf{G}_{I_{j}}^{l}\right)$. Must show this implies $\left(\exists l \in \Sigma_{L_{j}}^{*}\right) u l \in T$.

From (18) and (21), we also have $T=L_{m}\left(\mathcal{G}_{L_{j}}\right) \cap \bar{T}$

From (22), we have $\bar{T}=\Omega_{p 6_{j}}(\bar{T})$

$$
\begin{aligned}
& \Rightarrow \bar{T}=\left\{s \in \bar{T} \mid(\forall t \leq s) t \in L_{m}\left(\mathbf{G}_{I_{j}}^{l}\right) \Rightarrow\left(\exists l \in \Sigma_{L_{j}}^{*}\right) t l \in L_{m}\left(\mathcal{G}_{L_{j}}\right) \cap \bar{T}\right\} \\
& \Rightarrow u \in\left\{s \in \bar{T} \mid(\forall t \leq s) t \in L_{m}\left(\mathbf{G}_{I_{j}}^{l}\right) \Rightarrow\left(\exists l \in \Sigma_{L_{j}}^{*}\right) t l \in L_{m}\left(\mathcal{G}_{L_{j}}\right) \cap \bar{T}\right\}, \text { as } u \in \bar{T} \\
& \Rightarrow(\forall t \leq u)\left(t \in L_{m}\left(\mathbf{G}_{I_{j}}^{l}\right) \Rightarrow\left(\exists l \in \Sigma_{L_{j}}^{*}\right) t l \in L_{m}\left(\mathcal{G}_{L_{j}}\right) \cap \bar{T}\right) \\
& \Rightarrow u \in L_{m}\left(\mathbf{G}_{I_{j}}^{l}\right) \Rightarrow\left(\exists l \in \Sigma_{L_{j}}^{*}\right) u l \in L_{m}\left(\mathcal{G}_{L_{j}}\right) \cap \bar{T}, \quad \text { as } u \leq u \\
& \Rightarrow\left(\exists l \in \Sigma_{L_{j}}^{*}\right) u l \in L_{m}\left(\mathcal{G}_{L_{j}}\right) \cap \bar{T}, \quad \text { by assumption } u \in L_{m}\left(\mathbf{G}_{I_{j}}^{l}\right) \\
& \Rightarrow\left(\exists l \in \Sigma_{L_{j}}^{*}\right) u l \in T, \quad \text { by }(23)
\end{aligned}
$$

From (18), clearly, $T \subseteq L_{m}\left(\mathcal{G}_{L_{j}}\right)$.

\subsubsection{Computing the Greatest Fixpoint of $\Omega_{L_{j}}$}

From Proposition 4.20 , we know that we can compute $\sup \mathcal{C}_{L_{j}}\left(L_{m}\left(\mathcal{G}_{L_{j}}\right)\right)$ by computing the greatest fixpoint of $\Omega_{L_{j}}$. It is tempting to compute the greatest fixpoint by iteration of $\Omega_{L_{j}}$.

Proposition 4.21. For system $\boldsymbol{\Phi}$, the set theoretic limit $\lim _{i \rightarrow \infty}\left(L\left(\mathcal{G}_{L_{j}}\right)\right), i \in\{1,2, \ldots\}$ exists, and $\sup \mathcal{C}_{L_{j}}\left(L_{m}\left(\mathcal{G}_{L_{j}}\right) \subseteq \lim _{i \rightarrow \infty} \Omega_{L_{j}}^{i}\left(L\left(\mathcal{G}_{L_{j}}\right)\right)\right.$. 


\section{Master Thesis - R. Song - McMaster - Computing and Software}

\section{proof:}

Identical to the proof of Proposition 4.3 by substituting $\mathcal{G}_{H}$ with $\mathcal{G}_{L_{j}}$, sup $\mathcal{C}_{H}$ with $\sup \mathcal{C}_{L_{j}}, \Omega_{H}$ with $\Omega_{L_{j}}$, Proposition 4.1 with Proposition 4.19, and Proposition 4.2 with Proposition 4.20 .

Similar to the high-level, here we start the iteration from the closed language $L\left(\mathcal{G}_{L_{j}}\right)$. It remains to show the reverse $\lim _{i \rightarrow \infty} \Omega_{L_{j}}^{i}\left(L\left(\mathcal{G}_{L_{j}}\right)\right) \subseteq \sup \mathcal{C}_{L_{j}}\left(L_{m}\left(\mathcal{G}_{L_{j}}\right)\right)$ and that the limit will be reached after a finite number of iterations on $\Omega_{L_{j}}($ i.e. $i<\infty)$. Again, in general case, the reverse does not hold, but in our case where all the languages are regular, the reverse does hold and the number of iterations on $\Omega_{L_{j}}$ is finite, as we will show.

For a given predicate $P \in \operatorname{Pred}\left(Q_{L_{j}}\right)$, we already know the method to compute $\sup \mathcal{L P C}_{j}\left(L\left(\mathcal{G}_{L_{j}}, P\right)\right)$ from Proposition 4.18. We now show the method to compute $\Omega_{p 5_{j}}\left(L\left(\mathcal{G}_{L_{j}}, P\right)\right)$ and $\Omega_{p 6_{j}}\left(L\left(\mathcal{G}_{L_{j}}, P\right)\right)$.

Computing $\Omega_{p 5_{j}}\left(L\left(\mathcal{G}_{L_{j}}, P\right)\right)$

Definition 4.18. For system $\boldsymbol{\Phi}$, define the function $W_{p 5_{j}}: \operatorname{Pred}\left(Q_{L_{j}}\right) \rightarrow \operatorname{Pwr}\left(\Sigma_{I L_{j}}^{*}\right)$ according to

$$
\begin{aligned}
\left(\forall P \in \operatorname{Pred}\left(Q_{L_{j}}\right)\right) W_{p 5_{j}}(P):=\left\{s \in L\left(\mathcal{G}_{L_{j}}, P\right) \mid\left(\exists \rho \in \Sigma_{R_{j}}\right)\left(\exists \alpha \in \Sigma_{A_{j}}\right)\right. \\
\left.s \rho \alpha \in L\left(\mathbf{G}_{I_{j}}^{l}\right) \&\left(\forall l \in \Sigma_{L_{j}}^{*}\right) \operatorname{s\rho l} \alpha \notin L\left(\mathcal{G}_{L_{j}}, P\right)\right\}
\end{aligned}
$$

Clearly, for all $P \in \operatorname{Pred}\left(Q_{L_{j}}\right), W_{p 5_{j}}(P) \subseteq L\left(\mathcal{G}_{L_{j}}, P\right)$.

Lemma 4.16. For system $\boldsymbol{\Phi}$, the following holds:

$$
\begin{aligned}
& \left(\forall P \in \operatorname{Pred}\left(Q_{L_{j}}\right)\right)\left(\forall s, t \in L\left(\mathcal{G}_{L_{j}}, P\right)\right) \\
& \quad\left(s \in W_{p 5_{j}}(P) \&\left(\delta_{L_{j}}\left(q_{L j_{0}}, s\right)=\delta_{L_{j}}\left(q_{L j_{0}}, t\right)\right)\right) \Rightarrow t \in W_{p 5_{j}}(P) .
\end{aligned}
$$




\section{Master Thesis - R. Song-McMaster-Computing and Software}

\section{proof:}

Let $P \in \operatorname{Pred}\left(Q_{L_{j}}\right)$, and $s, t \in L\left(\mathcal{G}_{L_{j}}, P\right)$.

Assume $\delta\left(q_{L j_{0}}, s\right)=\delta\left(q_{L j_{0}}, t\right)$ and $s \in W_{p 5_{j}}(P)$.

Must show this implies $t \in W_{p 5_{j}}(P)$.

Let $\equiv_{L\left(\mathcal{G}_{L_{j}}, P\right)}, \equiv_{L\left(\mathbf{G}_{I_{j}}^{l}\right)}$ be the Nerode equivalence relation on $\Sigma_{I L_{j}}^{*}$ with respect to $L\left(\mathcal{G}_{L_{j}}, P\right)$ and $L\left(\mathbf{G}_{I_{j}}^{l}\right)$ respectively.

From (2), we have $\delta\left(q_{L j_{0}}, s\right)=\delta\left(q_{L j_{0}}, t\right)$

$\Rightarrow s \equiv{ }_{L\left(\mathcal{G}_{L_{j}}, P\right)} t$,

by Proposition 2.6

As $\mathcal{G}_{L_{j}}=\mathbf{E}_{L_{j}} \times \mathbf{G}_{L_{j}}^{p} \times \mathbf{G}_{I_{j}}^{l}$ and $\delta_{L_{j}}\left(q_{L j_{0}}, s\right)=\delta_{L_{j}}\left(q_{L j_{0}}, t\right)$, we have

$\xi_{j}^{l}\left(x_{j 0}^{l}, s\right)=\xi_{j}^{l}\left(x_{j 0}^{l}, t\right)$

$\Rightarrow s \equiv_{L\left(\mathbf{G}_{I_{j}}^{l}\right)} t$,

by Proposition 2.6 and fact $L\left(\mathbf{G}_{I_{j}}^{l}\right.$, true $)=L\left(\mathbf{G}_{I_{j}}^{l}\right)$.

From (2), we have $s \in W_{p 5_{j}}(P)$

$\Rightarrow\left(\exists \rho \in \Sigma_{R_{j}}\right)\left(\exists \alpha \in \Sigma_{A_{j}}\right) s \rho \alpha \in L\left(\mathbf{G}_{I_{j}}^{l}\right) \&\left(\forall l \in \Sigma_{L_{j}}^{*}\right) s \rho l \alpha \notin L\left(\mathcal{G}_{L_{j}}, P\right)$

$\Rightarrow\left(\exists \rho \in \Sigma_{R_{j}}\right)\left(\exists \alpha \in \Sigma_{A_{j}}\right) t \rho \alpha \in L\left(\mathbf{G}_{I_{j}}^{l}\right) \&\left(\forall l \in \Sigma_{L_{j}}^{*}\right)$ t $\rho l \alpha \notin L\left(\mathcal{G}_{L_{j}}, P\right)$, by (3)(4)

$\Rightarrow t \in W_{p 5_{j}}(P), \quad$ as $t \in L\left(\mathcal{G}_{L_{j}}, P\right)$.

Lemma 4.17. For system $\mathbf{\Phi}$, the following holds:

$\left.\left(\forall P \in \operatorname{Pred}\left(Q_{L_{j}}\right)\right)\left(\forall s \in L\left(\mathcal{G}_{L_{j}}, P\right)\right)\left((\exists t \leq s) t \in W_{p 5_{j}}(P)\right) \Leftrightarrow s \notin \Omega_{p 5_{j}}\left(L\left(\mathcal{G}_{L_{j}}, P\right)\right)\right)$.

\section{proof:}

Let $P \in \operatorname{Pred}\left(Q_{L_{j}}\right)$ and $s \in L\left(\mathcal{G}_{L_{j}}, P\right)$.

1. Show that $\left((\exists t \leq s) t \in W_{p 5_{j}}(P)\right) \Rightarrow s \notin \Omega_{p 5_{j}}\left(L\left(\mathcal{G}_{L_{j}}, P\right)\right)$.

Assume $(\exists t \leq s) t \in W_{p 5_{j}}(P)$. 
Master Thesis - R. Song - McMaster - Computing and Software

Must show this implies $s \notin \Omega_{p 5_{j}}\left(L\left(\mathcal{G}_{L_{j}}, P\right)\right)$.

From (2), we have

$$
\begin{aligned}
& (\exists t \leq s)\left(\exists \rho \in \Sigma_{R_{j}}\right)\left(\exists \alpha \in \Sigma_{A_{j}}\right) t \rho \alpha \in L\left(\mathbf{G}_{I_{j}}^{l}\right) \&\left(\forall l \in \Sigma_{L_{j}}^{*}\right) t \rho l \alpha \notin L\left(\mathcal{G}_{L_{j}}, P\right) \\
& \Rightarrow \neg\left((\forall t \leq s)\left(\forall \rho \in \Sigma_{R_{j}}\right)\left(\forall \alpha \in \Sigma_{A_{j}}\right)\right. \\
& \left.\quad t \rho \alpha \in L\left(\mathbf{G}_{I_{j}}^{l}\right) \Rightarrow\left(\exists l \in \Sigma_{L_{j}}^{*}\right) t \rho l \alpha \in L\left(\mathcal{G}_{L_{j}}, P\right)\right) \\
& \Rightarrow s \notin \Omega_{p 5_{j}}\left(L\left(\mathcal{G}_{L_{j}}, P\right)\right) .
\end{aligned}
$$

2. Show that $s \notin \Omega_{p 5_{j}}\left(L\left(\mathcal{G}_{L_{j}}, P\right)\right) \Rightarrow(\exists t \leq s) t \in W_{p 5_{j}}(P)$.

Assume $s \notin \Omega_{p 5_{j}}\left(L\left(\mathcal{G}_{L_{j}}, P\right)\right)$.

Must show this implies $(\exists t \leq s) t \in W_{p 5_{j}}(P)$.

From (1), we know $s \in L\left(\mathcal{G}_{L_{j}}, P\right)$. Combine this with (3), we thus have

$$
\begin{gathered}
\neg\left((\forall t \leq s)\left(\forall \rho \in \Sigma_{R_{j}}\right)\left(\forall \alpha \in \Sigma_{A_{j}}\right)\right. \\
\left.t \rho \alpha \in L\left(\mathbf{G}_{I_{j}}^{l}\right) \Rightarrow\left(\exists l \in \Sigma_{L_{j}}^{*}\right) t \rho l \alpha \in L\left(\mathcal{G}_{L_{j}}, P\right)\right) \\
\Rightarrow(\exists t \leq s)\left(\exists \rho \in \Sigma_{R_{j}}\right)\left(\exists \alpha \in \Sigma_{A_{j}}\right) \\
t \rho \alpha \in L\left(\mathbf{G}_{I_{j}}^{l}\right) \&\left(\forall l \in \Sigma_{L_{j}}^{*}\right) t \rho l \alpha \notin L\left(\mathcal{G}_{L_{j}}, P\right)
\end{gathered}
$$

As $s \in L\left(\mathcal{G}_{L_{j}}, P\right)$ and $L\left(\mathcal{G}_{L_{j}}, P\right)$ is closed, we know $t \in L\left(\mathcal{G}_{L_{j}}, P\right)$.

By (4) and (5), we thus have $(\exists t \leq s) t \in W_{p 5_{j}}(P)$.

Proposition 4.22. For system $\boldsymbol{\Phi}$, the following holds

$$
\left(\forall P \in \operatorname{Pred}\left(Q_{L_{j}}\right)\right) L\left(\mathcal{G}_{L_{j}}, P\right)-\Omega_{p 5_{j}}\left(L\left(\mathcal{G}_{L_{j}}, P\right)\right)=L^{W_{p 5_{j}}(P)}\left(\mathcal{G}_{L_{j}}, P\right)
$$

where $L^{W_{p 5_{j}}(P)}\left(\mathcal{G}_{L_{j}}, P\right)=\cup_{s \in W_{p 5_{j}}(P)} L^{s}\left(\mathcal{G}_{L_{j}}, P\right)$. 


\section{Master Thesis - R. Song - McMaster - Computing and Software}

\section{proof:}

Identical to the proof of Proposition 4.6 after substituting $\operatorname{Sub}\left(\mathcal{P}_{h a}\right)$ with $\operatorname{Pred}\left(Q_{L_{j}}\right)$, $\Omega_{H I C}$ with $\Omega_{p 5_{j}}, W_{H}(P)$ with $W_{p 5_{j}}(P), \mathcal{G}_{H}$ with $\mathcal{G}_{L_{j}}, \delta_{H}$ with $\delta_{L_{j}}, q_{H_{0}}$ with $q_{L j_{0}}$, Lemma 4.2 with Lemma 4.16 and Lemma 4.3 with Lemma 4.17.

Definition 4.19. For system $\Phi$, define the function $\Gamma_{p 5_{j}}: \operatorname{Pred}\left(Q_{L_{j}}\right) \rightarrow \operatorname{Pred}\left(Q_{L_{j}}\right)$ according to

$$
\begin{aligned}
& \left(\forall P \in \operatorname{Pred}\left(Q_{L_{j}}\right)\right) \\
& \quad \Gamma_{p 5_{j}}(P):=P-\operatorname{pr}\left(\left\{q \in Q_{L_{j}} \mid\left(\exists \rho \in \Sigma_{R_{j}}\right)\left(\exists \alpha \in \Sigma_{A_{j}}\right)\right.\right. \\
& \left.\left.\xi_{j}^{l}(x, \rho \alpha) ! \& \delta_{L_{j}}(q, \rho) \models \neg \mathcal{C} \mathcal{R}\left(\mathcal{G}_{L_{j}}, P_{\alpha}, \Sigma_{L_{j}}, P\right)\right\}\right) .
\end{aligned}
$$

where $P_{\alpha}:=\operatorname{pr}\left(\left\{q^{\prime} \in Q_{L_{j}} \mid \delta_{L_{j}}\left(q^{\prime}, \alpha\right) \models P\right\}\right)$ and

$$
q=(z, y, x) \text { as in equation } 4.2 \text { (Page 105). }
$$

The following lemma will be used later on.

Lemma 4.18. For system $\Phi, \Gamma_{p 5_{j}}$ is monotone with respect to $\preceq$, i.e.

$$
\left(\forall P_{1}, P_{2} \in \operatorname{Pred}\left(Q_{L_{j}}\right)\right) P_{1} \preceq P_{2} \Rightarrow \Gamma_{p 5_{j}}\left(P_{1}\right) \preceq \Gamma_{p 5_{j}}\left(P_{2}\right) .
$$

\section{proof:}

Let $P_{1}, P_{2} \in \operatorname{Pred}\left(Q_{L_{j}}\right)$. Assume $P_{1} \preceq P_{2}$.

Must show this implies $\Gamma_{p 5_{j}}\left(P_{1}\right) \preceq \Gamma_{p 5_{j}}\left(P_{2}\right)$.

$$
\begin{gathered}
\Gamma_{p 5_{j}}\left(P_{1}\right)=P_{1}-\operatorname{pr}\left(\left\{q \in Q_{L_{j}} \mid\left(\exists \rho \in \Sigma_{R_{j}}\right)\left(\exists \alpha \in \Sigma_{A_{j}}\right)\right.\right. \\
\left.\left.\xi_{j}^{l}(x, \rho \alpha) ! \& \delta_{L_{j}}(q, \rho) \models \neg \mathcal{C R}\left(\mathcal{G}_{L_{j}}, P_{\alpha}, \Sigma_{L_{j}}, P_{1}\right)\right\}\right) \\
=P_{1} \wedge \neg p r\left(\left\{q \in Q_{L_{j}} \mid\left(\exists \rho \in \Sigma_{R_{j}}\right)\left(\exists \alpha \in \Sigma_{A_{j}}\right)\right.\right. \\
\left.\left.\xi_{j}^{l}(x, \rho \alpha) ! \& \delta_{L_{j}}(q, \rho) \models \neg \mathcal{C R}\left(\mathcal{G}_{L_{j}}, P_{\alpha}, \Sigma_{L_{j}}, P_{1}\right)\right\}\right) \\
=P_{1} \wedge p r\left(\left\{q \in Q_{L_{j}} \mid\left(\forall \rho \in \Sigma_{R_{j}}\right)\left(\forall \alpha \in \Sigma_{A_{j}}\right)\right.\right. \\
\left.\left.\xi_{j}^{l}(x, \rho \alpha) ! \Rightarrow \delta_{L_{j}}(q, \rho) \models \mathcal{C R}\left(\mathcal{G}_{L_{j}}, P_{\alpha}, \Sigma_{L_{j}}, P_{1}\right)\right\}\right)
\end{gathered}
$$


Master Thesis - R. Song - McMaster - Computing and Software

$$
\begin{aligned}
& \preceq P_{2} \wedge \operatorname{pr}\left(\left\{q \in Q_{L_{j}} \mid\left(\forall \rho \in \Sigma_{R_{j}}\right)\left(\forall \alpha \in \Sigma_{A_{j}}\right)\right.\right. \\
& \left.\left.\xi_{j}^{l}(x, \rho \alpha) ! \Rightarrow \delta_{L_{j}}(q, \rho) \models \mathcal{C} \mathcal{R}\left(\mathcal{G}_{L_{j}}, P_{\alpha}, \Sigma_{L_{j}}, P_{2}\right)\right\}\right), \\
& \text { as } P_{1} \preceq P_{2} \text { and } \mathcal{C} \mathcal{R} \text { is monotone. } \\
& =P_{2} \wedge \neg p r\left(\left\{q \in Q_{L_{j}} \mid\left(\exists \rho \in \Sigma_{R_{j}}\right)\left(\exists \alpha \in \Sigma_{A_{j}}\right)\right.\right. \\
& \left.\left.\xi_{j}^{l}(x, \rho \alpha) ! \& \delta_{L_{j}}(q, \rho) \models \neg \mathcal{C R}\left(\mathcal{G}_{L_{j}}, P_{\alpha}, \Sigma_{L_{j}}, P_{2}\right)\right\}\right) \\
& =P_{2}-\operatorname{pr}\left(\left\{q \in Q_{L_{j}} \mid\left(\exists \rho \in \Sigma_{R_{j}}\right)\left(\exists \alpha \in \Sigma_{A_{j}}\right)\right.\right. \\
& \left.\left.\xi_{j}^{l}(x, \rho \alpha) ! \& \delta_{L_{j}}(q, \rho) \models \neg \mathcal{C R}\left(\mathcal{G}_{L_{j}}, P_{\alpha}, \Sigma_{L_{j}}, P_{2}\right)\right\}\right) \\
& =\Gamma_{p 5_{j}}\left(P_{2}\right)
\end{aligned}
$$

The following lemma will be used in the proof of next proposition.

Lemma 4.19. For system $\boldsymbol{\Phi}$, let $P \in \operatorname{Pred}\left(Q_{L_{j}}\right), w \in \Sigma_{I L_{j}}^{*}$ and $q \in Q_{L_{j}}$. If $q \models$ $R\left(\mathcal{G}_{L_{j}}, P\right)$ and $q=\delta_{L_{j}}\left(q_{L j_{0}}, w\right)$, then

$\left(\forall \rho \in \Sigma_{R_{j}}\right)\left(\forall \alpha \in \Sigma_{A_{j}}\right) \delta_{L_{j}}(q, \rho) \models \mathcal{C} \mathcal{R}\left(\mathcal{G}_{L_{j}}, P_{\alpha}, \Sigma_{L_{j}}, P\right)$ iff $\left(\exists l \in \Sigma_{L_{j}}^{*}\right)$ w $\rho l \alpha \in L\left(\mathcal{G}_{L_{j}}, P\right)$,

where $P_{\alpha}:=\operatorname{pr}\left(\left\{q^{\prime} \in Q_{L_{j}} \mid \delta_{L_{j}}\left(q^{\prime}, \alpha\right) \models P\right\}\right)$ as defined in Definition 4.19.

\section{proof:}

Assume $q \models R\left(\mathcal{G}_{L_{j}}, P\right)$ and $q=\delta_{L_{j}}\left(q_{L j_{0}}, w\right)$.

Let $\rho \in \Sigma_{R_{j}}$ and $\alpha \in \Sigma_{A_{j}}$.

$$
\begin{gathered}
\delta_{L_{j}}(q, \rho) \models \mathcal{C R}\left(\mathcal{G}_{L_{j}}, P_{\alpha}, \Sigma_{L_{j}}, P\right) \\
\Leftrightarrow(\exists k \in\{0,1, \ldots\})\left(\exists \sigma_{1}, \ldots, \sigma_{k} \in \Sigma_{L_{j}}\right)\left(\exists q_{1}, \ldots, q_{k+2} \in Q_{L_{j}}\right) \\
q_{1}=\delta_{L_{j}}(q, \rho) \\
q_{k+1} \models P_{\alpha} \\
q_{1}, \ldots, q_{k+2} \models P \\
q_{i+1}=\delta_{L_{j}}\left(q_{i}, \sigma_{i}\right), i=1,2, \ldots, k \\
q_{k+2}=\delta_{L_{j}}\left(q_{k+1}, \alpha\right),
\end{gathered}
$$

by the definitions of $\mathcal{C R}$ and $P_{\alpha}$. 


$$
\begin{aligned}
& \text { Master Thesis - R. Song - McMaster - Computing and Software } \\
& \Leftrightarrow(\exists k \in\{0,1, \ldots\})\left(\exists \sigma_{1}, \ldots, \sigma_{k} \in \Sigma_{L_{j}}\right) \\
& \qquad \delta_{L_{j}}\left(q, \rho \sigma_{1} \cdots \sigma_{k} \alpha\right) \models R\left(\mathcal{G}_{L_{j}}, P\right), \quad \text { as } q \models R\left(\mathcal{G}_{L_{j}}, P\right) \\
& \Leftrightarrow\left(\exists l \in \Sigma_{L_{j}}^{*}\right) w \rho l \alpha \in L\left(\mathcal{G}_{L_{j}}, P\right), \\
& \quad \text { by letting } l=\sigma_{1} \cdots \sigma_{k} \text { and the assumption } q \models R\left(\mathcal{G}_{L_{j}}, P\right) \text { and } q=\delta_{L_{j}}\left(q_{L j_{0}}, w\right) .
\end{aligned}
$$

Proposition 4.23. For system $\Phi$, the following holds:

$$
\begin{aligned}
& \left(\forall P \in \operatorname{Pred}\left(Q_{L_{j}}\right)\right) \\
& \quad\left((\forall q \models P)\left(\forall \rho \in \Sigma_{R_{j}}\right) \xi_{j}^{l}(x, \rho) ! \Rightarrow \delta_{L_{j}}(q, \rho) !\right) \Rightarrow\left(\Omega_{p 5_{j}}\left(L\left(\mathcal{G}_{L_{j}}, P\right)\right)=L\left(\mathcal{G}_{L_{j}}, \Gamma_{p 5_{j}}(P)\right)\right), \\
& \text { where } q=(z, y, x) \text { as in equation (4.2) (Page 105). }
\end{aligned}
$$

\section{proof:}

Let $P \in \operatorname{Pred}\left(Q_{L_{j}}\right)$.

Assume $(\forall q \models P)\left(\forall \rho \in \Sigma_{R_{j}}\right) \xi_{j}^{l}(x, \rho) ! \Rightarrow \delta_{L_{j}}(q, \rho)$ !.

Must show this implies $\Omega_{p 5_{j}}\left(L\left(\mathcal{G}_{L_{j}}, P\right)\right)=L\left(\mathcal{G}_{L_{j}}, \Gamma_{p 5_{j}}(P)\right)$.

We first claim the following holds by (1).

$(\forall q \models P)\left(\forall \rho \in \Sigma_{R_{j}}\right)\left(\forall \alpha \in \Sigma_{A_{j}}\right) \xi_{j}^{l}(x, \rho \alpha) ! \Rightarrow \delta_{L_{j}}(q, \rho) !$.

Proof of the claim:

Let $q \models P, \rho \in \Sigma_{R_{j}}$ and $\alpha \in \Sigma_{A_{j}}$.

Assume $\xi_{j}^{l}(x, \rho \alpha) !$.

Must show implies $\delta_{L_{j}}(q, \rho)$ !.

From (3) and the definition of $\xi_{j}^{l}$, we know that $\xi_{j}^{l}(x, \rho) !$ and $\xi_{j}^{l}\left(\xi_{j}^{l}(x, \rho), \alpha\right)$ !.

$\Rightarrow \xi_{j}^{l}(x, \rho)$ !

$\Rightarrow \delta_{L_{j}}(q, \rho) !, \quad$ by $(1)$

\section{Claim proven.}

We now prove this proposition by a series of transformation. 
Master Thesis - R. Song - McMaster - Computing and Software

$\Omega_{p 5_{j}}\left(L\left(\mathcal{G}_{L_{j}}, P\right)\right)$

$=L\left(\mathcal{G}_{L_{j}}, P\right)-L^{W_{p 5_{j}}(P)}\left(\mathcal{G}_{L_{j}}, P\right), \quad$ by Proposition 4.22

$=L\left(\mathcal{G}_{L_{j}}, P\right)-\cup_{w \in W_{p 5_{j}}(P)} L^{w}\left(\mathcal{G}_{L_{j}}, P\right)$

$=L\left(\mathcal{G}_{L_{j}}, P-\operatorname{pr}\left(\cup_{w \in W_{p 5_{j}}(P)}\left\{\delta_{L_{j}}\left(q_{L_{j 0}}, w\right)\right\}\right)\right), \quad$ by Proposition 2.7

$=L\left(\mathcal{G}_{L_{j}}, P-\operatorname{pr}\left(\left\{q \in R\left(\mathcal{G}_{L_{j}}, P\right) \mid \delta_{L_{j}}\left(q_{L_{j 0}}, w\right)=q\right.\right.\right.$ for some $\left.\left.\left.w \in W_{p 5_{j}}(P)\right\}\right)\right)$,

as $W_{p 5_{j}}(P) \subseteq L\left(\mathcal{G}_{L_{j}}, P\right)$ and by Proposition 2.5

$=L\left(\mathcal{G}_{L_{j}}, P-\operatorname{pr}\left(\left\{q \in R\left(\mathcal{G}_{L_{j}}, P\right) \mid\left(\exists w \in L\left(\mathcal{G}_{L_{j}}, P\right)\right)\left(\exists \rho \in \Sigma_{R_{j}}\right)\left(\exists \alpha \in \Sigma_{A_{j}}\right)\right.\right.\right.$

$\left.\left.\left.\delta_{L_{j}}\left(q_{L_{j 0}}, w\right)=q \& w \rho \alpha \in L\left(\mathbf{G}_{I_{j}}^{l}\right) \&\left(\forall l \in \Sigma_{L_{j}}^{*}\right) w \rho l \alpha \notin L\left(\mathcal{G}_{L_{j}}, P\right)\right\}\right)\right)$,

by definition of $W_{p 5_{j}}$

$=L\left(\mathcal{G}_{L_{j}}, P-\operatorname{pr}\left(\left\{q \in R\left(\mathcal{G}_{L_{j}}, P\right) \mid\left(\exists w \in L\left(\mathcal{G}_{L_{j}}, P\right)\right)\left(\exists \rho \in \Sigma_{R_{j}}\right)\left(\exists \alpha \in \Sigma_{A_{j}}\right)\right.\right.\right.$

$\delta_{L_{j}}\left(q_{L_{j 0}}, w\right)=q \& \xi_{j}^{l}(x, \rho \alpha) ! \&$

$\left.\left.\left.\left(\delta_{L_{j}}(q, \rho) ! \operatorname{or} \delta_{L_{j}}(q, \rho) \not !\right) \&\left(\forall l \in \Sigma_{L_{j}}^{*}\right) w \rho l \alpha \notin L\left(\mathcal{G}_{L_{j}}, P\right)\right\}\right)\right)$,

as $\left(\delta_{L_{j}}(q, \rho)\right.$ ! or $\left.\delta_{L_{j}}(q, \rho) \not \cdot\right)$ is always true, where $q=(z, y, x)$ as in equation (4.2)

$=L\left(\mathcal{G}_{L_{j}}, P-\operatorname{pr}\left(\left\{q \in R\left(\mathcal{G}_{L_{j}}, P\right) \mid\left(\exists w \in L\left(\mathcal{G}_{L_{j}}, P\right)\right)\left(\exists \rho \in \Sigma_{R_{j}}\right)\left(\exists \alpha \in \Sigma_{A_{j}}\right)\right.\right.\right.$

$\delta_{L_{j}}\left(q_{L_{j 0}}, w\right)=q \& \xi_{j}^{l}(x, \rho \alpha) ! \&$

$\left(\delta_{L_{j}}(q, \rho)\right.$ \% or $\left.\left.\left.\left.\left(\delta_{L_{j}}(q, \rho) ! \&\left(\forall l \in \Sigma_{L_{j}}^{*}\right) w \rho l \alpha \notin L\left(\mathcal{G}_{L_{j}}, P\right)\right)\right)\right\}\right)\right)$,

by logical distribution law and the fact that $\delta_{L_{j}}(q, \rho) \quad y \Rightarrow\left(\forall l \in \Sigma_{L_{j}}^{*}\right) w \rho l \alpha \notin L\left(\mathcal{G}_{L_{j}}, P\right)$.

$=L\left(\mathcal{G}_{L_{j}}, P-\operatorname{pr}\left(\left\{q \models R\left(\mathcal{G}_{L_{j}}, P\right) \mid\left(\exists \rho \in \Sigma_{R_{j}}\right)\left(\exists \alpha \in \Sigma_{A_{j}}\right)\right.\right.\right.$

$\xi_{j}^{l}(x, \rho \alpha) ! \&\left(\delta_{L_{j}}(q, \rho)\right.$ o or $\left.\left.\left.\left.\left(\delta_{L_{j}}(q, \rho) ! \& \delta_{L_{j}}(q, \rho) \not \models \mathcal{C R}\left(\mathcal{G}_{L_{j}}, P_{\alpha}, \Sigma_{L_{j}}, P\right)\right)\right)\right\}\right)\right)$,

by Lemma 4.19, where $P_{\alpha}:=\operatorname{pr}\left(\left\{q^{\prime} \in Q_{L_{j}} \mid \delta_{L_{j}}\left(q^{\prime}, \alpha\right) \models P\right\}\right)$ as defined in Definition 4.19.

$=L\left(\mathcal{G}_{L_{j}}, P-\operatorname{pr}\left(\left\{q \models R\left(\mathcal{G}_{L_{j}}, P\right) \mid\left(\exists \rho \in \Sigma_{R_{j}}\right)\left(\exists \alpha \in \Sigma_{A_{j}}\right)\right.\right.\right.$

$\xi_{j}^{l}(x, \rho \alpha) ! \&\left(\delta_{L_{j}}(q, \rho)\right.$ ٪ or $\left.\left.\left.\left.\delta_{L_{j}}(q, \rho) \models \neg \mathcal{C R}\left(\mathcal{G}_{L_{j}}, P_{\alpha}, \Sigma_{L_{j}}, P\right)\right)\right\}\right)\right)$,

as $\left(\delta_{L_{j}}(q, \rho) \not \models \mathcal{C R}\left(\mathcal{G}_{L_{j}}, P_{\alpha}, \Sigma_{L_{j}}, P\right)\right) \Leftrightarrow\left(\delta_{L_{j}}(q, \rho) ! \& \delta_{L_{j}}(q, \rho) \models \neg \mathcal{C R}\left(\mathcal{G}_{L_{j}}, P_{\alpha}, \Sigma_{L_{j}}, P\right)\right)$

$=L\left(\mathcal{G}_{L_{j}}, P-\operatorname{pr}\left(\left\{q \models R\left(\mathcal{G}_{L_{j}}, P\right) \mid\left(\exists \rho \in \Sigma_{R_{j}}\right)\left(\exists \alpha \in \Sigma_{A_{j}}\right)\right.\right.\right.$

$\left(\xi_{j}^{l}(x, \rho \alpha) ! \& \delta_{L_{j}}(q, \rho) \not y\right)$ or $\left.\left.\left.\left(\xi_{j}^{l}(x, \rho \alpha) ! \& \delta_{L_{j}}(q, \rho) \models \neg \mathcal{C} \mathcal{R}\left(\mathcal{G}_{L_{j}}, P_{\alpha}, \Sigma_{L_{j}}, P\right)\right)\right\}\right)\right)$,

by logical distribution law. 
Master Thesis - R. Song - McMaster - Computing and Software

$$
\begin{aligned}
=L\left(\mathcal{G}_{L_{j}},\right. & P-\operatorname{pr}\left(\left\{q \models R\left(\mathcal{G}_{L_{j}}, P\right) \mid\right.\right. \\
& \left.\left.\left.\left(\exists \rho \in \Sigma_{R_{j}}\right)\left(\exists \alpha \in \Sigma_{A_{j}}\right) \xi_{j}^{l}(x, \rho \alpha) ! \& \delta_{L_{j}}(q, \rho) \models \neg \mathcal{C} \mathcal{R}\left(\mathcal{G}_{L_{j}}, P_{\alpha}, \Sigma_{L_{j}}, P\right)\right\}\right)\right), \\
& \text { as }\left(\xi_{j}^{l}(x, \rho \alpha) ! \& \delta_{L_{j}}(q, \rho) \not .\right) \text { is always false by }(2) . \\
=L\left(\mathcal{G}_{L_{j}},\right. & P-\operatorname{pr}\left(\left\{q \in Q_{L_{j}} \mid\right.\right. \\
& \left.\left.\left.\left(\exists \rho \in \Sigma_{R_{j}}\right)\left(\exists \alpha \in \Sigma_{A_{j}}\right) \xi_{j}^{l}(x, \rho \alpha) ! \& \delta_{L_{j}}(q, \rho) \models \neg \mathcal{C R}\left(\mathcal{G}_{L_{j}}, P_{\alpha}, \Sigma_{L_{j}}, P\right)\right\}\right)\right),
\end{aligned}
$$$$
\text { as removing the states satisfying }\left(\text { true }-R\left(\mathcal{G}_{L_{j}}, P\right)\right) \text { from } P
$$$$
\text { does not change the resulting language, by Proposition } 2.5 \text {. }
$$

$$
=L\left(\mathcal{G}_{L_{j}}, \Gamma_{p 5_{j}}(P)\right)
$$

In this proposition, we put the assumption $(\forall q \models P)\left(\forall \rho \in \Sigma_{R_{j}}\right) \xi_{j}^{l}(x, \rho)$ ! $\Rightarrow$ $\delta_{L_{j}}(q, \rho)$ !, because in the function $\Omega_{L_{j}}$, the input language for the function $\Omega_{p 5_{j}}$ is always $j^{\text {th }}$ low-level P4 interface controllable. Algorithm 4.2 shows how to compute $\Gamma_{p 5_{j}}(P)$, where $P \in \operatorname{Pred}\left(Q_{L_{j}}\right)$.

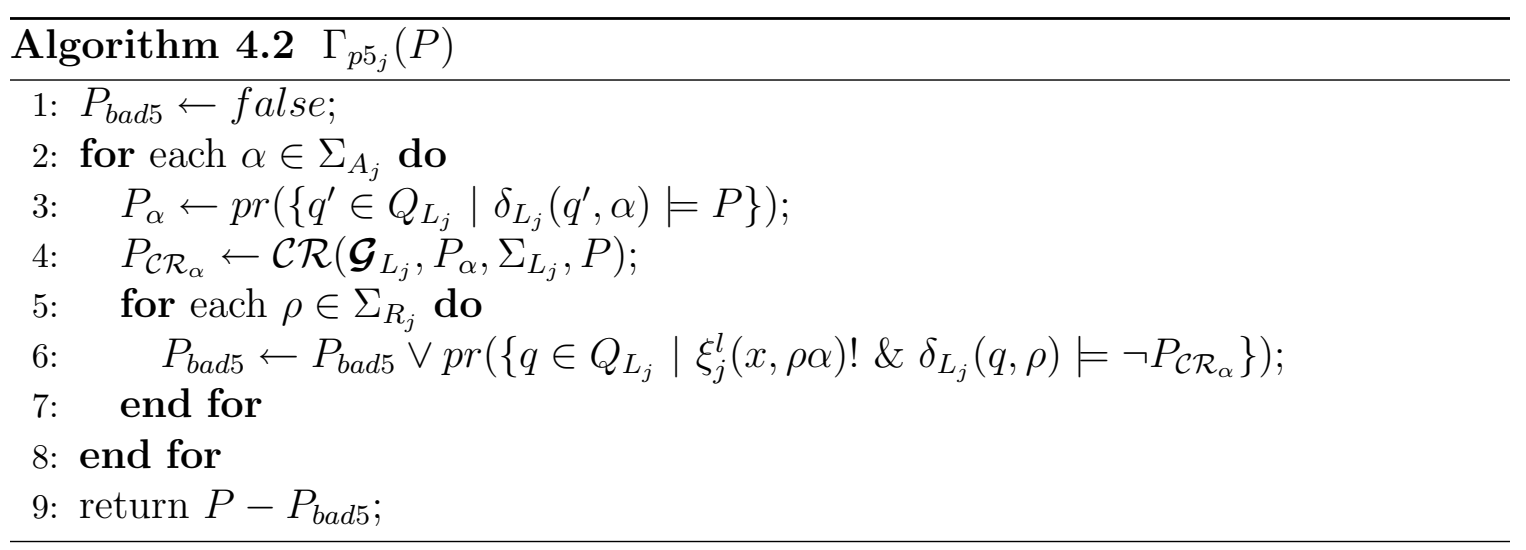

In Line $6, q=(z, y, x)$ is as in equation (4.2). In our BDD implementations, the computation of $P_{\mathcal{C R}_{\alpha}}$ is a very costly step. However, usually in an HISC system the number of answer events is small. Furthermore, as the computation of each answer event is independent, we can compute the $P_{b a d 5}$ for each answer event in parallel and then combine them together. 
Master Thesis - R. Song-McMaster - Computing and Software

Computing $\Omega_{p 6_{j}}\left(L\left(\mathcal{G}_{L_{j}}, P\right)\right)$

Definition 4.20. For system $\boldsymbol{\Phi}$, define the function $W_{p 6_{j}}: \operatorname{Pred}\left(Q_{L_{j}}\right) \rightarrow \operatorname{Pwr}\left(\Sigma_{I L_{j}}^{*}\right)$ according to

$\left(\forall P \in \operatorname{Pred}\left(Q_{L_{j}}\right)\right)$

$W_{p 6_{j}}(P):=\left\{s \in L\left(\mathcal{G}_{L_{j}}, P\right) \mid s \in L_{m}\left(\mathbf{G}_{I_{j}}^{l}\right) \&\left(\forall l \in \Sigma_{L_{j}}^{*}\right) s l \notin L_{m}\left(\mathcal{G}_{L_{j}}\right) \cap L\left(\mathcal{G}_{L_{j}}, P\right)\right\}$

Clearly, for all $P \in \operatorname{Pred}\left(Q_{L_{j}}\right), W_{p 6_{j}}(P) \subseteq L\left(\mathcal{G}_{L_{j}}, P\right)$.

Lemma 4.20. For system $\Phi$, the following holds:

$\left(\forall P \in \operatorname{Pred}\left(Q_{L_{j}}\right)\right)\left(\forall s, t \in L\left(\mathcal{G}_{L_{j}}, P\right)\right)$

$\left(s \in W_{p 6_{j}}(P) \&\left(\delta_{L_{j}}\left(q_{L j_{0}}, s\right)=\delta_{L_{j}}\left(q_{L j_{0}}, t\right)\right)\right) \Rightarrow t \in W_{p 6_{j}}(P)$.

proof:

Let $P \in \operatorname{Pred}\left(Q_{L_{j}}\right)$, and $s, t \in L\left(\mathcal{G}_{L_{j}}, P\right)$.

Assume $\delta_{L_{j}}\left(q_{L j_{0}}, s\right)=\delta_{L_{j}}\left(q_{L j_{0}}, t\right)$ and $s \in W_{p 6_{j}}(P)$.

Must show this implies $t \in W_{p 6_{j}}(P)$.

Let $\equiv_{L\left(\mathcal{G}_{L_{j}}, P\right)}, \equiv_{L_{m}\left(\mathbf{G}_{I_{j}}^{l}\right)}$, and $\equiv_{L_{m}\left(\mathcal{G}_{L_{j}}\right)}$ be the Nerode equivalence relation on $\Sigma_{I L_{j}}^{*}$ with respect to $L\left(\mathcal{G}_{L_{j}}, P\right), L_{m}\left(\mathbf{G}_{I_{j}}^{l}\right)$ and $L_{m}\left(\mathcal{G}_{L_{j}}\right)$ respectively.

From (2), we have $\delta\left(q_{L j_{0}}, s\right)=\delta\left(q_{L j_{0}}, t\right)$

$\Rightarrow s \equiv_{L\left(\mathcal{G}_{L_{j}}, P\right)} t \& s \equiv_{L_{m}\left(\mathcal{G}_{L_{j}}\right)} t$,

by Proposition 2.6 and fact $L_{m}\left(\mathcal{G}_{L_{j}}\right)=L_{m}\left(\mathcal{G}_{L_{j}}\right.$, true $)$.

As $\mathcal{G}_{L_{j}}=\mathbf{E}_{L_{j}} \times \mathbf{G}_{L_{j}}^{p} \times \mathbf{G}_{I_{j}}^{l}$ and $\delta_{L_{j}}\left(q_{L j_{0}}, s\right)=\delta_{L_{j}}\left(q_{L j_{0}}, t\right)$, we have

$\xi_{j}^{l}\left(x_{j 0}^{l}, s\right)=\xi_{j}^{l}\left(x_{j 0}^{l}, t\right)$

$\Rightarrow s \equiv_{L_{m}\left(\mathbf{G}_{I_{j}}^{l}\right)} t$,

by Proposition 2.6 and fact $L_{m}\left(\mathbf{G}_{I_{j}}^{l}\right)=L_{m}\left(\mathbf{G}_{I_{j}}^{l}\right.$, true $)$.

From (2), we have $s \in W_{p 6_{j}}(P)$ 


$$
\begin{aligned}
& \quad \text { Master Thesis }- \text { R. Song - McMaster - Computing and Software } \\
& \Rightarrow s \in L_{m}\left(\mathbf{G}_{I_{j}}^{l}\right) \&\left(\forall l \in \Sigma_{L_{j}}^{*}\right) s l \notin L_{m}\left(\mathcal{G}_{L_{j}}\right) \cap L\left(\mathcal{G}_{L_{j}}, P\right) \\
& \Rightarrow t \in L_{m}\left(\mathbf{G}_{I_{j}}^{l}\right) \&\left(\forall l \in \Sigma_{L_{j}}^{*}\right) t l \notin L_{m}\left(\mathcal{G}_{L_{j}}\right) \cap L\left(\mathcal{G}_{L_{j}}, P\right), \quad \text { by }(3)(4) \\
& \Rightarrow t \in W_{p 6_{j}}(P), \quad \text { as } t \in L\left(\mathcal{G}_{L_{j}}, P\right) .
\end{aligned}
$$

Lemma 4.21. For system $\boldsymbol{\Phi}$, the following holds:

$$
\left.\left(\forall P \in \operatorname{Pred}\left(Q_{L_{j}}\right)\right)\left(\forall s \in L\left(\mathcal{G}_{L_{j}}, P\right)\right)\left((\exists t \leq s) t \in W_{p 6_{j}}(P)\right) \Leftrightarrow s \notin \Omega_{p 6_{j}}\left(L\left(\mathcal{G}_{L_{j}}, P\right)\right)\right) .
$$

\section{proof:}

Let $P \in \operatorname{Pred}\left(Q_{L_{j}}\right)$ and $s \in L\left(\mathcal{G}_{L_{j}}, P\right)$.

1. Show that $\left((\exists t \leq s) t \in W_{p 6_{j}}(P)\right) \Rightarrow s \notin \Omega_{p 6_{j}}\left(L\left(\mathcal{G}_{L_{j}}, P\right)\right)$.

Assume $(\exists t \leq s) t \in W_{p 6_{j}}(P)$.

Must show this implies $s \notin \Omega_{p 6_{j}}\left(L\left(\mathcal{G}_{L_{j}}, P\right)\right)$.

From (2), we have

$$
\begin{aligned}
& (\exists t \leq s) t \in L_{m}\left(\mathbf{G}_{I_{j}}^{l}\right) \&\left(\forall l \in \Sigma_{L_{j}}^{*}\right) t l \notin L_{m}\left(\mathcal{G}_{L_{j}}\right) \cap L\left(\mathcal{G}_{L_{j}}, P\right) \\
& \Rightarrow \neg\left((\forall t \leq s) t \in L_{m}\left(\mathbf{G}_{I_{j}}^{l}\right) \Rightarrow\left(\exists l \in \Sigma_{L_{j}}^{*}\right) t l \in L_{m}\left(\mathcal{G}_{L_{j}}\right) \cap L\left(\mathcal{G}_{L_{j}}, P\right)\right) \\
& \Rightarrow s \notin \Omega_{p 6_{j}}\left(L\left(\mathcal{G}_{L_{j}}, P\right)\right)
\end{aligned}
$$

2. Show that $s \notin \Omega_{p 6_{j}}\left(L\left(\mathcal{G}_{L_{j}}, P\right)\right) \Rightarrow\left((\exists t \leq s) t \in W_{p 6_{j}}(P)\right)$.

Assume $s \notin \Omega_{p 6_{j}}\left(L\left(\mathcal{G}_{L_{j}}, P\right)\right)$.

Must show this implies $(\exists t \leq s) t \in W_{p 6_{j}}(P)$.

From (1), we know $s \in L\left(\mathcal{G}_{L_{j}}, P\right)$. Combine this with (3), we thus have

$$
\begin{aligned}
& \neg\left((\forall t \leq s) t \in L_{m}\left(\mathbf{G}_{I_{j}}^{l}\right) \Rightarrow\left(\exists l \in \Sigma_{L_{j}}^{*}\right) t l \in L_{m}\left(\mathcal{G}_{L_{j}}\right) \cap L\left(\mathcal{G}_{L_{j}}, P\right)\right) \\
& \Rightarrow(\exists t \leq s) t \in L_{m}\left(\mathbf{G}_{I_{j}}^{l}\right) \&\left(\forall l \in \Sigma_{L_{j}}^{*}\right) t l \notin L_{m}\left(\mathcal{G}_{L_{j}}\right) \cap L\left(\mathcal{G}_{L_{j}}, P\right)
\end{aligned}
$$


Master Thesis - R. Song - McMaster - Computing and Software

As $s \in L\left(\mathcal{G}_{L_{j}}, P\right)$ and $L\left(\mathcal{G}_{L_{j}}, P\right)$ is closed, we know $t \in L\left(\mathcal{G}_{L_{j}}, P\right)$.

By (4) and (5), we thus have $(\exists t \leq s) t \in W_{p 6_{j}}(P)$.

Proposition 4.24. For system $\mathbf{\Phi}$, the following holds

$$
\left(\forall P \in \operatorname{Pred}\left(Q_{L_{j}}\right)\right) L\left(\mathcal{G}_{L_{j}}, P\right)-\Omega_{p 6_{j}}\left(L\left(\mathcal{G}_{L_{j}}, P\right)\right)=L^{W_{p 6_{j}}(P)}\left(\mathcal{G}_{L_{j}}, P\right),
$$

where $L^{W_{p 6_{j}}(P)}\left(\mathcal{G}_{L_{j}}, P\right)=\cup_{s \in W_{p 6_{j}}(P)} L^{s}\left(\mathcal{G}_{L_{j}}, P\right)$.

proof: Identical to the proof of Proposition 4.6 after substituting $S u b\left(\mathcal{P}_{h a}\right)$ with $\operatorname{Pred}\left(Q_{L_{j}}\right), \Omega_{H I C}$ with $\Omega_{p 6_{j}}, W_{H}(P)$ with $W_{p 6_{j}}(P), \mathcal{G}_{H}$ with $\mathcal{G}_{L_{j}}, \delta_{H}$ with $\delta_{L_{j}}, q_{H_{0}}$ with $q_{L j_{0}}$, Lemma 4.2 with Lemma 4.20 , Lemma 4.3 with Lemma 4.21.

Definition 4.21. For system $\boldsymbol{\Phi}$, define the function $\Gamma_{p 6_{j}}: \operatorname{Pred}\left(Q_{L_{j}}\right) \rightarrow \operatorname{Pred}\left(Q_{L_{j}}\right)$ according to

$$
\begin{aligned}
& \left(\forall P \in \operatorname{Pred}\left(Q_{L_{j}}\right)\right) \Gamma_{p 6_{j}}(P):=P-\left(P_{X_{j_{m}}}-\mathcal{C R}\left(\mathcal{G}_{L_{j}}, P_{L_{j_{m}}}, \Sigma_{L_{j}}, P\right)\right), \\
& \text { where } P_{X_{j_{m}}}:=\operatorname{pr}\left(\left\{q \in Q_{L_{j}} \mid x \in X_{j_{m}}^{l}\right\}\right), P_{L_{j_{m}}}:=\operatorname{pr}\left(Q_{L_{j_{m}}}\right), \\
& \qquad q=(z, y, x) \text { as in equation } 4.2(\text { Page } 105), \\
& \text { and } X_{j_{m}}^{l} \text { and } Q_{L_{j_{m}}} \text { are the marker state sets of } \mathbf{G}_{I_{j}}^{l} \text { and } \mathcal{G}_{L_{j}} \text { respectively } \\
& \text { as defined in Section 4.1. }
\end{aligned}
$$

Note that for system $\boldsymbol{\Phi}, P_{X_{j_{m}}}$ and $P_{L_{j_{m}}}$ are constant. The following lemma will be used later on.

Lemma 4.22. For system $\boldsymbol{\Phi}, \Gamma_{p 6_{j}}$ is monotone with respect to $\preceq$, i.e.

$$
\left(\forall P_{1}, P_{2} \in \operatorname{Pred}\left(Q_{L_{j}}\right)\right) P_{1} \preceq P_{2} \Rightarrow \Gamma_{p 6_{j}}\left(P_{1}\right) \preceq \Gamma_{p 6_{j}}\left(P_{2}\right)
$$




\section{Master Thesis - R. Song - McMaster - Computing and Software}

\section{proof:}

Let $P_{1}, P_{2} \in \operatorname{Pred}\left(Q_{L_{j}}\right)$. Assume $P_{1} \preceq P_{2}$.

Must show this implies $\Gamma_{p 6_{j}}\left(P_{1}\right) \preceq \Gamma_{p 6_{j}}\left(P_{2}\right)$.

$$
\begin{aligned}
& \Gamma_{p 6_{j}}\left(P_{1}\right) \\
& =P_{1}-\left(P_{X_{j m}}-\mathcal{C} \mathcal{R}\left(\mathcal{G}_{L_{j}}, P_{L_{j m}}, \Sigma_{L_{j}}, P_{1}\right)\right) \text {, by definition of } \Gamma_{p 6_{j}}\left(P_{1}\right) \\
& =P_{1} \wedge \neg\left(P_{X_{j m}} \wedge \neg \mathcal{C R}\left(\mathcal{G}_{L_{j}}, P_{L_{j m}}, \Sigma_{L_{j}}, P_{1}\right)\right) \\
& =P_{1} \wedge\left(\neg P_{X_{j m}} \vee \mathcal{C R}\left(\mathcal{G}_{L_{j}}, P_{L_{j_{m}}}, \Sigma_{L_{j}}, P_{1}\right)\right) \\
& \preceq P_{2} \wedge\left(\neg P_{X_{j m}} \vee \mathcal{C R}\left(\mathcal{G}_{L_{j}}, P_{L_{j_{m}}}, \Sigma_{L_{j}}, P_{2}\right)\right) \text {, as } P_{1} \preceq P_{2} \text { and } \mathcal{C} \mathcal{R} \text { is monotone } \\
& =P_{2} \wedge \neg\left(P_{X_{j_{m}}} \wedge \neg \mathcal{C} \mathcal{R}\left(\mathcal{G}_{L_{j}}, P_{L_{j_{m}}}, \Sigma_{L_{j}}, P_{2}\right)\right) \\
& =P_{2}-\left(P_{X_{j m}}-\mathcal{C} \mathcal{R}\left(\mathcal{G}_{L_{j}}, P_{L_{j_{m}}}, \Sigma_{L_{j}}, P_{2}\right)\right) \\
& =\Gamma_{p 6_{j}}\left(P_{2}\right)
\end{aligned}
$$

In order to show the method to compute $\Omega_{p 6_{j}}$, we need the following proposition and lemma.

Proposition 4.25. For system $\boldsymbol{\Phi}$, the following holds:

$$
\left(\forall P \in \operatorname{Pred}\left(Q_{L_{j}}\right)\right) L_{m}\left(\mathcal{G}_{L_{j}}\right) \cap L\left(\mathcal{G}_{L_{j}}, P\right)=L_{m}\left(\mathcal{G}_{L_{j}}, C R\left(\mathcal{G}_{L_{j}}, P\right)\right)
$$

\section{proof:}

Identical to the proof of Proposition 4.10 after substituting $\mathcal{G}_{H}$ with $\mathcal{G}_{L_{j}}, Q_{H}$ with $Q_{L_{j}}, Q_{H_{m}}$ with $Q_{L_{j m}}, \delta_{H}$ with $\delta_{L_{j}}, \Sigma_{I H}$ with $\Sigma_{I L_{j}}$

Lemma 4.23. For system $\mathbf{\Phi}$, the following holds:

$$
\left(\forall P \in \operatorname{Pred}\left(Q_{L_{j}}\right)\right) L_{m}\left(\mathcal{G}_{L_{j}}\right) \cap L\left(\mathcal{G}_{L_{j}}, P\right)=L_{m}\left(\mathcal{G}_{L_{j}}, P\right)
$$

\section{proof:}

Let $P \in \operatorname{Pred}\left(Q_{L_{j}}\right)$. 
Master Thesis - R. Song - McMaster - Computing and Software

1. Show that $L_{m}\left(\mathcal{G}_{L_{j}}, P\right) \subseteq L_{m}\left(\mathcal{G}_{L_{j}}\right) \cap L\left(\mathcal{G}_{L_{j}}, P\right)$.

Clearly, $L_{m}\left(\mathcal{G}_{L_{j}}, P\right) \subseteq L_{m}\left(\mathcal{G}_{L_{j}}\right)$ and $L_{m}\left(\mathcal{G}_{L_{j}}, P\right) \subseteq L\left(\mathcal{G}_{L_{j}}, P\right)$, so $L_{m}\left(\mathcal{G}_{L_{j}}, P\right) \subseteq$ $L_{m}\left(\mathcal{G}_{L_{j}}\right) \cap L\left(\mathcal{G}_{L_{j}}, P\right)$.

2. Show that $L_{m}\left(\mathcal{G}_{L_{j}}\right) \cap L\left(\mathcal{G}_{L_{j}}, P\right) \subseteq L_{m}\left(\mathcal{G}_{L_{j}}, P\right)$.

By Proposition 4.25, we know $L_{m}\left(\mathcal{G}_{L_{j}}\right) \cap L\left(\mathcal{G}_{L_{j}}, P\right)=L_{m}\left(\mathcal{G}_{L_{j}}, C R\left(\mathcal{G}_{L_{j}}, P\right)\right)$.

By the definition of $C R$, we also know that $L_{m}\left(\mathcal{G}_{L_{j}}, C R\left(\mathcal{G}_{L_{j}}, P\right)\right) \subseteq L_{m}\left(\mathcal{G}_{L_{j}}, P\right)$.

So, $L_{m}\left(\mathcal{G}_{L_{j}}\right) \cap L\left(\mathcal{G}_{L_{j}}, P\right) \subseteq L_{m}\left(\mathcal{G}_{L_{j}}, P\right)$.

The following lemma will be used in the proof of next proposition.

Lemma 4.24. For system $\boldsymbol{\Phi}$, let $P \in \operatorname{Pred}\left(Q_{L_{j}}\right), w \in \Sigma_{I L_{j}}^{*}$ and $q \in Q_{L_{j}}$. If $q=$ $\delta\left(q_{L j_{0}}, w\right)$ and $q \models R\left(\mathcal{G}_{L_{j}}, P\right)$, then

$$
q \models \mathcal{C R}\left(\mathcal{G}_{L_{j}}, P_{L_{m}}, \Sigma_{L_{j}}, P\right) \text { if and only if }\left(\exists l \in \Sigma_{L_{j}}^{*}\right) w l \in L_{m}\left(\mathcal{G}_{L_{j}}, P\right)
$$

where $P_{L_{j m}}:=\operatorname{pr}\left(Q_{L_{j_{m}}}\right)$ as defined in Definition 4.21.

proof:

Assume $q=\delta\left(q_{L j_{0}}, w\right)$ and $q=R\left(\mathcal{G}_{L_{j}}, P\right)$.

$$
\begin{gathered}
q \models \mathcal{C} \mathcal{R}\left(\mathcal{G}_{L_{j}}, P_{L j_{m}}, \Sigma_{L_{j}}, P\right) \\
\Leftrightarrow(\exists k \in\{0,1, \ldots\})\left(\exists \sigma_{1}, \ldots, \sigma_{k} \in \Sigma_{L_{j}}\right)\left(\exists q_{1}, \ldots, q_{k+1} \in Q_{L_{j}}\right) \\
q_{1}=q \\
q_{k+1} \models P_{L j_{m}} \\
q_{1}, \ldots, q_{k+1} \models P \\
q_{i+1}=\delta_{L_{j}}\left(q_{i}, \sigma_{i}\right), i=1,2, \ldots, k
\end{gathered}
$$


Master Thesis - R. Song-McMaster - Computing and Software

$\Leftrightarrow(\exists k \in\{0,1, \ldots\})\left(\exists \sigma_{1}, \ldots, \sigma_{k} \in \Sigma_{L_{j}}\right)$

$\delta_{L_{j}}\left(q, \sigma_{1} \ldots \sigma_{k}\right) \models R\left(\mathcal{G}_{L_{j}}, P\right) \& \delta_{L_{j}}\left(q, \sigma_{1} \ldots \sigma_{k}\right) \in Q_{L j_{m}}$,

by $q \models R\left(\mathcal{G}_{L_{j}}, P\right)$ and the definition of $P_{L j_{m}}$.

$\Leftrightarrow\left(\exists l \in \Sigma_{L_{j}}^{*}\right) w l \in L_{m}\left(\mathcal{G}_{L_{j}}, P\right)$,

by letting $l=\sigma_{1} \ldots \sigma_{k}$ and the assumption $q=\delta\left(q_{L j_{0}}, w\right)$ and $q \models R\left(\mathcal{G}_{L_{j}}, P\right)$.

Proposition 4.26. For system $\boldsymbol{\Phi}$, the following holds:

$\left(\forall P \in \operatorname{Pred}\left(Q_{L_{j}}\right)\right) \Omega_{p 6_{j}}\left(L\left(\mathcal{G}_{L_{j}}, P\right)\right)=L\left(\mathcal{G}_{L_{j}}, \Gamma_{p 6_{j}}(P)\right)$.

\section{proof:}

Let $P \in \operatorname{Pred}\left(Q_{L_{j}}\right)$. We prove this proposition by a series of transformation.

$\Omega_{p 6_{j}}\left(L\left(\mathcal{G}_{L_{j}}, P\right)\right)$

$=L\left(\mathcal{G}_{L_{j}}, P\right)-L^{W_{p \sigma_{j}}(P)}\left(\mathcal{G}_{L_{j}}, P\right), \quad$ by Proposition 4.24

$=L\left(\mathcal{G}_{L_{j}}, P\right)-\cup_{w \in W_{p 6_{j}}(P)} L^{w}\left(\mathcal{G}_{L_{j}}, P\right)$

$=L\left(\mathcal{G}_{L_{j}}, P-\operatorname{pr}\left(\cup_{w \in W_{p 6_{j}}(P)}\left\{\delta_{L_{j}}\left(q_{L_{j 0}}, w\right)\right\}\right)\right), \quad$ by Proposition 2.7

$=L\left(\mathcal{G}_{L_{j}}, P-\operatorname{pr}\left(\left\{q \in R\left(\mathcal{G}_{L_{j}}, P\right) \mid \delta_{L_{j}}\left(q_{L_{j_{0}}}, w\right)=q\right.\right.\right.$ for some $\left.\left.\left.w \in W_{p 6_{j}}(P)\right\}\right)\right)$,

as $W_{p 6_{j}}(P) \subseteq L\left(\mathcal{G}_{L_{j}}, P\right)$ and by Proposition 2.5

$=L\left(\mathcal{G}_{L_{j}}, P-\operatorname{pr}\left(\left\{q \in R\left(\mathcal{G}_{L_{j}}, P\right) \mid\left(\exists w \in L\left(\mathcal{G}_{L_{j}}, P\right)\right) \delta_{L_{j}}\left(q_{L_{j_{0}}}, w\right)=q \&\right.\right.\right.$

$\left.\left.\left.w \in L_{m}\left(\mathbf{G}_{I_{j}}^{l}\right) \&\left(\forall l \in \Sigma_{L_{j}}^{*}\right) w l \notin L_{m}\left(\mathcal{G}_{L_{j}}\right) \cap L\left(\mathcal{G}_{L_{j}}, P\right)\right\}\right)\right)$,

by definition of $W_{p 6_{j}}(P)$

$=L\left(\mathcal{G}_{L_{j}}, P-\operatorname{pr}\left(\left\{q \in R\left(\mathcal{G}_{L_{j}}, P\right) \mid\left(\exists w \in L\left(\mathcal{G}_{L_{j}}, P\right)\right) \delta_{L_{j}}\left(q_{L_{j_{0}}}, w\right)=q \&\right.\right.\right.$

$\left.\left.\left.w \in L_{m}\left(\mathbf{G}_{I_{j}}^{l}\right) \&\left(\forall l \in \Sigma_{L_{j}}^{*}\right) w l \notin L_{m}\left(\mathcal{G}_{L_{j}}, P\right)\right\}\right)\right)$, by Lemma 4.23

$=L\left(\mathcal{G}_{L_{j}}, P-\operatorname{pr}\left(\left\{q \models R\left(\mathcal{G}_{L_{j}}, P\right) \mid x \in X_{j_{m}}^{l} \& q \not \models \mathcal{C R}\left(\mathcal{G}_{L_{j}}, P_{L_{j_{m}}}, \Sigma_{L_{j}}, P\right)\right\}\right)\right)$,

by Lemma 4.24 ,

where $P_{L_{j_{m}}}:=\operatorname{pr}\left(Q_{L_{j_{m}}}\right)$ as defined in Definition 4.21,

and $q=(z, y, x)$ as in Equation 4.2 (Page 105). 
Master Thesis - R. Song - McMaster - Computing and Software

$=L\left(\mathcal{G}_{L_{j}}, P-\operatorname{pr}\left(\left\{q \in Q_{L_{j}} \mid x \in X_{j_{m}}^{l} \& q \not \models \mathcal{C R}\left(\mathcal{G}_{L_{j}}, P_{L_{j_{m}}}, \Sigma_{L_{j}}, P\right)\right\}\right)\right)$, as removing the states satisfying $\left(\right.$ true $\left.-R\left(\mathcal{G}_{L_{j}}, P\right)\right)$

does not change the resulting language, by Proposition 2.5 .

$=L\left(\mathcal{G}_{L_{j}}, P-\left(\operatorname{pr}\left(\left\{q \in Q_{L_{j}} \mid x \in X_{j_{m}}^{l}\right\}\right)-\mathcal{C R}\left(\mathcal{G}_{L_{j}}, P_{L_{j_{m}}}, \Sigma_{L_{j}}, P\right)\right)\right)$

$=L\left(\mathcal{G}_{L_{j}}, P-\left(P_{X_{j_{m}}}-\mathcal{C} \mathcal{R}\left(\mathcal{G}_{L_{j}}, P_{L_{j_{m}}}, \Sigma_{L_{j}}, P\right)\right)\right)$, where $P_{X_{j_{m}}}$ is as in Definition 4.21.

$=L\left(\mathcal{G}_{L_{j}}, \Gamma_{p 6_{j}}(P)\right)$.

\subsubsection{The Algorithm to Compute $\sup \mathcal{C}_{L_{j}}\left(L_{m}\left(\mathcal{G}_{L_{j}}\right)\right)$}

For a predicate $P \in \operatorname{Pred}\left(Q_{L_{j}}\right)$, we know from Proposition 4.25 and the definition of $\Omega_{L N B_{j}}$ that

$$
\left.\Omega_{L N B_{j}}\left(L\left(\mathcal{G}_{L_{j}}, P\right)\right)=L_{m}\left(\mathcal{G}_{L_{j}}\right) \cap L_{(} \mathcal{G}_{L_{j}}, P\right)=L_{m}\left(\mathcal{G}_{L_{j}}, C R\left(\mathcal{G}_{L_{j}}, P\right)\right) .
$$

We now put all the predicate functions together to show the method to compute $\sup \mathcal{C}_{L_{j}}\left(L_{m}\left(\mathcal{G}_{L_{j}}\right)\right)$

Definition 4.22. For system $\boldsymbol{\Phi}$, define the function $\Gamma_{L_{j}}: \operatorname{Pred}\left(Q_{L_{j}}\right) \rightarrow \operatorname{Pred}\left(Q_{L_{j}}\right)$ according to

$$
\left(\forall P \in \operatorname{Pred}\left(Q_{L_{j}}\right)\right) \Gamma_{L_{j}}(P):=C R\left(\mathcal{G}_{L_{j}}, \Gamma_{p 6_{j}}\left(\Gamma_{p 5_{j}}\left(\operatorname{PLPC}_{j}(P)\right)\right)\right)
$$

By Lemma 4.12, $\mathrm{PLPC}_{j}$ is monotone; by Lemma 4.18, $\Gamma_{p 5_{j}}$ is monotone; by Lemma $4.22, \Gamma_{p 6_{j}}$ is monotone; the predicate transformer $C R\left(\mathcal{G}_{L_{j}},.\right)$ is monotone; thus the function $\Gamma_{L_{j}}$ is also monotone.

Lemma 4.25. For system $\boldsymbol{\Phi}$, the following holds:

$$
\left(\forall P \in \operatorname{Pred}\left(Q_{L_{j}}\right)\right) \overline{L_{m}\left(\mathcal{G}_{L_{j}}, C R\left(\mathcal{G}_{L_{j}}, P\right)\right)}=L\left(\mathcal{G}_{L_{j}}, C R\left(\mathcal{G}_{L_{j}}, P\right)\right)
$$




\section{Master Thesis - R. Song - McMaster - Computing and Software}

\section{proof:}

Identical to the proof of Lemma 4.6 by substituting $\mathcal{G}_{H}$ with $\mathcal{G}_{L_{j}}, Q_{H}$ with $Q_{L_{j}}$, $Q_{H_{m}}$ with $Q_{L_{j_{m}}}, \Sigma_{I H}$ with $\Sigma_{I L_{j}}, \delta_{H}$ with $\delta_{L_{j}}$.

Lemma 4.26. For system $\mathbf{\Phi}$, the following holds

$$
(\forall i \in\{1,2, \ldots\})\left(\forall P \in \operatorname{Pred}\left(Q_{L_{j}}\right)\right) \overline{L_{m}\left(\mathcal{G}_{L_{j}}, \Gamma_{L_{j}}^{i}(P)\right)}=L\left(\mathcal{G}_{L_{j}}, \Gamma_{L_{j}}^{i}(P)\right)
$$

\section{proof:}

Let $i \in\{1,2, \ldots\}$, and $P \in \operatorname{Pred}\left(Q_{L_{j}}\right)$. By definition of $\Gamma_{L_{j}}$, we have

$$
\begin{aligned}
\overline{L_{m}\left(\mathcal{G}_{L_{j}}, \Gamma_{L_{j}}^{i}(P)\right)} & =\overline{L_{m}\left(\mathcal{G}_{L_{j}}, \Gamma_{L_{j}}\left(\Gamma_{L_{j}}^{i-1}(P)\right)\right)} \\
& =\overline{L_{m}\left(\mathcal{G}_{L_{j}}, C R\left(\mathcal{G}_{L_{j}}, \Gamma_{p 6_{j}}\left(\Gamma_{p 5_{j}}\left(\operatorname{PLPC}_{j}\left(\Gamma_{L_{j}}^{i-1}(P)\right)\right)\right)\right)\right.},
\end{aligned}
$$

by definition of $\Gamma_{L_{j}}$

$$
=\overline{L_{m}\left(\mathcal{G}_{L_{j}}, C R\left(\mathcal{G}_{L_{j}}, P^{\prime}\right)\right)},
$$

by letting $P^{\prime}:=\Gamma_{p 6_{j}}\left(\Gamma_{p 5_{j}}\left(\operatorname{PLPC}_{j}\left(\Gamma_{L_{j}}^{i-1}(P)\right)\right)\right)$

$=L\left(\mathcal{G}_{L_{j}}, C R\left(\mathcal{G}_{L_{j}}, P^{\prime}\right)\right), \quad$ by Lemma 4.25

$$
=L\left(\mathcal{G}_{L_{j}}, C R\left(\mathcal{G}_{L_{j}}, \Gamma_{p 6_{j}}\left(\Gamma_{p 5_{j}}\left(\operatorname{PLPC}_{j}\left(\Gamma_{L_{j}}^{i-1}(P)\right)\right)\right)\right)\right)
$$$$
=L\left(\mathcal{G}_{L_{j}}, \Gamma_{L_{j}}^{i}(P)\right), \quad \text { by definition of } \Gamma_{L_{j}}
$$

Proposition 4.27. For system $\mathbf{\Phi}$, the following two points hold:

1. $\left(\forall P \in \operatorname{Pred}\left(Q_{L_{j}}\right)\right) \Omega_{L_{j}}\left(L\left(\mathcal{G}_{L_{j}}, P\right)\right)=L_{m}\left(\mathcal{G}_{L_{j}}, \Gamma_{L_{j}}(P)\right)$

2. $\left(\forall P \in \operatorname{Pred}\left(Q_{L_{j}}\right)\right)(\forall i \in\{1,2, \ldots\}) \Omega_{L_{j}}^{i}\left(L\left(\mathcal{G}_{L_{j}}, P\right)\right)=L_{m}\left(\mathcal{G}_{L_{j}}, \Gamma_{L_{j}}^{i}(P)\right)$

\section{proof:}

1. Show that $\left(\forall P \in \operatorname{Pred}\left(Q_{L_{j}}\right)\right) \Omega_{L_{j}}\left(L\left(\mathcal{G}_{L_{j}}, P\right)\right)=L_{m}\left(\mathcal{G}_{L_{j}}, \Gamma_{L_{j}}(P)\right)$ Let $P \in \operatorname{Pred}\left(Q_{L_{j}}\right)$. 
Master Thesis - R. Song - McMaster - Computing and Software

$$
\begin{aligned}
& \Omega_{L_{j}}\left(L\left(\mathcal{G}_{L_{j}}, P\right)\right) \\
& =\Omega_{L N B_{j}}\left(\Omega_{p 6_{j}}\left(\Omega_{p 5_{j}}\left(\mathrm{LPC}_{j}\left(L\left(\mathcal{G}_{L_{j}}, P\right)\right)\right)\right), \text { by definition of } \Omega_{L_{j}}\right. \\
& =L_{m}\left(\mathcal{G}_{L_{j}}\right) \cap \Omega_{p 6_{j}}\left(\Omega_{p 5_{j}}\left(\sup \mathcal{L} \mathcal{P} \mathcal{C}_{j}\left(\overline{L\left(\mathcal{G}_{L_{j}}, P\right)}\right)\right)\right) \text {, by definitions of } \mathrm{LPC}_{j} \text { and } \Omega_{L N B_{j}} \\
& =L_{m}\left(\mathcal{G}_{L_{j}}\right) \cap \Omega_{p 6_{j}}\left(\Omega_{p 5_{j}}\left(\sup \mathcal{L} \mathcal{P} \mathcal{C}_{j}\left(L\left(\mathcal{G}_{L_{j}}, P\right)\right)\right)\right) \text {, as } L\left(\mathcal{G}_{L_{j}}, P\right) \text { is closed } \\
& =L_{m}\left(\mathcal{G}_{L_{j}}\right) \cap \Omega_{p 6_{j}}\left(\Omega_{p 5_{j}}\left(L\left(\mathcal{G}_{L_{j}}, \operatorname{PLPC}_{j}(P)\right)\right)\right) \text {, by Proposition } 4.18 . \\
& =L_{m}\left(\mathcal{G}_{L_{j}}\right) \cap \Omega_{p 6_{j}}\left(L\left(\mathcal{G}_{L_{j}}, \Gamma_{p 5_{j}}\left(\operatorname{PLPC}_{j}(P)\right)\right)\right), \text { by Lemma } 4.13 \text { and Proposition } 4.23 . \\
& =L_{m}\left(\mathcal{G}_{L_{j}}\right) \cap L\left(\mathcal{G}_{L_{j}}, \Gamma_{p 6_{j}}\left(\Gamma_{p 5_{j}}\left(\mathrm{PLPC}_{j}(P)\right)\right)\right), \text { by Proposition } 4.26 \\
& =L_{m}\left(\mathcal{G}_{L_{j}}, C R\left(\mathcal{G}_{L_{j}}, \Gamma_{p 6_{j}}\left(\Gamma_{p 5_{j}}\left(\mathrm{PLPC}_{j}(P)\right)\right)\right)\right), \text { by Proposition } 4.25 \\
& =L_{m}\left(\mathcal{G}_{L_{j}}, \Gamma_{L_{j}}(P)\right)
\end{aligned}
$$

2. Show that $\left(\forall P \in \operatorname{Pred}\left(Q_{L_{j}}\right)\right)(\forall i \in\{1,2, \ldots\}) \Omega_{L_{j}}^{i}\left(L\left(\mathcal{G}_{L_{j}}, P\right)\right)=L_{m}\left(\mathcal{G}_{L_{j}}, \Gamma_{L_{j}}^{i}(P)\right)$

Let $P \in \operatorname{Pred}\left(Q_{L_{j}}\right)$. We now prove this by induction.

(a) Base Case: $i=1$

By Point 1 , we have $\Omega_{L_{j}}^{1}\left(L\left(\mathcal{G}_{L_{j}}, P\right)\right)=L_{m}^{1}\left(\mathcal{G}_{L_{j}}, \Gamma_{L_{j}}(P)\right)$

(b) Inductive step.

Let $k \in\{1,2, \ldots\}$. Assume $\Omega_{L_{j}}^{k}\left(L\left(\mathcal{G}_{L_{j}}, P\right)\right)=L_{m}\left(\mathcal{G}_{L_{j}}, \Gamma_{L_{j}}^{k}(P)\right)$.

Must show $\Omega_{L_{j}}^{k+1}\left(L\left(\mathcal{G}_{L_{j}}, P\right)\right)=L_{m}\left(\mathcal{G}_{L_{j}}, \Gamma_{L_{j}}^{k+1}(P)\right)$.

$$
\begin{aligned}
& \Omega_{L_{j}}^{k+1}\left(L\left(\mathcal{G}_{L_{j}}, P\right)\right)= \Omega_{L_{j}}\left(\Omega_{L_{j}}^{k}\left(L\left(\mathcal{G}_{L_{j}}, P\right)\right)\right) \\
&= \Omega_{L_{j}}\left(L_{m}\left(\mathcal{G}_{L_{j}}, \Gamma_{L_{j}}^{k}(P)\right)\right), \text { by inductive assumption } \\
&= \Omega_{L N B_{j}}\left(\Omega_{p 6_{j}}\left(\Omega_{p 5_{j}}\left(\sup \mathcal{L} \mathcal{P} \mathcal{C}_{j}\left(\overline{L_{m}\left(\mathcal{G}_{L_{j}}, \Gamma_{L_{j}}^{k}(P)\right)}\right)\right)\right),\right. \\
& \quad \text { by definition of } \Omega_{L_{j}} \\
&=\Omega_{L N B_{j}}\left(\Omega_{p 6_{j}}\left(\Omega_{p 5_{j}}\left(\sup \mathcal{L} \mathcal{P} \mathcal{C}_{j}\left(L\left(\mathcal{G}_{L_{j}}, \Gamma_{L_{j}}^{k}(P)\right)\right)\right)\right),\right.
\end{aligned}
$$

by Lemma 4.26 


$$
\begin{aligned}
\text { Master Thesis - } & \text {. Song - McMaster - Computing and Software } \\
& =\Omega_{L_{j}}\left(L\left(\mathcal{G}_{L_{j}}, \Gamma_{L_{j}}^{k}(P)\right)\right) \text {, by the definition of } \Omega_{L_{j}} . \\
& =L_{m}\left(\mathcal{G}_{L_{j}}, \Gamma_{L_{j}}^{k+1}(P)\right) \text {, by Point } 1 \text { of this proposition. }
\end{aligned}
$$

Theorem 4.2. For system $\mathbf{\Phi}$, the following two points hold:

1. There exists $k \in\{0,1, \ldots\}$ such that $k \leq\left|Q_{L_{j}}\right|$ and $\Gamma_{L_{j}}^{k}$ (true) is the greatest fixpoint of the function $\Gamma_{L_{j}}$ with respect to $\left(\operatorname{Pred}\left(Q_{L_{j}}\right), \preceq\right)$.

2. $\sup \mathcal{C}_{L_{j}}\left(L_{m}\left(\mathcal{G}_{L_{j}}\right)\right)=\sup \mathcal{C}_{L_{j}}\left(L_{m}\left(\mathcal{G}_{L_{j}}\right.\right.$, true $\left.)\right)=L_{m}\left(\mathcal{G}_{L_{j}}, \Gamma_{L_{j}}^{k}(\right.$ true $\left.)\right)$

\section{proof:}

1. Show that there exists $k \in\{0,1, \ldots\}$ such that $k \leq\left|Q_{L_{j}}\right|$ and $\Gamma_{L_{j}}^{k}($ true) is the greatest fixpoint of the function $\Gamma_{L_{j}}$ with respect to $\left(\operatorname{Pred}\left(Q_{L_{j}}\right), \preceq\right)$.

As $\Gamma_{L_{j}}$ is monotone and $\left|Q_{L_{j}}\right|$ is assumed to be finite (Section 4.1), we know immediately from Proposition 2.3 that there exists $k \in\{0,1, \ldots\}$ such that $k \leq\left|Q_{L_{j}}\right|$ and $\Gamma_{L_{j}}^{k}($ true $)$ is the greatest fixpoint of the function $\Gamma_{L_{j}}$.

2. Show that $\sup \mathcal{C}_{L_{j}}\left(L_{m}\left(\mathcal{G}_{L_{j}}\right)\right)=\sup \mathcal{C}_{L_{j}}\left(L_{m}\left(\mathcal{G}_{L_{j}}, \operatorname{true}\right)\right)=L_{m}\left(\mathcal{G}_{L_{j}}, \Gamma_{L_{j}}^{k}(\right.$ true $\left.)\right)$. $\sup \mathcal{C}_{L_{j}}\left(L_{m}\left(\mathcal{G}_{L_{j}}\right)\right)=\sup \mathcal{C}_{L_{j}}\left(L_{m}\left(\mathcal{G}_{L_{j}}\right.\right.$, true $\left.)\right)$ is automatic as $L_{m}\left(\mathcal{G}_{L_{j}}\right)=L_{m}\left(\mathcal{G}_{L_{j}}\right.$, true $)$

(a) Show that $\sup \mathcal{C}_{L_{j}}\left(L_{m}\left(\mathcal{G}_{L_{j}}, \operatorname{true}\right)\right) \subseteq L_{m}\left(\mathcal{G}_{L_{j}}, \Gamma_{L_{j}}^{k}(\right.$ true $\left.)\right)$

By Proposition 2.3, we know that

$$
\begin{aligned}
& (\forall i \in\{1,2, \ldots\}) i>k \Rightarrow L_{m}\left(\mathcal{G}_{L_{j}}, \Gamma_{L_{j}}^{i}(\text { true })\right)=L_{m}\left(\mathcal{G}_{L_{j}}, \Gamma_{L_{j}}^{k}(\text { true })\right) \\
& \Rightarrow \lim _{i \rightarrow \infty} L_{m}\left(\mathcal{G}_{L_{j}}, \Gamma_{L_{j}}^{i}(\text { true })\right)=L_{m}\left(\mathcal{G}_{L_{j}}, \Gamma_{L_{j}}^{k}(\text { true })\right) \\
& \Rightarrow \lim _{i \rightarrow \infty} \Omega_{L_{j}}^{i}\left(L\left(\mathcal{G}_{L_{j}}, \text { true }\right)\right)=L_{m}\left(\mathcal{G}_{L_{j}}, \Gamma_{L_{j}}^{k}(\text { true })\right), \text { by Proposition } 4.27 \\
& \Rightarrow \sup \mathcal{C}_{L_{j}}\left(L_{m}\left(\mathcal{G}_{L_{j}}, \text { true }\right)\right) \subseteq L_{m}\left(\mathcal{G}_{L_{j}}, \Gamma_{L_{j}}^{k}(\text { true })\right), \text { by Proposition } 4.21
\end{aligned}
$$


Master Thesis - R. Song-McMaster - Computing and Software

(b) Show that $L_{m}\left(\mathcal{G}_{L_{j}}, \Gamma_{L_{j}}^{k}(\right.$ true $\left.)\right) \subseteq \sup \mathcal{C}_{L_{j}}\left(L_{m}\left(\mathcal{G}_{L_{j}}\right.\right.$, true $\left.)\right)$.

Based on the value of $k$, we now show this in two cases:

- Case $1: k \in\{1,2, \ldots\}$

By Point 1 of this theorem and Proposition 2.3, we have

$\Gamma_{L_{j}}^{k}($ true $)=\Gamma_{L_{j}}^{k+1}($ true $)$

$\Rightarrow L_{m}\left(\mathcal{G}_{L_{j}}, \Gamma_{L_{j}}^{k}(\right.$ true $\left.)\right)=L_{m}\left(\mathcal{G}_{L_{j}}, \Gamma_{L_{j}}^{k+1}(\right.$ true $\left.)\right)$

$\Rightarrow L_{m}\left(\mathcal{G}_{L_{j}}, \Gamma_{L_{j}}^{k}(\right.$ true $\left.)\right)=\Omega_{L_{j}}^{k+1}\left(L\left(\mathcal{G}_{L_{j}}\right.\right.$, true $\left.)\right)$, by Proposition 4.27

$\Rightarrow L_{m}\left(\mathcal{G}_{L_{j}}, \Gamma_{L_{j}}^{k}(\right.$ true $\left.)\right)=\Omega_{L_{j}}\left(\Omega_{L_{j}}^{k}\left(L\left(\mathcal{G}_{L_{j}}\right.\right.\right.$, true $\left.\left.)\right)\right)$

$\Rightarrow L_{m}\left(\mathcal{G}_{L_{j}}, \Gamma_{L_{j}}^{k}(\right.$ true $\left.)\right)=\Omega_{L_{j}}\left(L_{m}\left(\mathcal{G}_{L_{j}}, \Gamma_{L_{j}}^{k}(\right.\right.$ true $\left.\left.)\right)\right)$,

by Proposition 4.27 and $k \in\{1,2, \ldots\}$

- Case 2: $k=0$

If $\Gamma_{L_{j}}^{0}($ true $)$ is the greatest fixpoint of $\Gamma_{L_{j}}$, then by Proposition 2.3 we know that

$$
\Gamma_{L_{j}}^{1}(\text { true })=\Gamma_{L_{j}}^{0}(\text { true }),
$$

so $\Gamma_{L_{j}}^{1}$ (true) is also the greatest fixpoint of $\Gamma_{L_{j}}$.

$\Rightarrow L_{m}\left(\mathcal{G}_{L_{j}}, \Gamma_{L_{j}}^{1}(\right.$ true $\left.)\right)=\Omega_{L_{j}}\left(L_{m}\left(\mathcal{G}_{L_{j}}, \Gamma_{L_{j}}^{1}(\right.\right.$ true $\left.\left.)\right)\right), \quad$ by Case 1

$\Rightarrow L_{m}\left(\mathcal{G}_{L_{j}}, \Gamma_{L_{j}}^{0}(\right.$ true $\left.)\right)=\Omega_{L_{j}}\left(L_{m}\left(\mathcal{G}_{L_{j}}, \Gamma_{L_{j}}^{0}(\right.\right.$ true $\left.\left.)\right)\right), \quad$ by $(1)$

From Case 1 and Case 2, we know that $L_{m}\left(\mathcal{G}_{L_{j}}, \Gamma_{L_{j}}^{k}(\right.$ true $\left.)\right)$ is always a fixpoint of the function $\Omega_{L_{j}}$.

From Proposition 4.20, $\sup \mathcal{C}_{L_{j}}\left(L_{m}\left(\mathcal{G}_{L_{j}}\right.\right.$, true $\left.)\right)$ is the greatest fixpoint of the function $\Omega_{L_{j}}$, so $L_{m}\left(\mathcal{G}_{L_{j}}, \Gamma_{L_{j}}^{k}(\right.$ true $\left.)\right) \subseteq \sup \mathcal{C}_{L_{j}}\left(L_{m}\left(\mathcal{G}_{L_{j}}\right.\right.$, true $\left.)\right)$.

Corollary 4.4. For system $\boldsymbol{\Phi}$, let $\mathbf{S}_{L_{j}}$ be a DES defined over event set $\Sigma_{I L_{j}}$ with $L_{m}\left(\mathbf{S}_{L_{j}}\right)=L_{m}\left(\mathcal{G}_{L_{j}}, \Gamma_{L_{j}}^{k}(\right.$ true $\left.)\right)$ and $L\left(\mathbf{S}_{L_{j}}\right)=L\left(\mathcal{G}_{L_{j}}, \Gamma_{L_{j}}^{k}(\right.$ true $\left.)\right)$, where $k \in\{0,1, \ldots\}$ 


\section{Master Thesis - R. Song-McMaster-Computing and Software}

and $\Gamma_{L_{j}}^{k}($ true $)$ is the greatest fixpoint of $\Gamma_{L_{j}}$ with respect to $\left(\operatorname{Pred}\left(Q_{L_{j}}\right), \preceq\right)$. Then for the $n^{\text {th }}$ degree parallel interface system composed of $\mathbf{G}_{H}^{p}, \mathbf{G}_{L_{1}}^{p}, \ldots, \mathbf{G}_{L_{n}}^{p}, \mathbf{G}_{I_{1}}, \ldots$, $\mathbf{G}_{I_{n}}, \mathbf{E}_{H}, \mathbf{E}_{L_{1}}, \ldots, \mathbf{E}_{L_{j-1}}, \mathbf{S}_{L_{j}}, \mathbf{E}_{L_{j+1}}, \ldots, \mathbf{E}_{L_{n}}$ with respect to the alphabet partition in Equation 3.1, $\mathbf{S}_{L_{j}}$ is a $j^{\text {th }}$ low-level proper supervisor.

\section{proof:}

We first note that by Theorem 4.2 , an appropriate $k \in\{0,1, \ldots\}$ exists such that $\Gamma_{L_{j}}^{k}($ true $)$ is the greatest fixpoint of $\Gamma_{L_{j}}$ with respect to $\left(\operatorname{Pred}\left(Q_{L_{j}}\right), \preceq\right)$.

1. Show that $\overline{L_{m}\left(\mathbf{S}_{L_{j}} \times \mathbf{G}_{L_{j}}^{p} \times \mathbf{G}_{I_{j}}^{l}\right)}=L\left(\mathbf{S}_{L_{j}} \times \mathbf{G}_{L_{j}}^{p} \times \mathbf{G}_{I_{j}}^{l}\right)$.

$$
\begin{aligned}
& L_{m}\left(\mathbf{S}_{L_{j}}\right)=L_{m}\left(\mathcal{G}_{L_{j}}, \Gamma_{L_{j}}^{k}(\text { true })\right) \\
& \Rightarrow L_{m}\left(\mathbf{S}_{L_{j}}\right) \subseteq L_{m}\left(\mathcal{G}_{L_{j}}\right) \\
& \Rightarrow L_{m}\left(\mathbf{S}_{L_{j}}\right) \subseteq L_{m}\left(\mathbf{E}_{L_{j}} \times \mathbf{G}_{L_{j}}^{p} \times \mathbf{G}_{I_{j}}^{l}\right) \\
& \Rightarrow L_{m}\left(\mathbf{S}_{L_{j}}\right) \subseteq L_{m}\left(\mathbf{G}_{L_{j}}^{p} \times \mathbf{G}_{I_{j}}^{l}\right) \\
& \Rightarrow L_{m}\left(\mathbf{S}_{L_{j}}\right)=L_{m}\left(\mathbf{S}_{L_{j}} \times \mathbf{G}_{L_{j}}^{p} \times \mathbf{G}_{I_{j}}^{l}\right)
\end{aligned}
$$

Similarly, we can show that $L\left(\mathbf{S}_{L_{j}}\right)=L\left(\mathbf{S}_{L_{j}} \times \mathbf{G}_{L_{j}}^{p} \times \mathbf{G}_{I_{j}}^{l}\right)$

If $k \in\{1,2, \ldots\}$ and $\Gamma_{L_{j}}^{k}($ true $)$ is the greatest fixpoint of $\Gamma_{L_{j}}$, then by Lemma 4.26 we know that $\overline{L_{m}\left(\mathbf{S}_{L_{j}}\right)}=L\left(\mathbf{S}_{L_{j}}\right)$.

If $k=0$ and $\Gamma_{L_{j}}^{0}$ (true) is the greatest fixpoint of $\Gamma_{L_{j}}$, then as $\left|Q_{L_{j}}\right|$ is assumed to be finite (Section 4.1), by Proposition 2.3 we know that $\Gamma_{L_{j}}^{1}($ true $)=\Gamma_{L_{j}}^{0}($ true).

$\Rightarrow L\left(\mathbf{S}_{L_{j}}\right)=L\left(\mathcal{G}_{L_{j}}, \Gamma_{L_{j}}^{1}(\right.$ true $\left.)\right)$ and $L_{m}\left(\mathbf{S}_{L_{j}}\right)=L_{m}\left(\mathcal{G}_{L_{j}}, \Gamma_{L_{j}}^{1}(\right.$ true $\left.)\right)$

$\Rightarrow \overline{L_{m}\left(\mathbf{S}_{L_{j}}\right)}=L\left(\mathbf{S}_{L_{j}}\right), \quad$ by Lemma 4.26 .

Therefore, we always have

$$
\overline{L_{m}\left(\mathbf{S}_{L_{j}}\right)}=L\left(\mathbf{S}_{L_{j}}\right)
$$

when $k \in\{0,1, \ldots\}$ and $\Gamma_{L_{j}}^{k}($ true $)$ is the greatest fixpoint of $\Gamma_{L_{j}}$. 
Master Thesis - R. Song - McMaster - Computing and Software

Combining (1), (2) and (3), we can conclude $\overline{L_{m}\left(\mathbf{S}_{L_{j}} \times \mathbf{G}_{L_{j}}^{p} \times \mathbf{G}_{I_{j}}^{l}\right)}=L\left(\mathbf{S}_{L_{j}} \times\right.$ $\left.\mathbf{G}_{L_{j}}^{p} \times \mathbf{G}_{I_{j}}^{l}\right)$.

2. Show that $\mathbf{S}_{L_{j}}$ is the $j^{\text {th }}$ low-level interface controllable.

By Part 1 and Definition 3.8, Definition 4.10, and Definition 4.16, it is sufficient to show that $L_{m}\left(\mathbf{S}_{L_{j}} \times \mathbf{G}_{L_{j}}^{p} \times \mathbf{G}_{I_{j}}^{l}\right)$ is $j^{\text {th }}$ low-level interface controllable.

From Theorem 4.2, we know that $L_{m}\left(\mathbf{S}_{L_{j}}\right)$ is $j^{\text {th }}$ low-level interface controllable. By (1), we can conclude $L_{m}\left(\mathbf{S}_{L_{j}} \times \mathbf{G}_{L_{j}}^{p} \times \mathbf{G}_{I_{j}}^{l}\right)$ is also $j^{\text {th }}$ low-level interface controllable.

The supervisor $\mathbf{S}_{L_{j}}$ can be constructed by removing states from $\mathcal{G}_{L_{j}}$ that do not satisfy $\Gamma_{L_{j}}^{k}$ (true). A trim supervisor DES $\mathbf{S}_{L_{j}}$ can be built by removing states from $\mathcal{G}_{L_{j}}$ that do not satisfy $R\left(\mathcal{G}_{L_{j}}, \Gamma_{L_{j}}^{k}(\right.$ true $\left.)\right)$.

Algorithm 4.3 shows how to compute $\Gamma_{L_{j}}^{k}($ true $)$, where $k \in\{0,1, \ldots\}$ and $\Gamma_{L_{j}}^{k}($ true $)$ is the greatest fixpoint of $\Gamma_{L_{j}}$ with respect to $\left(\operatorname{Pred}\left(Q_{L_{j}}\right), \preceq\right)$. By Point 1 of Theorem 4.2, we know the greatest fixpoint will be reached after finite number of iterations.

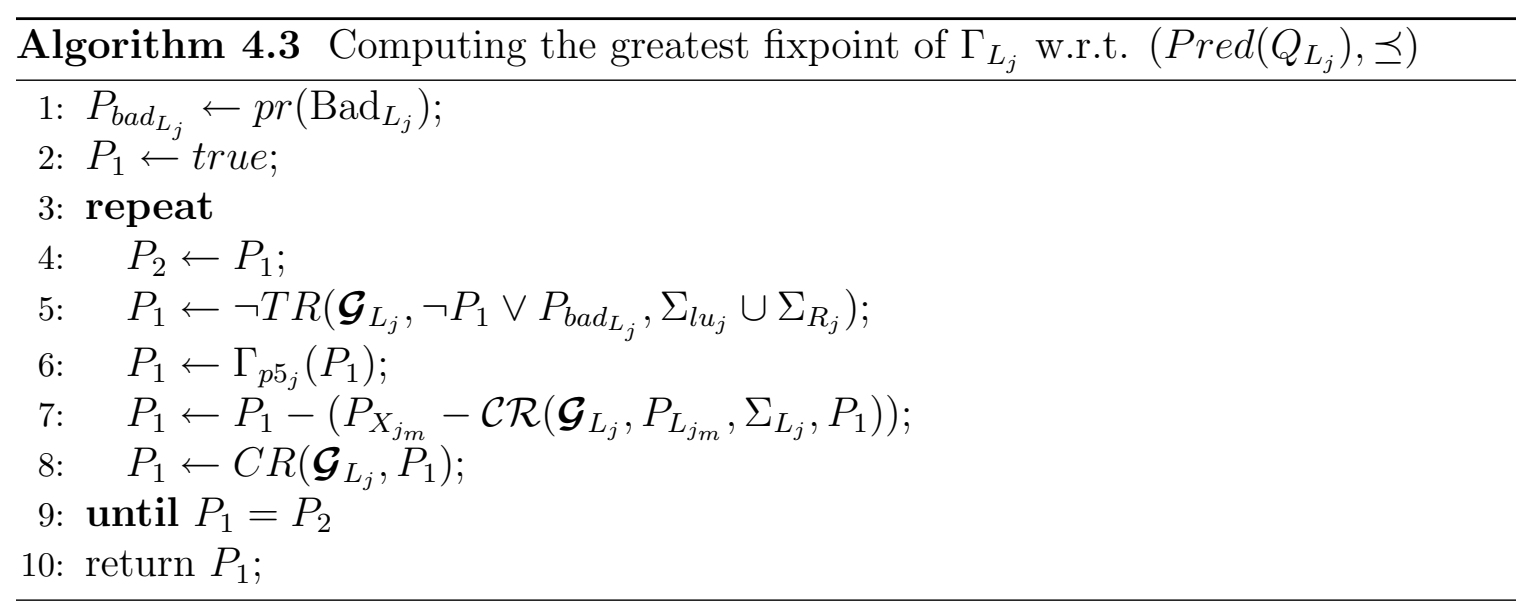

In the algorithm, $P_{X j_{m}}$ and $P_{L j_{m}}$ are as defined in Definition 4.21. Line 5 computes $\operatorname{PLPC}_{j}\left(P_{1}\right) . \Gamma_{p 5_{j}}\left(P_{1}\right)$ in Line 6 can be computed by using Algorithm 4.2. Line 7 computes $\Gamma_{p 6_{j}}\left(P_{1}\right)$. Line 8 is for the function $\Omega_{L N B_{j}}$. 


\section{Chapter 5}

\section{Verification of HISC}

In this chapter, we briefly discuss the method to verify a $n^{\text {th }}$ degree supervisor interface system and we give the verification algorithms.

In the previous chapter, we proved that for system $\mathbf{\Phi}$, we can synthesize a highlevel proper supervisor by trimming off states from $\mathcal{G}_{H}$ and the $j^{\text {th }}$ low-level proper supervisor by trimming off states from $\mathcal{G}_{L_{j}}$.

For the $n^{\text {th }}$ degree supervisor interface system that respects the alphabet partition given by 3.1 and is composed of plant components $\mathbf{G}_{H}^{p}, \mathbf{G}_{L_{1}}^{p}, \ldots, \mathbf{G}_{L_{n}}^{p}$, supervisors $\mathbf{S}_{H}, \mathbf{S}_{L_{1}}, \ldots, \mathbf{S}_{L_{n}}$, and interfaces $\mathbf{G}_{I_{1}}, \ldots, \mathbf{G}_{I_{n}}$, we can treat all the supervisors as their corresponding specifications (assuming the system is HISC-valid) and apply the synthesis algorithm to the system. If $\mathbf{S}_{H}$ is a high-level proper supervisor, then there would be no reachable state that must be removed from $\mathcal{G}_{H}$. Similarly, if $\mathbf{S}_{L_{j}}$ is a $j^{\text {th }}$ low-level proper supervisor $(j \in\{1, \ldots, n\})$, then there would be no reachable state that must be removed from $\mathcal{G}_{L_{j}}$.

Algorithm 5.1 shows the verification algorithm for the high-level. Bad $_{H}$ in Line 2 is a state subset as defined in Definition 4.7, and $\operatorname{pr}\left(\operatorname{Bad}_{H}\right)$ denotes the predicate identified by $\operatorname{Bad}_{H}$. 


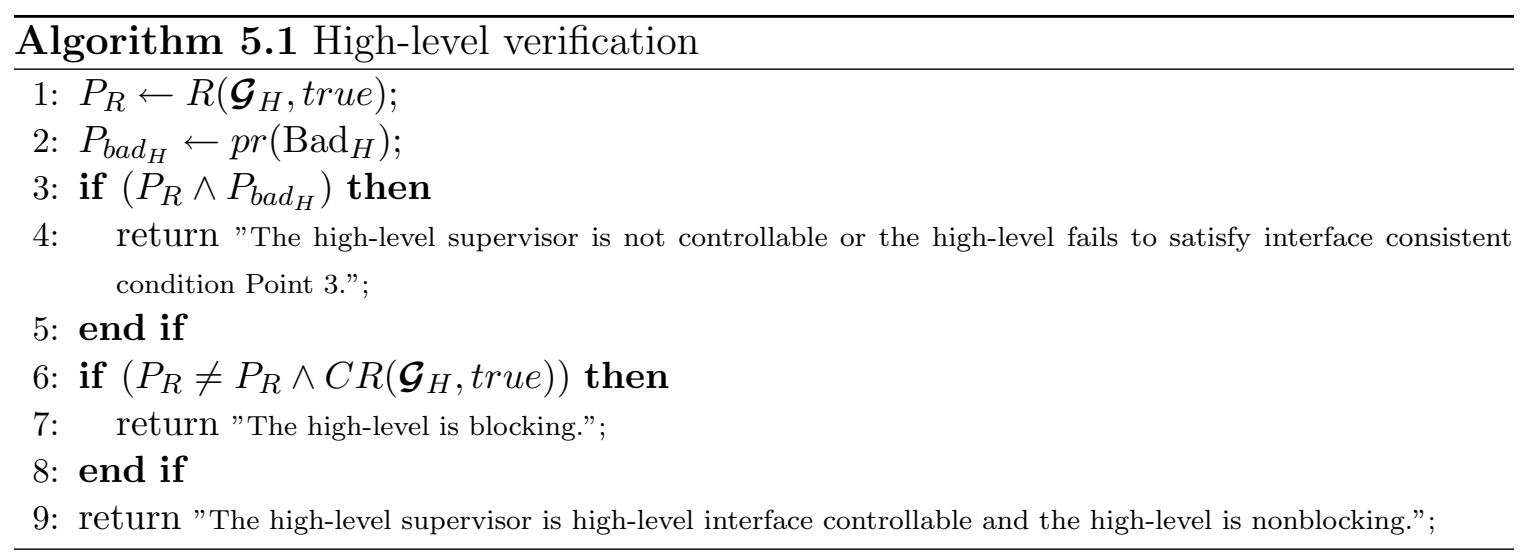

Algorithm 5.2 shows the verification algorithm for the $j^{\text {th }}$ low-level. Bad $_{L_{j}}$ in Line 2 is a state subset as defined in Definition 4.14, and $\operatorname{pr}\left(\operatorname{Bad}_{L_{j}}\right)$ is the predicate identified by $\operatorname{Bad}_{L_{j}} . \Gamma_{p 5_{j}}($ true) in Line 6 can be computed by using Algorithm 4.2. Line 9 verifies the interface consistent condition Point 6. Line 12 verifies the nonblocking property.

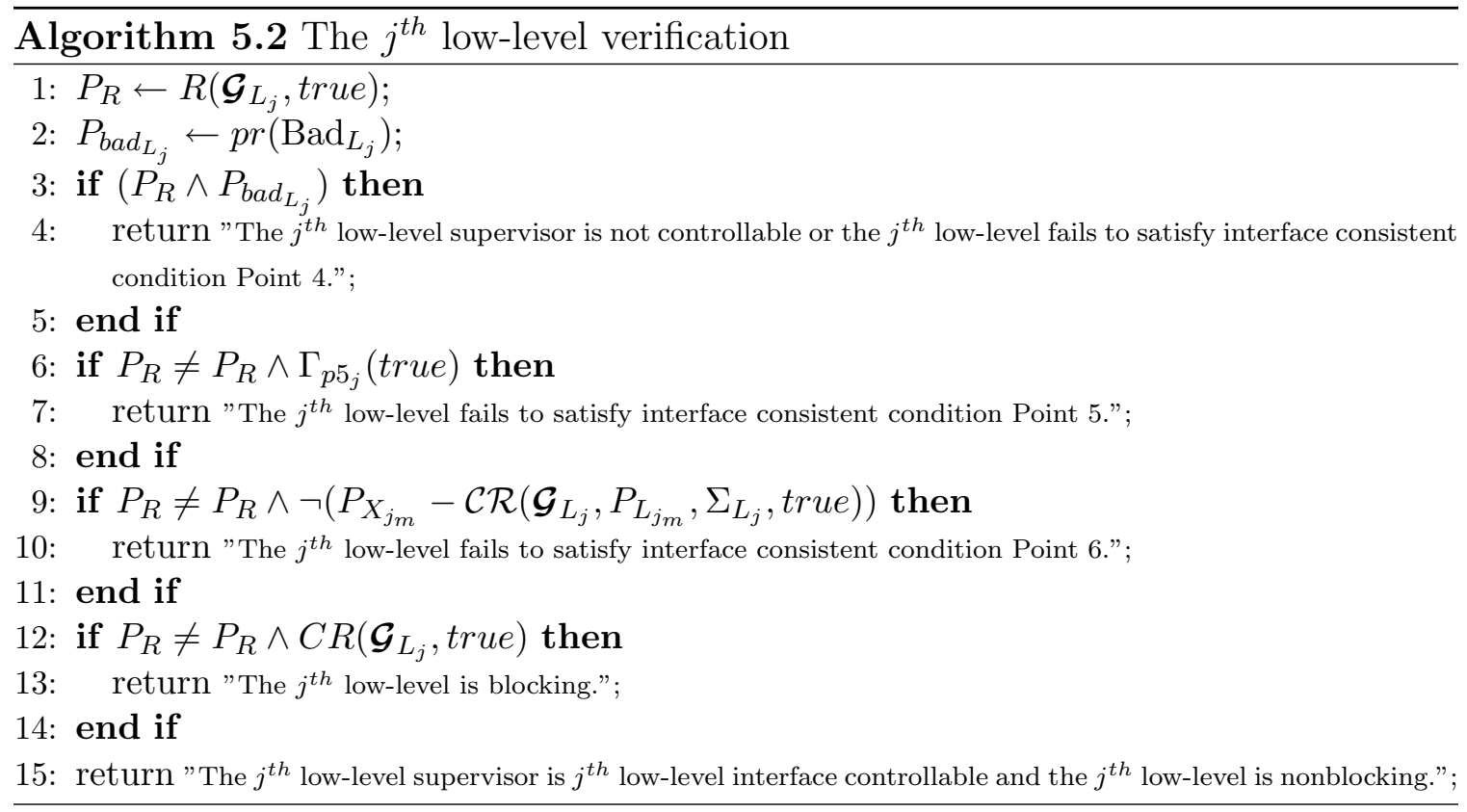

Usually, the verification process is faster than the synthesis process, because the verification algorithms do not need to compute the greatest fixpoints as Algorithm 4.1 and Algorithm 4.3 need to do. 


\section{Chapter 6}

\section{Symbolic Computation for HISC Synthesis and Verification}

In the previous two chapters, we developed the algorithms for the synthesis and verification of an HISC system. The efficiency of these algorithms is dominated by the computation of the four predicate transformers: $R, C R, T R$ and $\mathcal{C R}$. In this chapter, we first discuss how to use logic formulas to represent state subsets and transitions in a system, and then present how to compute the predicate transformers from the logic formulas. After that, we briefly introduce a method of using Reduced Ordered Binary Decision Diagram (ROBDD, or simply BDD) [6] to implement the algorithms. We then present an approach for controller implementation. Finally, we give a small tutorial example to demonstrate the algorithms.

Most of the ideas in this chapter are originally invented by Ma in [31] for the State Tree Structure (STS) or researchers in the model checking area. The approach to represent the state subsets and transitions for the HISC high-level or low-levels as logic formulas and BDD can be thought of as a special case of the approach for STS. The optimization technique using the tautology (Equation 6.2) to reduce the 


\section{Master Thesis - R. Song-McMaster - Computing and Software}

intermediate BDD is also applied in our implementations. What is new here is that we simplified the logic formula representation for a flat system composed of component DES and applied these ideas and techniques for the HISC synthesis and verification algorithms.

\subsection{Symbolic Representation of State Subsets and Transitions}

In Chapter 2, we discussed predicates defined on the state set of a DES, but we did not discuss how to define a predicate other than its corresponding state subset. From now on, we call a predicate that are defined on the state set of a DES as a state predicate. A state predicate essentially is a boolean function whose domain is the state set of the DES and range is $\{0,1\}$. If we use the identifying state subset to define a state predicate, then it is no better than directly using the state subset in our algorithms. In this section, for a given HISC system, we present how to define a state predicate as a logic formula, so the formula also represents a state subset. We say that the logic formula is a symbolic representation of the state subset.

In order to represent the high-level and low-levels in an HISC system symbolically, we also need to define predicates that are defined on the transitions of a DES, which we will call transition predicates. Then we will discuss how to use logic formulas to define the transition predicates.

The high-level or low-levels in an HISC system can be thought of as a special flat system which is composed of component DES: plant(s), supervisor(s)/specification(s) and interface(s). Because our symbolic representation for state subsets and transitions does not care about the type of a component DES, in this section, we only talk about 


\section{Master Thesis - R. Song - McMaster - Computing and Software}

the symbolic representation for a flat system composed of component DES.

\subsubsection{Symbolic Representation of State Subsets}

Let the system $\mathbf{G}:=\left(Q, \Sigma, \delta, q_{0}, Q_{m}\right)$ be the cross product of component DES $\mathbf{G}_{1}:=\left(Q_{1}, \Sigma, \delta_{1}, q_{1_{0}}, Q_{1_{m}}\right), \ldots, \mathbf{G}_{n}:=\left(Q_{n}, \Sigma, \delta_{n}, q_{n_{0}}, Q_{n_{m}}\right)$, where $n \in\{1,2, \ldots\}$. i.e. $\mathbf{G}:=\mathbf{G}_{1} \times \cdots \times \mathbf{G}_{n}$.

Let $q \in Q$, then there exists a unique $q_{1} \in Q_{1}, \ldots, q_{n} \in Q_{n}$ such that $q=$ $\left(q_{1}, \ldots, q_{n}\right)$. We say $q_{1}$ is the first component part of $q ; \ldots ; q_{n}$ is the $n^{t h}$ component part of $q$.

Definition 6.1. For the system $\mathbf{G}$ composed of components $\mathbf{G}_{1}, \ldots, \mathbf{G}_{n}$, the state variable $v_{i}(i \in\{1, \ldots, n\})$ for the $i^{\text {th }}$ component DES $\mathbf{G}_{i}$ is a variable whose domain is $Q_{i}$. The state variable vector $\mathbf{v}$ for $\mathbf{G}$ is $\left(v_{1}, \ldots, v_{n}\right)$, where $v_{1}, \ldots, v_{n}$ are the state variables for each component.

Let $i \in\{1, \ldots, n\}$ and $q_{i} \in Q_{i}, v_{i}=q_{i}$ returns 1 if the state variable $v_{i}$ has been assigned value $q_{i}$, otherwise it returns 0 . Let $A \subseteq Q$ be a state subset, then the predicate $P_{A}$ for $A$ can be written by ${ }^{1}$

$$
P_{A}(\mathbf{v}):=\bigvee_{q \in A}\left(v_{1}=q_{1} \wedge v_{2}=q_{2} \wedge \cdots \wedge v_{n}=q_{n}\right)
$$

For convenience, if $\mathbf{v}$ is understood, we write $P_{A}$ instead of $P_{A}(\mathbf{v})$. Clearly, for all $q \in Q, q \in A$ if and only if $P_{A}(q) \equiv 1$. Here we use $\equiv$ to stand for logical equivalence, because $=$ has been used to test if $v_{i}$ has been assigned value $q_{i}$.

\footnotetext{
${ }^{1}$ As a flat system composed of component DES is a special case of STS, we will not give a soundness and completeness proof for the logic formula representation, interested readers can find a proof for STS in [31].
} 


\section{Master Thesis - R. Song - McMaster - Computing and Software}

For all $i \in\{1, \ldots, n\}$, if we treat $v_{i}=q_{i}$ as a propositional atom, then the formula generated by Equation 6.1 is a propositional logic formula. This is a very important aspect, because a predicate logic formula can not be represented as a BDD.

This formula does not look promising, but if we notice the following tautology, we will see it is possible that the formula can be greatly simplified as the following examples show.

$$
\left(\bigvee_{q_{i} \in Q_{i}}\left(v_{i}=q_{i}\right)\right) \equiv 1
$$

Figure 6.1 is the simplified small factory example from [47] with buffer size two. The event set for the system is $\Sigma=\left\{\alpha_{1}, \alpha_{2}, \beta_{1}, \beta_{2}\right\}$. All the states in each component should also be added selfloops with events not appearing on the component.

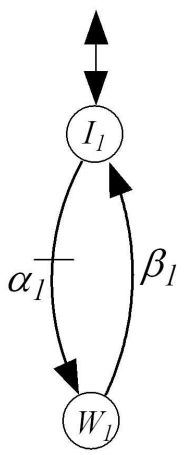

$\mathbf{M}_{1}$

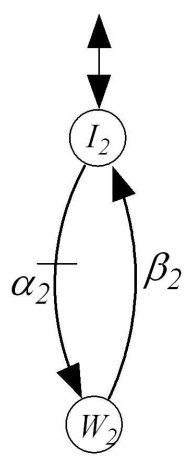

$\mathbf{M}_{\mathbf{2}}$

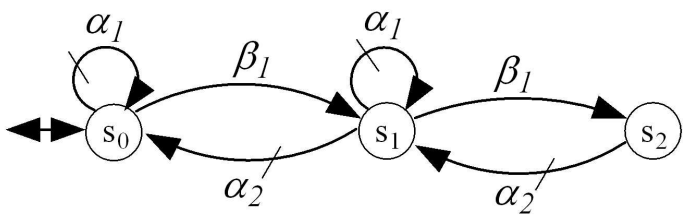

BUF

Figure 6.1: Small factory example

Let $v_{M_{1}}, v_{M_{2}}, v_{B U F}$ be the state variables for the component DES $\mathbf{M}_{1}, \mathbf{M}_{2}$ and BUF respectively, then the state variable vector for the small factory is $\mathbf{v}=\left(v_{M_{1}}\right.$, $\left.v_{M_{2}}, v_{B U F}\right)$. Let $A \subseteq\left\{I_{1}, W_{1}\right\} \times\left\{I_{2}, W_{2}\right\} \times\left\{s_{0}, s_{1}, s_{2}\right\}$ be a state subset of the small factory. Let $P_{A}$ be the state predicate for $A$.

First let $A:=\left\{\left(I_{1}, I_{2}, s_{0}\right)\right\}$, then by Equation 6.1 , we have $P_{A}(\mathbf{v}):=\left(v_{M_{1}}=\right.$ $\left.I_{1} \wedge v_{M_{2}}=I_{2} \wedge v_{B U F}=s_{0}\right)$. This formula for the predicate $P_{A}$ is not simpler than a state subset. 


$$
\text { Master Thesis - R. Song - McMaster - Computing and Software }
$$

Now let $A:=\left\{\left(I_{1}, I_{2}, s_{0}\right),\left(W_{1}, I_{2}, s_{0}\right),\left(I_{1}, I_{2}, s_{1}\right),\left(W_{1}, I_{2}, s_{1}\right),\left(I_{1}, I_{2}, s_{2}\right),\left(W_{1}, I_{2}, s_{2}\right)\right\}$, then by Equation 6.1, we have

$$
\begin{aligned}
P_{A}(\mathbf{v}):= & \left(v_{M_{1}}=I_{1} \wedge v_{M_{2}}=I_{2} \wedge v_{B U F}=s_{0}\right) \vee\left(v_{M_{1}}=W_{1} \wedge v_{M_{2}}=I_{2} \wedge v_{B U F}=s_{0}\right) \vee \\
& \left(v_{M_{1}}=I_{1} \wedge v_{M_{2}}=I_{2} \wedge v_{B U F}=s_{1}\right) \vee\left(v_{M_{1}}=W_{1} \wedge v_{M_{2}}=I_{2} \wedge v_{B U F}=s_{1}\right) \vee \\
& \left(v_{M_{1}}=I_{1} \wedge v_{M_{2}}=I_{2} \wedge v_{B U F}=s_{2}\right) \vee\left(v_{M_{1}}=W_{1} \wedge v_{M_{2}}=I_{2} \wedge v_{B U F}=s_{2}\right) \\
\equiv & \left(v_{M_{1}}=I_{1} \vee v_{M_{1}}=W_{1}\right) \wedge\left(v_{M_{2}}=I_{2}\right) \wedge\left(v_{B U F}=s_{0} \vee v_{B U F}=s_{1} \vee v_{B U F}=s_{2}\right),
\end{aligned}
$$

by the distributive law of propositional logic

$$
\begin{aligned}
& \equiv 1 \wedge\left(v_{M_{2}}=I_{2}\right) \wedge 1 \\
& \equiv\left(v_{M_{2}}=I_{2}\right)
\end{aligned}
$$

We can see that $P_{A}(\mathbf{v}):=\left(v_{M_{2}}=I_{2}\right)$ is much simpler than the state subset $A$. Furthermore, the formula clearly says that $A$ includes all the states whose $\mathbf{M}_{\mathbf{2}}$ part is $I_{2}$.

With the given formula representation for a state predicate, we can easily check if a state is in the corresponding state subset. Let $P_{A}(\mathbf{v}):=\left(v_{M_{2}}=I_{2}\right)$ be a predicate for the small factory system. For example, the state $\left(I_{1}, I_{2}, s_{0}\right) \in A$ because

$$
P_{A}\left(I_{1}, I_{2}, s_{0}\right):=\left(I_{2}=I_{2}\right) \equiv 1
$$

and the state $\left(I_{1}, W_{2}, s_{1}\right) \notin A$ because

$$
P_{A}\left(I_{1}, W_{2}, s_{1}\right):=\left(W_{2}=I_{2}\right) \equiv 0
$$

With the formula representation for a state predicate, the set operations such as intersection, union and complement can be done by the logic operations $\wedge, \vee$ and $\neg$ on formulas. We now give examples for the small factory system.

Let $A_{1}, A_{2} \subseteq\left\{I_{1}, W_{1}\right\} \times\left\{I_{2}, W_{2}\right\} \times\left\{s_{0}, s_{1}, s_{2}\right\}$ be two state subsets of the small factory, and

$$
A_{1}=\left\{\left(I_{1}, I_{2}, s_{0}\right),\left(W_{1}, I_{2}, s_{0}\right),\left(I_{1}, I_{2}, s_{2}\right),\left(W_{1}, I_{2}, s_{2}\right)\right\},
$$


Master Thesis - R. Song - McMaster - Computing and Software

$$
\begin{aligned}
A_{2}=\{ & \left(I_{1}, I_{2}, s_{0}\right),\left(I_{1}, I_{2}, s_{1}\right),\left(I_{1}, W_{2}, s_{0}\right),\left(I_{1}, W_{2}, s_{1}\right),\left(W_{1}, I_{2}, s_{0}\right),\left(W_{1}, I_{2}, s_{1}\right), \\
& \left.\left(W_{1}, W_{2}, s_{0}\right),\left(W_{1}, W_{2}, s_{1}\right)\right\} .
\end{aligned}
$$

Let $P_{A_{1}}$ and $P_{A_{2}}$ be the state predicates for $A_{1}$ and $A_{2}$ respectively, then by Equation 6.1, we have the simplified formulas as

$$
\begin{gathered}
P_{A_{1}}(\mathbf{v}):=\left(v_{M_{2}}=I_{2}\right) \wedge\left(v_{B U F}=s_{0} \vee v_{B U F}=s_{2}\right) \\
P_{A_{2}}(\mathbf{v}):=\left(v_{B U F}=s_{0} \vee v_{B U F}=s_{1}\right) .
\end{gathered}
$$

- $A_{1} \cap A_{2}$

By set intersection, $A_{1} \cap A_{2}=\left\{\left(I_{1}, I_{2}, s_{0}\right),\left(W_{1}, I_{2}, s_{0}\right)\right\}$, and

$$
\begin{aligned}
P_{A_{1}} \wedge P_{A_{2}} & \equiv\left(v_{M_{2}}=I_{2}\right) \wedge\left(v_{B U F}=s_{0} \vee v_{B U F}=s_{2}\right) \wedge\left(v_{B U F}=s_{0} \vee v_{B U F}=s_{1}\right) \\
& \equiv\left(v_{M_{2}}=I_{2}\right) \wedge\left(v_{B U F}=s_{0}\right) .
\end{aligned}
$$

One can see that $P_{A_{1}} \wedge P_{A_{2}}$ really represents the state subset $A_{1} \cap A_{2}$.

- $A_{1} \cup A_{2}$

By set union,

$$
\begin{aligned}
A_{1} \cup A_{2}=\{ & \left(I_{1}, I_{2}, s_{0}\right),\left(W_{1}, I_{2}, s_{0}\right),\left(I_{1}, I_{2}, s_{2}\right),\left(W_{1}, I_{2}, s_{2}\right),\left(I_{1}, I_{2}, s_{1}\right),\left(I_{1}, W_{2}, s_{0}\right), \\
& \left.\left(I_{1}, W_{2}, s_{1}\right),\left(W_{1}, I_{2}, s_{1}\right),\left(W_{1}, W_{2}, s_{0}\right),\left(W_{1}, W_{2}, s_{1}\right)\right\},
\end{aligned}
$$

and

$$
\begin{aligned}
P_{A_{1}} \vee P_{A_{2}} & \equiv\left(\left(v_{M_{2}}=I_{2}\right) \wedge\left(v_{B U F}=s_{0} \vee v_{B U F}=s_{2}\right)\right) \vee\left(v_{B U F}=s_{0} \vee v_{B U F}=s_{1}\right) \\
& \equiv\left(v_{M_{2}}=I_{2}\right) \vee\left(v_{B U F}=s_{0} \vee v_{B U F}=s_{1}\right) .
\end{aligned}
$$

$P_{A_{1}} \vee P_{A_{2}}$ also represents the state subset $A_{1} \cup A_{2}$.

- $\overline{A_{2}}$

By set complement,

$$
\overline{A_{2}}=\left\{\left(I_{1}, I_{2}, s_{2}\right),\left(I_{1}, W_{2}, s_{2}\right),\left(W_{1}, I_{2}, s_{2}\right),\left(W_{1}, W_{2}, s_{2}\right)\right\},
$$




\section{Master Thesis - R. Song-McMaster-Computing and Software}

and

$$
\begin{aligned}
\neg P_{A_{2}} & \equiv \neg\left(v_{B U F}=s_{0} \vee v_{B U F}=s_{1}\right) \\
& \equiv\left(v_{B U F}=s_{2}\right) .
\end{aligned}
$$

So, $\neg P_{A_{2}}$ represents the state subset $\overline{A_{2}}$.

\subsubsection{Symbolic Representation of Transitions}

Let $\mathbf{G}:=\left(Q, \Sigma, \delta, q_{0}, Q_{m}\right)$ be the system in Section 6.1.1 composed of $\mathbf{G}_{1}, \ldots, \mathbf{G}_{n}$. For a given event $\sigma \in \Sigma$, the transition function $\delta$ is essentially a subset of the set $Q \times Q$.

Definition 6.2. For the system $\mathbf{G}$ composed of components $\mathbf{G}_{1}, \ldots, \mathbf{G}_{n}$, for a given event $\sigma \in \Sigma$, a transition predicate $N_{\sigma}$ defined on $Q \times Q$ is a boolean function $N_{\sigma}: Q \times Q \rightarrow\{1,0\}$ according to

$$
\left(\forall q, q^{\prime} \in Q\right) N_{\sigma}\left(q, q^{\prime}\right):= \begin{cases}1, & \delta(q, \sigma) ! \& \delta(q, \sigma)=q^{\prime} \\ 0, & \text { otherwise. }\end{cases}
$$

Let $\sigma \in \Sigma$ be an event, then $N_{\sigma}$ identifies all the transitions for $\sigma$ in $\mathbf{G}$. Like the representation of a state subset, we can also use the a logic formula to represent the subset of $Q \times Q$ (thus a transition predicate), but now we need two different sets of state variables for each component DES. One variable will be for the source state of a transition, the other will be for the target state of a transition.

Definition 6.3. For the system $\mathbf{G}$ composed of components $\mathbf{G}_{1}, \ldots, \mathbf{G}_{n}$, the normal state variable $v_{i},(i \in\{1, \ldots, n\})$ and the prime state variable $v_{i}^{\prime}$ for each component DES $\mathbf{G}_{i}$ are two variables whose domains both are $Q_{i}$. The normal state variable 


\section{Master Thesis - R. Song - McMaster - Computing and Software}

vector $\mathbf{v}$ is $\left(v_{1}, \ldots, v_{n}\right)$ and the prime state variable vector $\mathbf{v}^{\prime}$ is $\left(v_{1}^{\prime}, \ldots, v_{n}^{\prime}\right)$, where $v_{1}, \ldots, v_{n}$ are normal state variables for each component in $\mathbf{G}$, and $v_{1}^{\prime}, \ldots, v_{n}^{\prime}$ are prime state variables for each component in $\mathbf{G}$.

For a given event $\sigma \in \Sigma$ in the system $\mathbf{G}$ composed of components $\mathbf{G}_{1}, \ldots, \mathbf{G}_{n}$, the transition predicate for $N_{\sigma}$ can be written as

$$
N_{\sigma}\left(\mathbf{v}, \mathbf{v}^{\prime}\right):=\bigwedge_{i \in\{1,2, \ldots, n\}}\left(\bigvee_{\begin{array}{c}
\left(q_{i}, q_{i}^{\prime}\right) \in Q_{i} \times Q_{i} \\
\delta_{i}\left(q_{i}, \sigma\right)=q_{i}^{\prime}
\end{array}}\left(v_{i}=q_{i} \wedge v_{i}^{\prime}=q_{i}^{\prime}\right)\right)
$$

Note the following fact. Let $i \in\{1, \ldots, n\}$ and $\sigma \in \Sigma$. If for all $q_{i} \in Q_{i}, \delta_{i}\left(q_{i}, \sigma\right) \not$., then

$$
\left(\bigvee_{\begin{array}{c}
\left(q_{i}, q_{i}^{\prime}\right) \in Q_{i} \times Q_{i}, \\
\delta_{i}\left(q_{i}, \sigma\right)=q_{i}^{\prime}
\end{array}}\left(v_{i}=q_{i} \wedge v_{i}^{\prime}=q_{i}^{\prime}\right)\right) \equiv 0
$$

Therefore, $N_{\sigma}\left(\mathbf{v}, \mathbf{v}^{\prime}\right) \equiv 0$. This is in line with that the system will block an event if one of the component DES blocks the event.

Equation 6.3 assumes that every component DES is defined on the event set $\Sigma$. However, when we design a system with multiple component DES, each component DES is defined on its own event set. The system event set is the union of all the component event set and the system is the synchronous product of all the component DES. Therefore, Equation 6.3 requires that each component DES must be selflooped with all the events that are not in its own event set, on each state. This not only creates a lot of clutter, but also makes the formula to represent the transitions much more complicated.

To solve this problem, for each $\sigma \in \Sigma$, we use the transition tuple $\left(\boldsymbol{v}_{\sigma}, \boldsymbol{v}_{\sigma}^{\prime}, N_{\sigma}\right)$ to 


\section{Master Thesis - R. Song-McMaster-Computing and Software}

represent the transitions on $\sigma$, where $\boldsymbol{v}_{\sigma}$ is a normal state variable set including all the normal state variables whose corresponding component DES event set contains $\sigma$, and $\boldsymbol{v}_{\sigma}^{\prime}$ is a prime state variable set including all the corresponding prime state variables of the normal state variables in $\boldsymbol{v}_{\sigma}$. For those state variables that are not in the set $\boldsymbol{v}_{\sigma}$, we know that $\sigma$ must be selflooped on each state of their corresponding component DES.

For the system $\mathbf{G}$ composed of components $\mathbf{G}_{1}, \ldots, \mathbf{G}_{n}$, let $\mathbf{G}_{1}^{\prime}:=\left(Q_{1}, \Sigma_{1}, \delta_{1}^{\prime}\right.$, $\left.q_{1_{0}}, Q_{1_{m}}\right), \ldots, \mathbf{G}_{n}^{\prime}:=\left(Q_{n}, \Sigma_{n}, \delta_{n}^{\prime}, q_{n_{0}}, Q_{n_{m}}\right)$ be the component DES such that $\mathbf{G}_{1}=$ $\operatorname{selfloop}\left(\mathbf{G}_{1}^{\prime}, \Sigma-\Sigma_{1}\right), \ldots, \mathbf{G}_{n}=\operatorname{selfloop}\left(\mathbf{G}_{n}^{\prime}, \Sigma-\Sigma_{n}\right)$. So, we have $\mathbf{G}=\mathbf{G}_{1}^{\prime}\|\cdots\|$ $\mathbf{G}_{n}^{\prime}$ and $\Sigma=\Sigma_{1} \cup \cdots \cup \Sigma_{n}$.

For a given event $\sigma \in \Sigma$ in the system $\mathbf{G}$ with components $\mathbf{G}_{1}^{\prime}, \ldots, \mathbf{G}_{n}^{\prime}$, the tuple $\left(\boldsymbol{v}_{\sigma}, \boldsymbol{v}_{\sigma}^{\prime}, N_{\sigma}\right)$ can be written by ${ }^{2}$

$$
\begin{gathered}
\boldsymbol{v}_{\sigma}:=\left\{v_{i} \in \mathbf{v} \mid \sigma \in \Sigma_{i}\right\}, \quad \boldsymbol{v}_{\sigma}^{\prime}:=\left\{v_{i}^{\prime} \in \mathbf{v}^{\prime} \mid \sigma \in \Sigma_{i}\right\} \\
N_{\sigma}\left(\mathbf{v}, \mathbf{v}^{\prime}\right):=\bigwedge_{\left\{i \in\{1,2, \ldots, n\} \mid \sigma \in \Sigma_{i}\right\}}\left(\bigvee_{\begin{array}{c}
\left(q_{i}, q^{\prime}\right) \in Q_{i} \times Q_{i} \\
\delta_{i}^{\prime}\left(q_{i}, \sigma\right)=q_{i}^{\prime}
\end{array}}^{\bigvee}\left(v_{i}=q_{i} \wedge v_{i}^{\prime}=q_{i}^{\prime}\right)\right) .
\end{gathered}
$$

Although the tuple $\left(\boldsymbol{v}_{\sigma}, \boldsymbol{v}_{\sigma}^{\prime}, N_{\sigma}\right)$ are created from unselflooped components, note that the tuple actually expresses the selfloop information, which means that by creating the tuple we automatically selfloop $\sigma$ for each component whose own event set does not contain $\sigma$. Note also that Equation 6.4 and 6.5 can be used for creating the transition tuples for selflooped components as well.

We now create the transition tuples for all the events in the small factory example shown in Figure 6.1 to demonstrate how to use Equation 6.4 and 6.5.

\footnotetext{
${ }^{2}$ Here we are abusing the notation a bit, as $\mathbf{v}$ and $\mathbf{v}^{\prime}$ should not be treated as state variable sets.
} 
Master Thesis - R. Song - McMaster - Computing and Software

- $\left(\boldsymbol{v}_{\alpha_{1}}, \boldsymbol{v}_{\alpha_{1}}^{\prime}, N_{\alpha_{1}}\right)$

$\boldsymbol{v}_{\alpha_{1}}:=\left\{v_{M_{1}}, v_{B U F}\right\}, \boldsymbol{v}_{\alpha_{1}}^{\prime}:=\left\{v_{M_{1}}^{\prime}, v_{B U F}^{\prime}\right\}$

$N_{\alpha_{1}}:=\left(v_{M_{1}}=I_{1} \wedge v_{M_{1}}^{\prime}=W_{1}\right) \wedge$

$\left(\left(v_{B U F}=s_{0} \wedge v_{B U F}^{\prime}=s_{0}\right) \vee\left(v_{B U F}=s_{1} \wedge v_{B U F}^{\prime}=s_{1}\right)\right)$

- $\left(\boldsymbol{v}_{\beta_{1}}, \boldsymbol{v}_{\beta_{1}}^{\prime}, N_{\beta_{1}}\right)$

$\boldsymbol{v}_{\beta_{1}}:=\left\{v_{M_{1}}, v_{B U F}\right\}, \boldsymbol{v}_{\beta_{1}}^{\prime}:=\left\{v_{M_{1}}^{\prime}, v_{B U F}^{\prime}\right\}$

$N_{\beta_{1}}:=\left(v_{M_{1}}=W_{1} \wedge v_{M_{1}}^{\prime}=I_{1}\right) \wedge$

$\left(\left(v_{B U F}=s_{0} \wedge v_{B U F}^{\prime}=s_{1}\right) \vee\left(v_{B U F}=s_{1} \wedge v_{B U F}^{\prime}=s_{2}\right)\right)$

- $\left(\boldsymbol{v}_{\alpha_{2}}, \boldsymbol{v}_{\alpha_{2}}^{\prime}, N_{\alpha_{2}}\right)$

$\boldsymbol{v}_{\alpha_{2}}:=\left\{v_{M_{2}}, v_{B U F}\right\}, \boldsymbol{v}_{\alpha_{2}}^{\prime}:=\left\{v_{M_{2}}^{\prime}, v_{B U F}^{\prime}\right\}$

$N_{\alpha_{2}}:=\left(v_{M_{2}}=I_{2} \wedge v_{M_{2}}^{\prime}=W_{2}\right) \wedge$

$\left(\left(v_{B U F}=s_{1} \wedge v_{B U F}^{\prime}=s_{0}\right) \vee\left(v_{B U F}=s_{2} \wedge v_{B U F}^{\prime}=s_{1}\right)\right)$

- $\left(\boldsymbol{v}_{\beta_{2}}, \boldsymbol{v}_{\beta_{2}}^{\prime}, N_{\beta_{2}}\right)$

$\boldsymbol{v}_{\beta_{2}}:=\left\{v_{M_{2}}\right\}, \boldsymbol{v}_{\beta_{2}}^{\prime}:=\left\{v_{M_{2}}^{\prime}\right\}$

$N_{\beta_{2}}:=\left(v_{M_{2}}=W_{2} \wedge v_{M_{2}}^{\prime}=I_{2}\right)$

\subsection{Symbolic Computation of Predicate Transform-}

\section{ers}

With the logic formula representation for state subsets and transitions, we now talk about how to compute the four predicate transformers $T R, R, C R$ and $\mathcal{C R}$. Because $C R$ is a special case of $\mathcal{C R}$, we only discuss $\mathcal{C R}$ in this section. 


\section{Master Thesis - R. Song-McMaster-Computing and Software}

In this section, we will use the system $\mathbf{G}$ composed of components $\mathbf{G}_{1}^{\prime}, \ldots, \mathbf{G}_{n}^{\prime}$ defined in Section 6.1.2.

\subsubsection{Computation of Transition and Inverse Transition}

For the system $\mathbf{G}:=\left(Q, \Sigma, \delta, q_{0}, Q_{m}\right)$ composed of components $\mathbf{G}_{1}^{\prime}, \ldots, \mathbf{G}_{n}^{\prime}$, to compute the predicate transformer $R$, we must know how to compute $\delta(q, \sigma)$, where $q \in Q$ and $\sigma \in \Sigma$. A state predicate $P \in \operatorname{Pred}(Q)$ can represent a state subset $Q_{P} \subseteq Q$. To compute all the states that can be reached by a $\sigma$ transition from any state in $Q_{P}$, we can compute $\cup_{q \in Q_{P}}\{\delta(q, \sigma)\}$. However, this method is rather time consuming, especially when the state space of a system is large. We hope we

can instead directly compute the function $\widehat{\delta}: \operatorname{Pred}(Q) \times \Sigma \rightarrow \operatorname{Pred}(Q)$, defined as follows:

$$
(\forall P \in \operatorname{Pred}(Q))(\forall \sigma \in \Sigma) \widehat{\delta}(P, \sigma):=\operatorname{pr}\left(\left\{q^{\prime} \in Q \mid(\exists q \models P) \delta(q, \sigma)=q^{\prime}\right\}\right) .
$$

For the system $\mathbf{G}$, to compute the predicate transformers $T R$ and $\mathcal{C R}$, we define a function $\delta^{-1}: Q \times \Sigma \rightarrow P w r(Q)$ according to

$$
(\forall q \in Q)(\sigma \in \Sigma) \delta^{-1}(q, \sigma):=\left\{q^{\prime} \in Q \mid \delta\left(q^{\prime}, \sigma\right)=q\right\}
$$

Let $P \in \operatorname{Pred}(Q)$ be a predicate. To compute all the states that have a $\sigma$ transition to any state satisfying $P$, we can compute $\cup_{q \models P} \delta^{-1}(q, \sigma)$. Similarly, we hope we can instead directly compute the function $\widehat{\delta^{-1}}: \operatorname{Pred}(Q) \times \Sigma \rightarrow \operatorname{Pred}(Q)$, defined as follows:

$$
(\forall P \in \operatorname{Pred}(Q))(\forall \sigma \in \Sigma) \widehat{\delta^{-1}}(P, \sigma):=\operatorname{pr}(\{q \in Q \mid \delta(q, \sigma) \models P\}) .
$$

As in [31], we will use a method similar to the relational product, developed by the researchers in the model checking area [11], to compute $\widehat{\delta}(P, \sigma)$ and $\widehat{\delta^{-1}}(P, \sigma)$. 
Definition 6.4. For the system $\mathbf{G}$ composed of components $\mathbf{G}_{1}^{\prime}, \ldots, \mathbf{G}_{n}^{\prime}$, for all $\sigma \in \Sigma$, let $\left(\boldsymbol{v}_{\sigma}, \boldsymbol{v}_{\sigma}^{\prime}, N_{\sigma}\right)$ be the transition tuple for $\sigma$ in $\mathbf{G}$.

For all $i \in\{1, \ldots, n\}$, if $v_{i} \in \boldsymbol{v}_{\sigma}, v_{i}^{\prime} \in \boldsymbol{v}_{\sigma}^{\prime}$, then define $\exists v_{i} N_{\sigma}$ and $\exists v_{i}^{\prime} N_{\sigma}$ as

$$
\begin{aligned}
& \exists v_{i} N_{\sigma}:=\bigvee_{q_{i} \in Q_{i}} N_{\sigma}\left[q_{i} / v_{i}\right], \\
& \exists v_{i}^{\prime} N_{\sigma}:=\bigvee_{q_{i} \in Q_{i}} N_{\sigma}\left[q_{i} / v_{i}^{\prime}\right],
\end{aligned}
$$

where $N_{\sigma}\left[q_{i} / v_{i}\right]$ is the resulting predicate by assigning $q_{i}$ to $v_{i} ; N_{\sigma}\left[q_{i} / v_{i}^{\prime}\right]$ is the resulting predicate by assigning $q_{i}$ to $v_{i}^{\prime}$.

The above definition of $\exists v_{i} N_{\sigma}$ and $\exists v_{i}^{\prime} N_{\sigma}$ is based on the existential quantifier elimination method for finite domain [2]. That is, $\exists v_{i}$ and $\exists v_{i}^{\prime}$ eliminate the variable $v_{i}$ and $v_{i}^{\prime}$ from $N_{\sigma}$ respectively. The results of $\exists v_{i} N_{\sigma}$ and $\exists v_{i}^{\prime} N_{\sigma}$ are still propositional logic formulas, which can be represented by BDD.

Let $\sigma \in \Sigma$ and $\left(\boldsymbol{v}_{\sigma}, \boldsymbol{v}_{\sigma}^{\prime}, N_{\sigma}\right)$ be the transition tuple for $\sigma$ in G. Assume $\boldsymbol{v}_{\sigma}=$ $\left\{\widehat{v}_{1}, \widehat{v}_{2}, \ldots \widehat{v}_{m}\right\}$ and $\boldsymbol{v}_{\sigma}^{\prime}=\left\{\widehat{v}_{1}^{\prime}, \widehat{v}_{2}^{\prime}, \ldots \widehat{v}_{m}^{\prime}\right\}$, where $m \in\{1, \ldots, n\}$. For convenience, we define the shorthand $\exists \boldsymbol{v}_{\sigma} N_{\sigma}$ and $\exists \boldsymbol{v}_{\sigma}^{\prime} N_{\sigma}$ as follows:

$$
\begin{aligned}
& \exists \boldsymbol{v}_{\sigma} N_{\sigma}:=\exists \widehat{v}_{1}\left(\exists \widehat{v}_{2} \cdots\left(\exists \widehat{v}_{m} N_{\sigma}\right) \cdots\right), \\
& \exists \boldsymbol{v}_{\sigma}^{\prime} N_{\sigma}:=\exists \widehat{v}_{1}^{\prime}\left(\exists \widehat{v}_{2}^{\prime} \cdots\left(\exists \widehat{v}_{m}^{\prime} N_{\sigma}\right) \cdots\right) .
\end{aligned}
$$

The resulting logic formula for $\exists \boldsymbol{v}_{\sigma} N_{\sigma}$ contains only the prime variables in $\boldsymbol{v}_{\sigma}^{\prime}$. If we replace all the prime variables as normal variables, denoted it as $\exists \boldsymbol{v}_{\sigma} N_{\sigma}\left[\boldsymbol{v}_{\sigma}^{\prime} \rightarrow \boldsymbol{v}_{\sigma}\right]$, then the resulting predicate $\exists \boldsymbol{v}_{\sigma} N_{\sigma}\left[\boldsymbol{v}_{\sigma}^{\prime} \rightarrow \boldsymbol{v}_{\sigma}\right]$ represents the target state set of all the $\sigma$ transitions in $\mathbf{G}$ (i.e. Each state in the target state set has a $\sigma$ transition entering it). We need to do the variable replacement because we use the normal state variables to express the logic formula of a state subset predicate. 


\section{Master Thesis - R. Song - McMaster - Computing and Software}

As the resulting logic formula for $\exists \boldsymbol{v}_{\sigma}^{\prime} N_{\sigma}$ contains only the normal variables in $\boldsymbol{v}_{\sigma}$, we do not need to do the variable replacement. $\exists \boldsymbol{v}_{\sigma}^{\prime} N_{\sigma}$ represents the source state set of all the $\sigma$ transitions in $\mathbf{G}$ (i.e. Each state in the target state set has a $\sigma$ transition leaving it).

We now give an example to demonstrate $\exists \boldsymbol{v}_{\sigma} N_{\sigma}$ and $\exists \boldsymbol{v}_{\sigma}^{\prime} N_{\sigma}$. Figure 6.2 shows a system with only one component $\mathbf{G}_{E}$. Let $v_{E}, v_{E}^{\prime}$ be the normal and prime state variable for the component $\mathbf{G}_{E}$.

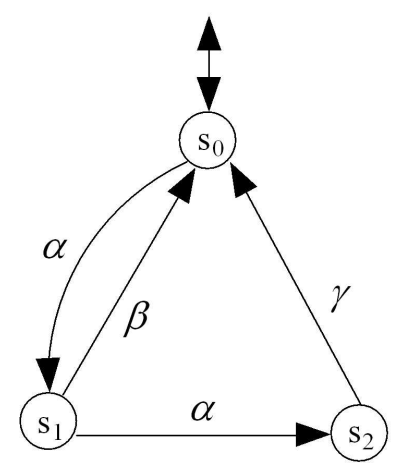

Figure 6.2: Example DES $\mathbf{G}_{E}$

- $\exists \boldsymbol{v}_{\alpha} N_{\alpha}, \exists \boldsymbol{v}_{\alpha}^{\prime} N_{\alpha}$

For the event $\alpha$, we have

$$
\begin{aligned}
& \boldsymbol{v}_{\alpha}:=\left\{v_{E}\right\}, \boldsymbol{v}_{\alpha}^{\prime}:=\left\{v_{E}^{\prime}\right\} \\
& N_{\alpha}:=\left(v_{E}=s_{0} \wedge v_{E}^{\prime}=s_{1}\right) \vee\left(v_{E}=s_{1} \wedge v_{E}^{\prime}=s_{2}\right) . \\
& \exists \boldsymbol{v}_{\alpha} N_{\alpha}:=\exists v_{E} N_{\alpha} \\
& \equiv\left(s_{0}=s_{0} \wedge v_{E}^{\prime}=s_{1}\right) \vee\left(s_{0}=s_{1} \wedge v_{E}^{\prime}=s_{2}\right) \vee \\
& \quad\left(s_{1}=s_{0} \wedge v_{E}^{\prime}=s_{1}\right) \vee\left(s_{1}=s_{1} \wedge v_{E}^{\prime}=s_{2}\right) \vee \\
& \quad\left(s_{2}=s_{0} \wedge v_{E}^{\prime}=s_{1}\right) \vee\left(s_{2}=s_{1} \wedge v_{E}^{\prime}=s_{2}\right) \\
& \equiv v_{E}^{\prime}=s_{1} \vee v_{E}^{\prime}=s_{2}
\end{aligned}
$$


Master Thesis - R. Song - McMaster - Computing and Software

$\exists \boldsymbol{v}_{\alpha} N_{\alpha}\left[\boldsymbol{v}_{\alpha}^{\prime} \rightarrow \boldsymbol{v}_{\alpha}\right]:=v_{E}=s_{1} \vee v_{E}=s_{2}$

The logic formula $v_{E}=s_{1} \vee v_{E}=s_{2}$ represents the state subset $\left\{s_{1}, s_{2}\right\}$, which exactly includes the target states of all the $\alpha$ transitions in $\mathbf{G}_{E}$.

$$
\begin{aligned}
\exists \boldsymbol{v}_{\alpha}^{\prime} N_{\alpha}:= & \exists v_{E}^{\prime} N_{\alpha} \\
\equiv & \left(v_{E}=s_{0} \wedge s_{0}=s_{1}\right) \vee\left(v_{E}=s_{1} \wedge s_{0}=s_{2}\right) \vee \\
& \left(v_{E}=s_{0} \wedge s_{1}=s_{1}\right) \vee\left(v_{E}=s_{1} \wedge s_{1}=s_{2}\right) \vee \\
& \left(v_{E}=s_{0} \wedge s_{2}=s_{1}\right) \vee\left(v_{E}=s_{1} \wedge s_{2}=s_{2}\right) \\
\equiv & v_{E}=s_{0} \vee v_{E}=s_{1}
\end{aligned}
$$

The logic formula $v_{E}=s_{0} \vee v_{E}=s_{1}$ represents the state subset $\left\{s_{0}, s_{1}\right\}$, which exactly includes the source states of all the $\alpha$ transitions in $\mathbf{G}_{E}$.

- $\exists \boldsymbol{v}_{\beta} N_{\beta}, \exists \boldsymbol{v}_{\beta}^{\prime} N_{\beta}$

For the event $\beta$, we have

$$
\begin{aligned}
& \boldsymbol{v}_{\beta}:=\left\{v_{E}\right\}, \boldsymbol{v}_{\beta}^{\prime}:=\left\{v_{E}^{\prime}\right\} \\
& N_{\beta}:=\left(v_{E}=s_{1} \wedge v_{E}^{\prime}=s_{0}\right) . \\
& \exists \boldsymbol{v}_{\beta} N_{\beta}:=\exists v_{E} N_{\beta} \\
& \quad \equiv\left(s_{0}=s_{1} \wedge v_{E}^{\prime}=s_{0}\right) \vee\left(s_{1}=s_{1} \wedge v_{E}^{\prime}=s_{0}\right) \vee\left(s_{2}=s_{1} \wedge v_{E}^{\prime}=s_{0}\right) \\
& \quad \equiv v_{E}^{\prime}=s_{0} \\
& \exists \boldsymbol{v}_{\beta} N_{\beta}\left[\boldsymbol{v}_{\beta}^{\prime} \rightarrow \boldsymbol{v}_{\beta}\right]:=v_{E}=s_{0} . \\
& \exists \boldsymbol{v}_{\beta}^{\prime} N_{\beta}:=\exists v_{E}^{\prime} N_{\beta} \\
& \quad \equiv\left(v_{E}=s_{1} \wedge s_{0}=s_{0}\right) \vee\left(v_{E}=s_{1} \wedge s_{1}=s_{0}\right) \vee\left(v_{E}=s_{1} \wedge s_{2}=s_{0}\right) \\
& \quad \equiv v_{E}=s_{1}
\end{aligned}
$$




\section{Master Thesis - R. Song-McMaster - Computing and Software}

We now give an example to compute the state set consisting of target states of all the $\beta_{1}$ transitions in the small factory example shown in Figure 6.1.

$$
\begin{gathered}
\boldsymbol{v}_{\beta_{1}}:=\left\{v_{M_{1}}, v_{B U F}\right\}, \boldsymbol{v}_{\beta_{1}}^{\prime}:=\left\{v_{M_{1}}^{\prime}, v_{B U F}^{\prime}\right\} \\
N_{\beta_{1}}:=\left(v_{M_{1}}=W_{1} \wedge v_{M_{1}}^{\prime}=I_{1}\right) \wedge \\
\left(\left(v_{B U F}=s_{0} \wedge v_{B U F}^{\prime}=s_{1}\right) \vee\left(v_{B U F}=s_{1} \wedge v_{B U F}^{\prime}=s_{2}\right)\right) \\
\exists \boldsymbol{v}_{\beta_{1}} N_{\beta_{1}}:=\exists v_{M_{1}}\left(\exists v_{B U F} N_{\beta_{1}}\right) \\
\equiv \exists v_{M_{1}}\left(\left(v_{M_{1}}=W_{1} \wedge v_{M_{1}}^{\prime}=I_{1}\right) \wedge\right. \\
\quad\left(\left(s_{0}=s_{0} \wedge v_{B U F}^{\prime}=s_{1}\right) \vee\left(s_{0}=s_{1} \wedge v_{B U F}^{\prime}=s_{2}\right) \vee\right. \\
\quad\left(s_{1}=s_{0} \wedge v_{B U F}^{\prime}=s_{1}\right) \vee\left(s_{1}=s_{1} \wedge v_{B U F}^{\prime}=s_{2}\right) \vee \\
\left.\left.\quad\left(s_{2}=s_{0} \wedge v_{B U F}^{\prime}=s_{1}\right) \vee\left(s_{2}=s_{1} \wedge v_{B U F}^{\prime}=s_{2}\right)\right)\right) \\
\equiv \exists v_{M_{1}}\left(\left(v_{M_{1}}=W_{1} \wedge v_{M_{1}}^{\prime}=I_{1}\right) \wedge\left(v_{B U F}^{\prime}=s_{1} \vee v_{B U F}^{\prime}=s_{2}\right)\right) \\
\equiv\left(\left(I_{1}=W_{1} \wedge v_{M_{1}}^{\prime}=I_{1}\right) \vee\left(W_{1}=W_{1} \wedge v_{M_{1}}^{\prime}=I_{1}\right)\right) \wedge \\
\left(v_{B U F}^{\prime}=s_{1} \vee v_{B U F}^{\prime}=s_{2}\right) \\
\equiv\left(v_{M_{1}}^{\prime}=I_{1}\right) \wedge\left(v_{B U F}^{\prime}=s_{1} \vee v_{B U F}^{\prime}=s_{2}\right) \\
\exists \boldsymbol{v}_{\beta_{1} N_{\beta_{1}}}\left[\boldsymbol{v}_{\beta_{1}}^{\prime} \rightarrow \boldsymbol{v}_{\beta_{1}}\right]:=\left(v_{M_{1}}=I_{1}\right) \wedge\left(v_{B U F}=s_{1} \vee v_{B U F}=s_{2}\right) .
\end{gathered}
$$

For the system $\mathbf{G}$ composed of components $\mathbf{G}_{1}^{\prime}, \ldots, \mathbf{G}_{n}^{\prime}$, let $\sigma \in \Sigma$. From the above definitions and examples, it is clear that $\exists \boldsymbol{v}_{\sigma} N_{\sigma}\left[\boldsymbol{v}_{\sigma}^{\prime} \rightarrow \boldsymbol{v}_{\sigma}\right]$ exactly computes the predicate representing the state subset $\left\{q^{\prime} \in Q \mid(\exists q \in Q) \delta(q, \sigma)=q^{\prime}\right\}$ and $\exists \boldsymbol{v}_{\sigma}^{\prime} N_{\sigma}$ exactly computes the predicate representing the state subset $\{q \in Q \mid \delta(q, \sigma) !\}$.

With the help of existential quantifier elimination method, we now can compute $\widehat{\delta}$ and $\widehat{\delta^{-1}}$ symbolically. For system $\mathbf{G}$, let $P \in \operatorname{Pred}(Q)$ and $\sigma \in \Sigma . \widehat{\delta}(P, \sigma)$ requires computing the predicate representing the state subset $\left\{q^{\prime} \in Q \mid(\exists q \models P) \delta(q, \sigma)=\right.$ $\left.q^{\prime}\right\}$, while $\widehat{\delta^{-1}}(P, \sigma)$ requires computing the predicate representing the state subset 
Master Thesis - R. Song - McMaster - Computing and Software

$\{q \in Q \mid \delta(q, \sigma) \models P\}$. So, $\widehat{\delta}(P, \sigma)$ and $\widehat{\delta^{-1}}(P, \sigma)$ can be computed as follows:

$$
\begin{gathered}
\widehat{\delta}(P, \sigma):=\left(\exists \boldsymbol{v}_{\sigma}\left(N_{\sigma} \wedge P\right)\right)\left[\boldsymbol{v}_{\sigma}^{\prime} \rightarrow \boldsymbol{v}_{\sigma}\right] \\
\widehat{\delta^{-1}}(P, \sigma):=\exists \boldsymbol{v}_{\sigma}^{\prime}\left(N_{\sigma} \wedge\left(P\left[\boldsymbol{v}_{\sigma} \rightarrow \boldsymbol{v}_{\sigma}^{\prime}\right]\right)\right)
\end{gathered}
$$

In Equation 6.6, by first computing $N_{\sigma} \wedge P$, we restrict the $\sigma$ transitions to those whose source states satisfy $P$.

In Equation 6.7, note that $P$ is first transformed to the prime variable format, because we want to restrict the $\sigma$ transitions to those whose target states satisfy $P$.

For convenience, we omit the hat on $\widehat{\delta}(P, \sigma)$ and $\widehat{\delta^{-1}}(P, \sigma)$, because from the parameters we can clearly tell what the functions are.

We now give examples again for the small factory example shown in Figure 6.1 to demonstrate the computing of $\delta(P, \sigma)$ and $\delta^{-1}(P, \sigma)$.

Let $P$ be a state predicate defined on the state set of the small factory example shown in Figure 6.1, and let $P:=\left(v_{M_{1}}=W_{1} \wedge v_{B U F}=s_{1}\right)$. We now demonstrate how to compute $\delta\left(P, \alpha_{2}\right)$ and $\delta^{-1}\left(P, \alpha_{1}\right)$.

- $\delta\left(P, \alpha_{2}\right)$

$$
\begin{aligned}
& N_{\alpha_{2}} \wedge P:=\left(v_{M_{2}}=I_{2} \wedge v_{M_{2}}^{\prime}=W_{2}\right) \wedge \\
&\left(\left(v_{B U F}=s_{1} \wedge v_{B U F}^{\prime}=s_{0}\right) \vee\left(v_{B U F}=s_{2} \wedge v_{B U F}^{\prime}=s_{1}\right)\right) \wedge \\
&\left(v_{M_{1}}=W_{1} \wedge v_{B U F}=s_{1}\right) . \\
& \equiv\left(v_{M_{2}}=I_{2} \wedge v_{M_{2}}^{\prime}=W_{2}\right) \wedge \\
&\left(v_{B U F}=s_{1} \wedge v_{B U F}^{\prime}=s_{0} \wedge v_{M_{1}}=W_{1}\right) . \\
& \exists \boldsymbol{v}_{\alpha_{2}}\left(N_{\alpha_{2}} \wedge P\right):=\exists v_{M_{2}}\left(\exists v _ { B U F } \left(\left(v_{M_{2}}=I_{2} \wedge v_{M_{2}}^{\prime}=W_{2}\right) \wedge\right.\right. \\
&\left.\left.\quad\left(v_{B U F}=s_{1} \wedge v_{B U F}^{\prime}=s_{0} \wedge v_{M_{1}}=W_{1}\right)\right)\right) \\
& \equiv \exists v_{M_{2}}\left(\left(v_{M_{2}}=I_{2} \wedge v_{M_{2}}^{\prime}=W_{2}\right) \wedge\left(v_{B U F}^{\prime}=s_{0} \wedge v_{M_{1}}=W_{1}\right)\right)
\end{aligned}
$$


Master Thesis - R. Song - McMaster - Computing and Software

$$
\begin{gathered}
\equiv\left(v_{M_{2}}^{\prime}=W_{2}\right) \wedge\left(v_{B U F}^{\prime}=s_{0}\right) \wedge\left(v_{M_{1}}=W_{1}\right) \\
\delta\left(P, \alpha_{2}\right):=\left(\exists \boldsymbol{v}_{\alpha_{2}}\left(N_{\alpha_{2}} \wedge P\right)\right)\left[\boldsymbol{v}_{\alpha_{2}}^{\prime} \rightarrow \boldsymbol{v}_{\alpha_{2}}\right] \\
\equiv\left(\left(v_{M_{2}}^{\prime}=W_{2}\right) \wedge\left(v_{B U F}^{\prime}=s_{0}\right) \wedge\left(v_{M_{1}}=W_{1}\right)\right)\left[\boldsymbol{v}_{\alpha_{2}}^{\prime} \rightarrow \boldsymbol{v}_{\alpha_{2}}\right] \\
\equiv\left(v_{M_{2}}=W_{2}\right) \wedge\left(v_{B U F}=s_{0}\right) \wedge\left(v_{M_{1}}=W_{1}\right)
\end{gathered}
$$

- $\delta^{-1}\left(P, \alpha_{1}\right)$

$$
\begin{aligned}
& N_{\alpha_{1}} \wedge\left(P\left[\boldsymbol{v}_{\alpha_{1}} \rightarrow \boldsymbol{v}_{\alpha_{1}}^{\prime}\right]\right):=\left(v_{M_{1}}=I_{1} \wedge v_{M_{1}}^{\prime}=W_{1}\right) \wedge \\
&\left(\left(v_{B U F}=s_{0} \wedge v_{B U F}^{\prime}=s_{0}\right) \vee\left(v_{B U F}=s_{1} \wedge v_{B U F}^{\prime}=s_{1}\right)\right) \wedge \\
&\left(v_{M_{1}}^{\prime}=W_{1} \wedge v_{B U F}^{\prime}=s_{1}\right) \\
& \equiv\left(v_{M_{1}}=I_{1}\right) \wedge\left(v_{M_{1}}^{\prime}=W_{1}\right) \wedge\left(v_{B U F}=s_{1}\right) \wedge\left(v_{B U F}^{\prime}=s_{1}\right) \\
& \delta^{-1}\left(P, \alpha_{1}\right):=\exists \boldsymbol{v}_{\alpha_{1}}^{\prime}\left(N_{\alpha_{1}} \wedge\right.\left.\left(P\left[\boldsymbol{v}_{\alpha_{1}} \rightarrow \boldsymbol{v}_{\alpha_{1}}^{\prime}\right]\right)\right) \\
& \equiv \exists v_{M_{1}}^{\prime}\left(\exists v_{B U F}^{\prime}\left(\left(v_{M_{1}}=I_{1}\right) \wedge\left(v_{M_{1}}^{\prime}=W_{1}\right) \wedge\left(v_{B U F}=s_{1}\right) \wedge\left(v_{B U F}^{\prime}=s_{1}\right)\right)\right) \\
& \equiv\left(v_{M_{1}}=I_{1}\right) \wedge\left(v_{B U F}=s_{1}\right)
\end{aligned}
$$

\subsubsection{Computation of $R$}

For the system $\mathbf{G}$ composed of components $\mathbf{G}_{1}^{\prime}, \ldots, \mathbf{G}_{n}^{\prime}$, let $P \in \operatorname{Pred}(Q)$. From the definition of $R(\mathbf{G},$.$) , we can write a straightforward algorithm for R(\mathbf{G}, P)$ as shown in Algorithm 6.1.

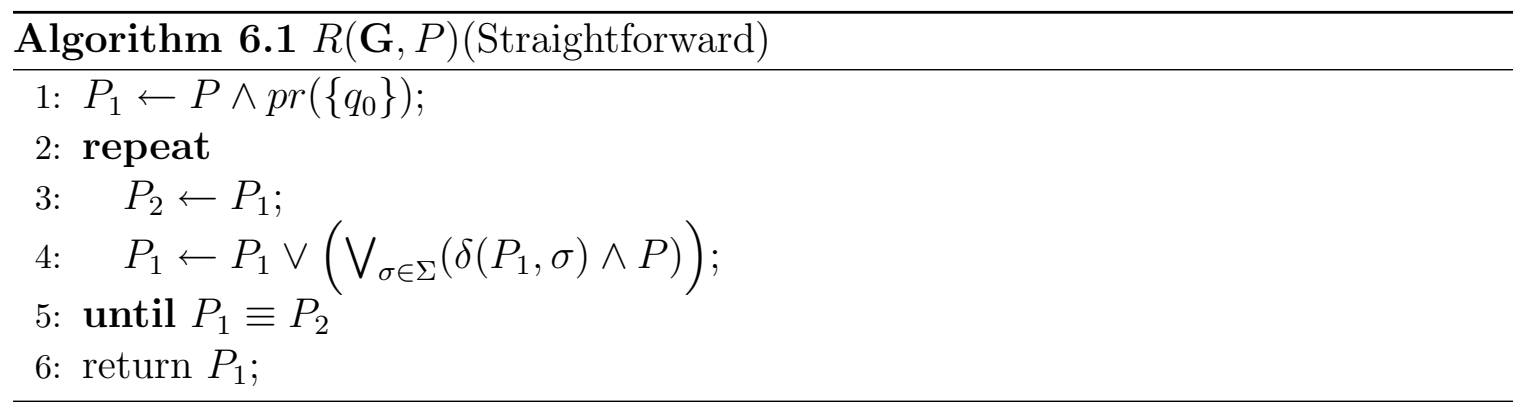




\section{Master Thesis - R. Song - McMaster - Computing and Software}

In Line 4 of the algorithm, we never remove states from $P_{1}$. As the number of states in $\mathbf{G}$ is finite, after finite number of steps we will have $P 1 \equiv P 2$; thus the algorithm will terminate.

Algorithm 6.1 works like a breadth first search. As found by Ma in [31], a breath first search method could generate a very complicated intermediate result, even though the final result is quite simple because of the tautology in Equation 6.2. An algorithm (shown below) working like a depth first search can greatly reduce the intermediate result. The following example is almost directly taken from [31] for the sake of content completeness, the difference is that the example there is used for explaining the algorithm of a predicate transformer similar to our $T R$ predicate transformer.
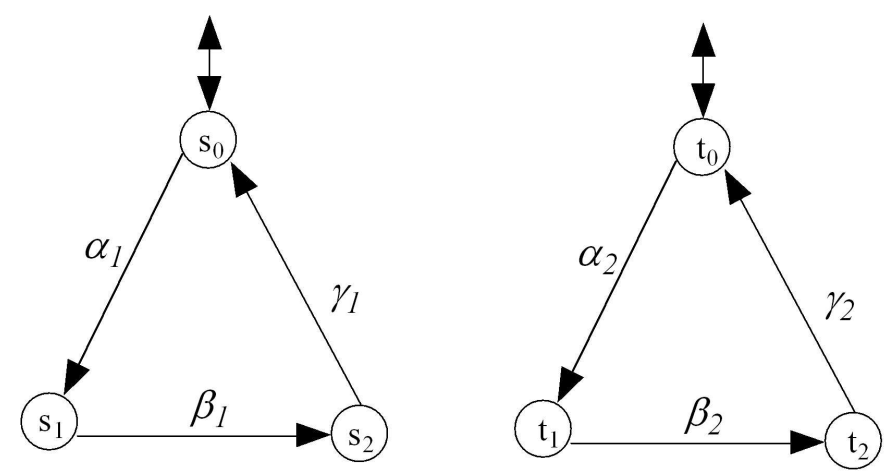

Figure 6.3: Example system M

Figure 6.3 shows a system $\mathbf{M}$ composed of two component DES $\mathbf{M}_{1}$ and $\mathbf{M}_{2}$. For simplicity, let $P:=$ true be the input predicate of $R(\mathbf{M},$.$) , i.e. we now compute$ $R(\mathbf{M}$, true $)$. Let $v_{M_{1}}$ and $v_{M_{2}}$ be the state variables for $\mathbf{M}_{1}$ and $\mathbf{M}_{2}$ respectively.

Initially, $P_{1} \equiv\left(v_{M_{1}}=s_{0} \wedge v_{M_{2}}=t_{0}\right)$;

- First iteration

$$
P_{1}:=P_{1} \vee\left(v_{M_{1}}=s_{1} \wedge v_{M_{2}}=t_{0}\right) \vee\left(v_{M_{1}}=s_{0} \wedge v_{M_{2}}=t_{1}\right)
$$




$$
\begin{aligned}
& \text { Master Thesis }- \text { R. Song - McMaster - Computing and Software } \\
& \equiv\left(v_{M_{1}}=s_{1} \wedge v_{M_{2}}=t_{0}\right) \vee\left(v_{M_{1}}=s_{0} \wedge\left(v_{M_{2}}=t_{0} \vee v_{M_{2}}=t_{1}\right)\right)
\end{aligned}
$$

- Second iteration

$$
\begin{aligned}
P_{1} & :=P_{1} \vee\left(v_{M_{1}}=s_{1} \wedge v_{M_{2}}=t_{1}\right) \vee\left(v_{M_{1}}=s_{2} \wedge v_{M_{2}}=t_{0}\right) \vee\left(v_{M_{1}}=s_{0} \wedge v_{M_{2}}=t_{2}\right) \\
& \equiv\left(v_{M_{1}}=s_{0}\right) \vee\left(v_{M_{1}}=s_{1} \wedge\left(v_{M_{2}}=t_{0} \vee v_{M_{2}}=t_{1}\right)\right) \vee\left(v_{M_{1}}=s_{2} \wedge v_{M_{2}}=t_{0}\right)
\end{aligned}
$$

- Third iteration

$$
\begin{aligned}
P_{1} & :=P_{1} \vee\left(v_{M_{1}}=s_{2} \wedge v_{M_{2}}=t_{1}\right) \vee\left(v_{M_{1}}=s_{1} \wedge v_{M_{2}}=t_{2}\right) \\
& \equiv\left(v_{M_{1}}=s_{0}\right) \vee\left(v_{M_{1}}=s_{1}\right) \vee\left(v_{M_{1}}=s_{2} \wedge\left(v_{M_{2}}=t_{0} \vee v_{M_{2}}=t_{1}\right)\right) \\
& \equiv\left(v_{M_{1}}=s_{0}\right) \vee\left(v_{M_{1}}=s_{1}\right) \vee\left(v_{M_{1}}=s_{2} \wedge\left(v_{M_{2}}=t_{0} \vee v_{M_{2}}=t_{1}\right)\right)
\end{aligned}
$$

- Fourth iteration

$$
\begin{aligned}
P_{1} & :=P_{1} \vee\left(v_{M_{1}}=s_{2} \wedge v_{M_{2}}=t_{2}\right) \\
& \equiv 1
\end{aligned}
$$

Although the result can be represented as 1 , one can see that the intermediate logic formula is much more complicated than the result during the computation.

To reduce the intermediate result, we can take a look at equation 6.2. Only when a state variable can be assigned to all the values in its domain, the part related to the state variable can be reduced to 1 . In the above example, for instance, if we first compute the transitions $\alpha_{1}, \beta_{1}, \gamma_{1}$, then $v_{M_{1}}$ can be assigned to all the states in its domain $\left\{s_{0}, s_{1}, s_{2}\right\}$, so this part can be reduced to 1 .

For the system $\mathbf{G}$ composed of components $\mathbf{G}_{1}^{\prime}, \ldots, \mathbf{G}_{n}^{\prime}$, Algorithm 6.2 formally states the above idea. The algorithm chooses the events component by component.

Note that in Line 7 , the event set is $\Sigma_{i}$, but the transition function is $\delta$. This algorithm makes great use of the tautology of Equation 6.2 to reduce the intermediate result. The difference between this algorithm and the previous one is the order of 


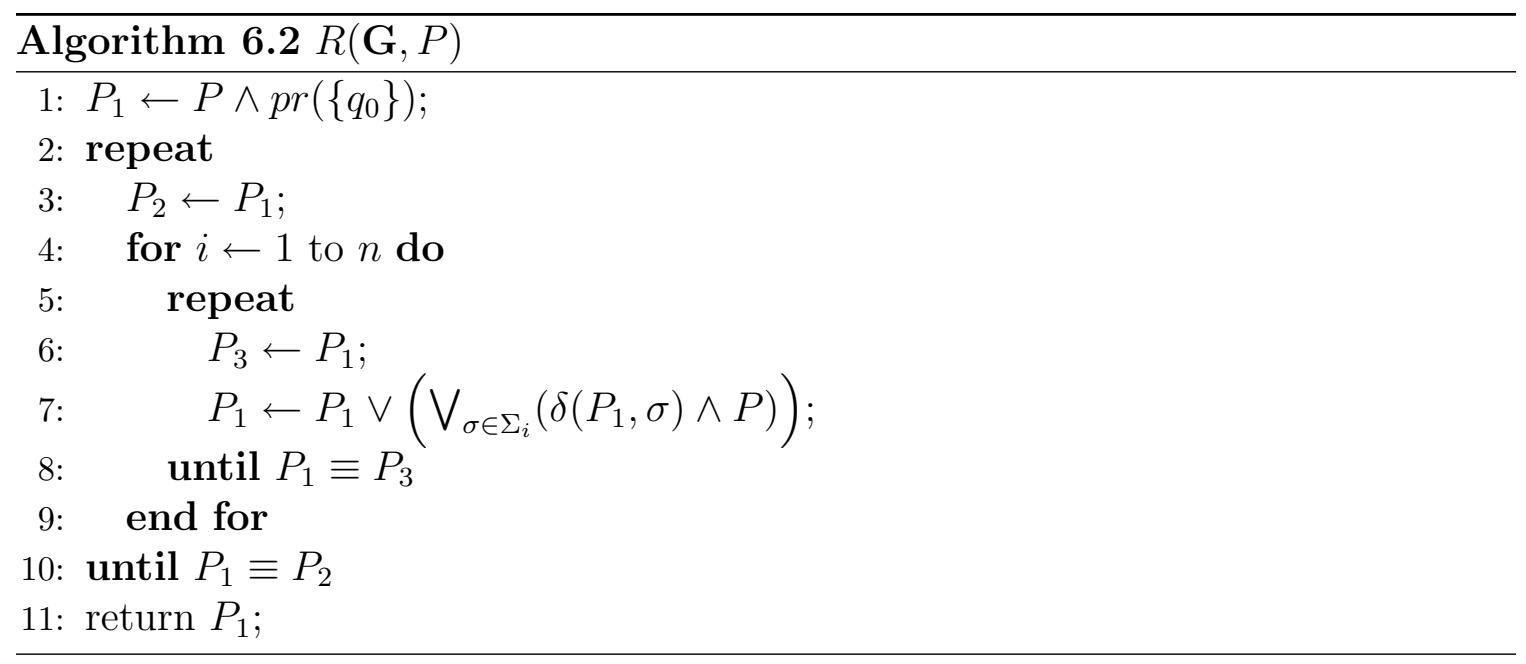

choosing events. In Line 7 of the algorithm, we never remove states from $P_{1}$. As the number of states in $\mathbf{G}$ is finite, after finite number of steps we will have $P 1 \equiv P 3$ for the repeat loop from Line 5 to Line 8 and $P 1 \equiv P 2$ for the repeat loop from Line 2 to Line 10; thus the algorithm will terminate.

Now we compute $R(\mathbf{M}$, true $)$ according to Algorithm 6.2 , where $\mathbf{M}$ is shown in Figure 6.3. The phrase "outer repeat" means the one on Line 2, and "inner repeat" means the one on Line 5 .

Initially, $P_{1} \equiv\left(v_{M_{1}}=s_{0} \wedge v_{M_{2}}=t_{0}\right)$;

- First outer repeat iteration $i:=1 ; / /$ For the component $\mathbf{M}_{1}$

- First inner repeat iteration

$$
\begin{aligned}
P_{1} & :=P_{1} \vee\left(v_{M_{1}}=s_{1} \wedge v_{M_{2}}=t_{0}\right) \\
& \equiv\left(v_{M_{1}}=s_{0} \vee v_{M_{1}}=s_{1}\right) \wedge v_{M_{2}}=t_{0}
\end{aligned}
$$

- Second inner repeat iteration

$$
\begin{aligned}
P_{1} & :=P_{1} \vee\left(v_{M_{1}}=s_{2} \wedge v_{M_{2}}=t_{0}\right) \\
& \equiv v_{M_{2}}=t_{0}
\end{aligned}
$$

- Third inner repeat iteration

$$
P_{1}:=v_{M_{2}}=t_{0}
$$

$i:=2 ; / /$ For the component $\mathbf{M}_{2}$ 
Master Thesis - R. Song - McMaster - Computing and Software

- First inner repeat iteration

$$
\begin{aligned}
P_{1} & :=P_{1} \vee\left(v_{M_{2}}=t_{1}\right) \\
& \equiv\left(v_{M_{2}}=t_{0} \vee v_{M_{2}}=t_{1}\right)
\end{aligned}
$$

- Second inner repeat iteration

$$
\begin{aligned}
P_{1} & :=P_{1} \vee\left(v_{M_{2}}=t_{2}\right) \\
& \equiv 1
\end{aligned}
$$

- Third inner repeat iteration

$$
P_{1}:=1
$$

- Second outer repeat iteration

$P_{1}:=1$

During the computation, we can see the intermediate predicates are much shorter than those of the previous algorithm. This is very important for our BDD-based algorithms, because the running time of BDD operations directly depends on the number of $\mathrm{BDD}$ nodes, and the number of nodes a $\mathrm{BDD}$ contains is proportional to the length of its corresponding logic formula (simplified).

For the system $\mathbf{G}$ composed of components $\mathbf{G}_{1}^{\prime}, \ldots, \mathbf{G}_{n}^{\prime}$, let $P \in \operatorname{Pred}(Q)$. We now discuss another optimization technique for the computation of $R(\mathbf{G}, P)$.

Notice that during the computation of $R(\mathbf{G}, P)$, we always compute the new reachable states from all the states that have been reached. This is obviously not efficient in an automata-based algorithm, because we can compute the new reachable states from the states that were found most recently (or in last loop). In our symbolic algorithms, however, computing from all the states that have reached could make the algorithm run faster because the tautology could dramatically reduce the size of the intermediate logic formulas.

However, in our experience, under some cases, computing the new reachable states from the states most recently found is faster than computing them from all the states that have been reached. For instance, in the AIP example in next chapter, computing from the most recently found states is faster. 


\section{Master Thesis - R. Song - McMaster - Computing and Software}

Algorithm 6.3 shows how to compute $R(\mathbf{G}, P)$ from the most recently found states. Again, In Line 9 of the algorithm, we never remove states from $P_{1}$. As the number of states in $\mathbf{G}$ is finite, after finite number of steps we will have $P 1 \equiv P 3$ for the repeat loop from Line 6 to Line 10 and $P 1 \equiv P 2$ for the repeat loop from Line 3 to Line 12 ; thus the algorithm will terminate.

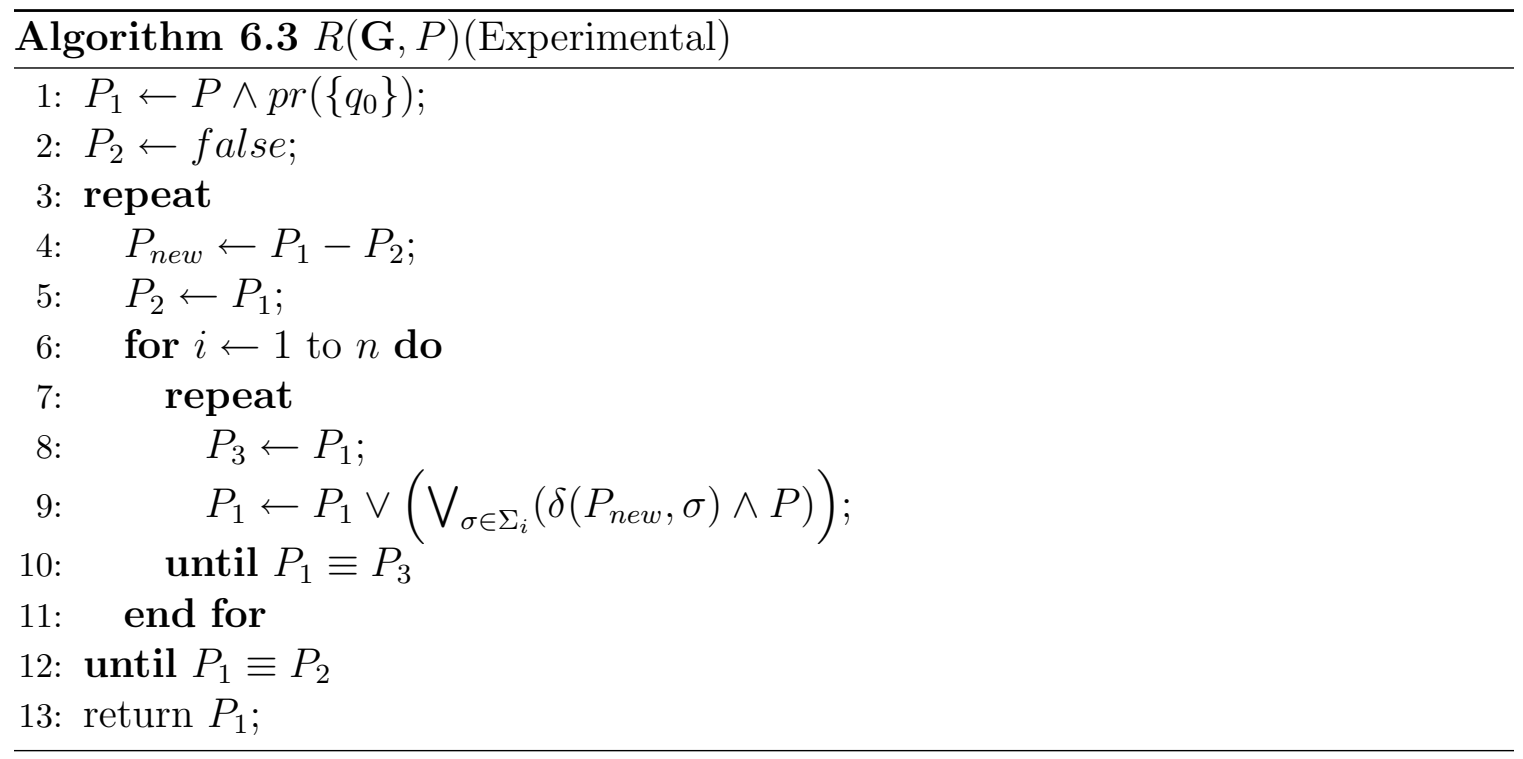

As this technique does not always make the algorithm work more efficient, we only give the algorithm for the predicate transformer $R$. For predicate transformers $T R$ and $\mathcal{C R}$, the algorithms applying this technique can easily be obtained. Furthermore, when we say the algorithm for the predicate transformer $R$ later on, we mean Algorithm 6.2.

As we need to do synthesis and verification on the AIP example in the next chapter, in the current version of our software tool, we use Algorithm 6.3 to compute the $R$ predicate transformer. Similarly, we also apply this optimization technique to compute $T R$ and $\mathcal{C} \mathcal{R}$ in our current software tool. 


\section{Master Thesis - R. Song-McMaster-Computing and Software}

\subsubsection{Computation of $T R$}

For the system $\mathbf{G}$ composed of components $\mathbf{G}_{1}^{\prime}, \ldots, \mathbf{G}_{n}^{\prime}$, let $\Sigma^{\prime} \subseteq \Sigma$ be a fixed event subset, $P \in \operatorname{Pred}(Q)$. From the definition of $T R\left(\mathbf{G}, ., \Sigma^{\prime}\right)$, we can write a straightforward algorithm for $T R\left(\mathbf{G}, \Sigma^{\prime}, P\right)$ as shown in Algorithm 6.4. Like the algorithm for $R(\mathbf{G}, P)$, this algorithm will terminate in finite number of steps.

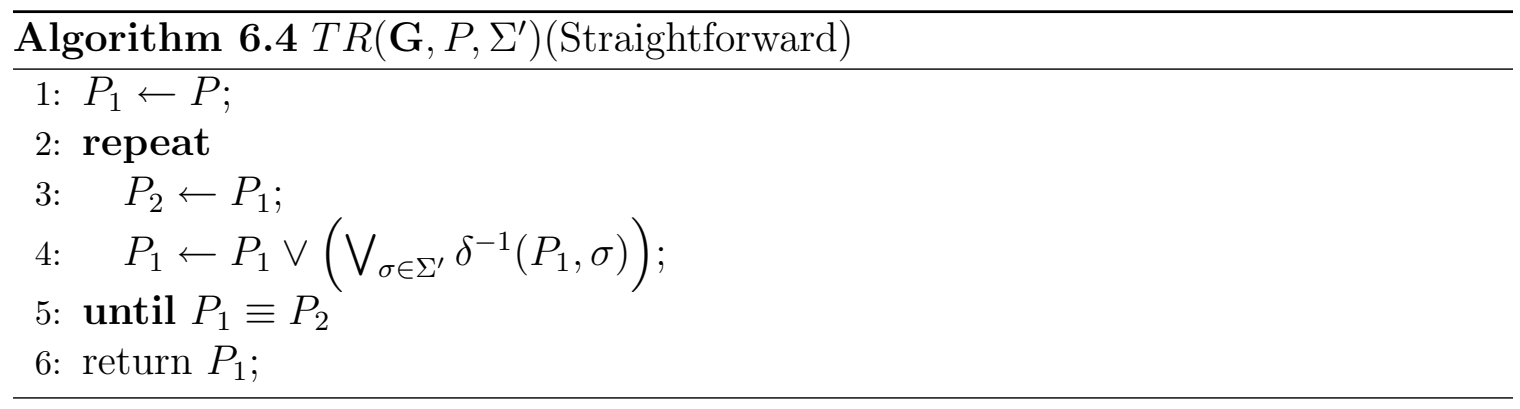

An improved algorithm using the tautology of Equation 6.2 is given in Algorithm 6.5.

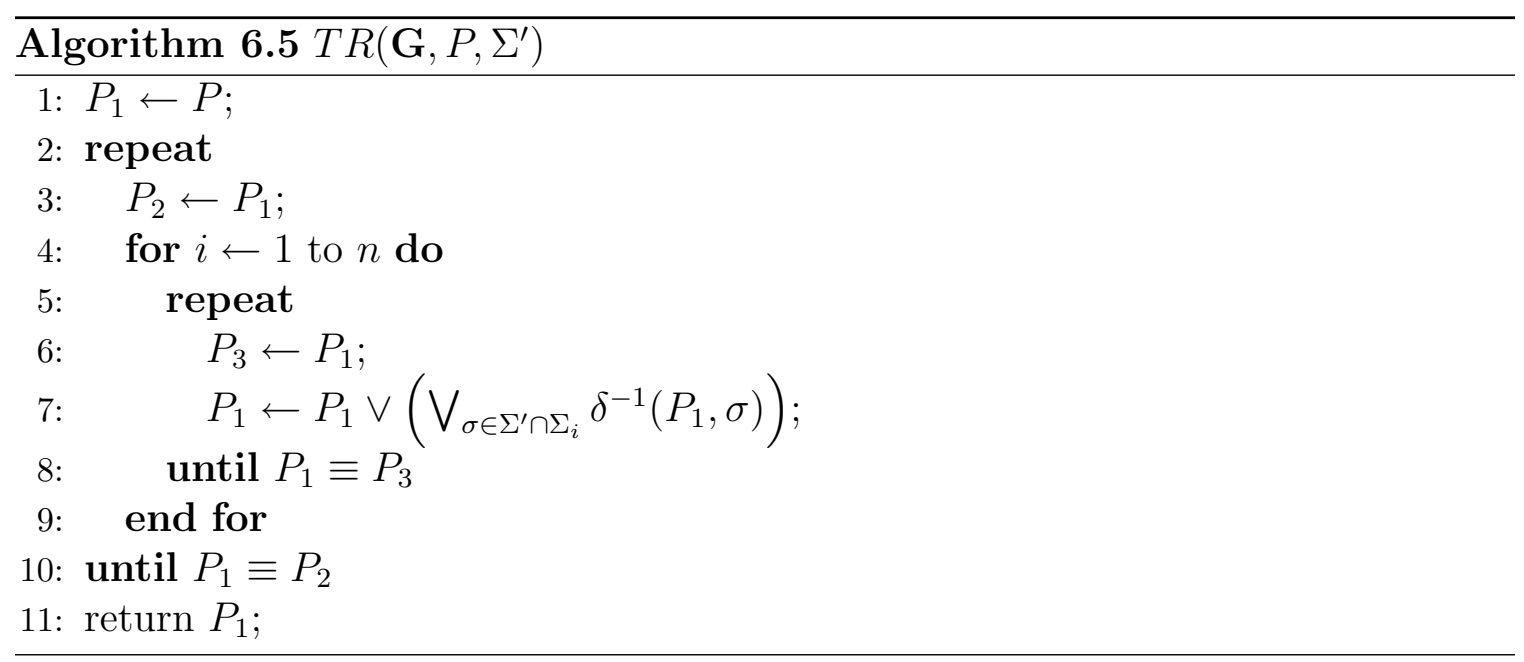

\subsubsection{Computation of $\mathcal{C R}$}

For the system $\mathbf{G}$ composed of components $\mathbf{G}_{1}^{\prime}, \ldots, \mathbf{G}_{n}^{\prime}$, let $\Sigma^{\prime} \subseteq \Sigma$ and $P^{\prime} \in$ $\operatorname{Pred}(Q)$ be fixed, and let $P \in \operatorname{Pred}(Q)$. From the definition of $\mathcal{C R}\left(\mathbf{G}, P^{\prime}, \Sigma^{\prime},.\right)$, we 
can write a straightforward algorithm for $\mathcal{C R}\left(\mathbf{G}, P^{\prime}, \Sigma^{\prime}, P\right)$ as shown in Algorithm 6.6. Like the algorithm for $R(\mathbf{G}, P)$, this algorithm will terminate in finite number of steps.

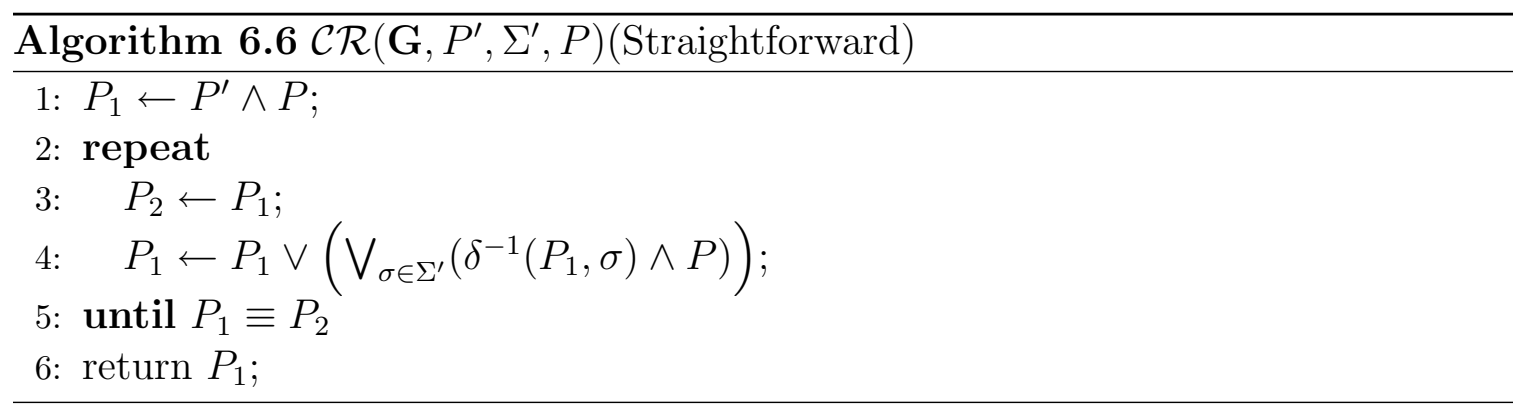

An improved algorithm using the tautology of Equation 6.2 is given in Algorithm 6.7.

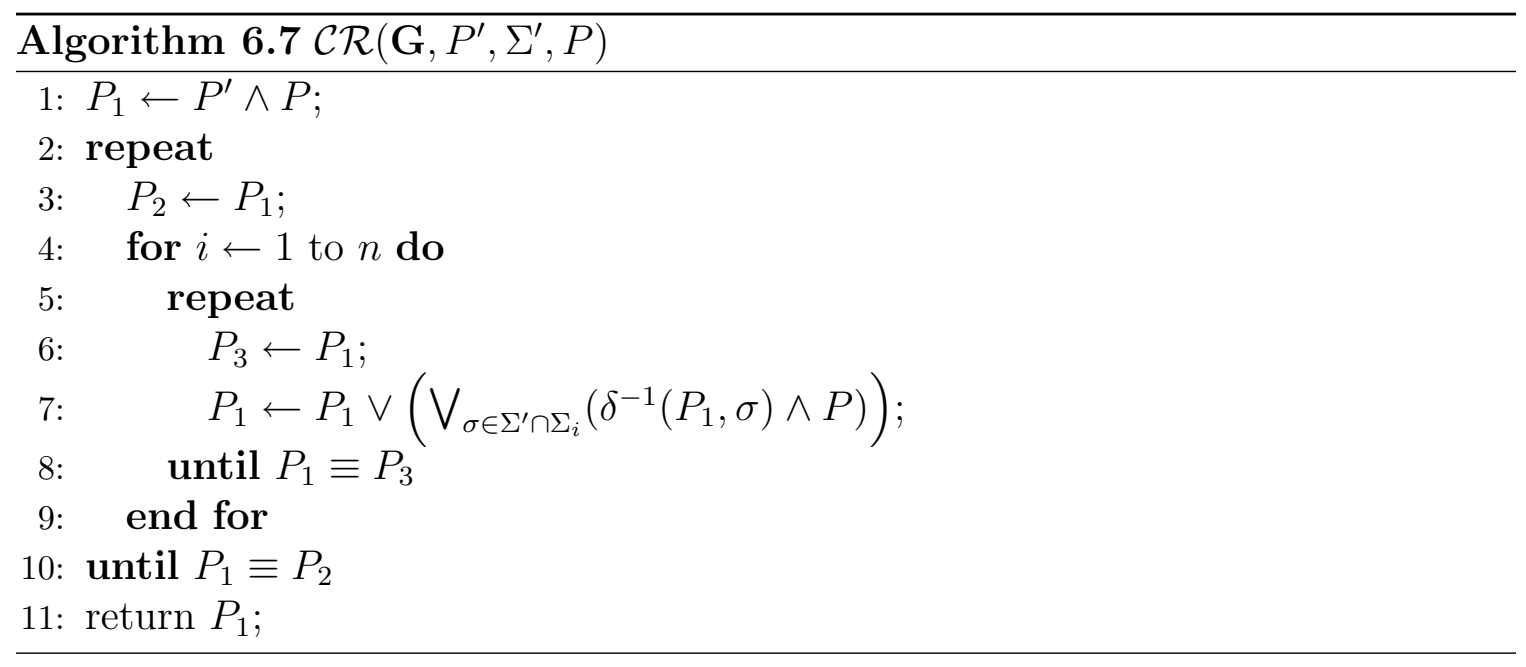

\subsection{Miscellaneous Computation for HISC Synthe- sis and Verification}

Let $\Phi$ be the $n^{\text {th }}$ degree interface system that respects the alphabet partition given by Equation 3.1 and is composed of plant components $\mathbf{G}_{H}^{p}, \mathbf{G}_{L_{1}}^{p}, \ldots, \mathbf{G}_{L_{n}}^{p}$, specifica- 


\section{Master Thesis - R. Song-McMaster-Computing and Software}

tions $\mathbf{E}_{H}, \mathbf{E}_{L_{1}}, \ldots, \mathbf{E}_{L_{n}}$, and interfaces $\mathbf{G}_{I_{1}}, \ldots, \mathbf{G}_{I_{n}}$. Let $j$ be an index with range $\{1, \ldots, n\}$. For system $\boldsymbol{\Phi}$, from Section 6.2 we already know how to symbolically compute the predicate transformers that we used in the algorithms we defined in the previous two chapters. However, to complete the full task of HISC synthesis and verification symbolically, we also have to compute $\operatorname{pr}\left(\operatorname{Bad}_{H}\right)$ for the high-level and $\operatorname{pr}\left(\operatorname{Bad}_{L_{j}}\right)$ for the $j^{\text {th }}$ low-level for each $j \in\{1, \ldots, n\}$, to verify that all the interface DES are command-pair interface and to verify that system $\boldsymbol{\Phi}$ respects the alphabet partition given by Equation 3.1.

\subsubsection{Computation of $\operatorname{pr}\left(\operatorname{Bad}_{H}\right)$}

For system $\boldsymbol{\Phi}$, we now copy the definition of $\operatorname{pr}\left(\operatorname{Bad}_{H}\right)$ from Chapter 4 here for convenience.

$$
\begin{aligned}
\operatorname{Bad}_{H}: & =\left\{q \in Q_{H} \mid\right. \\
& \left(\left(\exists \sigma_{u} \in \Sigma_{h u}\right)\left(\eta_{H} \times \xi^{h}\left((y, x), \sigma_{u}\right) ! \& \quad \zeta_{H}\left(z, \sigma_{u}\right) \not y\right)\right. \text { or } \\
& \left(( \exists j \in \{ 1 , \ldots , n \} ) ( \exists \sigma _ { a _ { j } } \in \Sigma _ { A _ { j } } ) \left(\xi_{j}^{h}\left(x_{j}, \sigma_{a_{j}}\right) ! \&\right.\right. \\
& \left.\left.\zeta_{H} \times \eta_{H} \times \xi_{1}^{h} \times \cdots \times \xi_{j-1}^{h} \times \xi_{j+1}^{h} \times \cdots \times \xi_{n}^{h}\left(\left(z, y, x_{1}, \ldots, x_{j-1}, x_{j+1}, \ldots, x_{n}\right), \sigma_{a_{j}}\right) \not . \cdot\right)\right\},
\end{aligned}
$$

where $q=(z, y, x)=\left(z, y, x_{1}, \ldots, x_{n}\right)$ as in Equation 4.1 (Page 79).

For system $\boldsymbol{\Phi}$, let $\mathbf{v}_{I H}$ and $\mathbf{v}_{I H}^{\prime}$ be the normal state variable vector and the prime state variable vector for the high-level $\mathcal{G}_{H}$, respectively.

For system $\boldsymbol{\Phi}$, for each $\sigma \in \Sigma_{I H}$, let $\left(\boldsymbol{v}_{\sigma}, \boldsymbol{v}_{\sigma}^{\prime}, N_{\sigma}\right)$ be the transition tuple for $\sigma$ in the high level $\mathcal{G}_{H}$; For each $\sigma_{u} \in \Sigma_{h u}$, let $\left(\boldsymbol{v}_{p I, \sigma_{u}}, \boldsymbol{v}_{p I, \sigma_{u}}^{\prime}, N_{p I, \sigma_{u}}\right)$ be the transition tuple for the component DES of $\mathbf{G}_{H}^{p} \times \mathbf{G}_{I}^{h}$; For each $\sigma_{u} \in \Sigma_{h u}$, let $\left(\boldsymbol{v}_{s, \sigma_{u}}, \boldsymbol{v}_{s, \sigma_{u}}^{\prime}, N_{s, \sigma_{u}}\right)$ be the transition tuple for the component DES of $\mathbf{E}_{H}$; For each $j \in\{1, \ldots, n\}$ and each $\alpha \in \Sigma_{A_{j}}$, let $\left(\boldsymbol{v}_{\left(H-I_{j}\right), \alpha}, \boldsymbol{v}_{\left(H-I_{j}\right), \alpha}^{\prime}, N_{\left(H-I_{j}\right), \alpha}\right)$ be the transition tuple for the component DES of $\mathbf{E}_{H} \times \mathbf{G}_{H}^{p} \times \mathbf{G}_{I_{1}}^{h} \times \cdots \times \mathbf{G}_{I_{j-1}}^{h} \times \mathbf{G}_{I_{j+1}}^{h} \times \cdots \times \mathbf{G}_{I_{n}}^{h}$. For each $j \in\{1, \ldots, n\}$ 


\section{Master Thesis - R. Song-McMaster - Computing and Software}

and each $\alpha \in \Sigma_{A_{j}}$, let $v_{I_{j}}$ and $v_{I_{j}}^{\prime}$ respectively be the normal and prime state variable for the interface $\mathbf{G}_{I_{j}}$ and $N_{I_{j}, \alpha}$ be the transition predicate for $\alpha$ in the interface DES $\mathbf{G}_{I_{j}}$.

Now, for system $\boldsymbol{\Phi}$, we can compute $\operatorname{pr}\left(\operatorname{Bad}_{H}\right)$ symbolically by

$$
\begin{aligned}
\operatorname{pr}\left(\operatorname{Bad}_{H}\right):= & \left(\bigvee_{\sigma_{u} \in \Sigma_{h u}}\left(\exists \boldsymbol{v}_{p I, \sigma_{u}}^{\prime} N_{p I, \sigma_{u}} \wedge \neg\left(\exists \boldsymbol{v}_{s, \sigma_{u}}^{\prime} N_{s, \sigma_{u}}\right)\right)\right) \vee \\
& \left(\bigvee_{j \in\{1, \ldots, n\}} \bigvee_{\alpha \in \Sigma_{A_{j}}}\left(\exists v_{I_{j}}^{\prime} N_{I_{j}, \alpha} \wedge \neg\left(\exists \boldsymbol{v}_{\left(H-I_{j}\right), \alpha}^{\prime} N_{\left(H-I_{j}\right), \alpha}\right)\right)\right)
\end{aligned}
$$

In order to save memory space, for $\sigma_{u} \in \Sigma_{h u}$, we do not define $N_{s, \sigma_{u}}$ in our software tool directly. We compute $\exists\left(\boldsymbol{v}_{p I, \sigma_{u}} \cup \boldsymbol{v}_{p I, \sigma_{u}}^{\prime}\right) N_{\sigma_{u}}$ instead of directly defining $N_{s, \sigma_{u}}$. In most cases, $\exists\left(\boldsymbol{v}_{p I, \sigma_{u}} \cup \boldsymbol{v}_{p I, \sigma_{u}}^{\prime}\right) N_{\sigma_{u}}$ represents the transition predicate for $\sigma_{u}$ in $\mathbf{E}_{H}$, i.e. $N_{s, \sigma_{u}}$. However, if $\sigma_{u}$ is blocked by DES $\mathbf{G}_{H}^{p} \times \mathbf{G}_{I}^{h}$ (e.g. $\sigma_{u}$ is in the event set of a component DES, but there is no $\sigma_{u}$ transition in that DES) and there exist $\sigma_{u}$ transitions in the component DES of $\mathbf{E}_{H}$ and $\sigma_{u}$ is never blocked by any component DES or combinations of component DES of $\mathbf{E}_{H}$, then by Equation 6.3, we have $N_{p I, \sigma_{u}} \equiv 0, N_{\sigma_{u}} \equiv 0$ and $N_{s, \sigma_{u}} \not \equiv 0$, but $\exists\left(\boldsymbol{v}_{p I, \sigma_{u}} \cup \boldsymbol{v}_{p I, \sigma_{u}}^{\prime}\right) N_{\sigma_{u}} \equiv 0$. However, because $N_{p I, \sigma_{u}} \equiv 0$, we have $\exists \boldsymbol{v}_{p I, \sigma_{u}}^{\prime} N_{p I, \sigma_{u}} \equiv 0$. Then we always have

$$
\exists \boldsymbol{v}_{p I, \sigma_{u}}^{\prime} N_{p I, \sigma_{u}} \wedge \neg\left(\exists \boldsymbol{v}_{s, \sigma_{u}}^{\prime} N_{s, \sigma_{u}}\right) \equiv 0
$$

regardless of the value of $\neg\left(\exists \boldsymbol{v}_{s, \sigma_{u}}^{\prime} N_{s, \sigma_{u}}\right)$. Therefore, by replacing $N_{s, \sigma_{u}}$ with $\exists\left(\boldsymbol{v}_{p I, \sigma_{u}} \cup\right.$ $\left.\boldsymbol{v}_{p I, \sigma_{u}}^{\prime}\right) N_{\sigma_{u}}$ does not change the result of $\operatorname{pr}\left(\operatorname{Bad}_{H}\right)$.

Similarly, for $j \in\{1, \ldots, n\}$ and $\alpha \in \Sigma_{A_{j}}$, we compute $\exists v_{I_{j}}\left(\exists v_{I_{j}}^{\prime} N_{\alpha}\right)$ instead of directly defining $N_{\left(H-I_{j}\right), \alpha}$. For the similar reason as above, this replacement does not affect the result of $\operatorname{pr}\left(\operatorname{Bad}_{H}\right)$. 


\section{Master Thesis - R. Song - McMaster - Computing and Software}

\subsubsection{Computation of $\operatorname{pr}\left(\operatorname{Bad}_{L_{j}}\right)$}

For system $\boldsymbol{\Phi}$ with $j \in\{1, \ldots, n\}$, we now discuss how to compute $\operatorname{pr}\left(\operatorname{Bad}_{L_{j}}\right)$ for the $j^{\text {th }}$ low-level in system $\boldsymbol{\Phi}$.

For convenience, we reprint the definition of $\operatorname{Bad}_{L_{j}}$ from Chapter 4 here.

$$
\begin{array}{r}
\operatorname{Bad}_{L_{j}}:=\left\{q \in Q_{L_{j}} \mid\left(\left(\exists \sigma_{l u_{j}} \in \Sigma_{l u_{j}}\right) \eta_{L_{j}}\left(y, \sigma_{l u_{j}}\right) ! \& \zeta_{L_{j}} \times \xi_{j}^{l}\left((z, x), \sigma_{l u_{j}}\right) \not y\right)\right. \text { or } \\
\left.\left(\left(\exists \rho \in \Sigma_{R_{j}}\right) \xi_{j}^{l}(x, \rho) ! \& \zeta_{L_{j}} \times \eta_{L_{j}}((z, y), \rho) \not\right)\right\},
\end{array}
$$

where $q=(z, y, x)$ as in Equation 4.2 (Page 105).

For system $\boldsymbol{\Phi}$, let $\mathbf{v}_{I L_{j}}$ and $\mathbf{v}_{I L_{j}}^{\prime}$ be the normal state variable vector and the prime state variable vector for the $j^{\text {th }}$ low-level $\mathcal{G}_{L_{j}}$, respectively.

For system $\boldsymbol{\Phi}$, for each $\sigma \in \Sigma_{I L_{j}}$, let $\left(\boldsymbol{v}_{\sigma}, \boldsymbol{v}_{\sigma}^{\prime}, N_{\sigma}\right)$ be the transition tuple for $\sigma$ in the $j^{\text {th }}$ low-level $\mathcal{G}_{L_{j}}$; For each $\sigma_{u} \in \Sigma_{l u_{j}}$, let $\left(\boldsymbol{v}_{p, \sigma_{u}}, \boldsymbol{v}_{p, \sigma_{u}}^{\prime}, N_{p, \sigma_{u}}\right)$ be the transition tuple for the component DES of $\mathbf{G}_{L_{j}}^{p}$; For each $\sigma_{u} \in \Sigma_{l u_{j}}$, let $\left(\boldsymbol{v}_{s I, \sigma_{u}}, \boldsymbol{v}_{s I, \sigma_{u}}^{\prime}, N_{s I, \sigma_{u}}\right)$ be the transition tuple for the component DES of $\mathbf{E}_{L_{j}} \times \mathbf{G}_{I_{j}}^{l}$; For each $\rho \in \Sigma_{R_{j}}$, let $\left(\boldsymbol{v}_{\left(L_{j}-I_{j}\right), \rho}, \boldsymbol{v}_{\left(L_{j}-I_{j}\right), \rho}^{\prime}, N_{\left(L_{j}-I_{j}\right), \rho}\right)$ be the transition tuple for the component DES of $\mathbf{E}_{L_{j}} \times \mathbf{G}_{L_{j}}^{p}$; For each $\rho \in \Sigma_{R_{j}}$, let $v_{I_{j}}$ and $v_{I_{j}}^{\prime}$ respectively be the normal and prime state variable for the interface $\mathbf{G}_{I_{j}}$ and $N_{I_{j}, \rho}$ be the transition predicate for $\rho$ in the interface DES $\mathbf{G}_{I_{j}}$.

For the $j^{\text {th }}$ low-level in system $\boldsymbol{\Phi}$, we can now compute $\operatorname{pr}\left(\operatorname{Bad}_{L_{j}}\right)$ symbolically by

$$
\begin{aligned}
\operatorname{pr}\left(\operatorname{Bad}_{L_{j}}\right):= & \left(\bigvee_{\sigma_{u} \in \Sigma_{l u_{j}}}\left(\exists \boldsymbol{v}_{p, \sigma_{u}}^{\prime} N_{p, \sigma_{u}} \wedge \neg\left(\exists \boldsymbol{v}_{s I, \sigma_{u}}^{\prime} N_{s I, \sigma_{u}}\right)\right)\right) \vee \\
& \left(\bigvee_{\rho \in \Sigma_{R_{j}}}\left(\exists v_{I_{j}}^{\prime} N_{I_{j}, \rho} \wedge \neg\left(\exists \boldsymbol{v}_{\left(L_{j}-I_{j}\right), \rho}^{\prime} N_{\left(L_{j}-I_{j}\right), \rho}\right)\right)\right)
\end{aligned}
$$

Similarly to how we compute $\operatorname{pr}\left(\operatorname{Bad}_{H}\right)$, for $\sigma_{u} \in \Sigma_{l u_{j}}$, we compute $\exists\left(\boldsymbol{v}_{p, \sigma_{u}} \cup\right.$ $\left.\boldsymbol{v}_{p, \sigma_{u}}^{\prime}\right) N_{\sigma_{u}}$ instead of directly defining $N_{s I, \sigma_{u}}$, and for $\rho \in \Sigma_{R_{j}}$, we compute $\exists v_{I_{j}}\left(\exists v_{I_{j}}^{\prime} N_{\rho}\right)$ 
instead of directly defining $N_{\left(L_{j}-I_{j}\right), \rho}$. For similar reasons as for the computation of $\operatorname{pr}\left(\operatorname{Bad}_{H}\right)$, we know that these replacements will not change the result of $\operatorname{pr}\left(\operatorname{Bad}_{L_{j}}\right)$.

\subsubsection{Miscellaneous Computation in Algorithm 4.2}

For system $\Phi$ with $j \in\{1, \ldots, n\}$, we now show how to compute $P_{\alpha}$ for $\alpha \in \Sigma_{A_{j}}$ and $P \in \operatorname{Pred}\left(Q_{L_{j}}\right)$ in Algorithm 4.2 and how to compute $\operatorname{pr}\left(\left\{q \in Q_{L_{j}} \mid \xi_{j}^{l}(x, \rho \alpha) ! \&\right.\right.$ $\left.\left.\delta_{L_{j}}(q, \rho) \models \neg P_{\mathcal{C R}_{\alpha}}\right\}\right)$ in Line 6 of Algorithm 4.2, where $q=(z, y, x)$ as in Equation 4.2 (Page 105).

For convenience, we copy here $P_{\alpha}$ in Definition 4.19 here

$$
P_{\alpha}:=\operatorname{pr}\left(\left\{q^{\prime} \in Q_{L_{j}} \mid \delta_{L_{j}}\left(q^{\prime}, \alpha\right) \models P\right\}\right)
$$

For system $\boldsymbol{\Phi}$, let $\mathbf{v}_{I L_{j}}$ and $\mathbf{v}_{I L_{j}}^{\prime}$ be the normal state variable vector and the prime state variable vector for the $j^{\text {th }}$ low-level $\mathcal{G}_{L_{j}}$ respectively.

For system $\boldsymbol{\Phi}$, for each $\sigma \in \Sigma_{I L_{j}}$, let $\left(\boldsymbol{v}_{\sigma}, \boldsymbol{v}_{\sigma}^{\prime}, N_{\sigma}\right)$ be the transition tuple for $\sigma$ in the $j^{\text {th }}$ low-level $\mathcal{G}_{L_{j}}$; For each $\sigma_{I_{j}} \in \Sigma_{I_{j}}$, let $v_{I_{j}}$ and $v_{I_{j}}^{\prime}$ respectively be the normal and prime state variable for the interface $\mathbf{G}_{I_{j}}$ and $N_{I_{j}, \sigma_{I_{j}}}$ be the transition predicate for $\sigma_{I_{j}}$ in the interface DES $\mathbf{G}_{I_{j}}$.

For system $\boldsymbol{\Phi}$, let $\alpha \in \Sigma_{A_{j}}$ and $P \in \operatorname{Pred}\left(Q_{L_{j}}\right)$. From the definition of $P_{\alpha}$, we know $P_{\alpha}=\delta_{L_{j}}^{-1}(P, \alpha)$. So, we can compute $P_{\alpha}$ symbolically by

$$
P_{\alpha}:=\exists \boldsymbol{v}_{\alpha}^{\prime}\left(N_{\alpha} \wedge\left(P\left[\boldsymbol{v}_{\alpha} \rightarrow \boldsymbol{v}_{\alpha}^{\prime}\right]\right)\right)
$$

For system $\boldsymbol{\Phi}$, let $\rho \in \Sigma_{R_{j}}, \alpha \in \Sigma_{A_{j}}$ and $P_{\mathcal{C R}_{\alpha}} \in \operatorname{Pred}\left(Q_{L_{j}}\right)$. We can compute $\operatorname{pr}\left(\left\{q \in Q_{L_{j}} \mid \xi_{j}^{l}(x, \rho \alpha) ! \& \delta_{L_{j}}(q, \rho) \models \neg P_{\mathcal{C R}_{\alpha}}\right\}\right)$ symbolically by

$$
\begin{gathered}
\exists v_{I_{j}}^{\prime}\left(N_{I_{j}, \rho} \wedge\left(\left(\exists v_{I_{j}}^{\prime} N_{I_{j}, \alpha}\right)\left[v_{I_{j}} \rightarrow v_{I_{j}}^{\prime}\right]\right)\right) \wedge \\
\exists \boldsymbol{v}_{\rho}^{\prime}\left(N_{\rho} \wedge\left(\left(\neg P_{\mathcal{C} \mathcal{R}_{\alpha}}\right)\left[\boldsymbol{v}_{\rho} \rightarrow \boldsymbol{v}_{\rho}^{\prime}\right]\right)\right) .
\end{gathered}
$$




\section{Master Thesis - R. Song-McMaster-Computing and Software}

$\exists v_{I_{j}}^{\prime} N_{I_{j}, \alpha}$ computes the state predicate including all the states in $Q_{L_{j}}$ with $\alpha$ transition defined. So, $\exists v_{I_{j}}^{\prime}\left(N_{I_{j}, \rho} \wedge\left(\left(\exists v_{I_{j}}^{\prime} N_{I_{j}, \alpha}\right)\left[v_{I_{j}} \rightarrow v_{I_{j}}^{\prime}\right]\right)\right)$ computes the state predicate including all the states in $Q_{L_{j}}$ with $\rho$ transitions defined, and the target states of these $\rho$ transitions are the source states of $\alpha$ transitions in $Q_{L_{j}}$. That is to say, $\exists v_{I_{j}}^{\prime}\left(N_{I_{j}, \rho} \wedge\left(\left(\exists v_{I_{j}}^{\prime} N_{I_{j}, \alpha}\right)\left[v_{I_{j}} \rightarrow v_{I_{j}}^{\prime}\right]\right)\right)$ computes the predicate identifying the set $\left\{q \in Q_{L_{j}} \mid \xi_{j}^{l}(x, \rho \alpha) !\right\}$.

\subsubsection{Verifying Event Partition}

One of the most fundamental properties in the HISC architecture is that an HISC system must respect the event partition defined in Equation 3.1.

As it is usually the case that the number of events in an HISC system is very limited compared to the number of states (e.g. only hundreds in the AIP example in next chapter), the efficiency of the algorithm to verify this property is not critical.

The method used in our software tool is as follows. For each event in the system, we encode it as an integer value. From the integer value, we are able to know which event set the event belongs to. We save the event name and its encoded integer value for all events in a balanced binary search tree ${ }^{3}$ with the event name as the key.

Initially, the tree is empty. When we read a component DES file, we can determine which event partition each event belongs to based on the information in the DES file and where the DES file belongs in the system (e.g. part of the high-level, the $j^{\text {th }}$ interface, the $j^{\text {th }}$ low-level, etc.). Thus we can encode it as an integer value. Then we search for this event in the tree. If the event does not exist, we insert this event into the tree. Otherwise, we compare the integer value associated with the event in the tree and the value we just encoded. If they are the same, then there is no problem. Otherwise, it means that this event is in two different event partitions. After we finish

\footnotetext{
${ }^{3}$ The data structure used in our software tool is GNU C++ STL map, which is a red-black tree.
} 


\section{Master Thesis - R. Song - McMaster - Computing and Software}

reading all the DES, if there are no two events having the same name but different integer values, then we know the system respects the event partition.

As the data structure to save all the events is a balanced binary search tree, the worst-case searching and insertion time for each event are both $O\left(\log _{2} n\right)$, where $n$ is the number of events in the system. Thus the total time for handling each event is still $O\left(\log _{2} n\right)$, and the worst-case verification time for event partition is $O\left(m n \log _{2} n\right)$, where $m$ is the number of DES components in the system.

\subsubsection{Verifying Command-pair Interfaces}

For the HISC system $\boldsymbol{\Phi}$, we also need to verify if all the interfaces in the system are command-pair interfaces.

Let $j \in\{1, \ldots, n\}$. We now discuss the algorithm to verify the $j^{\text {th }}$ interface DES $\mathbf{G}_{I_{j}}:=\left(X_{j}, \Sigma_{I_{j}}, \xi_{j}, x_{j_{0}}, X_{j_{m}}\right)$. Because $\mathbf{G}_{I_{j}}$ is usually composed of single component DES, efficiency is not the main concern for the verification algorithm.

For all $\sigma \in \Sigma_{I_{j}}$, let $N_{I_{j}, \sigma}$ be the transition predicate for $\sigma$ in $\mathbf{G}_{I_{j}}$. Let $v_{I_{j}}$ and $v_{I_{j}}^{\prime}$ be the normal state variable and prime state variable for $\mathbf{G}_{I_{j}}$ respectively.

Algorithm 6.8 shows how to verify if the $j^{\text {th }}$ interface DES $\mathbf{G}_{I_{j}}$ is a command-pair interface.

Line 1 to 4 says that $\mathbf{G}_{I_{j}}$ can not be an empty DES. Line 5 computes the predicate including all the reachable states. Line 6 computes the predicate including all the reachable marker states.

Line 7 and 8 computes the predicate including all the reachable states which are the target states of answer event transitions plus the initial state. The states satisfying $P_{R_{j}}$ should only be the source states of request event transitions.

Line 9 and 10 computes the predicate including all the reachable states which are 


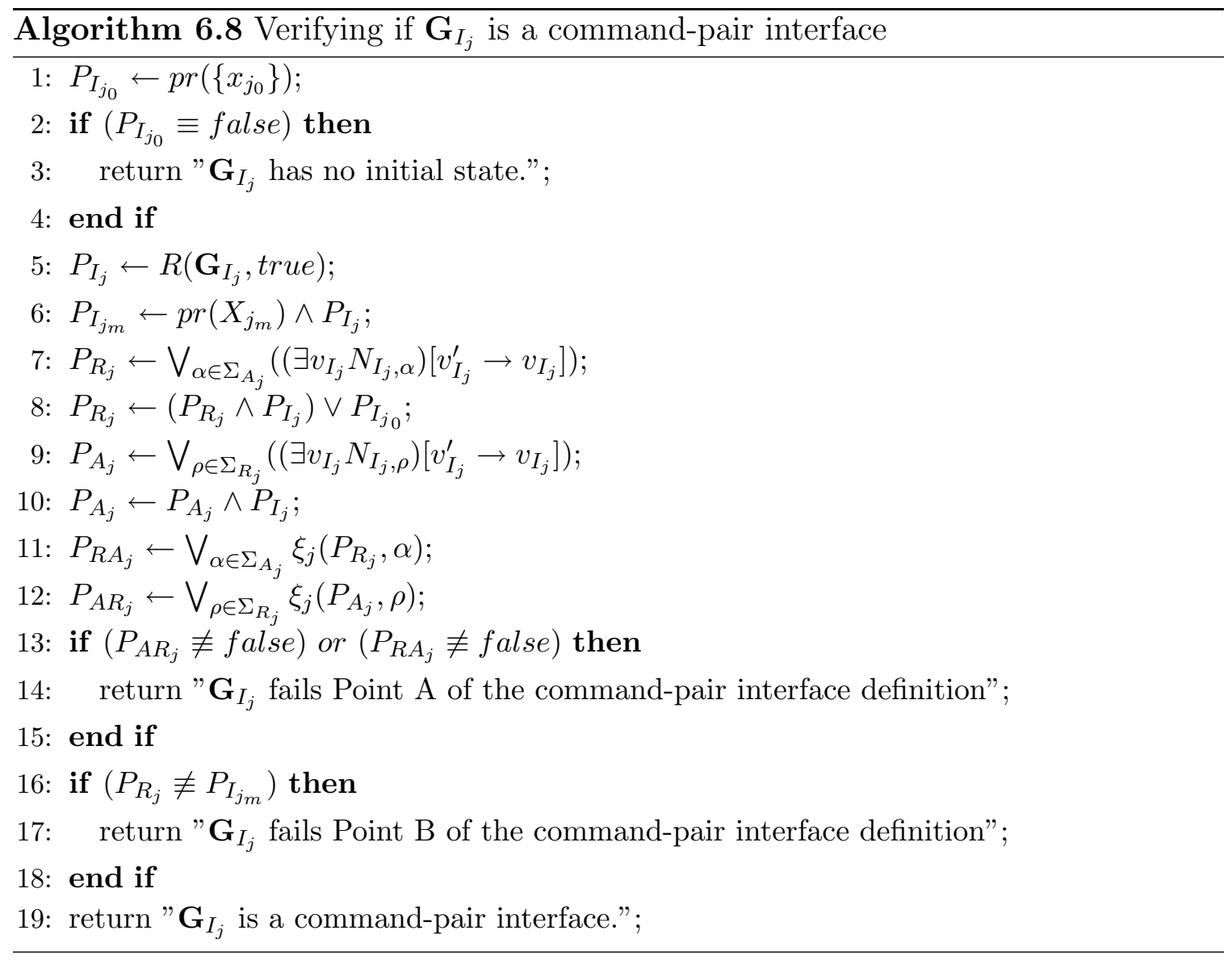

the target states of request event transitions. The states satisfying $P_{A_{j}}$ should only be the source states of answer event transitions.

Line 11 computes the predicate including all the reachable states which are the target states of answer event transitions with source states satisfying $P_{R_{j}}$.

Line 12 computes the predicate including all the reachable states which are the target states of request event transitions with source states satisfying $P_{A_{j}}$.

Now we informally explain the correctness of the algorithm.

From Line 1 to 4 , the algorithm makes sure that $\mathbf{G}_{I_{j}}$ is not empty.

In the following, we always assume $\mathbf{G}_{I_{j}}$ is not empty.

We first discuss Point A of Definition 3.1. To show the correctness of the algorithm 
for Point A, it is sufficient to show that

$\left(\forall s \in L\left(\mathbf{G}_{I_{j}}\right)\right) s \in \overline{\left(\Sigma_{R_{j}} \cdot \Sigma_{A_{j}}\right)^{*}}$ if and only if $P_{R A_{j}} \equiv$ false and $P_{A R_{j}} \equiv$ false.

Equivalently, we can show that $\left(\exists s \in L\left(\mathbf{G}_{I_{j}}\right)\right) s \notin \overline{\left(\Sigma_{R_{j}} \cdot \Sigma_{A_{j}}\right)^{*}}$ if and only if $P_{R A_{j}} \not \equiv$ false or $P_{A R_{j}} \not \equiv$ false.

1. Show $\left(\left(\exists s \in L\left(\mathbf{G}_{I_{j}}\right)\right) s \notin \overline{\left(\Sigma_{R_{j}} \cdot \Sigma_{A_{j}}\right)^{*}}\right) \Rightarrow P_{R A_{j}} \not \equiv$ false or $P_{A R_{j}} \not \equiv$ false.

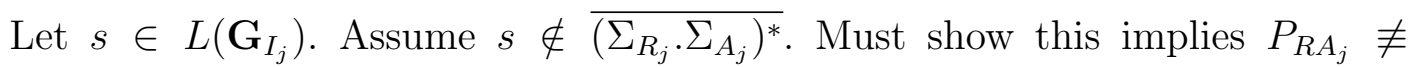
false or $P_{A R_{j}} \not \equiv$ false.

From $s \notin \overline{\left(\Sigma_{R_{j}} . \Sigma_{A_{j}}\right)^{*}}$, we know that one or more of the following three conditions must be satisfied.

(a) $s \in \Sigma_{I_{j}}^{*} \cdot \Sigma_{A_{j}} \cdot \Sigma_{A_{j}} \cdot \Sigma_{I_{j}}^{*}$

$\Rightarrow\left(\exists u, v \in \Sigma_{I_{j}}^{*}\right)\left(\exists \alpha_{1}, \alpha_{2} \in \Sigma_{A_{j}}\right) s=u \alpha_{1} \alpha_{2} v$.

From Line 7 and 8 , we know that $\xi_{j}\left(x_{j_{0}}, u \alpha_{1}\right) \models P_{R_{j}}$.

From Line 11, we know that $\xi_{j}\left(x_{j_{0}}, u \alpha_{1} \alpha_{2}\right) \models P_{R A_{j}}$, so $P_{R A_{j}} \not \equiv$ false.

(b) $s \in \Sigma_{I_{j}}^{*} \cdot \Sigma_{R_{j}} \cdot \Sigma_{R_{j}} \cdot \Sigma_{I_{j}}^{*}$

$\Rightarrow\left(\exists u, v \in \Sigma_{I_{j}}^{*}\right)\left(\exists \rho_{1}, \rho_{2} \in \Sigma_{R_{j}}\right) s=u \rho_{1} \rho_{2} v$.

From Line 9 and 10, we know that $\xi_{j}\left(x_{j_{0}}, u \rho_{1}\right) \models P_{A_{j}}$.

From Line 12, we know that $\xi_{j}\left(x_{j_{0}}, u \rho_{1} \rho_{2}\right) \models P_{A R_{j}}$, so $P_{A R_{j}} \not \equiv$ false.

(c) $s \in \Sigma_{A_{j}} . \Sigma_{I_{j}}^{*}$

$\Rightarrow\left(\exists \alpha \in \Sigma_{A_{j}}\right)\left(\exists u \in \Sigma_{I_{j}}^{*}\right) s=\alpha u$.

From Line 8, we know that $x_{j_{0}} \models P_{R_{j}}$.

From Line 11, we know that $\xi_{j}\left(x_{j_{0}}, \alpha\right) \models P_{R A_{j}}$, so $P_{R A_{j}} \not \equiv$ false.

From (a), (b) and (c), we know that Point 1 holds. 


\section{Master Thesis - R. Song - McMaster - Computing and Software}

2. Show $P_{R A_{j}} \not \equiv$ false or $P_{A R_{j}} \not \equiv$ false $\Rightarrow\left(\left(\exists s \in L\left(\mathbf{G}_{I_{j}}\right)\right) s \notin \overline{\left(\Sigma_{R_{j}} \cdot \Sigma_{A_{j}}\right)^{*}}\right)$.

From Line 7,8 and 11, we know that if $P_{R A_{j}} \not \equiv$ false then $\left(\exists s \in L\left(\mathbf{G}_{I_{j}}\right)\right) s \in$ $\Sigma_{I_{j}}^{*} \cdot \Sigma_{A_{j}} \cdot \Sigma_{A_{j}} \cdot \Sigma_{I_{j}}^{*}$ or $s \in \Sigma_{A_{j}} \cdot \Sigma_{I_{j}}^{*}$.

From Line 9, 10 and 12, we know that if $P_{A R_{j}} \not \equiv$ false then $\left(\exists s \in L\left(\mathbf{G}_{I_{j}}\right)\right) s \in$ $\Sigma_{I_{j}}^{*} \cdot \Sigma_{R_{j}} \cdot \Sigma_{R_{j}} \cdot \Sigma_{I_{j}}^{*}$.

We can thus conclude that Point 2 holds.

We now discuss Point B of Definition 3.1. Assuming Point A of Definition 3.1 holds, to show the correctness of Point $\mathrm{B}$, it is sufficient to show that $\mathbf{G}_{I_{j}}$ satisfies Point B if and only if $P_{R_{j}} \equiv P_{I_{j_{m}}}$.

From Point B, we know $\mathbf{G}_{I_{j}}$ satisfies Point B if and only if all the target states (reachable) of all answer event transitions in $\mathbf{G}_{I_{j}}$ and the initial state of $\mathbf{G}_{I_{j}}$ must be marked (i.e. $P_{R_{j}} \preceq P_{I_{j_{m}}}$.), and there is no other states being marked (i.e. $P_{I_{j_{m}}} \preceq$ $P_{R_{j}}$.). Therefore, we have $\mathbf{G}_{I_{j}}$ satisfies Point B if and only if $P_{R_{j}} \equiv P_{I_{j_{m}}}$.

\subsection{BDD Implementations of Algorithms}

So far, we have shown how to represent an HISC system as logic formulas and the method to implement the HISC synthesis and verification algorithms from the logic formulas. The logic formulas we used can be directly represented as an Integer Decision Diagram(IDD) $[16,51]$. We use Binary Decision Diagram (BDD) [6] to represent the formulas, as a BDD software package is readily available now. The BDD software package we used is BuDDy 2.4 developed by Jørn Lind-Nielsen. Due to space and time constraints, we will not give an introduction for BDD or the software package here. Readers are referred to [19] for an introduction to BDD and its application to symbolic model checking, and to [1] for the BDD package implementation details. 


\section{Master Thesis - R. Song-McMaster - Computing and Software}

Each state variable in our logic formula is a finite domain variable (simply means that a variable can only be assigned finite number of values), which can be represented by a block of binary variables. For instance, a state variable for a 3-state DES can be represented by two binary variables, and a state variable for a 5-state DES can be represented by three binary variables. Assume the number of states in a DES is $m$, by replacing the finite domain state variable for this DES with a block of binary variables, we actually extend the variable domain to $2^{k}$, where $k=\left\lceil\log _{2} m\right\rceil$. That is, we actually extend the number of states in the DES to $2^{k}$. However, because of the transition predicate, those extended states will be treated as unreachable states, which do not affect the language result at all.

All the logic operations such as $\wedge, \vee, \neg$ and $\exists$ can be done by corresponding BDD operations directly. For the $\exists$ operation of a finite domain variable, we have to do $\exists$ operation on all of its corresponding binary variables. For example, a state variable $v$ with domain $\{0,1,2\}$ is represented by two binary variables $v_{1}$ and $v_{2}$. The operation $\exists v$ can be done by $\exists v_{1}\left(\exists v_{2}\right)$ instead. A very good aspect in the software package $B u D D y$ is that it provides a series of functions with prefix " $f d d_{-}$", which allow developers to be able to implement the logic operations on finite domain variables directly. In other words, we can implement operations in terms of finite domain variables (state variables in our case), and $B u D D y$ package will automatically translate the operations into ones on their corresponding binary variables. Please refer to the source code of our software tool in Appendix A for more details.

\subsubsection{State Variable Ordering}

A well-known fact of BDD is that the order of variables in a BDD can dramatically change the number of the BDD nodes. Because the efficiency of all the BDD 


\section{Master Thesis - R. Song-McMaster-Computing and Software}

operations directly depends on the number of the BDD nodes, the variable order in a BDD plays an important role in the algorithm's actual efficiency. However, to find the optimal order for BDD is NP-Complete [4], so heuristics on variable orders are usually used to reduce the BDD size.

In our case, we always keep binary variables in a block for each finite domain variable together because of the tautology in Equation 6.2. Therefore, we only care about the order of the finite domain variables, i.e. the state variables.

Although the software package provides a so-called dynamic BDD variable ordering function [38] to optimize the order, an initial variable order is critical, otherwise the dynamic ordering function will not have as much effect as expected.

The method we used is that two state variables should be kept closer if their corresponding DES have more shared events. This method was found by Zhang in [51]. The central idea behind this method is that a BDD can be reduced more if two state variables that share more information are kept closer.

Assuming there are $k$ component DES in the high-level or a low-level of an HISC system, for example, we can arrange the state variables for all the DES by trying to make the value of Cross in the following expression as small as possible. The $C$ in the expression is a matrix storing the number of shared events between the $i^{\text {th }}$ component and the $j^{\text {th }}$ component in the current order. ${ }^{4}$

$$
\text { Cross }:=\sum_{i=1}^{k} \sum_{j=i+2}^{k} C[i, j] *(j-i-1)
$$

For a high-level or low-level with $k$ DES, there are $k$ ! different orders. It is impractical to compute Cross for all the possible orders on a personal computer for a even moderately large $k$ (e.g. $\left.k=50, k !=3 \times 10^{64}\right)$. Therefore, we use the sifting

\footnotetext{
${ }^{4}$ This expression is quite experimental, but it works well in our experience.
} 


\section{Master Thesis - R. Song - McMaster - Computing and Software}

algorithm [38] to compute only a very small number of the orders, and then choose the order with the smallest value for Cross among them as the initial order. Here we only adopt how the sifting algorithm swaps the variables. We then compute Cross for the order after each swapping. As a result, it has much less computation than to swap two variables in a BDD and then count the number of BDD nodes.

\subsection{Controller Implementation}

Let $\boldsymbol{\Phi}$ be the $n^{\text {th }}$ degree HISC-valid specification interface system that respects the alphabet partition given by Equation 3.1 and is composed of plant components $\mathbf{G}_{H}^{p}, \mathbf{G}_{L_{1}}^{p}, \ldots, \mathbf{G}_{L_{n}}^{p}$, specifications $\mathbf{E}_{H}, \mathbf{E}_{L_{1}}, \ldots, \mathbf{E}_{L_{n}}$, and interfaces $\mathbf{G}_{I_{1}}, \ldots, \mathbf{G}_{I_{n}}$. From Chapter 4 , we can synthesize a high-level proper supervisor $\mathbf{S}_{H}$ by making $\mathbf{S}_{H}$ represent $L_{m}\left(\mathcal{G}_{H}, \Gamma_{H}^{k}(\right.$ true $\left.)\right)$, where $k \in\{0,1, \ldots\}$ and $\Gamma_{H}^{k}($ true $)$ is the greatest fixpoint of $\Gamma_{H}$ with respect to $\left(\operatorname{Pred}\left(Q_{H}\right), \preceq\right)$, and synthesize a $j^{\text {th }}$ low-level proper supervisor $\mathbf{S}_{L_{j}}$ by making $\mathbf{S}_{L_{j}}$ represent $L_{m}\left(\mathcal{G}_{L_{j}}, \Gamma_{L_{j}}^{k_{j}}(\right.$ true $\left.)\right)$ for all $j \in\{1, \ldots, n\}$, where $k_{j} \in\{0,1, \ldots\}$ and $\Gamma_{L_{j}}^{k_{j}}($ true $)$ is the greatest fixpoint of $\Gamma_{L_{j}}$ with respect to $\left(\operatorname{Pred}\left(Q_{L_{j}}\right), \preceq\right)$. The supervisor SUP for the whole system would be SUP := $\mathbf{S}_{H}\left\|\mathbf{S}_{L_{1}}\right\| \cdots\left\|\mathbf{S}_{L_{n}}\right\| \mathbf{G}_{I_{1}}\|\cdots\| \mathbf{G}_{I_{n}}$. However, the automata-based supervisors $\mathbf{S}_{H}, \mathbf{S}_{L_{1}}, \ldots, \mathbf{S}_{L_{n}}$ could easily be very large, so that they are very difficult to implement. For example, $\mathbf{S}_{H}$ for the 3-2 AIP example in the next chapter has a state space size on the order of $10^{12}$.

From the synthesis algorithms in Chapter 4 , we can see that $\mathbf{S}_{H}$ can be obtained by trimming off states from the high-level $\mathcal{G}_{H}$, and $\mathbf{S}_{L_{j}}$ can be obtained by trimming off states from the $j^{\text {th }}$ low-level $\mathcal{G}_{L_{j}}, j \in\{1, \ldots, n\}$. Therefore, we can implement the supervisor for system $\boldsymbol{\Phi}$ in another way as follows.

For system $\boldsymbol{\Phi}$, let $P_{H}$ be the resulting predicate of the Algorithm 4.1 for the high- 


\section{Master Thesis - R. Song-McMaster-Computing and Software}

level, i.e. $P_{H}:=\Gamma_{H}^{k}($ true $)$ and $P_{L_{j}}$ be the result predicate of the Algorithm 4.3 for the $j^{\text {th }} \operatorname{low}-\operatorname{level}(j \in\{1, \ldots, n\})$, i.e. $P_{L_{j}}:=\Gamma_{L_{j}}^{k_{j}}($ true $)$. Assume ${ }^{5} P_{H} \not \equiv$ false and $P_{L_{j}} \not \equiv$ false for all $j \in\{1, \ldots, n\}$. In the following, $j$ is always an index with range $\{1, \ldots, n\}$.

Let $\mathbf{G}:=\left(Q, \Sigma, \delta, q_{0}, Q_{m}\right)$ be the DES tuple for the synchronous product of all the DES in system $\boldsymbol{\Phi}$, then $\Sigma$ is defined as in Equation 3.1 and $\Sigma:=\Sigma_{c} \dot{\cup} \Sigma_{u}$. A state $q \in Q$ can be represented as a tuple

$$
\left(z_{H}, y_{H}, z_{L_{1}}, \ldots, z_{L_{n}}, y_{L_{1}}, \ldots, y_{L_{n}}, x_{1}, \ldots, x_{n}\right)
$$

where $z_{H} \in Z_{H}, y_{H} \in Y_{H}, z_{L_{1}} \in Z_{L_{1}}, \ldots, z_{L_{n}} \in Z_{L_{n}}, y_{L_{1}} \in Y_{L_{1}}, \ldots, y_{L_{n}} \in Y_{L_{n}}, x_{1} \in$ $X_{1}, \ldots, x_{n} \in X_{n}$ (see Section 4.1 for more information).

For each $\sigma \in \Sigma$, define the control predicate $f_{\sigma} \in \operatorname{Pred}(Q)$ for $\sigma$ as following:

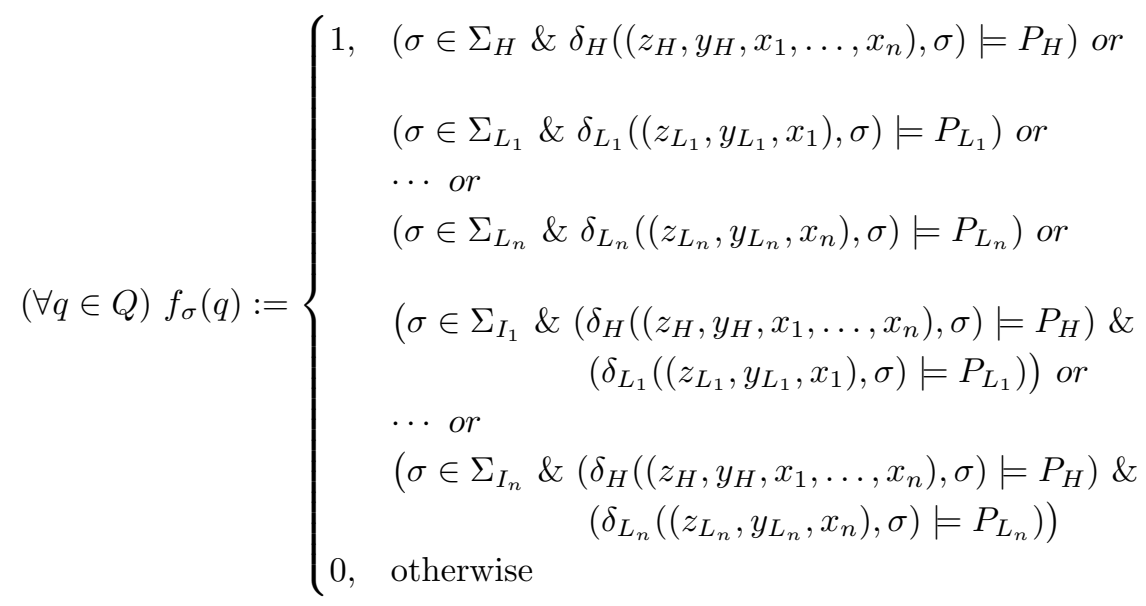

Equation 6.9 is based on the event partition in Equation (3.1), and the fact that $\mathcal{G}_{H}$ is defined over $\Sigma_{I H}$ and $\mathcal{G}_{L_{j}}$ is defined over $\Sigma_{I L_{j}}$.

For $q \in Q$ and $\sigma \in \Sigma, \sigma$ should then be enabled at state $q$ if $f_{\sigma}(q)=1$. Therefore, from the control predicate $f_{\sigma}$ for all $\sigma \in \Sigma$, we can decide the control action. To

\footnotetext{
${ }^{5}$ If $P_{H}$ or any of $P_{L_{j}}(j \in\{1, \ldots, n\})$ are equal to false, this would mean no supervisor exists, so we would have nothing to implement.
} 


\section{Master Thesis - R. Song-McMaster - Computing and Software}

implement, we only need to use the predicates for $\sigma \in \Sigma_{c}$, as our supervisor is controllable by our synthesis process and Theorem 3.2. Thus it will never try to disable $\sigma \in \Sigma_{u}$ when $\sigma$ is possible in the plant. Note that each of these predicates can also be represented as a BDD, and usually the BDD representation is much smaller than the automata representation. However, to evaluate $f_{\sigma}$, we need to know the current state of $\mathbf{G}$.

We will use all the component automata models (component DES) $\mathbf{G}_{H}^{p}, \mathbf{G}_{L_{1}}^{p}, \ldots$, $\mathbf{G}_{L_{n}}^{p}, \mathbf{E}_{H}, \mathbf{E}_{L_{1}}, \ldots, \mathbf{E}_{L_{n}}, \mathbf{G}_{I_{1}}, \ldots, \mathbf{G}_{I_{n}}$ in system $\mathbf{\Phi}$ to trace the state of the physical plant. In other words, we will use the automata models as a kind of observer to provide our controller with the needed system state information. Note that the plant DES and specification DES are usually the synchronous product of component DES again. For example, the high-level plant in the AIP example in the next chapter is composed of 19 component DES. Although the synchronous product of them could be very large, the individual DES are usually very small.

Let $\Sigma_{c}=\left\{\sigma_{1}, \sigma_{2}, \ldots, \sigma_{k}\right\}(k \in\{0,1, \ldots\})$. Figure 6.4 shows the overall picture of what our controller will look like. In the diagram, the plant models $\mathbf{G}_{H}^{p}, \mathbf{G}_{L_{1}}^{p}, \ldots, \mathbf{G}_{L_{n}}^{p}$ and specification models $\mathbf{E}_{H}, \mathbf{E}_{L_{1}}, \ldots, \mathbf{E}_{L_{n}}$ would be replaced by their own component DES. We also assume that all the events exist as part of the physical plant. There are many issues about the controller implementations which are beyond the scope of this thesis; here we only want to show that we do not need to build the automata supervisors $\mathbf{S}_{H}$ and $\mathbf{S}_{L_{j}}(j \in\{1, \ldots, n\})$ to implement our controller. Interested readers are referred to [20] to see an example.

Equation 6.9 can actually be simplified. Notice that Point 3 and Point 4 in the interface consistent definition are very similar to the definitions of the controllable language. As the supervisor of the system never disable the uncontrollable events, the supervisor for the high-level should never disable the answer events, and the 
Master Thesis - R. Song - McMaster - Computing and Software

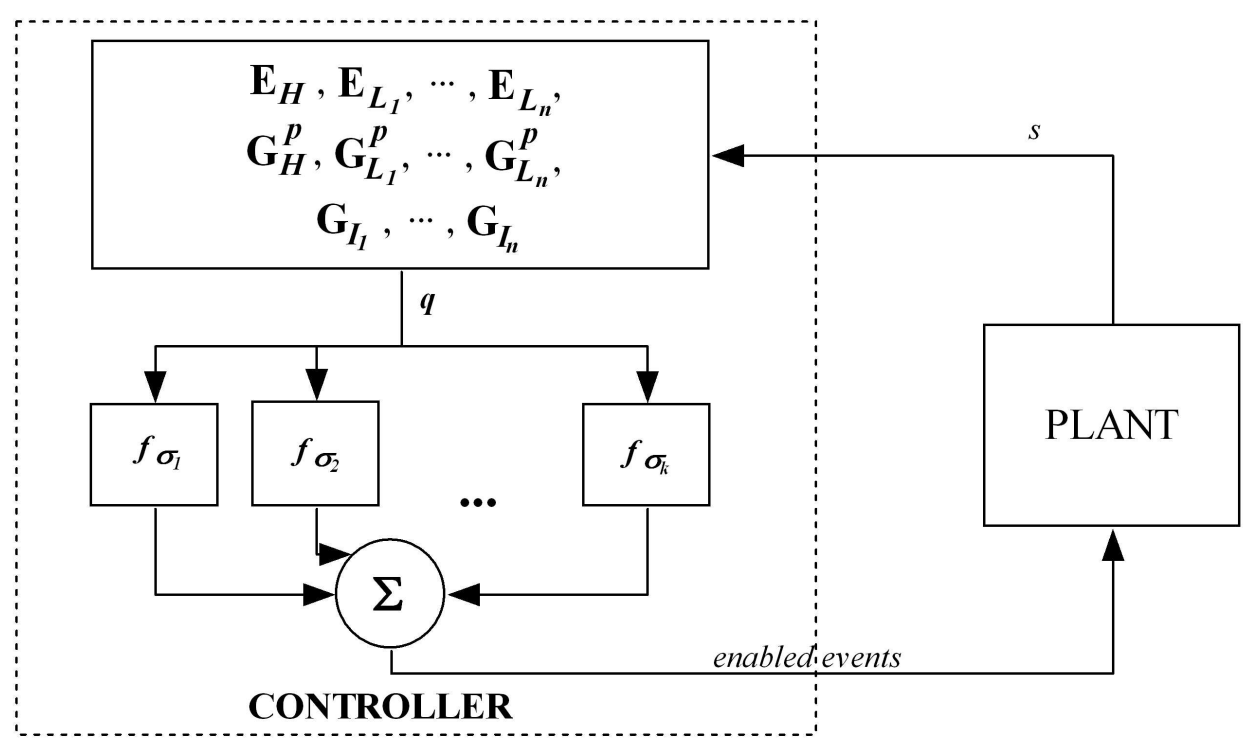

Figure 6.4: Control diagram

supervisor for the $j^{\text {th }}$ low-level should never disable the request events in the $j^{\text {th }}$ low-level.

Proposition 6.1. For system $\mathbf{\Phi}$, let $\mathbf{G}:=\left(Q, \Sigma, \delta, q_{0}, Q_{m}\right)$ be the DES tuple for the synchronous product of all the DES in system $\mathbf{\Phi}$. Let $q \in Q$ be a reachable state with a tuple as in Equation 6.8, $s \in \Sigma^{*}$ and $q=\delta\left(q_{0}, s\right)$. If the following three points are true:

- $s:=\sigma_{1} \sigma_{2} \cdots \sigma_{k}$, where $k \in\{0,1, \ldots\}(k=0$ means $s=\epsilon)$ and $\sigma_{1}, \sigma_{2}, \ldots, \sigma_{k} \in \Sigma$;

- $q_{1}:=\delta\left(q_{0}, \sigma_{1}\right), q_{2}:=\delta\left(q_{1}, \sigma_{2}\right), \ldots, q=q_{k}:=\delta\left(q_{k-1}, \sigma_{k}\right)$, where $q_{1}, q_{2}, \ldots, q_{k} \in Q$;

- $f_{\sigma_{1}}\left(q_{0}\right) \equiv 1, f_{\sigma_{2}}\left(q_{1}\right) \equiv 1, \ldots, f_{\sigma_{k}}\left(q_{k-1}\right) \equiv 1$,

then the following two points hold:

1. $(\forall j \in\{1, \ldots, n\})\left(\forall \rho \in \Sigma_{R_{j}}\right)$ $\left(\delta_{H}\left(\left(z_{H}, y_{H}, x_{1}, \ldots, x_{n}\right), \rho\right) \models P_{H}\right) \Rightarrow\left(\delta_{L_{j}}\left(\left(z_{L_{j}}, y_{L_{j}}, x_{j}\right), \rho\right) \models P_{L_{j}}\right)$ 
Master Thesis - R. Song - McMaster - Computing and Software

2. $(\forall j \in\{1, \ldots, n\})\left(\forall \alpha \in \Sigma_{A_{j}}\right)$

$$
\left(\delta_{L_{j}}\left(\left(z_{L_{j}}, y_{L_{j}}, x_{j}\right), \alpha\right) \models P_{L_{j}}\right) \Rightarrow\left(\delta_{H}\left(\left(z_{H}, y_{H}, x_{1}, \ldots, x_{n}\right), \alpha\right) \models P_{H}\right)
$$

\section{proof:}

1. Show Point 1 holds.

Let $j \in\{1, \ldots, n\}, \rho \in \Sigma_{R_{j}}$.

Assume $\delta_{H}\left(\left(z_{H}, y_{H}, x_{1}, \ldots, x_{n}\right), \rho\right) \models P_{H}$.

Must show this implies $\delta_{L_{j}}\left(\left(z_{L_{j}}, y_{L_{j}}, x_{j}\right), \rho\right) \models P_{L_{j}}$.

Let function $\mathrm{PRJ}_{I L j}: \Sigma^{*} \rightarrow \Sigma_{I L_{j}}^{*}$ be a natural projection.

From (2), we have $\delta_{H}\left(\left(z_{H}, y_{H}, x_{1}, \ldots, x_{n}\right), \rho\right) \models P_{H}$

$\Rightarrow \delta_{H}\left(\left(z_{H}, y_{H}, x_{1}, \ldots, x_{n}\right), \rho\right) !$

$\Rightarrow \xi_{j}^{h}\left(x_{j}, \rho\right) !, \quad$ as $\mathcal{G}_{H}:=\mathbf{E}_{H} \times \mathbf{G}_{H}^{p} \times \mathbf{G}_{I_{1}}^{h} \times \cdots \times \mathbf{G}_{I_{n}}^{h}$

$\Rightarrow \xi_{j}\left(x_{j}, \rho\right) !, \quad$ by definition of $\mathbf{G}_{I_{j}}^{h}$ (see Section 3.2) and the fact $\rho \in \Sigma_{R_{j}}$

$\Rightarrow \xi_{j}^{l}\left(x_{j}, \rho\right) !, \quad$ by definition of $\mathbf{G}_{I_{j}}^{l}$

$\Rightarrow \operatorname{PRJ}_{I L j}(s) \rho \in L\left(\mathbf{G}_{I_{j}}^{l}\right), \quad$ by (1) and definition of $\mathbf{G}$

From the definition in Equation 6.9 and (1), we have

$$
\operatorname{PRJ}_{I L j}(s) \in L\left(\mathcal{G}_{L_{j}}, P_{L_{j}}\right) .
$$

By Theorem 4.2, we know that $L_{m}\left(\mathcal{G}_{L_{j}}, P_{L_{j}}\right)$ is $j^{\text {th }}$ low-level interface controllable. From Definition 4.16 , we know that $L_{m}\left(\mathcal{G}_{L_{j}}, P_{L_{j}}\right)$ is $j^{\text {th }}$ low-level P4 interface controllable. From Definition 4.10, we know that $\overline{L_{m}\left(\mathcal{G}_{L_{j}}, P_{L_{j}}\right)}$ is $j^{\text {th }}$ low-level P4 interface controllable. By Corollary 4.4, we have $\overline{L_{m}\left(\mathcal{G}_{L_{j}}, P_{L_{j}}\right)}=$ $L\left(\mathcal{G}_{L_{j}}, P_{L_{j}}\right)$. Thus we know that $L\left(\mathcal{G}_{L_{j}}, P_{L_{j}}\right)$ is $j^{\text {th }}$ low-level P4 interface controllable. By Definition 4.10, (3) and (4), we now have 
Master Thesis - R. Song - McMaster - Computing and Software

$\operatorname{PRJ}_{I L j}(s) \rho \in L\left(\mathcal{G}_{L_{j}}, P_{L_{j}}\right)$

$\Rightarrow \delta_{L_{j}}\left(\left(z_{L_{j}}, y_{L_{j}}, x_{j}\right), \rho\right) \models P_{L_{j}}$

2. Show Point 2 holds.

Let $j \in\{1, \ldots, n\}, \alpha \in \Sigma_{A_{j}}$.

Assume $\delta_{L_{j}}\left(\left(z_{L_{j}}, y_{L_{j}}, x_{j}\right), \alpha\right) \models P_{L_{j}}$.

Must show this implies $\delta_{H}\left(\left(z_{H}, y_{H}, x_{1}, \ldots, x_{n}\right), \alpha\right) \models P_{H}$.

Let function $\mathrm{PRJ}_{I H}: \Sigma^{*} \rightarrow \Sigma_{I H}^{*}$ be a natural projection.

From (5), we have $\delta_{L_{j}}\left(\left(z_{L_{j}}, y_{L_{j}}, x_{j}\right), \alpha\right) \models P_{L_{j}}$

$\Rightarrow \delta_{L_{j}}\left(\left(z_{L_{j}}, y_{L_{j}}, x_{j}\right), \alpha\right) !$

$\Rightarrow \xi_{j}^{l}\left(x_{j}, \alpha\right) !, \quad$ as $\mathcal{G}_{L_{j}}:=\mathbf{E}_{L_{j}} \times \mathbf{G}_{L_{j}}^{p} \times \mathbf{G}_{I_{j}}^{l}$

$\Rightarrow \xi_{j}\left(x_{j}, \alpha\right) !, \quad$ by definition of $\mathbf{G}_{I_{j}}^{l}$ (see Section 3.2) and the fact $\alpha \in \Sigma_{A_{j}}$

$\Rightarrow \xi_{j}^{h}\left(x_{j}, \alpha\right) !, \quad$ by definition of $\mathbf{G}_{I_{j}}^{h}$

$\Rightarrow \operatorname{PRJ}_{I H}(s) \alpha \in L\left(\mathbf{G}_{I_{j}}^{h}\right), \quad$ by (1) and definition of $\mathbf{G}$

From the definition in Equation 6.9 and (1), we have

$$
\operatorname{PRJ}_{I H}(s) \in L\left(\mathcal{G}_{H}, P_{H}\right) .
$$

By Theorem 4.1, we know that $L_{m}\left(\mathcal{G}_{H}, P_{H}\right)$ is high-level interface controllable. By Definition 4.2, we know that $\overline{L_{m}\left(\mathcal{G}_{H}, P_{H}\right)}$ is high-level interface controllable. By Corollary 4.2, we have $\overline{L_{m}\left(\mathcal{G}_{H}, P_{H}\right)}=L\left(\mathcal{G}_{H}, P_{H}\right)$. Thus we have $L\left(\mathcal{G}_{H}, P_{H}\right)$ is high-level interface controllable. By Definition 4.2, (6) and (7), we now have $\operatorname{PRJ}_{I H}(s) \alpha \in L\left(\mathcal{G}_{H}, P_{H}\right)$ $\Rightarrow \delta_{H}\left(\left(z_{H}, y_{H}, x_{1}, \ldots, x_{n}\right), \alpha\right) \models P_{H}$. 


\section{Master Thesis - R. Song - McMaster - Computing and Software}

From the above proposition, now for all $\sigma \in \Sigma_{c}$, we can redefine the predicate $f_{\sigma} \in \operatorname{Pred}(Q)$ as following:

$$
(\forall q \in Q) f_{\sigma}(q):= \begin{cases}1, \quad\left(\sigma \in \Sigma_{H} \cup \Sigma_{R_{1}} \cup \cdots \cup \Sigma_{R_{n}} \& \delta_{H}\left(\left(z_{H}, y_{H}, x_{1}, \ldots, x_{n}\right), \sigma\right) \models P_{H}\right) \text { or } \\ \quad\left(\sigma \in \Sigma_{L_{1}} \cup \Sigma_{A_{1}} \& \delta_{L_{1}}\left(\left(z_{L_{1}}, y_{L_{1}}, x_{1}\right), \sigma\right) \models P_{L_{1}}\right) \text { or } \\ \cdots \text { or } \\ \quad\left(\sigma \in \Sigma_{L_{n}} \cup \Sigma_{A_{n}} \& \delta_{L_{n}}\left(\left(z_{L_{n}}, y_{L_{n}}, x_{n}\right), \sigma\right) \models P_{L_{n}}\right) \\ 0, \quad \text { otherwise }\end{cases}
$$

This allows us to replace the set of global control predicates $f_{\sigma}$ with the set of local control predicates $f_{H_{\sigma}}$ and $f_{L_{j_{\sigma}}}$.

For each $\sigma \in \Sigma_{c} \cap\left(\Sigma_{H} \cup \Sigma_{R_{1}} \cup \cdots \cup \Sigma_{R_{n}}\right)$, we define the predicate $f_{H \sigma} \in \operatorname{Pred}\left(Q_{H}\right)$ as

$$
(\forall q \in Q) f_{H \sigma}\left(z_{H}, y_{H}, x_{1}, \ldots, x_{n}\right):= \begin{cases}1, & \delta_{H}\left(\left(z_{H}, y_{H}, x_{1}, \ldots, x_{n}\right), \sigma\right) \models P_{H} \\ 0, & \text { otherwise }\end{cases}
$$

For each $j \in\{1, \ldots, n\}, \sigma \in \Sigma_{c} \cap\left(\Sigma_{L_{j}} \cup \Sigma_{A_{j}}\right)$, we define the predicate $f_{L_{j_{\sigma}}} \in$ $\operatorname{Pred}\left(Q_{L_{j}}\right)$ as

$$
(\forall q \in Q) f_{L_{j} \sigma}\left(z_{L_{j}}, y_{L_{j}}, x_{j}\right):= \begin{cases}1, & \delta_{L_{j}}\left(\left(z_{L_{j}}, y_{L_{j}}, x_{j}\right), \sigma\right) \models P_{L_{j}} \\ 0, & \text { otherwise }\end{cases}
$$

Let $\Sigma_{c} \cap\left(\Sigma_{H} \cup \Sigma_{R_{1}} \cup \cdots \cup \Sigma_{R_{n}}\right):=\left\{\sigma_{1}, \sigma_{2}, \ldots, \sigma_{k_{H}}\right\}\left(k_{H} \in\{0,1, \ldots\}\right)$ and $\Sigma_{c} \cap$ $\left(\Sigma_{L_{j}} \cup \Sigma_{A_{j}}\right):=\left\{\sigma_{j-1}, \sigma_{j-2}, \ldots, \sigma_{j-k_{j}}\right\}\left(k_{j} \in\{0,1, \ldots\}\right)$ for $j \in\{1, \ldots, n\}$. The control diagram in Figure 6.4 can be modified as shown in Figure 6.5. Again, we assume all the events in the system exist as part of the physical plant.

For system $\boldsymbol{\Phi}$, for each $\sigma \in \Sigma_{c} \cap\left(\Sigma_{H} \cup \Sigma_{R_{1}} \cup \cdots \cup \Sigma_{R_{n}}\right)$, the predicate $f_{H \sigma}$ can be computed by

$$
f_{H \sigma}:=\delta_{H}^{-1}\left(P_{H}, \sigma\right)
$$


Master Thesis - R. Song - McMaster - Computing and Software

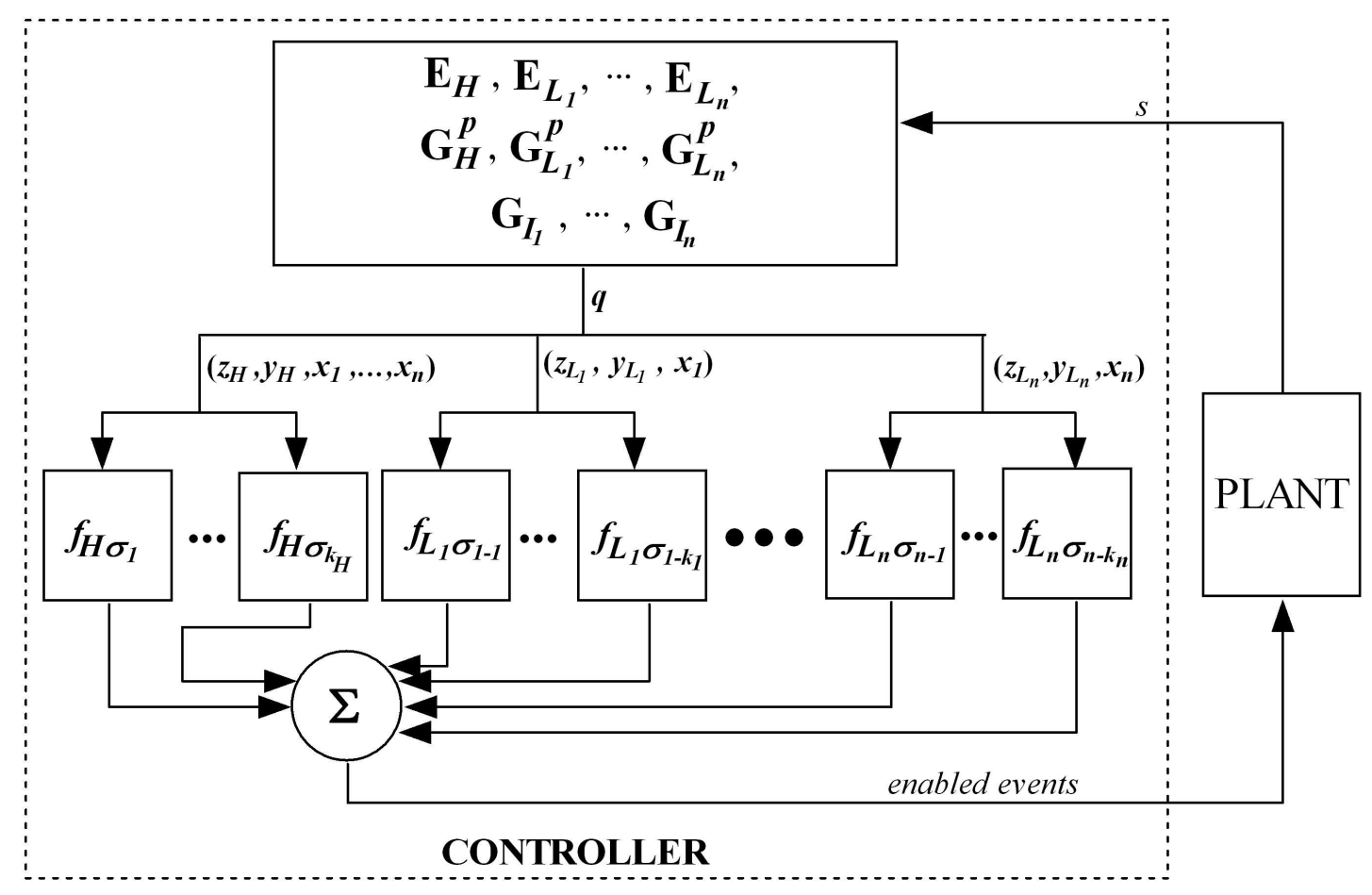

Figure 6.5: New control diagram

Let $\left(\boldsymbol{v}_{H_{\sigma}}, \boldsymbol{v}_{H_{\sigma}}^{\prime}, N_{H_{\sigma}}\right)$ be the transition tuple for $\sigma$ in the high-level $\mathcal{G}_{H}$, then the predicate $f_{H \sigma}$ can be computed symbolically by

$$
f_{H \sigma}=\exists \boldsymbol{v}_{H_{\sigma}}^{\prime}\left(N_{H_{\sigma}} \wedge\left(P_{H}\left[\boldsymbol{v}_{H_{\sigma}} \rightarrow \boldsymbol{v}_{H_{\sigma}}^{\prime}\right]\right)\right) .
$$

For system $\boldsymbol{\Phi}$, for each $j \in\{1, \ldots, n\}, \sigma \in \Sigma_{c} \cap\left(\Sigma_{L_{j}} \cup \Sigma_{A_{j}}\right)$, the predicate $P_{L_{j_{\sigma}}}$ can be computed by

$$
f_{L_{j_{\sigma}}}:=\delta_{L_{j}}^{-1}\left(P_{L_{j}}, \sigma\right)
$$

Let $\left(\boldsymbol{v}_{L_{j_{\sigma}}}, \boldsymbol{v}_{L_{j_{\sigma}}}^{\prime}, N_{L_{j_{\sigma}}}\right)$ be the transition tuple for $\sigma$ in the $j^{\text {th }}$ low-level $\mathcal{G}_{L_{j}}$, then the predicate $f_{L_{j} \sigma}$ can be computed symbolically by

$$
f_{L_{j} \sigma}=\exists \boldsymbol{v}_{L_{j_{\sigma}}}^{\prime}\left(N_{L_{j_{\sigma}}} \wedge\left(P_{L_{j}}\left[\boldsymbol{v}_{L_{j_{\sigma}}} \rightarrow \boldsymbol{v}_{L_{j_{\sigma}}}^{\prime}\right]\right)\right) .
$$


Master Thesis - R. Song - McMaster - Computing and Software

\subsubsection{Simplifying Control Predicates}

Let $\mathbf{G}:=\left(Q, \Sigma, \delta, q_{0}, Q_{m}\right)$ be the DES tuple for the synchronous product of all the DES in system $\boldsymbol{\Phi}$. If we use local control predicates as in Figure 6.5, then a state $q \in Q$ (in the form of Equation 6.8) can be reached in the controlled system only if $\left(z_{H}, y_{H}, x_{1}, \ldots, x_{n}\right) \models P_{H}$ and for all $j \in\{1, \ldots, n\},\left(z_{L_{j}}, y_{L_{j}}, x_{j}\right) \models P_{L_{j}}$. Based on this fact, we can simplify the control predicate for all $\sigma \in \Sigma_{c}$ as follows:

1. $\sigma \in \Sigma_{c} \cap\left(\Sigma_{H} \cup \Sigma_{R_{1}} \cup \cdots \cup \Sigma_{R_{n}}\right)$

If $\sigma \in \Sigma_{c} \cap\left(\Sigma_{H} \cup \Sigma_{R_{1}} \cup \cdots \cup \Sigma_{R_{n}}\right), f_{H_{\sigma}}$ is required only for the state set $\left\{q \in Q \mid\left(z_{H}, y_{H}, x_{1}, \ldots, x_{n}\right) \models P_{H}\right\}$. For the other states in $Q$, the return value of $f_{H_{\sigma}}$ can either be 0 or 1 , as these states are unreachable.

For a state $q \in Q$ in the form of Equation 6.8, let $q_{H}:=\left(z_{H}, y_{H}, x_{1}, \ldots, x_{n}\right)$ be the high-level part of $q$. Let $d_{H_{\sigma}} \in \operatorname{Pred}\left(Q_{H}\right)$ and $d_{H_{\sigma}}:=P_{H}$. A predicate $f_{H_{\sigma}}^{\prime} \in \operatorname{Pred}\left(Q_{H}\right)$ satisfying $d_{H_{\sigma}} \wedge f_{H_{\sigma}}^{\prime} \equiv d_{H_{\sigma}} \wedge f_{H_{\sigma}}$ will have the same effect as $f_{H_{\sigma}}$ has, because for state $q$, if $d_{H_{\sigma}}\left(q_{H}\right) \equiv 1$, then $f_{H_{\sigma}} \equiv f_{H_{\sigma}}^{\prime}$, otherwise we do not care the value of $f_{H_{\sigma}}\left(q_{H}\right)$ as $q$ is unreachable. In other words, $f_{H_{\sigma}}^{\prime}$ and $f_{H_{\sigma}}$ are equal on the domain defined by the constraint $d_{H_{\sigma}}[12]$.

Predicate $f_{H_{\sigma}}^{\prime}$ can be computed by the function bdd_simplify in the BDD package we are using when passed $d_{H_{\sigma}}$ and $f_{H_{\sigma}}$ as arguments. ${ }^{6}$

2. $\sigma \in \Sigma_{c} \cap\left(\Sigma_{L_{j}} \cup \Sigma_{A_{j}}\right), j \in\{1, \ldots, n\}$

If $\sigma \in \Sigma_{c} \cap\left(\Sigma_{L_{j}} \cup \Sigma_{A_{j}}\right)$, then $f_{L_{j \sigma}}$ is required only for the state set $\{q \in$ $\left.Q \mid\left(z_{L_{j}}, y_{L_{j}}, x_{L_{j}}\right) \models P_{L_{j}}\right\}$. For the other states in $Q$, the return value of $f_{L_{j_{\sigma}}}$ can either be 0 or 1 , as those states are unreachable.

\footnotetext{
${ }^{6}$ Usually, the BDD for $f_{H_{\sigma}}^{\prime}$ is smaller than the BDD for $f_{H_{\sigma}}$, but according to the documentation of the $B u D D y$ package, it is not guaranteed.
} 


\section{Master Thesis - R. Song-McMaster-Computing and Software}

For a state $q \in Q$ in the form of Equation 6.8, let $q_{L_{j}}:=\left(z_{L_{j}}, y_{L_{j}}, x_{L_{j}}\right)$ be the $j^{\text {th }}$ low-level part of $q$. Let $d_{L_{j_{\sigma}}} \in \operatorname{Pred}\left(Q_{L_{j}}\right)$ and $d_{L_{j_{\sigma}}}:=P_{L_{j}}$. A predicate $f_{L_{j \sigma}}^{\prime} \in \operatorname{Pred}\left(Q_{L_{j}}\right)$ satisfying $d_{L_{j_{\sigma}}} \wedge f_{L_{j \sigma}}^{\prime} \equiv d_{L_{j_{\sigma}}} \wedge f_{L_{j \sigma}}$ will have the same effect as $f_{L_{j \sigma}}$ has, because for state $q$, if $d_{L_{j \sigma}}\left(q_{L_{j}}\right) \equiv 1$, then $f_{L_{j \sigma}} \equiv f_{L_{j \sigma}}^{\prime}$, otherwise we do not care the value of $f_{L_{j \sigma}}\left(q_{L_{j}}\right)$ as $q$ is unreachable. That is, $f_{L_{j \sigma}}^{\prime}$ and $f_{L_{j \sigma}}$ are equal on the domain defined by the constraint $d_{L_{j \sigma}}$.

Predicate $f_{L_{j \sigma}}^{\prime}$ can be computed by the function bdd_simplify in the BDD package we are using when passed $d_{L_{j \sigma}}$ and $f_{L_{j \sigma}}$ as arguments.

For system $\boldsymbol{\Phi}$, let $\sigma \in \Sigma_{c}$. If $\sigma$ is not permitted at a state in a plant component means that $\sigma$ can not happen at that state physically, then the control predicates $f_{H \sigma}$ or $f_{L_{j_{\sigma}}}(j \in\{1, \ldots, n\})$ can be further simplified. In the control diagram in Figure 6.5, if $\sigma \in \Sigma_{c} \cap\left(\Sigma_{H} \cup \Sigma_{R_{1}} \cup \cdots \cup \Sigma_{R_{n}}\right), f_{H \sigma}$ has an actual effect only at a state $q \in Q$ (in the form of Equation 6.8) with $\eta_{H}\left(y_{H}, \sigma\right)$ !, i.e. $\sigma$ transition is defined at the high-level plant part of $q$. Also if $\sigma \in \Sigma_{c} \cap\left(\Sigma_{L_{j}} \cup \Sigma_{A_{j}}\right)$, then $f_{L_{j_{\sigma}}}$ has an actual effect only when a state $q \in Q$ (of the form of Equation 6.8) with $\eta_{L_{j}}\left(y_{L_{j}}, \sigma\right)$ ! i.e. $\sigma$ transition is defined at the $j^{\text {th }}$ low-level plant part of $q$.

For system $\boldsymbol{\Phi}$, let $\sigma \in \Sigma_{c}$. Assume $\sigma$ can not happen if a plant component does not permit it. By the above analysis, we have the following:

1. $\sigma \in \Sigma_{c} \cap\left(\Sigma_{H} \cup \Sigma_{R_{1}} \cup \cdots \cup \Sigma_{R_{n}}\right)$

If $\sigma \in \Sigma_{c} \cap\left(\Sigma_{H} \cup \Sigma_{R_{1}} \cup \cdots \cup \Sigma_{R_{n}}\right), f_{H_{\sigma}}$ is required only for the state set $\left\{q \in Q \mid\left(z_{H}, y_{H}, x_{1}, \ldots, x_{n}\right) \models P_{H} \& \eta_{H}\left(y_{H}, \sigma\right) !\right\}$. For the other states in $Q$, the return value of $f_{H_{\sigma}}$ can either be 0 or 1 as either the states are unreachable or $\sigma$ can not physically occur in the high-level plant.

For a state $q \in Q$ in the form of Equation 6.8 , let $q_{H}:=\left(z_{H}, y_{H}, x_{1}, \ldots, x_{n}\right)$ be the high-level part of $q$. Let $d_{H_{\sigma}}^{\prime} \in \operatorname{Pred}\left(Q_{H}\right)$ and $d_{H_{\sigma}}^{\prime}:=P_{H} \wedge \operatorname{pr}\left(\left\{q_{H} \in\right.\right.$ 
Master Thesis - R. Song - McMaster - Computing and Software

$\left.\left.Q_{H} \mid \eta_{H}\left(y_{H}, \sigma\right) !\right\}\right)$. A predicate $f_{H_{\sigma}}^{\prime \prime} \in \operatorname{Pred}\left(Q_{H}\right)$ satisfying $d_{H_{\sigma}}^{\prime} \wedge f_{H_{\sigma}}^{\prime \prime} \equiv d_{H_{\sigma}}^{\prime} \wedge f_{H_{\sigma}}$ will have the same effect as $f_{H_{\sigma}}$ has, because for the state $q$, if $d_{H_{\sigma}}^{\prime}\left(q_{H}\right) \equiv 1$, then $f_{H_{\sigma}} \equiv f_{H_{\sigma}}^{\prime \prime}$, otherwise we do not care the value of $f_{H_{\sigma}}\left(q_{H}\right)$ as either $q$ is unreachable or $\sigma$ can not physically occur in the high-level plant. That is, $f_{H_{\sigma}}^{\prime \prime}$ and $f_{H_{\sigma}}$ are equal on the domain defined by the constraint $d_{H_{\sigma}}^{\prime}$.

We can also simplify $f_{H_{\sigma}}$ as $f_{H_{\sigma}}^{\prime \prime \prime}$ in the following proposition.

Proposition 6.2. For system $\boldsymbol{\Phi}$, let $\sigma \in \Sigma_{c} \cap\left(\Sigma_{H} \cup \Sigma_{R_{1}} \cup \cdots \cup \Sigma_{R_{n}}\right)$. For each $q \in Q$ in the form of Equation 6.8, let $q_{H}:=\left(z_{H}, y_{H}, x_{1}, \ldots, x_{n}\right)$ be the high-level part of q. Let $d_{H_{\sigma}}^{\prime \prime} \in \operatorname{Pred}\left(Q_{H}\right)$ and $d_{H_{\sigma}}^{\prime \prime}:=P_{H} \wedge \operatorname{pr}\left(\left\{q_{H} \in Q_{H} \mid \delta_{H}\left(q_{H}, \sigma\right) !\right\}\right)$. Let $F_{H_{\sigma}} \in \operatorname{Pred}\left(Q_{H}\right)$ satisfying $d_{H_{\sigma}}^{\prime \prime} \wedge F_{H_{\sigma}} \equiv d_{H_{\sigma}}^{\prime \prime} \wedge f_{H_{\sigma}}$, then $f_{H_{\sigma}}^{\prime \prime \prime}:=F_{H_{\sigma}} \wedge \operatorname{pr}\left(\left\{q_{H} \in\right.\right.$ $\left.\left.Q_{H} \mid \zeta_{H} \times \xi_{1}^{h} \times \ldots \times \xi_{n}^{h}\left(\left(z_{H}, x_{1}, \ldots, x_{n}\right), \sigma\right) !\right\}\right)$ and $f_{H_{\sigma}}$ are also equal on the domain defined by the constraint $d_{H_{\sigma}}^{\prime}$.

\section{proof:}

Sufficient to show that $d_{H_{\sigma}}^{\prime} \wedge f_{H_{\sigma}}^{\prime \prime \prime} \equiv d_{H_{\sigma}}^{\prime} \wedge f_{H_{\sigma}}$. We show this by a series of transformations starting from the left hand side of the equation.

$$
\begin{aligned}
& d_{H_{\sigma}}^{\prime} \wedge f_{H_{\sigma}}^{\prime \prime \prime} \\
& \equiv d_{H_{\sigma}}^{\prime \prime} \wedge F_{H_{\sigma}} \wedge \operatorname{pr}\left(\left\{q_{H} \in Q_{H} \mid \zeta_{H} \times \xi_{1}^{h} \times \ldots \times \xi_{n}^{h}\left(\left(z_{H}, x_{1}, \ldots, x_{n}\right), \sigma\right) !\right\}\right) \\
& \equiv P_{H} \wedge \operatorname{pr}\left(\left\{q_{H} \in Q_{H} \mid \eta_{H}\left(y_{H}, \sigma\right) !\right\}\right) \wedge F_{H_{\sigma}} \wedge \\
& \quad \operatorname{pr}\left(\left\{q_{H} \in Q_{H} \mid \zeta_{H} \times \xi_{1}^{h} \times \ldots \times \xi_{n}^{h}\left(\left(z_{H}, x_{1}, \ldots, x_{n}\right), \sigma\right) !\right\}\right), \text { by definition of } d_{H_{\sigma}}^{\prime} \\
& \equiv P_{H} \wedge \operatorname{pr}\left(\left\{q_{H} \in Q_{H} \mid \delta_{H}\left(\left(z_{H}, y_{H}, x_{1}, \ldots, x_{n}\right), \sigma\right) !\right\}\right) \wedge F_{H_{\sigma}} \\
& \equiv d_{H_{\sigma}}^{\prime \prime} \wedge F_{H_{\sigma}}, \quad \text { by definition of } d_{H_{\sigma}}^{\prime \prime} \\
& \equiv d_{H_{\sigma}}^{\prime \prime} \wedge f_{H_{\sigma}}, \quad \text { as } d_{H_{\sigma}}^{\prime \prime} \wedge F_{H_{\sigma}} \equiv d_{H_{\sigma}}^{\prime \prime} \wedge f_{H_{\sigma}} \\
& \equiv P_{H} \wedge \operatorname{pr}\left(\left\{q_{H} \in Q_{H} \mid \delta_{H}\left(q_{H}, \sigma\right) !\right\}\right) \wedge f_{H_{\sigma}}, \quad \text { by definition of } d_{H_{\sigma}}^{\prime \prime}
\end{aligned}
$$




\section{Master Thesis - R. Song-McMaster-Computing and Software}

By the definition of $f_{H_{\sigma}}$, we know that for all $q_{H} \in Q_{H}, f_{H_{\sigma}}\left(q_{H}\right) \equiv 1$ if and only if $\delta_{H}\left(q_{H}, \sigma\right) \models P_{H}$, which implies that $\delta_{H}\left(q_{H}, \sigma\right)$ !. By the definition of $\preceq$, we thus have

$$
f_{H_{\sigma}} \preceq \operatorname{pr}\left(\left\{q_{H} \in Q_{H} \mid \delta_{H}\left(q_{H}, \sigma\right) !\right\}\right)
$$

By (2), we can conclude that (1) is equivalent to $P_{H} \wedge f_{H_{\sigma}}$

We know that for all $q_{H} \in Q_{H}, \delta_{H}\left(q_{H}, \sigma\right) ! \Rightarrow \eta_{H}\left(y_{H}, \sigma\right) !$, so we have

$$
\operatorname{pr}\left(\left\{q_{H} \in Q_{H} \mid \delta_{H}\left(q_{H}, \sigma\right) !\right\}\right) \preceq \operatorname{pr}\left(\left\{q_{H} \in Q_{H} \mid \eta_{H}\left(y_{H}, \sigma\right) !\right\}\right)
$$

By (2) and (4), we have

$$
f_{H_{\sigma}} \preceq \operatorname{pr}\left(\left\{q_{H} \in Q_{H} \mid \eta_{H}\left(y_{H}, \sigma\right) !\right\}\right) .
$$

By (5), we know that (3) is equivalent to

$P_{H} \wedge \operatorname{pr}\left(\left\{q_{H} \in Q_{H} \mid \eta_{H}\left(y_{H}, \sigma\right) !\right\}\right) \wedge f_{H_{\sigma}}$ $\equiv d_{H_{\sigma}}^{\prime} \wedge f_{H_{\sigma}}, \quad$ by definition of $d_{H_{\sigma}}^{\prime}$.

2. $\sigma \in \Sigma_{c} \cap\left(\Sigma_{L_{j}} \cup \Sigma_{A_{j}}\right), j \in\{1, \ldots, n\}$

If $\sigma \in \Sigma_{c} \cap\left(\Sigma_{L_{j}} \cup \Sigma_{A_{j}}\right)$, then $f_{L_{j \sigma}}$ is required only for the state set $\{q \in$ $\left.Q \mid\left(z_{L_{j}}, y_{L_{j}}, x_{L_{j}}\right) \models P_{L_{j}} \& \eta_{L_{j}}\left(y_{L_{j}}, \sigma\right) !\right\}$. For the other states in $Q$, the return value of $f_{L_{j_{\sigma}}}$ can either be 0 or 1 , as either the states are unreachable or $\sigma$ can not physically occur in the $j^{\text {th }}$ low-level plant.

For a state $q \in Q$ in the form of Equation 6.8 , let $q_{L_{j}}:=\left(z_{L_{j}}, y_{L_{j}}, x_{L_{j}}\right)$ be the $j^{\text {th }}$ low-level part of $q$. Let $d_{L_{j_{\sigma}}}^{\prime} \in \operatorname{Pred}\left(Q_{L_{j}}\right)$ and $d_{L_{j_{\sigma}}}^{\prime}:=P_{L_{j}} \wedge \operatorname{pr}\left(\left\{q_{L_{j}} \in\right.\right.$ $\left.\left.Q_{L_{j}} \mid \eta_{L_{j}}\left(y_{L_{j}}, \sigma\right) !\right\}\right)$. A predicate $f_{L_{j \sigma}}^{\prime \prime} \in \operatorname{Pred}\left(Q_{L_{j}}\right)$ satisfying $d_{L_{j_{\sigma}}}^{\prime} \wedge f_{L_{j \sigma}}^{\prime \prime} \equiv d_{L_{j_{\sigma}}}^{\prime} \wedge$ 
Master Thesis - R. Song - McMaster - Computing and Software

$f_{L_{j \sigma}}$ will have the same effect as $f_{L_{j \sigma}}$ has, because for the state $q$, if $d_{L_{j \sigma}}^{\prime}\left(q_{L_{j}}\right) \equiv 1$, then $f_{L_{j \sigma}} \equiv f_{L_{j \sigma}}^{\prime \prime}$, otherwise we do not care the value of $f_{L_{j \sigma}}\left(q_{L_{j}}\right)$ as either $q$ is unreachable or $\sigma$ can not physically occur in the $j^{\text {th }}$ low-level plant. That is, $f_{L_{j \sigma}}^{\prime \prime}$ and $f_{L_{j \sigma}}$ are equal on the domain defined by the constraint $d_{L_{j \sigma}}^{\prime}$.

We can also simplify $f_{L_{j \sigma}}$ as $f_{L_{j \sigma}}^{\prime \prime \prime}$ in the following proposition.

Proposition 6.3. For system $\boldsymbol{\Phi}$, let $j \in\{1, \ldots, n\}$ and $\sigma \in \Sigma_{c} \cap\left(\Sigma_{L_{j}} \cup \Sigma_{A_{j}}\right)$. For each $q \in Q$ in the form of Equation 6.8, let $q_{L_{j}}:=\left(z_{L_{j}}, y_{L_{j}}, x_{L_{j}}\right)$ be the $j^{\text {th }}$ low-level part of q. Let $d_{L_{j \sigma}}^{\prime \prime}:=P_{L_{j}} \wedge \operatorname{pr}\left(\left\{q_{L_{j}} \in Q_{L_{j}} \mid \delta_{L_{j}}\left(q_{L_{j}}, \sigma\right) !\right\}\right)$. Let $F_{L_{j_{\sigma}}} \in \operatorname{Pred}\left(Q_{L_{j}}\right)$ satisfying $d_{L_{j \sigma}}^{\prime \prime} \wedge F_{L_{j_{\sigma}}} \equiv d_{L_{j \sigma}}^{\prime \prime} \wedge f_{L_{j_{\sigma}}}$, then $f_{L_{j_{\sigma}}}^{\prime \prime \prime}:=F_{L_{j_{\sigma}}} \wedge$ $\operatorname{pr}\left(\left\{q_{L_{j}} \in Q_{L_{j}} \mid \zeta_{L_{j}} \times \xi_{j}^{l}\left(\left(z_{L_{j}}, x_{j}\right), \sigma\right) !\right\}\right)$ and $f_{L_{j_{\sigma}}}$ are also equal on the domain defined by the constraint $d_{L_{j \sigma}}^{\prime}$.

\section{proof:}

Identical to the proof of Proposition 6.2 by substituting all the high-level predicates with their corresponding $j^{\text {th }}$ low-level predicates, $\delta_{H}$ with $\delta_{L_{j}}, \zeta_{H}$ with $\zeta_{L_{j}}, \eta_{H}$ with $\eta_{L_{j}}$, and $\xi_{1}^{h} \times \cdots \xi_{n}^{h}$ with $\xi_{j}^{l}$.

Our software tool (source code in Appendix A) produces $f_{H_{\sigma}}, f_{H_{\sigma}}^{\prime}$ and $f_{H_{\sigma}}^{\prime \prime \prime}$ for all events in $\Sigma_{c} \cap\left(\Sigma_{H} \cup \Sigma_{R_{1}} \cup \cdots \cup \Sigma_{R_{n}}\right)$, and $f_{L_{j_{\sigma}}}, f_{L_{j_{\sigma}}}^{\prime}$ and $f_{L_{j_{\sigma}}}^{\prime \prime \prime}$ for all events in $\Sigma_{c} \cap\left(\Sigma_{L_{j}} \cup \Sigma_{A_{j}}\right)$ for all $j \in\{1, \ldots, n\}$. In the rest of this thesis, we will refer to $f_{H_{\sigma}}^{\prime}$ and $f_{L_{j_{\sigma}}}^{\prime}$ as prime simplified control predicates and refer to $f_{H_{\sigma}}^{\prime \prime \prime}$ and $f_{L_{j_{\sigma}}}^{\prime \prime \prime}$ as triple-prime simplified control predicates. 


\section{Master Thesis - R. Song-McMaster-Computing and Software}

\subsection{A Small Example}

In this section, we present a small example for HISC synthesis and provide the final automata supervisors and control predicates. This example is only for the purpose of demonstrating algorithms, so it is a bit contrived.

This example is inspired from the transfer line example from [47]. The system diagram is shown in Figure 6.6. Components $m 1$ and $m 2$ are two machines. Component $t u$ is a test unit. Components $b 1$ and $b 2$ are two buffers with capacity 1.

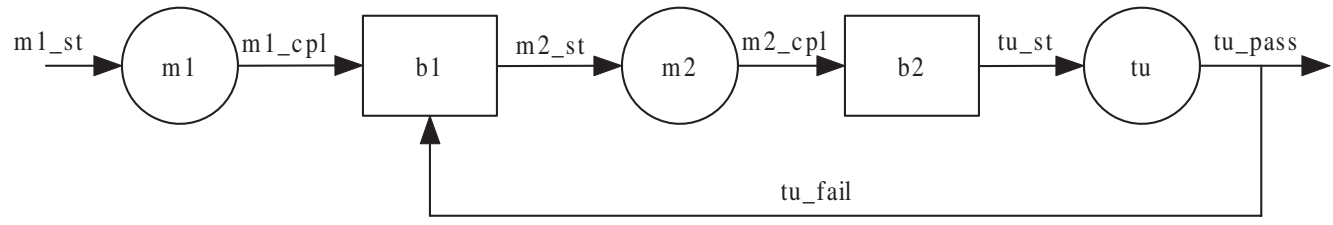

Figure 6.6: System diagram for small example

We model the system as an HISC system as follows. We treat $m 1$ and $m 2$ as two low-level subsystems with interfaces intfm1 and intfm2 as shown in Figure 6.7(e) and Figure 6.7(f), respectively.

The low-level subsystem for low-level $m 1$ is composed of one plant component, low_m1 (Figure 6.7(g)), and the low-level subsystem for low-level $m 2$ is composed of one plant component, low_m2(Figure 6.7(h)).

The high-level subsystem is composed of one plant component high_tu as shown in Figure 6.7(d) and three specification components high_m2, high_b1 and high_b2 as shown in Figure 6.7(c), Figure 6.7(a) and Figure 6.7(b) respectively. The specification high $\_\mathbf{m} 2$ tells that a part must be completely processed by $m 2$. The specification high_b1 and high_b2 are used to control the underflow and overflow of buffer b1 and buffer b2.

The event partition is shown as follows: 
Master Thesis - R. Song-McMaster - Computing and Software

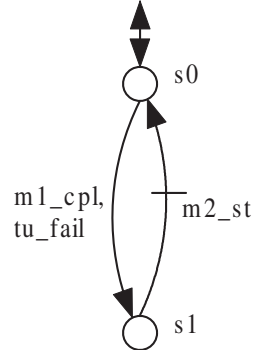

(a) high_b1 (spec)

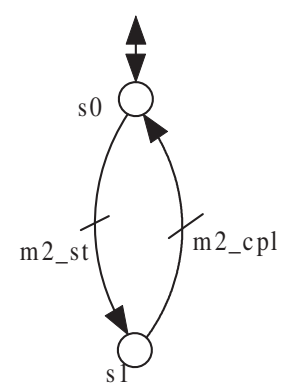

(c) high $\_$2 (spec)

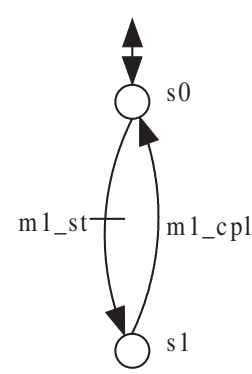

(e) Interface to low level $\mathrm{m} 1$

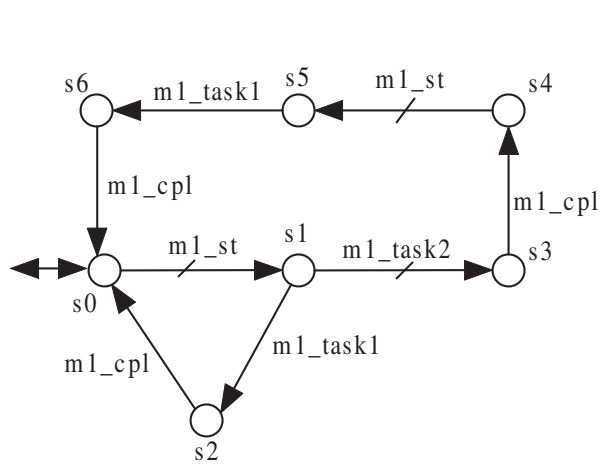

(g) low_m1 (plant)

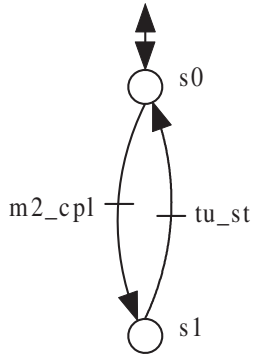

(b) high_b2 (spec)

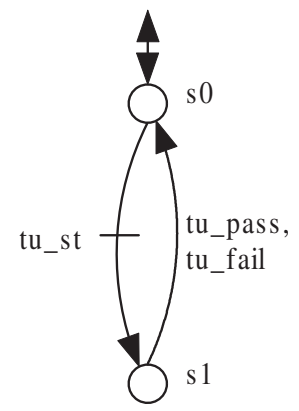

(d) high_tu (plant)

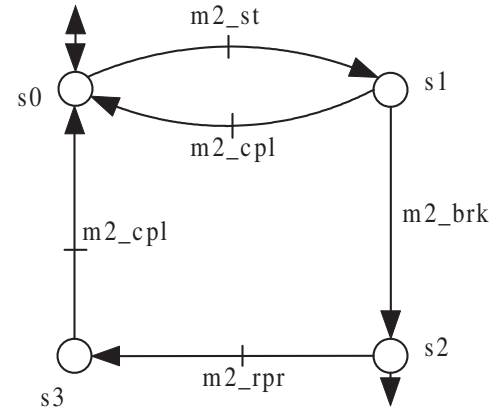

(f) Interface to low level m2

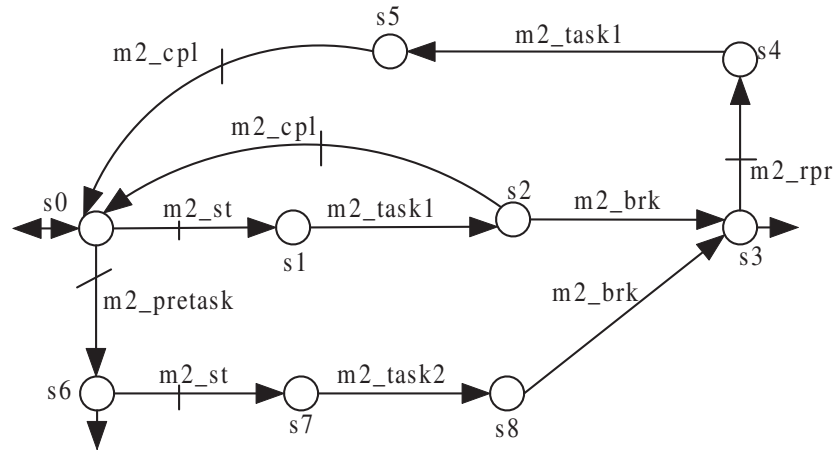

(h) low_m2 (plant)

Figure 6.7: The small example 


$$
\begin{aligned}
& \text { Master Thesis }-R . \text { Song }- \text { McMaster }- \text { Computing and Software } \\
\Sigma_{H}:= & \left\{t u \_s t, t u \_p a s s, t u \_f a i l\right\} \\
\Sigma_{R_{1}}:= & \left\{m 1 \_s t\right\}, \Sigma_{A_{1}}:=\left\{m 1 \_c p l\right\} \\
\Sigma_{R_{2}}:= & \left\{m 2 \_s t, m 2 \_r p r\right\}, \Sigma_{A_{2}}:=\left\{m 2 \_c p l, m 2 \_b r k\right\} \\
\Sigma_{L_{1}}:= & \left\{m 1 \_t a s k 1, m 1 \_t a s k 2\right\}, \Sigma_{L_{2}}:=\left\{m 2 \_p r e t a s k, m 2 \_t a s k 1, m 2 \_t a s k 2\right\}
\end{aligned}
$$

By using our software tool, we synthesized trim automata supervisors for the high-level and low-levels $m 1$ and $m 2$. They are shown in Figure 6.8, Figure 6.9, and Figure 6.10 respectively.

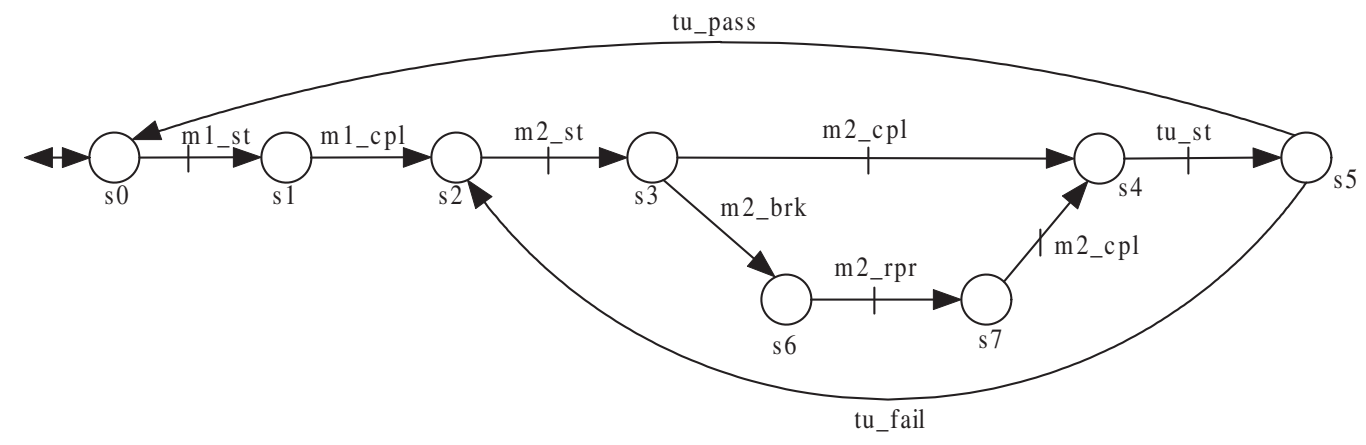

Figure 6.8: Synthesized high-level proper supervisor

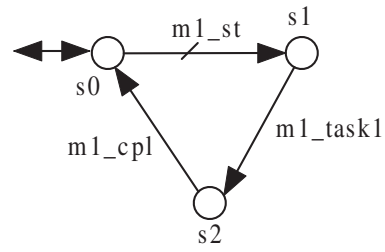

Figure 6.9: Synthesized low-level proper supervisor for low-level $m 1$

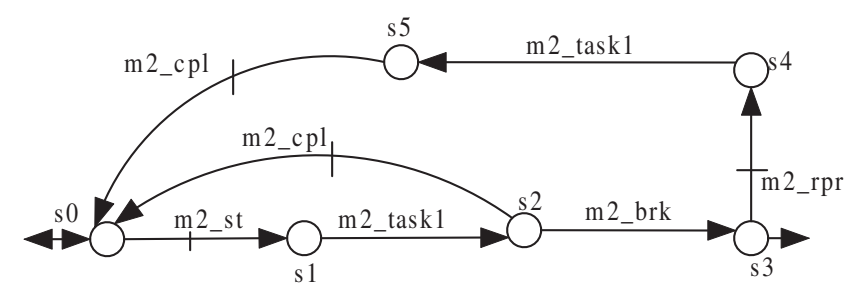

Figure 6.10: Synthesized low-level proper supervisor for low-level $m 2$ 


\section{Master Thesis - R. Song - McMaster - Computing and Software}

Although event $m 2 \_c p l$ is controllable, notice that the high-level supervisor can not disable it when intfm2 is at state $s 1$ or $s 3$. This is because $m 2 \_c p l$ is an answer event and the high-level containing this supervisor must satisfy Point 3 of the interfaceconsistent condition (Definition 3.5). However, if we treat this HISC system as a flat system, a synthesized supervisor could allow event $m 2$ st when buffer $b 2$ is full. In order to prevent the overflow of buffer $b 2$, the supervisor could then disable event $m 2 \_c p l$. This would have the effect of allowing the low-level to do the actual operation but not reporting the result. Thus in general, we can not use a normal flat synthesis algorithm to synthesize a flat supervisor for an HISC system model.

From the low-level DES low_m1 and interface DES intfm1, it is clear that low $\_\mathbf{m} 1 \|$ intfm1 $=$ low $\_\mathbf{m} 1$. By inspecting the DES low_m1, we see that a string reaching state $s 4$ can not reach a marker state by a string composed of only lowlevel $(m 1)$ events. Therefore, we need to trim off state $s 4$. The resulting trim DES is the final supervisor for low-level $m 1$.

From the low-level DES low_m2 and interface DES intfm2, we also have low_m2 $\|$ intfm2 $=$ low $\_\mathbf{m} 2$. By inspecting the DES low $\mathbf{m} \mathbf{2}$, we can not find a string $l$ composed of only low-level $(m 2)$ events such that $m 2$ pretask $m 2 \_s t l m 2 \_c p l$ belongs to the closed language of low-level $m 2$. Therefore, $s 6$ must be trimmed off. The resulting trim DES is the final supervisor for low-level $m 2$.

The BDD representations for control predicates $f_{H_{m 1 \_s t}}, f_{H_{m 2 \_s t}}, f_{H_{m 2 \_r p r}}, f_{H_{t u \_s t}}$ are shown in Figure 6.11. In the diagrams, dotted line means the variable at its source is assigned to be 0 and solid line means the variable at its source is assigned to be 1. The states in each of the component DES are encoded as the number in their names.(e.g. s0 is encoded as 0 , s1 is encoded as $1, \ldots$ ). As intfm2 contains four states, we need two binary variables for it. The binary value for a state is encoded with least significant bit first (e.g. $s 1$ is encoded as $10($ intfm2_0 $=1$, intfm2_1 = 0) 


\section{Master Thesis - R. Song - McMaster - Computing and Software}

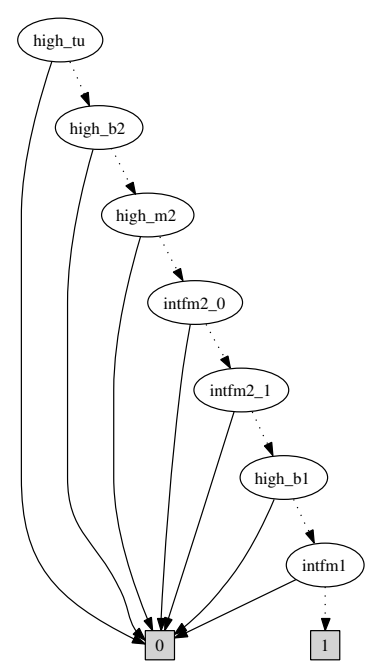

(a) $f_{H_{m 1-s t}}$

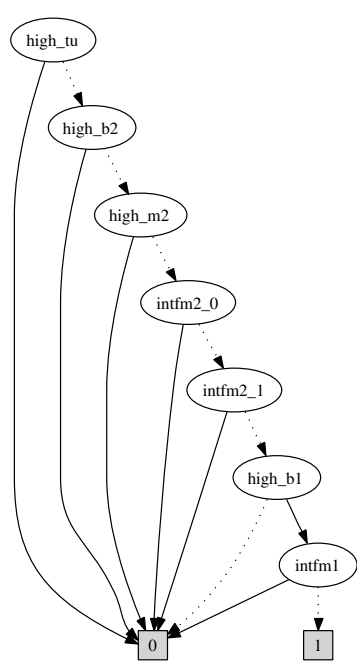

(b) $f_{H_{m 2-s t}}$

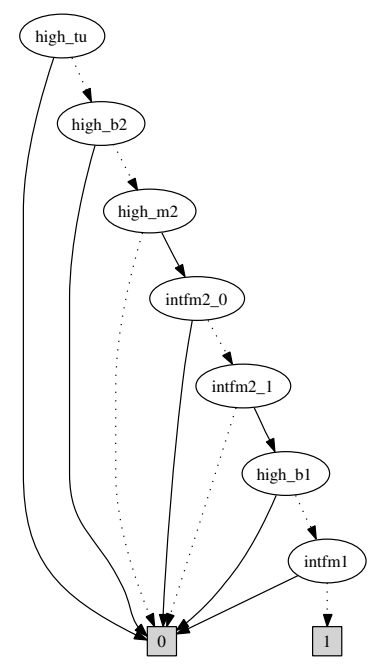

(c) $f_{H_{m 2}-r p r}$

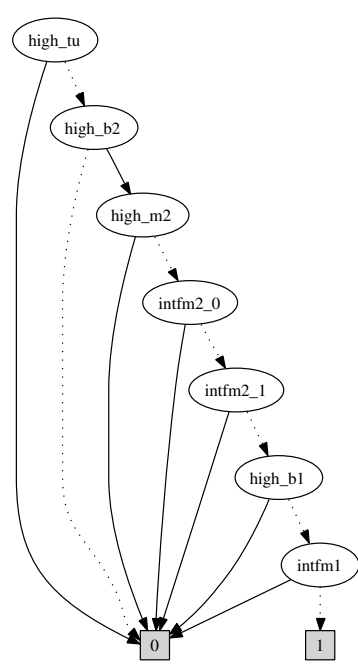

(d) $f_{H_{t u-s t}}$

Figure 6.11: Control predicates for $m 1 \_s t, m 2 \_s t, m 2 \_r p r, t u \_s t$

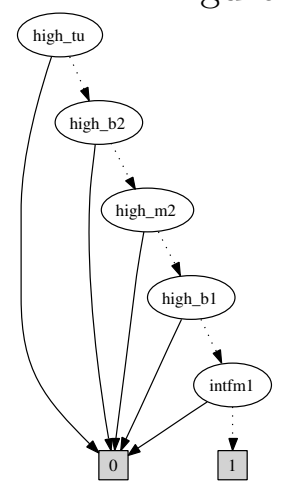

(a) $f_{H_{m 1 \_s t}}^{\prime}$

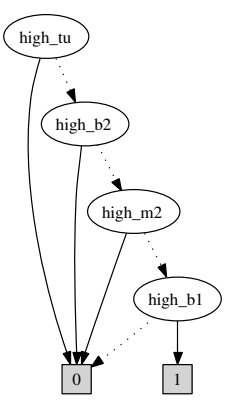

(b) $f_{H_{m 2-s t}}^{\prime}$

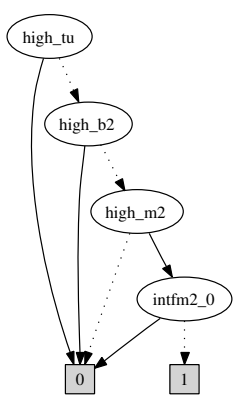

(c) $f_{H_{m 2-r p r}}^{\prime}$

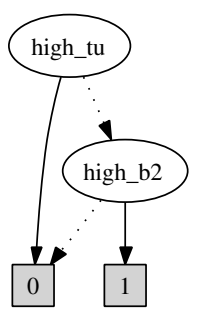

(d) $f_{H_{t u_{-} s t}}^{\prime}$

Figure 6.12: Prime simplified control predicates for $m 1 \_s t, m 2 \_s t, m 2 \_r p r, t u \_s t$

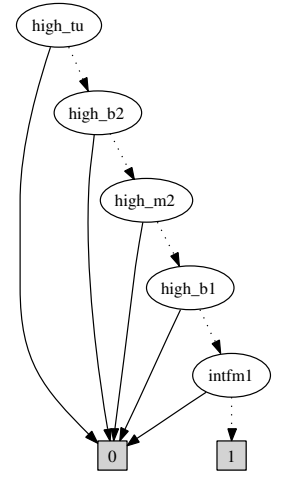

(a) $f_{H_{m 1-s t}}^{\prime \prime \prime}$

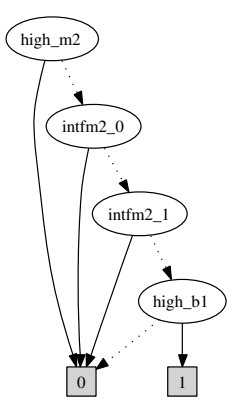

(b) $f_{H_{m 2} s t}^{\prime \prime \prime}$

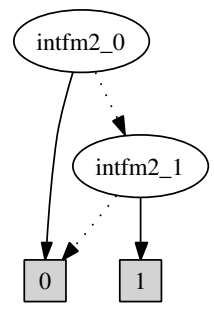

(c) $f_{H_{m 2-r p r}}^{\prime \prime \prime}$

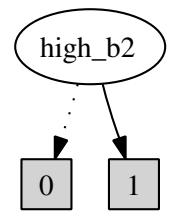

(d) $f_{H_{t u \_s t}^{\prime \prime \prime}}^{\prime \prime \prime}$

Figure 6.13: Triple-prime simplified control predicates for $m 1 \_s t, m 2 \_s t, m 2 \_r p r, t u \_s t$ 


\section{Master Thesis - R. Song - McMaster - Computing and Software}

and $s 2$ is encoded as 01 (intfm2 $\_0=0$, intfm2_1 = 1) in DES intfm2).

The BDD representations for prime simplified control predicates and triple-prime simplified control predicates are shown in Figure 6.12 and Figure 6.13, respectively.

For low-level $m 1$, the only control predicate is $f_{L_{1 m 1 \_t a s k 2}}$, which is always equal to false, so are $f_{L_{1 m 1 \_t a s k 2}}^{\prime}$ and $f_{L_{11_{-} \text {task2 }}}^{\prime \prime \prime}$.

For low-level $m 2$, the control predicate $f_{L_{2 m 2-p r e t a s k}}$ is also always false. The BDD representations for the control predicate $f_{L_{2 m 2-c p l}}$ and its simplified versions $f_{L_{2 m 2 \_c p l}}^{\prime}$ and $f_{L_{2 m 2} \text { cpl }}^{\prime \prime \prime}$ are shown in Figure 6.14.

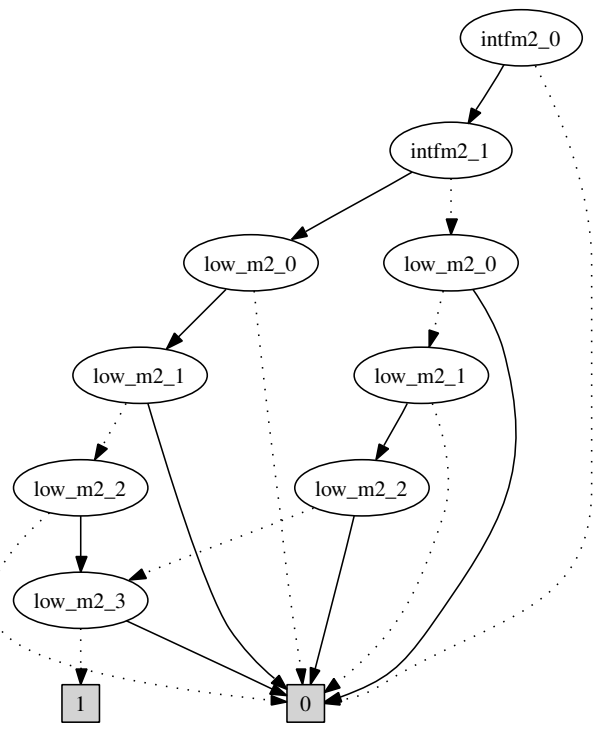

(a) $f_{L_{2 m 2-c p l}}$

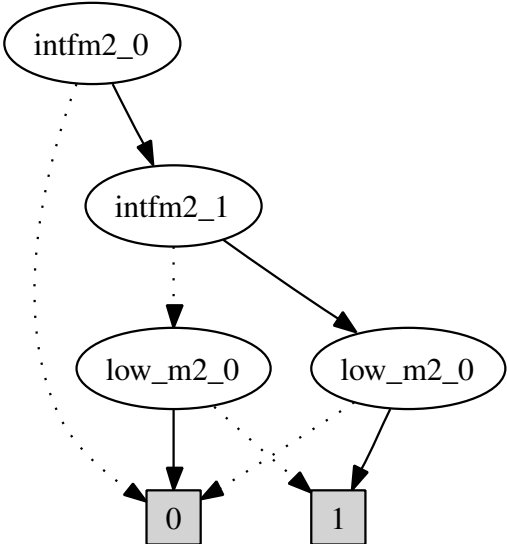

(b) $f_{L_{2 m 2_{-} c p l}}^{\prime}$

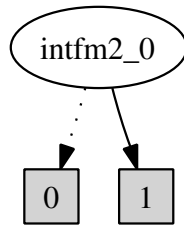

(c) $f_{L_{2 m 2}-p p l}^{\prime \prime \prime}$

Figure 6.14: The control predicates for $m 2_{-} c p l$. 


\section{Chapter 7}

\section{The AIP Example}

In order to demonstrate our approach, in this chapter we give an example with a large and complex high-level subsystem, which is modified from the AIP example ${ }^{1}$ in $[21,23,26]$. First we give an introduction of the AIP and our new control specifications, which are extensions of the one used in the original example. Next we present a set of plant DES and a set of modular supervisor DES and then verify each subsystem satisfies its corresponding conditions.After that, we relax the system by removing one restriction of the modular supervisors in the high-level and then synthesize a highlevel proper supervisor. Finally, we report our results. The reason we focus on the high-level is that it is usually the limiting factor as it is often more complex than the low-levels and usually increases in complexity as we add new low-levels to an existing system. In next chapter, we will give an example with large and complex low-level subsystems.

\footnotetext{
${ }^{1}$ In this chapter, for convenience we cite some diagrams directly or with some minor changes from $[21,23,26]$ with permission.
} 
Master Thesis - R. Song - McMaster - Computing and Software

\subsection{Introduction of the AIP}

The AIP is a highly automated manufacturing system, and was first modeled as a discrete event system using modular supervisory control theory in [5] (Brandin and Charbonnier) and $[7]($ Charbonnier $) .{ }^{2}$ Leduc then modeled the AIP with more detailed behavior added in $[21,23,26]$ and verified the properties of controllability and nonblocking using the HISC method.

The AIP system includes a central loop (CL) conveyor, and four external loop (EL) conveyors. Between each external loop and the central loop, there is a transport

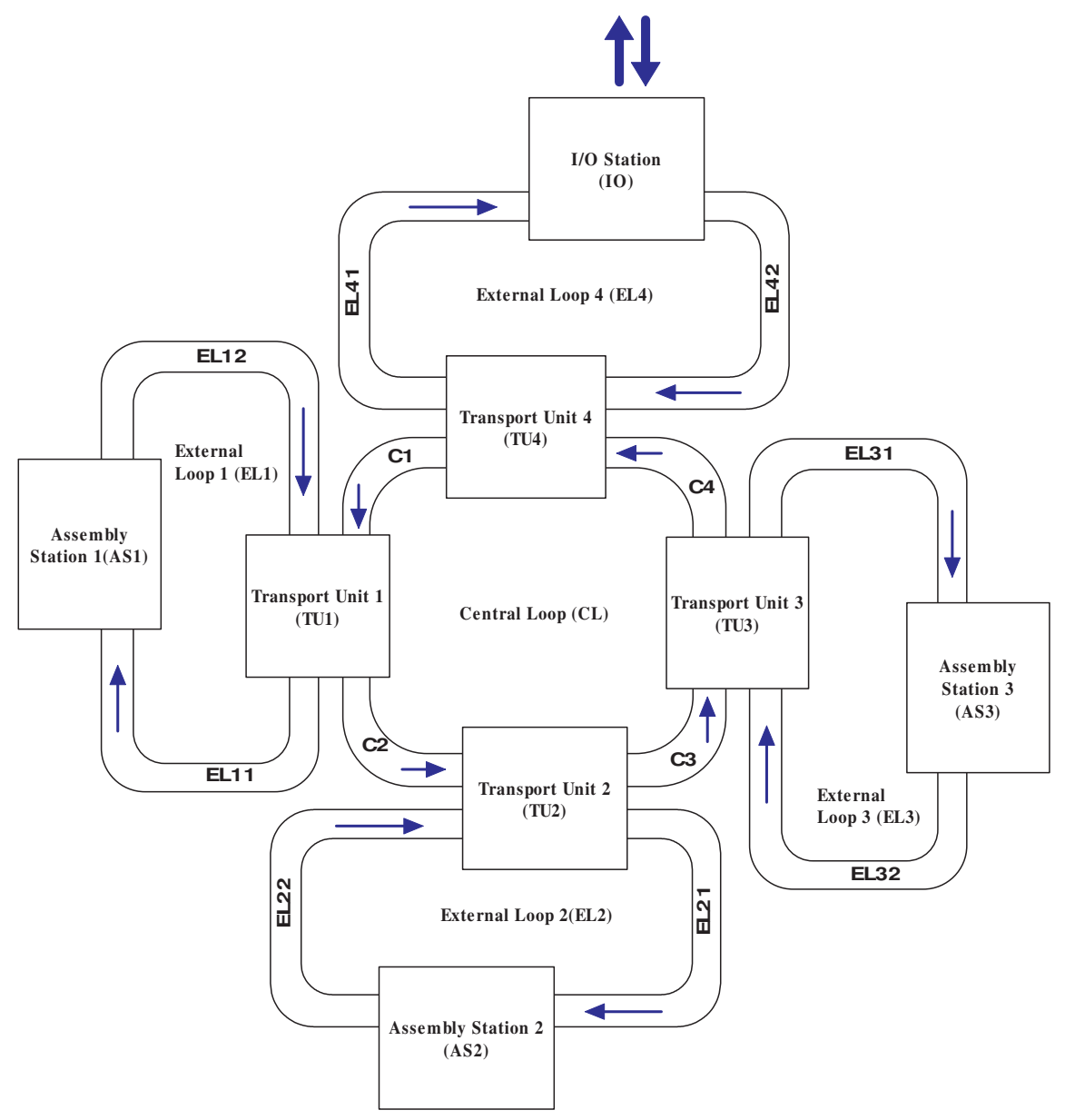

Figure 7.1: The AIP system architecture(from [21])

\footnotetext{
${ }^{2}$ What the author really read is an English version translated by R. Leduc.
} 


\section{Master Thesis - R. Song - McMaster - Computing and Software}

unit (TU) to transport pallets between them. At external loops 1, 2 and 3, they each have an assembly station (AS) including a robot to process pallets. At external loop 4, an Input/Output (I/O) station is there to allow pallets to enter and leave the system. Two types of pallets, Type1 and Type2, can be processed by the AIP system. The system architecture is shown in Figure 7.1. In the diagram, the arrows indicate the direction a pallet can move on a given loop.

The four transport units separate the central loop into four areas: C1, C2, C3 and C4. For each external loop conveyor, there are also two areas separated by either an assembly station (external loop 1, 2 and 3) or by an I/O station (external loop 4). These areas could be thought of as a buffer area. See Figure 7.1 for labels for these areas.

All three assembly stations have the same components and structure (see Figure 7.2) but different functions. The assembly station AS1 is capable of doing Task1A

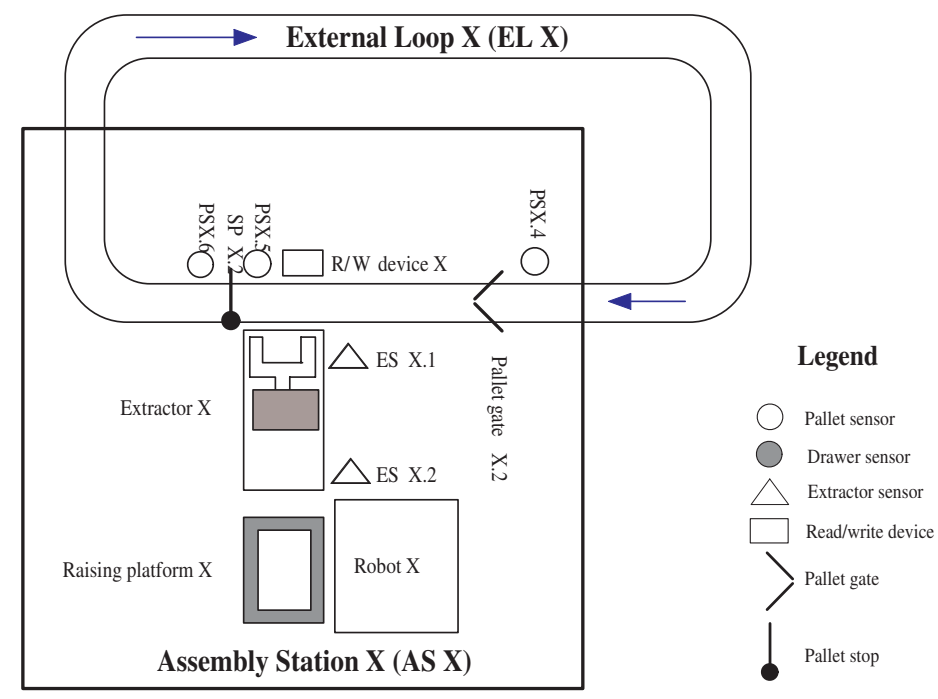

Figure 7.2: Assembly station of external loop $\mathrm{X}=1,2,3$ (from [21])

and Task1B, and AS2 is capable of doing Task2A and Task2B, while AS3 can do all the tasks. AS3 also repairs assembly errors to pallets. AS1 and AS2 can break down, 


\section{Master Thesis - R. Song - McMaster - Computing and Software}

while AS3 is assumed to never break down. AS3 can also substitute for AS1 and AS2 when they break down. In an assembly station, there are three pallet sensors (PS X.4, PS X.5, PS X.6) to detect a pallet arriving at the pallet gate, arriving at the pallet stop, or leaving the assembly station, respectively. Pallet gate X.2 can prevent other pallets from entering the assembly station while the station is busy. $\mathrm{R} / \mathrm{W}$ device $\mathrm{X}$ can read and write information on the label of a pallet. Pallet stop SP X.2 is used to prevent the pallet from leaving the station when it has not yet been processed. Extractor $\mathrm{X}$ is able to transfer a pallet between the conveyor and the raising platform. Two extractor sensors (ES X.1, ES X.2) are used to detect the location of the extractor. Raising platform $\mathrm{X}$ is used to feed the pallet to the robot (Robot X) to be processed, and move the processed pallet to the extractor.

In a transport unit (see Figure 7.3), there are three pallet sensors (PS 5.X.1, PS 5.X.2, PS5.X.3) close to the central loop side to detect a pallet arriving at pallet

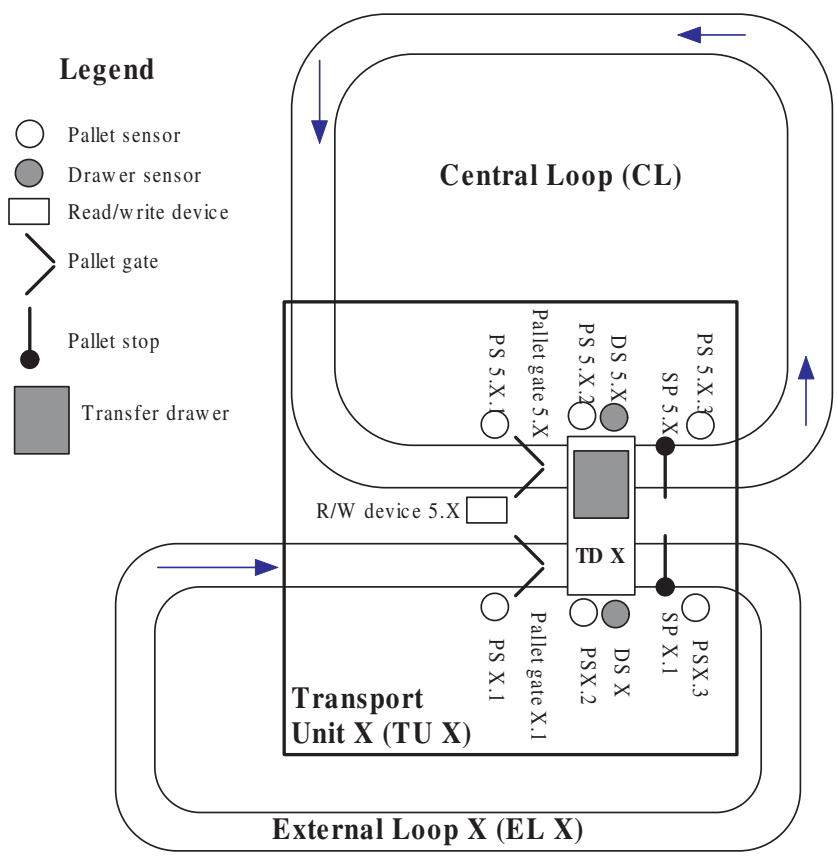

Figure 7.3: Transport unit for external loop $\mathrm{X}=1,2,3,4$ (from [21]) 


\section{Master Thesis - R. Song-McMaster-Computing and Software}

gate 5.X, arriving at the transfer drawer (TD X) and leaving the transport unit to next area of the central loop, respectively. There are another three pallet sensors (PS X.1, PS X.2, PS X.3) close to the external loop X side to detect a pallet arriving at pallet gate X.1, arriving at transfer drawer (TD X), and leaving the transfer unit to the external loop X, respectively. A pallet coming from the central loop can be transferred to the external loop $\mathrm{X}$ or liberated to the next area of the central loop, while a pallet coming from the external loop can only be transferred to the central loop. Pallet gate 5.X and pallet gate X.1 can prevent a pallet from entering the transfer unit when it is busy. Two pallet stops (SP5.X, SP X.1) are used to control pallets leaving the transfer unit. Transfer drawer (TD X) moves pallets between the central loop and the external loop X. Two drawer sensors can detect the location of the drawer.

The I/O station should have similar components and structure as an assembly station except there is no robot, extractor and raising platform. Due to lack of detailed information and time limit, here we assume that there is a sensor to detect if a pallet is ready to enter the system and a sensor to detect if a pallet is ready to leave the system.

\subsection{Control Specifications}

Our control specifications for the AIP system are extended from the specifications in $[21,23]$. For convenience, here we list all the specifications but the new ones begin with a '*'. We also assume that initially the AIP system is empty (i.e. no pallet in the system).

1. *Input: The type of the pallets entering the system must alternate, starting with Type1. 
Master Thesis - R. Song - McMaster - Computing and Software

2. Output: The type of the pallets leaving the system must alternate starting with Type1.

3. Routing: Type1 pallet must first go to AS1 to undergo Task1 and then go to AS2 to undergo Task2. Type2 pallet must first go to AS2 to undergo Task2 and then go to AS1 to undergo Task1. Both types of pallets are allowed to leave the system only when both tasks are done.

4. Assembly errors: When the robot in AS1 or AS2 makes an assembly error, the $\mathrm{R} / \mathrm{W}$ device in AS1 or AS2 will write assembly error information on the pallet label, and then AS3 will repair the pallet and AS1 and AS2 can do assembly task again.

5. Assembly station breakdown: When either AS1 or AS2 breaks down, all the pallets for that station will be routed to AS3 for assembly. However, when AS1 or AS2 is repaired, all the pallets not already in external loop 3 are rerouted to the original station.

6. Assembly task ordering: Assembly tasks are performed in a different order for pallets of different types. For a Type1 pallet, Task1A is performed before Task1B, and Task2A is performed before Task2B. For a Type2 pallet, Task1B is performed before task $1 \mathrm{~A}$, and Task2B is performed before task $2 \mathrm{~A}$.

7. Maximum capacity of assembly stations: At any time, only one pallet is allowed in a given assembly station.

8. *Maximum capacity of I/O station: At any time, only one pallet is allowed in the I/O station. 
Master Thesis - R. Song - McMaster - Computing and Software

9. *Maximum capacity of external loops: At each area of each external loop, the maximum allowed number of pallets at a given time is three.

10. *Maximum capacity of the central loops: At each area of the central loop, the maximum allowed number of pallets at a given time is two.

\subsection{System Structure}

In $[21,23]$, the AIP system is modeled as a bi-level HISC system with one highlevel and seven low-levels. The low-levels represent AS1, AS2, AS3, TU1, TU2, TU3, and TU4. In our example, we keep all the low-levels and add one more low-level for the I/O station. Therefore, in total we have one high-level and eight low-levels. The system structure is shown in Figure 7.4. As a large portion of our example will be the same as $[21,23,26]$, we will only present what is new and direct readers to $[21,23,26]$ for the remaining details.

The high-level is composed of the high-level subsystem $\mathbf{G}_{H}$ and 8 interfaces $\mathbf{G}_{I_{j}}, j \in\{1, \ldots, 8\}$. Each low-level $j$ contains the $j^{\text {th }}$ low-level subsystem $\mathbf{G}_{L_{j}}$ and

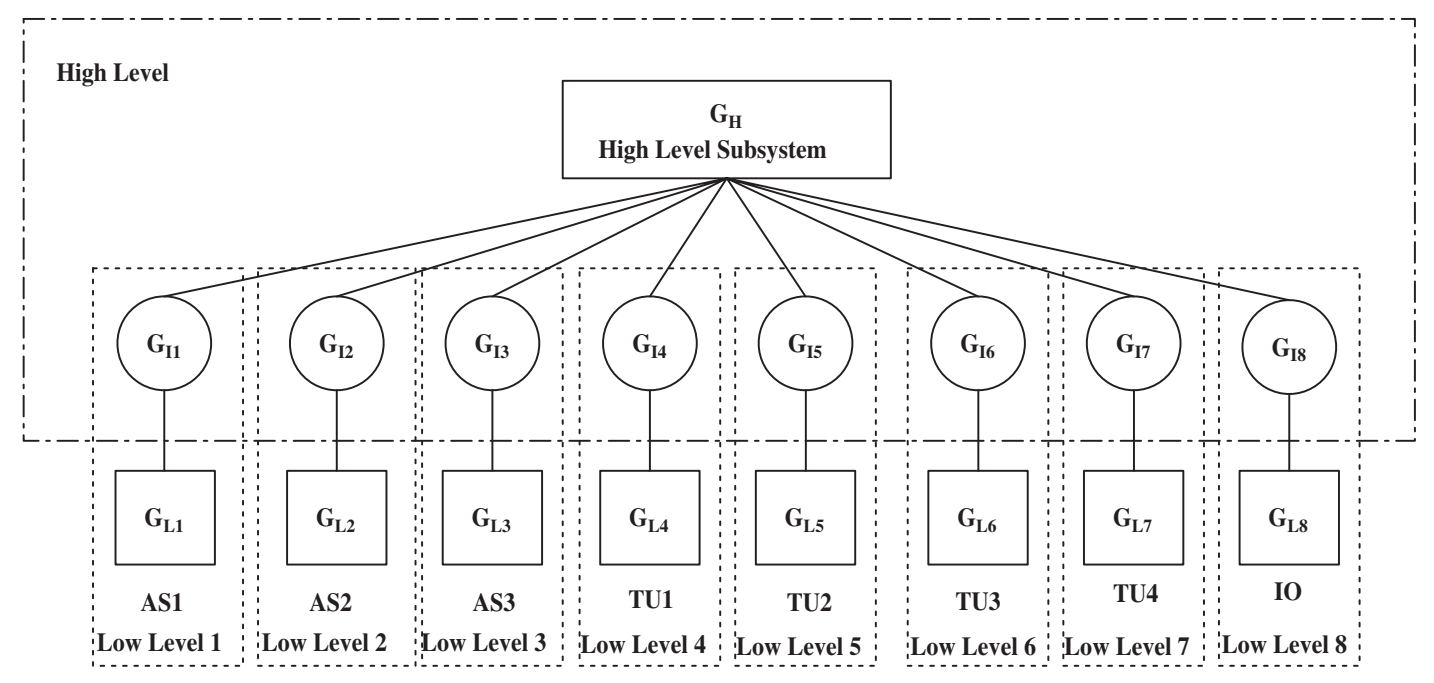

Figure 7.4: The AIP system structure 
Master Thesis - R. Song - McMaster - Computing and Software

the $j^{\text {th }}$ interface $\mathbf{G}_{I_{j}}$.

For the I/O station, we model the two sensors to detect that pallets are ready to enter or leave the system in the high-level. As we do not have the internal structure of the I/O station, we only provide an interface DES for the low-level 8 (I/O station) and have the low-level 8 subsystem containing only one plant component DES and set $\Sigma_{L_{8}}=\varnothing$. The plant DES contains only an initial state, which is also marked.

The event set for the whole AIP system $\Sigma:=\dot{\cup}_{j \in\{1, \ldots, 8\}}\left[\Sigma_{L_{j}} \dot{\cup} \Sigma_{R_{j}} \dot{\cup} \Sigma_{A_{j}}\right] \dot{\cup} \Sigma_{H}$ is defined based on the event partition given in [21]. The primed event set below stands for the corresponding event set from the AIP example in [21].

$$
\begin{aligned}
& \Sigma_{H}:=\left(\Sigma_{H}^{\prime}-\{\text { PalletArvGEL_2.AS3\}) } \cup\right. \\
& \text { \{QPalletOut.IO, IsPalletOut.IO, NoPalletOut.IO, QPalletType1In.IO, } \\
& \text { QPalletType2In.IO, IsPalletType1In.IO, IsPalletType2In.IO, } \\
& \text { NoPalletType1In.IO, NoPalletType2In.IO\} } \\
& \Sigma_{L_{i}}:=\Sigma_{L_{i}}^{\prime} \text {, where } i \in\{1,2,4,5,6,7\} \\
& \Sigma_{L_{3}}:=\Sigma_{L_{3}}^{\prime} \cup\{\text { PalletArvGEL_2.AS3\} } \\
& \Sigma_{L_{8}}:=\varnothing \\
& \Sigma_{R_{k}}:=\Sigma_{R_{k}}^{\prime} \text {, where } k \in\{1, \ldots, 7\} \\
& \Sigma_{R_{8}}:=\{\text { MvInType1Pallet.IO, MvInType2Pallet.IO, MvOutPallet.IO }\} \\
& \Sigma_{A_{k}}:=\Sigma_{A_{k}}^{\prime} \text {, where } k \in\{1, \ldots, 7\} \\
& \Sigma_{A_{8}}:=\{C p l M v I n \text { Pallet.IO, CplMvOutPallet.IO }\}
\end{aligned}
$$

In the DES diagrams in this chapter, initial states are identified by a thick circle, and marker states are filled in with gray. Uncontrollable events are shown in italic font, and controllable events are shown in normal font. For a given DES, its event set is taken to be that of the event labels shown on transitions in the diagram unless explicitly state otherwise. 


\section{Master Thesis - R. Song-McMaster-Computing and Software}

\subsection{The Interface DES}

For low-level 1 to 7 , we use the same interface as in $[23,26]$. The interfaces for low-level 1 and 2 are shown in Figure 7.5 with $\mathrm{k}=\mathrm{AS} 1$, AS2. The interface for low-level 3 (AS3) is shown in Figure 7.6. The interfaces for low-levels 4, 5, and 7 are shown in Figure 7.7 with $\mathrm{q}=$ TU1, TU2, TU4. The interface for low-level 6 (TU3) is shown in Figure 7.8. Finally, the interface for low-level 8 (I/O) is shown in Figure 7.9.

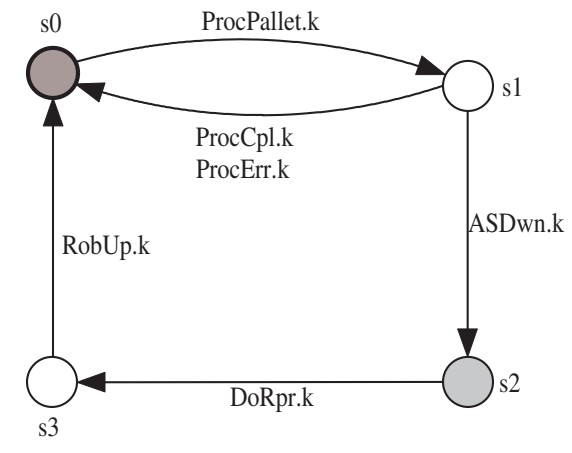

Figure 7.5: Interface to low-level $w=$ $1,2($ from $[23])$

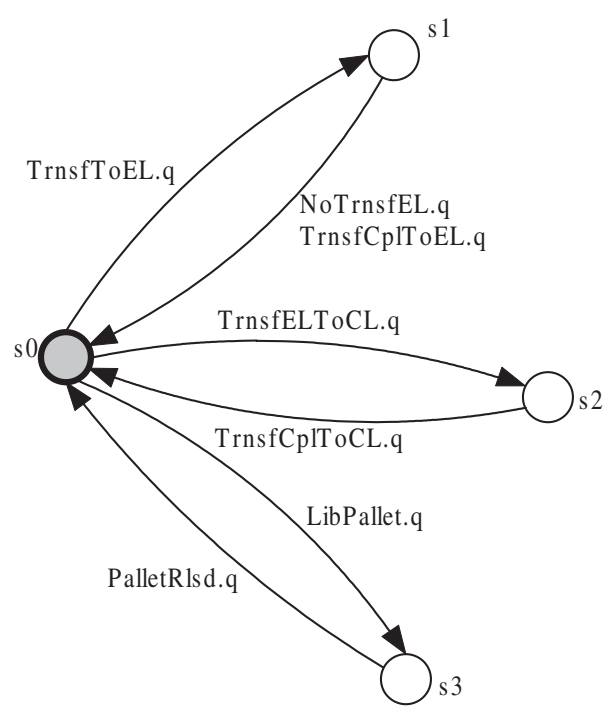

Figure 7.7: Interface to low-level $v=$ $4,5,7$ (from [23])

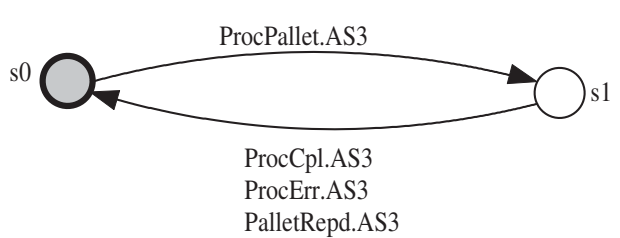

Figure 7.6: Interface to low-level 3 (from [23])

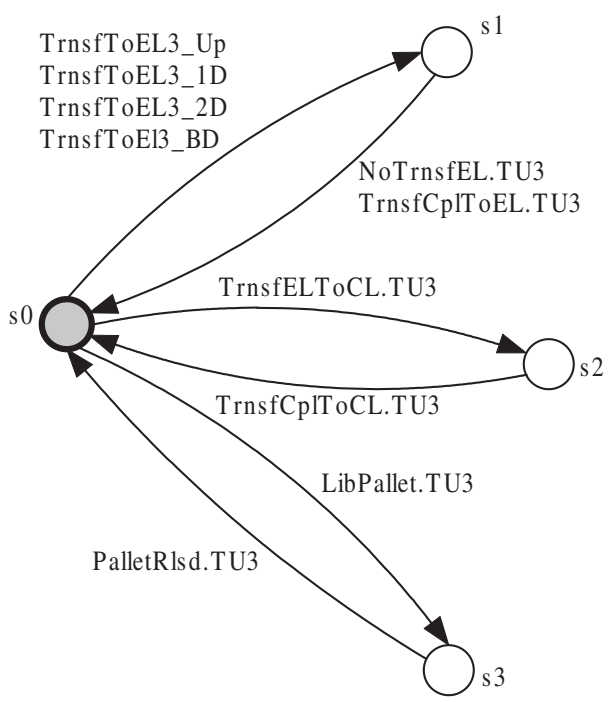

Figure 7.8: Interface to low-level 6 (from [23]) 
Master Thesis - R. Song - McMaster - Computing and Software

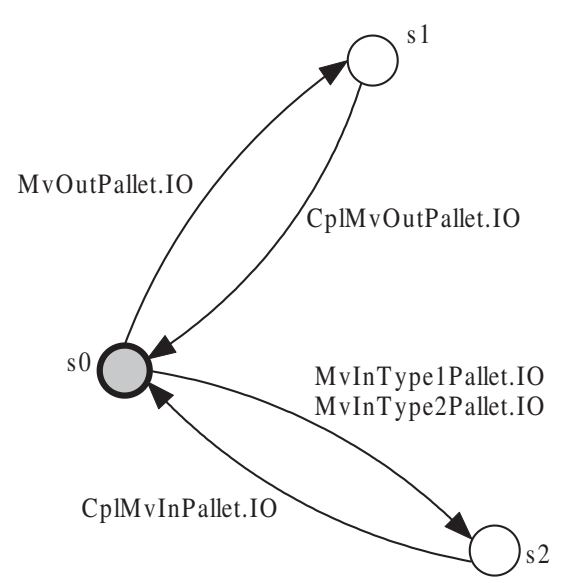

Figure 7.9: Interface to low-level 8

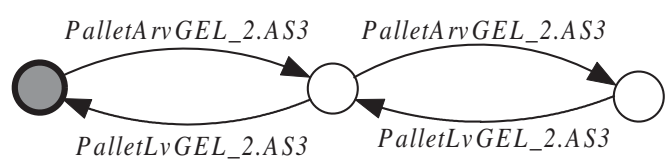

Figure 7.10: CapGateEL_2.AS3

\subsection{Low-level Subsystems}

For low-level 1 (AS1), 2 (AS2), 4 (TU1), 5 (TU2), 6 (TU3) and 7 (TU4), we use exactly the same models as in $[23,26]$. For low-level 3 (AS3), we make some minor modifications. The reason for these modifications is that there was no capacity restriction on external loop 3 in [23]. In order to show the relationship between the event ProcPallet.AS3 and PalletArvGEL_2.AS3, Leduc created a high-level plant component DES that was in Figure 12.3 in [21] (PalletArvGateSenEL $\_$2.AS3). However, in this thesis, we will enforce a capacity restriction on external loop 3. This allows us to move this functionality to low-level 3 (AS3). The following shows how we modified the models for low-level 3.

- Replace the plant component CapGateEL_2.AS3 by the one in Figure 7.10.

- Replace the supervisor component OperateGateEL_2.AS3 by the one in Figure 7.11.

- Add a plant component PalletArvGateSenEL_2.AS3 as shown in Figure 7.12. 


\section{Master Thesis - R. Song - McMaster - Computing and Software}

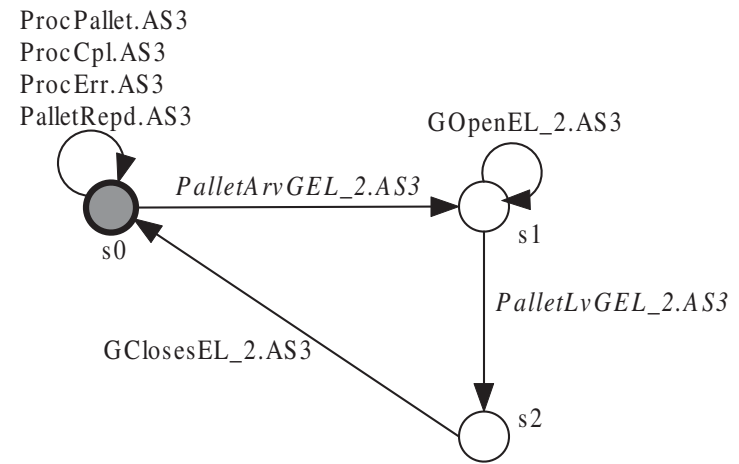

Figure 7.11: OperateGateEL_2.AS3

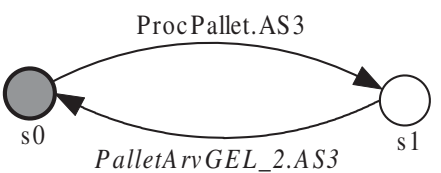

Figure $\quad 7.12$ : PalletArvGateSenEL_2.AS3

For low-level 8 (I/O), as described in section 7.3, we lack the information and time needed to model the subsystem in more detail, so we model its subsystem containing only a one-state plant DES as shown in Figure 7.13

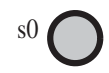

Figure 7.13: Low-level 8 Subsystem

\subsection{The High-level Subsystem}

In this section, we present the high-level subsystem $\mathbf{G}_{H}$ for the AIP. The high-level controls the global behavior of the system, such as controlling the capacity of each area of the central loop and external loops, detecting the status of AS1 and AS2 and reporting the status to other low-levels. As our newly added control specifications primarily focus on the capacity of the conveyor areas, the high-level is significantly larger and more complicated than the one in [23].

To demonstrate our high-level verification algorithms and synthesis algorithms, we designed all the modular supervisors by hand to meet the control specifications and then verified that the system under the control of these supervisors satisfies all the 


\section{Master Thesis - R. Song - McMaster - Computing and Software}

high-level conditions (e.g. level-wise controllable, level-wise nonblocking and interface consistent). In the next section, we will relax the restrictions and then synthesize a supervisor to satisfy the control specifications.

The AIP high-level subsystem $\mathbf{G}_{H}$ is composed of 19 plant components and 21 supervisor components, as shown in Figure 7.14. The high-level plant $\mathbf{G}_{H}^{p}$ and supervisor $\mathbf{S}_{H}$ are defined to be the synchronous product of the indicated automata.

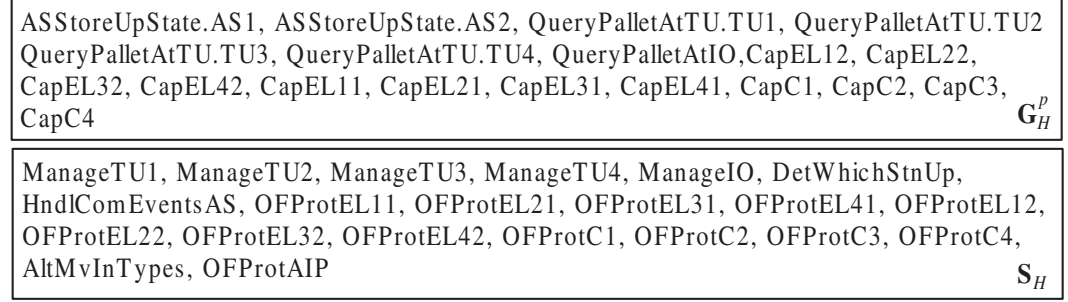

Figure 7.14: Component DES in the AIP high-level

\subsubsection{Plant Components}

We start from the set of DES ASStoreUpSate. $k$, where $k=$ AS1, AS2, shown in Figure 7.15. These two DES are used to check the status of AS1 and AS2 and tell when the high-level is allowed to send the requests ProcPallet.k and DoRpr.k. ${ }^{3}$

The next set of DES we introduce are QueryPalletAtTU.i, where $i=\mathrm{TU} 1$, TU2, TU3, TU4, shown in Figure 7.16. The DES QueryPalletAtTU. $i$ can tell if a pallet is waiting to enter the transport unit $i$ from the central loop (by detecting the gate sensor PS 5.X.1 ( $\mathrm{X}=1$ when $i=\mathrm{TU} 1, \ldots, \mathrm{X}=4$ when $i=\mathrm{TU} 4))$ or the related external loop (by detecting the gate sensor PS X.1).

\footnotetext{
${ }^{3}$ Actually we could remove those selfloop transitions on these two DES, since we now use command-pair interface for AS1 and AS2. The interfaces to low-level 1 and low-level 2 guarantee when the request events ProcPallet.k and DoRpr.k can happen.
} 
Master Thesis - R. Song - McMaster - Computing and Software

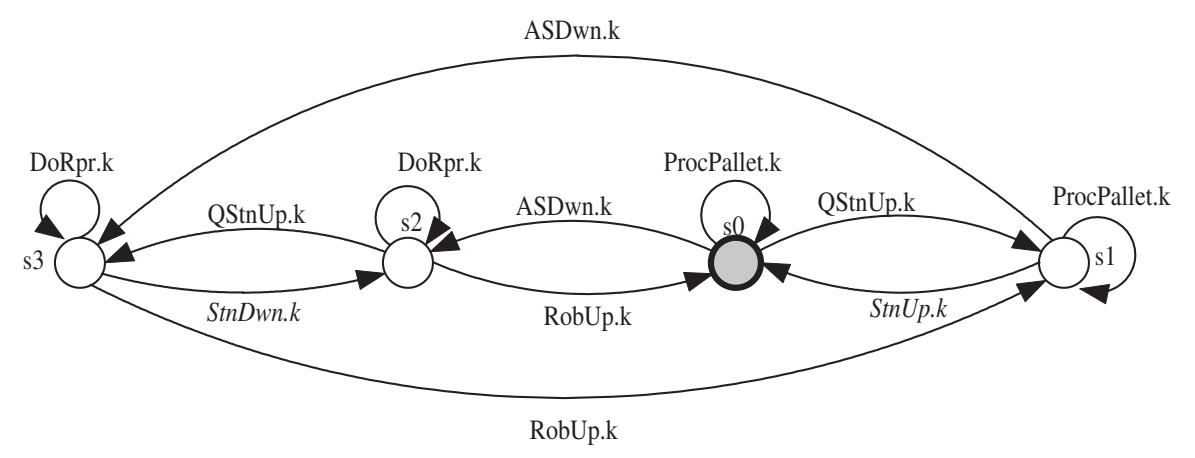

Figure 7.15: ASStoreUpState. $k=$ AS1, AS2 (from [23])

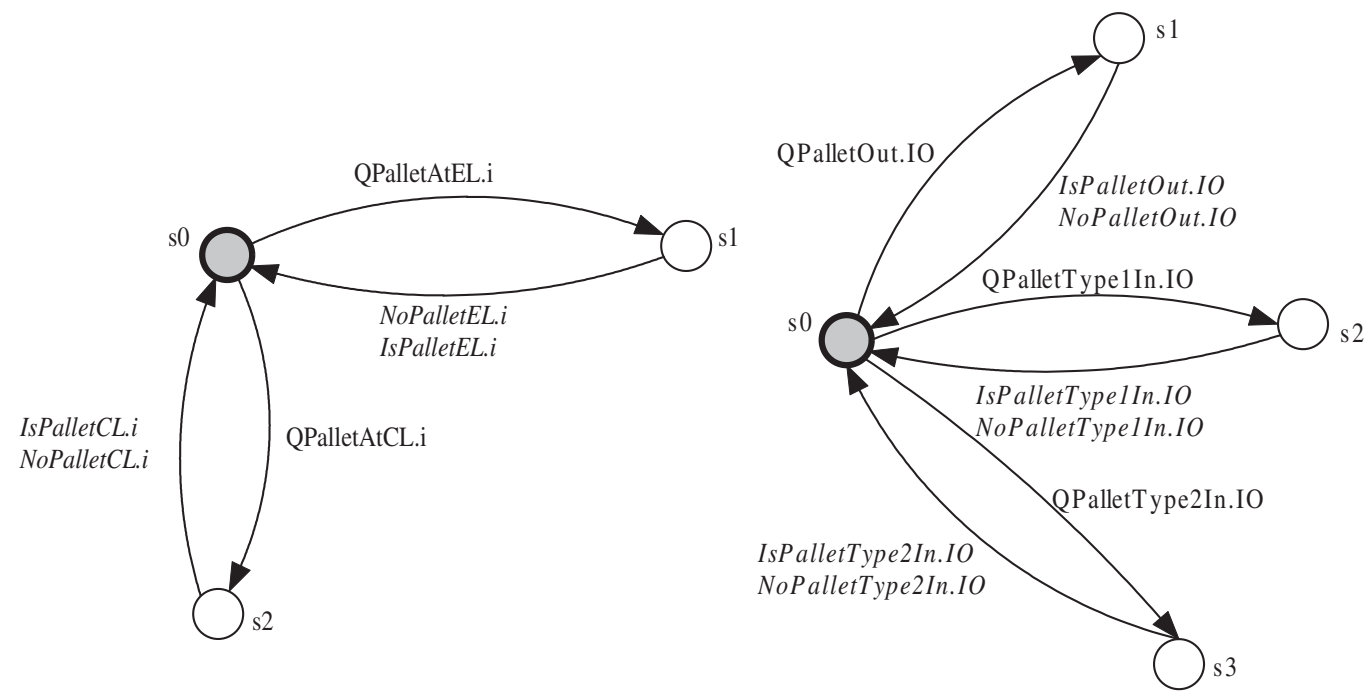

Figure 7.16: QueryPalletAtTU.i, $i=\mathrm{TU} 1, \mathrm{TU} 2, \mathrm{TU} 3, \mathrm{TU} 4 \quad$ Figure 7.17: QueryPalletAtIO

We now describe the DES QueryPalletAtIO, shown in Figure 7.17. This DES models the behavior of the the sensors in the $\mathrm{I} / \mathrm{O}$ station. It provides a way to determine if a pallet is ready to leave the system or if a Type1 or Type2 pallet is ready to enter the system.

The next series of DES represent the fact that a pallet on an external loop can arrive at a transport unit only if it has been processed by the associated assembly station or brought in from the I/O station. They are CapEL12, CapEL22, CapEL32 and CapEL42 as shown in Figure 7.18. Theoretically, each of the four DES should 


\section{Master Thesis - R. Song - McMaster - Computing and Software}

have infinite states. However, because the supervisors will limit the capacity of each of the conveyor areas EL12, EL22, EL32 and EL42 to three, we are safe to make each of them contain five states. Readers might ask why these DES have capacity of four, this is because we would like to show that the supervisors really prevent the fourth pallet from entering any of the above areas.

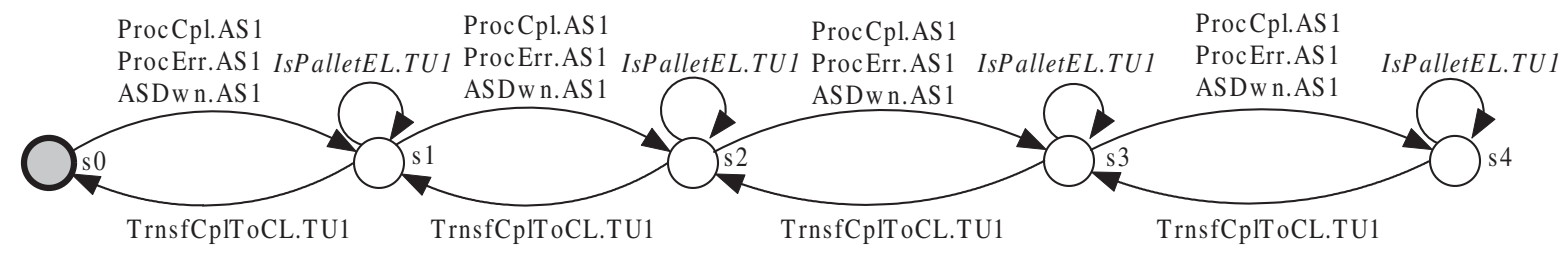

(a) CapEL12

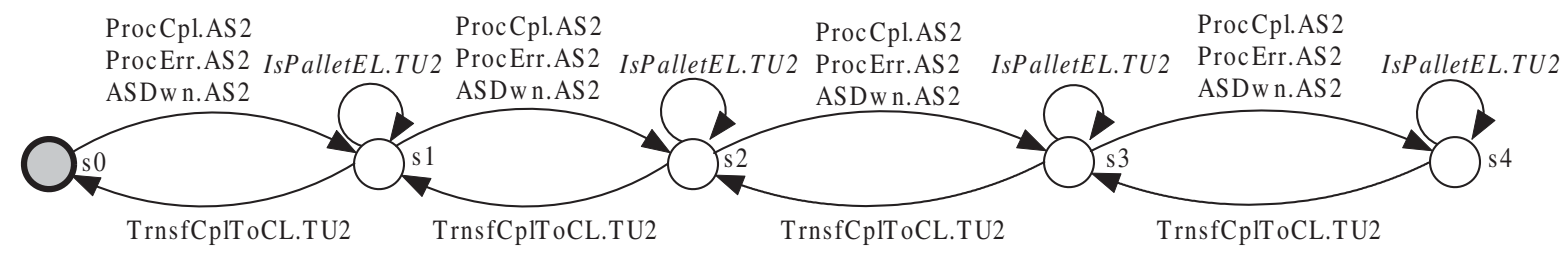

(b) CapEL22

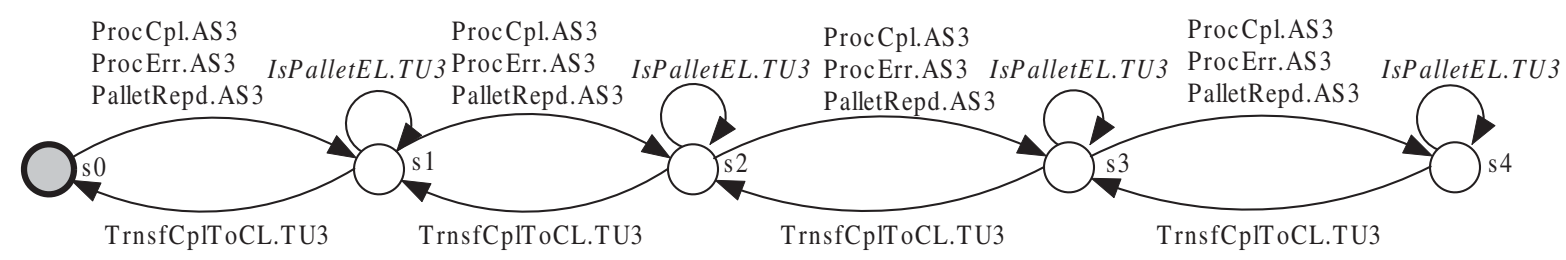

(c) CapEL32

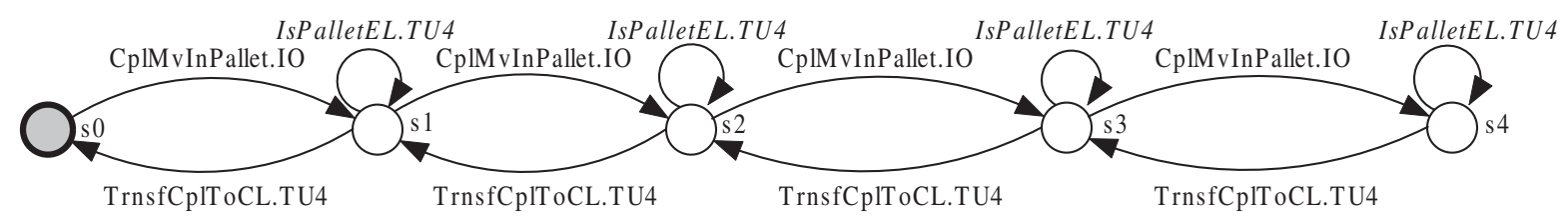

(d) CapEL42

Figure 7.18: CapEL12, CapEL22, CapEL32, CapEL42

Now we describe the series of DES representing the fact that a pallet on the center loop can arrive at a transport unit from the central loop only if it has been liberated or 


\section{Master Thesis - R. Song - McMaster - Computing and Software}

transferred from the external loop by the previous transport unit. They are $\mathbf{C a p C 1}$, CapC2, CapC2 and CapC4 as shown in Figure 7.19. Each of them should also have infinite states, but because the supervisors will limit the capacity of each central loop area to two, we are safe to make each of them contain only 4 states.

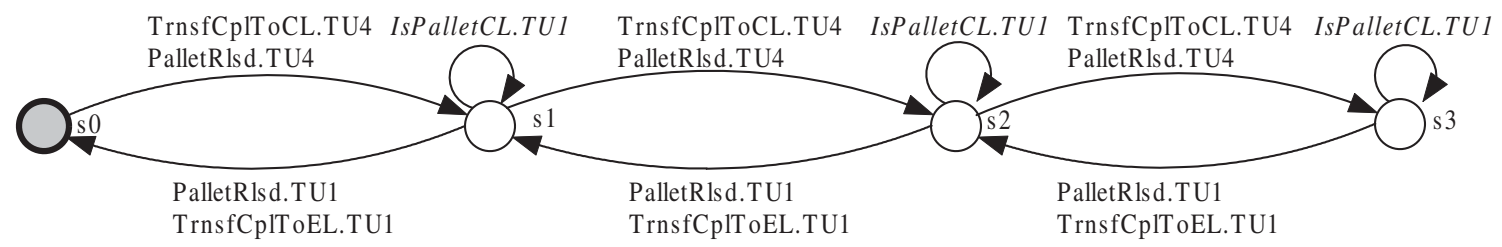

(a) $\mathrm{CapC1}$

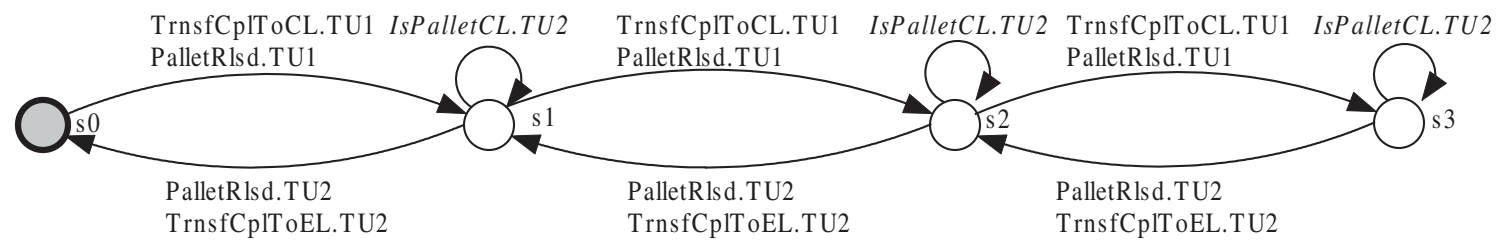

(b) $\mathrm{CapC} 2$

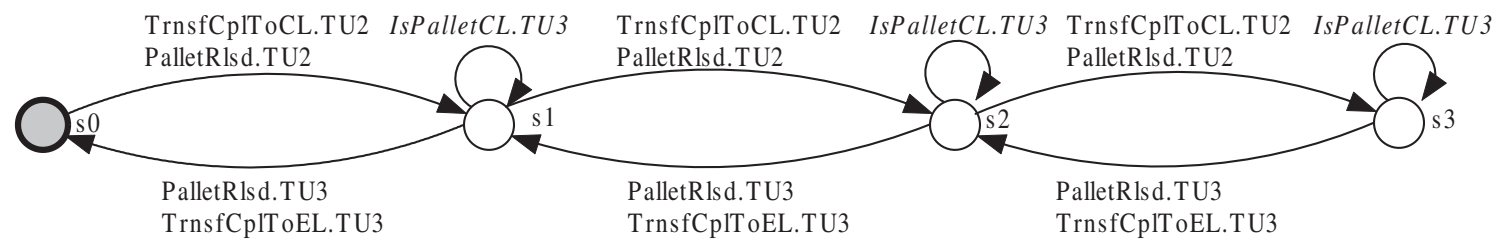

(c) $\mathrm{CapC} 3$

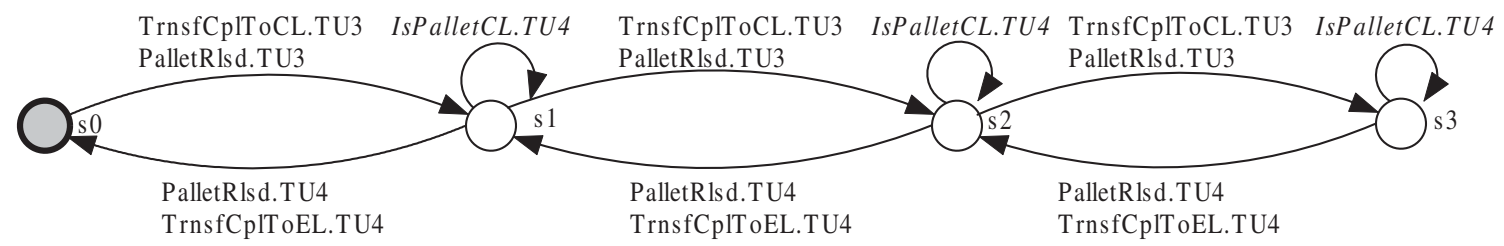

(d) $\mathrm{CapC} 4$

Figure 7.19: CapC1, CapC2, CapC3, CapC4

The plant components CapEL11, CapEL21 and CapEL31 (Figure 7.20(a), $7.20(\mathrm{~b}), 7.20(\mathrm{c}))$ represents the fact that a pallet can be processed by an assembly station only if it has been transferred to the associated external loop. The plant 
component CapEL41 (Figure 7.20(d)) represents the fact that a pallet can arrive at the I/O station only if it has been transferred to external loop 4 by transport unit TU4. Again, these DES should have infinite states theoretically, but the supervisors will limit the capacity of these areas to 3 , so we are safe to make it contain only 5 states.

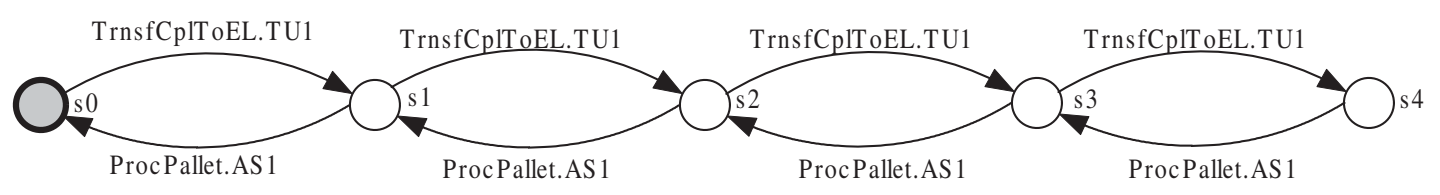

(a) CapEL11

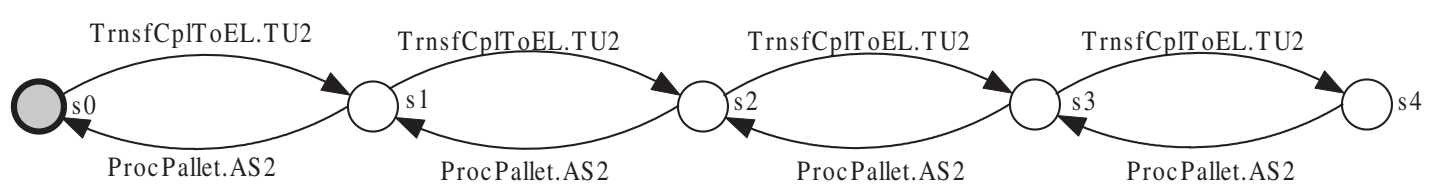

(b) CapEL21

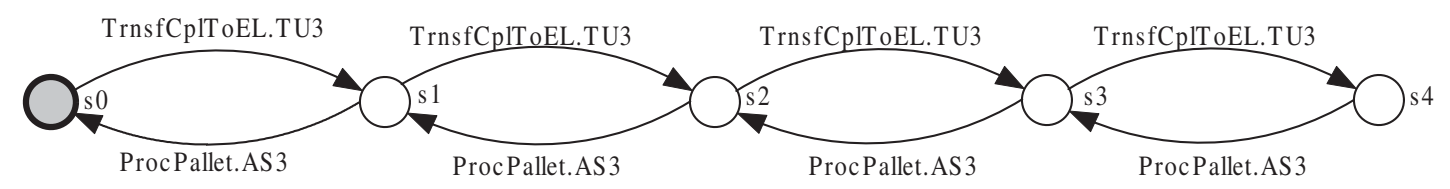

(c) CapEL31

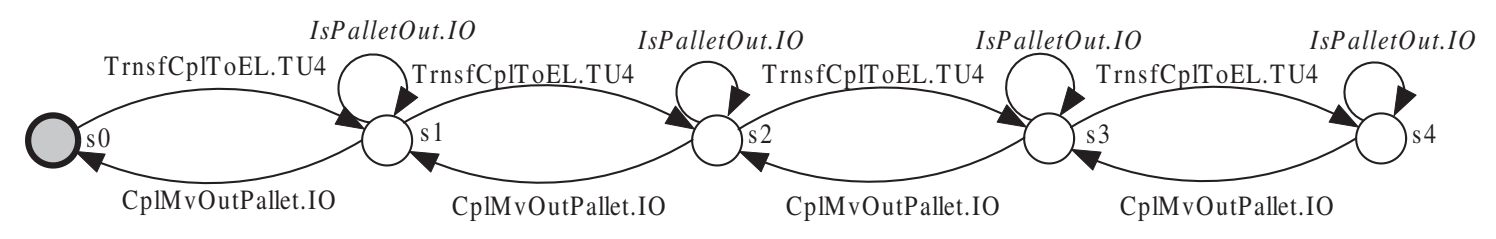

(d) CapEL41

Figure 7.20: CapEL11, CapEL21, CapEL31, CapEL41

\subsubsection{Supervisor Components}

We now discuss the supervisor components for the AIP high-level subsystem. We first introduce the group of four supervisors which control the transport units, Man- 


$$
\text { Master Thesis - R. Song - McMaster - Computing and Software }
$$

ageTU1, ManageTU2, ManageTU3 and ManageTU4. The following explains what they do.

- ManageTU1 : If a pallet appears before TU1 at the central loop, it could be transferred to external loop 1 or liberated. It can be liberated if AS1 breaks down, or the conveyor area EL11 is at capacity (i.e. 3, determined by OFProtEL11), or low-level 4 (TU1) says to do so through the event NoTransfEL.TU1. If a pallet appears before TU1 at the external loop 1, then it must be transferred to the central loop. See Figure 7.21.

- ManageTU2: If a pallet appears before TU2 at the central loop, it could be transferred to external loop 2 or liberated. It can be liberated if AS2 breaks down, or the conveyor area EL21 is at capacity (i.e. 3, determined by OFProtEL21), or low-level 5 (TU2) says to do so through the event NoTransfEL.TU2. If a pallet appears before TU2 at the external loop 2, then it must be transferred to the central loop. See Figure 7.22.

- ManageTU3: If a pallet appears before TU3 at the central loop, it could be transferred to external loop 3 or liberated. If the conveyor area EL31 is at capacity (i.e. 3, determined by OFProtEL31), then the pallet should be liberated. Otherwise the break down status of AS1 and AS2 will be checked through supervisor component DetWhichStnUp. Then a corresponding request event with the status of AS1 and AS2 encoded into it will be sent to low-level 6 (TU3). The pallet should be liberated if low-level 5 responds with a NoTransfEL.TU3 event. If a pallet appears before TU3 at external loop 3, then it must be transferred to the central loop. See Figure 7.23.

- ManageTU4: If a pallet appears before TU4 at the central loop, it could 
be transferred to external loop 4 or liberated. It can be liberated if the conveyor area EL41 is at capacity (i.e. 3, determined by OFProtEL41), or lowlevel 7 (TU4) says to do so through the event NoTransfEL.TU4 (e.g. not all tasks have been performed on the pallet). If a pallet appears before TU4 at external loop 4, then it must be transferred to the central loop. See Figure 7.24.

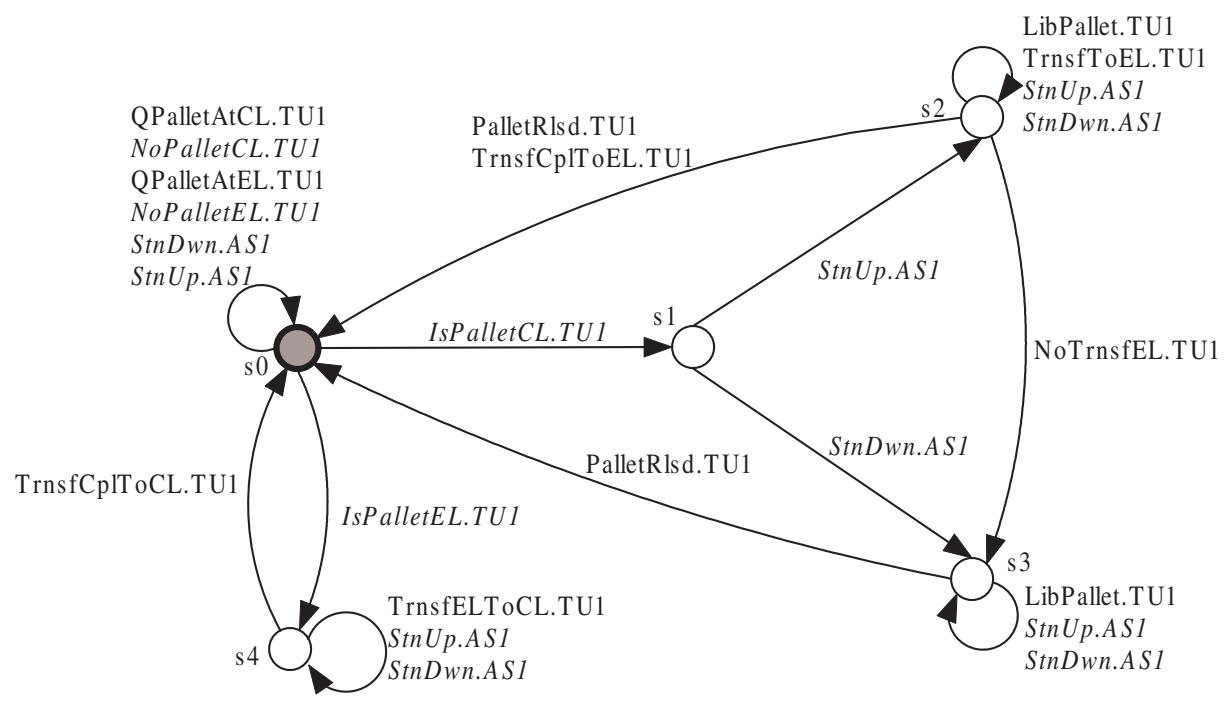

Figure 7.21: ManageTU1

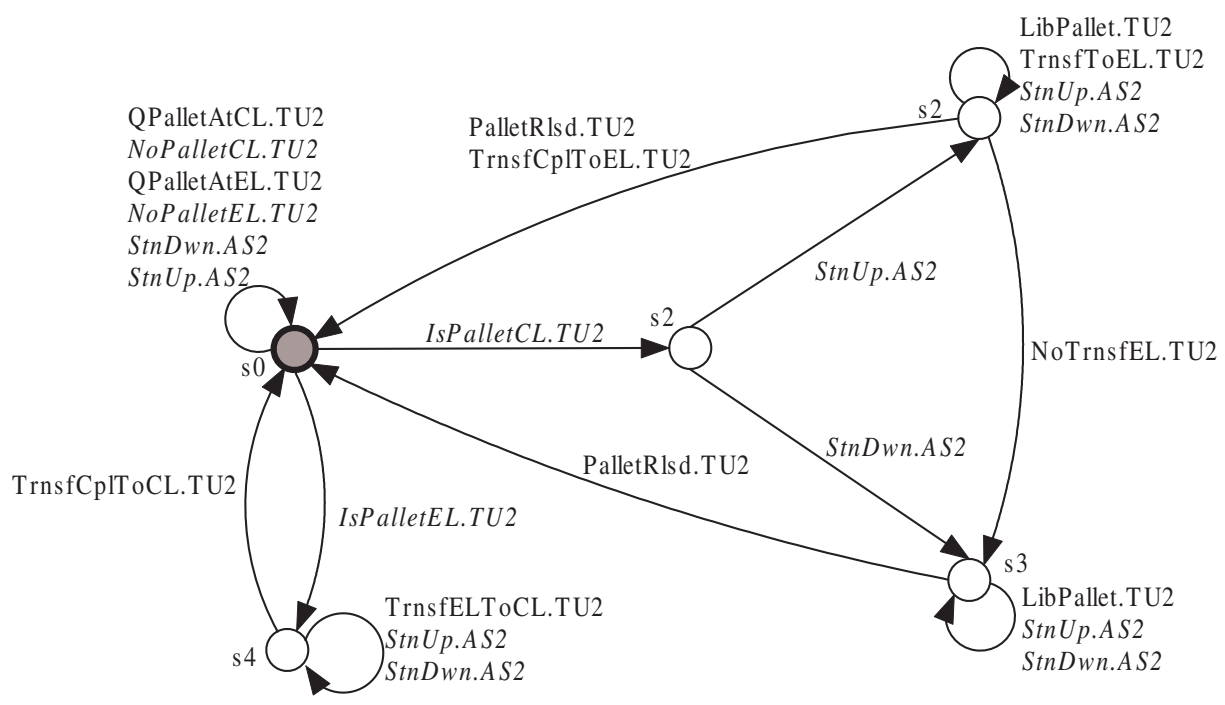

Figure 7.22: ManageTU2 


\section{Master Thesis - R. Song - McMaster - Computing and Software}

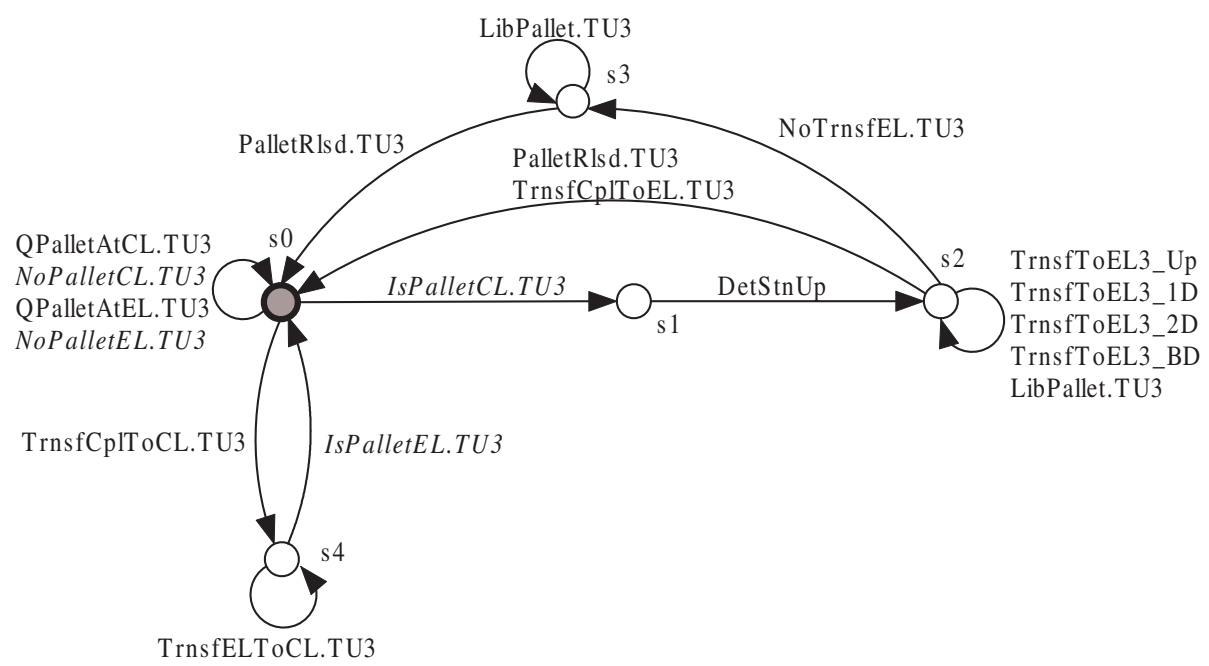

Figure 7.23: ManageTU3

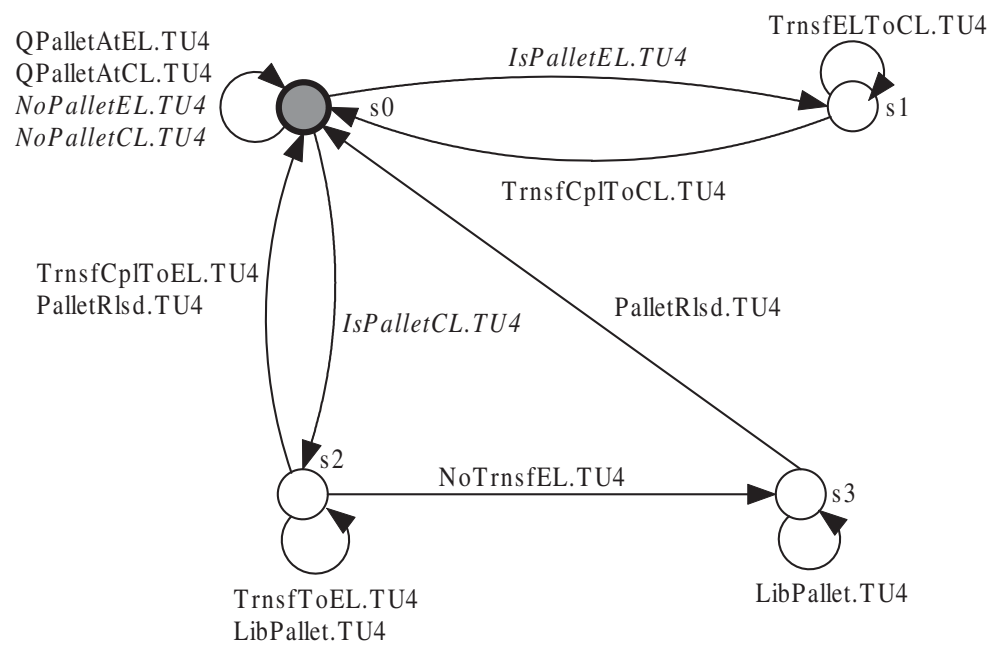

Figure 7.24: ManageTU4

Next we introduce two supervisor DES directly obtained from [21]. The first is DetWhichStnUp, shown in Figure 7.25. This supervisor is used to detect the breakdown status of $\mathrm{AS} 1$ and $\mathrm{AS} 2$, and then the corresponding request event that encodes this status will be chosen to be sent to low-level 6 (TU3). For example, if AS1 breaks down and AS2 does not, then request event LibPallet.TU3 or TrnsfToEL3_1D 
will be chosen. The second DES is HndlComEventsAS, shown in Figure 7.26. This supervisor is used to solve a blocking problem among the supervisors ManageTU1, ManageTU2 and DetWhichStnUp due to controllable events they use in common.

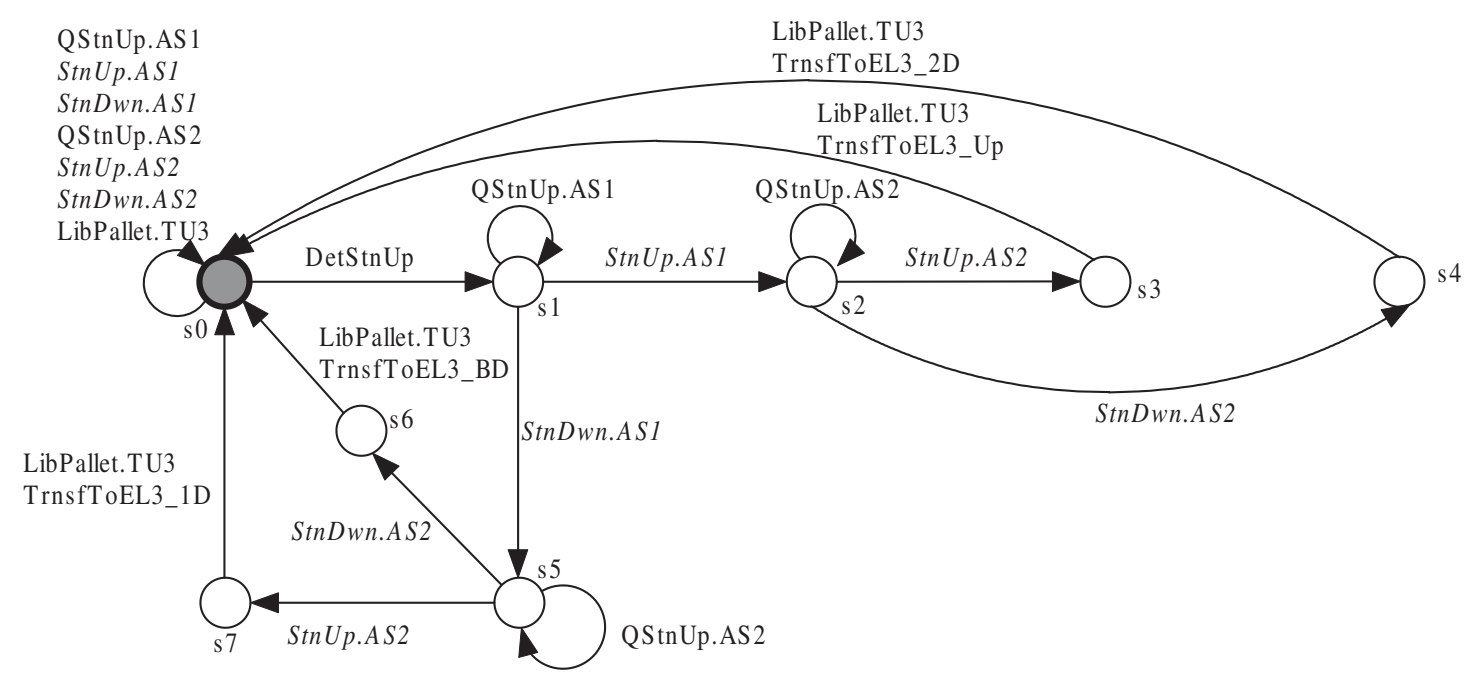

Figure 7.25: DetWhichStnUp (from [21])

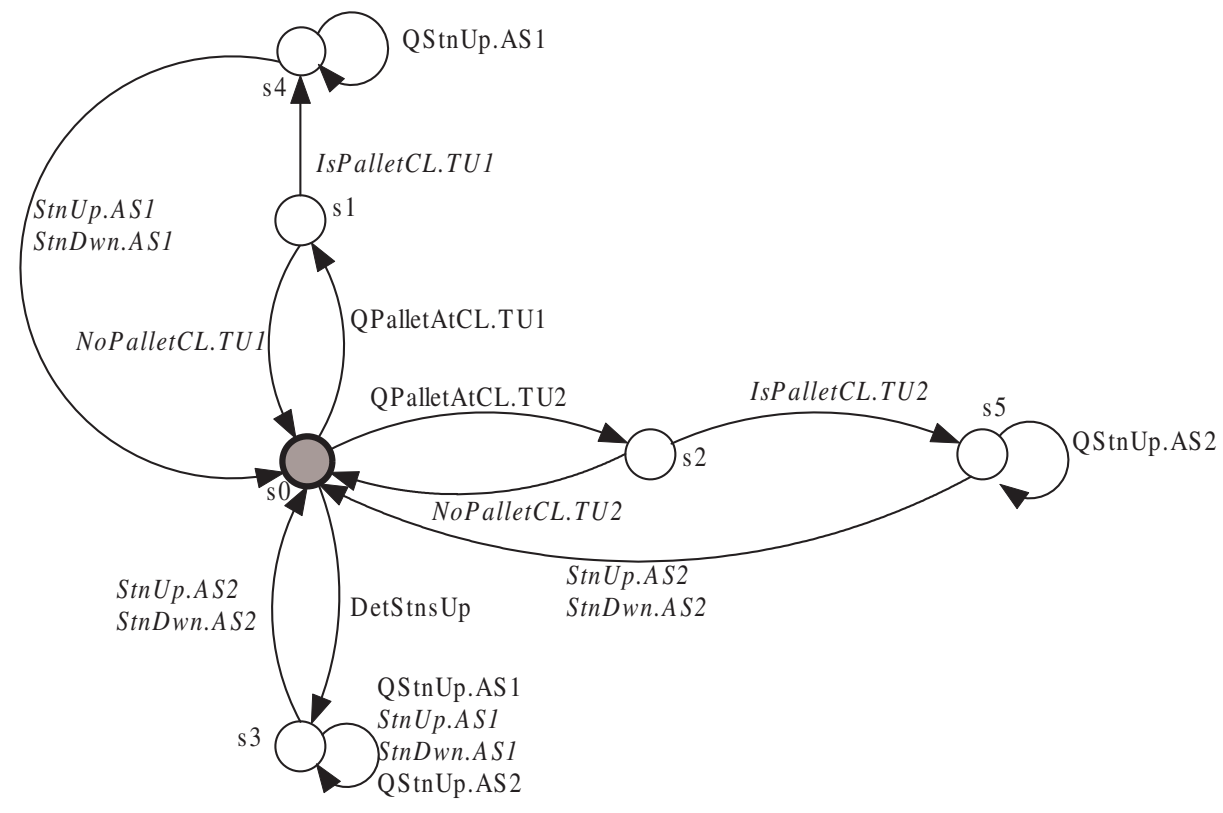

Figure 7.26: HndlComEventsAS(from [21]) 


\section{Master Thesis - R. Song - McMaster - Computing and Software}

We now discuss the supervisors for preventing overflow of conveyor areas EL11, EL21, EL31 and EL41. OFProtEL11 (see Figure 7.27) is used to prevent overflow of area EL11, but it also enforces that a pallet can not be processed by AS1 until it has been transferred to EL11. OFProtEL21 (see Figure 7.28) is identical to OFProtEL11 up to relabeling. OFProtEL31 (see Figure 7.29) is similar to the previous two. It prevents overflow of area EL31 and enforces that a pallet can not be processed or repaired by AS3 until it has been transferred to EL31. OFProtEL41(see Figure 7.30) prevents overflow of area EL41. However, it does not have the selfloop transitions of event MvOutPallet.IO at all states except $s 0$ and $s 10$, because the plant component CapEL41 (Figure 7.20(d)) and the component supervisor ManageIO (Figure 7.34) will ensure that the event MvOutPallet.IO will not happen at state $s 0$ and $s 10$.

The next four component supervisors are used to prevent overflow of conveyor areas EL12, EL22, EL32 and EL42, shown in Figure 7.31. OFProtEL12, OFProtEL22, OFProtEL32 are identical up to relabeling. OFProtEL42 disables events QPalletType1In.IO and QPalletType2In.IO at state s3 instead of event MvInPallet.IO, because of the supervisor component ManageIO(Figure 7.34). If we use event MvInType1Pallet.IO(or MvInType2Pallet.IO), then the system will be blocked when the area EL42 is full (at state $s 3$ ) and ManageIO is at state $s 1$ (or $s 2$ ).

The four supervisors OFProtC1, OFProtC2, OFProtC3, and OFProtC4 are used to prevent overflow of the conveyor areas C1, C2, C3 and C4 in the central loop, respectively. They are identical up to relabeling and are shown in Figure 7.32.

We now describe two supervisors for the I/O station. AltMvInTypes is used to enforce that the type of pallets entering the system must alternates, starting with Type1 pallets. It is shown in Figure 7.33. Note that both of the states are marked, because the last pallet entering the system can be either Type1 or Type2. ManageIO 
Master Thesis - R. Song - McMaster - Computing and Software
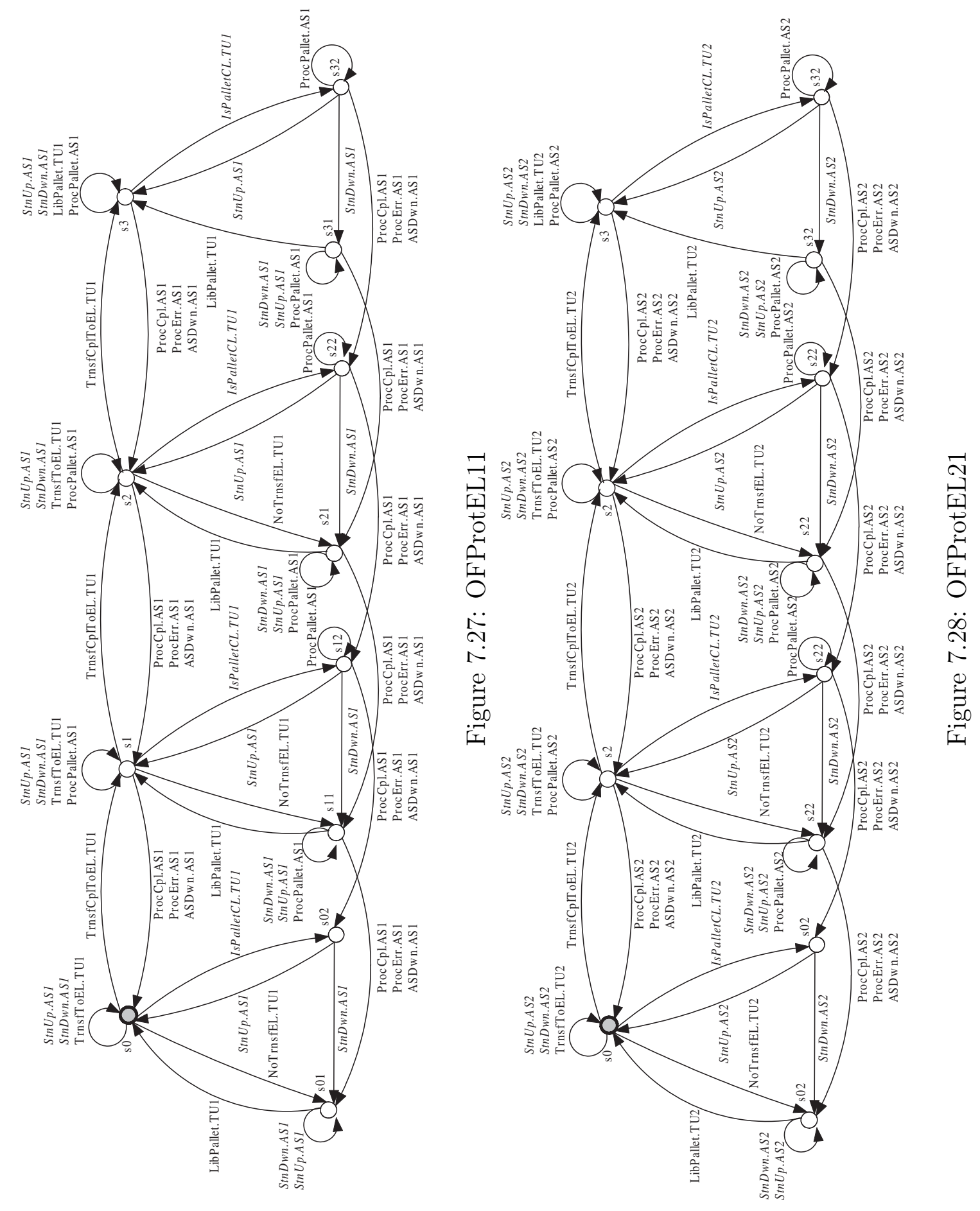
Master Thesis - R. Song-McMaster-Computing and Software
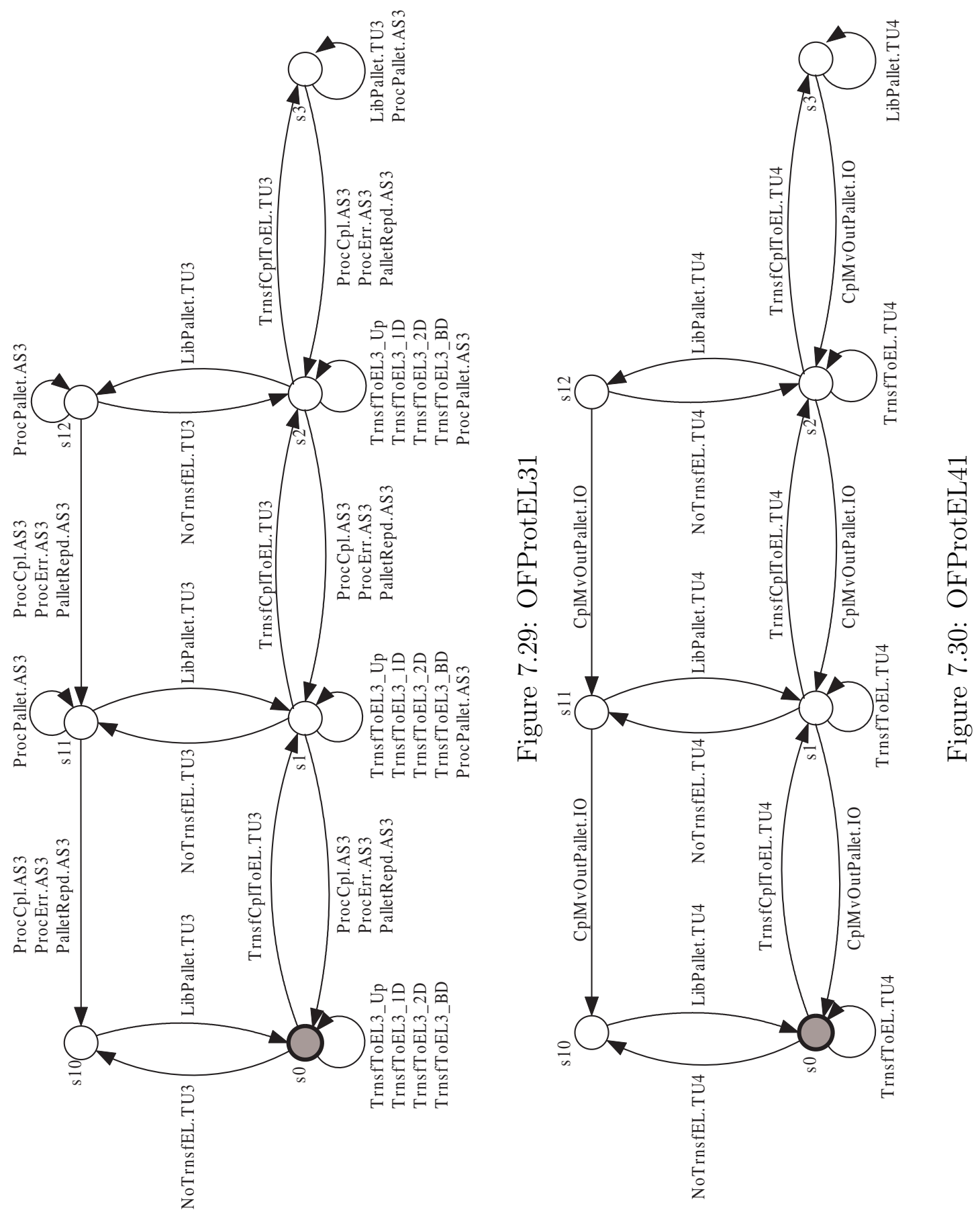
Master Thesis - R. Song - McMaster - Computing and Software

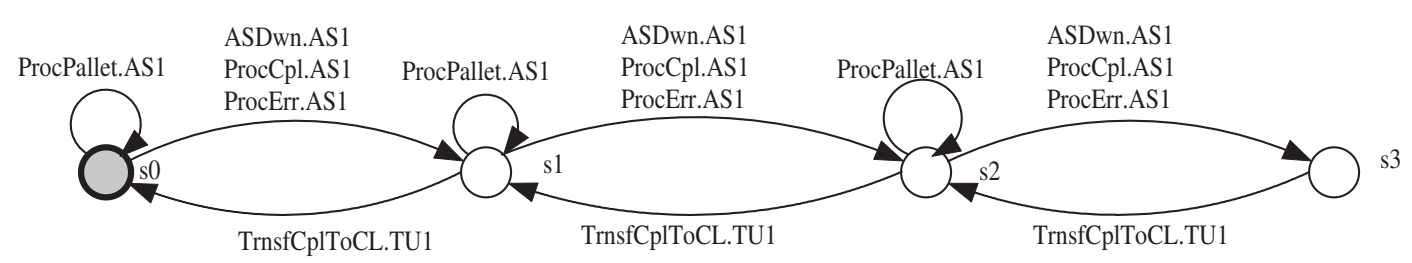

(a) OFProtEL12

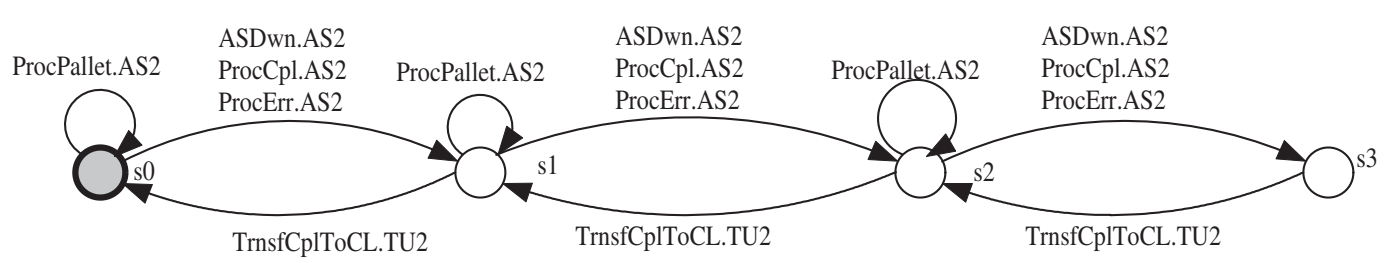

(b) OFProtEL22

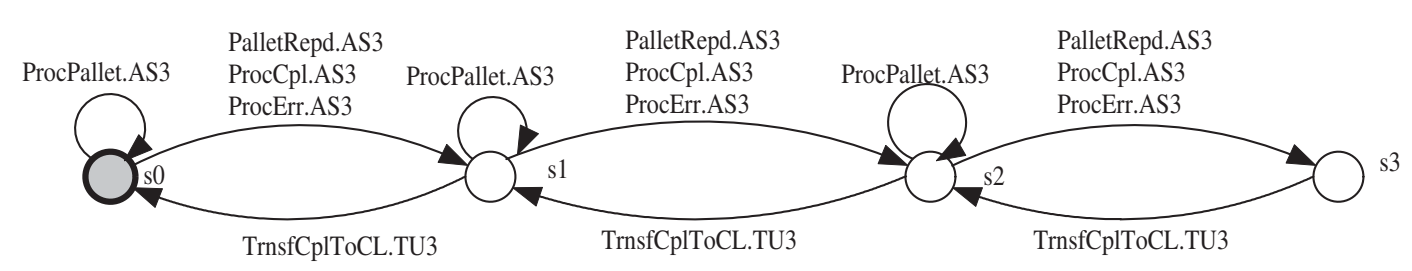

(c) OFProtEL32

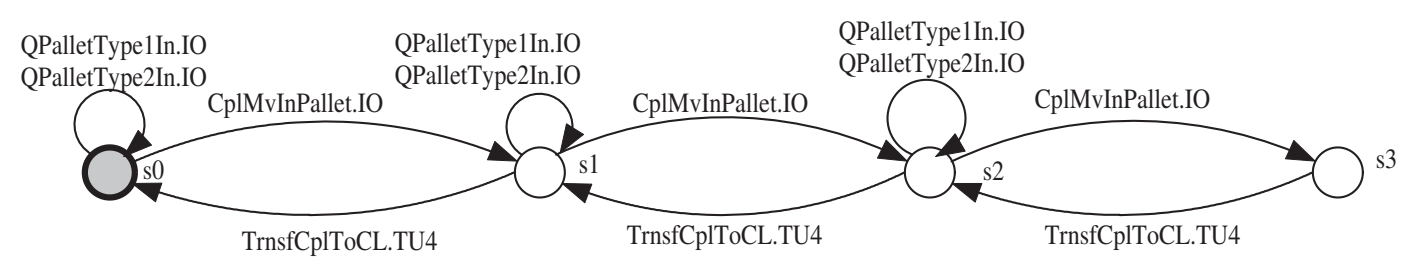

(d) OFProtEL42

Figure 7.31: OFProtEL12, OFProtEL22, OFProtEL32, OFProtEL42

is used to control that the $\mathrm{I} / \mathrm{O}$ station can only either move out a pallet or move in a pallet at a given time. ManageIO is shown in Figure 7.34.

\subsection{Verifying Properties}

So far, we have discussed all the necessary supervisors to meet the control specifications described in Section 7.2. However, when we put everything together and 
Master Thesis - R. Song - McMaster - Computing and Software

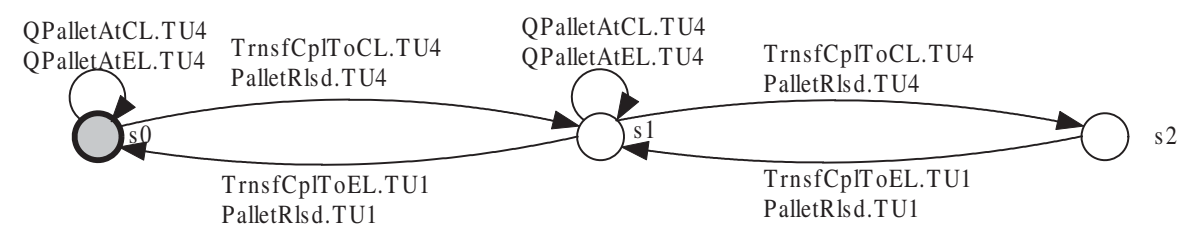

(a) OFProtC1

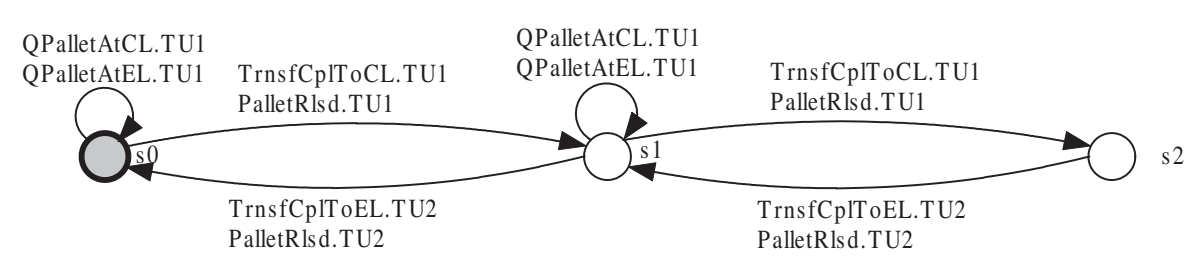

(b) OFProtC2

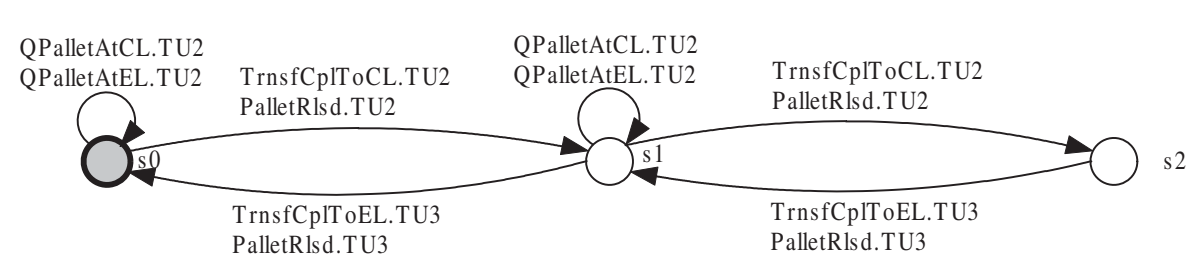

(c) OFProtC3

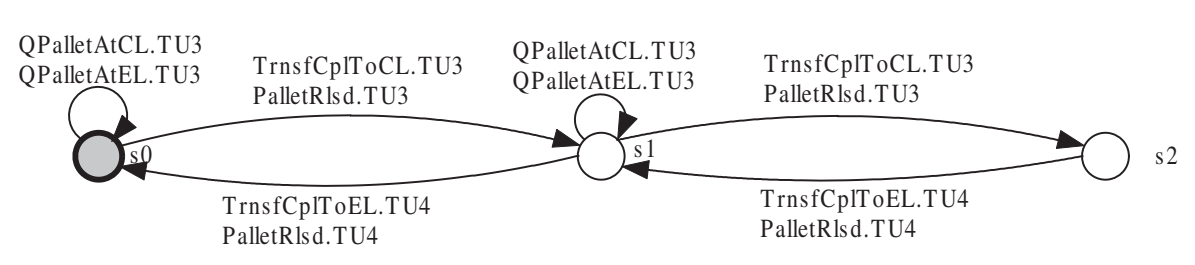

(d) OFProtC4

Figure 7.32: OFProtC1, OFProtC2, OFProtC3, OFProtC4

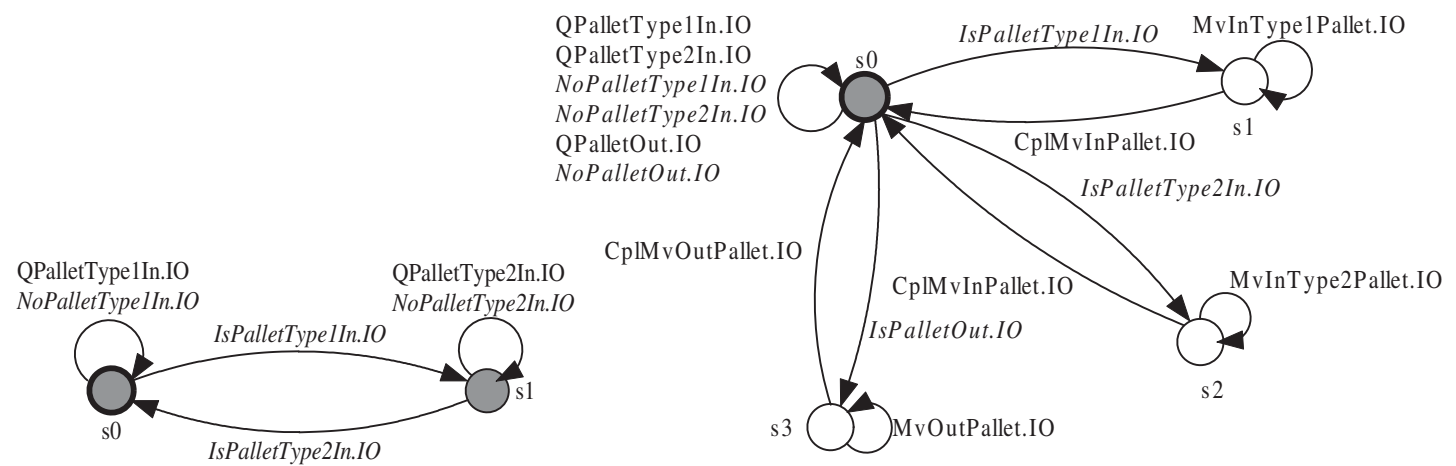

Figure 7.33: AltMvInTypes

Figure 7.34: ManageIO 


\section{Master Thesis - R. Song - McMaster - Computing and Software}

use our software tool to verify the high-level and all the low-levels, we find that the high-level is blocking. For example, when there are two pallets in each area of the central loop, the system will be blocked, because all the events QPalletCL.X and QPalletEL.X (where X = TU1, TU2, TU3, TU4) have been disabled.

Therefore, we add one more component supervisor OFProtAIP to restrict the number of pallets in the system (excluding external loop 4) at once to seven, shown in Figure 7.35 .

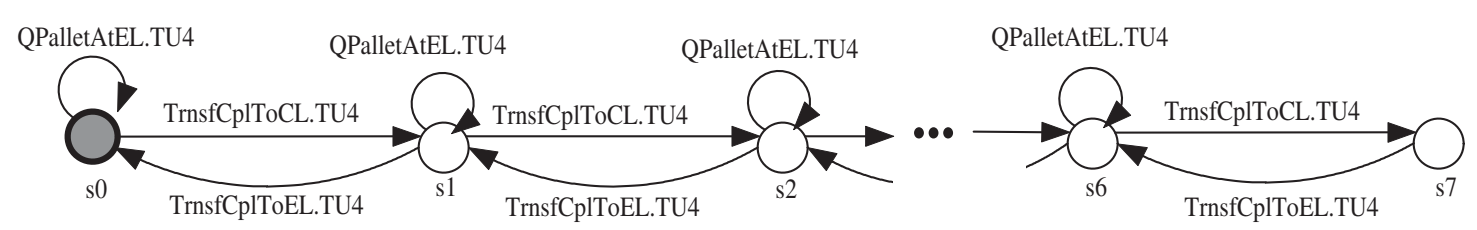

Figure 7.35: OFProtAIP

We then verified the system again using our program, and found that it satisfied all the conditions (level-wise nonblocking, level-wise controllable and interface consistent). We thus can conclude that the flat system is nonblocking by Theorem 3.1 and the flat supervisor of the system is controllable with respect to the flat plant of the system by Theorem 3.2. The system was verified on a Dell Dimension 3000 desktop PC (Pentium 42.8 GHz CPU, 512MB DDR 400 memory) running Fedora Core 2. It took 151 seconds to verify the high-level and less than 1 second to verify each low-level. All the computational time data in the rest of this thesis is based on this machine. The reachable state space sizes of the high-level and low-levels are listed in Table 7.1 under the column 3-2 AIP system ${ }^{4}$. The total estimated worst-case state space $\operatorname{size}^{5}$ of the AIP system is $3.61 \times 10^{25}$. The program initializes the size of the

\footnotetext{
${ }^{4} 3-2$ comes from the capacity $(=3)$ of each external loop conveyor area and the capacity $(=2)$ of each each central loop conveyor area

${ }^{5}$ The estimated size is calculated by multiplying the high-level state space size and all the lowlevel state space sizes together. We use this way to estimate the system state space size because the whole system is too large to be verified as a flat system. It is likely that the actual size of the system is much smaller.
} 


\section{Master Thesis - R. Song-McMaster-Computing and Software}

BDD node table as 2,000,000 and the cache table size as 1,000,000 for this example, and it did not increase both sizes during the verification computing. As each BDD node and each entry in the cache table in the BDD package we used occupies 20 bytes of memory, and the package uses 6 such cache tables for BDD operations, we actually used $(2,000,000+1,000,000 \times 6) \times 20=160 \mathrm{MB}$ memory $^{6}$. We also treated this AIP example as a flat system and tried to verify it using our BDD-based flat system tool. The program soon used up all the memory (RAM) by increasing the node and cache table and began to use the swap space, so the speed dramatically decreased. The program ran for 24 hours and still couldn't finish the computation so we thought there is no need to let it run any more.

\begin{tabular}{|c|c|c|c|c|c|c|}
\hline \multirow[b]{2}{*}{ Level/System } & \multicolumn{3}{|c|}{ 3-2 AIP system } & \multicolumn{3}{|c|}{ 5-4 AIP system } \\
\hline & $\begin{array}{l}\text { Number of } \\
\text { Reachable } \\
\text { States }\end{array}$ & $\begin{array}{c}\text { Computing } \\
\text { Time (s) }\end{array}$ & $\begin{array}{c}\text { Memory for } \\
\text { BDD node and } \\
\text { cache Table } \\
\text { (MB) }\end{array}$ & $\begin{array}{l}\text { Number of } \\
\text { Reachable } \\
\text { States }\end{array}$ & $\begin{array}{l}\text { Computing } \\
\text { Time (s) }\end{array}$ & $\begin{array}{c}\text { Memory for } \\
\text { BDD node and } \\
\text { cache Table } \\
\text { (MB) }\end{array}$ \\
\hline High-level & $2.65 * 10^{10}$ & 151 & 160 & $5.16 * 10^{13}$ & 1543 & 160 \\
\hline Low-level 1(AS1) & 120 & $<1$ & 160 & 120 & $<1$ & 160 \\
\hline Low-level 2(AS2) & 120 & $<1$ & 160 & 120 & $<1$ & 160 \\
\hline Low-level 3(AS3) & 106 & $<1$ & 160 & 106 & $<1$ & 160 \\
\hline Low-level 4(TU1) & 98 & $<1$ & 160 & 98 & $<1$ & 160 \\
\hline Low-level 5(TU2) & 98 & $<1$ & 160 & 98 & $<1$ & 160 \\
\hline Low-level 6(TU3) & 204 & $<1$ & 160 & 204 & $<1$ & 160 \\
\hline Low-level 7(TU4) & 152 & $<1$ & 160 & 152 & $<1$ & 160 \\
\hline Low-level 8(IO) & 3 & $<1$ & 160 & 3 & $<1$ & 160 \\
\hline Total Estimated & $3.61 * 10^{25}$ & & & $7.04 * 10^{28}$ & & \\
\hline
\end{tabular}

Table 7.1: The AIP example data (verification)

However, our BDD-based flat system tool can verify the original HISC model of the AIP system in [23] using 160MB memory (RAM) and taking 246 seconds. The size of the total reachable state space for this system was $7.32 \times 10^{13}$. Our BDD-based

\footnotetext{
${ }^{6}$ Actually, our software tool always initializes the size of BDD node table as 2,000,000 and the size of cache table size as $1,000,000$, because a personal computer with more than $160 \mathrm{MB}$ was very common at the time the author was writing this thesis. For some small subsystems(e.g. the low-level subsystems of the 3-2 AIP system here), one can decrease the initial size and may not affect the speed of the computation at all.
} 


\section{Master Thesis - R. Song - McMaster - Computing and Software}

HISC tool can verify the original high-level in 2 seconds. The size of the reachable state space for the original high-level is $3.31 \times 10^{6}$.

We also extend the system by increasing the capacity of each area in the central loop to 4 and the capacity of each area in each of the external loop to 5. To do this, we need to extend plant DES CapEL $X 1$, CapEL $X 2$, CapC $X$, and supervisor DES OFProtEL $X \mathbf{1}$, OFProtEL $X \mathbf{2}$, OFProtC $X$, where $X=1,2,3,4$. We then extend the supervisor OFProtAIP to restrict the number of pallets in the system (excluding external loop 4) at once to be 15. The method to extend these DES to the new capacity should be obvious. We call this system as the 5-4 AIP system. For the 5-4 AIP system, the reachable state space size for the high-level was $5.16 \times 10^{13}$, and the total estimated worst-case reachable state space size was $7.04 \times 10^{28}$ (See Table 7.1). We do not present the 5-4 AIP system in this thesis in detail because some of the DES diagrams for it are too large to be put on a single page.

\subsection{Synthesizing Supervisors}

In the previous section, we added a supervisor OFProtAIP to restrict the number of pallets in the AIP system (excluding external loop 4) to ensure the high-level was nonblocking. This supervisor obviously restricts the behavior of the system. However, it is difficult to design supervisors by hand to solve the blocking problem and not to restrict the behavior in the mean time as the high-level is so large. Therefore, we can use our synthesis method to produce the control predicates by treating all the supervisor components in the system as specifications. We of course exclude OFProtAIP from this system.

The reachable state space size for the high-level of the 3-2 AIP system under the synthesized control predicates is $1.10 \times 10^{12}$, and it took 1415 seconds (24 minutes) to 


\section{Master Thesis - R. Song-McMaster-Computing and Software}

complete the synthesis process for the high-level. This is much longer than the time to verify the 3-2 AIP system. One reason is that the high-level of this system has a bigger state space. Another reason is that the verification algorithm (Algorithm 5.1) does not have a similar repeat loop as the synthesis algorithm (Algorithm 4.1) has. The total estimated worst-case reachable state space size of the system under the control predicates is $1.50 \times 10^{27}$. Table 7.2 shows all the related data for the $3-2$ AIP system and the 5-4 AIP system.

\begin{tabular}{|c|c|c|c|c|c|c|}
\hline \multirow{2}{*}{ Level/System } & \multicolumn{3}{|c|}{ 3-2 AIP system } & \multicolumn{3}{|c|}{ 5-4 AIP system } \\
\cline { 2 - 7 } & $\begin{array}{c}\text { Number of } \\
\text { Reachable } \\
\text { States }\end{array}$ & $\begin{array}{c}\text { Computing } \\
\text { Time (s) }\end{array}$ & $\begin{array}{c}\text { Memory for } \\
\text { BDD node } \\
\text { and cache } \\
\text { Table (MB) }\end{array}$ & $\begin{array}{c}\text { Number of } \\
\text { Reachable } \\
\text { States }\end{array}$ & $\begin{array}{c}\text { Memory for } \\
\text { Computing } \\
\text { Time (s) }\end{array}$ & $\begin{array}{c}\text { BDD node and } \\
\text { cache Table } \\
\text { (MB) }\end{array}$ \\
\hline High-level & $1.10^{*} 10^{12}$ & 1415 & 160 & $1.14^{*} 10^{15}$ & 7679 & 160 \\
\hline Low-level 1(AS1) & 120 & $<1$ & 160 & 120 & $<1$ & 160 \\
\hline Low-level 2(AS2) & 120 & $<1$ & 160 & 120 & $<1$ & 160 \\
\hline Low-level 3(AS3) & 106 & $<1$ & 160 & 106 & $<1$ & 160 \\
\hline Low-level 4(TU1) & 98 & $<1$ & 160 & 98 & $<1$ & 160 \\
\hline Low-level 5(TU2) & 98 & $<1$ & 160 & 98 & $<1$ & 160 \\
\hline Low-level 6(TU3) & 204 & $<1$ & 160 & 204 & $<1$ & 160 \\
\hline Low-level 7(TU4) & 152 & $<1$ & 160 & 152 & $<1$ & 160 \\
\hline Low-level 8(IO) & 3 & $<1$ & 160 & 3 & $<1$ & 160 \\
\hline Total Estimated & $1.50 * 10^{27}$ & & & $1.51 * 10^{30}$ & & \\
\hline
\end{tabular}

Table 7.2: The AIP example data (synthesis)

In total there are 37 controllable high-level and request events in the AIP system. For the 3-2 AIP system, the biggest BDD for the prime simplified control predicates (see description in section 6.5.1) is for event QPalletAtEL.TU4 $\left(f_{H_{\text {QPalletAtEL.TU4 }}^{\prime}}\right)$, which includes 516 BDD nodes. The BDD for the triple-prime simplified control predicate for this event includes $200 \mathrm{BDD}$ nodes $\left(f_{H_{Q P a l l e t A t E L . T U 4}}^{\prime \prime \prime}\right)$. For the 5 -4 AIP system, the biggest BDD for the prime control predicates is also for event QPalletAtEL.TU4, which includes 715 BDD nodes. The BDD for the triple-prime control predicate for this event includes 266 BDD nodes.

There are 206 controllable low-level and answer events in total. Since all the 


\section{Master Thesis - R. Song - McMaster - Computing and Software}

low-levels already satisfy their corresponding conditions, the BDD for the control predicates are rather small. For the 3-2 AIP system (or the 5-4 AIP system), the biggest BDD representation for the prime simplified control predicates is for event tu3_DwnOpNeeded.TU3 $\left(f_{L_{6 t u 3 \_D w n O p N e e d e d . T U 3}}^{\prime}\right)$, which includes only 28 BDD nodes. The BDD representation of the triple-prime simplified control predicate for this event includes $20 \mathrm{BDD}$ nodes $\left(f_{L_{6 t u 3} \prime \prime \text { Dwn OpNeeded.TU3 }}^{\prime \prime \prime}\right)$.

\subsection{Results For the AIP Example}

In this chapter, we extended the AIP example from $[21,23]$ by adding more control requirements. The extended system is much bigger and more complicated than the original system which has a state space on the order of $10^{13}$. The high-level in the 5-4 AIP system alone has a state space on the order of $10^{15}$, and its estimated worst-case state space is on the order of $10^{30}$. In particular, the capacity restrictions of conveyor areas make the system highly coupled. If we extend the STS of the AIP example in [31] to reflect the capacity restrictions of conveyor areas, the specification DES for them would likely need to be put on level 1 of the STS. Consequently, the STS will become much flatter, and it will make the STS less structured.

Even though our predicate-based algorithm with BDD implementation can handle a larger system than an automata-based algorithm, our BDD-based software tool for a flat system can not handle the whole 3-2 AIP system. However, with the HISC method, we can easily verify the 5-4 AIP system and synthesize supervisors on a common personal computer. 


\section{Chapter 8}

\section{Multiple AIP Example}

To demonstrate the ability of our low-level synthesis and verification algorithms, we construct a system as shown in Figure 8.1, where each MAIP- $j(j \in\{1,2,3\})$ is the high-level of the 3-2 AIP system in the previous chapter with appropriate event relabeling ${ }^{1}$ but used as low-levels (we will discuss how to relabel events in Section 8.1). Other than the MAIPs, the system also includes a testing unit, a packaging unit and three $4 \times 6$ buffers. By $4 \times 6$ buffers, we mean that each buffer has 4 slots, and the capacity of each slot is 6 (pallets). One slot must be filled or emptied completely, which means that pallets can only be loaded into or taken out of a MAIP six at a time. We also assume that the system always puts three Type1 pallets and three Type2 Pallets into the input buffer each time. This example is based upon the parallel manufacturing example in [21].

As in the previous chapter, we first give a system with supervisors so that we can examine our verification algorithms, then we relax one of the restrictions to perform synthesis.

\footnotetext{
${ }^{1}$ We do not use the whole AIP, because our software can not handle one subsystem as large as the whole 3-2 AIP.
} 
Master Thesis - R. Song - McMaster - Computing and Software

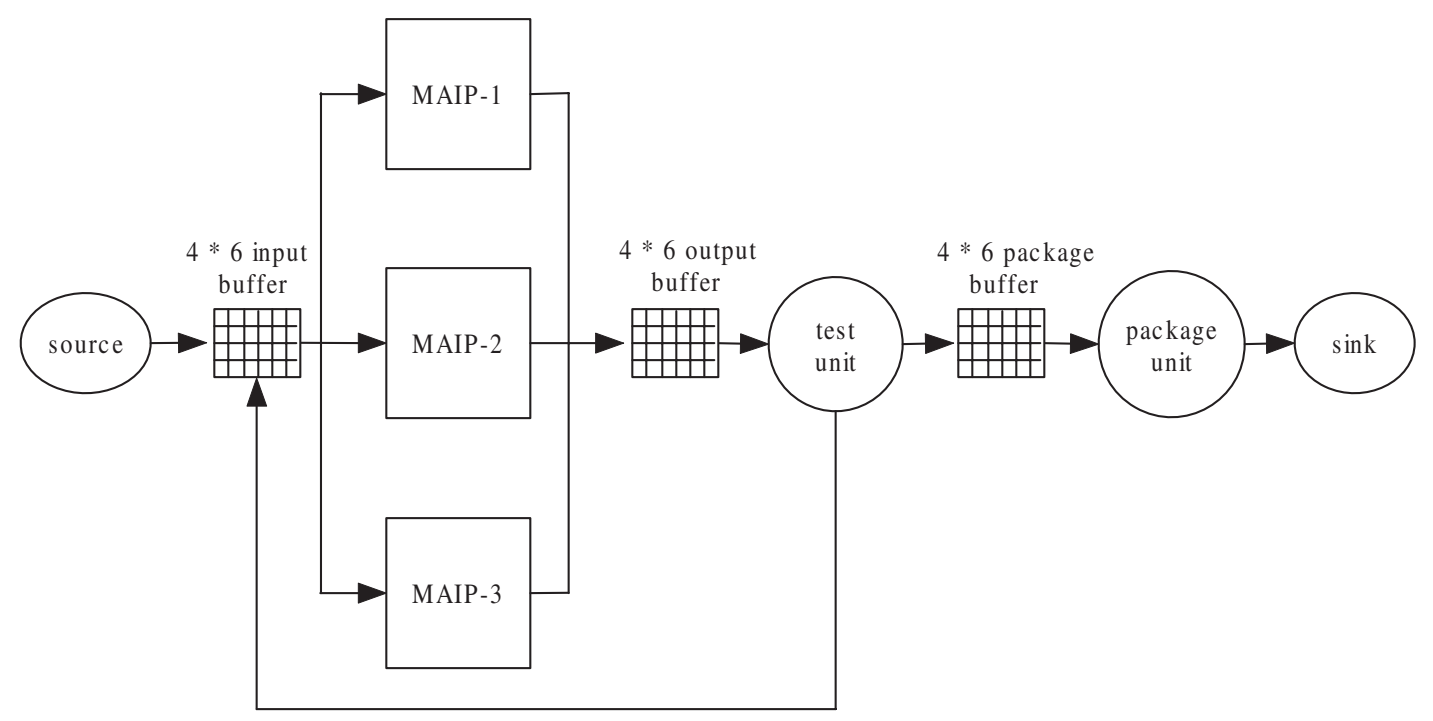

Figure 8.1: Block diagram of multiple AIPs

\subsection{System Model}

We model this system as a bi-level HISC system with three low-levels MAIP1, MAIP-2, and MAIP-3. As all the low-levels are identical up to relabeling, their interfaces are as well. The interface for MAIP- $j(j \in\{1,2,3\})$ is shown in Figure 8.2. Event startpallets $-j$ means that MAIP takes six pallets from an input buffer slot and starts processing them. Event finishpallets $-j$ means that six processed pallets are put into an output buffer slot.

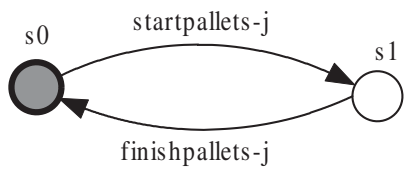

Figure 8.2: $\operatorname{IntfAIP-j}(\mathrm{j}=1,2,3)$

As we said before, the low-level MAIP- $j(j \in\{1,2,3\})$ here is the high-level of the 3-2 AIP system in the previous chapter with event relabeling. All the events(highlevel, request and answer events) in the original high-level of the 3-2 AIP system 


\section{Master Thesis - R. Song - McMaster - Computing and Software}

are treated as low-level events in MAIP- $j$. We also treat all the answer events in the original system as uncontrollable low-level events in MAIP- $j$ to capture the fact that we can not just disable them. The other events keep their original controllable or uncontrollable classification. Each event name in MAIP- $j$ is suffixed with $-j$ to capture the fact that events in each MAIP actually are different events, and so that the event partition requirements are satisfied.

The high-level plant components and all the interfaces in the original 3-2 AIP system are treated as low-level plant components in MAIP- $j$, and the high-level supervisors in the original system are treated as low-level supervisors except that we do not include OFProtAIP in MAIP- $j$. We also add three plant components PalletType1In- $j$, PalletType2In- $j$ and PalletOut- $j$ in low-level MAIP- $j$, as shown in Figure 8.3. These plant components state that MAIP- $j$ processes six pallets at a time.

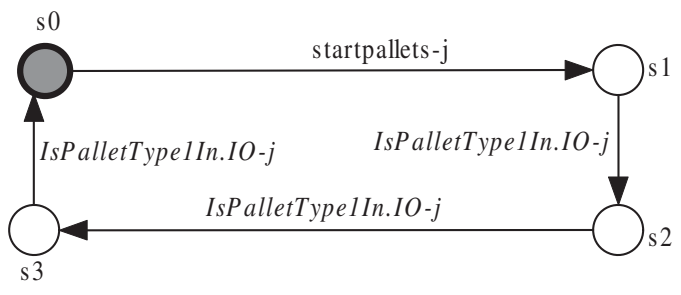

(a) PalletType1In- $j(j \in\{1,2,3\})$

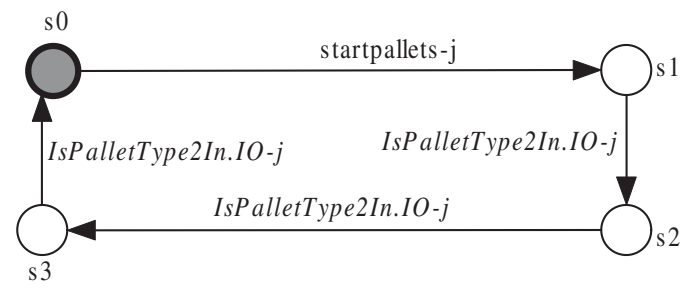

(b) PalletType2In- $j(j \in\{1,2,3\})$

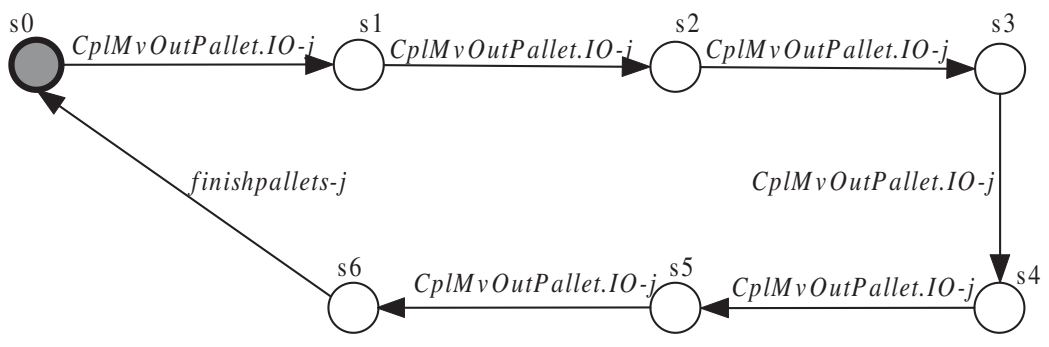

(c) PalletOut- $j(j \in\{1,2,3\})$

Figure 8.3: New low-level plants in 3-2 MAIP system(verfication)

In total, we have 30 plant components and 20 supervisor components in the low- 


\section{Master Thesis - R. Song - McMaster - Computing and Software}

level MAIP- $j$. The components in MAIP-j are shown in Figure 8.4. The plant $\mathbf{G}_{L_{j}}^{p}$ and supervisor $\mathbf{S}_{L_{j}}$ of MAIP-j are defined to be the synchronous product of the indicated automata.

\begin{tabular}{|c|c|}
\hline $\begin{array}{l}\text { ASS toreUpState.AS1-j, ASStoreUpState.AS2-j, QueryPalletAtTU.TU1-j, } \\
\text { QueryPalletAtTU.TU2-j, QueryPalletAtTU.TU3-j, QueryPalletAtTU.TU4-j, } \\
\text { QueryPalletAtIO-j, CapEL11-j, CapEL21-j, CapEL31-j, CapEL41-j, } \\
\text { CapEL12-j, CapEL22-j, CapEL32-j, CapEL42-j, CapC1-j, CapC2-j, CapC3-j, } \\
\text { CapC4-j, PalletType1In-j, PalletType2In-j, PalletOut-j, } \\
\text { IntfAssmbStn_AS1-j, IntfAssmbStn_AS2-j, IntfAssmbStn_AS3-j, } \\
\text { IntfTU_TU1-j, IntfTU_TU2-j, IntfTU_TU3-j, IntfTU_TU4-j, IntfIO-j }\end{array}$ & $\mathbf{G}_{L j}$ \\
\hline $\begin{array}{l}\text { ManageTU1-j, ManageTU2-j, ManageTU3-j, ManageTU4-j, ManageIO-j, } \\
\text { DetWhichStnUp-j, HndlComEvents AS-j, OFProtEL11-j, OFProtEL21-j, } \\
\text { OFProtEL31-j, OFProtEL41-j, OFProtEL12-j,OFProtEL22-j, OFProtEL32-j, } \\
\text { OFProtEL42-j, OFProtC1-j, OFProtC2-j, OFProtC3-j, OFProtC4-j, AltMvInTypes-j }\end{array}$ & \\
\hline
\end{tabular}

Figure 8.4: Components in low-level subsystem MAIP- $j,(j=1,2,3)$

The high-level plant components are shown in Figure 8.5. Plant component Source contains only one event, new_pallets, which means that six new pallets are put into one input buffer slot. The test unit (TestUnit) takes six pallets from the output buffer and then tests if the assembly of those pallets is correct. If any of the six pallets can not pass the test, information will be written on the labels of the failed pallets and the six pallets altogether must be sent back to the input buffer. For those pallets in the group that have passed the test, they will just go through the central loop of the MAIP- $j$ undergoing no assembly operations as their labels were not altered and are allowed to exist via the I/O station. If all six pallets pass the test, they are placed in the package buffer. Plant component PackSys is straightforward. Event take_pallets means that the package unit takes out all of the six successfully processed pallets in one package buffer slot. Plant component Sink also contains only one event, allow_exits, which means that the system allows the six packaged pallets to exit from the system.

The high-level supervisors are shown in Figure 8.6. InBuf, OutBuf and PackBuf are designed to prevent their corresponding buffers from overflow and underflow. 


\section{Master Thesis - R. Song - McMaster - Computing and Software}

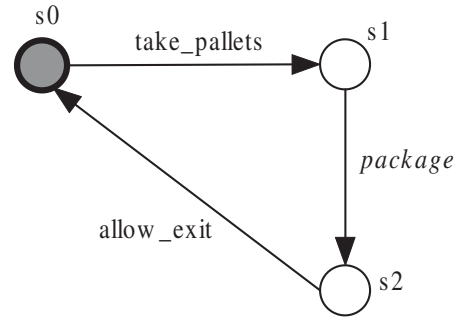

(a) PackSys

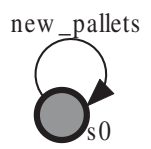

(c) Source

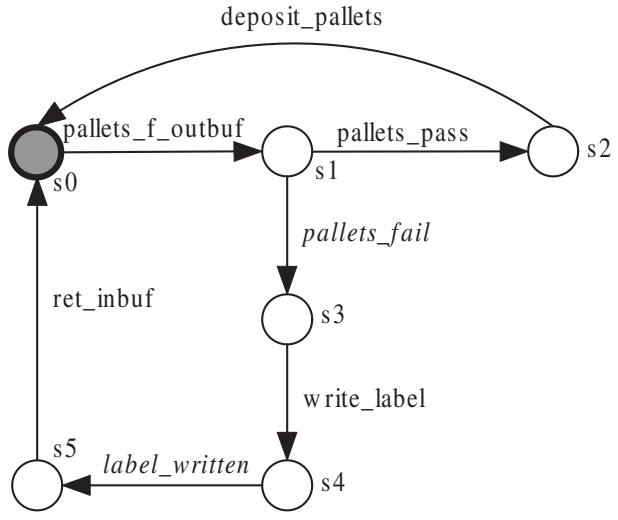

(b) TestUnit

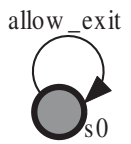

(d) Sink

Figure 8.5: High-level plant components

NoBlock is to make sure that the system is nonblocking.

The event partition is defined as follows, where $j=1,2,3, \Sigma_{H}^{\prime \prime}$ is the high-level event set, $\Sigma_{R_{1}}^{\prime \prime}, \ldots, \Sigma_{R_{8}}^{\prime \prime}$ are the request event sets, and $\Sigma_{A_{1}}^{\prime \prime}, \ldots, \Sigma_{A_{8}}^{\prime \prime}$ are the answer event sets for the 3-2 AIP example in the previous chapter. $\sigma-j$ means that the name of event $\sigma$ is suffixed with " $-j "$.

$$
\begin{aligned}
& \Sigma_{H}:=\{\text { take_pallets,package,allow_exit,new_pallets,pallets_f_outbuf }, \\
& \text { pallets_pass, deposit_pallets, pallets_fail,write_label, label_written, } \\
& \text { ret_inbuf\} } \\
& \Sigma_{R_{j}}:=\{\text { startpallets }-j\} \\
& \Sigma_{A_{j}}:=\{\text { finishpallets- } j\} \\
& \Sigma_{L_{j}}:=\left\{\sigma-j \mid \sigma \in\left\{\Sigma_{H}^{\prime \prime} \cup \Sigma_{R_{1}}^{\prime \prime} \cup \cdots \cup \Sigma_{R_{8}}^{\prime \prime} \cup \Sigma_{A_{1}}^{\prime \prime} \cup \cdots \cup \Sigma_{A_{8}}^{\prime \prime}\right\}\right\}
\end{aligned}
$$


Master Thesis - R. Song - McMaster - Computing and Software

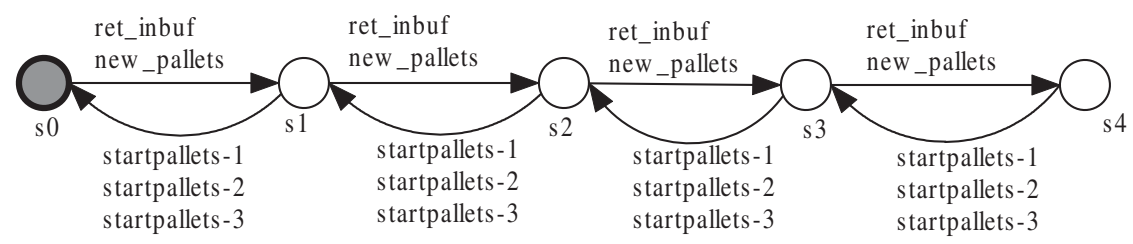

(a) InBuf

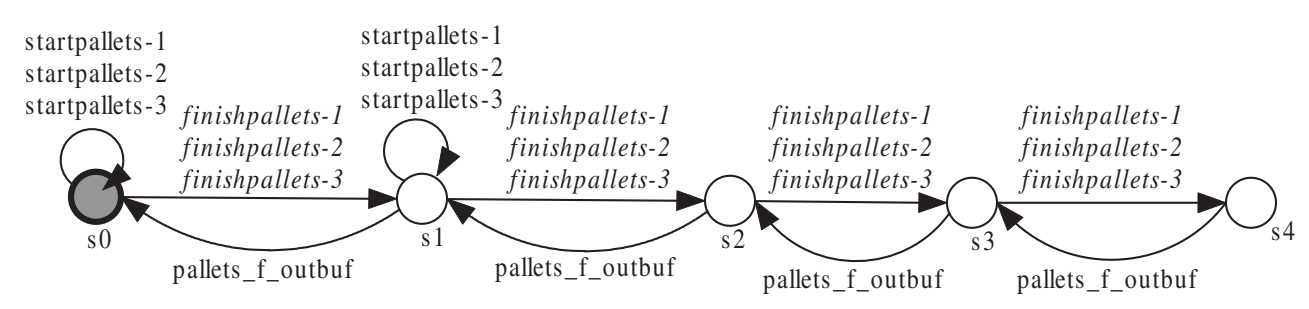

(b) OutBuf

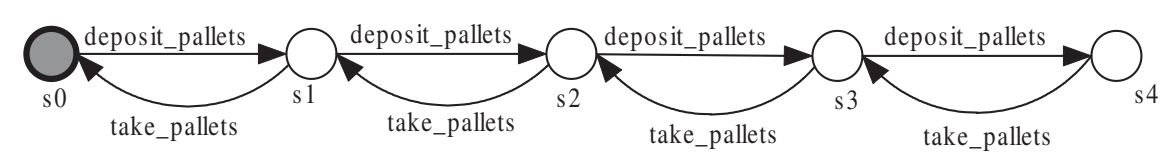

(c) PackBuf

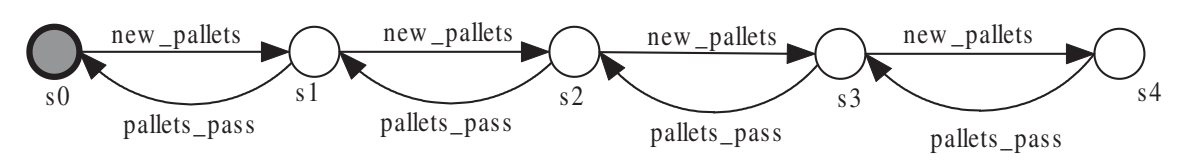

(d) NoBlock

Figure 8.6: High-level supervisor components

\subsection{Verifying Properties}

We now use our software tool to verify this system, and find that the system satisfies all the HISC local conditions (level-wise nonblocking, level-wise controllable and interface consistent). We thus can conclude that the flat system is nonblocking by Theorem 3.1 and the flat supervisor of the system is controllable with respect to the flat plant of the system by Theorem 3.2. The resulting computational data is shown in Table 8.1 under the column 3-2 MAIP system. We also treat this system as a flat system and use our BDD-based software tool for flat systems to verify it. The tool soon used up all the available memory (RAM) and there was still no result after 


\section{Master Thesis - R. Song-McMaster-Computing and Software}

24 hours.

\begin{tabular}{|c|c|c|c|c|c|c|}
\hline \multirow{2}{*}{ Level/System } & \multicolumn{3}{|c|}{$3-2$ MAIP system } & \multicolumn{3}{c|}{$5-4$ MAIP system } \\
\cline { 2 - 7 } & $\begin{array}{c}\text { Number of } \\
\text { States }\end{array}$ & $\begin{array}{c}\text { Computing } \\
\text { Time }(\mathrm{s})\end{array}$ & $\begin{array}{c}\text { Memory for } \\
\text { BDD node } \\
\text { and cache } \\
\text { Table (MB) }\end{array}$ & $\begin{array}{c}\text { Number of } \\
\text { Reachable } \\
\text { States }\end{array}$ & $\begin{array}{c}\text { Computing } \\
\text { Time (s) }\end{array}$ & $\begin{array}{c}\text { Memory for } \\
\text { BDD node } \\
\text { and cache } \\
\text { Table (MB) }\end{array}$ \\
\hline High-level & 4260 & $<1$ & 160 & 4260 & $<1$ & 160 \\
\hline $\begin{array}{c}\text { Low-level MAIP-j } \\
\mathrm{j}=1,2,3\end{array}$ & $6.88 * 10^{8}$ & 66 & 160 & $3.04 * 10^{12}$ & 1397 & 160 \\
\hline Total Estimated & $1.39 * 10^{30}$ & & & $1.20 * 10^{41}$ & & \\
\hline
\end{tabular}

Table 8.1: The multiple AIP example data(verification)

We also built a system by reusing the high-level in the 5-4 AIP system in the previous chapter in a similar fashion. We refer to this system as 5-4 MAIP system. For the 5-4 MAIP system, we increase the size of each buffer to $4 \times 14$ (pallets), and assume that the system always put seven Type1 pallets and seven Type2 Pallets into a 14-pallet slot of the input buffer each time. The high-level model and interfaces of the 5-4 MAIP system are the same as the high-level model and interfaces of the 3-2 MAIP system. The low-levels can be built by using the same method of building low-levels in the 3-2 MAIP system. The plant components PalletType1In- $j$, PalletType2In- $j$ and PalletOut- $j$ in low-level MAIP- $j$ of the 5-4 MAIP system are shown in Figure 8.7. The event partition is same as that of the 3-2 MAIP system. This 5-4 MAIP system also satisfies all the HISC local conditions (level-wise nonblocking, level-wise controllable and interface consistent). We thus can conclude that the flat system is nonblocking and the flat supervisor of the system is controllable with respect to the flat plant of the system. The resulting computational data is shown in Table 8.1 under the column 5-4 MAIP system. 
Master Thesis - R. Song - McMaster - Computing and Software

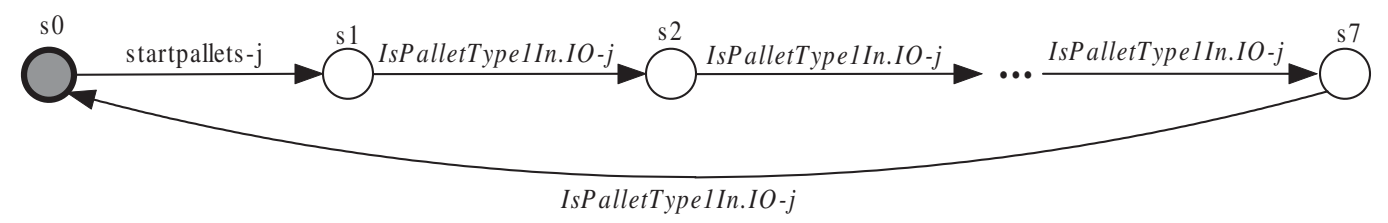

(a) PalletType1In- $j(j \in\{1,2,3\})$

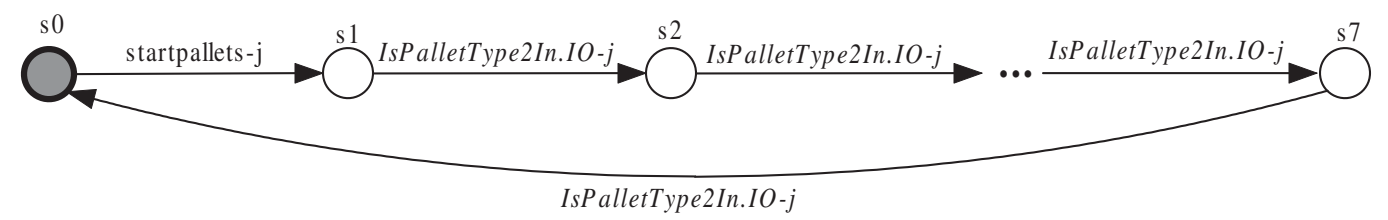

(b) PalletType2In- $j(j \in\{1,2,3\})$

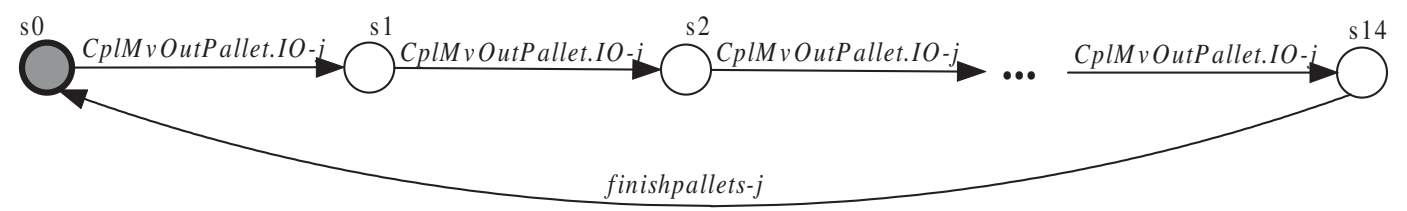

(c) PalletOut- $j(j \in\{1,2,3\})$

Figure 8.7: New low-level plants for the 5-4 MAIP system(verification)

\subsection{Synthesizing Supervisors}

In the above 3-2 MAIP system, the low-level MAIP- $j(j \in\{1,2,3\})$ processes pallets six at a time. In the previous chapter, the supervisor component OFProtAIP was used to make sure that the number of the pallets in the 3-2 AIP system (excluding external loop 4) is at most seven at any given time. Here we require that the above 3-2 MAIP system processes six pallets at a time, so it respects the requirement of the component OFProtAIP in the 3-2 AIP system. We set the size of each slot as six because we want the number of Type1 pallets and the number of Type2 pallets in each slot to be equal.

Now we increase the capacity of each slot in each buffer in the 3-2 MAIP system ${ }^{2}$

\footnotetext{
${ }^{2}$ Recall that in the 3-2 AIP system in the previous chapter, the capacity of each area in the central loop is 2 and the capacity of each area in an external loop is 3 , so in total the capacity of the conveyors is $2 \times 4+3 \times 2 \times 4=32$.
} 


\section{Master Thesis - R. Song-McMaster-Computing and Software}

to 32. Consequently, the plant component PalletType1In- $j$ should have 16 continuous IsPalletType1In.IO- $j$ transitions; the plant component PalletType2In- $j$ should have 16 continuous IsPalletType2In.IO-j transitions, and the plant component PalletOut- $j$ should have 32 continuous CplMvOutPallet.IO- $j$ transitions. They are shown in Figure 8.8.

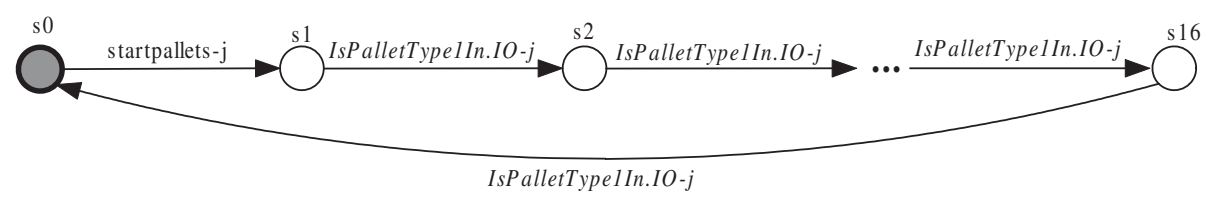

(a) PalletType1In- $j(j \in\{1,2,3\})$

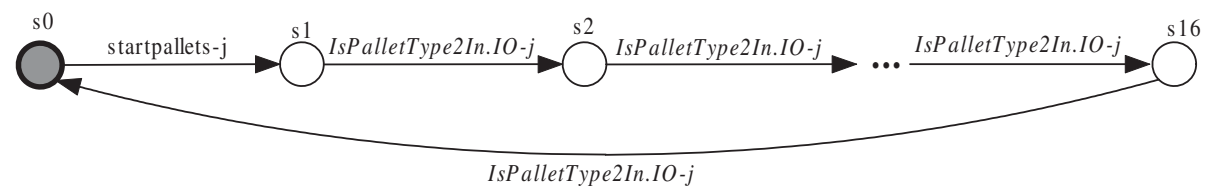

(b) PalletType2In- $j(j \in\{1,2,3\})$

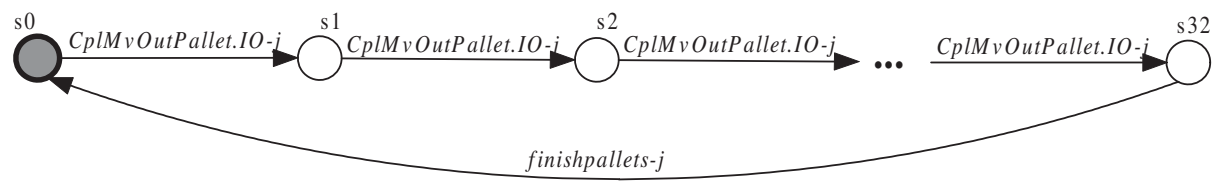

(c) PalletOut- $j(j \in\{1,2,3\})$

Figure 8.8: New low-level plants for the 3-2 MAIP system(synthesis)

When we use our software tool to verify this system, we find that it each lowlevel MAIP- $j(j \in\{1,2,3\})$ blocks. We then synthesize a low-level proper supervisor and its corresponding control predicates for each low-level MAIP- $j$. The resulting computational data for this system is shown in Table 8.2 under the column 3-2 MAIP system.

Similarly, we also increase the capacity of each slot in each buffer to 56 for the 5-4 MAIP system. Therefore, the plant component PalletType1In- $j$ should have 28 continuous IsPalletType1In.IO- $j$ transitions; the plant component PalletType2In$j$ should have 28 continuous IsPalletType2In.IO- $j$ transitions, and the plant com- 
Master Thesis - R. Song - McMaster - Computing and Software

ponent PalletOut- $j$ should have 56 continuous $C p l M v O u t P a l l e t . I O-j$ transitions. One can easily draw the diagrams for them from Figure 8.8. The low-levels of this 5-4 MAIP system also block. Therefore, we can synthesize a low-level proper supervisor and its corresponding control predicates for each low-level MAIP- $j$. The resulting computational data for this system is shown in Table 8.2 under the column 5-4 MAIP system. Note that the memory for BDD node and cache table for synthesizing the low-level MAIP- $j$ in this system has increased to 220MB, and all the other systems we examined in this thesis never increased the predefined BDD node and cache table size. Note also that the computing time is very long (26.7 hours). We decided to let the synthesizing process run this long time because it did not use up all the available memory (RAM) in our computer (512MB).

\begin{tabular}{|c|c|c|c|c|c|c|}
\hline \multirow[b]{2}{*}{ Level/System } & \multicolumn{3}{|c|}{ 3-2 MAIP system } & \multicolumn{3}{|c|}{ 5-4 MAIP system } \\
\hline & $\begin{array}{l}\text { Number of } \\
\text { Reachable } \\
\text { States }\end{array}$ & $\begin{array}{l}\text { Computing } \\
\text { Time (s) }\end{array}$ & $\begin{array}{l}\text { Memory for } \\
\text { BDD node } \\
\text { and cache } \\
\text { Table (MB) }\end{array}$ & $\begin{array}{c}\text { Number of } \\
\text { Reachable } \\
\text { States }\end{array}$ & $\begin{array}{l}\text { Computing } \\
\text { Time (s) }\end{array}$ & $\begin{array}{l}\text { Memory for } \\
\text { BDD node } \\
\text { and cache } \\
\text { Table (MB) }\end{array}$ \\
\hline High-level & 4260 & $<1$ & 160 & 4260 & $<1$ & 160 \\
\hline $\begin{array}{l}\text { Low-level MAIP-j } \\
\quad j=1,2,3\end{array}$ & $9.48 * 10^{12}$ & $\begin{array}{c}16453 \\
\text { (4.57 hours) }\end{array}$ & 160 & $1.61 * 10^{16}$ & $\begin{array}{c}96126 \\
\text { (26.7 hours) }\end{array}$ & 220 \\
\hline Total Estimated & $3.63 * 10^{42}$ & & & $1.78 * 10^{52}$ & & \\
\hline
\end{tabular}

Table 8.2: The multiple AIP example data(synthesis) 


\section{Chapter 9}

\section{Conclusions and Future Work}

\subsection{Conclusions}

In this thesis, by applying symbolic method to an HISC system, we have developed algorithms for synthesis and verification. We also gave large examples (e.g. the 5-4 AIP system has worst-case estimated state space $1.51 \times 10^{30}$ ) to demonstrate the potential abilities of the algorithms. We now discuss some conclusions and contributions of this thesis.

For an HISC system, a per level supervisor synthesis method based on predicate operations was proposed. This was not provided by Leduc et al. in [21-25,27] before, as they mainly focused on the verification method.

With the help of symbolic computation, we can handle much larger individual subsystems for verification and synthesis (e.g. the synthesized high-level supervisor has $1.14 \times 10^{15}$ states in the 5-4 AIP system and can be computed with less than $160 \mathrm{MB}$ of RAM) than before. With explicit state and transition listings, a software typically can only handle subsystems as large as $10^{7}$ states with 1 GB of RAM.

For the synthesized supervisors, a controller implementation scheme for an HISC 


\section{Master Thesis - R. Song - McMaster - Computing and Software}

system was provided based on a group of small local control predicates which can be represented as BDDs (The largest prime simplified control predicate for the 5-4 AIP system has 715 BDD nodes). As each control predicate is fairly small, the speed of this BDD-based controller can be guaranteed.

Note here that although the system containing the synthesized supervisors has locally maximally permissible behavior, the behavior of the flat system containing these supervisors in general is not globally maximally permissive. This is because of the HISC conditions, and this is the price we pay for synthesizing supervisors that respect the interface conditions. However, it is worthwhile to do so because we could design a very large system with great scalability.

In this thesis, we have talked about both synthesis and verification methods for an HISC system. With the synthesis method, a system designer can mainly focus on modeling system requirements and then compute the controller automatically. However, the synthesized supervisor is often too large to be understood by simple inspection by designers, especially for large systems (e.g. the AIP examples). With the hand-designed supervisor, nevertheless, one is much more confident about the behavior of a system. Furthermore, to verify a system is usually faster than to synthesize a supervisor for the system, as there is no need to compute the greatest fixpoint by repeatedly applying functions. However, sometimes it is very difficult to design all the supervisors by hand directly and not to restrict the system behavior too much in the mean time. For example, to make the 3-2 AIP example (verification version) nonblocking, we had to add a supervisor (OFProtAIP, See Figure 7.35) to restrict the system behavior. In the AIP examples we designed, we mainly design supervisors to reflect the control requirements, but to solve the blocking problem, we prefer to use the synthesis method. By designing system this way, we are confident that the controlled system behavior is what we expect but not to restrict the system 


\section{Master Thesis - R. Song - McMaster - Computing and Software}

too much.

\section{$9.2 \quad$ Future Work}

We now discuss some future works of using symbolic method for HISC systems.

\subsubsection{Intermediate BDD Size Problem}

In Algorithm 4.1 and Algorithm 4.3, there is a repeat loop. We only proved that the algorithm can terminate after finite number of iterations which is no more than the size of the state space in the high-level or low-levels. However, for practical systems, the number of iterations is likely to be very small. For example, the 3-2 AIP example in Chapter 7 only takes 3 iterations.

However, once the number of iterations gets bigger, we will have an intermediate BDD size problem. For example, in a system we worked on, the number of iterations was 26. For this system, the size of the BDD for predicate $P_{1}$ during iterations 1118 was much bigger than its size in other iterations, although the number of states satisfying $P_{1}$ was becoming smaller. Figure 9.1 shows how the number of BDD nodes changed, and the time to complete the $C R$ operation at each iteration in the synthesis process of that system. We can see that the time to complete each middle iteration is much longer than the time to complete each iteration at the beginning or end.

Usually the specification DES is composed of many specification components. For future work, we may synthesize supervisors first using only part of the specification

components, then use these intermediate supervisors as specifications to synthesize a final supervisor. By doing things this way, we may be able to control the size of intermediate BDD. This idea was inspired by Exercise 3.7.13 in [47]. 
Master Thesis - R. Song - McMaster - Computing and Software

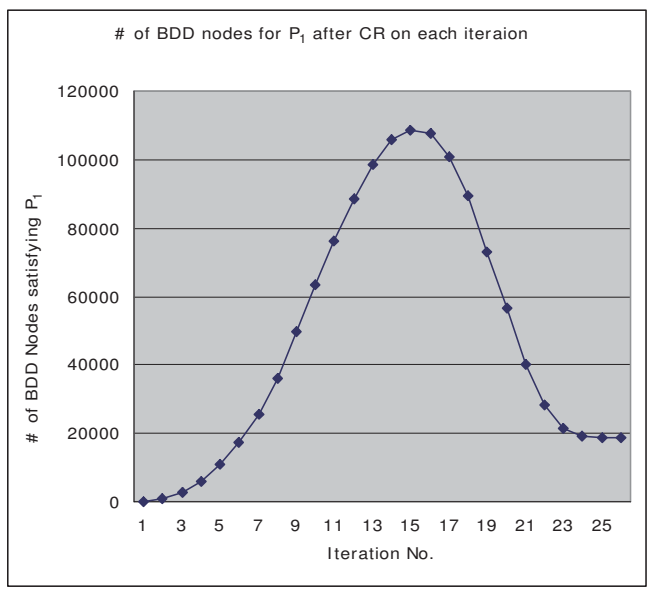

(a) Number of BDD nodes

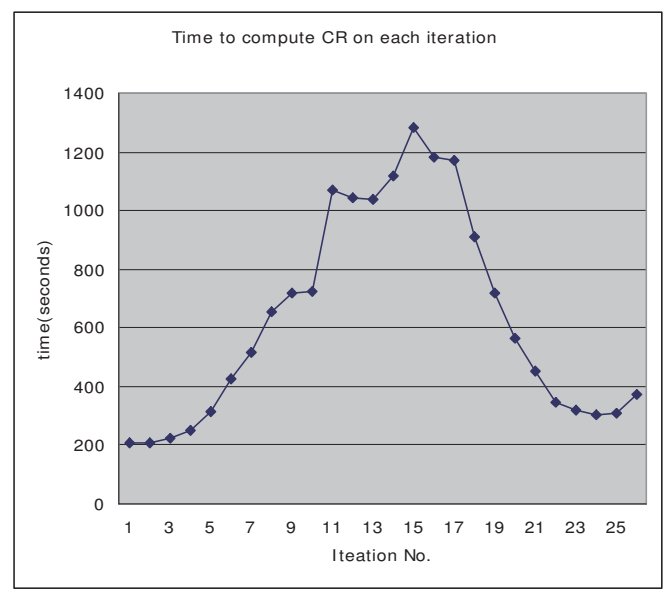

(b) Time to complete $C R$ operation

Figure 9.1: Number of BDD nodes and time for $C R$ operation at each iteration

\subsubsection{Complexity Analysis of the Algorithms}

Although we use BDD to represent a subsystem of the HISC system and use BDD operations to do the computations, the worst case of the computational complexity is still based on the state space size of the subsystem, which is exponential on the number of components. It is worthwhile to do a more detailed complexity analysis, although the actual computing time of a BDD implementation of the algorithms is highly dependent on the system being examined.

\subsubsection{Symbolic Computation for Timed HISC System}

Currently, the HISC method is only for untimed discrete event systems. As timed systems usually have much bigger state-spaces, we may apply the idea of the HISC to design a method for timed system and then develop the algorithms suitable for symbolic synthesis and verification. By combining these two methods, we may be able to handle more complicated timed systems. 


\section{Bibliography}

[1] H. R. Andersen. An Introduction to Binary Decision Diagrams. Lecture Notes, IT University of Copenhagen. [ONLINE] Available: http://www.itu.dk/people/hra.

[2] D. S. Arnon. A bibliography of quantifier elimination for real closed fields. Journal of Symbolic Computation, 5(1-2):267-274, February/April 1988.

[3] G. Barrett and S. Lafortune. Decentralized supervisory control with communicating controllers. IEEE Trans. Automatic Control, 45(9):1620-1638, 2000.

[4] B. Bollig and I. Wegener. Improving the Variable Ordering of OBDDs is NPComplete. IEEE Trans. Computers, 45(9):993-1002, Sept. 1996.

[5] B. A. Brandin and F. Charbonnier. The supervisory control of the automated manufacturing system of the AIP. In Proc. Rensselaer's 1994 Fourth International Conference on Computer Integrated Manufacturing and Automation Technology, pages 319-324, Troy, Oct 1994.

[6] R. E. Bryant. Graph-Based algorithm for boolean function manipulation. IEEE Transactions on Computers, 35(8):677-691, Aug. 1986.

[7] F. Charbonnier. Commande par supervision des systèmes à événements discrets: application à un site expérimental l'Altelier Inter-établissement de Productique. 
Master Thesis - R. Song - McMaster - Computing and Software

Technical report, Laboratoire d'Automatique de Grenoble, Grenoble, France, 1994.

[8] H. Chen and H.-M. Hanisch. Model aggregation for hierarchical control synthesis of discrete event systems. In Proc. 39th Conf. Decision Contr., pages 418-423, Sydney, Australia, December 2000.

[9] S.-L. Chen. Existence and design of supervisors for vector discrete event systems. Master's thesis, Department of Electrical Engineering, University of Toronto, Toronto, Ont, 1992.

[10] S.-L. Chen. Control of Discrete-Event Systems of Vector and Mixed Structural Type. PhD thesis, Department of Electrical and Computer Engineering, University of Toronto, Toronto, Ont, 1996.

[11] Edmund M. Clarke, Orna Grumberg, and Doron A. Peled. Model Checking. The MIT Press, Jan. 2000.

[12] O. Coudert, C. Berthet, and J. C. Madre. Verification of synchronous sequential machines based on symbolic execution. In Proceedings of the Workshop on Automatic Verification Methods for Finite State Systems, volume 407 of Lecture Notes in Computer Science, pages 365-373, J. Sifakis, editor, Berlin, Germany, June 1989. Springer-Verlag.

[13] M. Courvoisier, M.Combacau, and A. de Bonneval. Control and monitoring of large discrete event systems: a generic approach. In Proc. of ISIE 93, pages 571-576, Budapest, 1993. 


$$
\text { Master Thesis - R. Song - McMaster - Computing and Software }
$$

[14] Pengcheng Dai. Synthesis method for hierarchical interface-based supervisory control. Master's thesis (to appear), Department of Computing and Software, McMaster University, Hamilton, Ont.

[15] B.A. Davey and H.A. Priestley. Introduction to Lattices and Order. Cambridge University Press, 1990.

[16] Johan Gunnarsson. Symbolic Methods and Tools for Discrete Dynamic Systems. Ph.D. Thesis, Linkoping Studies in Science and Technology, 1997.

[17] D. Harel. Statecharts: A visual formalism for complex systems. Science of Computer Programming, 8(3):231-274, Jun. 1987.

[18] P. Hubbard and P.E. Caines. Dynamic consistency in hierarchical supervisory control. IEEE Transactions On Automatic Control, 47(1):37-52, Jan. 2002.

[19] M. Huth and M. Ryan. Logic in Computer Science: Modelling and Reasoning about Systems. Cambridge University Press, Nov. 1999.

[20] R. J. Leduc. PLC implementation of a DES supervisor for a manufacturing testbed: An implementation perspective. Master's thesis, Department of Electrical and Computer Engineering, University of Toronto, Toronto, Ont., 1996.

[21] R. J. Leduc. Hierarchical Interface-based Supervisory Control. PhD thesis, Department of Electrical and Computer Engineering, University of Toronto, Toronto, Ont., 2002. [ONLINE] Available: http://www.cas.mcmaster.ca/ leduc.

[22] R. J. Leduc, B.A. Brandin, M. Lawford, and W. Murray Wonham. Hierarchical interface-based supervisory control, part I: Serial case. IEEE Trans. Automatic Control, 50(9):1322-1335, Sept. 2005. See also SQRL Report No. 12, Dept. 
Master Thesis - R. Song - McMaster - Computing and Software

of Computing and Software, McMaster University, Hamilton, ON. [ONLINE] http://www.cas.mcmaster.ca/sqrl/sqrl_reports.html.

[23] R. J. Leduc and M. Lawford. Hierarchical interface-based supervisory control of flexible manufacturing system. Submitted to the IEEE Transactions on Control Systems Technology Journal, Jan. 2004.

[24] R. J. Leduc, M. Lawford, and P. Dai. Hierarchical interface-based supervisory control of a flexible manufacturing system. Technical Report No. 32, Software Quality Research Laboratory, Dept. of Computing and Software, McMaster University, Hamilton, ON, Canada, Dec. 2005.

[25] R. J. Leduc, M. Lawford, and W. Murray Wonham. Hierarchical interfacebased supervisory control,part II: Parallel case. IEEE Trans. Automatic Control, 50(9):1336-1348, Sept. 2005. See also SQRL Report No. 13, Dept. of Computing and Software, McMaster University, Hamilton, ON. [ONLINE] http://www.cas.mcmaster.ca/sqrl/sqrl_reports.html.

[26] R.J. Leduc. Hierarchical interface-based supervisory control: Command-pair interfaces (see extended version). In Proc. of the Third International DCDIS Conference on Engineering Applications and Computational Algorithms, pages 323-329, Guelph, Ontario, Canada, May 15-18, 2003. [ONLINE] Available: http://www.cas.mcmaster.ca/ ${ }^{\sim} l e d u c$.

[27] R.J. Leduc, W.M. Wonham, and M. Lawford. Hierarchical interface-based supervisory control: Bi-level systems. Technical report No. 0103, Systems Control Group, University of Toronto, Toronto, ON, Canada, Nov. 2001.

[28] Y. Li and W. M. Wonham. Control of vector discrete-event systems: I - the base model. IEEE Transactions on Automatic Control, 38(8):1214-1227, Aug. 1993. 


$$
\text { Master Thesis - R. Song - McMaster - Computing and Software }
$$

[29] Y. Li and W. M. Wonham. Control of vector discrete-event systems: II- controller synthesis. IEEE Transactions on Automatic Control, 39(3):512-531, Mar. 1994.

[30] F. Lin and W. M. Wonham. Decentralized control and coordination of discreteevent systems with partial observations. IEEE Transacitons on Automatic Control, 35(122):1330-1337, 1990.

[31] Chuan Ma. Nonblocking Supervisory Control of State Tree Structures. PhD thesis, Department of Electrical and Computer Engineering, University of Toronto, Toronto, Ont., 2004.

[32] John O. Moody and Panos J. Antsaklis. Supervisory Control of Discrete Event Systems using Petri Nets. Kluwer Academic Publishers, 1998.

[33] Ken Qian Pu. Modeling and control of discrete-event systems with hierarchical abstraction. Master's thesis, Dept. of Electrical and Computer Engineering, University of Toronto, Toronto, Ont, 2000.

[34] M. Queiroz and J. Cury. Modular supervisory control of large scale discrete event systems. In Proceedings of WODES 2000, pages 103-110, Ghent, Belgium, Aug 2000.

[35] P. J. Ramadge and W. M. Wonham. Modular feedback logic for discrete event systems. SIAM Journal on Control Optimization, 25(5):1202-1218, Sep. 1987.

[36] P. J. Ramadge and W. M. Wonham. Supervisory control of a class of discreteevent processes. SIAM Journal on Control Optimization, 25(1):206-230, Jan. 1987. 
Master Thesis - R. Song - McMaster - Computing and Software

[37] J.H. Richter and F. Wenck. Hierachical interface-based supervisory control of a bottling plant. In Proceedings of the 16th IFAC World Congress, Prague, Czech Republic, July 2005.

[38] R. Rudell. Dynamic variable ordering for ordered binary decision diagrams. In IEEE/ACM International Conference on CAD, pages 42-47, Santa Clara, California, Nov. 1993. ACM/IEEE, IEEE Computer Society Press.

[39] Karen Rudie and W.M. Wonham. Think globally, act locally: Decentralized control. IEEE Transactions on Automatic Control, 37(11):1692-1708, 1992.

[40] Alfred Tarski. A lattice-theoretical fixpoint theorem and its applications. Pacific Journal of Mathematics, 5(2):285-309, 1955.

[41] César R. C. Torrico and José E. R. Cury. Hierarchical supervisory control of discrete-event systems based on state aggregation. In Proc. of Fifteenth Triennial World Congress of the International Federation of Automatic Control, Barcelona, Spain, July 2002.

[42] M. Uzam. Petri-net-based Supervisory Control of Discrete Event Systems and their ladder logic diagram implementations. PhD thesis, University of Salford, Salford, UK, 1998.

[43] M. Uzam. An optimal deadlock prevention policy for flexible manufacturing systems using Petri net models with resources and the theory of regions. Int. J. Adv. Manuf. Technol., 19:192-208, 2002.

[44] Bing Wang. Top-down design for RW supervisory control theory. Master's thesis, Department of Electrical and Computer Engineering, University of Toronto, Toronto, Ont., 1995. 


$$
\text { Master Thesis - R. Song - McMaster - Computing and Software }
$$

[45] K. C. Wong and J. H. van Schuppen. Decentralized supervisory control of discrete event systems with communication. In Proc. of WODES 1996, pages 284-289, Edinburgh, UK, Aug 1996.

[46] K.C. Wong and W. M. Wonham. Hierarchical control of discrete-event systems. Discrete-Event Dynamic Systems: Theory and Applications, 6(3):241-273, July 1996.

[47] W. M. Wonham. Supervisory Control of Discrete-Event Systems. Dept. of Electrical and Computer Engineering, University of Toronto, Jul. 2005. Monograph and TCT software can be downloaded at http://www.control.toronto.edu/DES/.

[48] W. M. Wonham and P. J. Ramadge. On the supremal controllable sublanguage of a given language. SIAM Journal on Control Optimization, 25(3):637-659, May. 1987.

[49] W. M. Wonham and P.J. Ramadge. Modular supervisory control of discrete event systems. Mathematics of Control, Signal and Systems, 1(1):13-30, 1988.

[50] T. Yoo and S. Lafortune. A general architecture for decentralized supervisory control of discrete-event systems. In Proc. of WODES 2000, pages 111-118, Ghent, Belgium, Aug 2000.

[51] Zhonghua Zhang. Smart TCT (STCT): An efficient algorithm for supervisory control design. Master's thesis, Department of Electrical and Computer Engineering, University of Toronto, Toronto, Ont., Apr. 2001.

[52] H. Zhong and W. M. Wonham. On the consistency of hiearchical supervision in discrete-event systems. IEEE Transactions on Automatic Control, 35(10):1125$1134,1990$. 
Master Thesis - R. Song - McMaster - Computing and Software 


\section{Appendix A}

\section{HISC Software Program}

In this appendix, we first briefly introduce the running and development environment, and then we list all the source code of the program.

\section{A.1 Introduction of HISC Software Program}

The HISC software program is implemented by using $\mathrm{C}++$ and STL (Standard Template Library), and it is compiled by using GNU g++ 3.3.3. The software was developed by using Eclipse 3.0 with CDT (C/C++ Development Tools) plug-in on Fedora Core 2. The BDD package we used is BuDDy 2.4 developed by Jørn LindNielsen.

The HISC software program is implemented as a function library. For the interface

of the library, please see BddHisc.h in the source code list. All the function interfaces are compatible with $\mathrm{C}$ or $\mathrm{C}++$.

A very simple text user interface to demonstrate how to use the functions provided by the library is listed in main.cpp.

Table A.1 and Table A.2 are the lists of all the source code files. 
Master Thesis - R. Song-McMaster - Computing and Software

\begin{tabular}{|r|l|l|r|}
\hline No. & File Name & Description & Page \\
\hline 1 & BddHisc.h & The interface of all the functions in the library & 253 \\
2 & BddHisc.cpp & Implementation of the functions in BddHisc.h & 255 \\
3 & Project.h & Header file of Project.cpp & 260 \\
4 & Project.cpp & Process Project files (.prj) & 261 \\
5 & Sub.h & Header file for Sub*.cpp & 267 \\
6 & Sub.cpp & Process high-level or low-level subsystem file(.sub) & 269 \\
7 & Sub1.cpp & Reorder DES BDD variables in high-level or low-level & 273 \\
8 & Sub2.cpp & Save synthesized automata-based supervisor, & 279 \\
& & or synthesized local control predicates, & \\
9 & HighSub.h & Header file for HighSub*.cpp & \\
10 & HighSub.cpp & Read high-level DES files and initialize high-level BDDs & 286 \\
11 & HighSub1.cpp & Some misc functions for high-level such as printing & 294 \\
12 & HighSub2.cpp & Verification and synthesis functions for high-level & 298 \\
13 & LowSub.h & Header file for LowSub*.cpp & 308 \\
14 & LowSub.cpp & Read high-level DES files and initialize high-level BDDs & 309 \\
15 & LowSub1.cpp & Some misc functions for low-level such as printing & 317 \\
16 & LowSub2.cpp & Verify low-level interface (Command-pair) & 321 \\
17 & LowSub3.cpp & Verification and synthesis functions for high-level & 323 \\
18 & DES.h & Header file for DES.cpp & 334 \\
19 & DES.cpp & Process DES files & 335 \\
20 & type.h & self-defined data types & 344 \\
21 & errmsg.h & Error Code symbolic constants & 345 \\
22 & pubfunc.h & Header file for pubfunc.cpp & 346 \\
23 & pubfunc.cpp & Contains utility functions used in the program & 347 \\
\hline
\end{tabular}

Table A.1: Source code files in the software library

\begin{tabular}{|r|l|l|r|}
\hline No. & File Name & Description & Page \\
\hline 1 & main.h & Header file for main.cpp & 352 \\
2 & main.cpp & A text user interface example to show how to use & 353 \\
& & functions in BDD HISC library & \\
\hline
\end{tabular}

Table A.2: Source code files for the library usage example 


\section{Master Thesis - R. Song - McMaster - Computing and Software}

\section{A.2 Source Code}

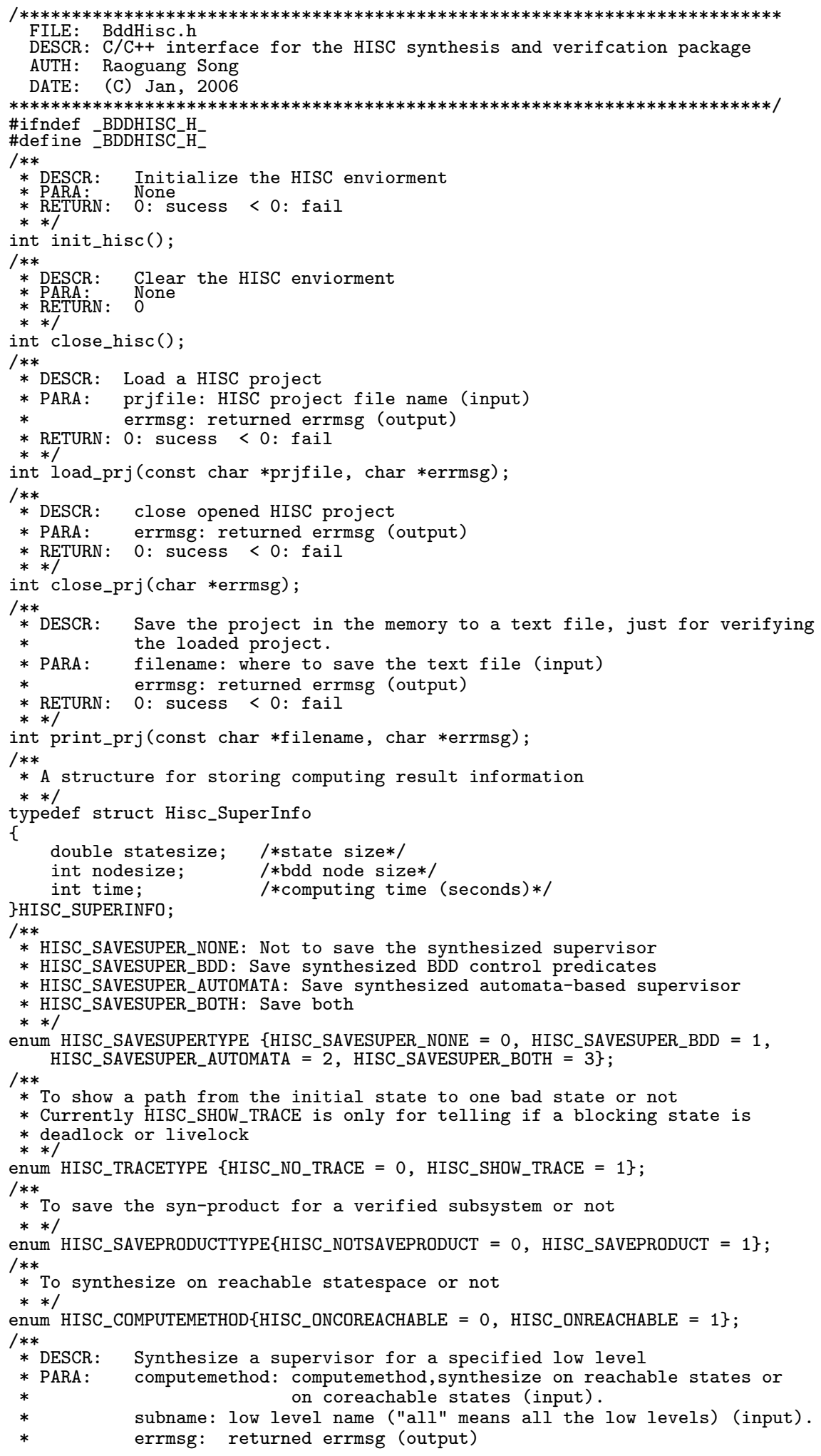




\section{Master Thesis - R. Song - McMaster - Computing and Software}

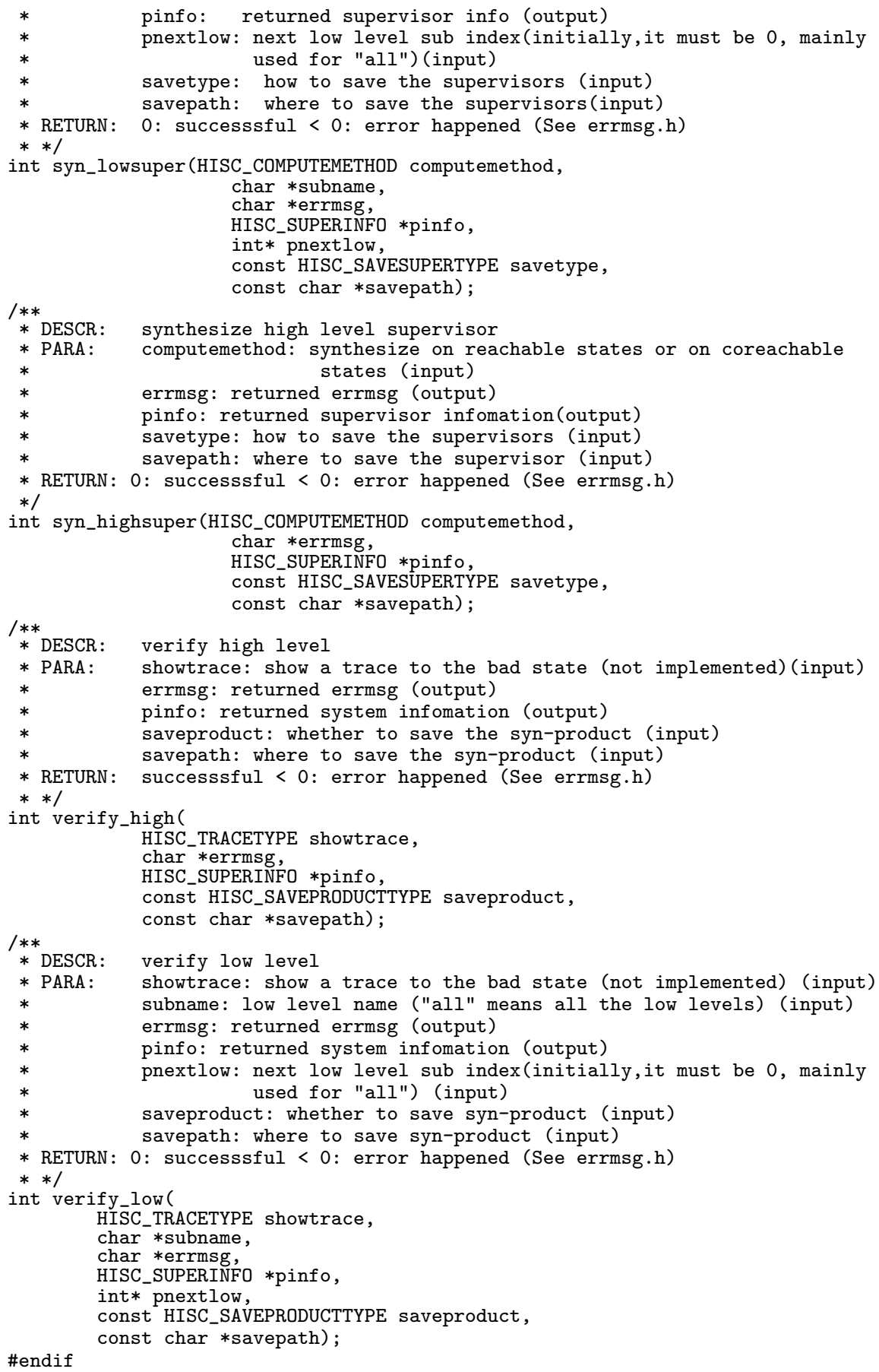




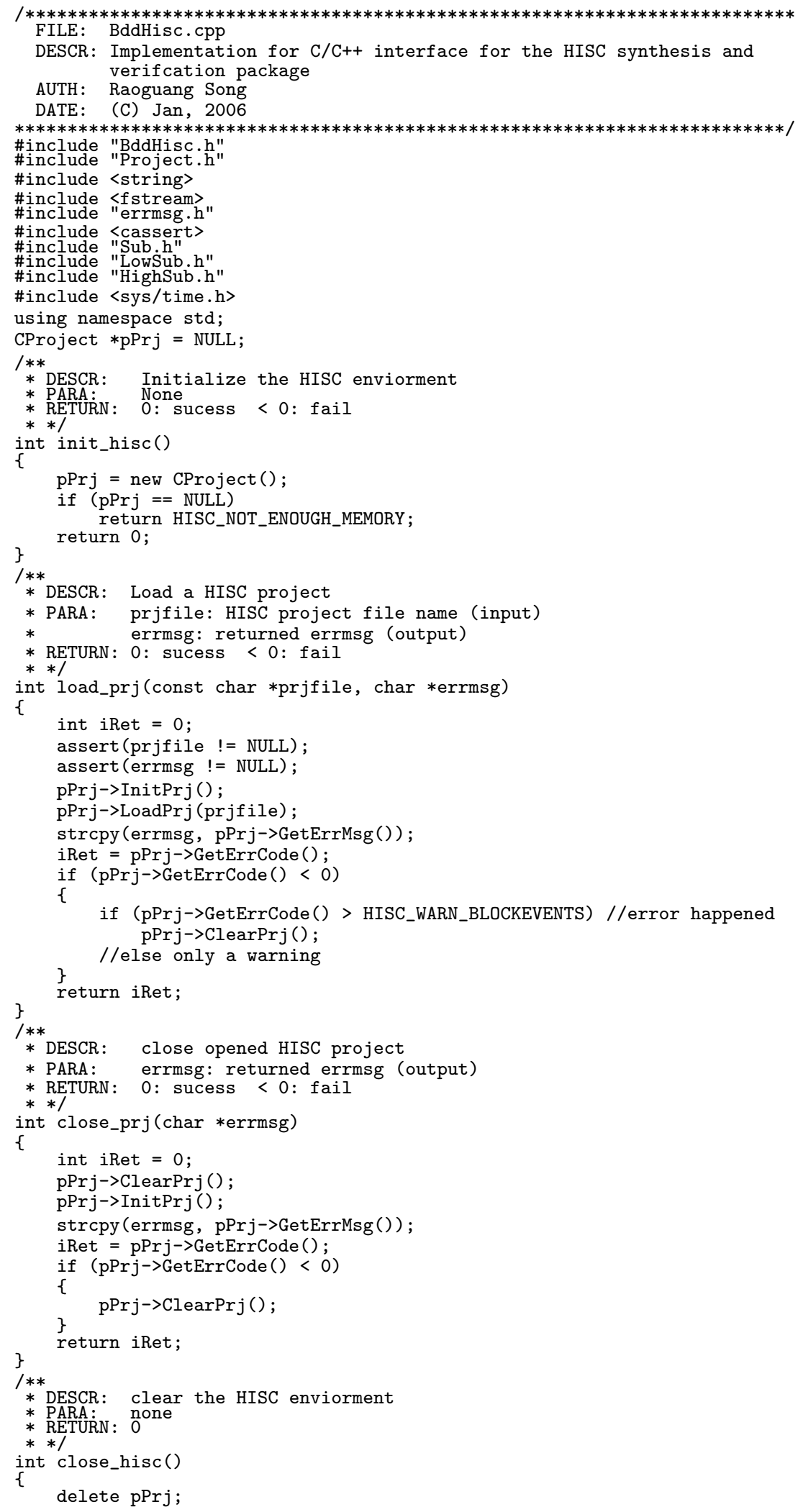




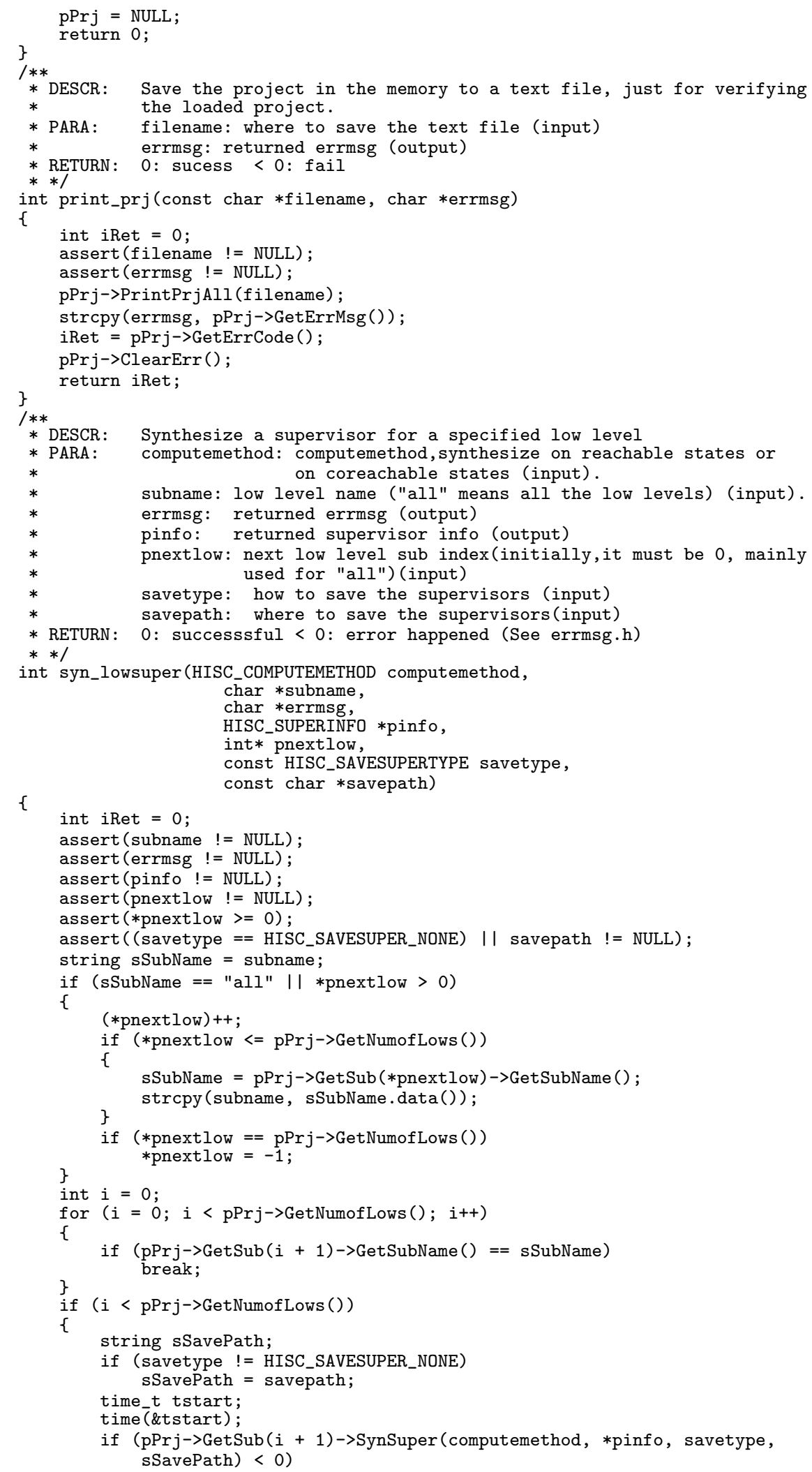




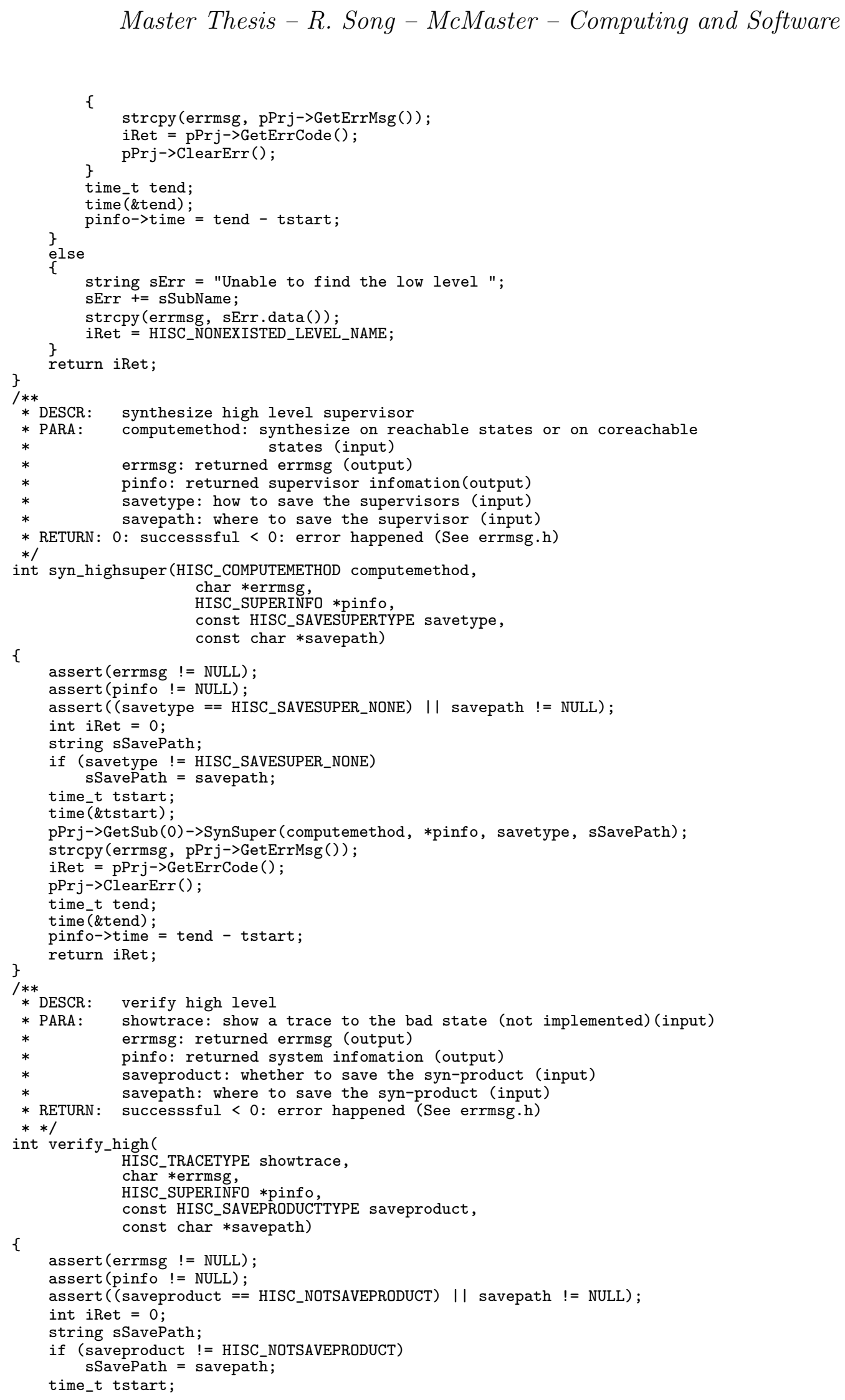




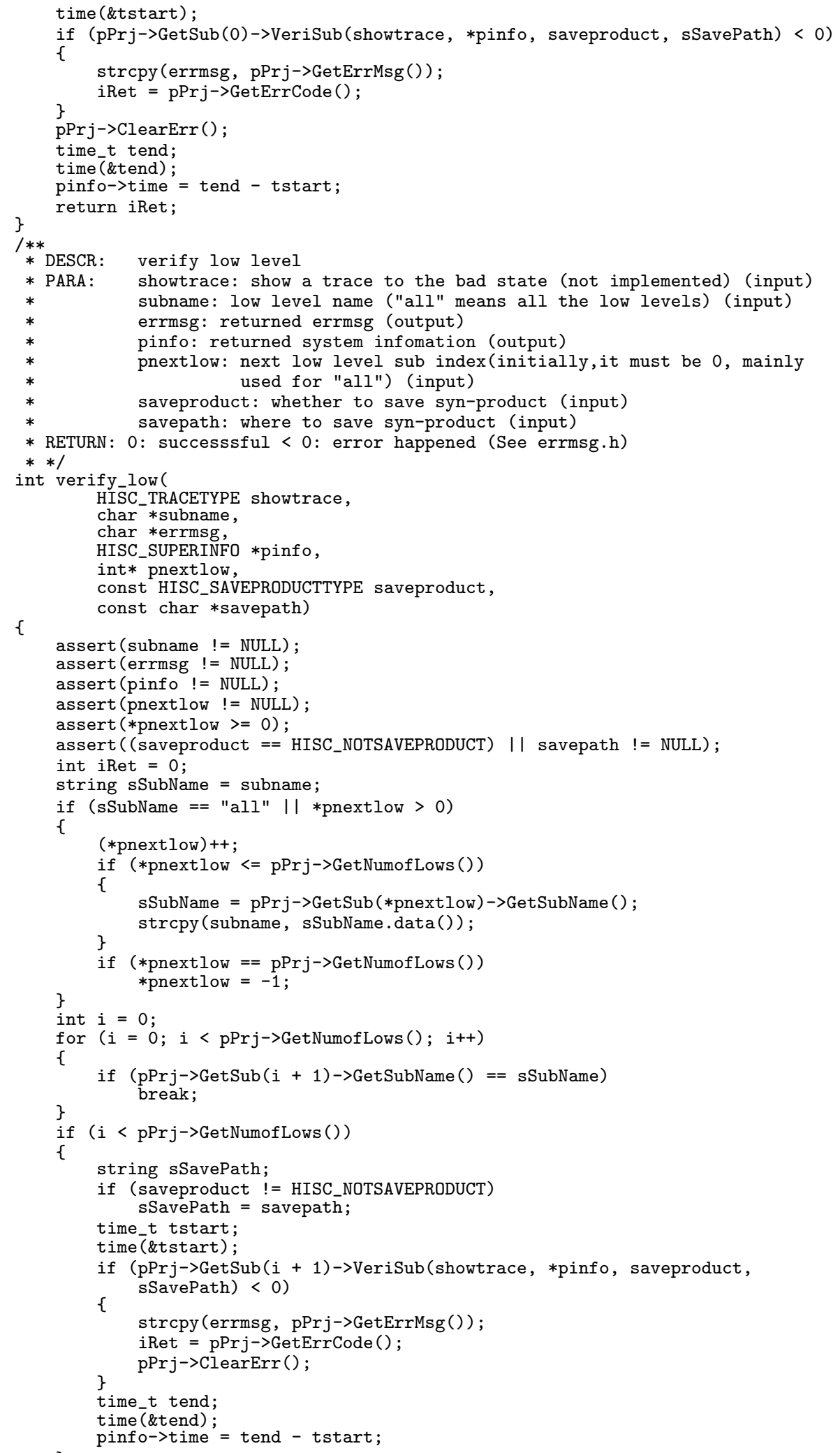


Master Thesis - R. Song-McMaster-Computing and Software

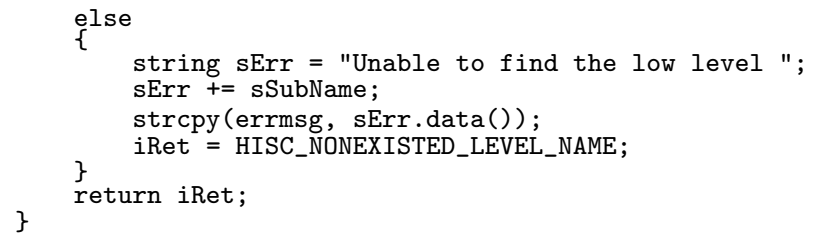




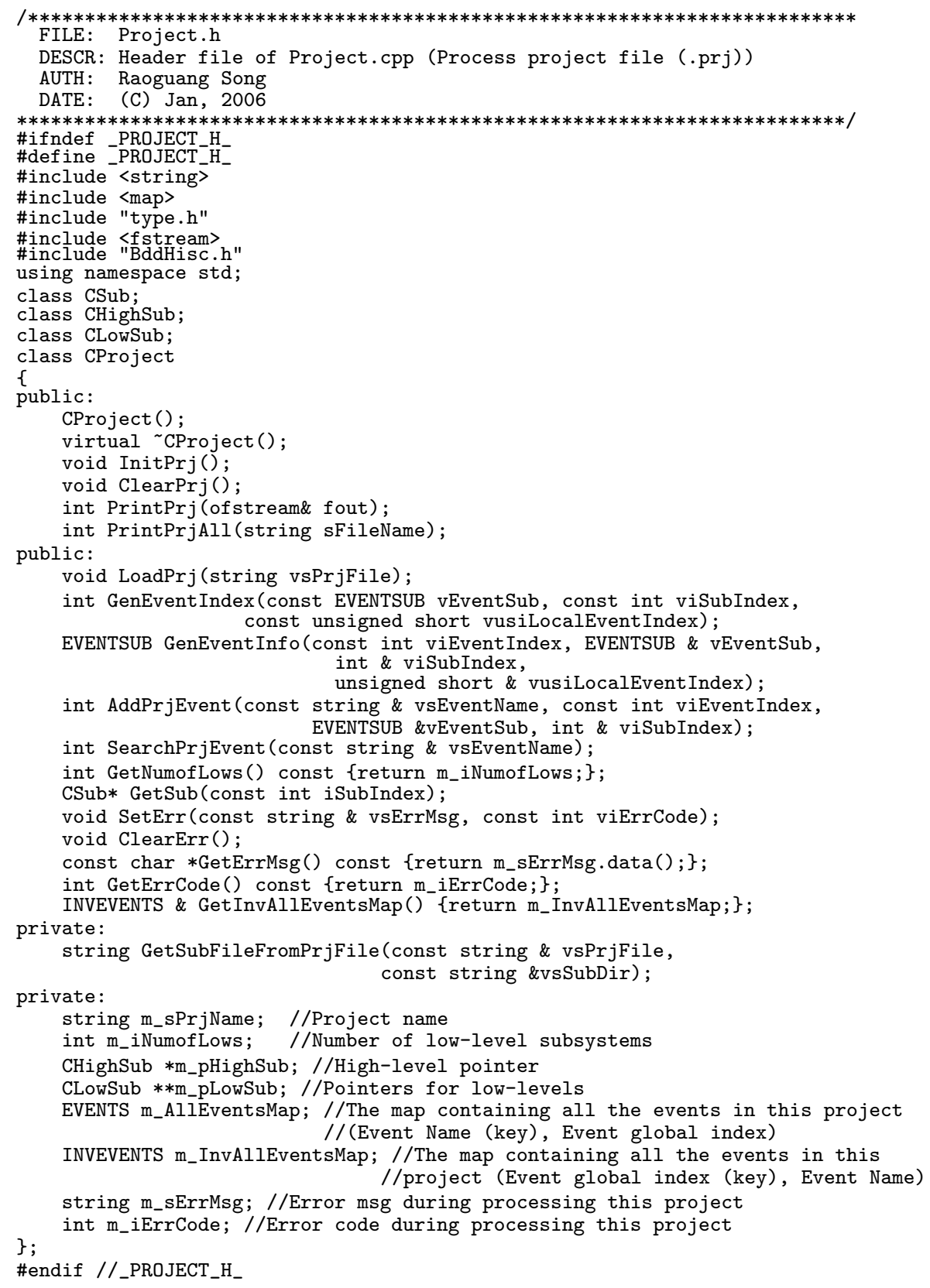




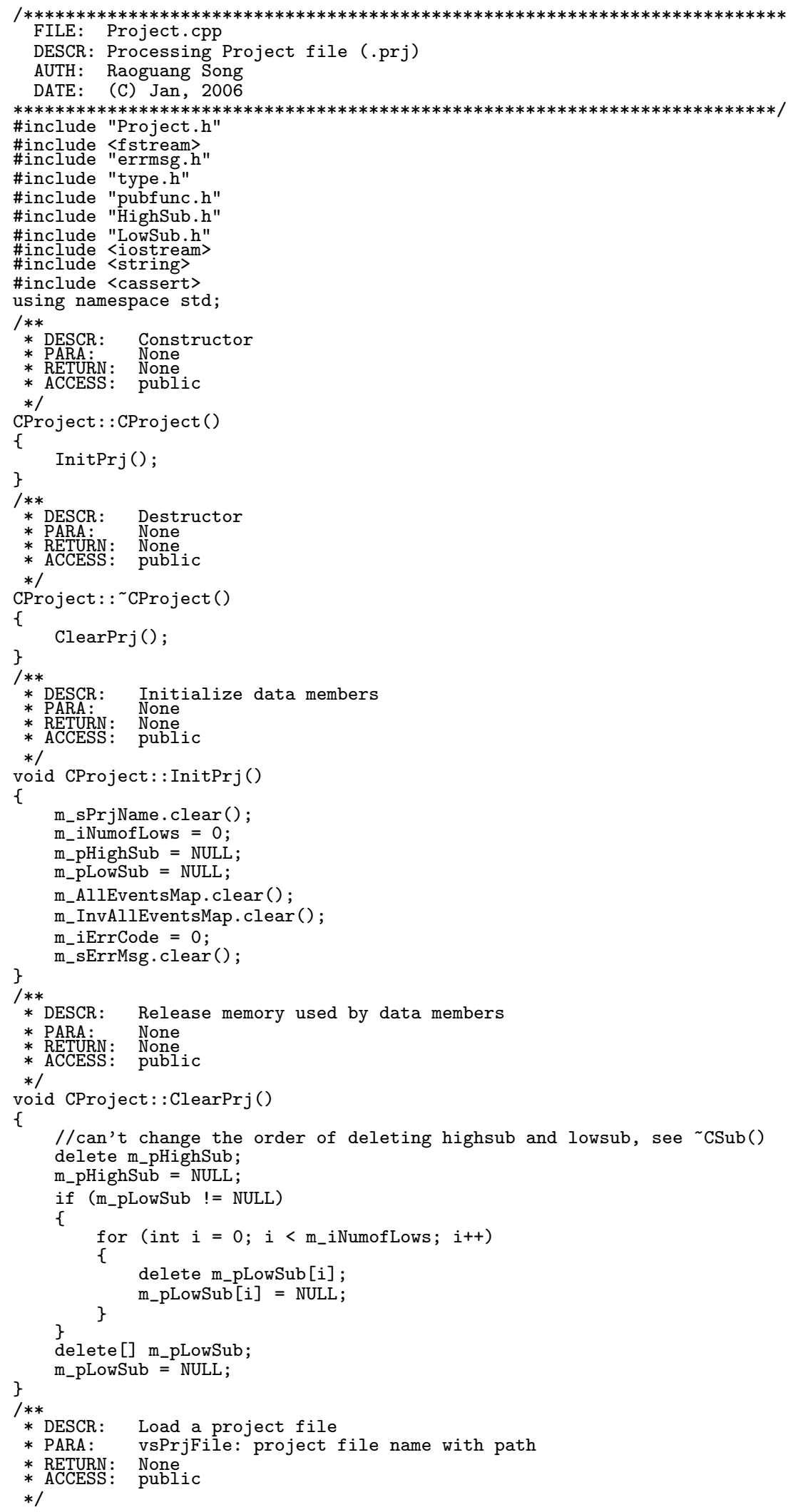




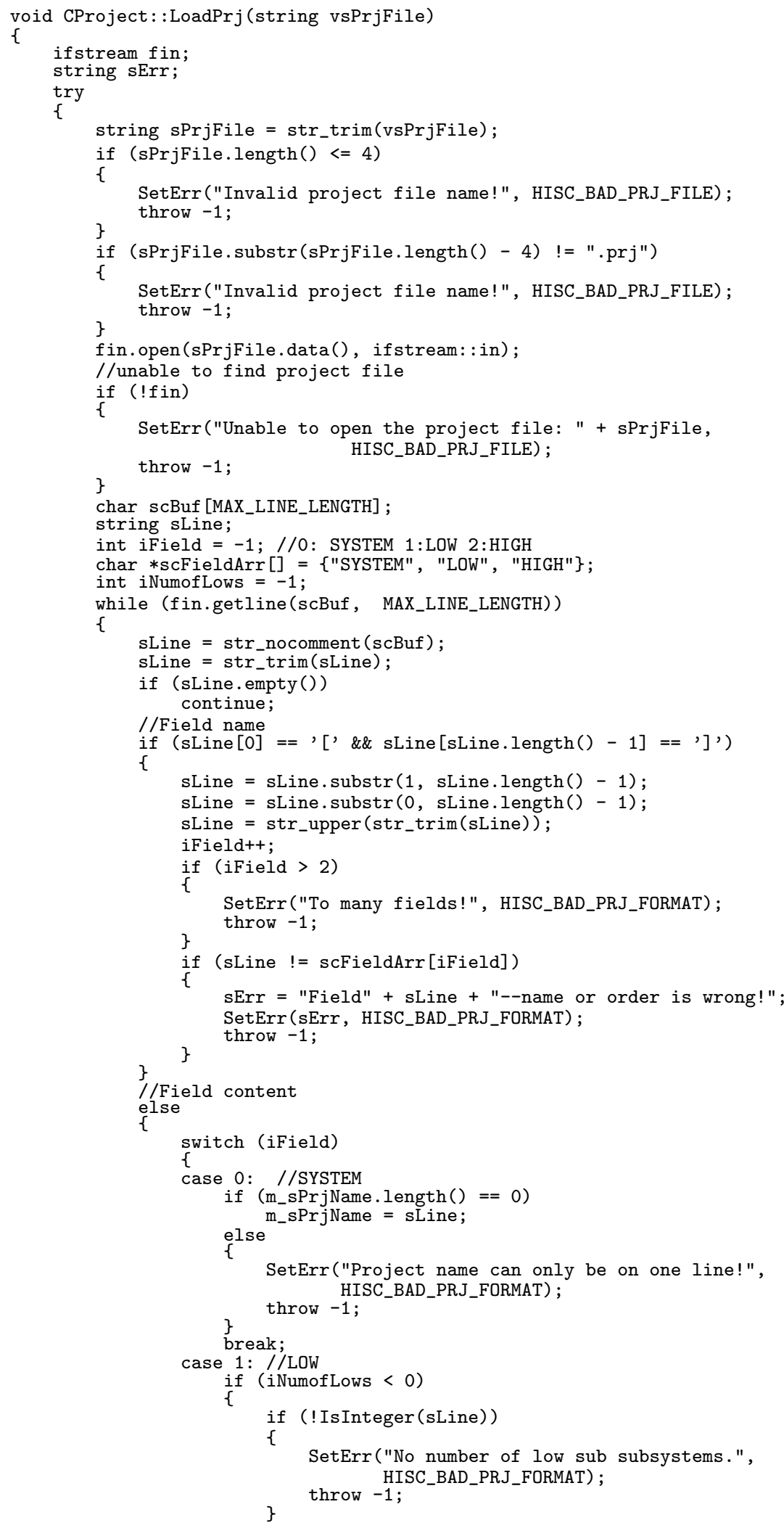




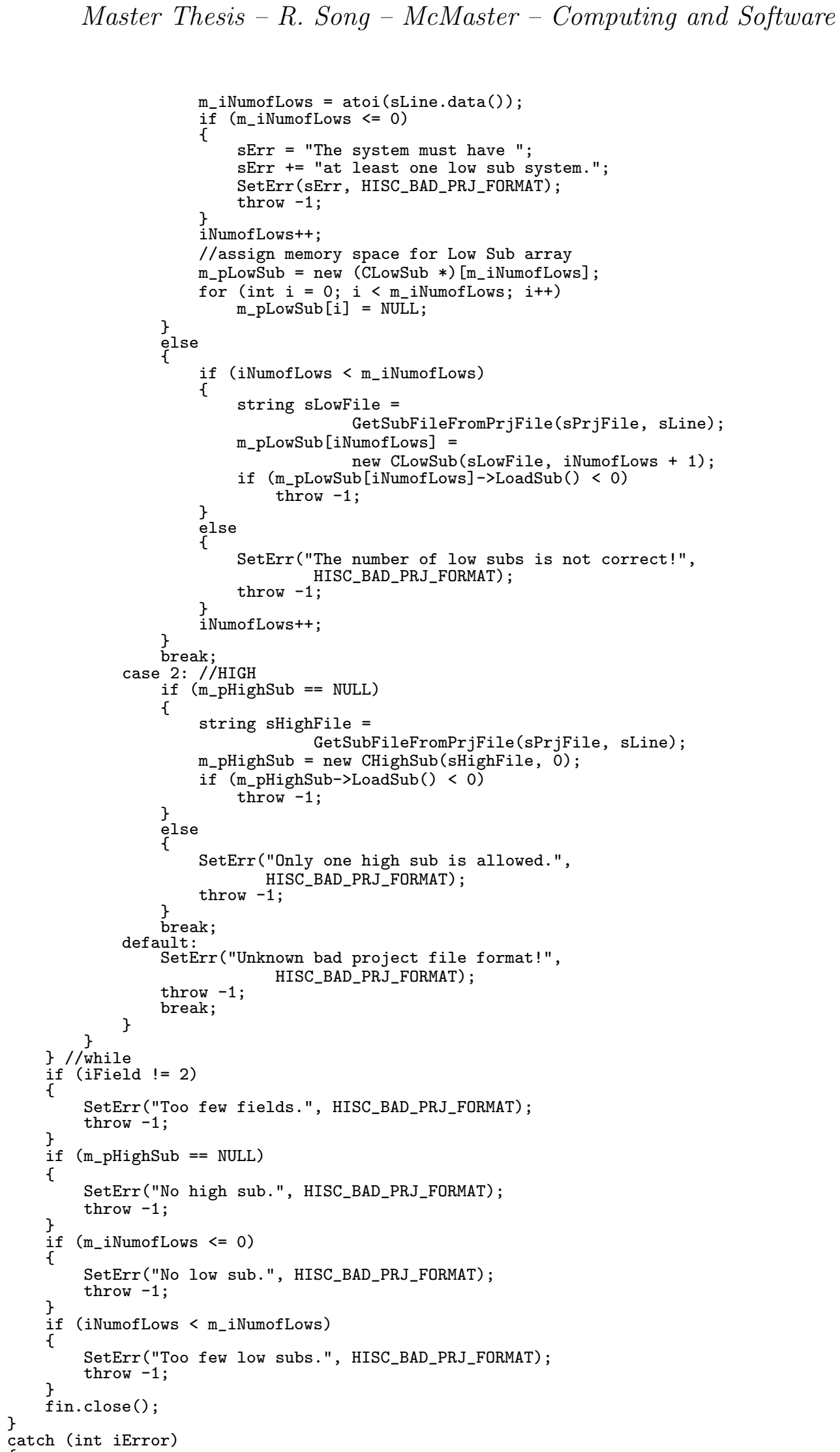




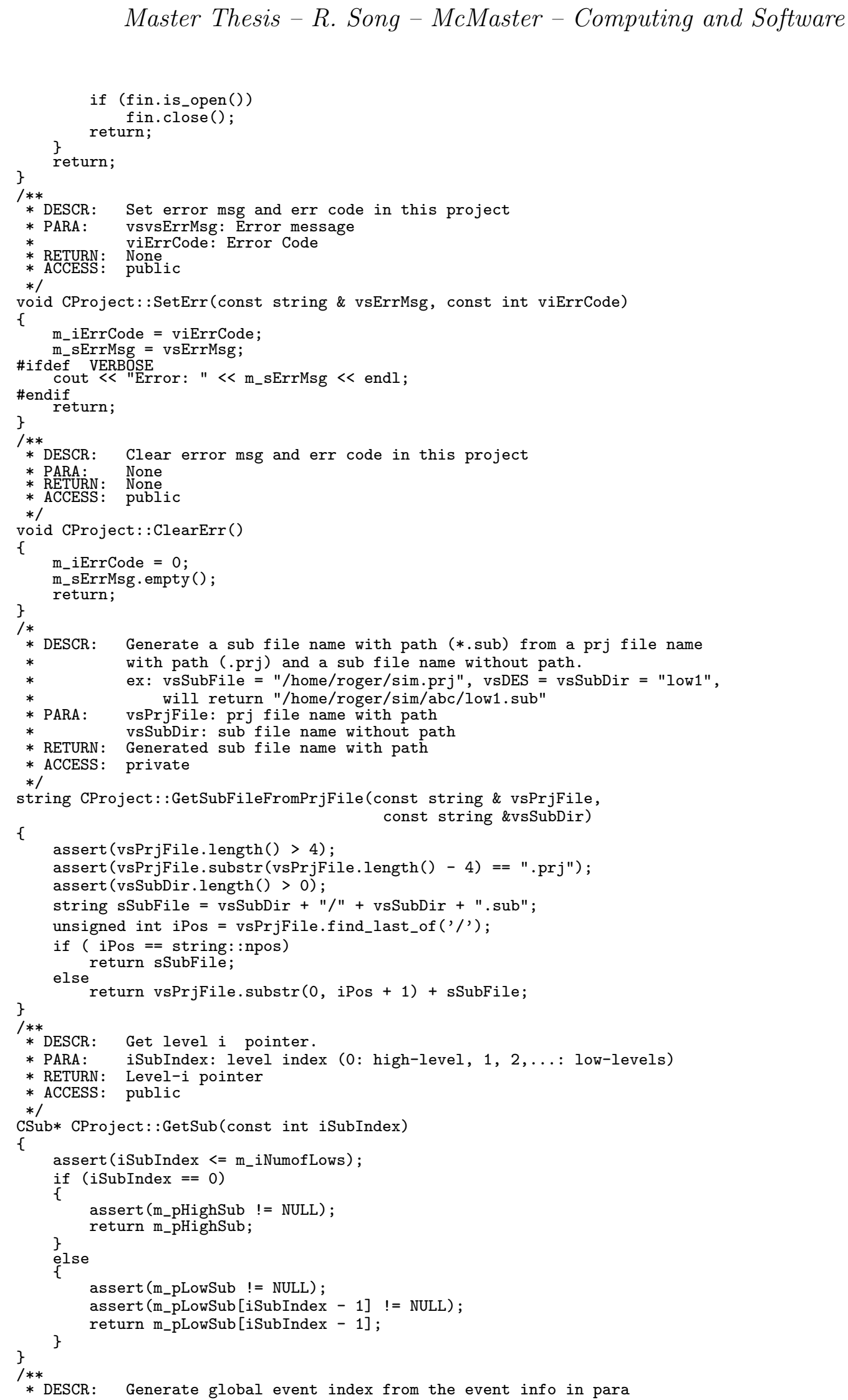




\section{Master Thesis - R. Song - McMaster - Computing and Software}

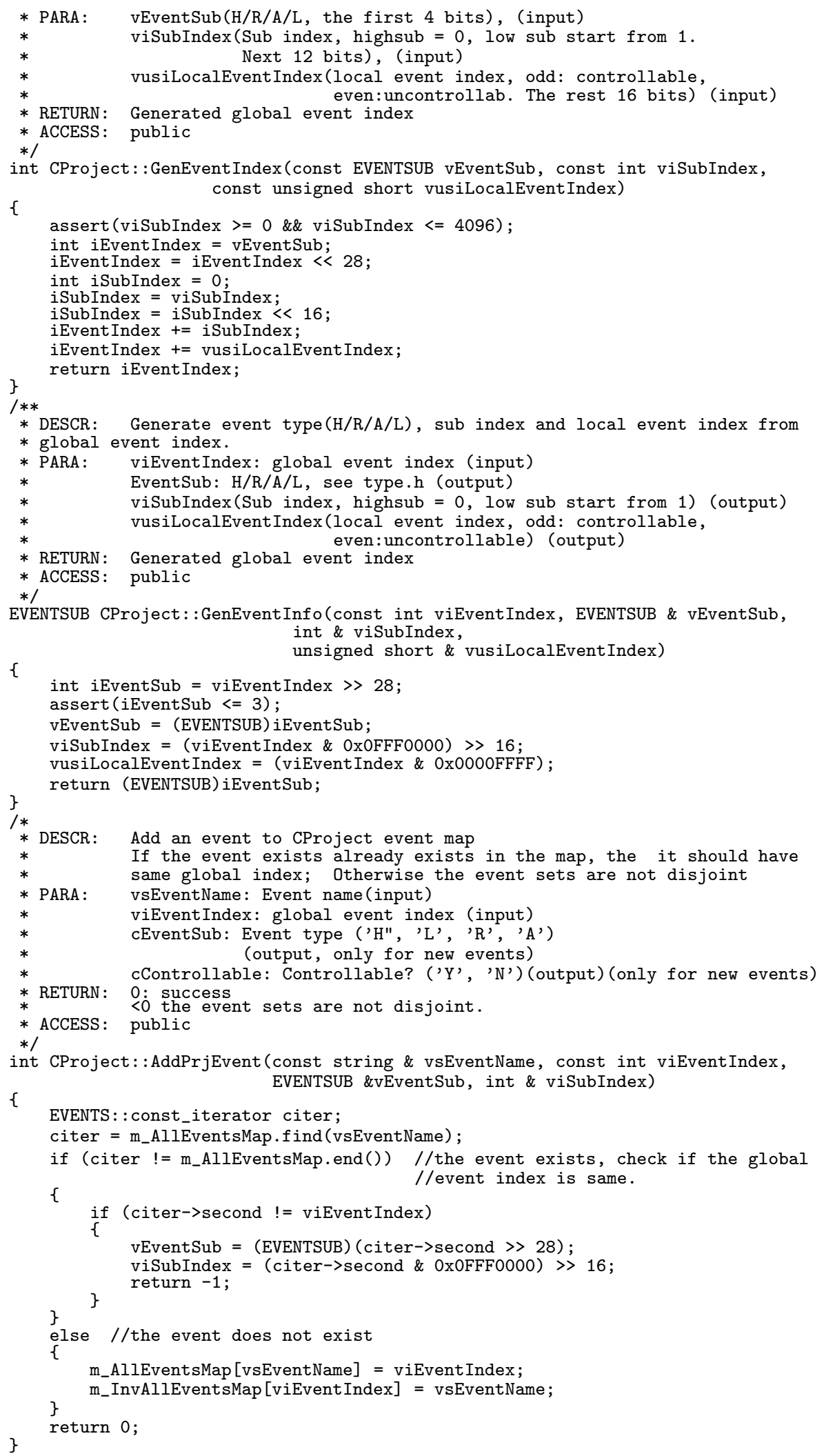




\section{Master Thesis - R. Song-McMaster - Computing and Software}

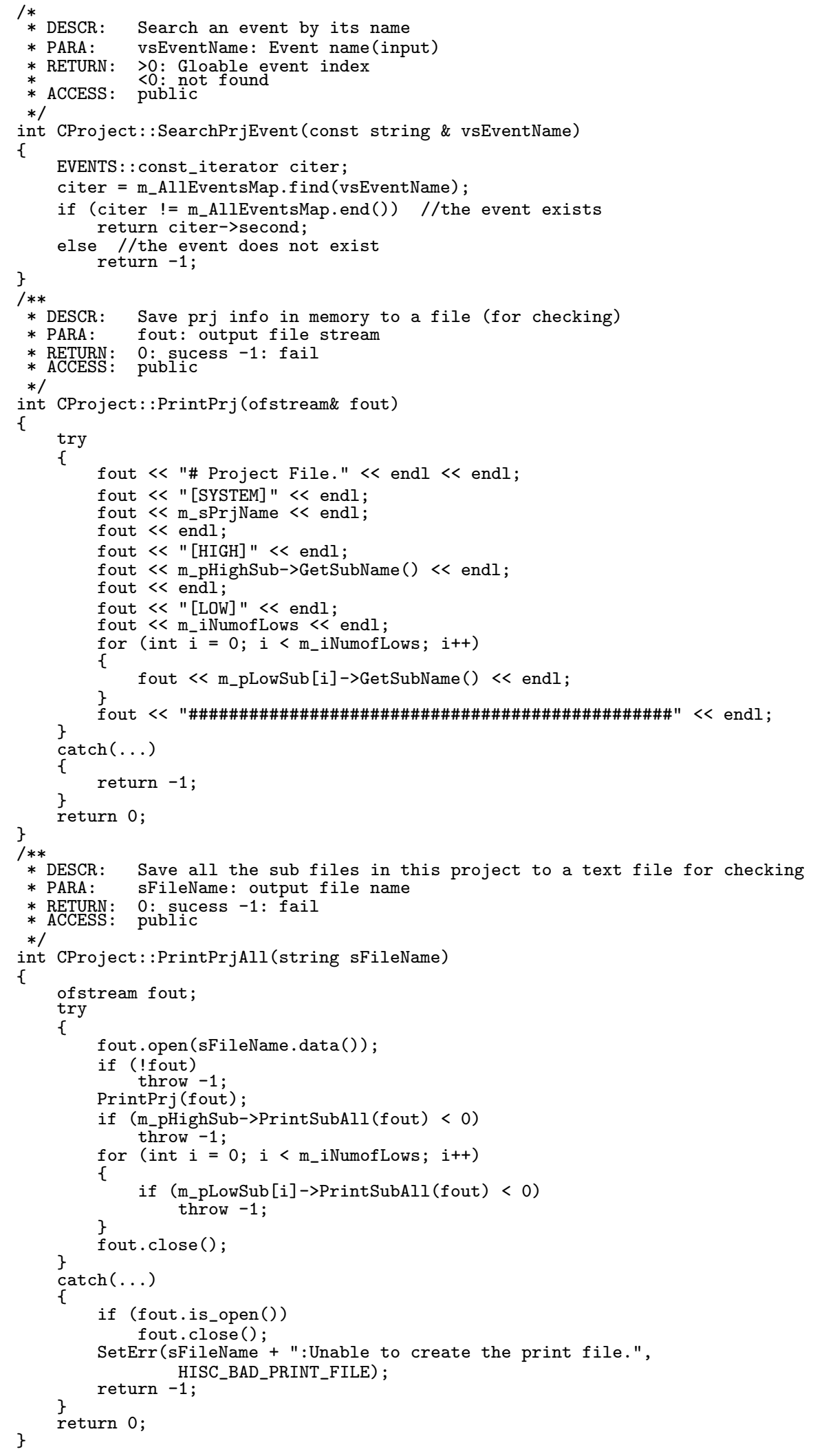




\section{Master Thesis - R. Song-McMaster - Computing and Software}

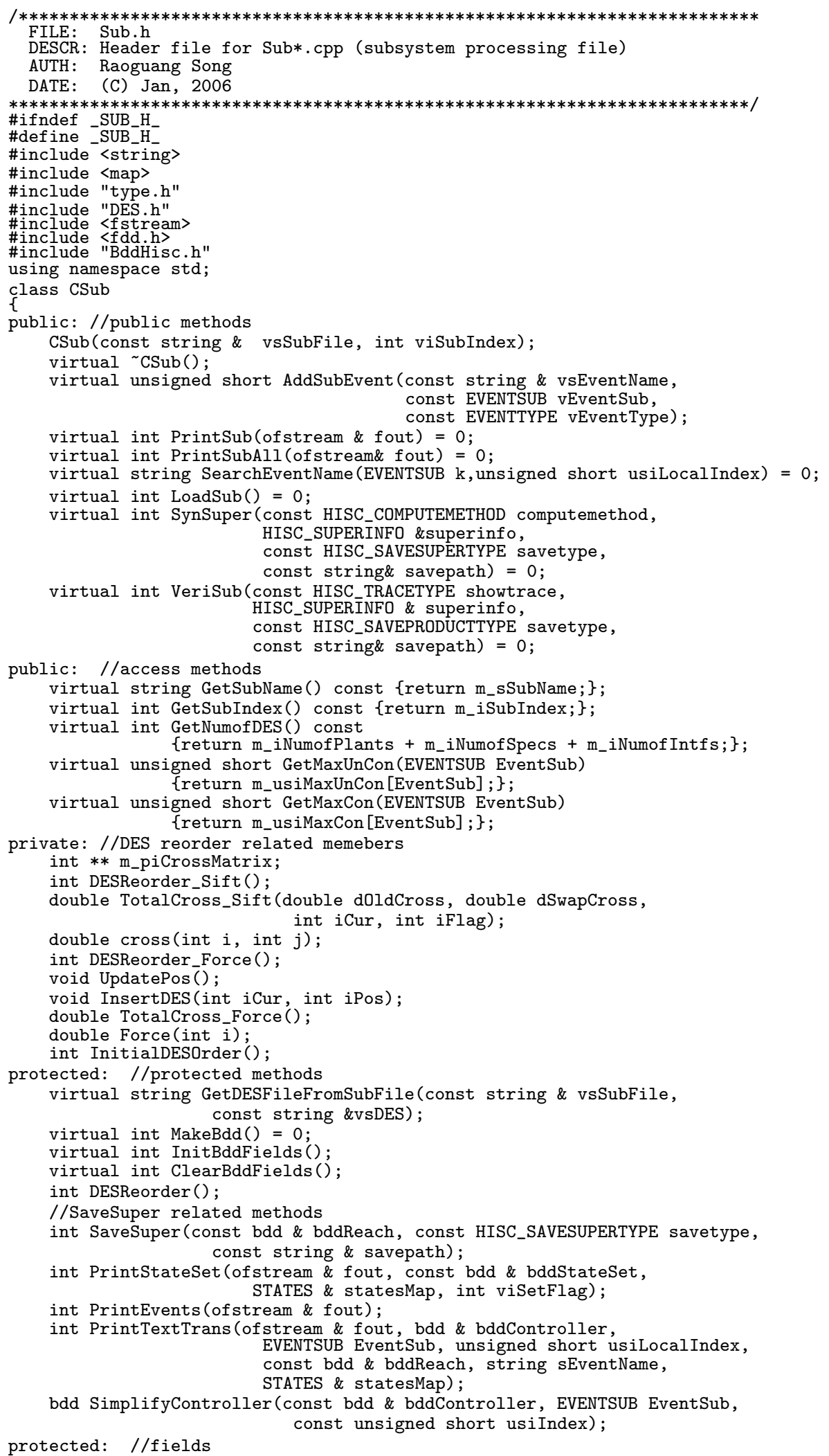




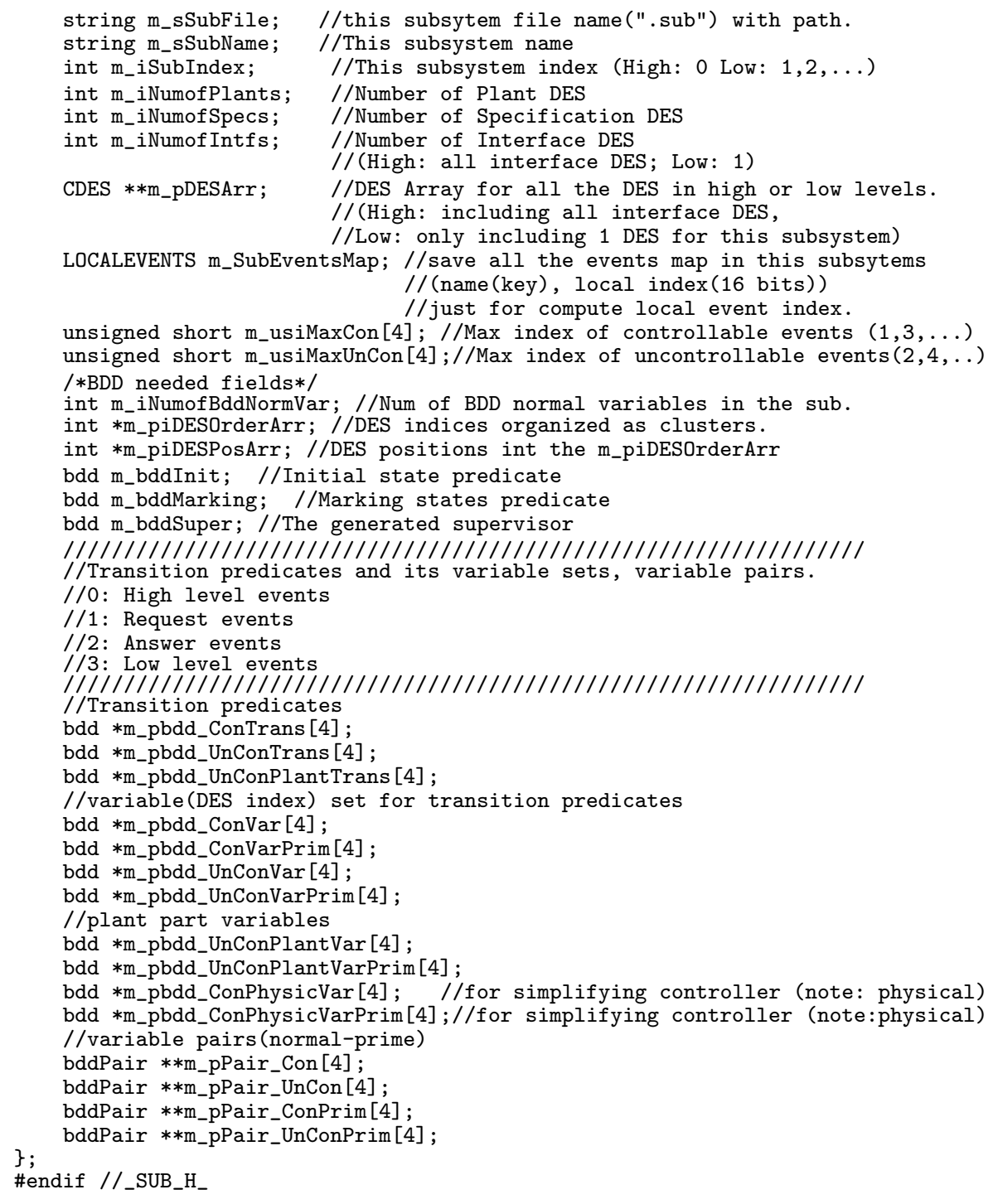




\section{Master Thesis - R. Song-McMaster - Computing and Software}

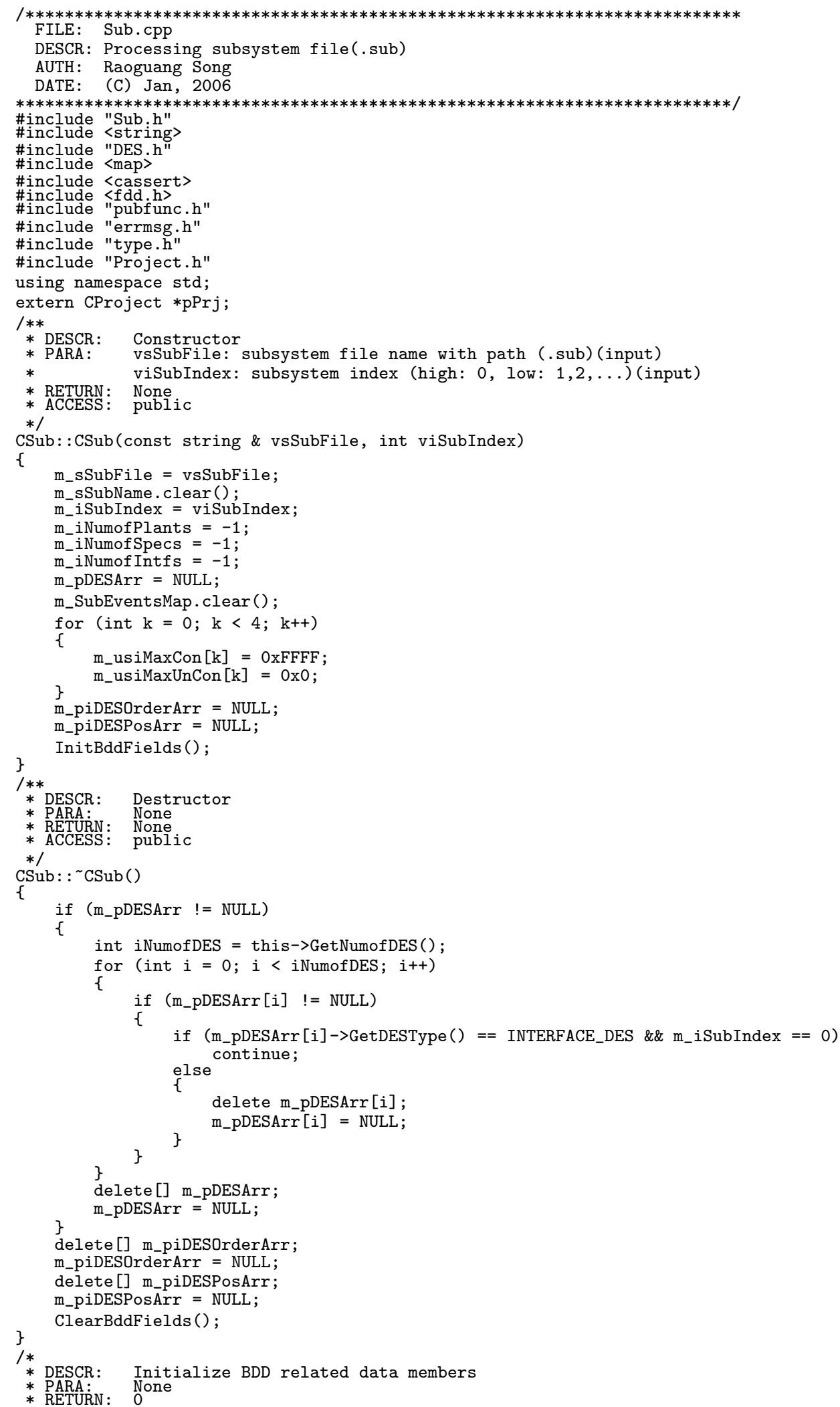


Master Thesis - R. Song - McMaster - Computing and Software

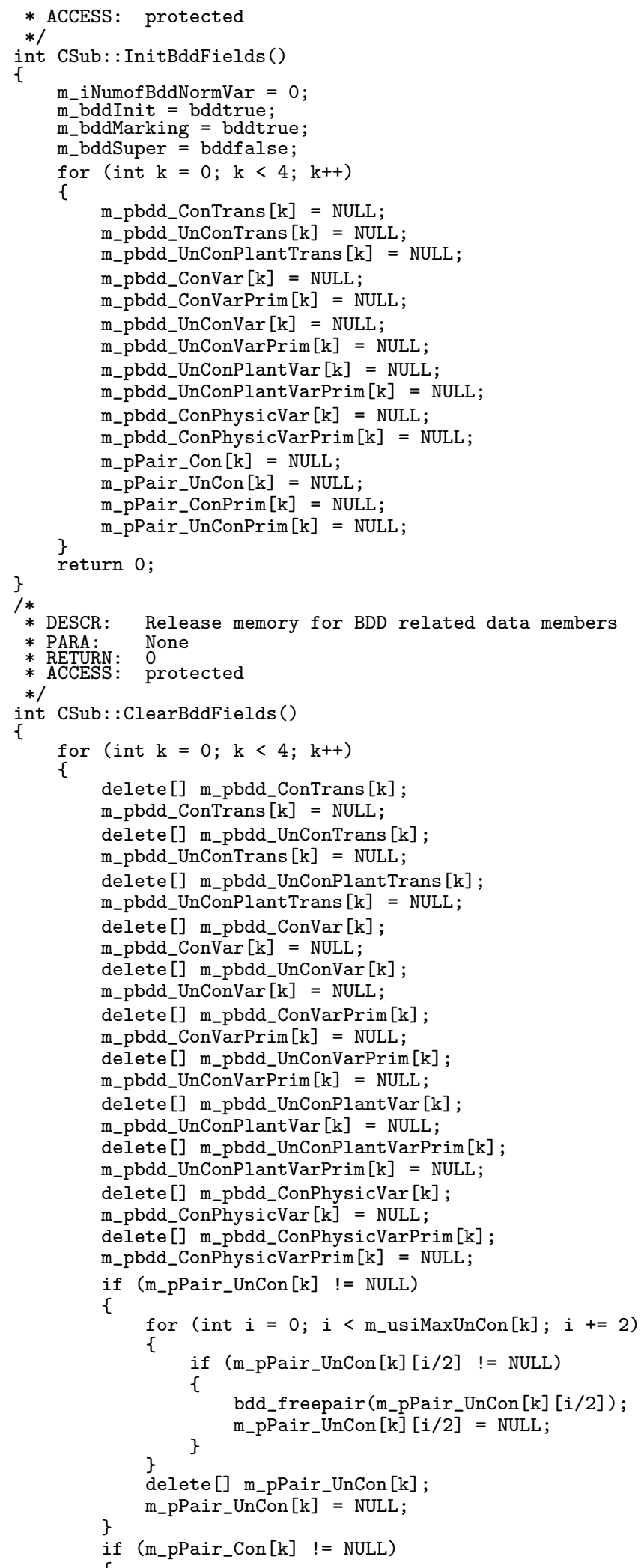




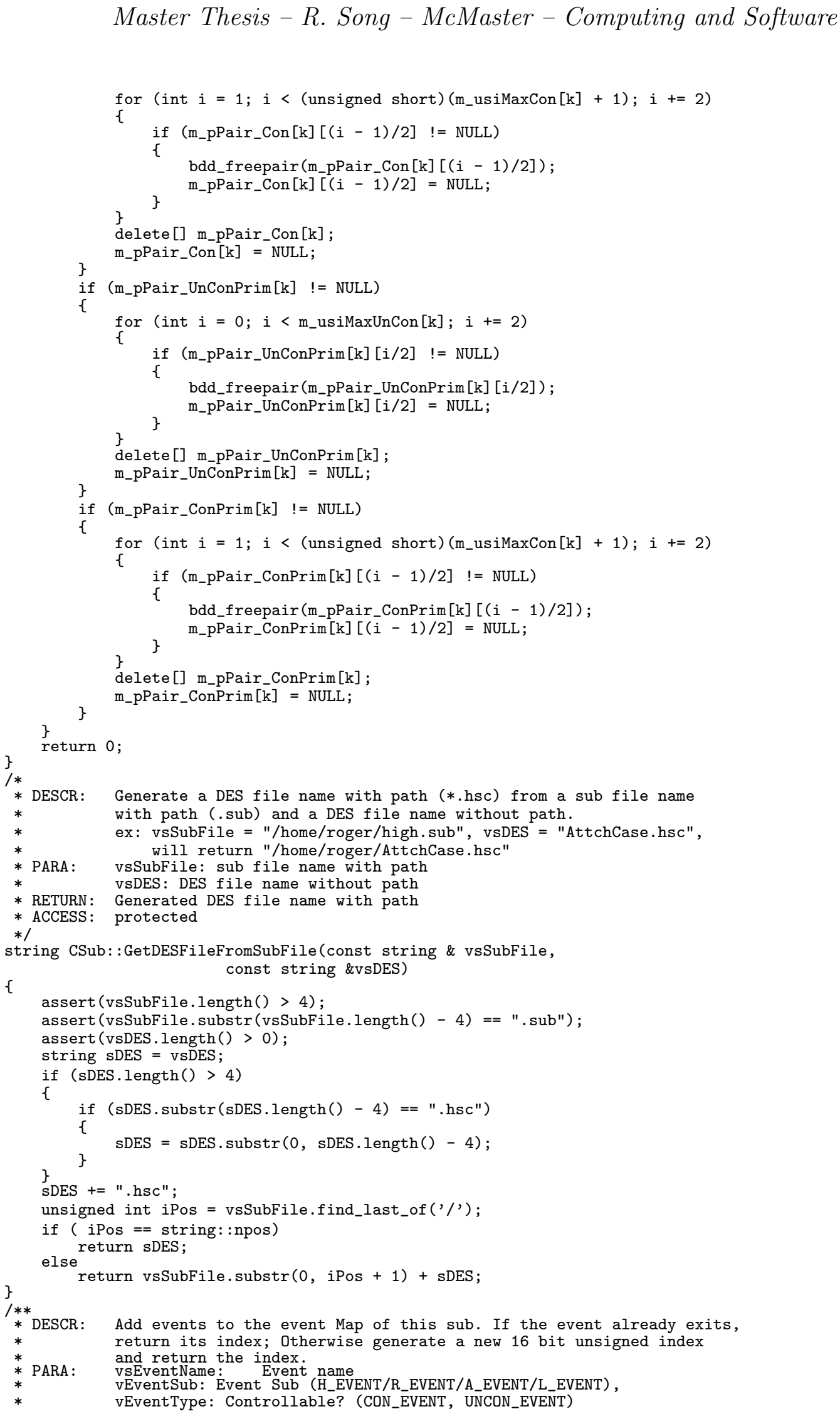


Master Thesis - R. Song - McMaster - Computing and Software

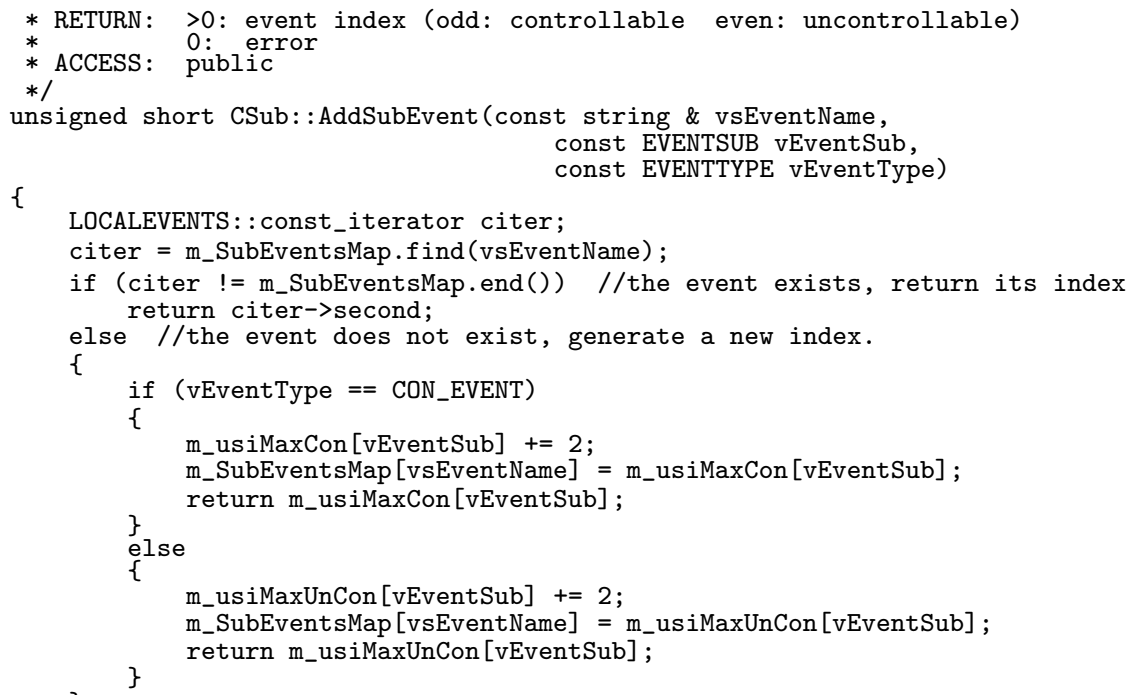




\section{Master Thesis - R. Song - McMaster - Computing and Software}

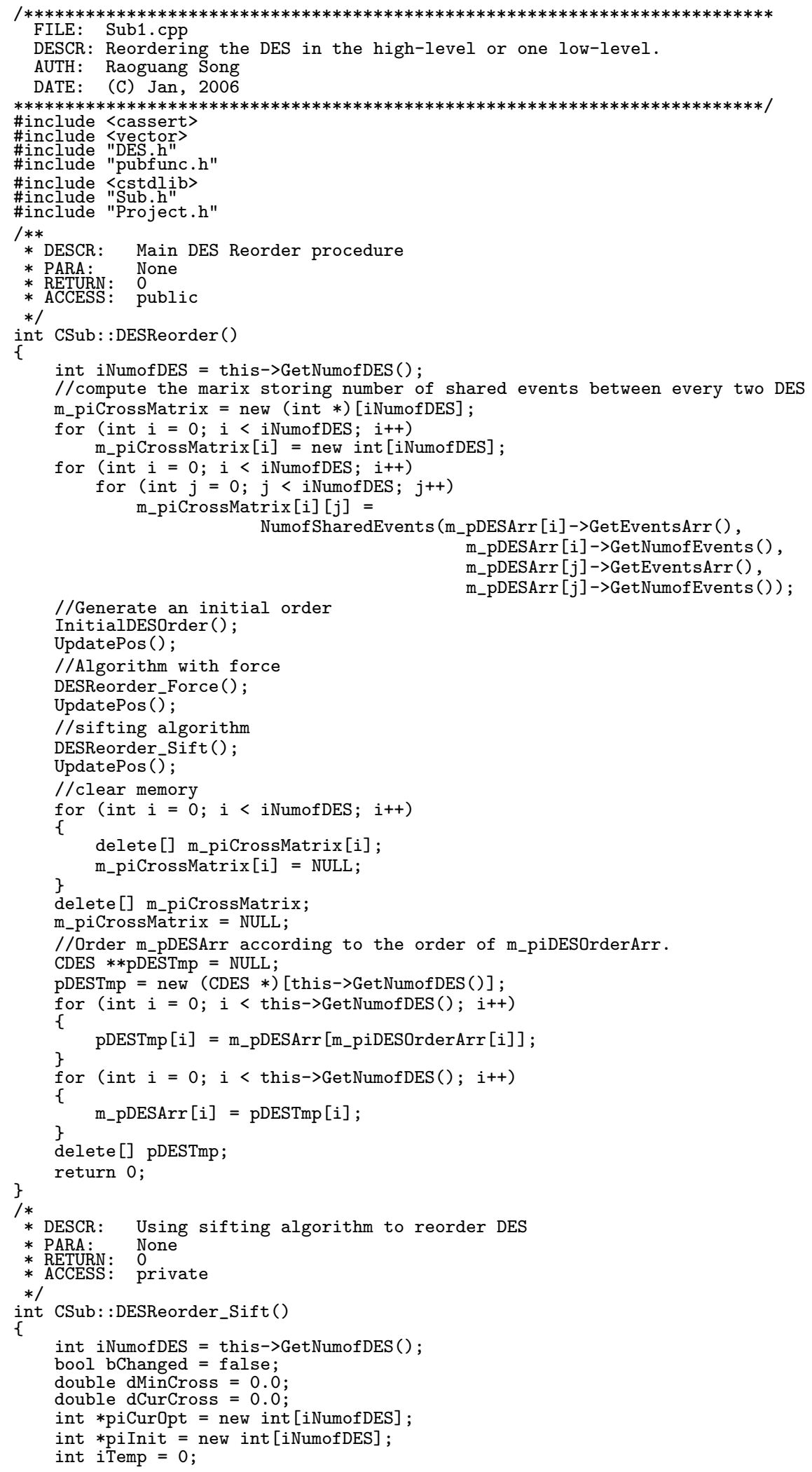




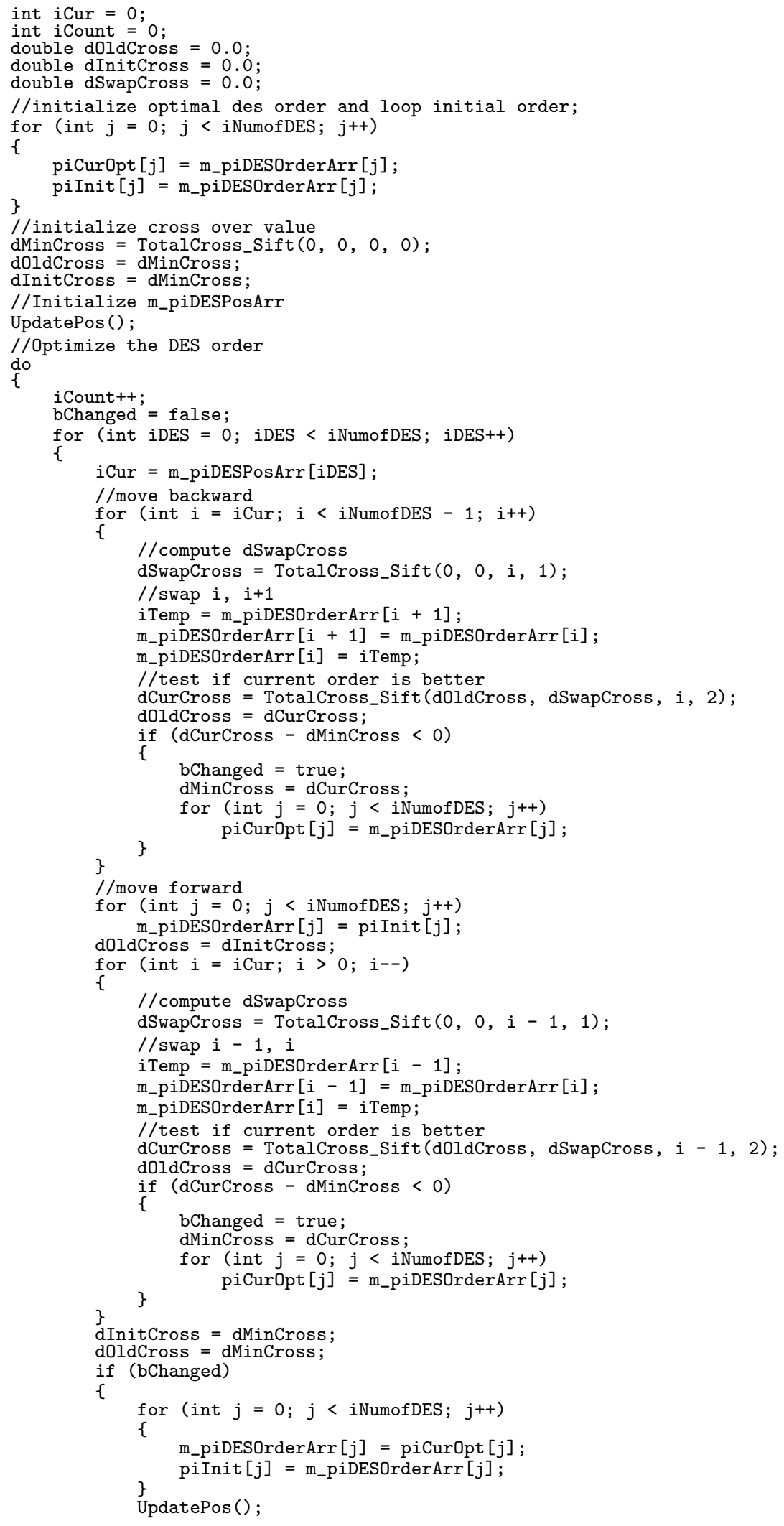




\section{Master Thesis - R. Song-McMaster - Computing and Software}

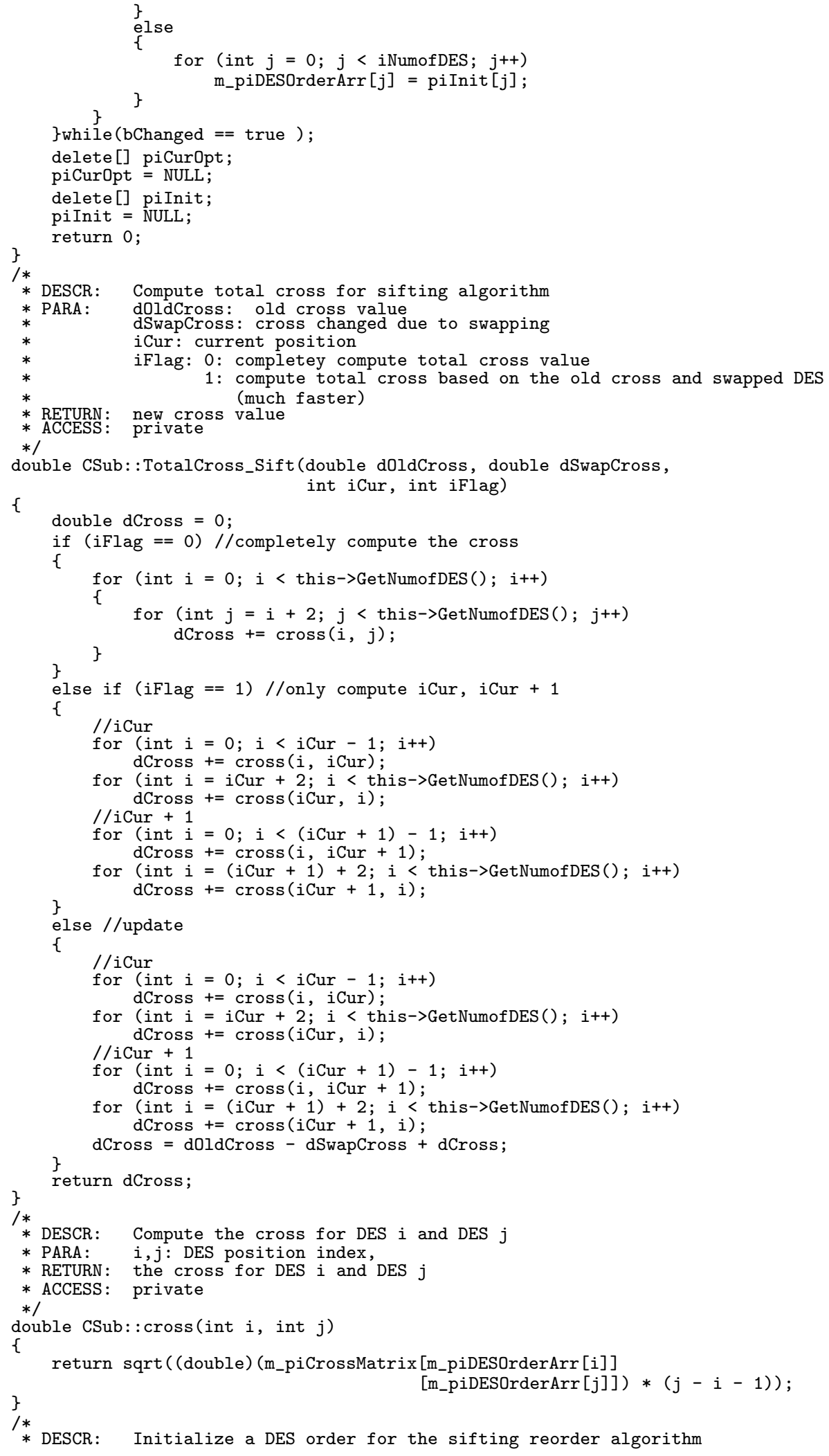




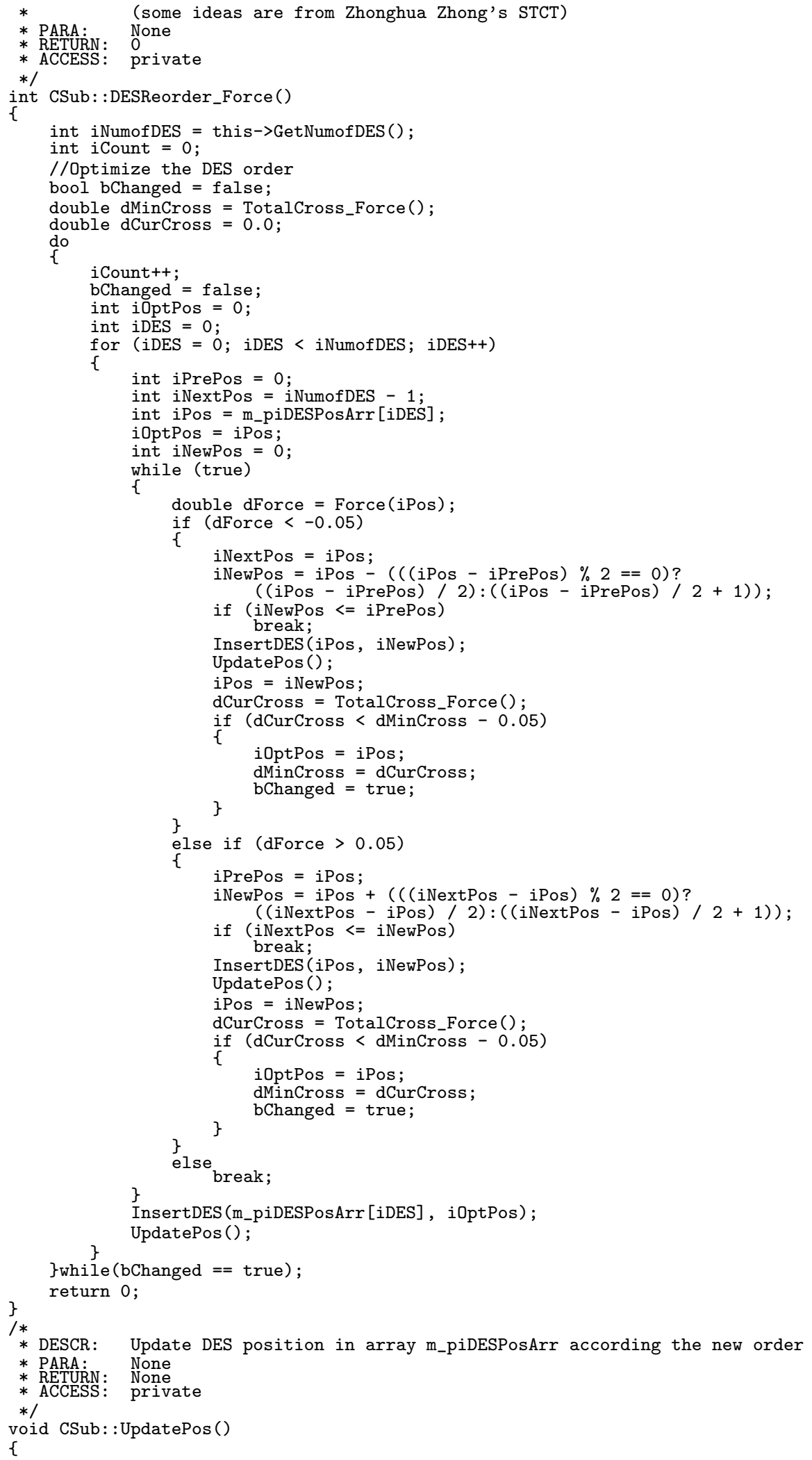


\}

for (int $i=0 ; i<$ this->GetNumofDES(); $i++$ )

m_piDESPosArr [m_piDESOrderArr $[i]]=i$; return;

* DESCR: Swap variables in m_piDESOrderArr for DESReorder_Force()

* PARA: iCur: current variable position

* iPos: destinate variable position

* RETURN: None

ACCESS: private

$*$

void CSub: : InsertDES(int iCur, int iPos)

int $i D E S=m$ piDESOrderArr [iCur $]$

if (iCur < iPos)

for (int $i=i$ Cur $+1 ; i<=i P o s ; i++$ )

m_piDESOrderArr $[i-1]=$ m_piDESOrderArr $[i]$;

m_piDESOrderArr [iPos] = iDES

else if (iCur > iPos)

for (int $i=i$ Cur $-1 ; i>=i P o s ; i--$ )

m_piDESOrderArr $[i+1]=m_{-}$piDESOrderArr $[i]$;

\} m_piDESOrderArr [iPos $]=$ iDES;

\}

return;

* DESCR: Compute total cross for DESReorder_Force()

* PARA: None

* RETURN: total cross

$* /$

double CSub: :TotalCross_Force()

\{

double dCross $=0$

for (int $i=0 ; i<$ this->GetNumofDES(); $i++)$

\{

for (int $j=i+2 ; j<$ this->GetNumofDES(); $j++$ )

dCross $+=\operatorname{cross}(i, j)$

\}

return dCross

* DESCR: Decide to move DES_i left or right. (< 0 : move left; >0 move right)

* for DESReorder_Force (

* PARA: $\quad$ i: position in m_piDESOrderArr

* RETURN: returned force

$* /$

double CSub: :Force(int i)

$\{$ double dForce $=0$;

for (int $j=0 ; j<i-1 ; j++$ )

dForce $+=\operatorname{sqrt}(($ double) $m$ piCrossMatrix[m_piDESOrderArr [i]]

for (int $j=i+2 ; j<$ this $->\operatorname{Get} \operatorname{NumofDES}() ; j++$ )

$[$ m_piDESOrderArr $[j]] *(j-i+1))$;

dForce $+=\operatorname{sqrt}(($ double)m piCrossMatrix [m piDESOrderArr [i]]

\}

return dForce

[m_piDESOrderArr $[j]] *(j-i-1))$;

* DESCR: Initialize a DES order

* PARA: None

* ACCESS: private

$* /$

int CSub: : InitialDESOrder()

int $i=0$;

int $j=0$;

int $\mathrm{k}=0$;

int iNumofDES $=$ this $->$ GetNumofDES () ;

//There is no DES at all

if (iNumofDES <= 0)

return 0;

//Only one DES

m_piDESOrderArr [0] = 0 ;

if (iNumofDES <= 1)

return 0 ; 


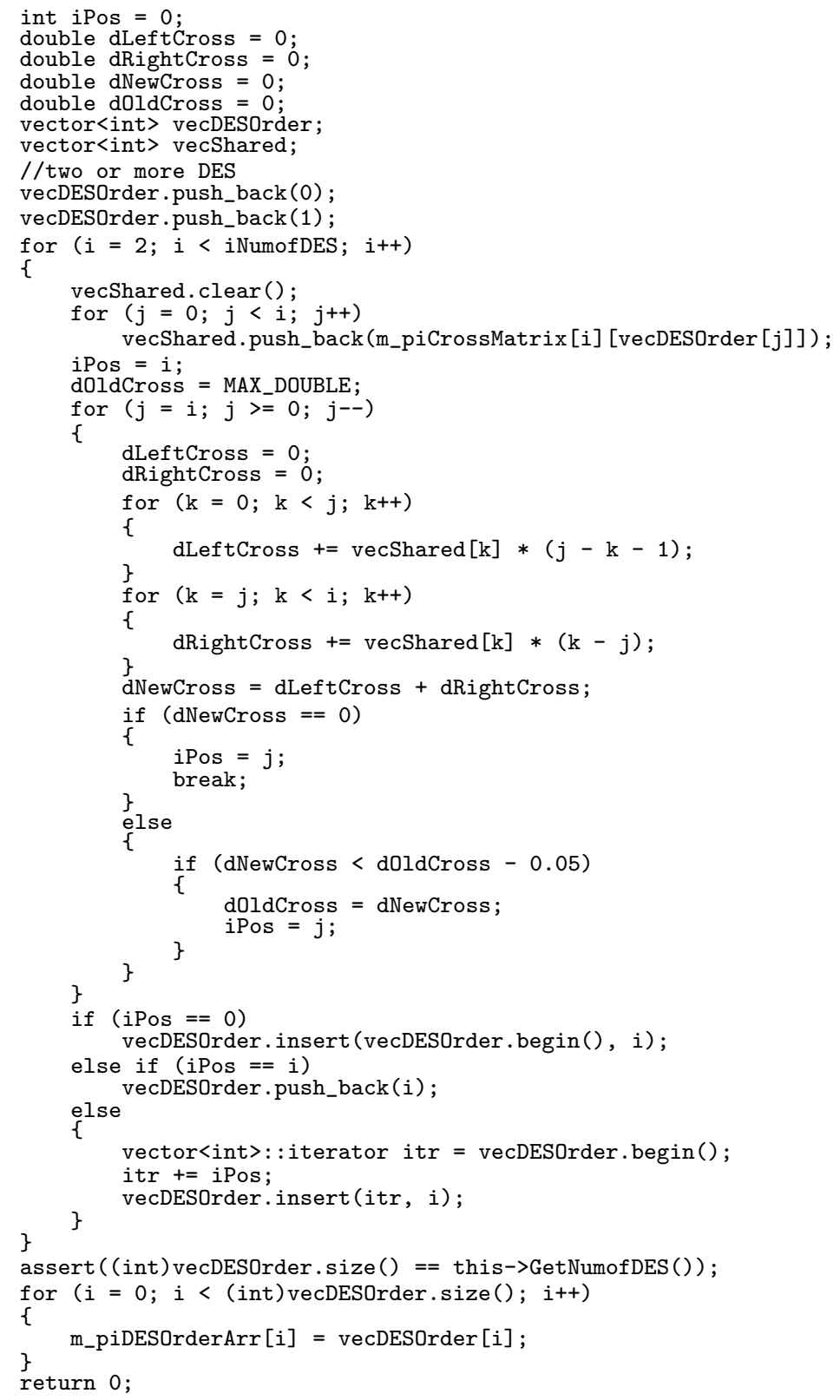




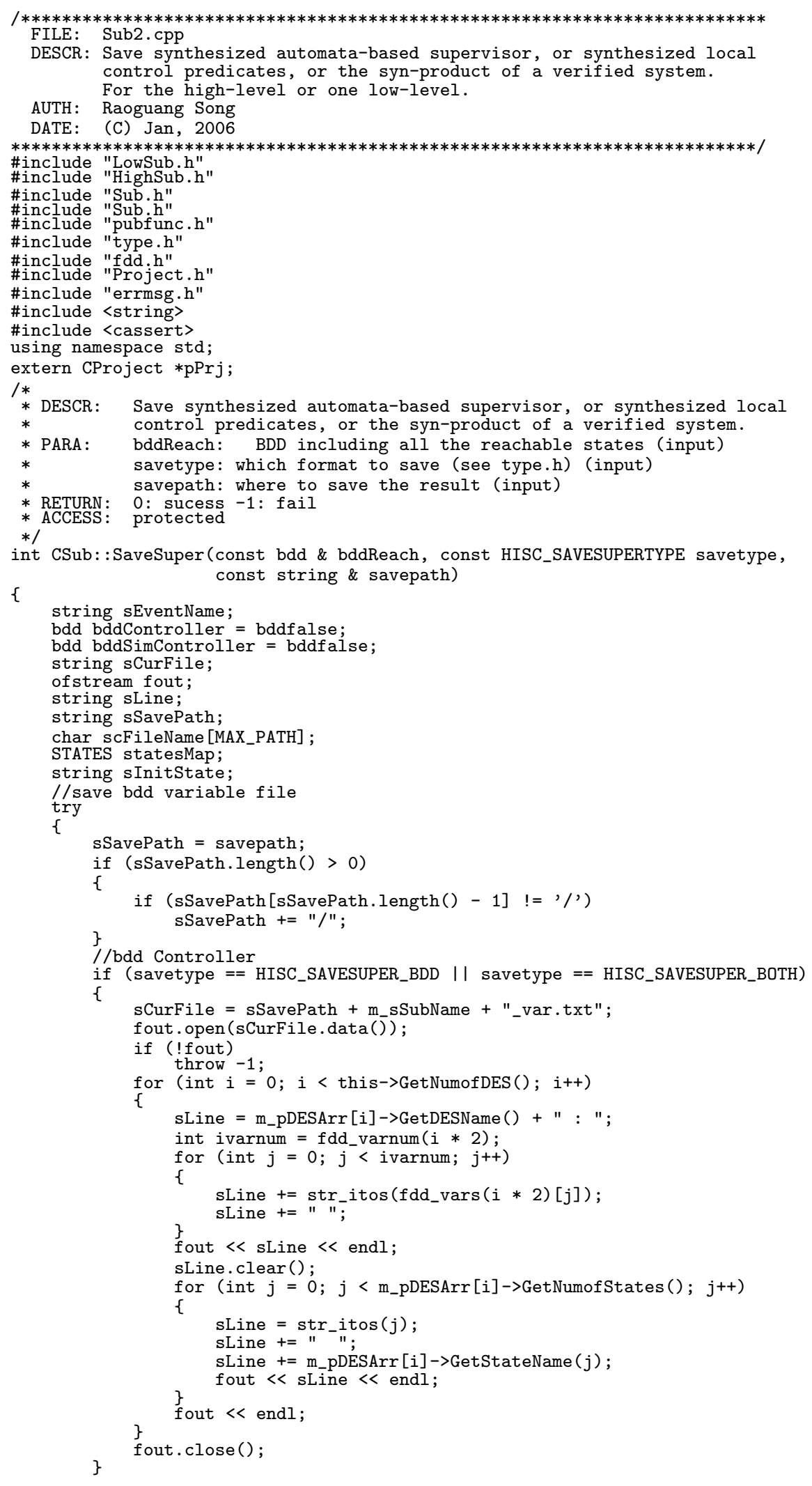


Master Thesis - R. Song - McMaster - Computing and Software

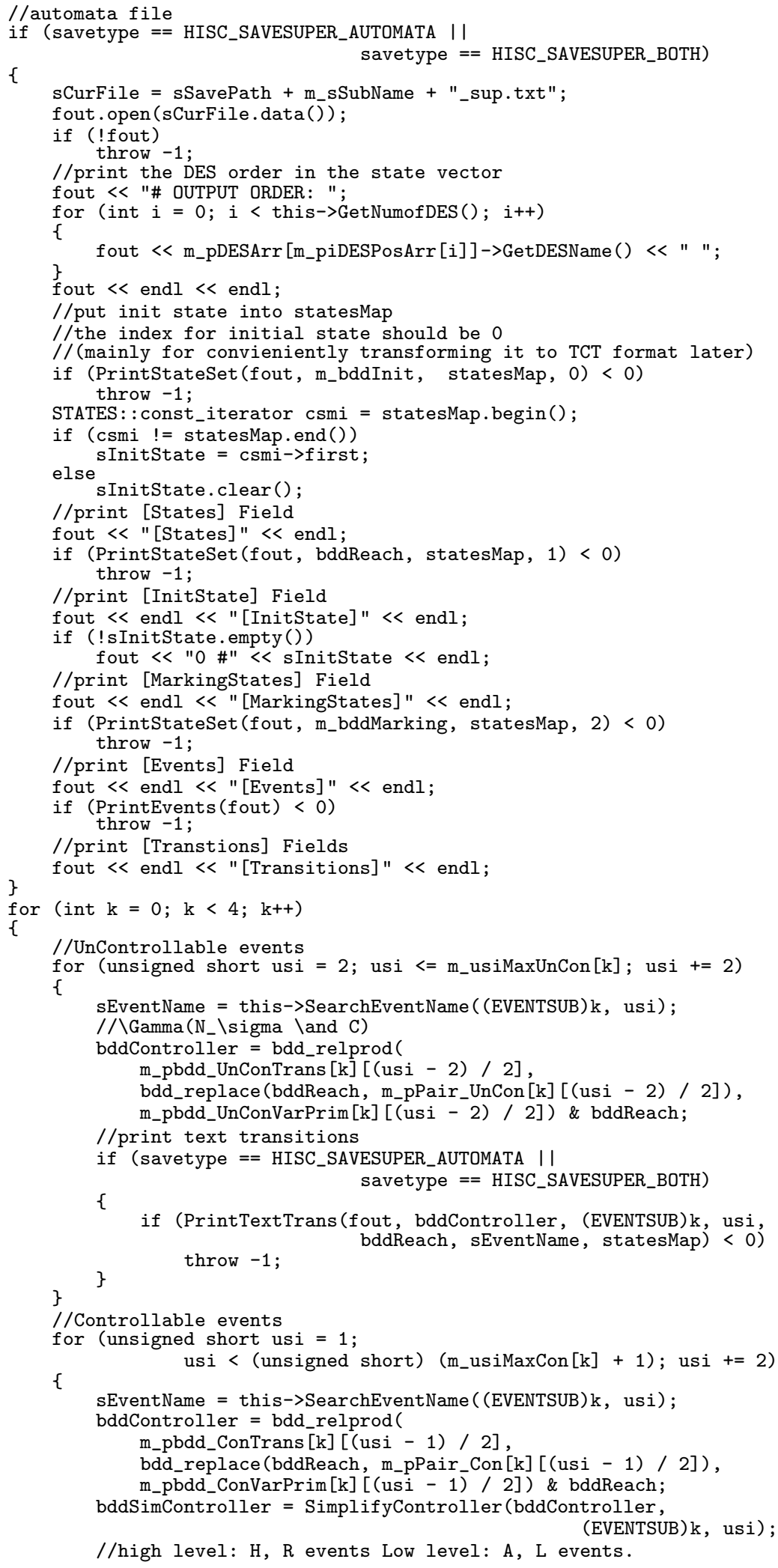




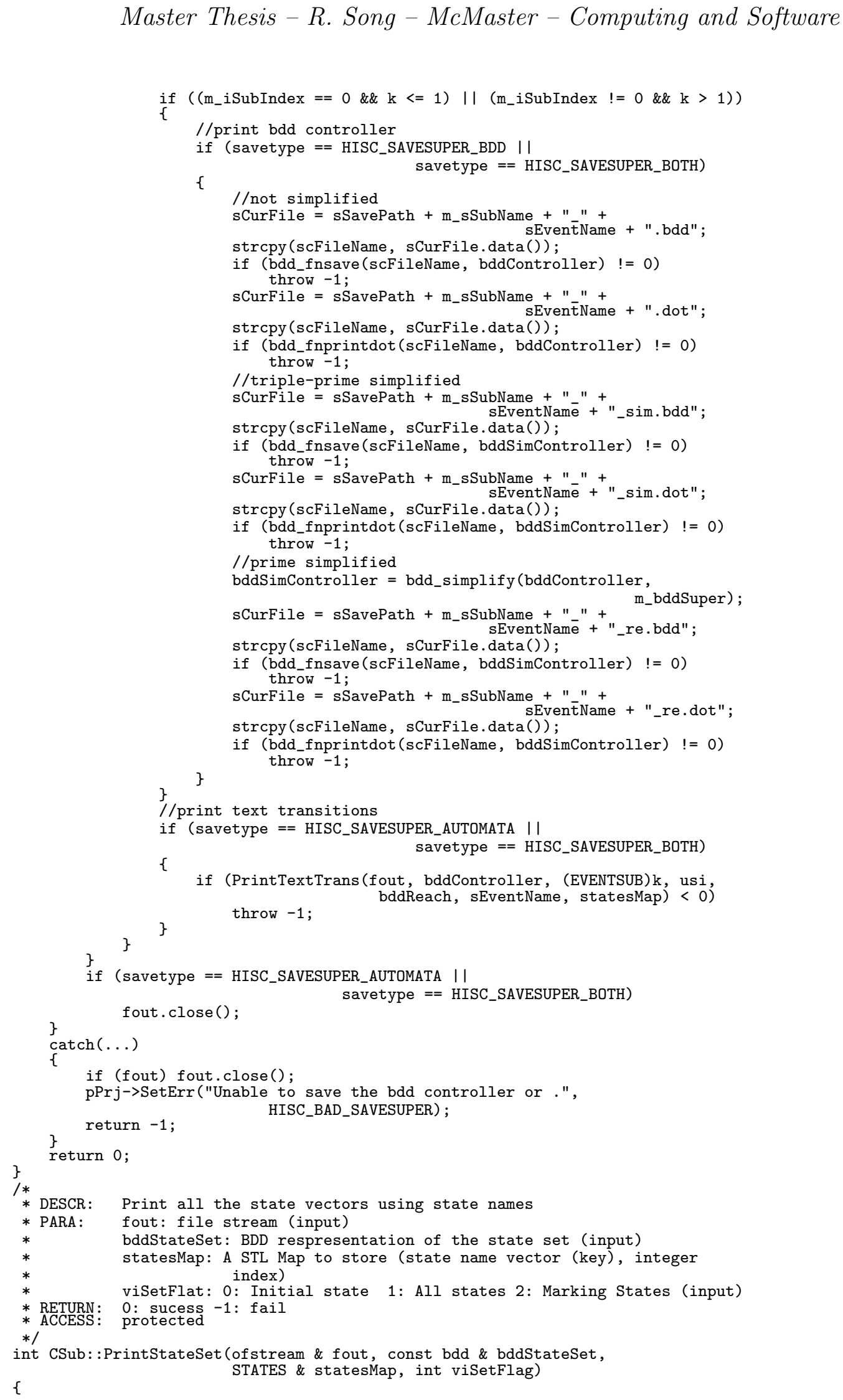




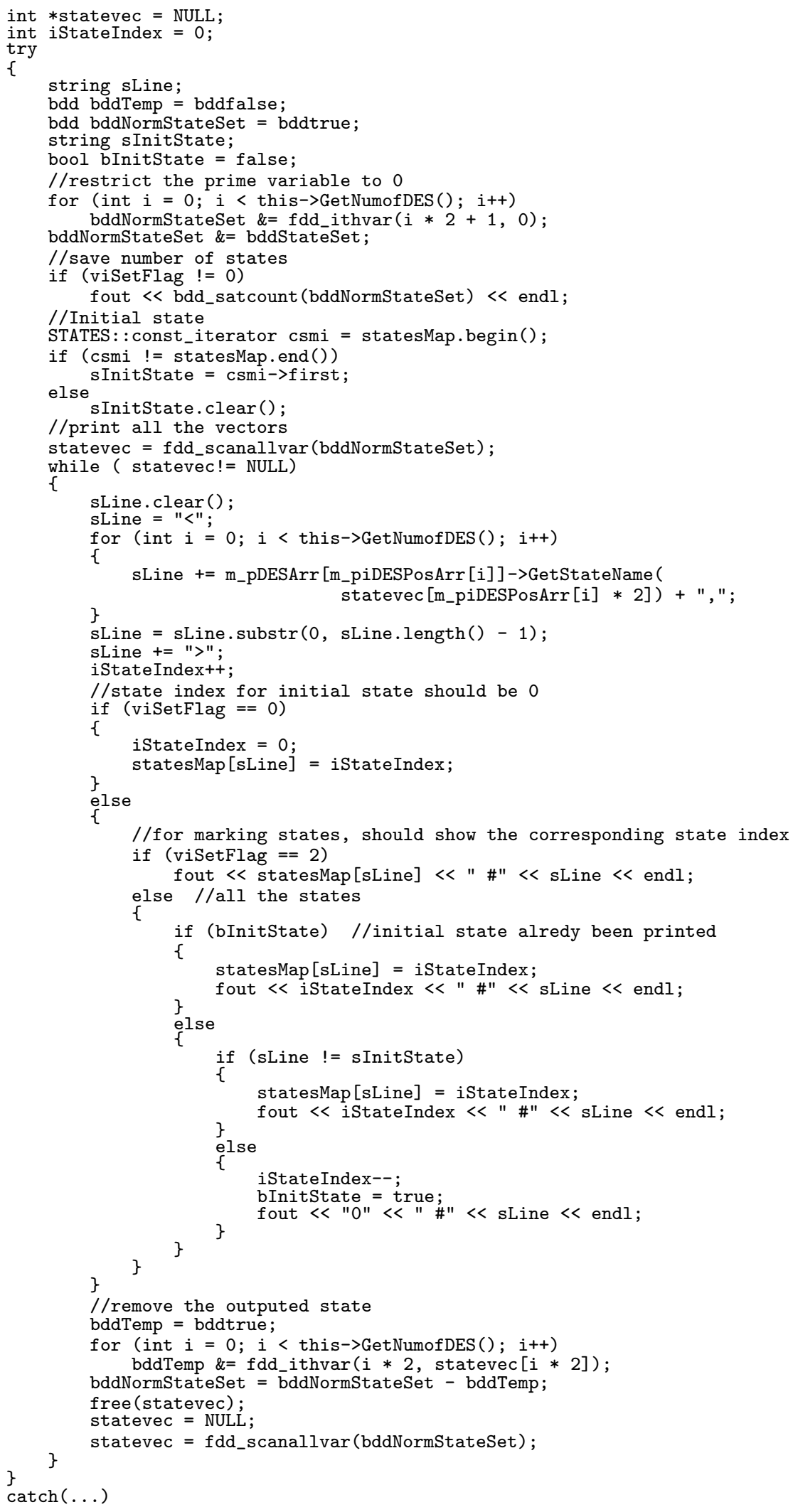




\section{Master Thesis - R. Song - McMaster - Computing and Software}

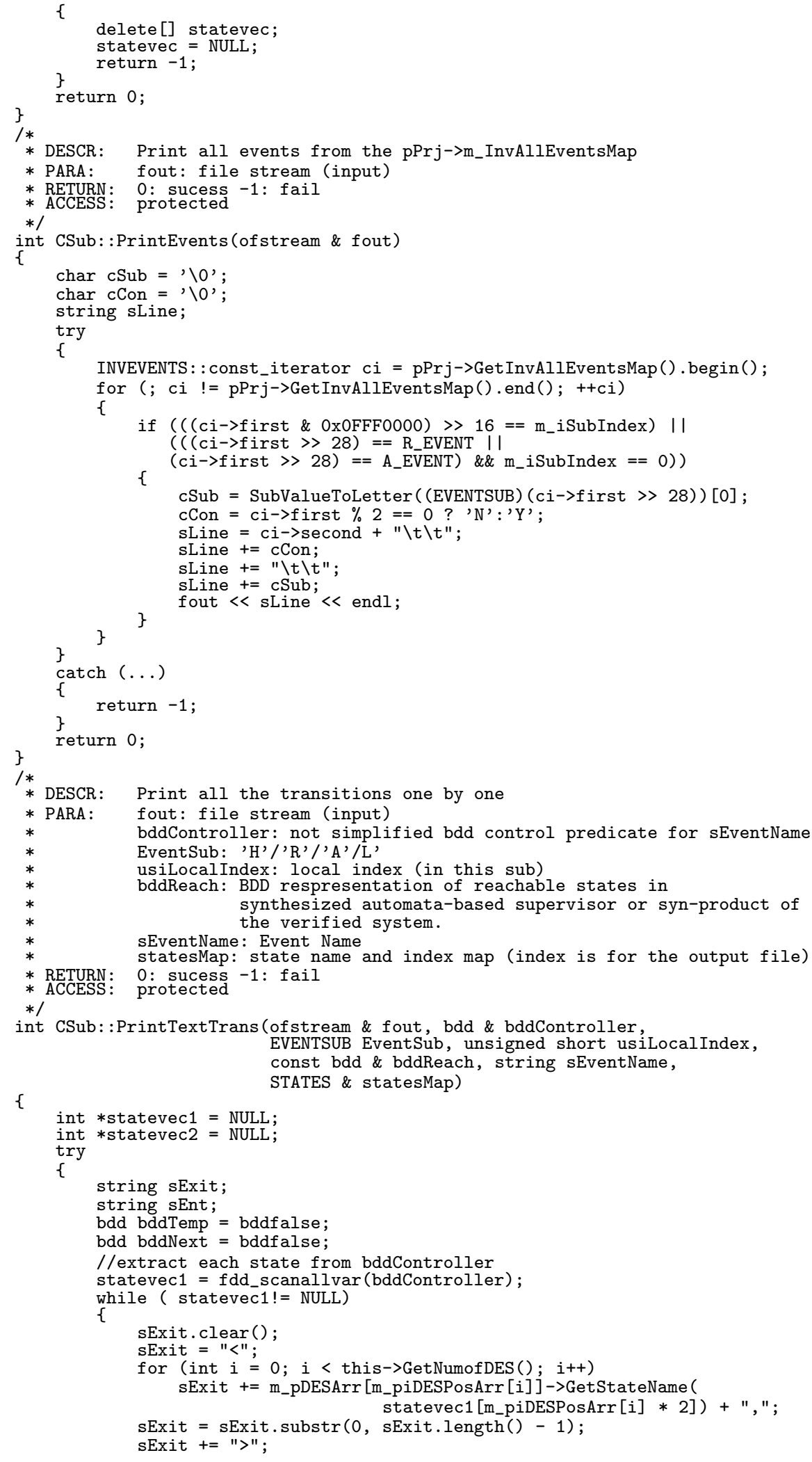


Master Thesis - R. Song - McMaster - Computing and Software

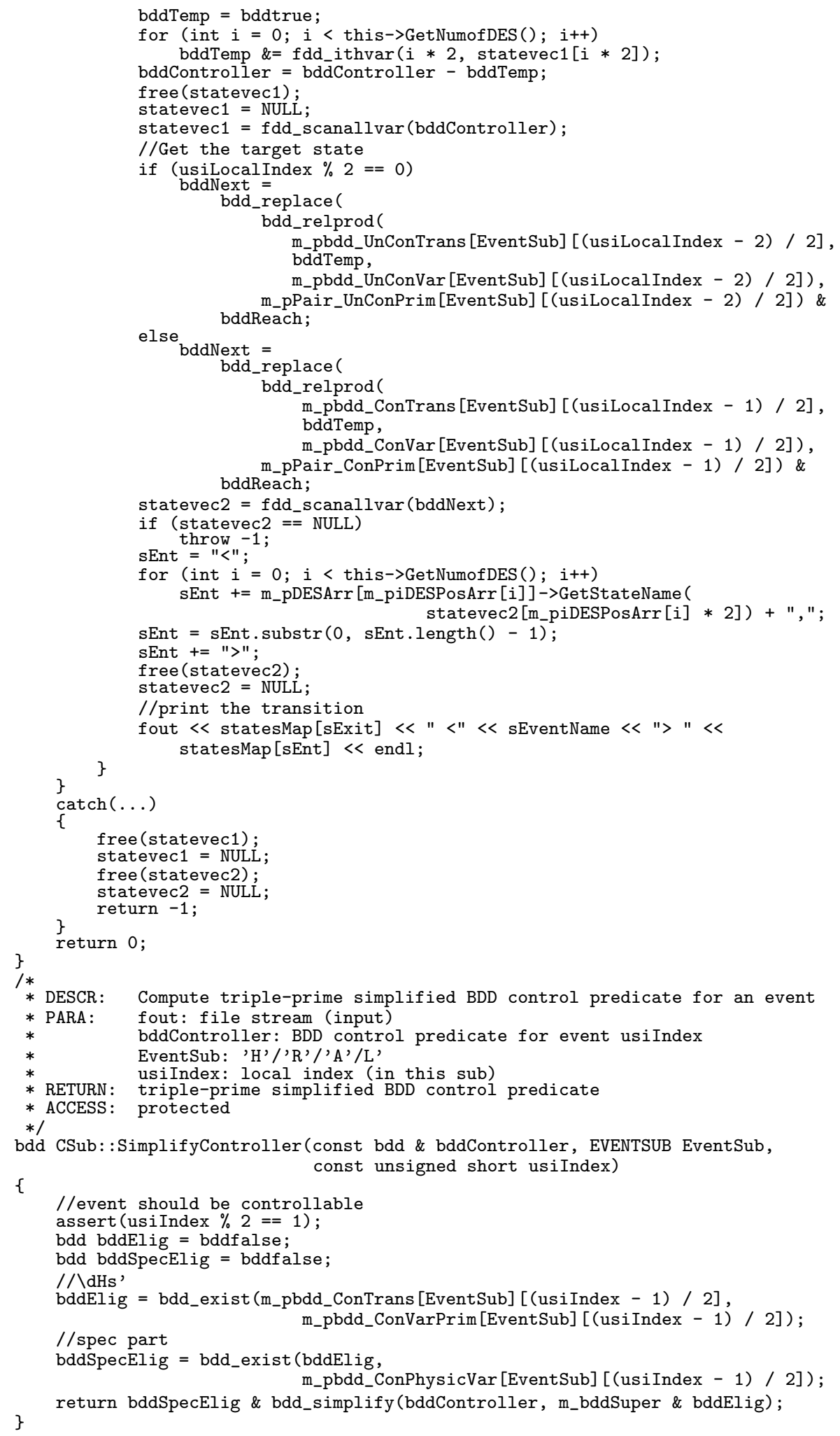




\section{Master Thesis - R. Song - McMaster - Computing and Software}

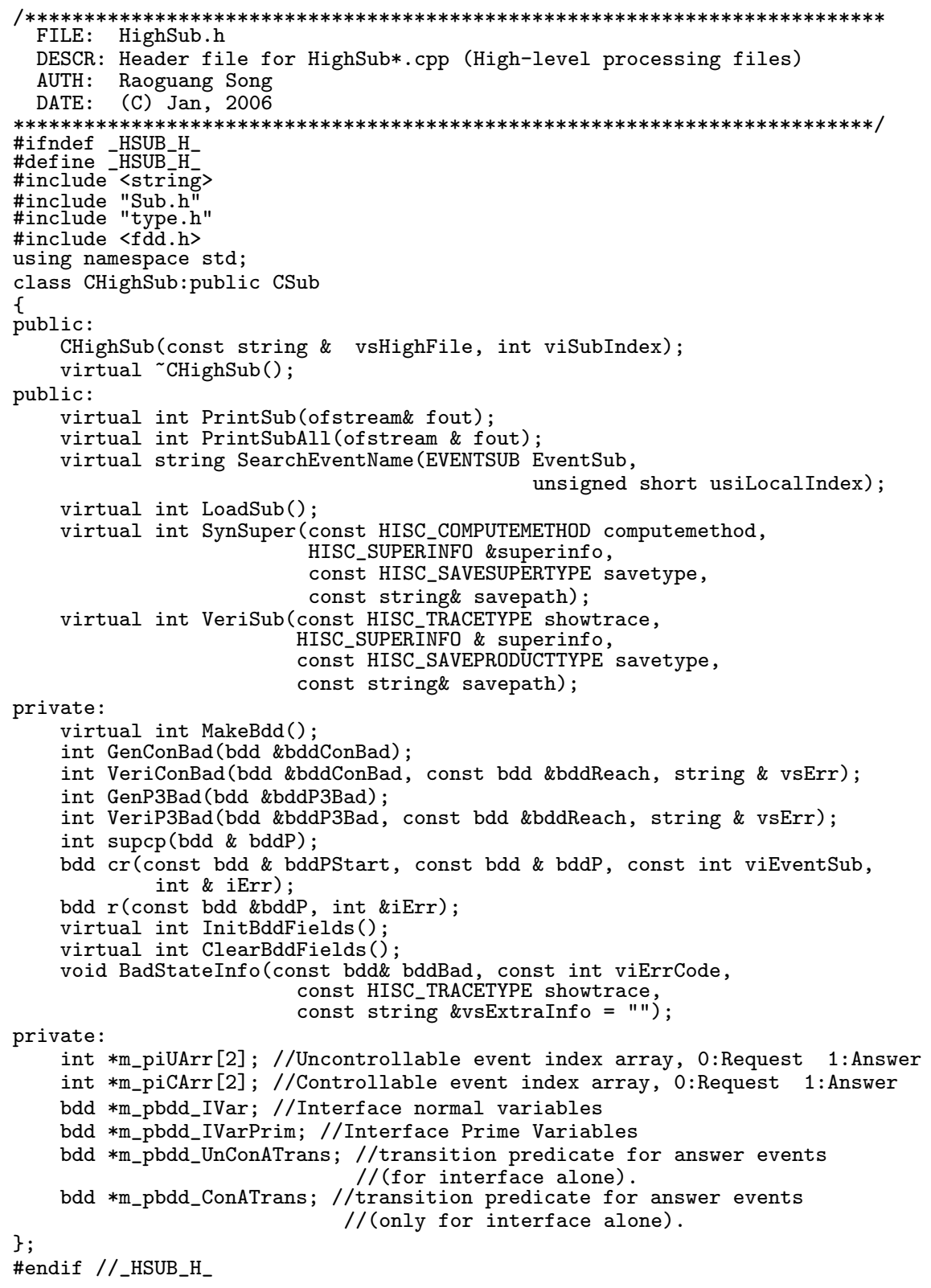




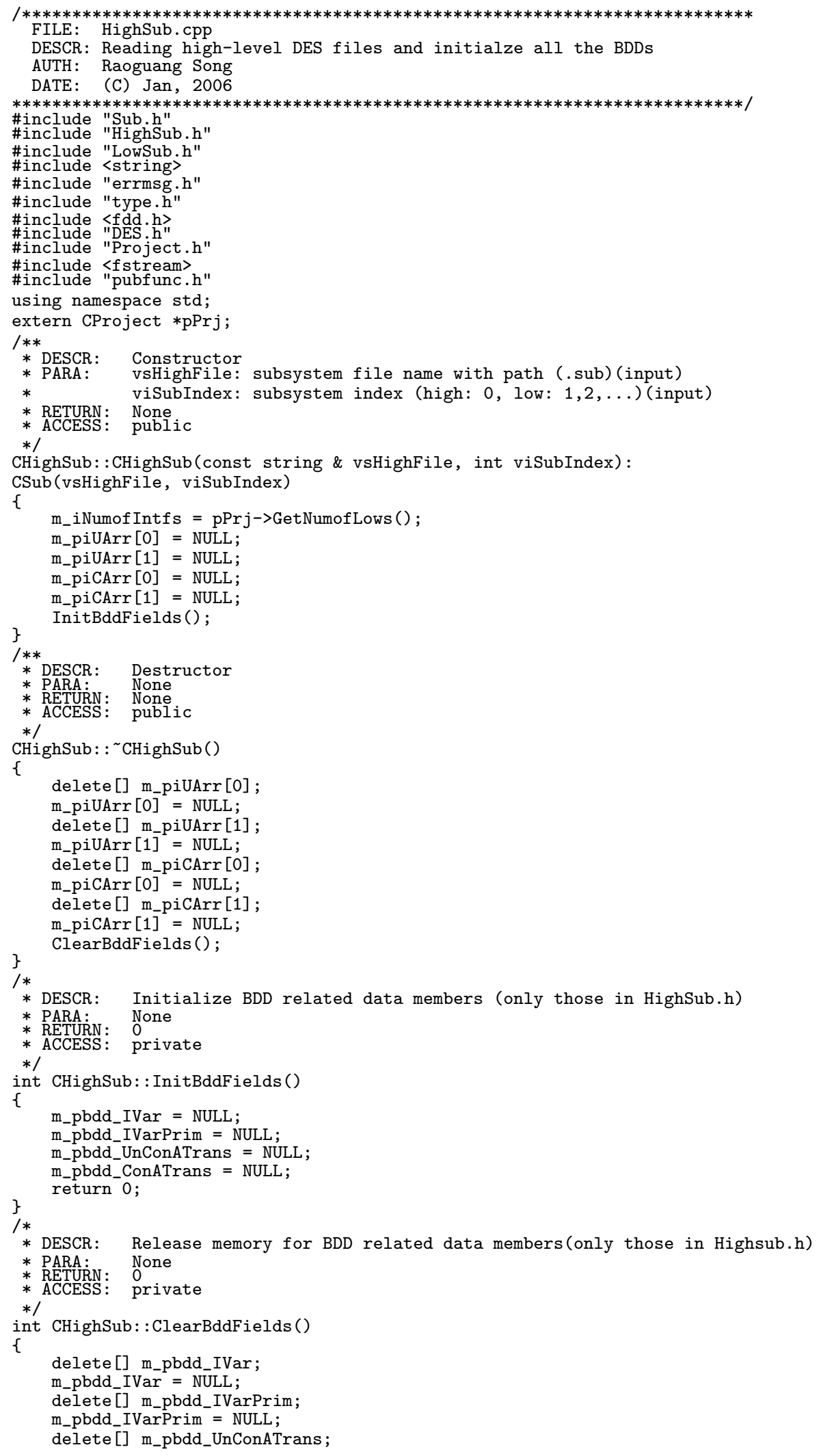




\section{Master Thesis - R. Song - McMaster - Computing and Software}

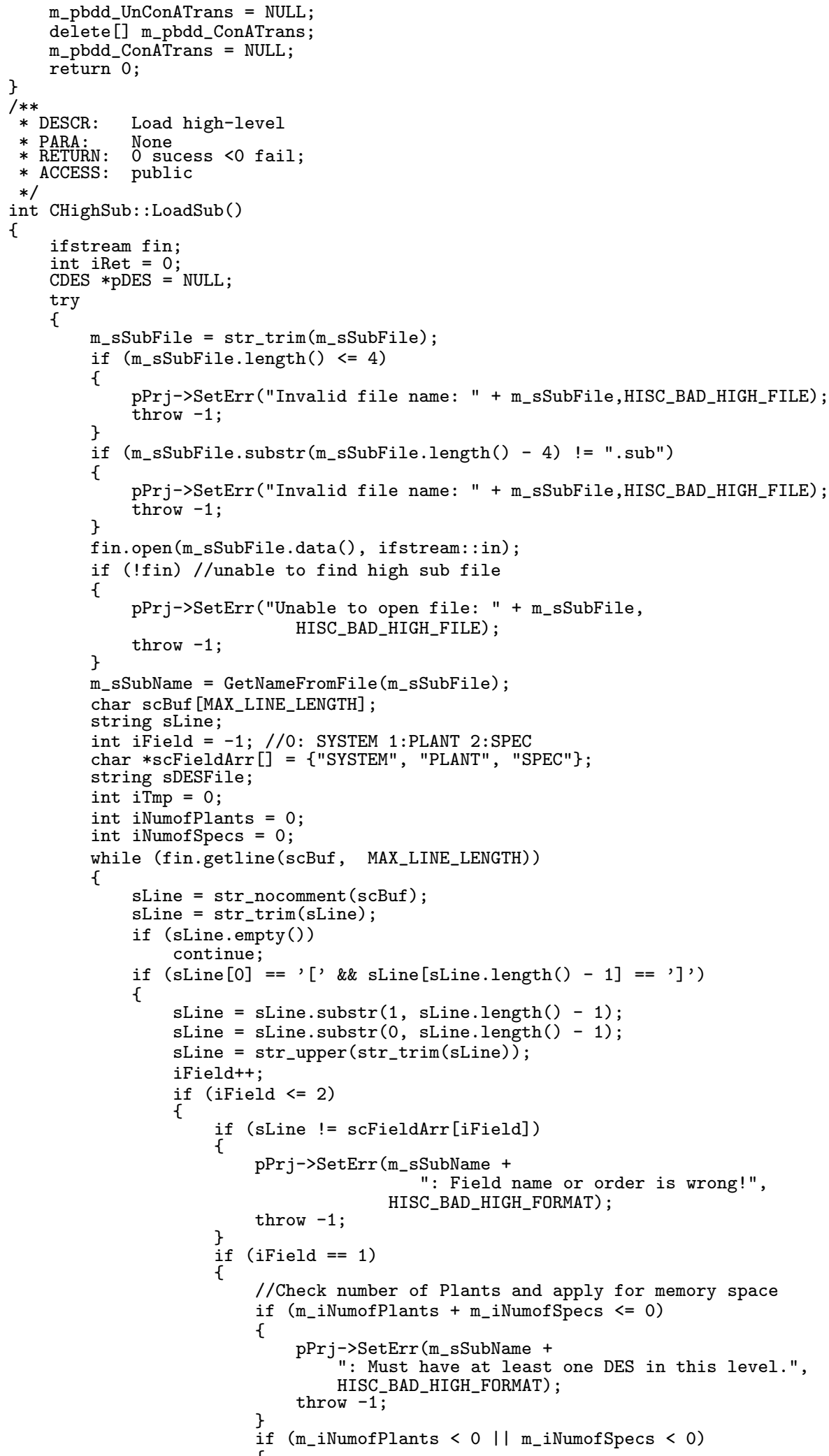


Master Thesis - R. Song - McMaster - Computing and Software

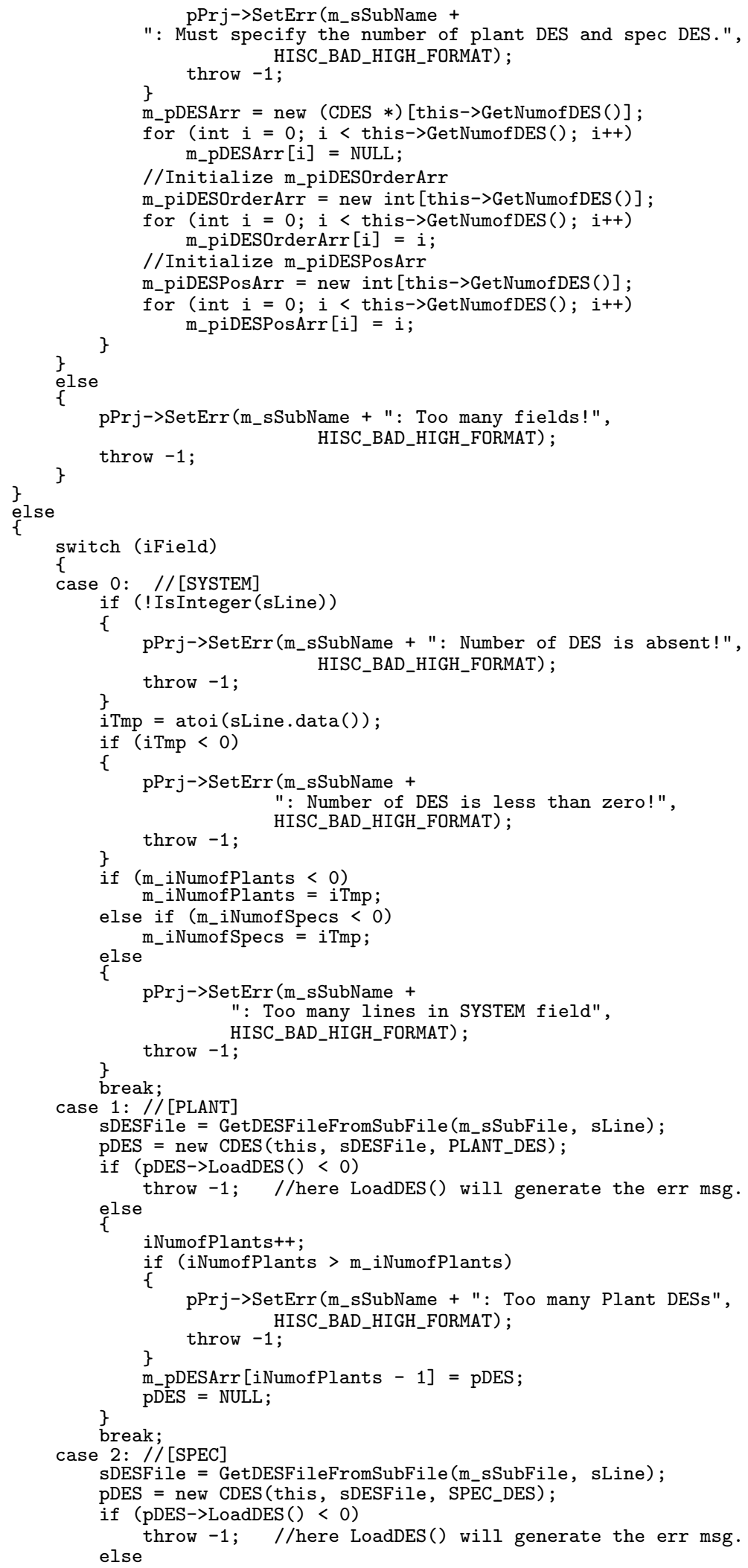




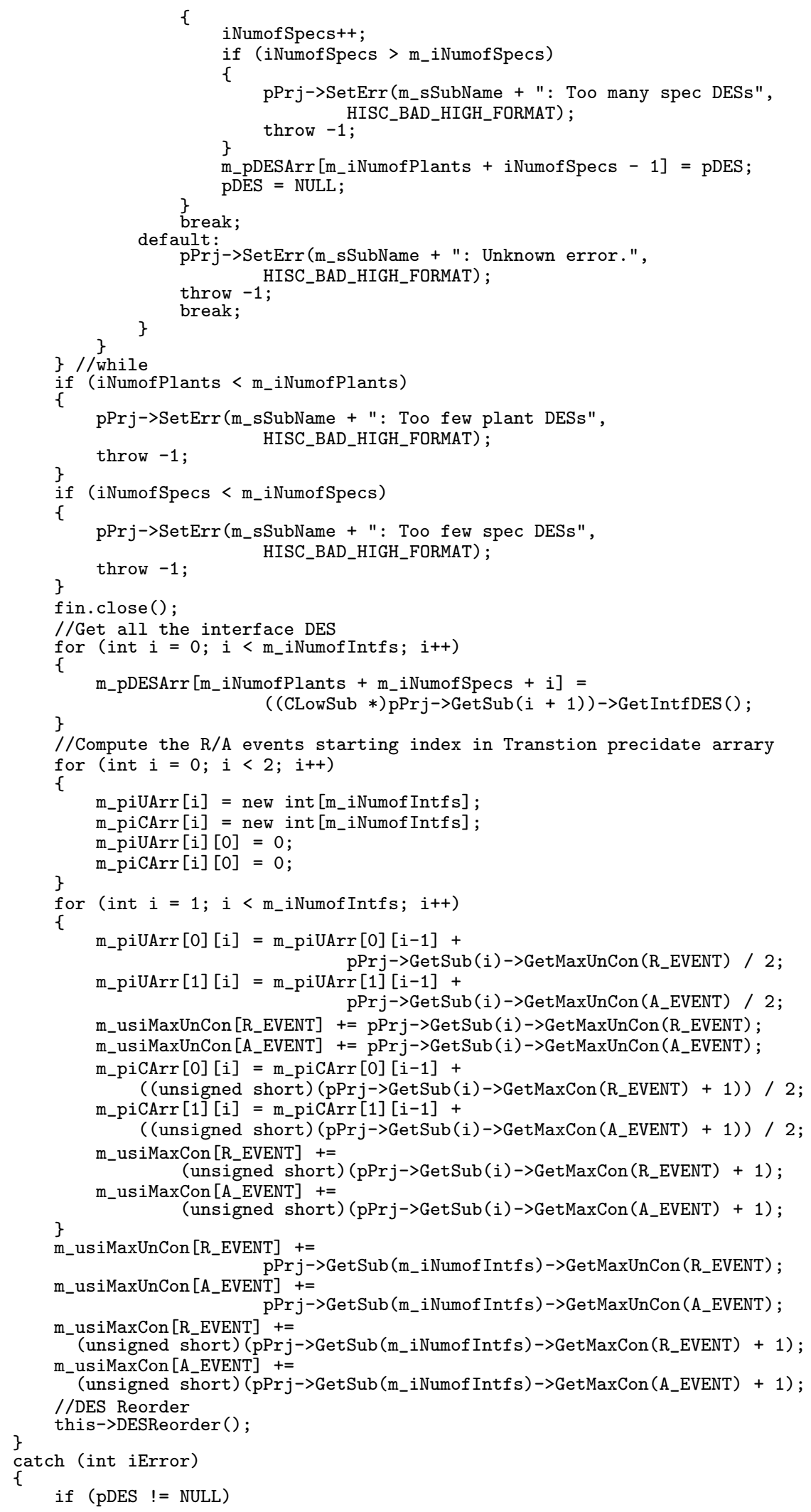




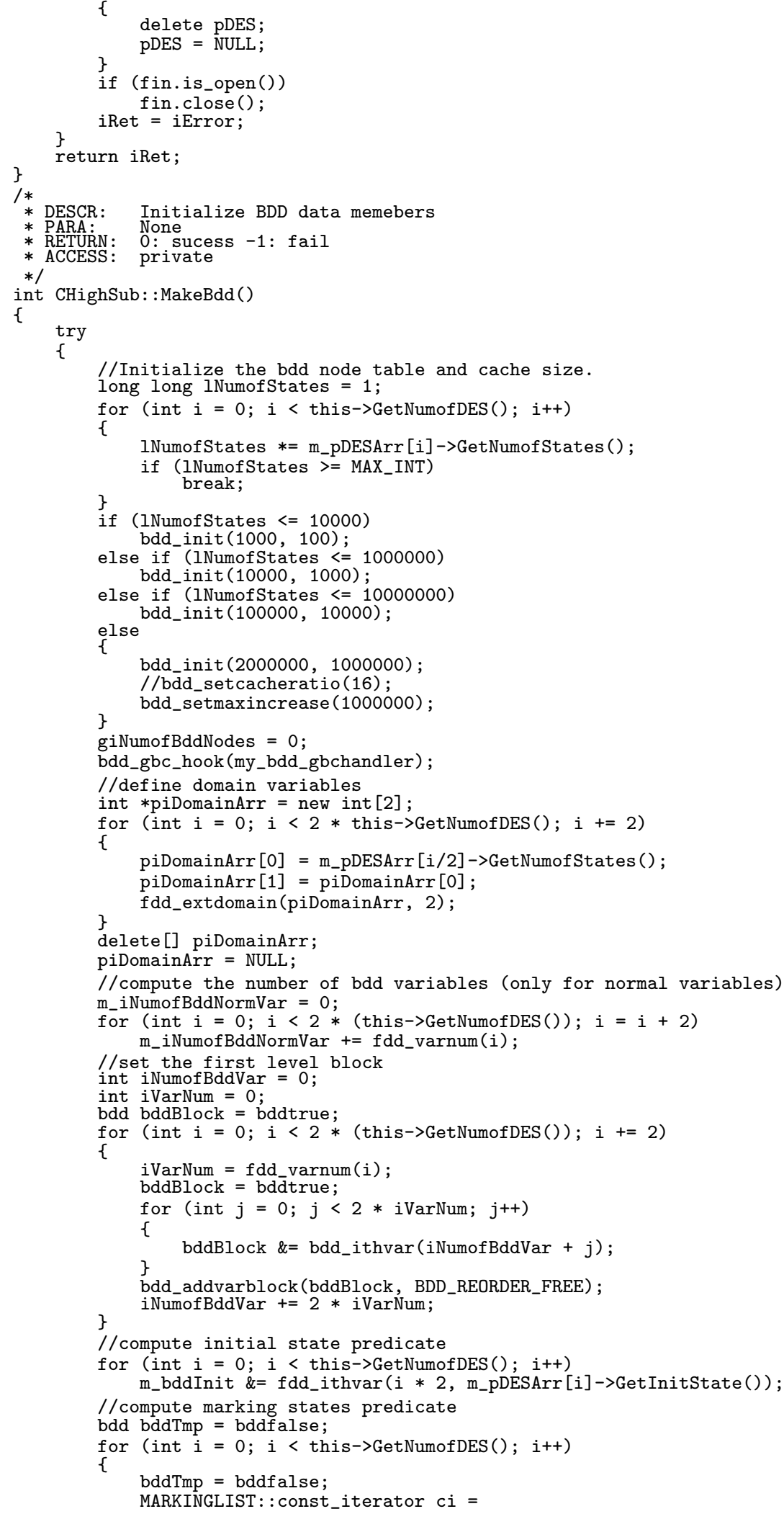




\section{Master Thesis - R. Song - McMaster - Computing and Software}

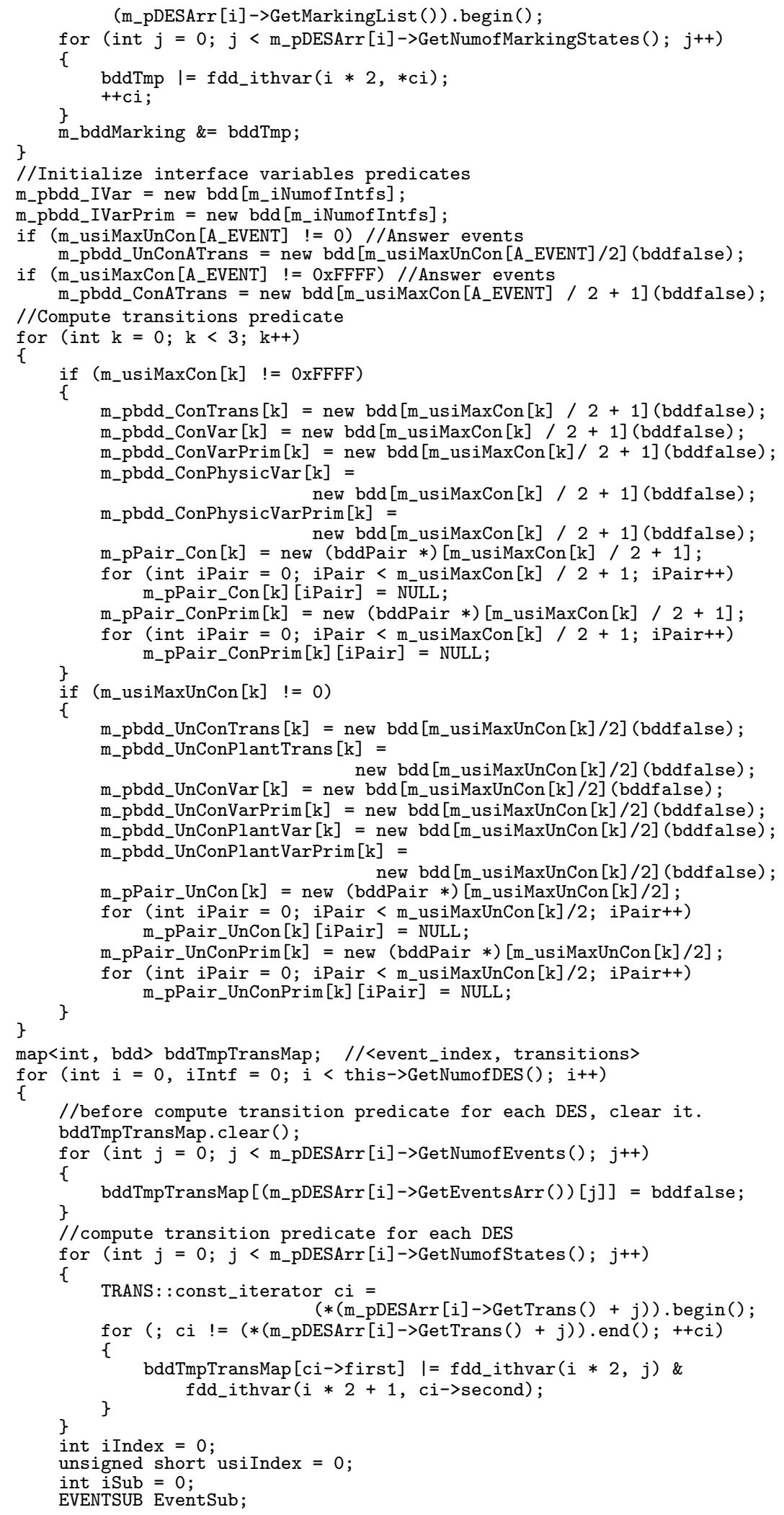




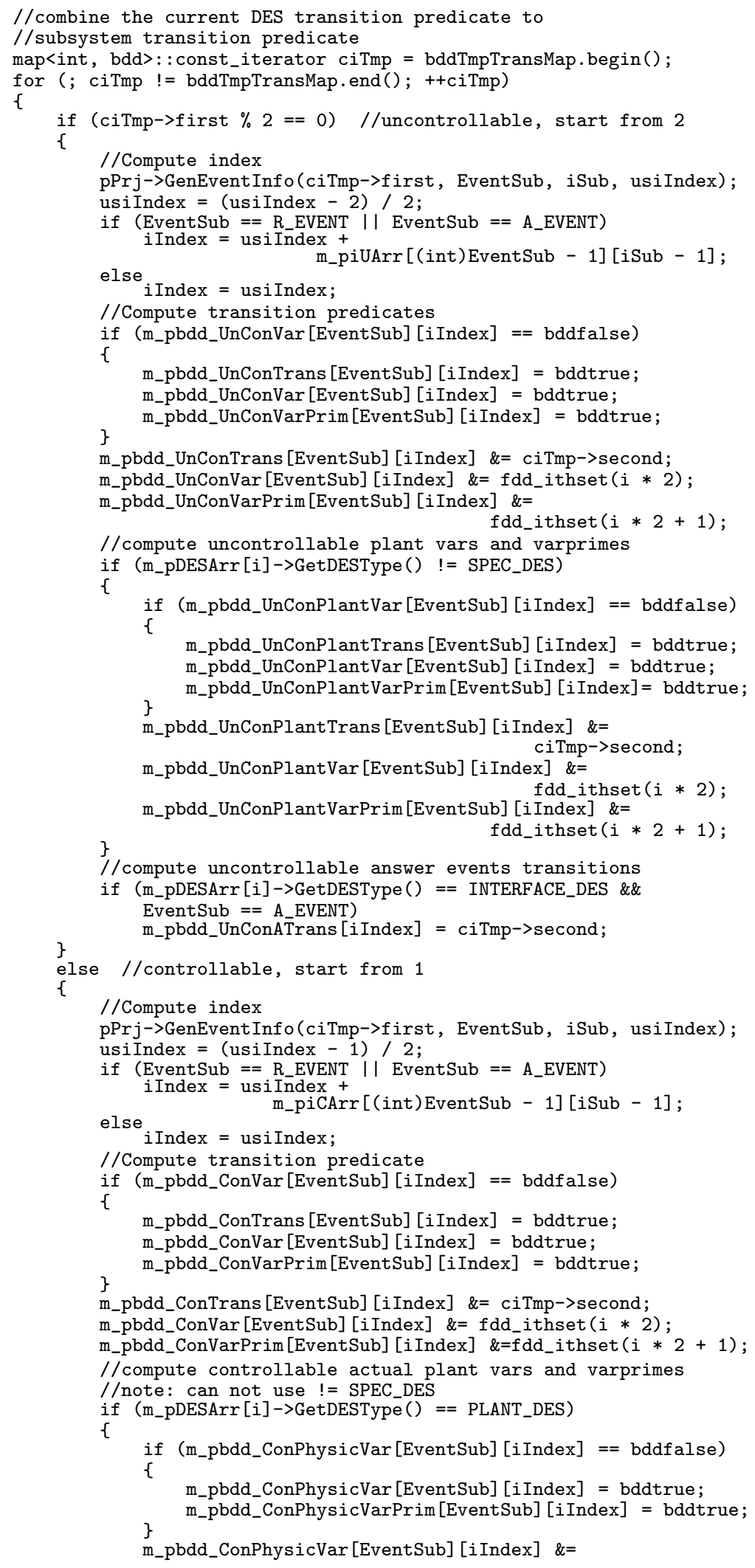




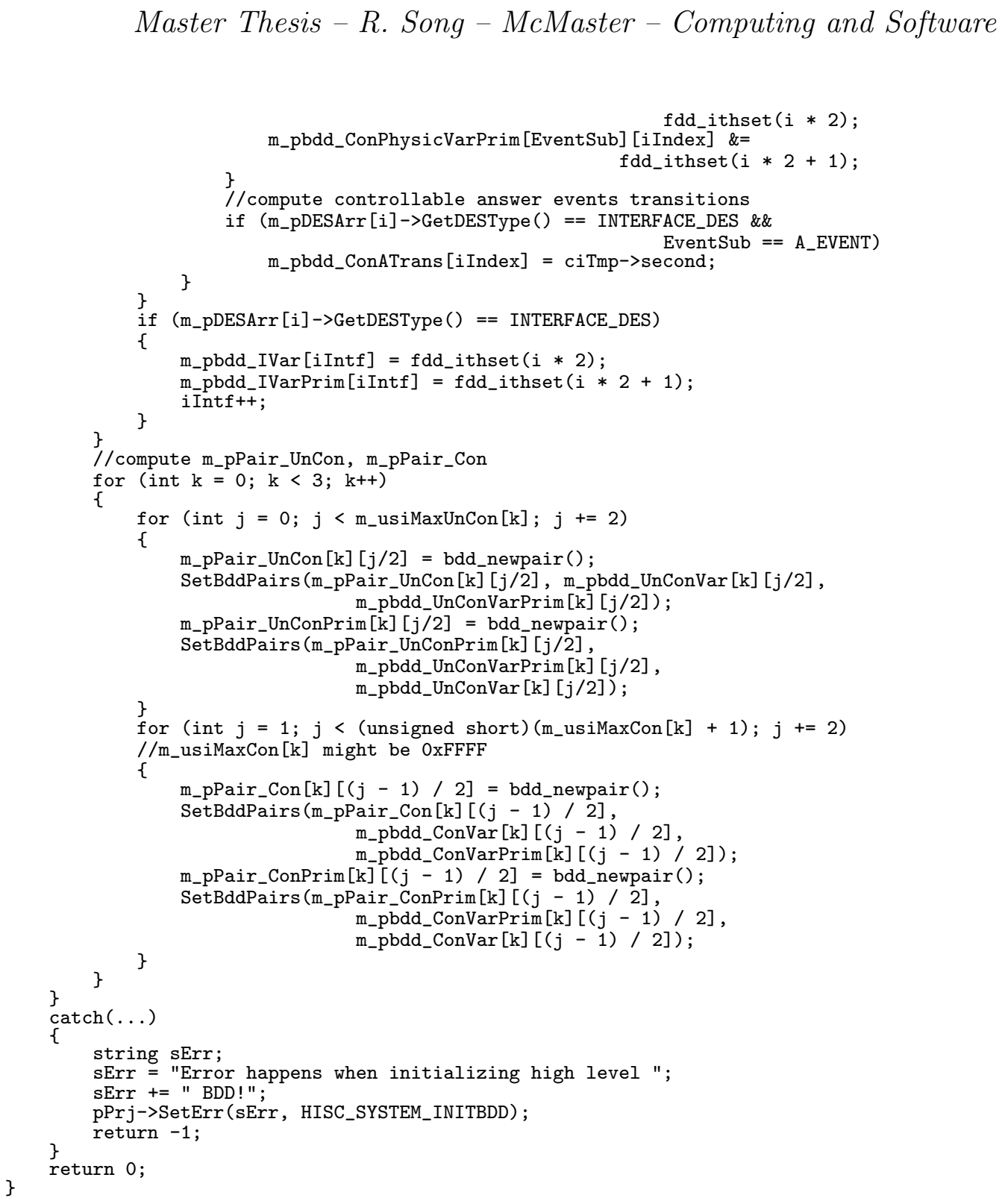




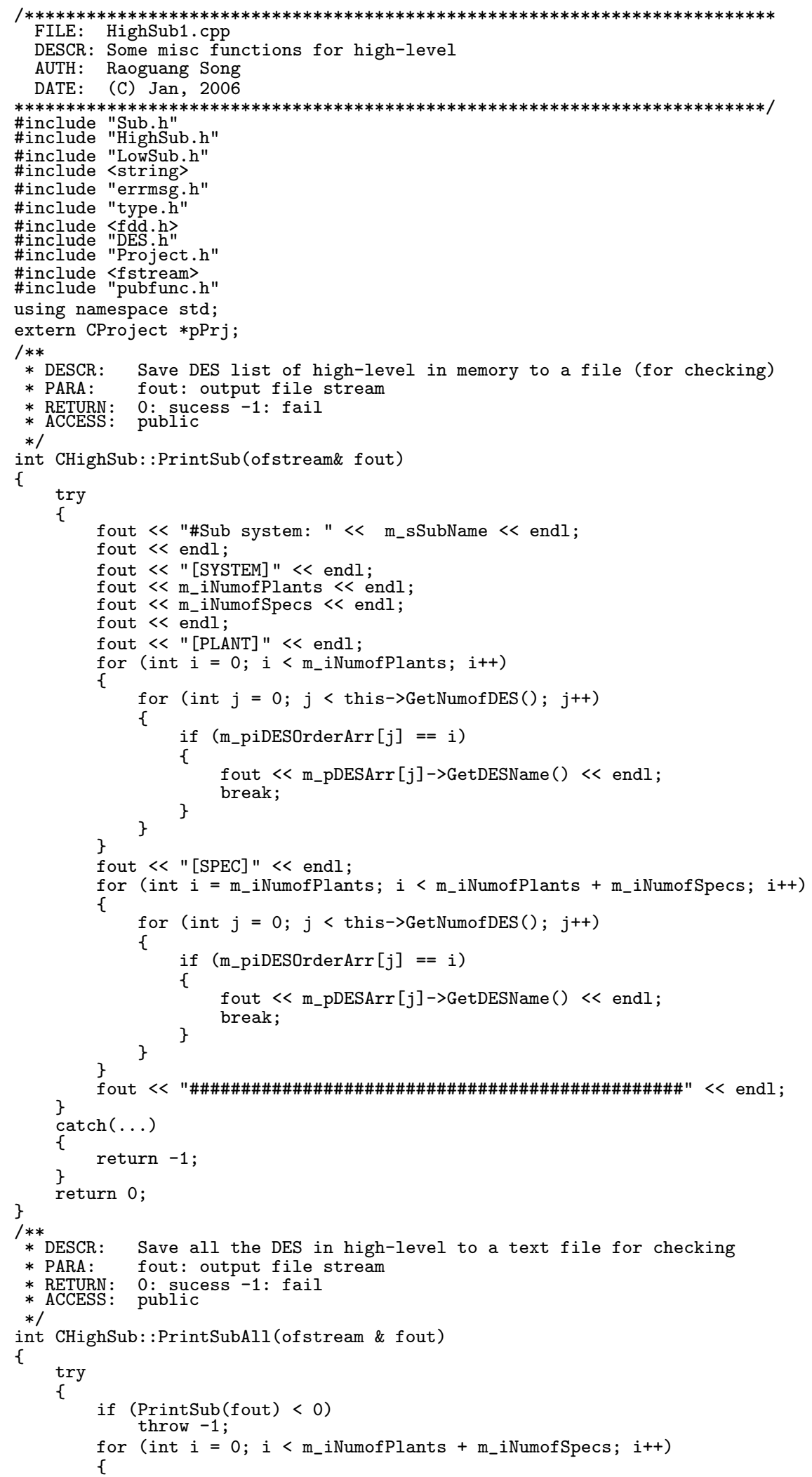




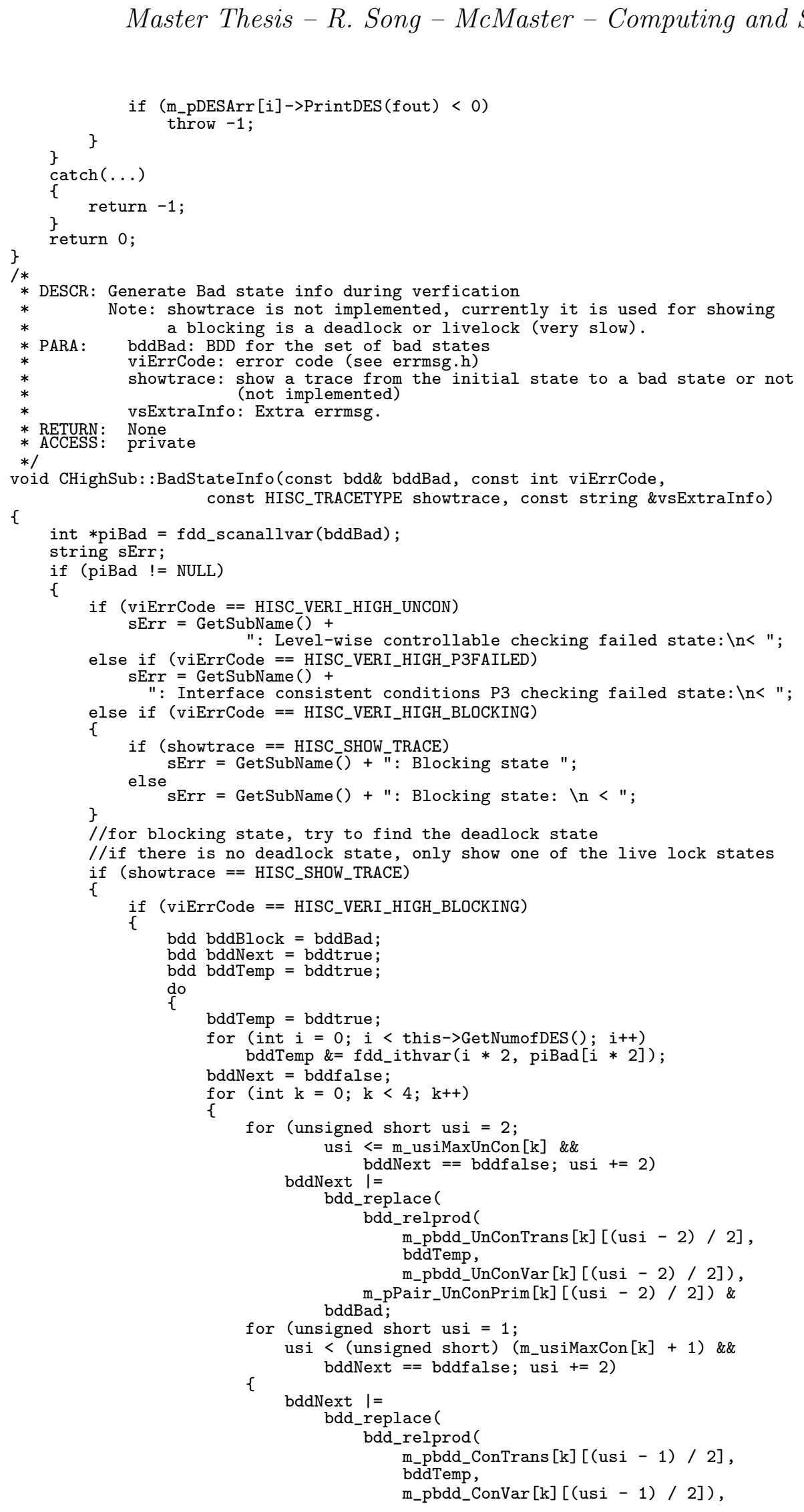


Master Thesis - R. Song - McMaster - Computing and Software

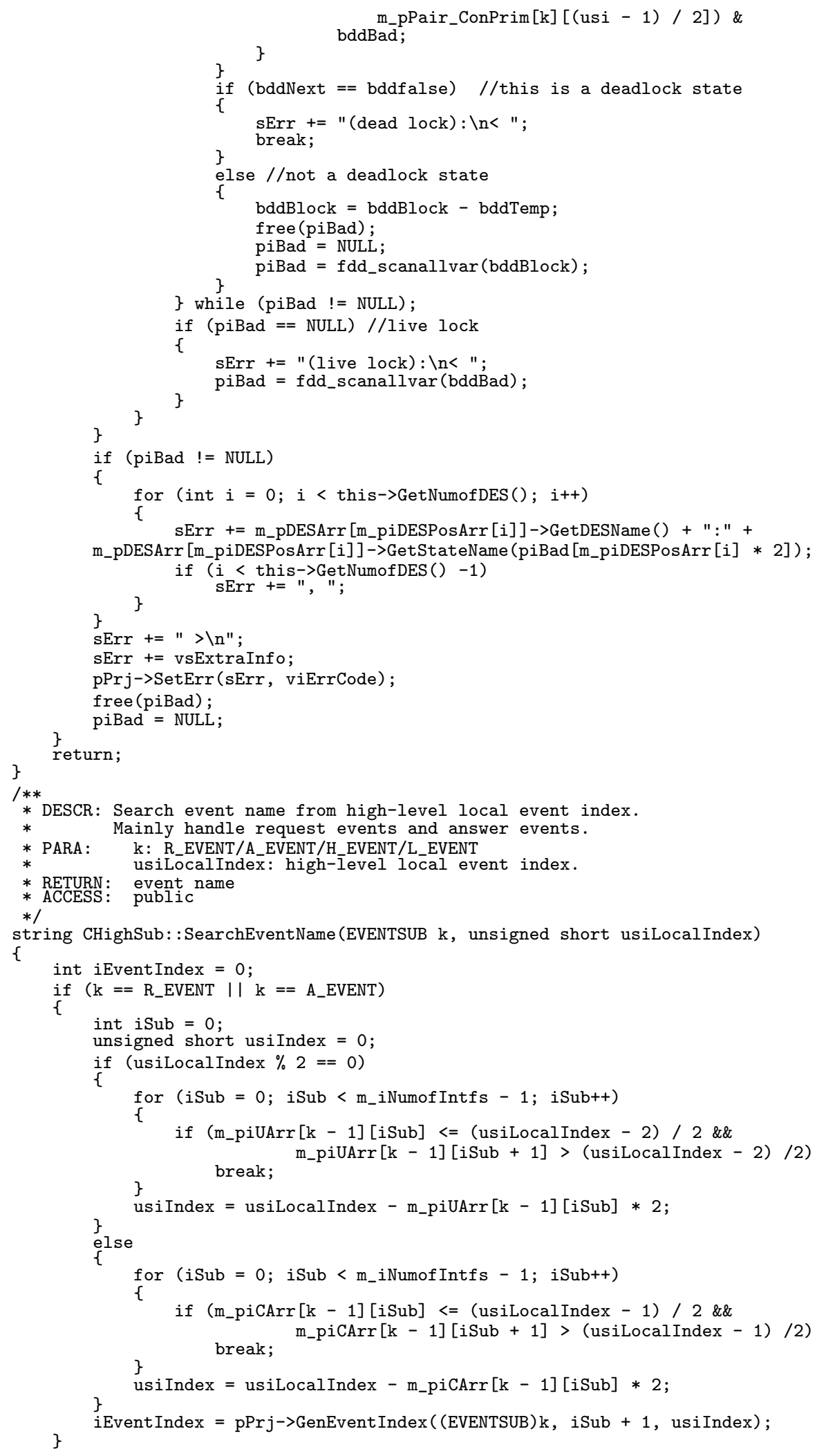




$$
\text { Master Thesis - R. Song - McMaster - Computing and Software }
$$

else

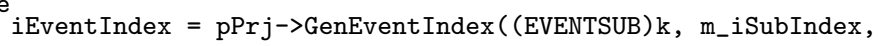

return (pPrj->GetInvAllEventsMap ()) [iEventIndex];

usiLocalIndex);

\} 


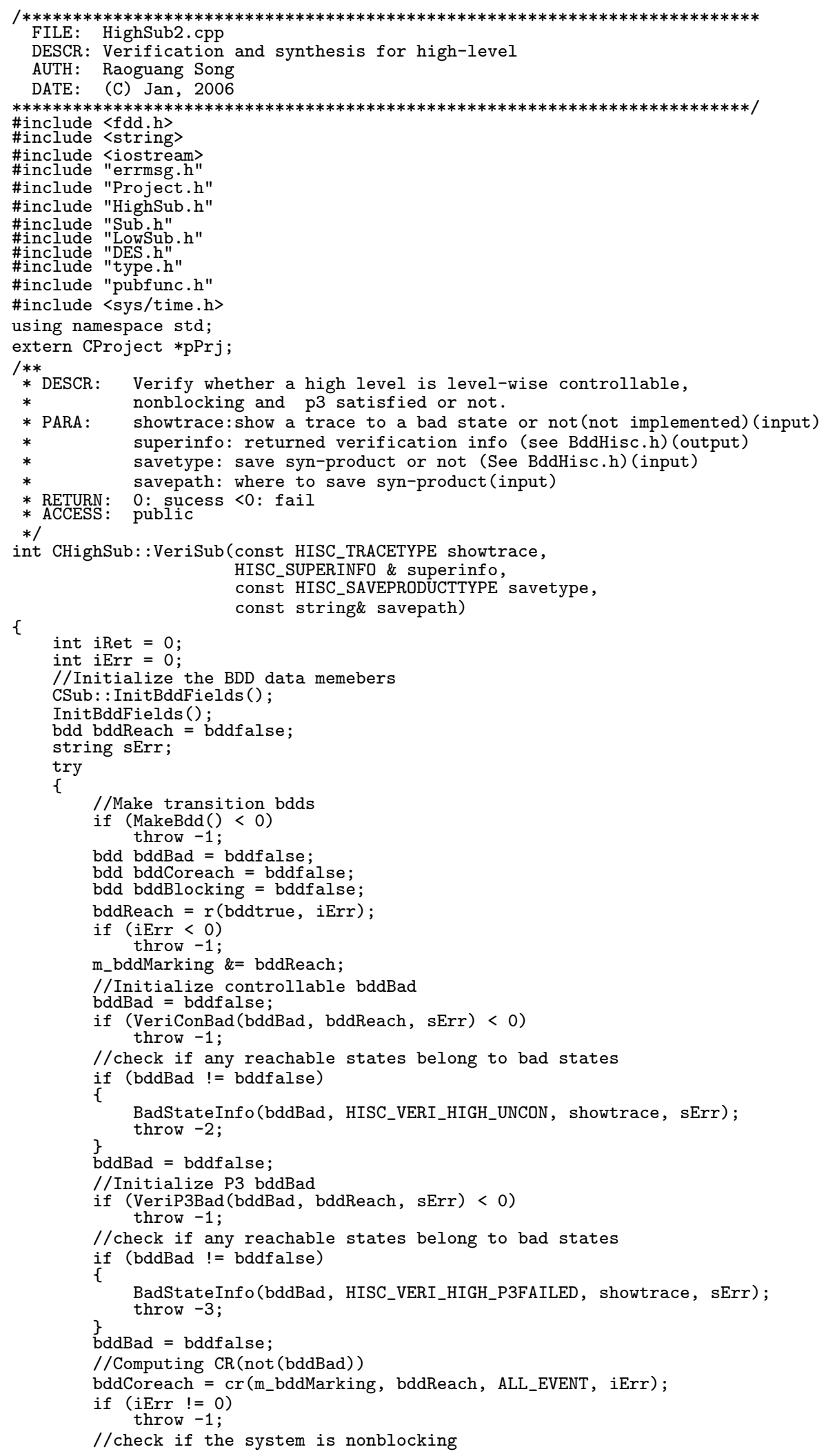




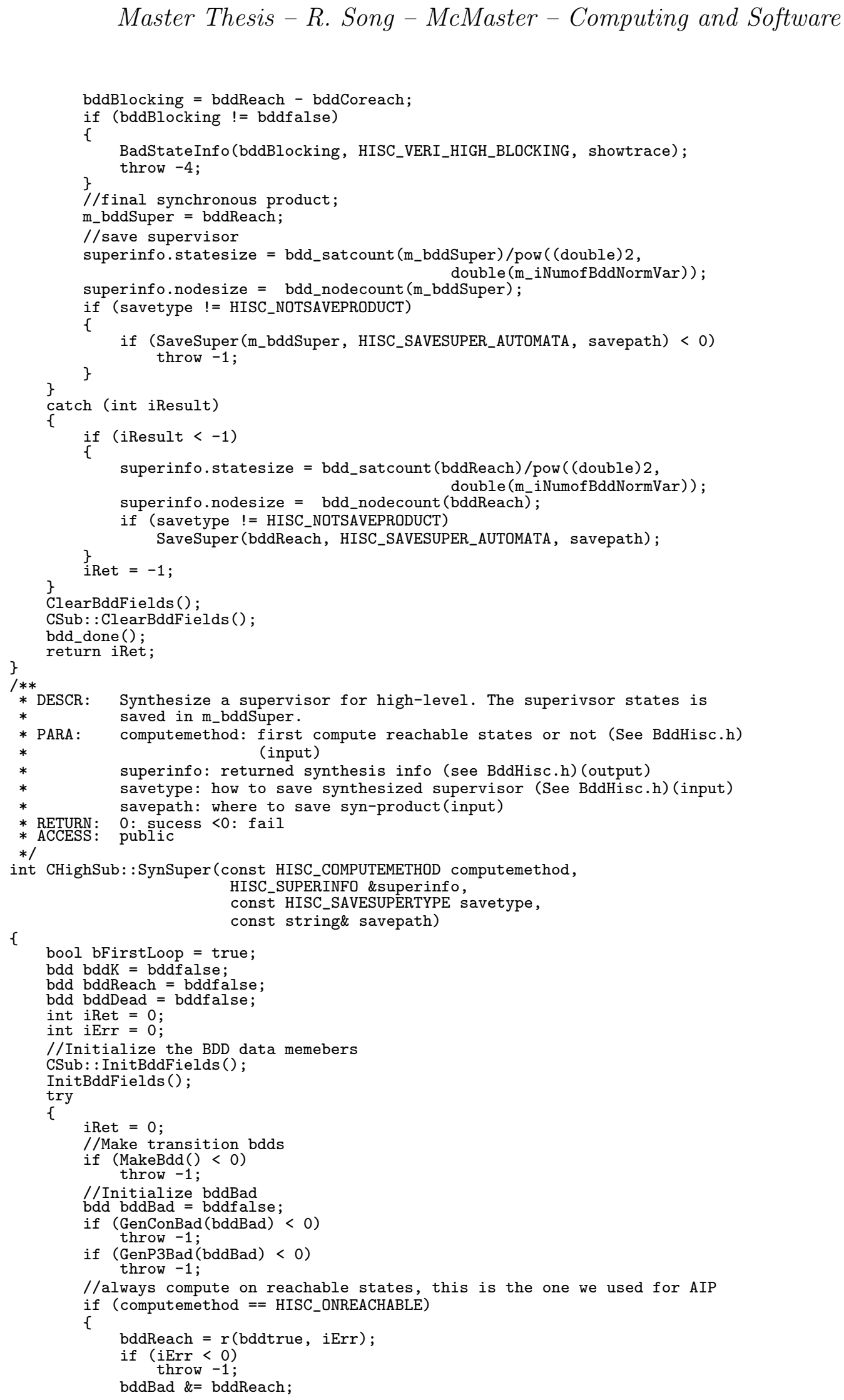


Master Thesis - R. Song - McMaster - Computing and Software

//compute controllable, p3, nonblocking fixpoint

do

bddK $=$ bddBad;

if $(\operatorname{supcp}($ bddBad $)<0)$

throw -1 ;

bddBad \& = bddReach;

if (bddK $==$ bddBad \&\& bFirstLoop $==$ false)

break;

//Computing $\mathrm{CR}()$

bdd bddTemp = bddReach - bddBad;

bddBad = bdd_not (cr (m_bddMarking, bddTemp, ALL_EVENT, iErr));

if (iErr $!=0$ )

$\begin{aligned} & \text { throw }-1 \text {; } \\ & \text { bddBad \& }=\text { bddReach; }\end{aligned}$

bFirstLoop = false;

\} while (bddBad != bddK);

$\mathrm{m}_{-}$bddSuper $=r($ bddReach - bddBad, iErr $)$;

\}

if $($ iErr $<0)$.

else //first compute on coreachable states, then do reachable operation

\{

//compute controllable, p3, nonblocking fixpoint

do

bddK = bddBad;

if $(\operatorname{supcp}(\mathrm{bddBad})<0)$

throw -1 ;

if (bddK $==$ bddBad \&\& bFirstLoop $==$ false)

break;

//Computing CR(not(bddBad))

bddBad = bdd_not (cr (m_bddMarking, bdd_not (bddBad),

if (iErr $!=0$ )

throw -1 ;

bFirstLoop = false;

\} while (bddBad != bddK);

m_bddSuper $=r($ bdd_not $($ bddBad $)$, iErr $)$;

if $(i \operatorname{Err}<0)$

\}

throw -1

m_bddMarking \& = m_bddSuper;

m_bddInit \& = m_bddSuper;

\#ifdef PRINTDEBUG

PrintBdd ();

\#endif

//save supervisor

superinfo.statesize $=$ bdd_satcount $\left(\mathrm{m}_{-}\right.$bddSuper $) /$pow $(($double $) 2$,

double (m iNumofBddNormVar)):

superinfo.nodesize $=$ bdd_nodecount (m_bddSuper);

if (savetype != HISC_SAVESUPER_NONE)

\{

if (SaveSuper (m_bddSuper, savetype, savepath) $<0$ )

\}

\} throw -1 ;

catch ( int iErr)

\}

iRet $=-1$;

ClearBddFields ();

CSub: : ClearBddFields ();

bdd_done();

\}

return iRet;

**

* DESCR: Compute the initial bad states (Bad_H) (uncontorlalble event part)

* PARA: bddConBad: BDD containing all the bad states (output)

* RETURN: 0: sucess -1 : fail

* ACCESS: private

$* /$

int CHighSub: :GenConBad(bdd \&bddConBad)

\{

$\operatorname{try}$

bdd bddPlantTrans = bddfalse;

bdd bddSpecTrans = bddfalse;

for (int $\mathrm{k}=0 ; \mathrm{k}<3 ; \mathrm{k}++$ ) 
Master Thesis - R. Song - McMaster - Computing and Software

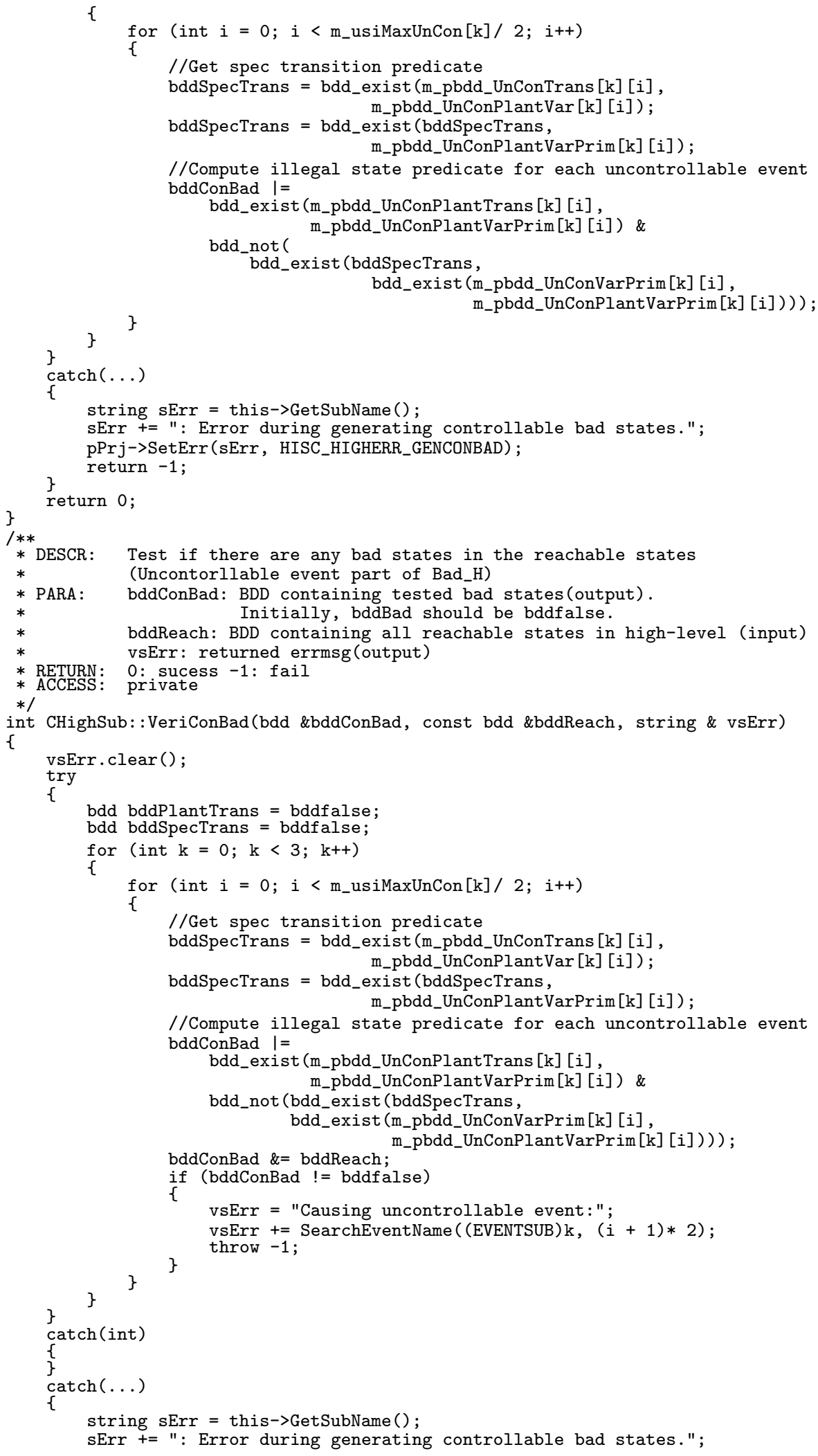




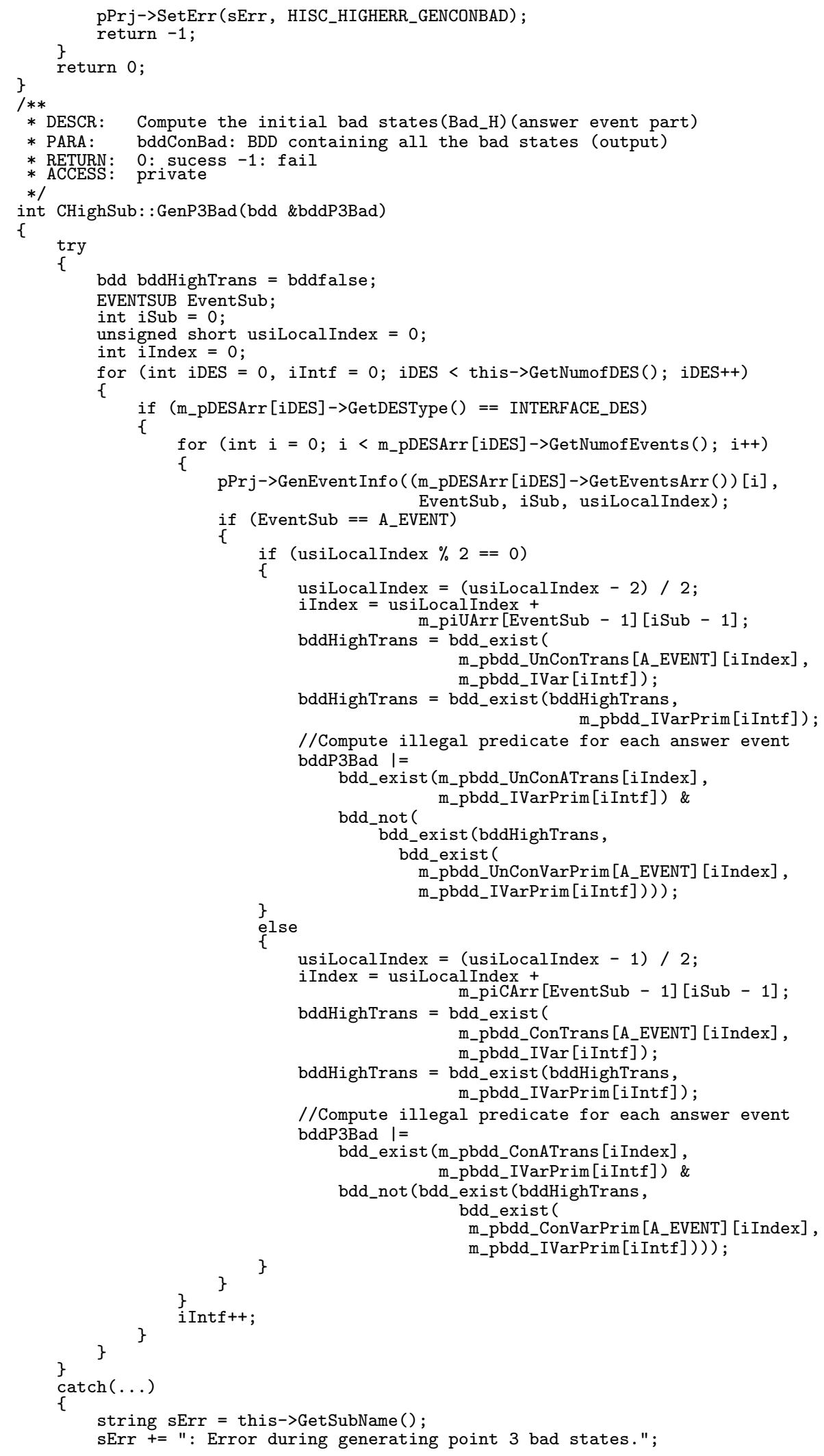




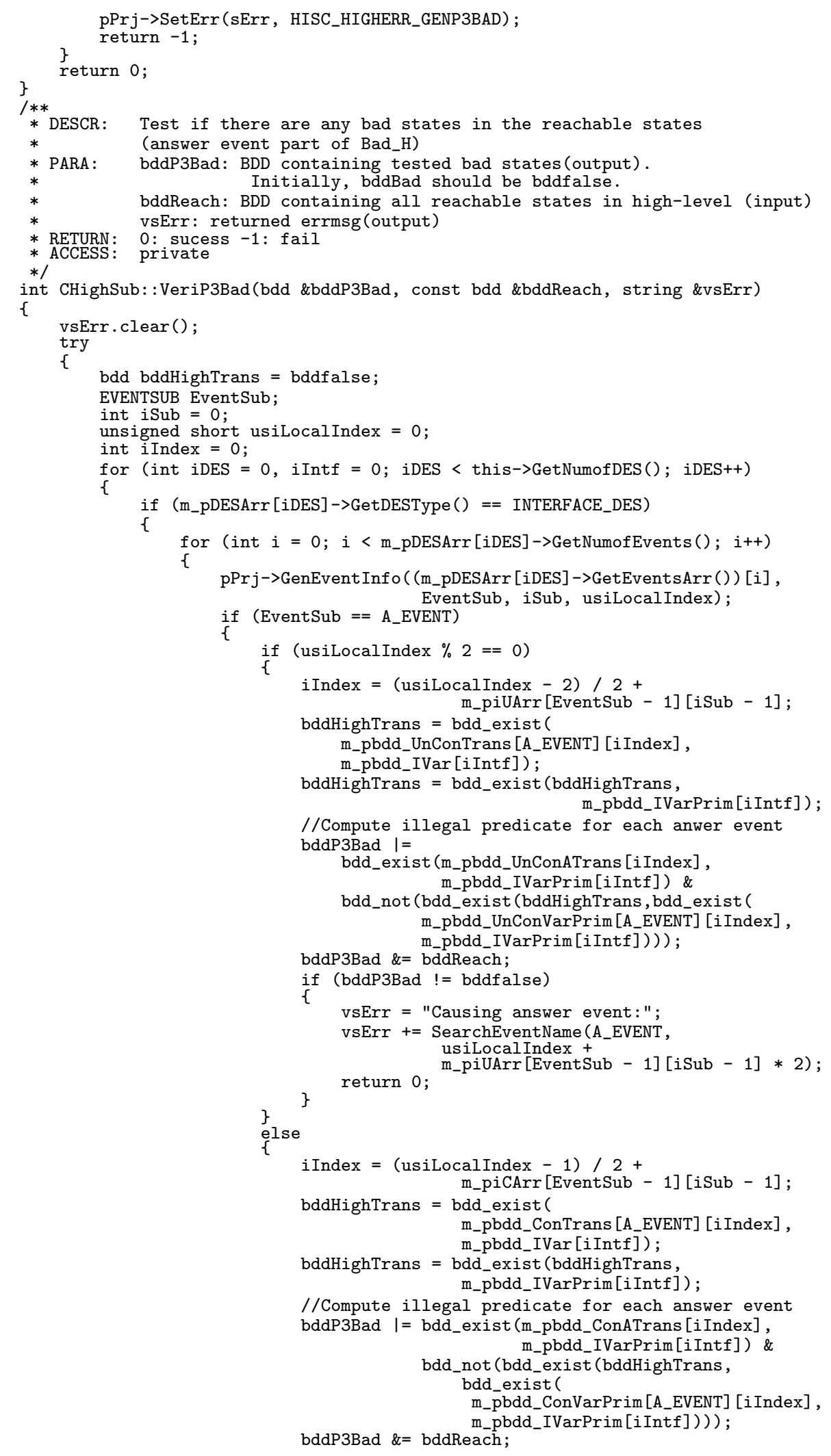


Master Thesis - R. Song - McMaster - Computing and Software

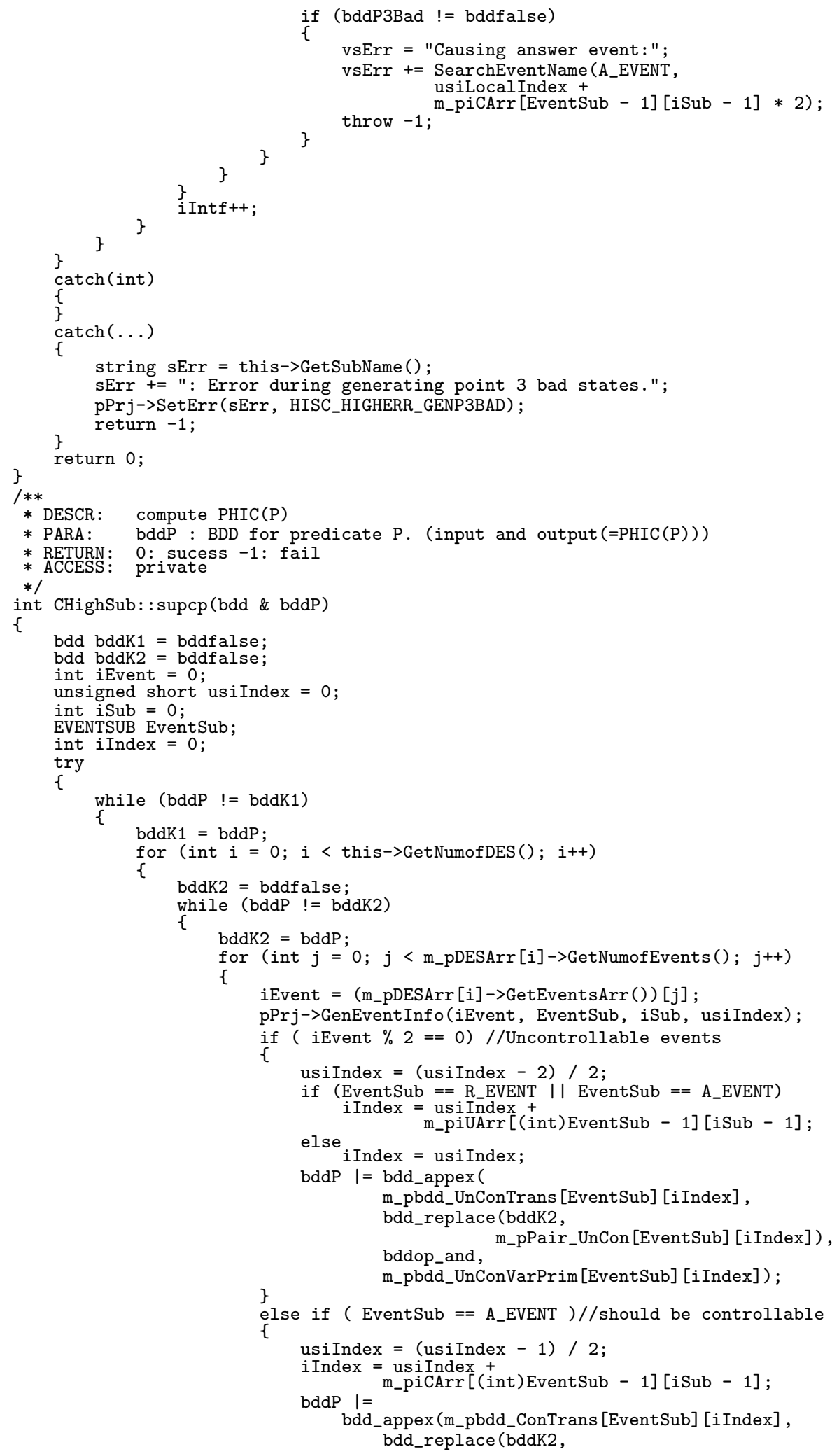




\section{Master Thesis - R. Song - McMaster - Computing and Software}

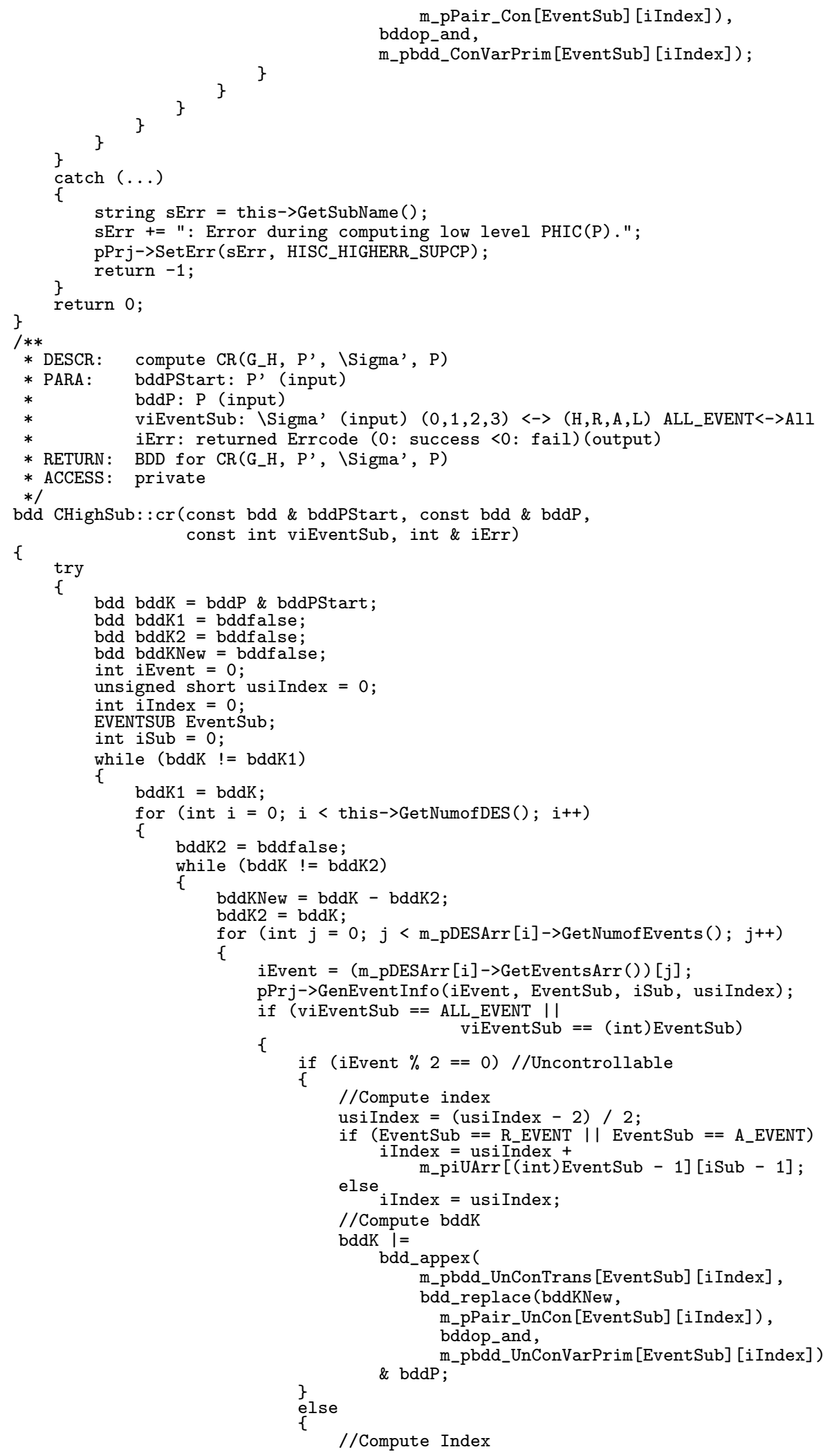


Master Thesis - R. Song - McMaster - Computing and Software

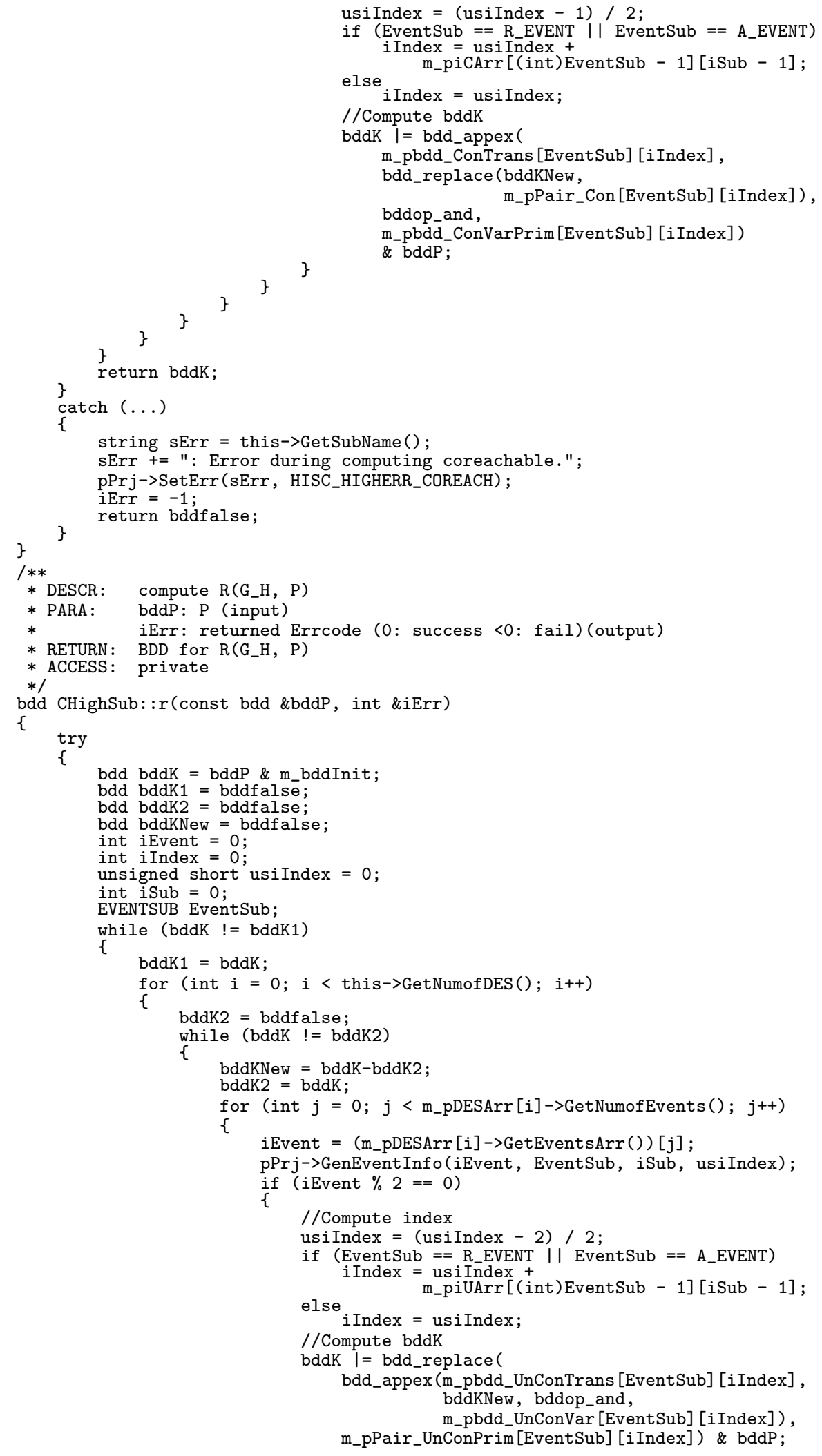




\section{Master Thesis - R. Song - McMaster - Computing and Software}

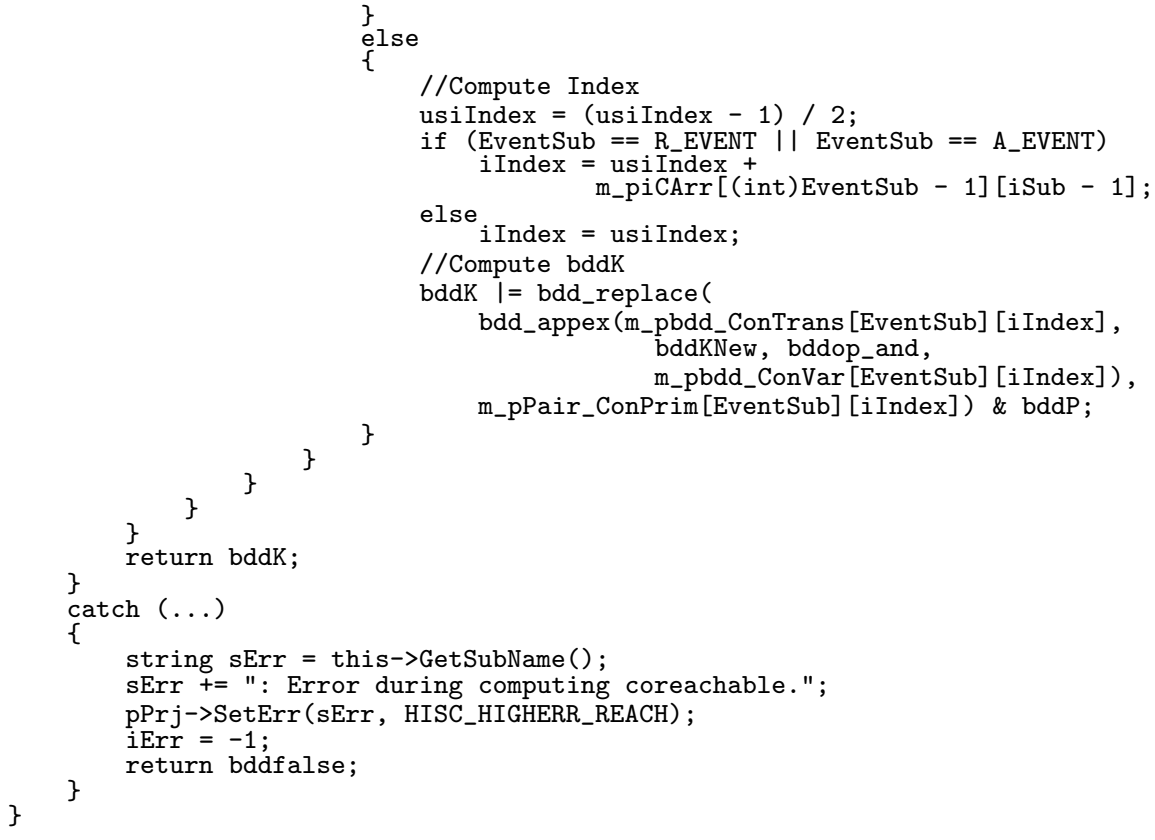




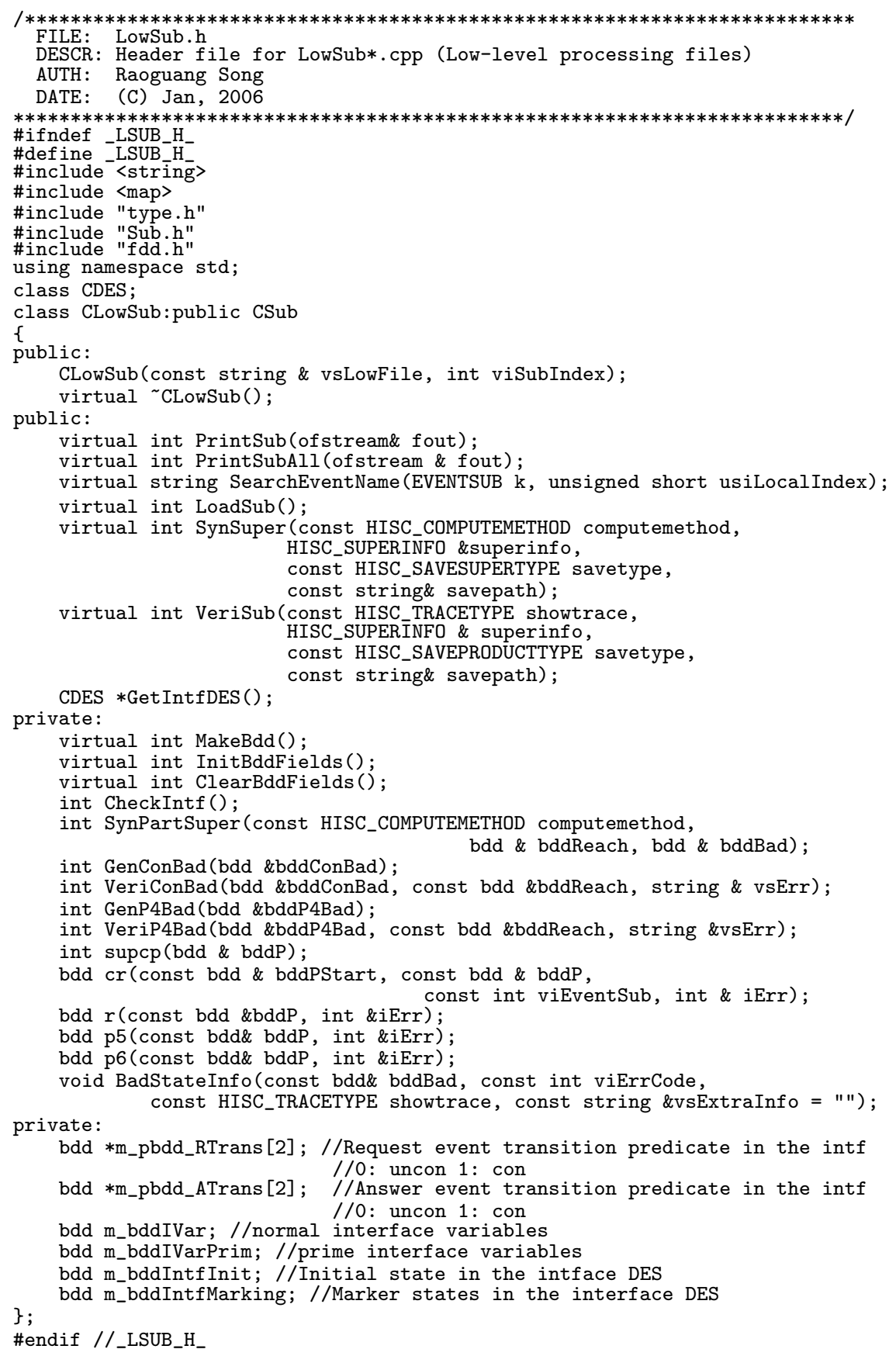




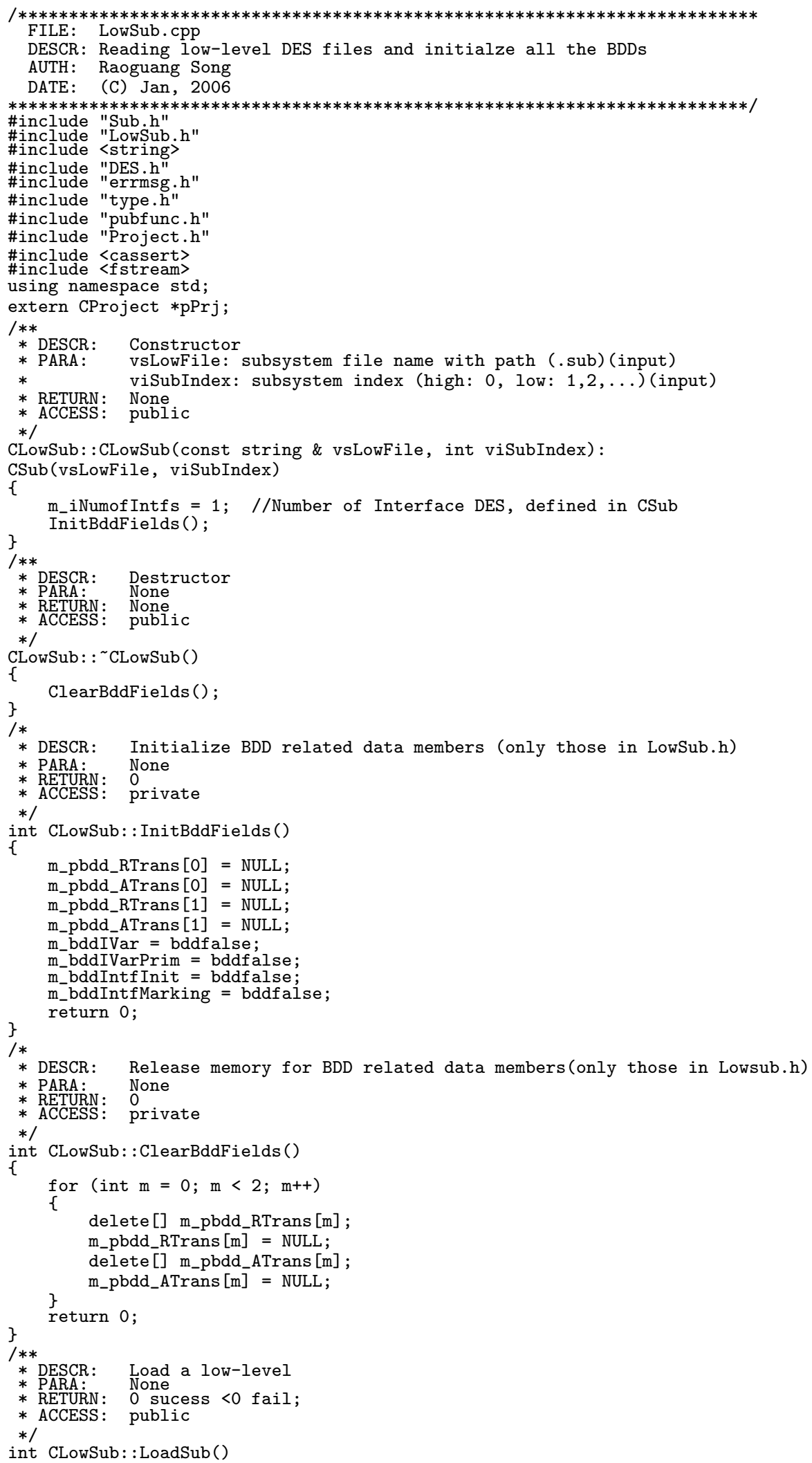




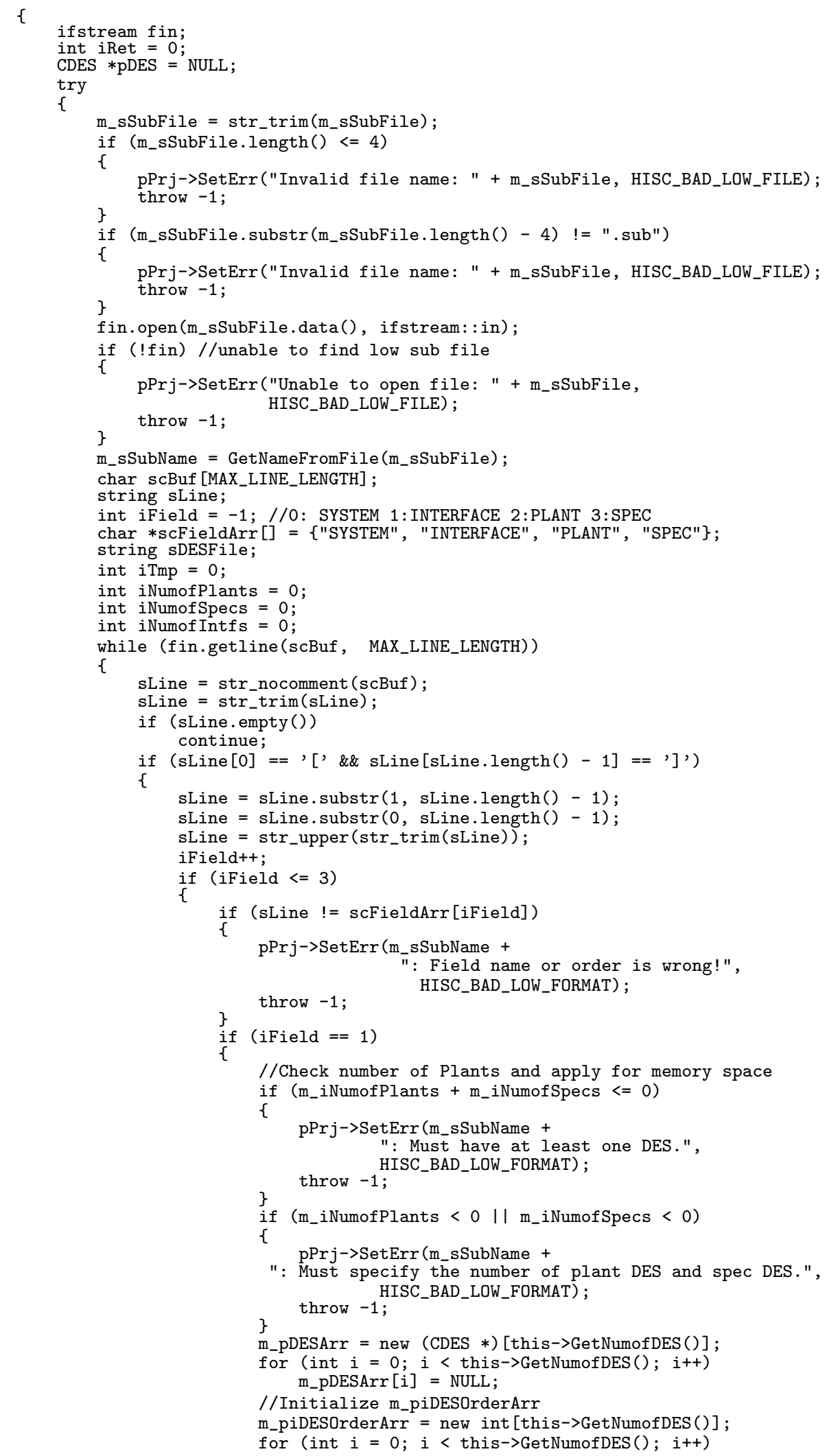




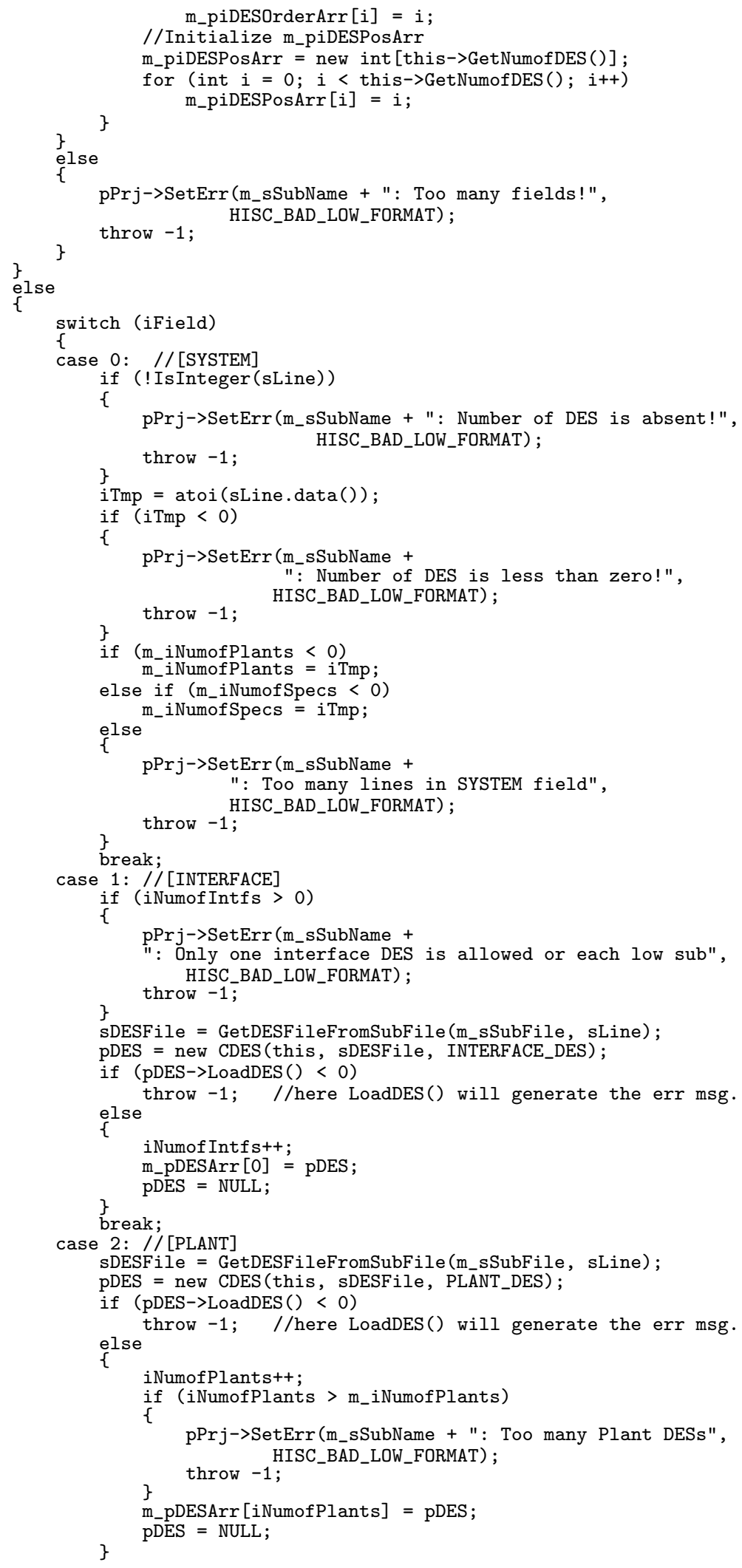


Master Thesis - R. Song - McMaster - Computing and Software

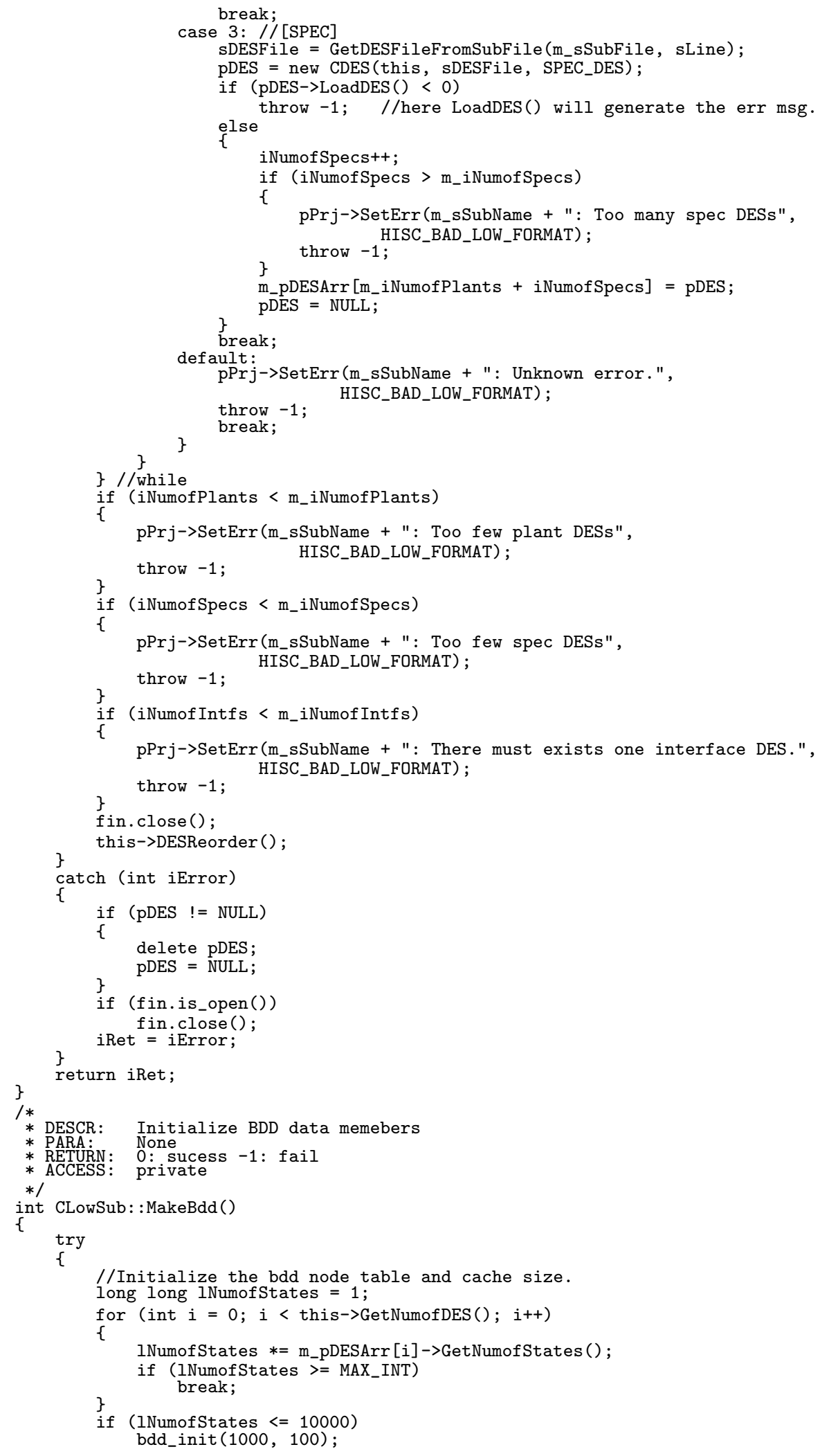




\section{Master Thesis - R. Song - McMaster - Computing and Software}

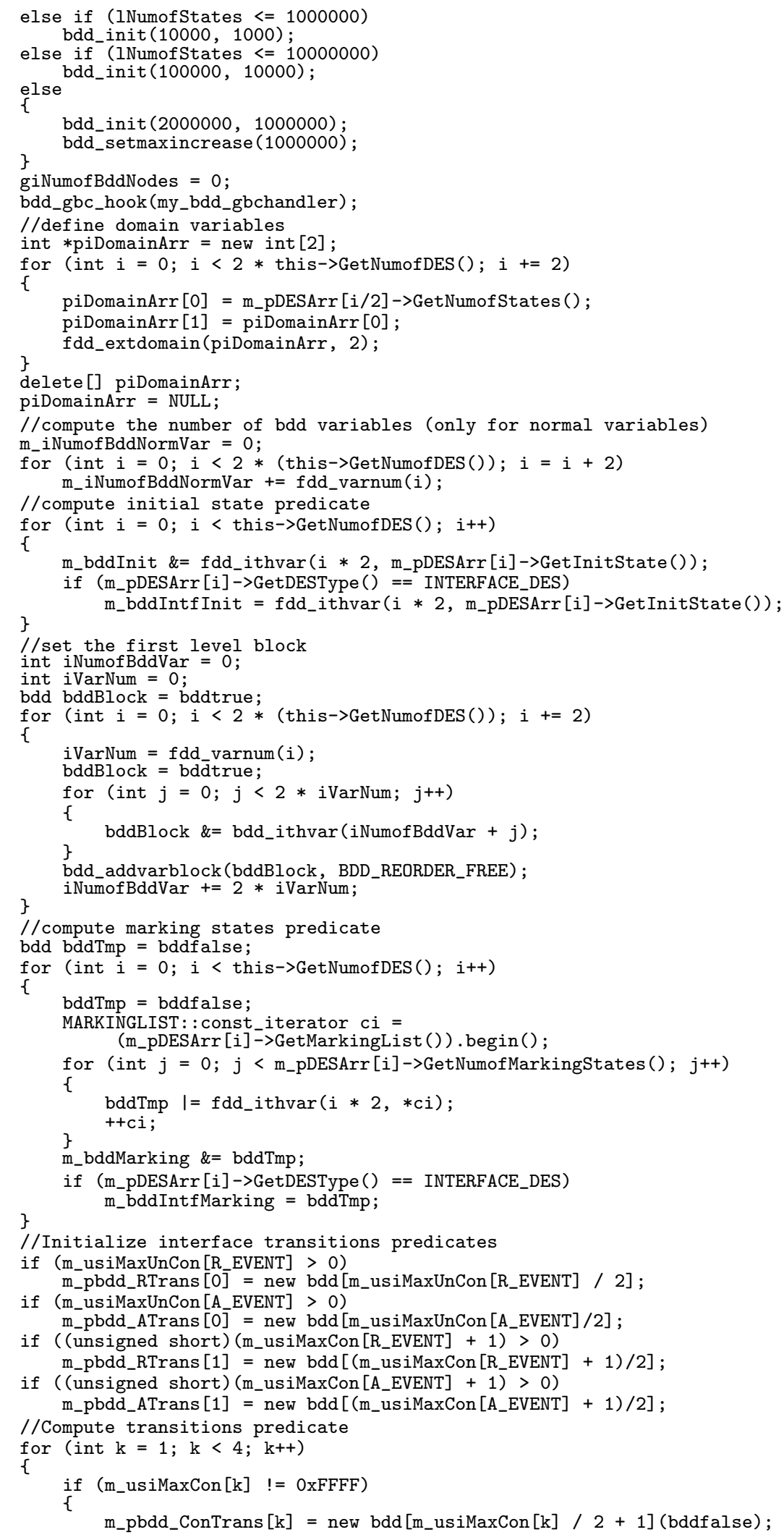


Master Thesis - R. Song - McMaster - Computing and Software

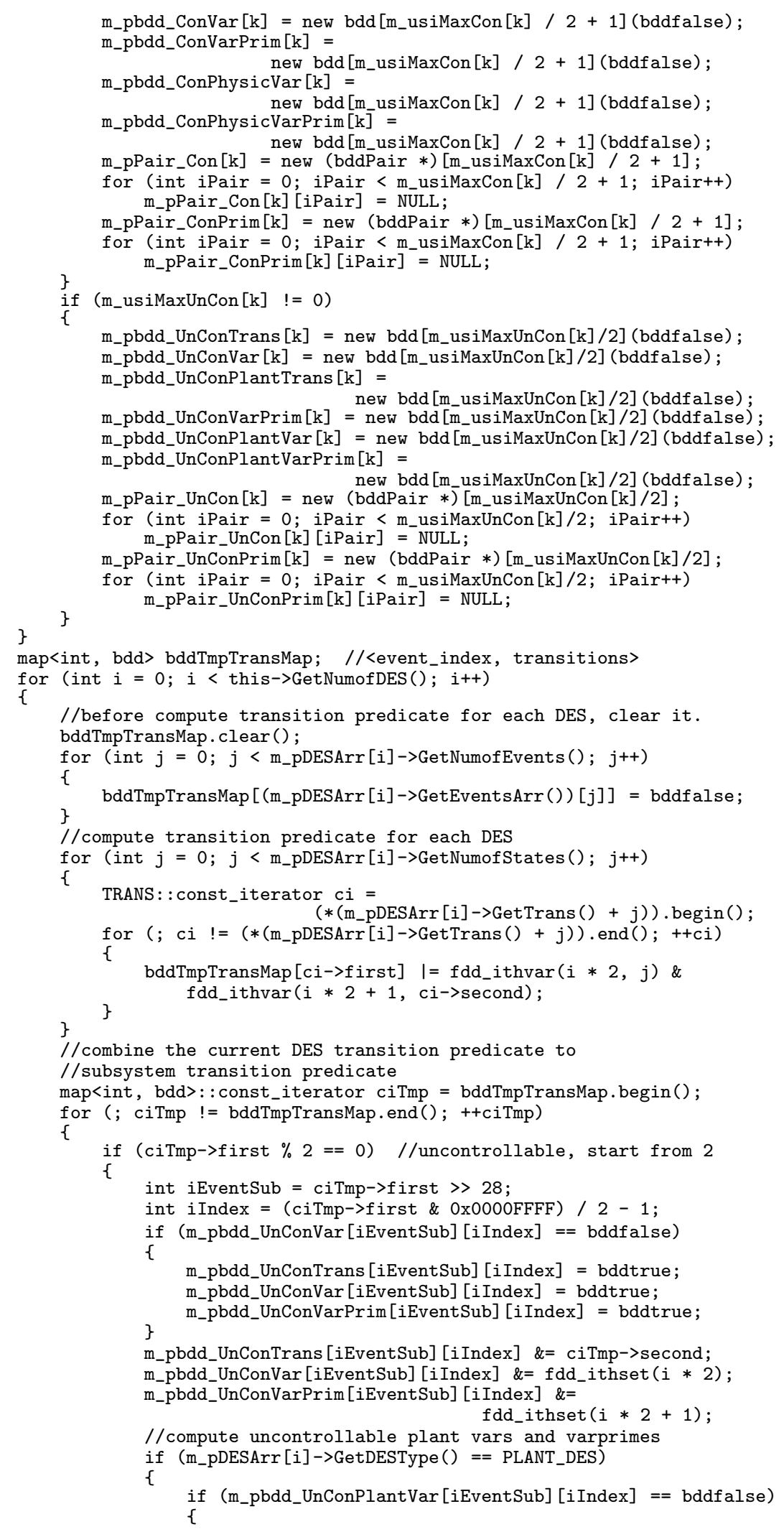


Master Thesis - R. Song - McMaster - Computing and Software

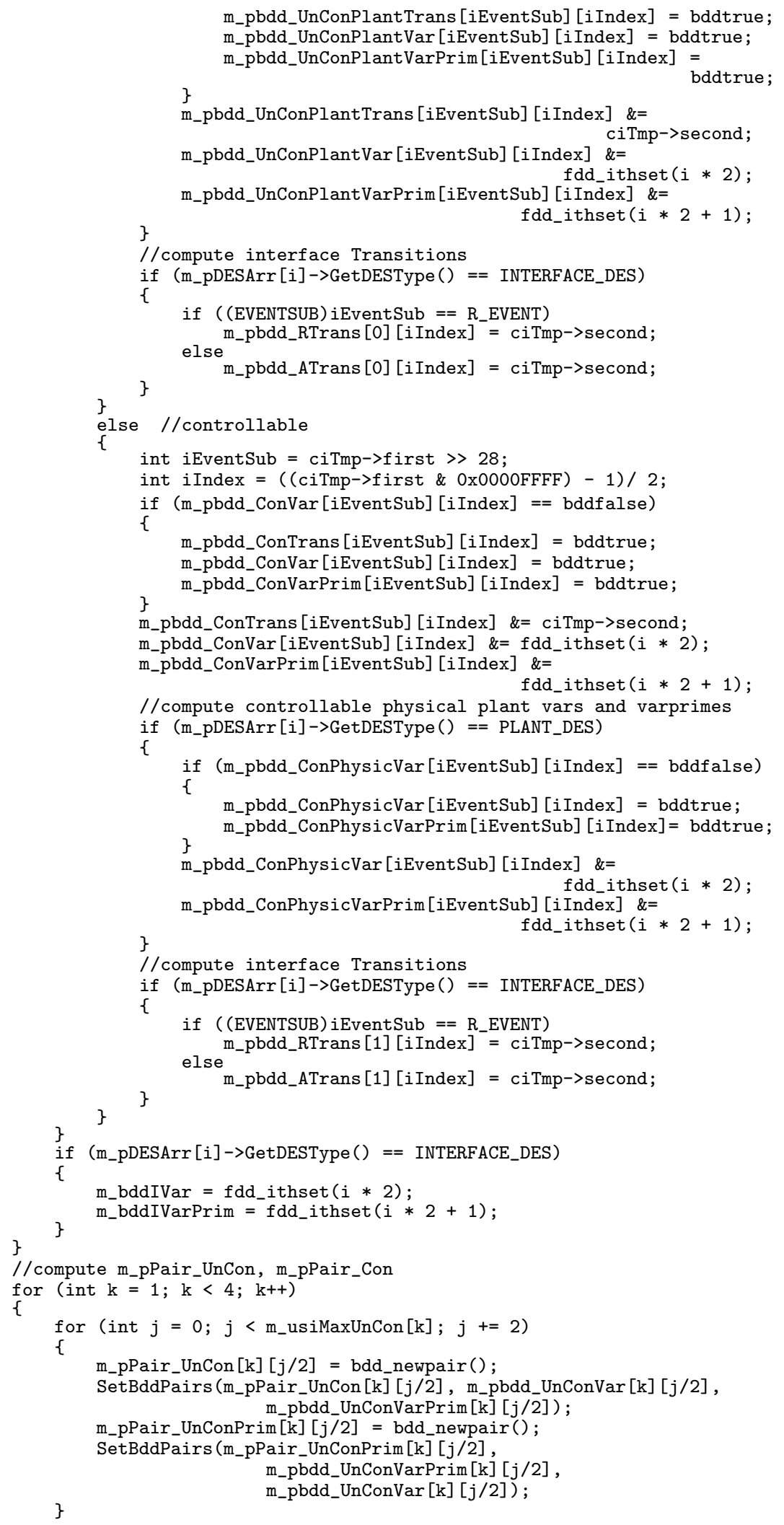




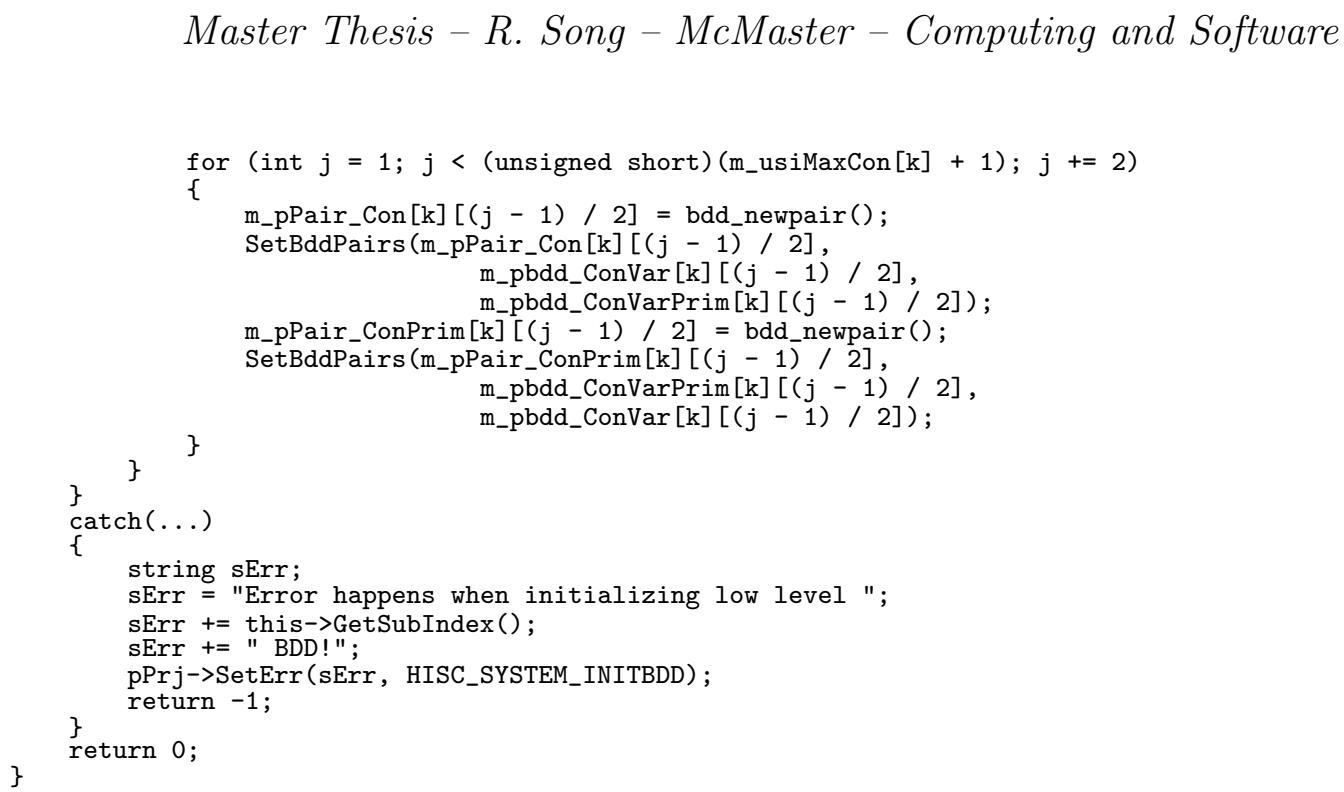




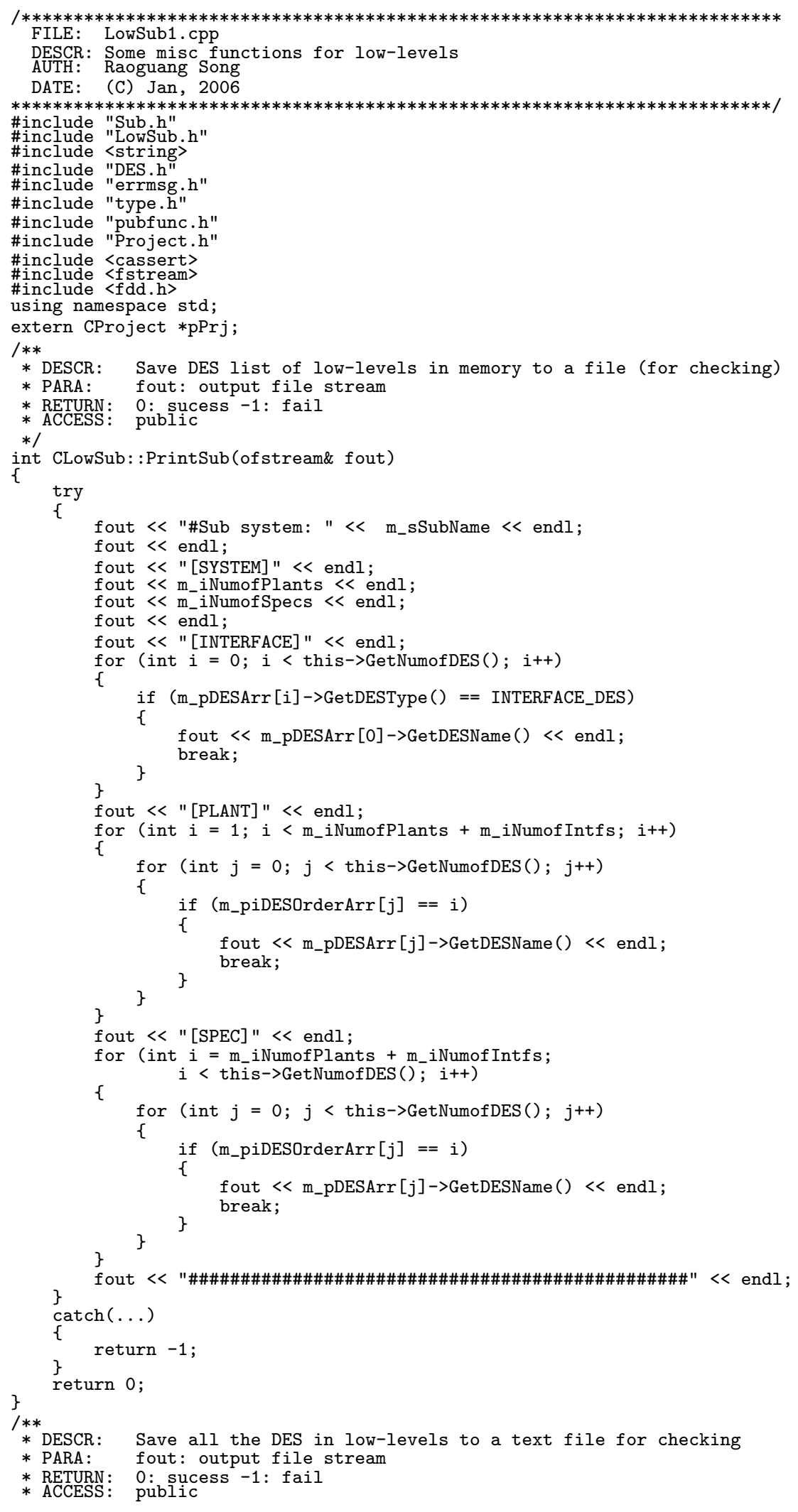




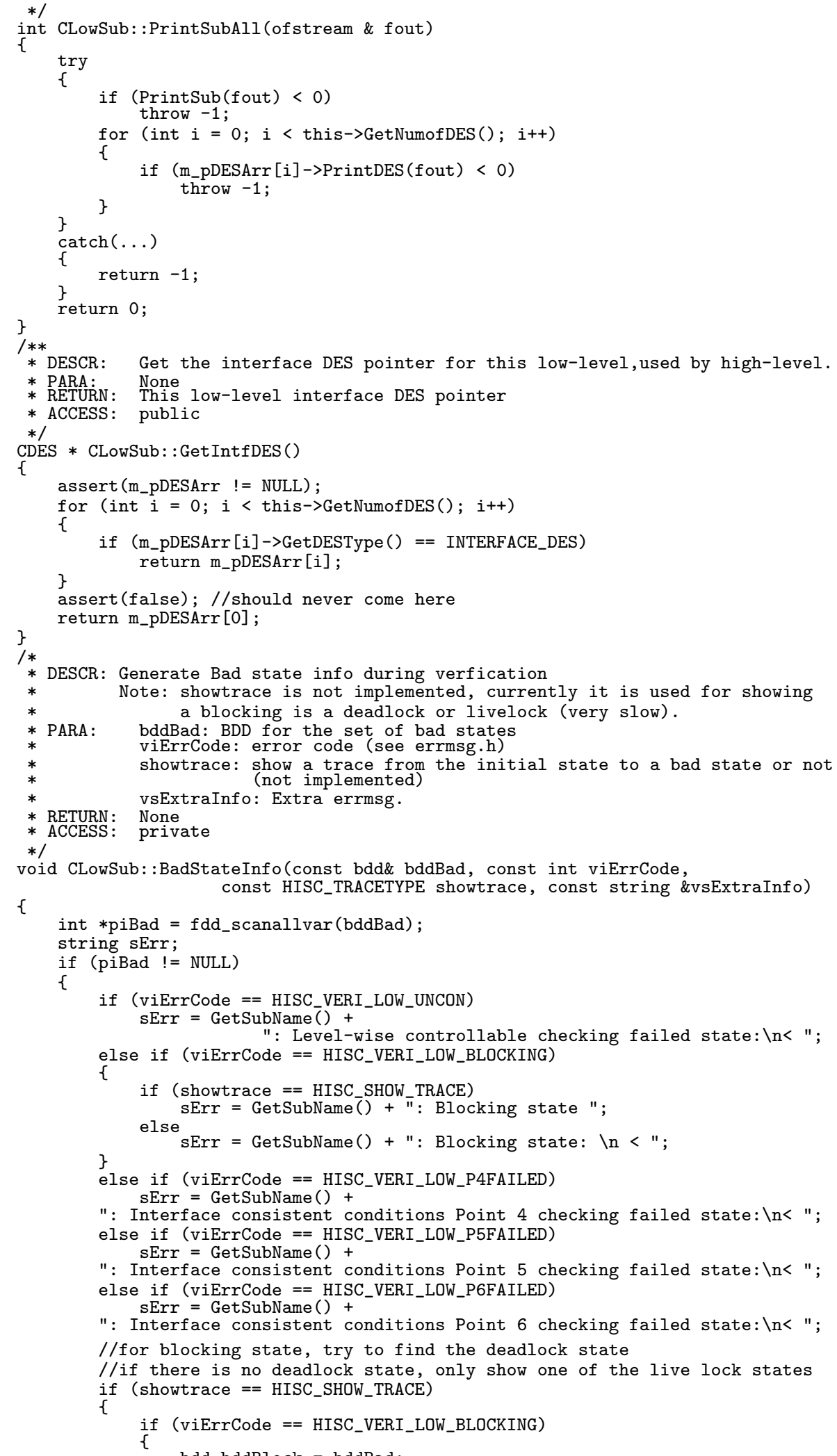




\section{Master Thesis - R. Song-McMaster - Computing and Software}

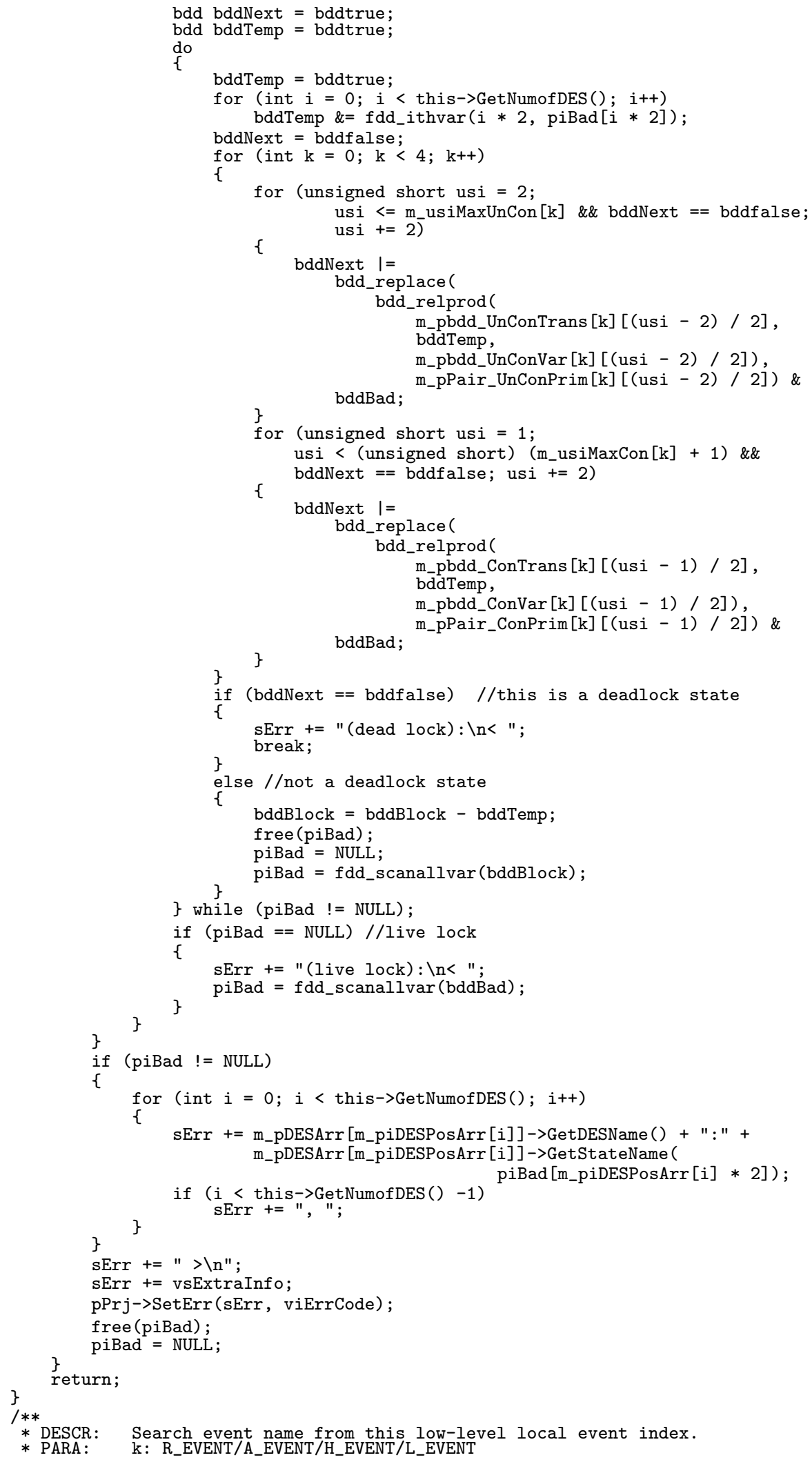


Master Thesis - R. Song - McMaster - Computing and Software

usiLocalIndex: this low-level local event index.

* RETURN: usiLocalinder

* ACCESS: public

*/

string CLowSub: :SearchEventName(EVENTSUB k, unsigned short usiLocalIndex)

\{

int iEventIndex $=0$;

iEventIndex $=\operatorname{pPrj}->\operatorname{GenEventIndex}\left((\right.$ EVENTSUB $) \mathrm{k}, \mathrm{m} \_i \operatorname{SubIndex}$, usiLocalIndex $)$;

\} return (pPrj->GetInvAllEventsMap ()) [iEventIndex]; 


\section{Master Thesis - R. Song-McMaster - Computing and Software}

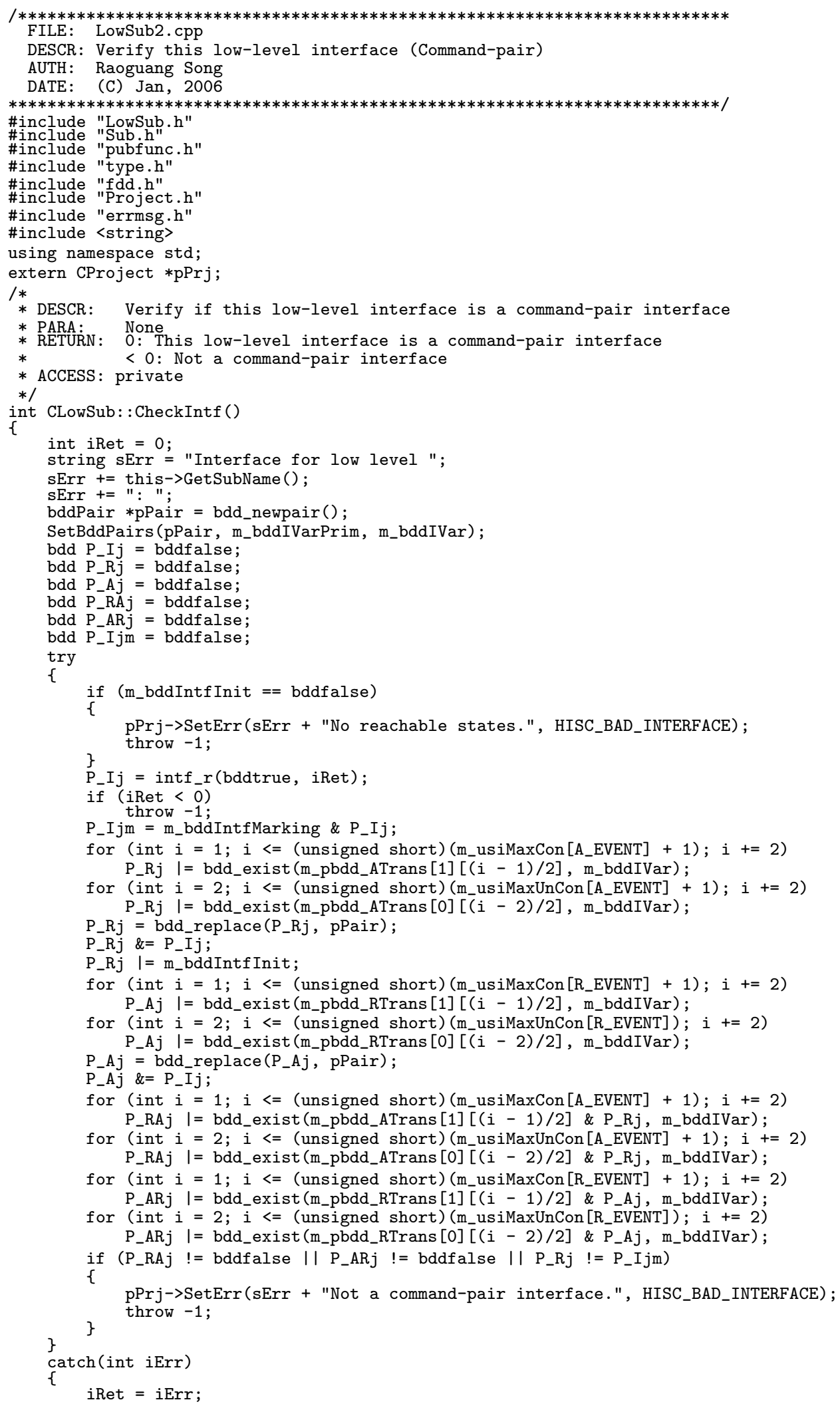




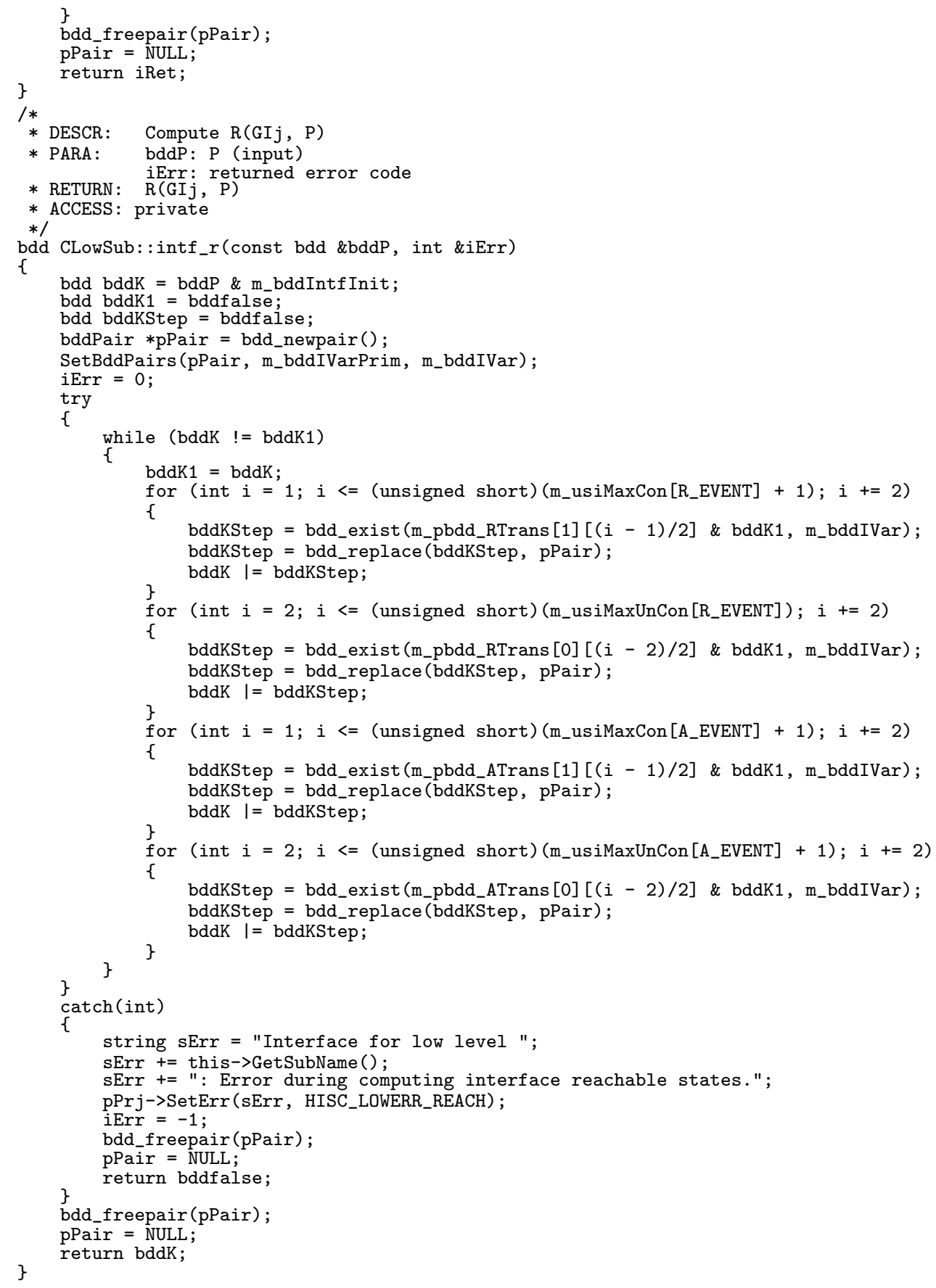




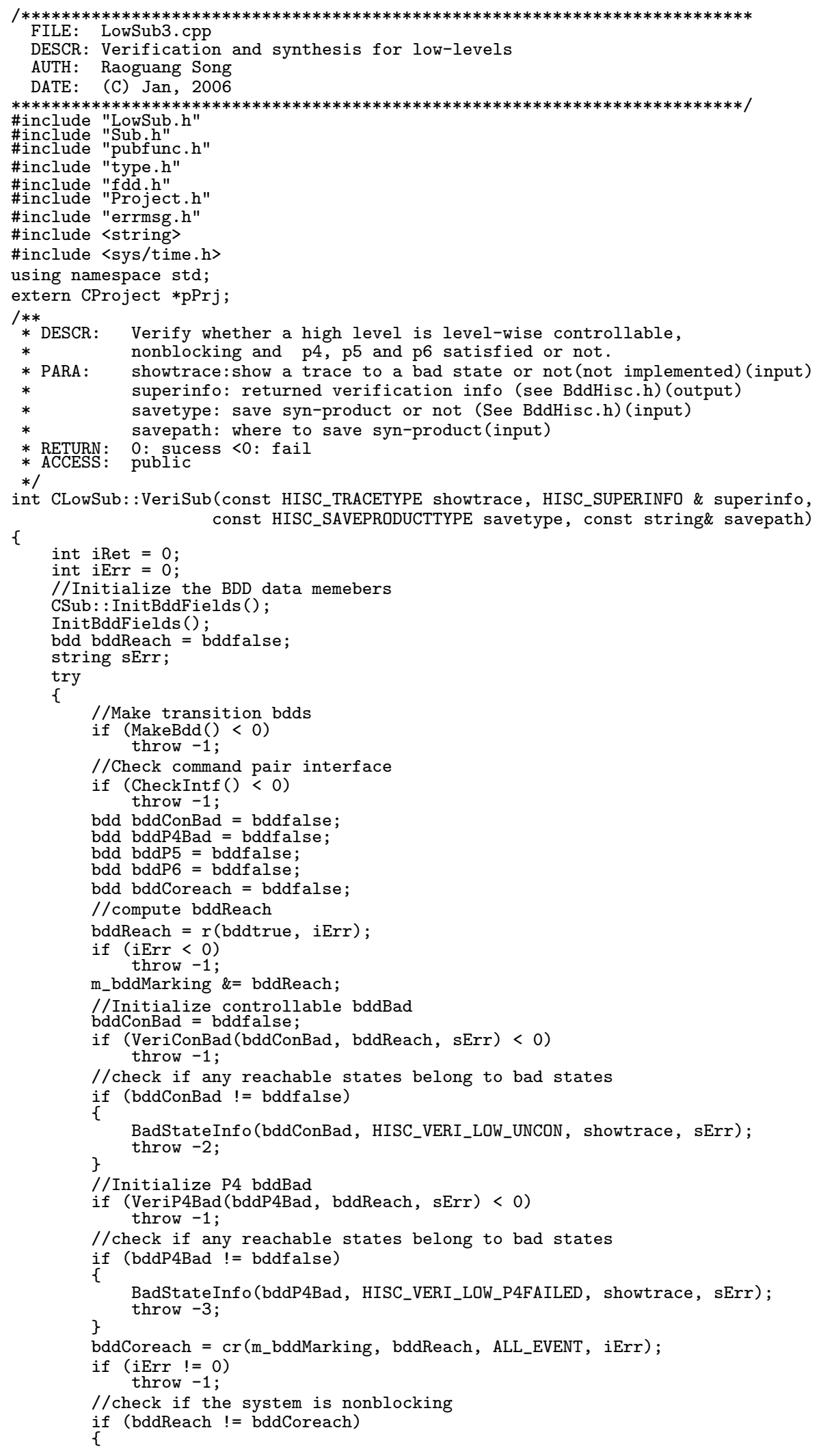




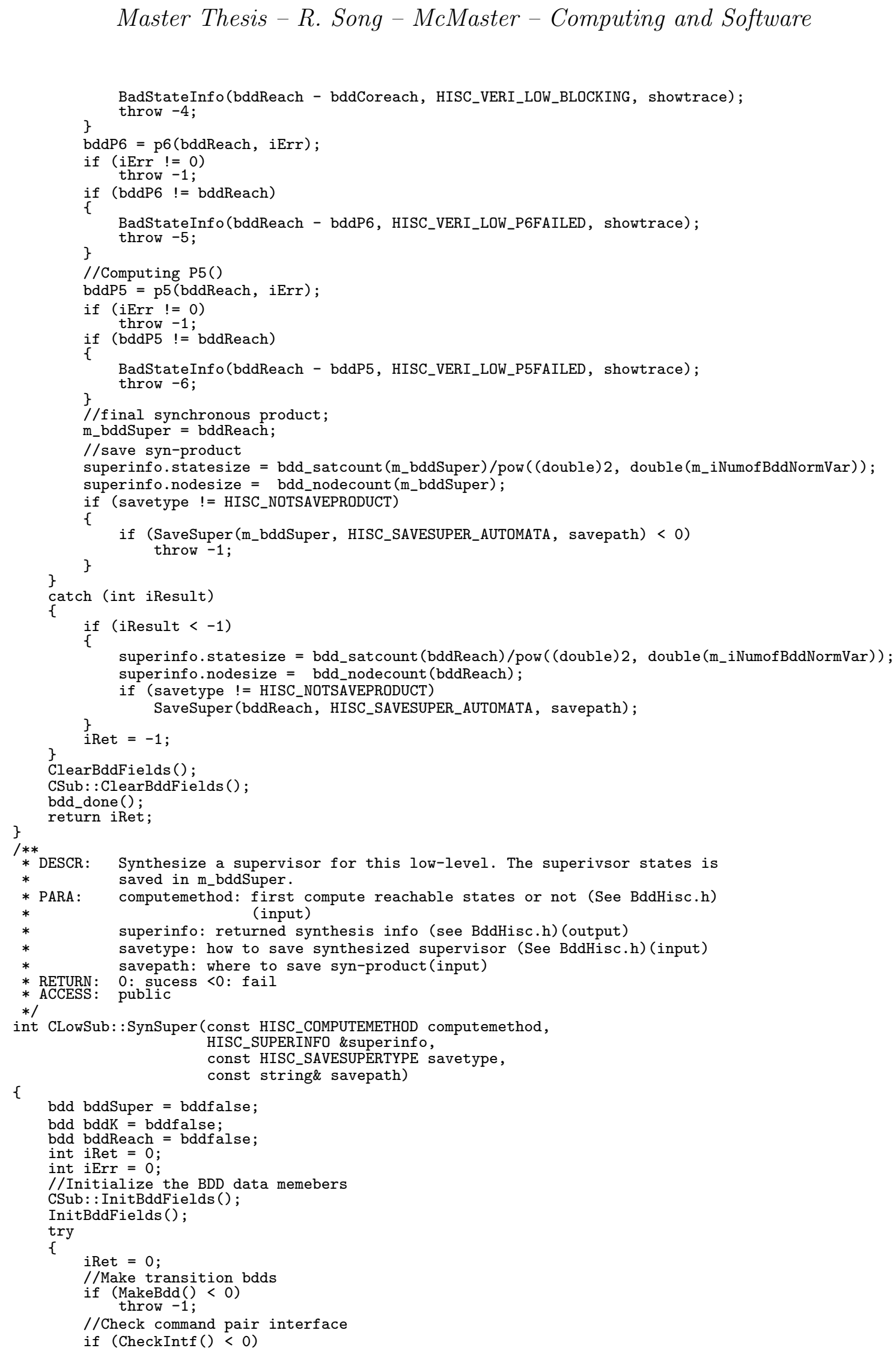




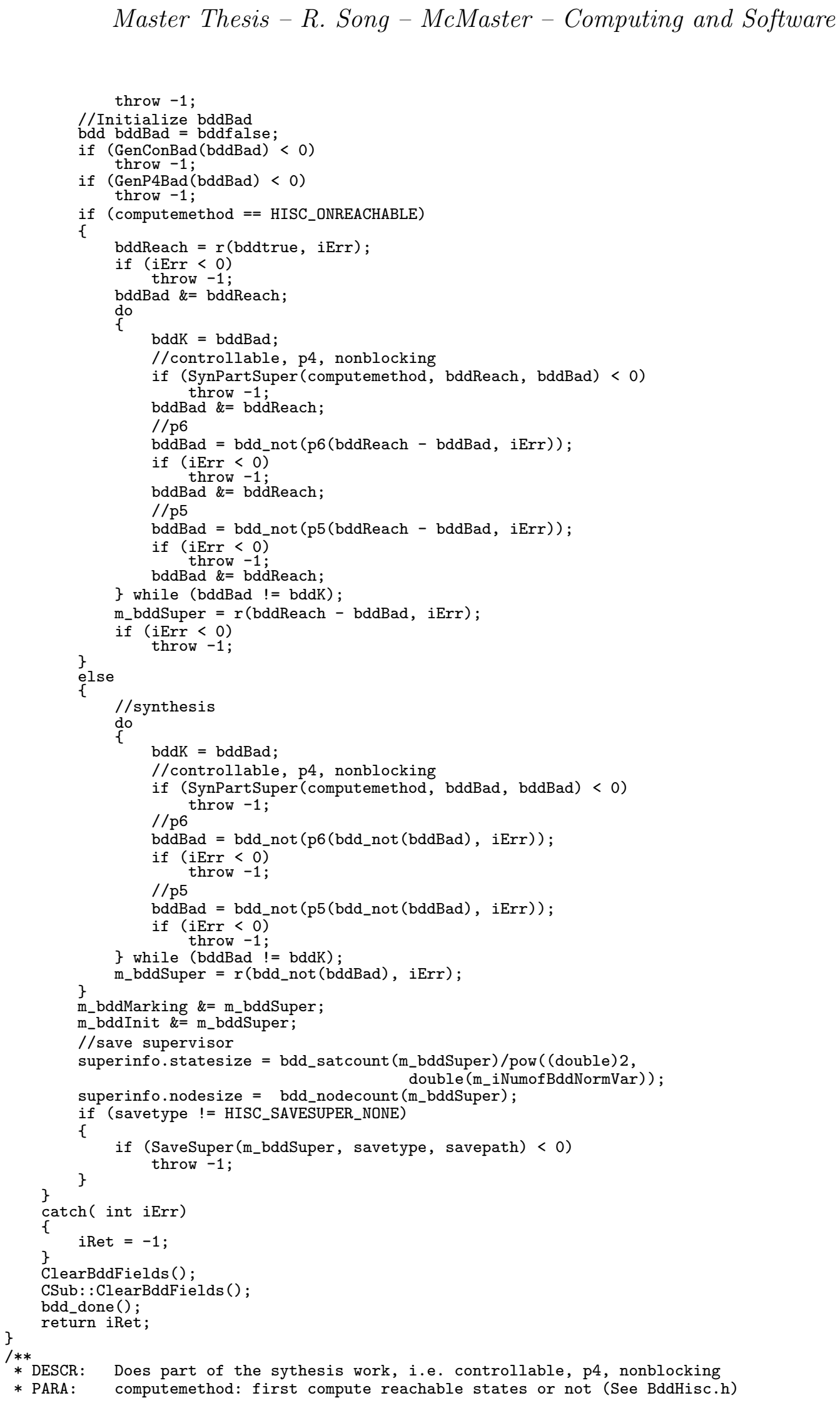




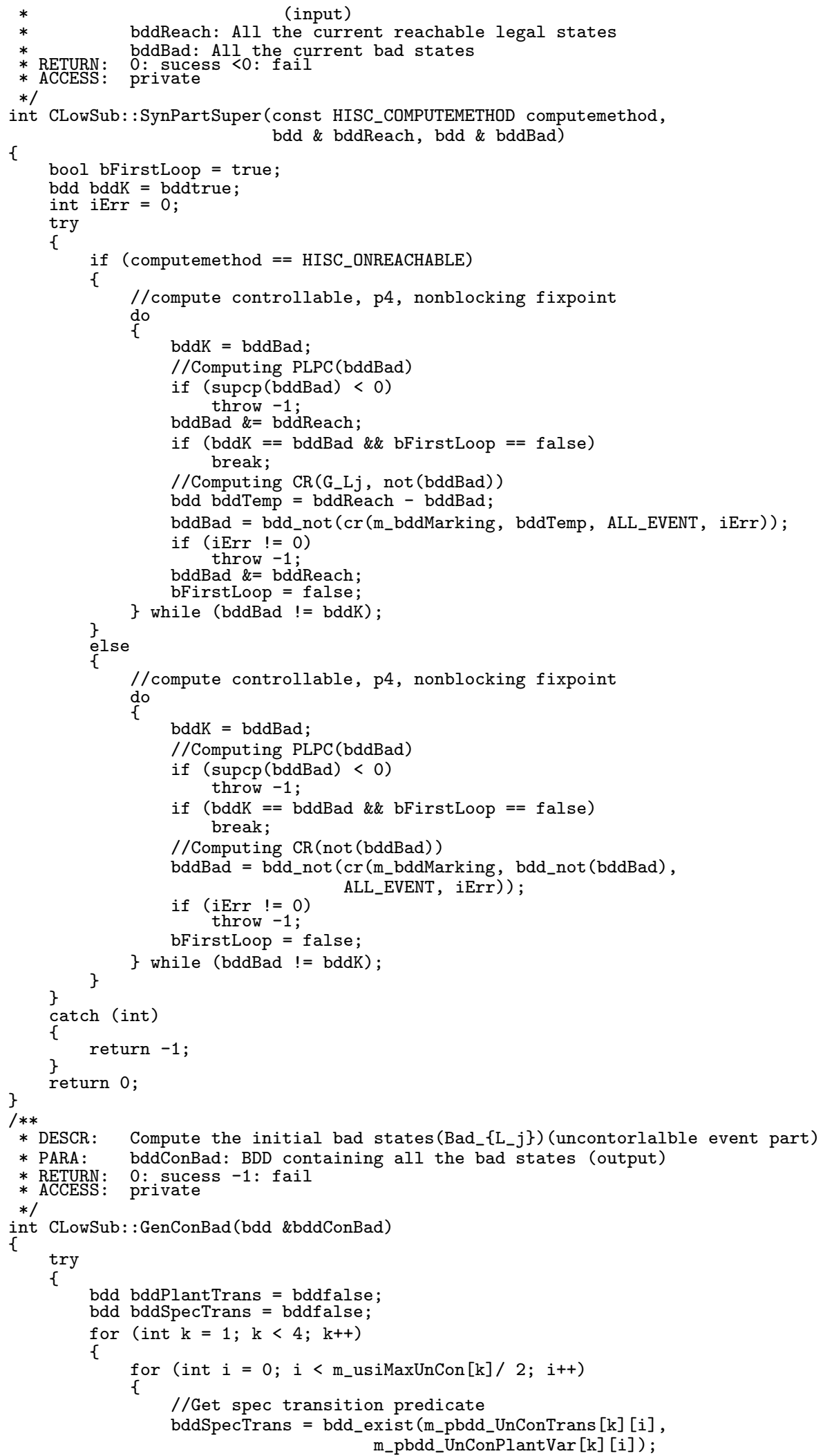




\section{Master Thesis - R. Song - McMaster - Computing and Software}

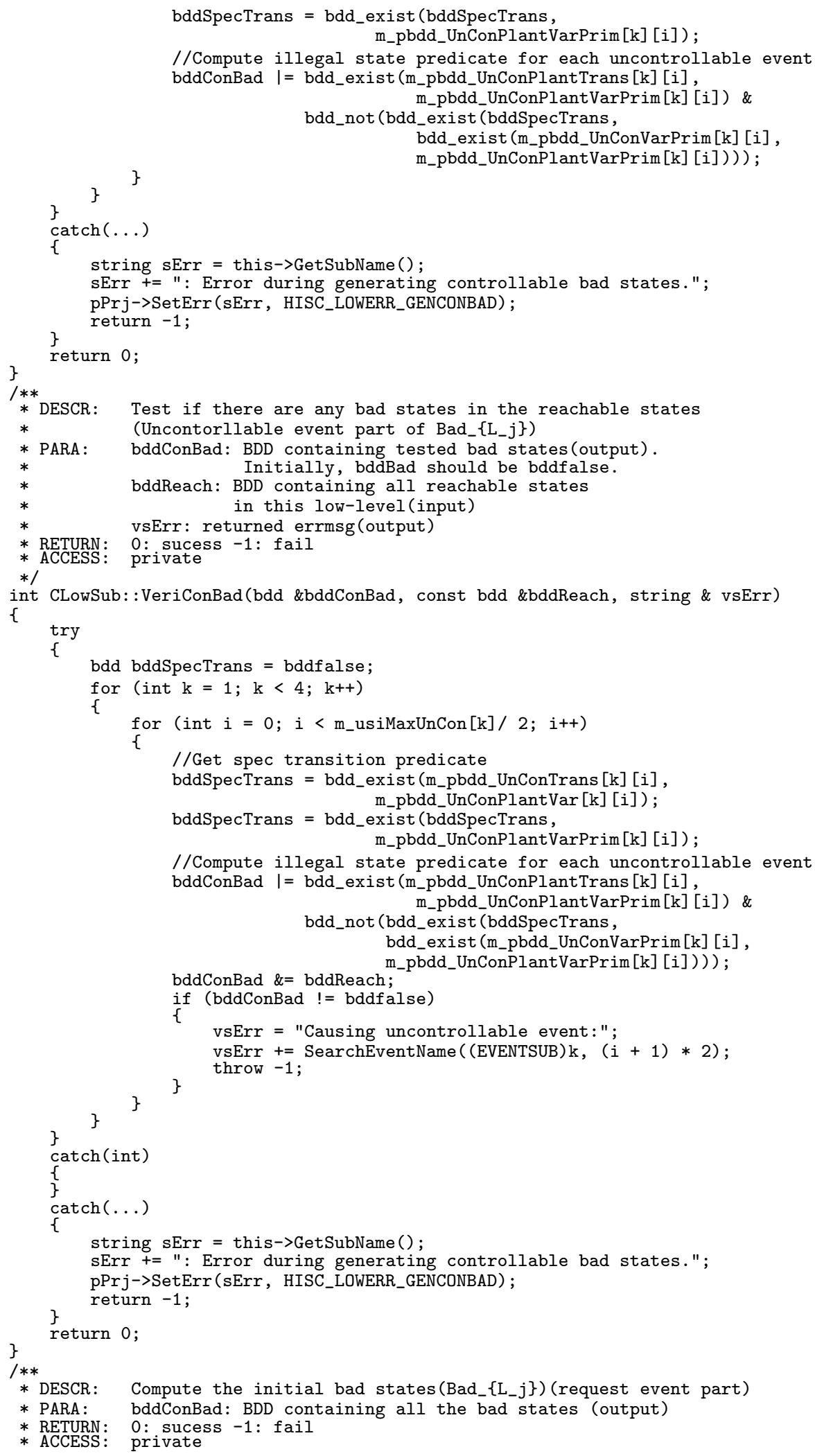




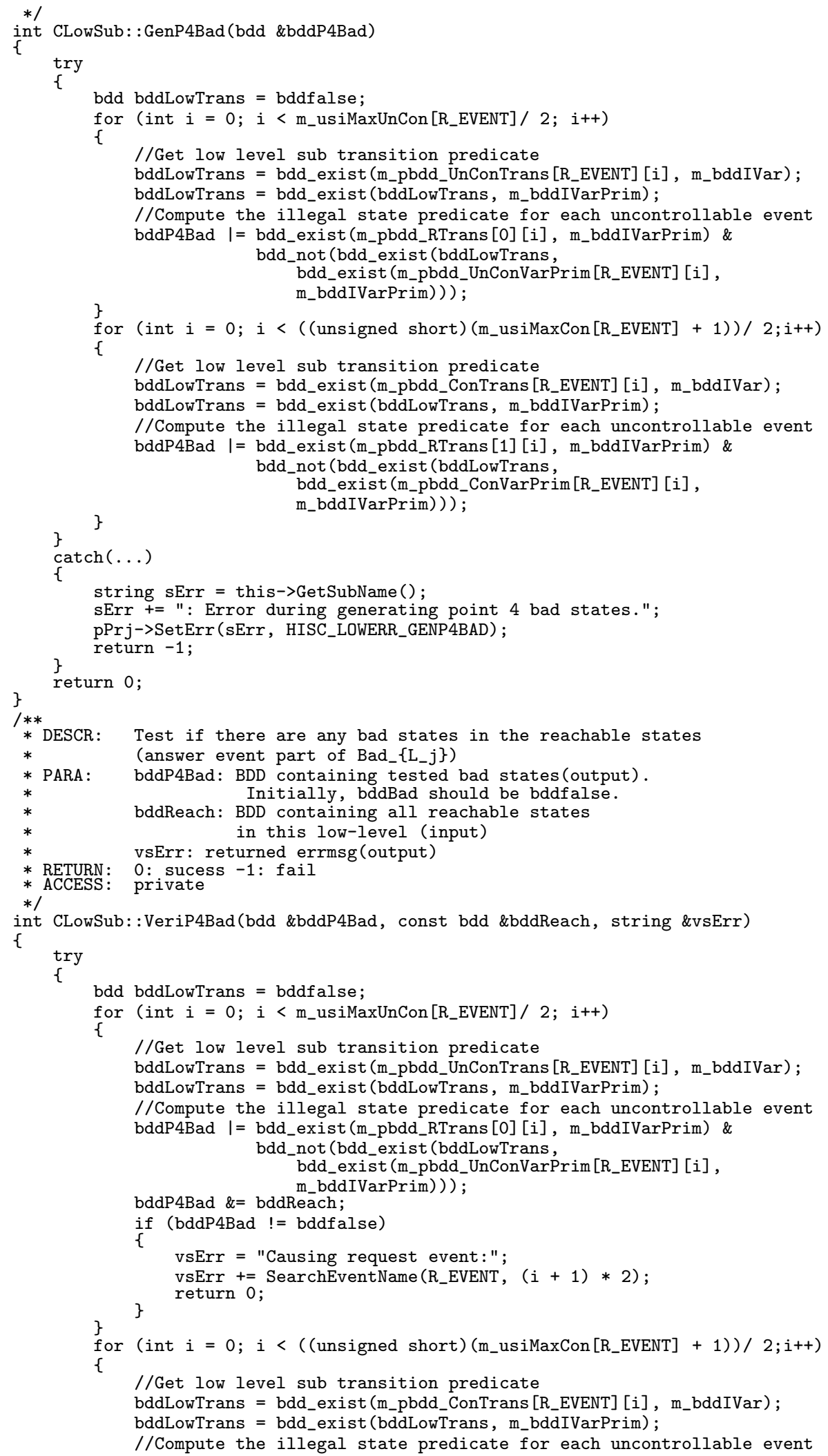




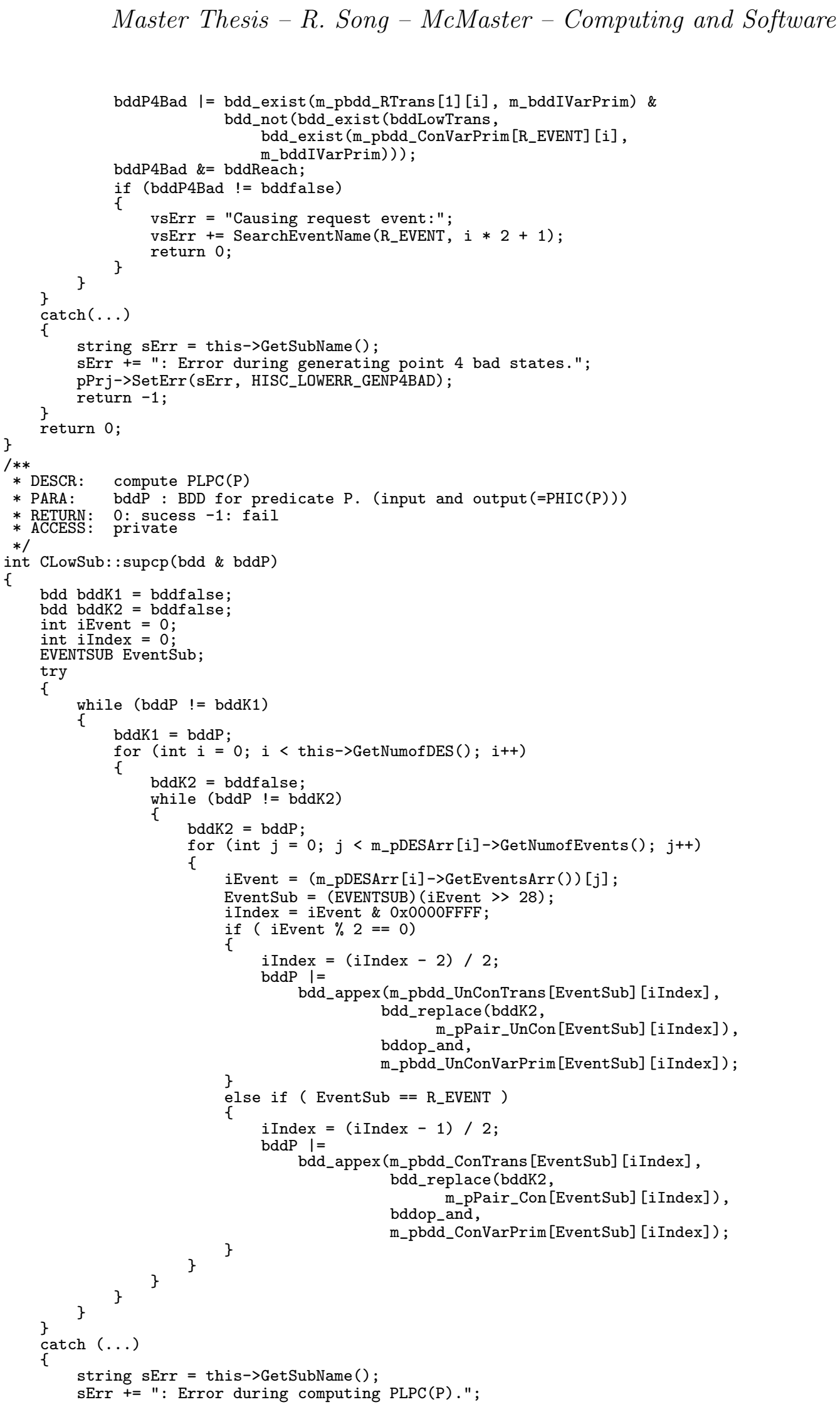


Master Thesis - R. Song - McMaster - Computing and Software

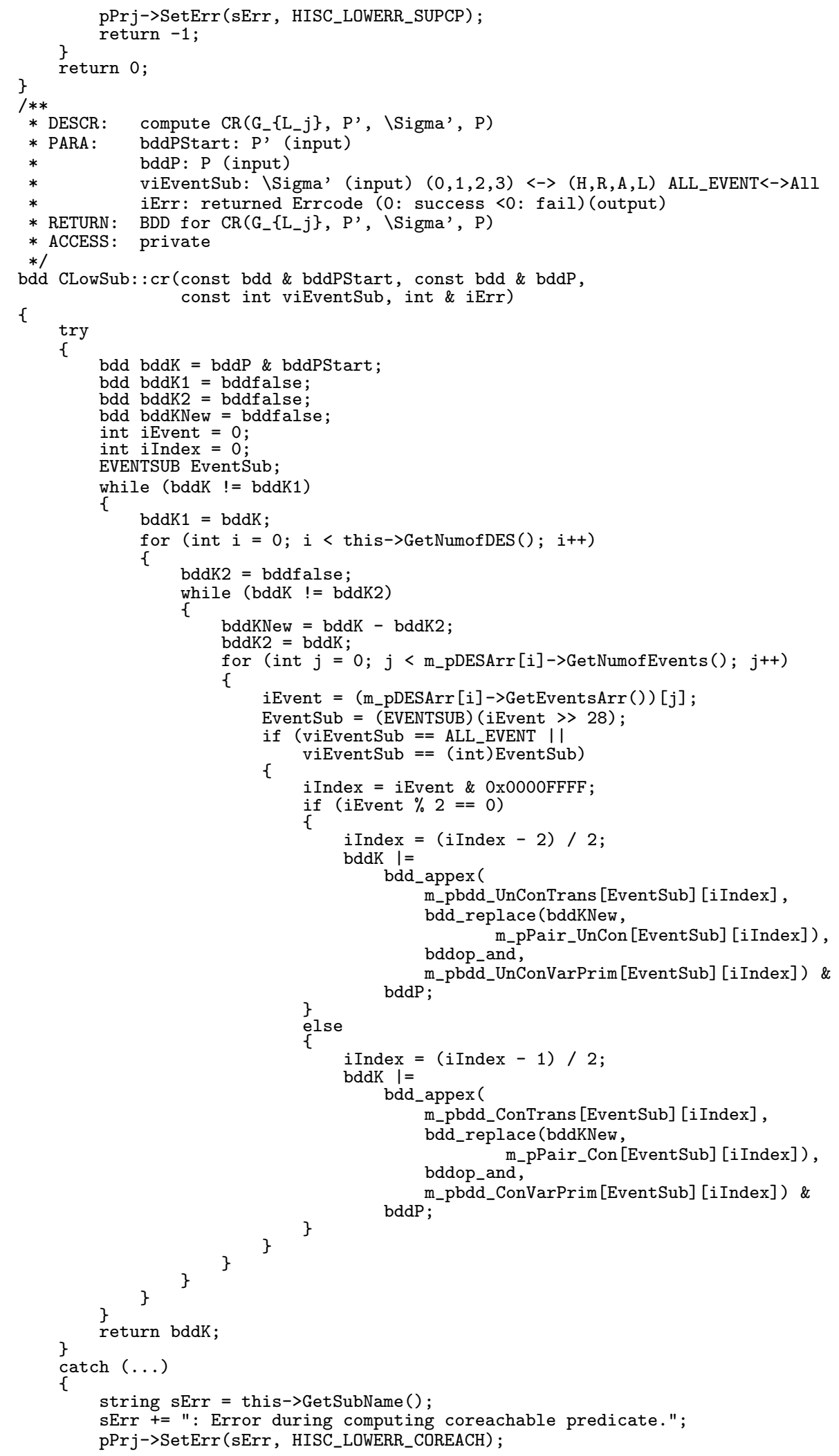




\section{Master Thesis - R. Song - McMaster - Computing and Software}

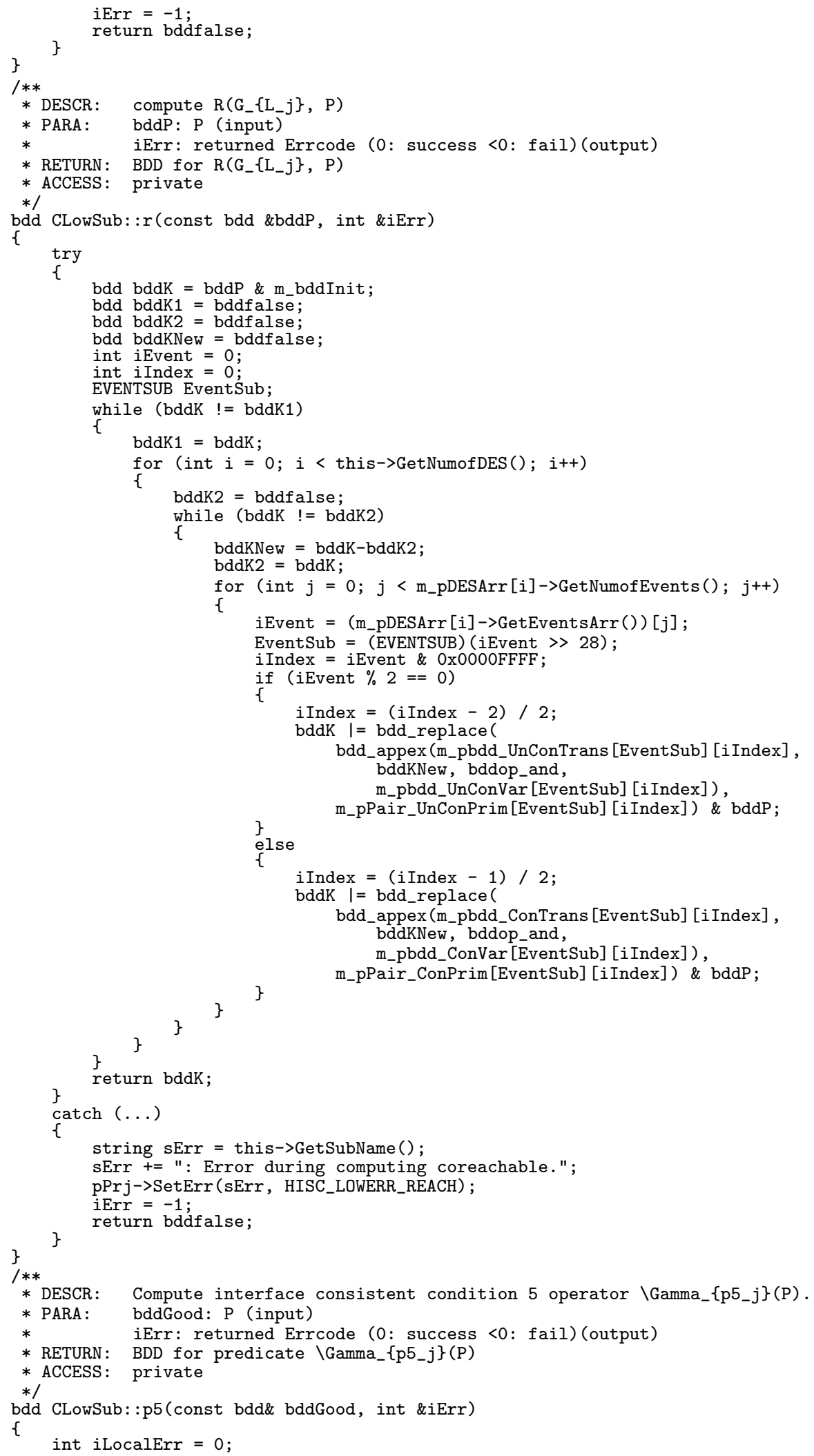




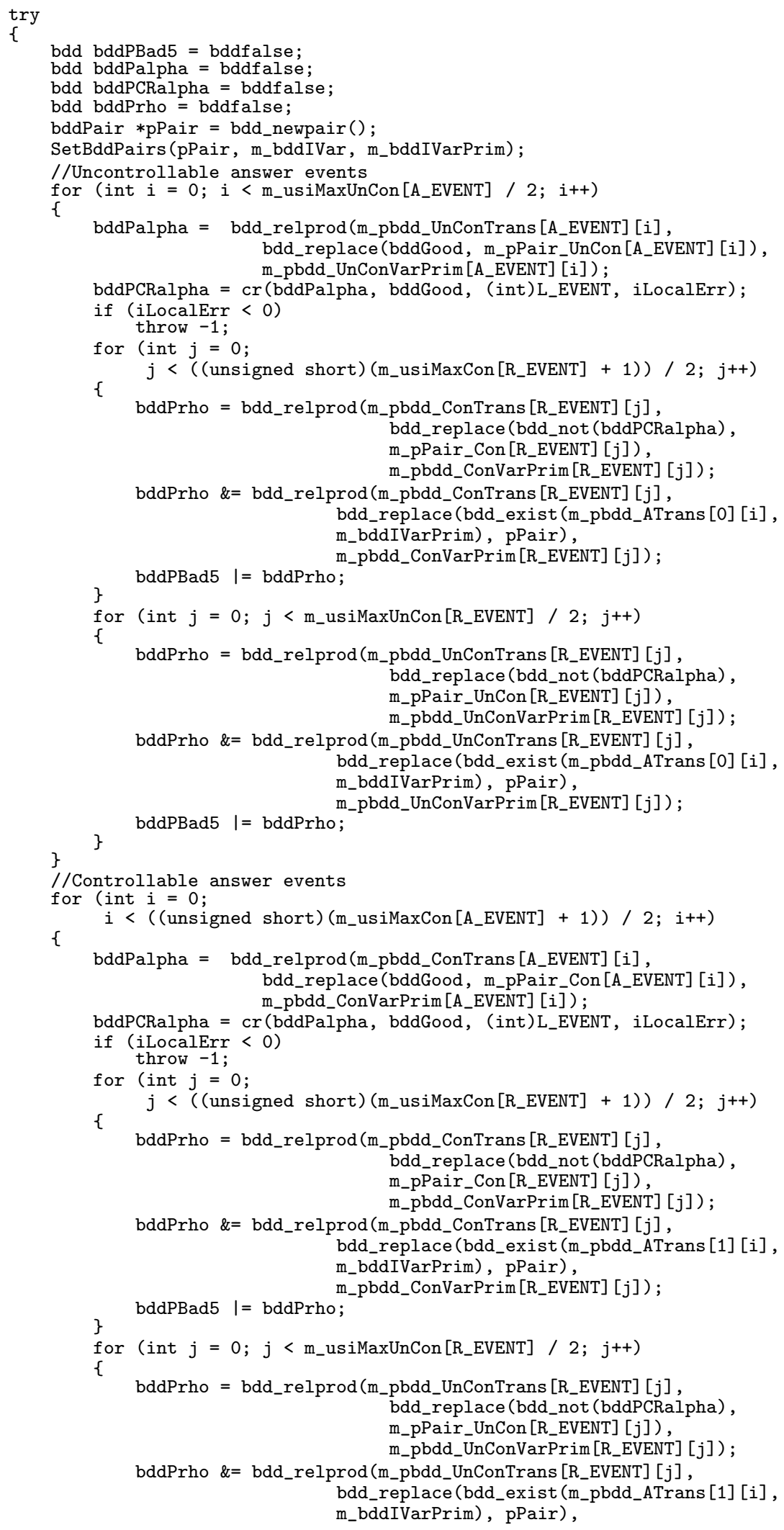




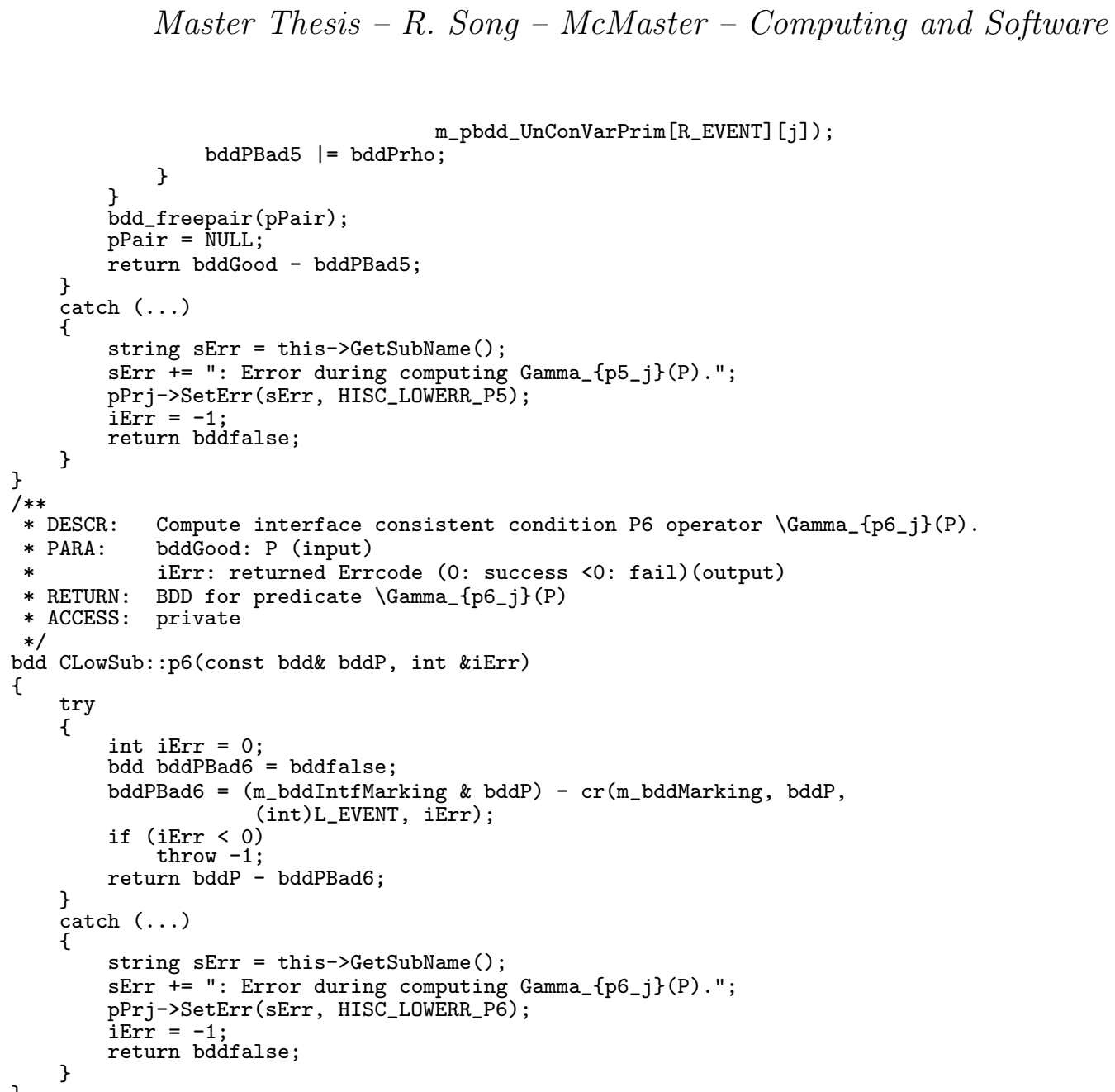




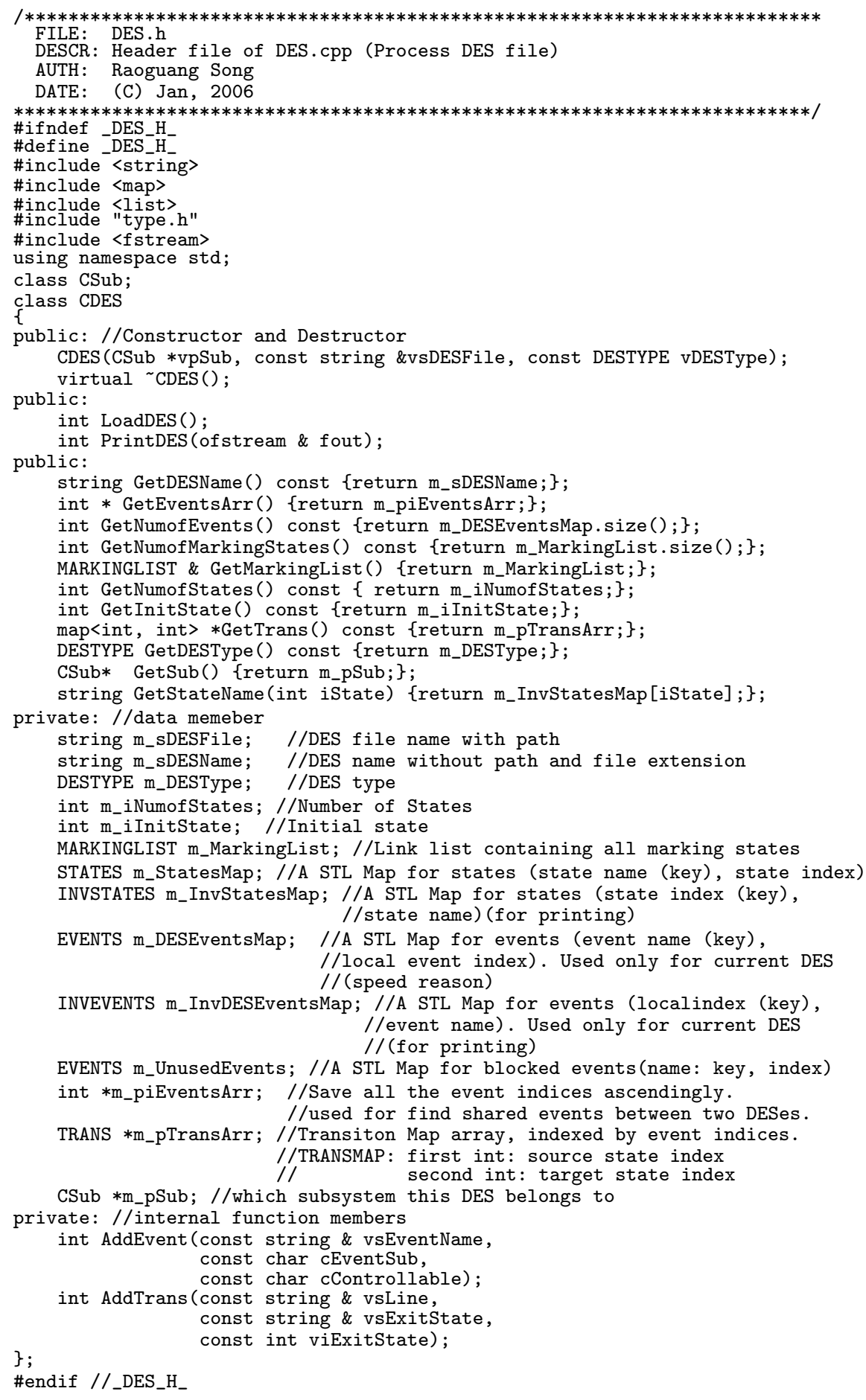




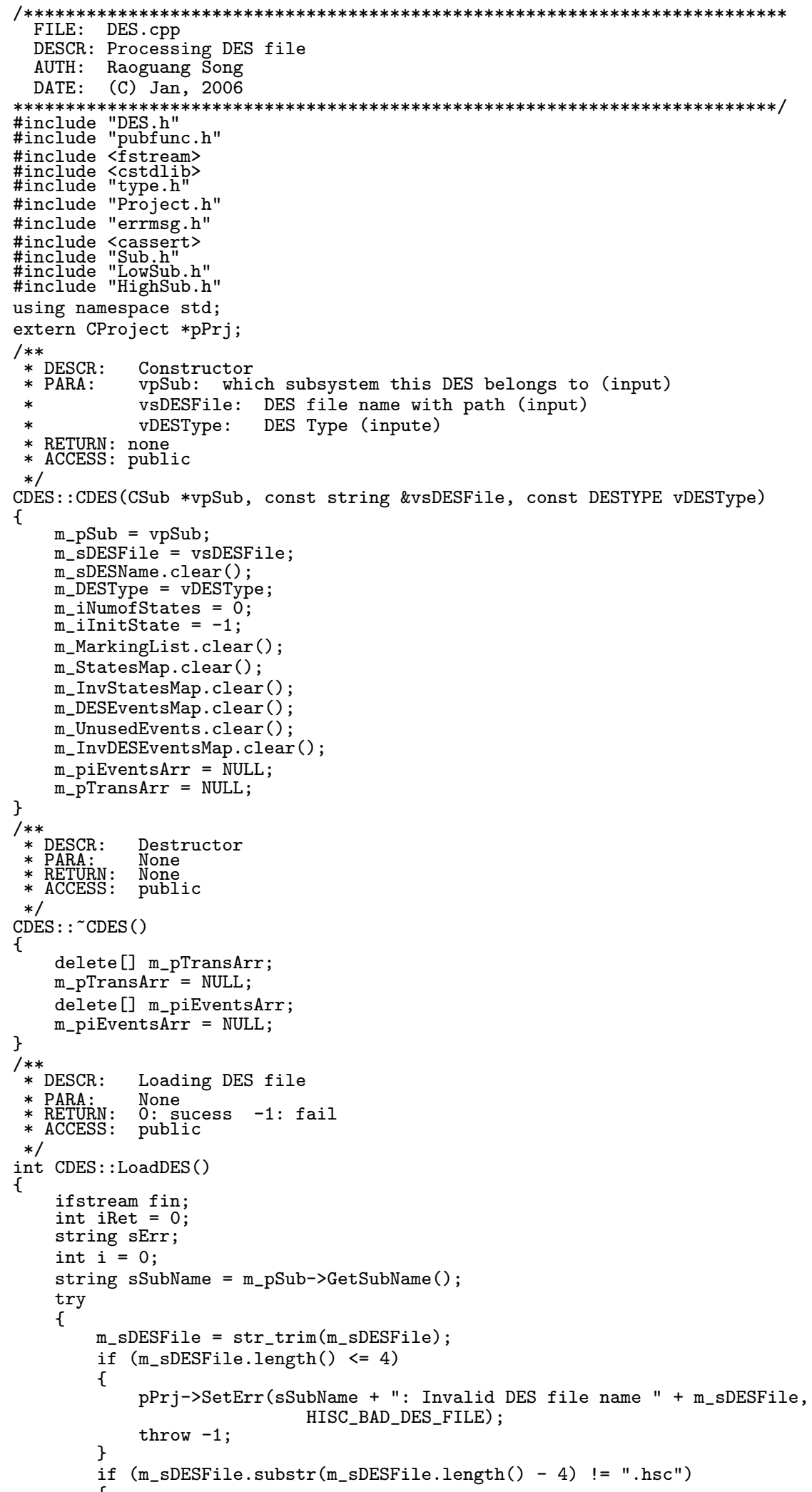




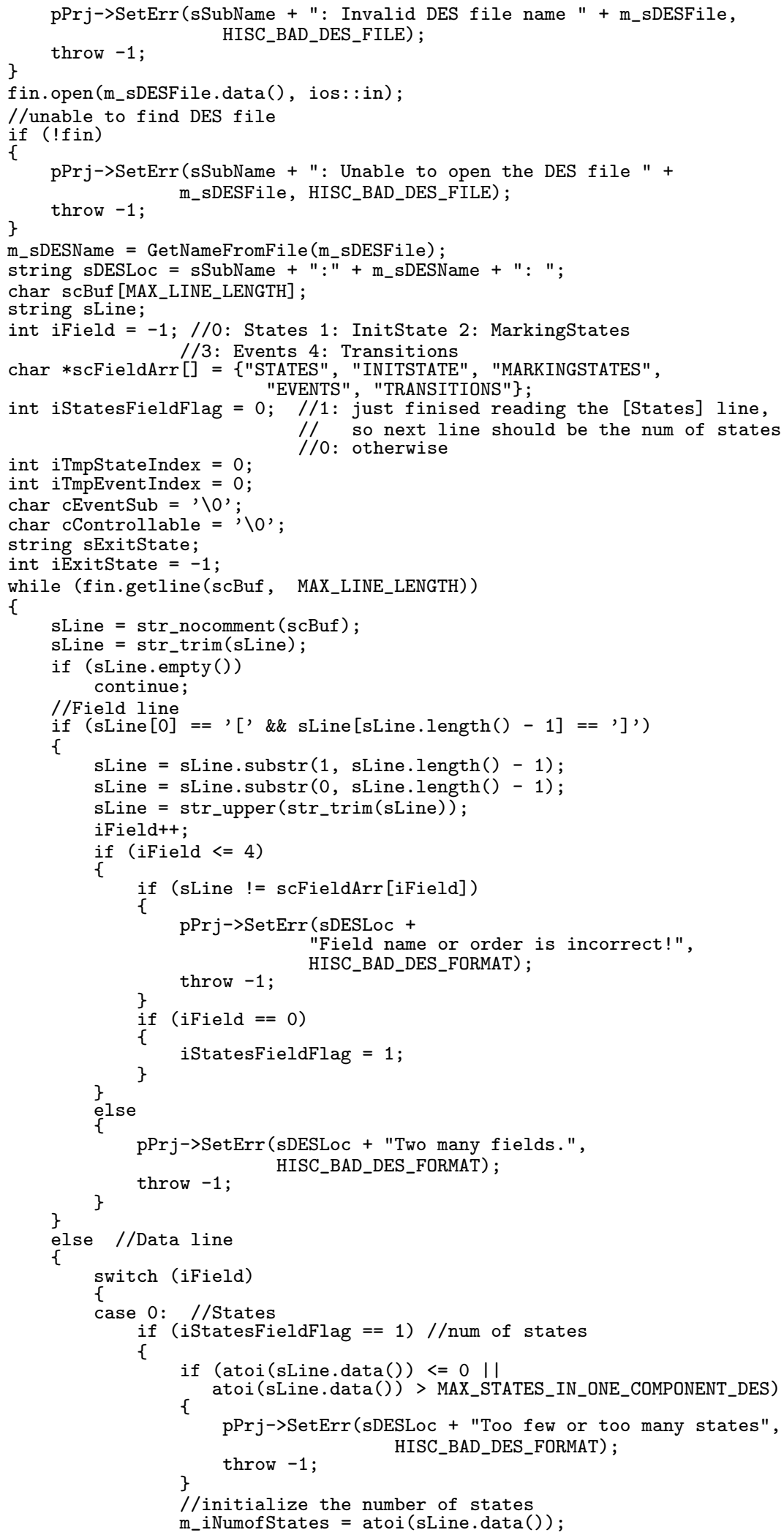


Master Thesis - R. Song - McMaster - Computing and Software

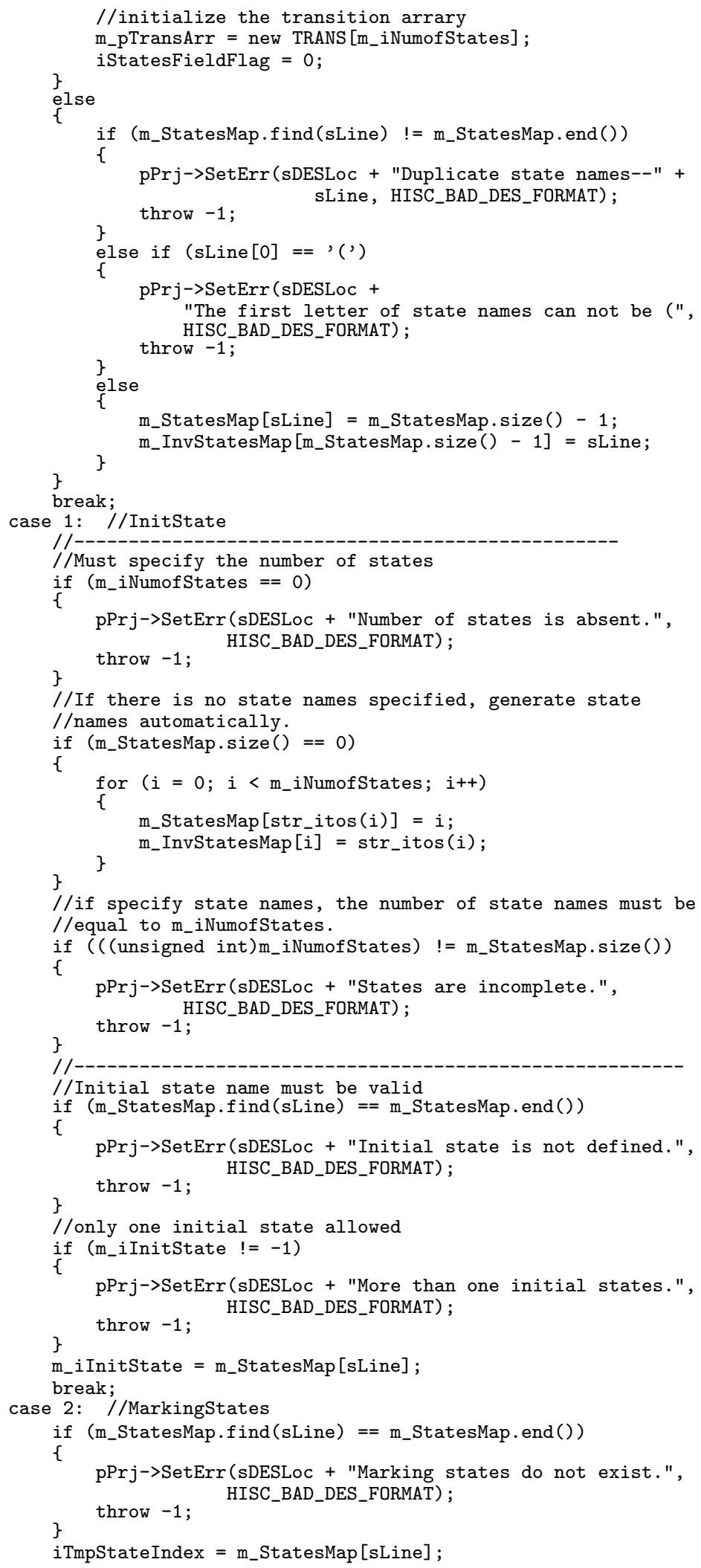


Master Thesis - R. Song - McMaster - Computing and Software

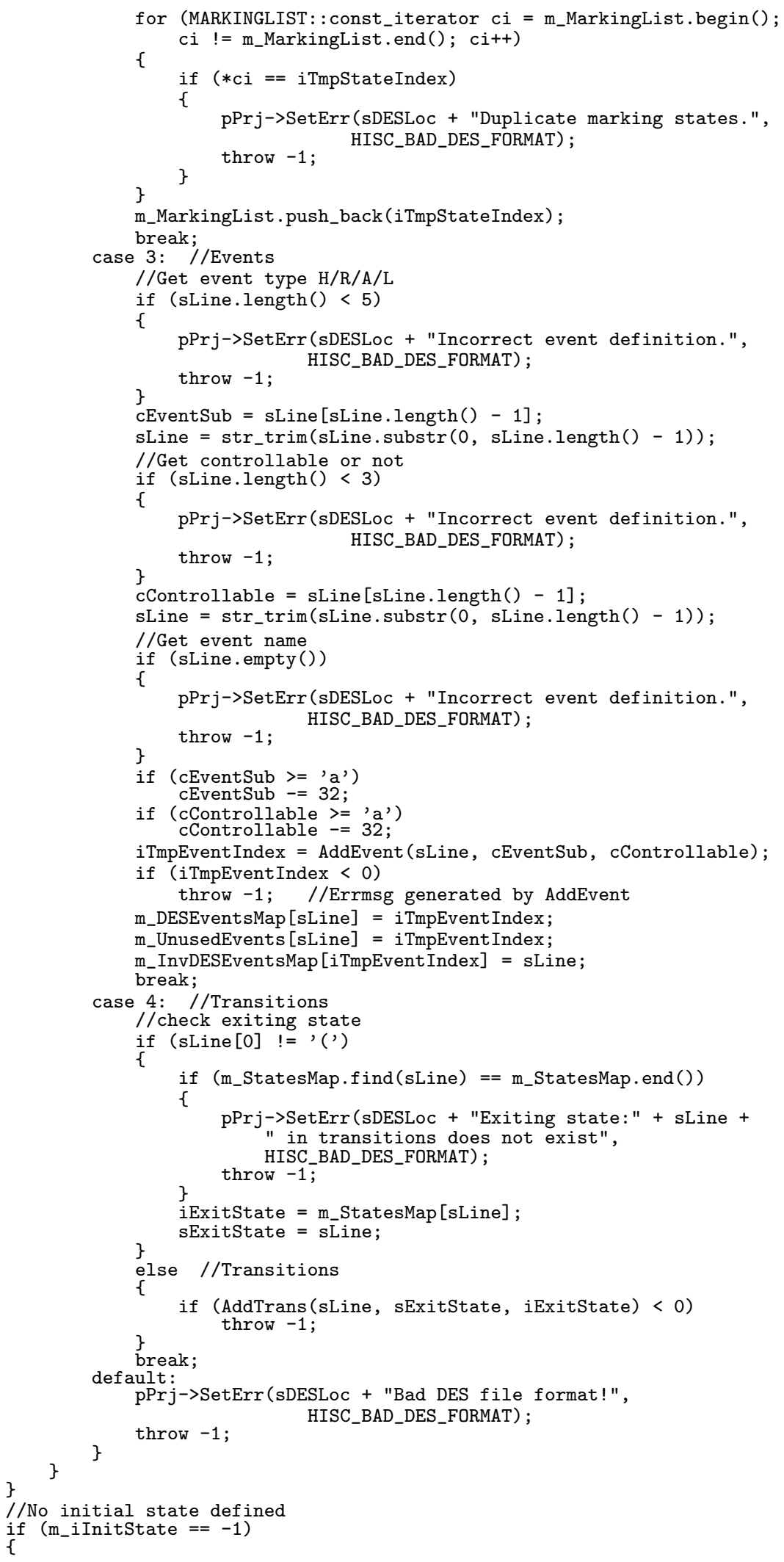




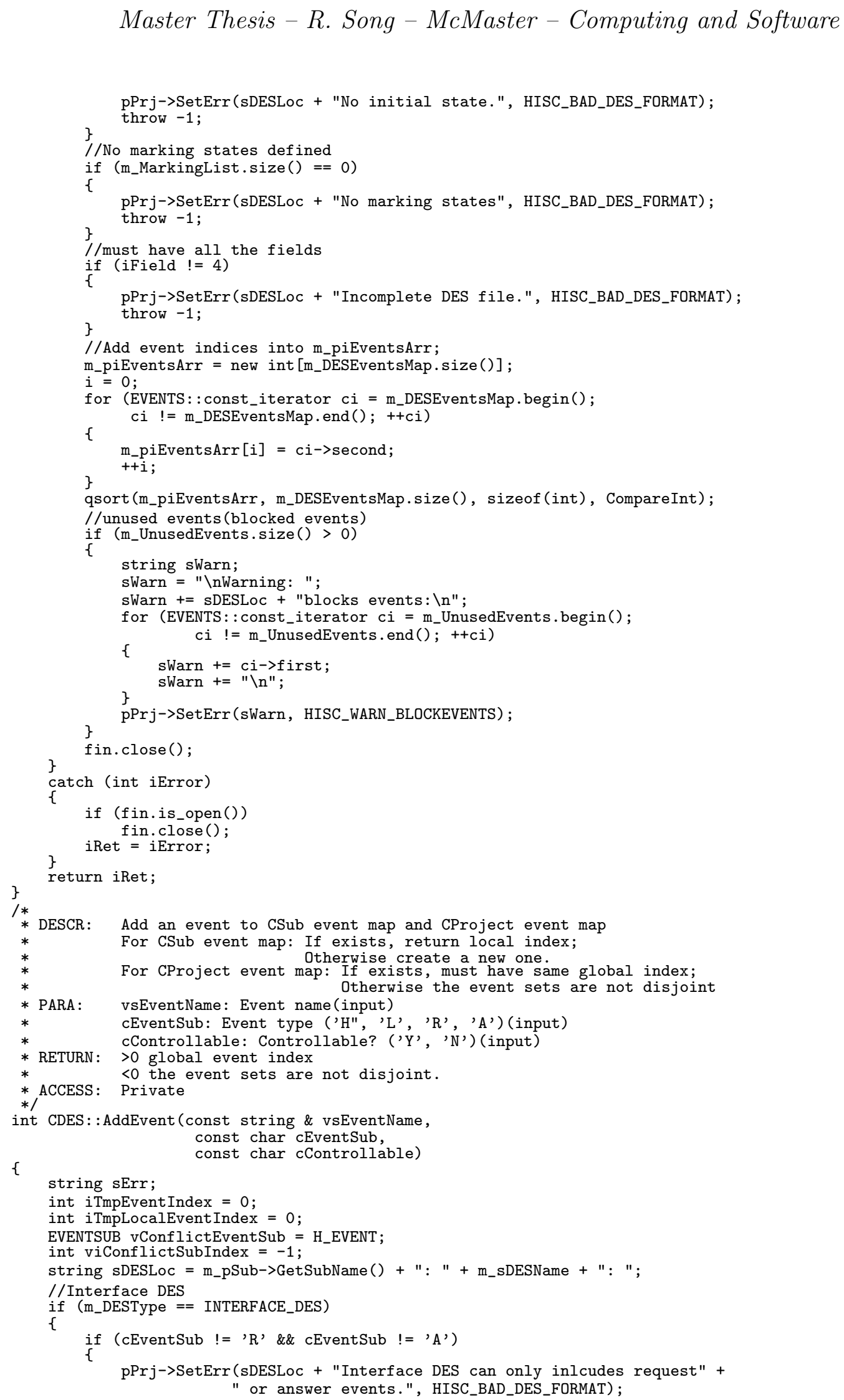




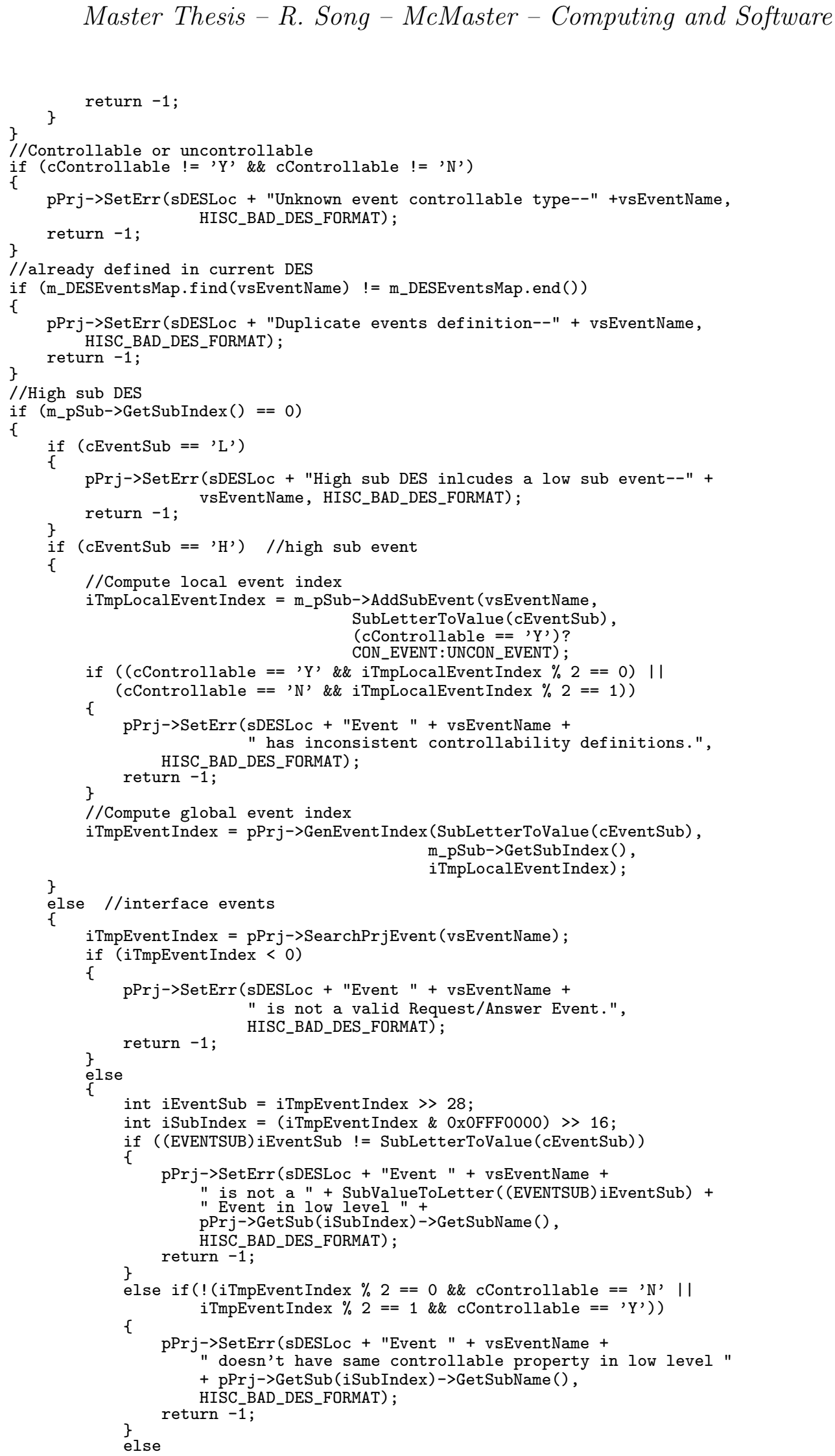


Master Thesis - R. Song - McMaster - Computing and Software

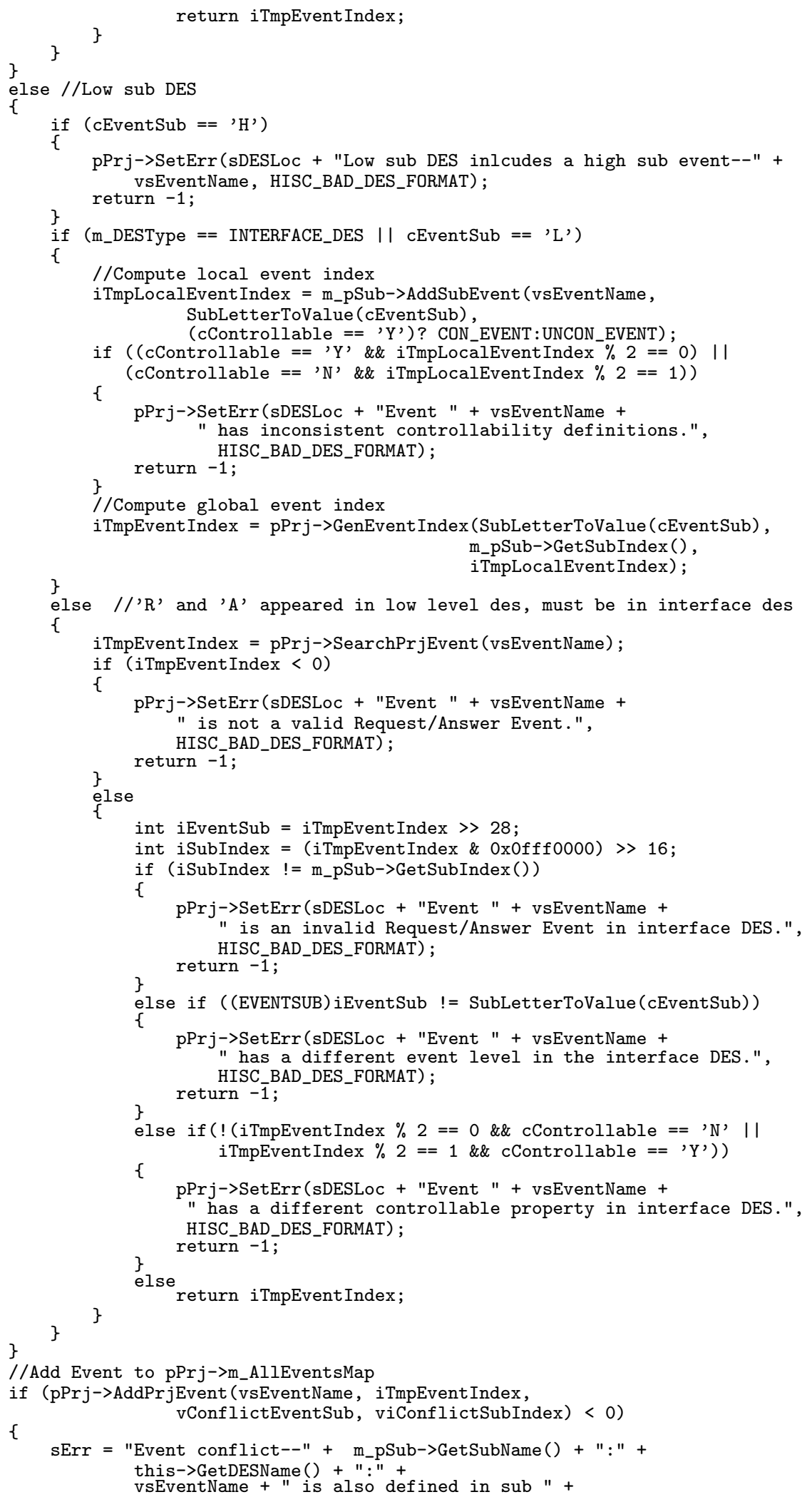




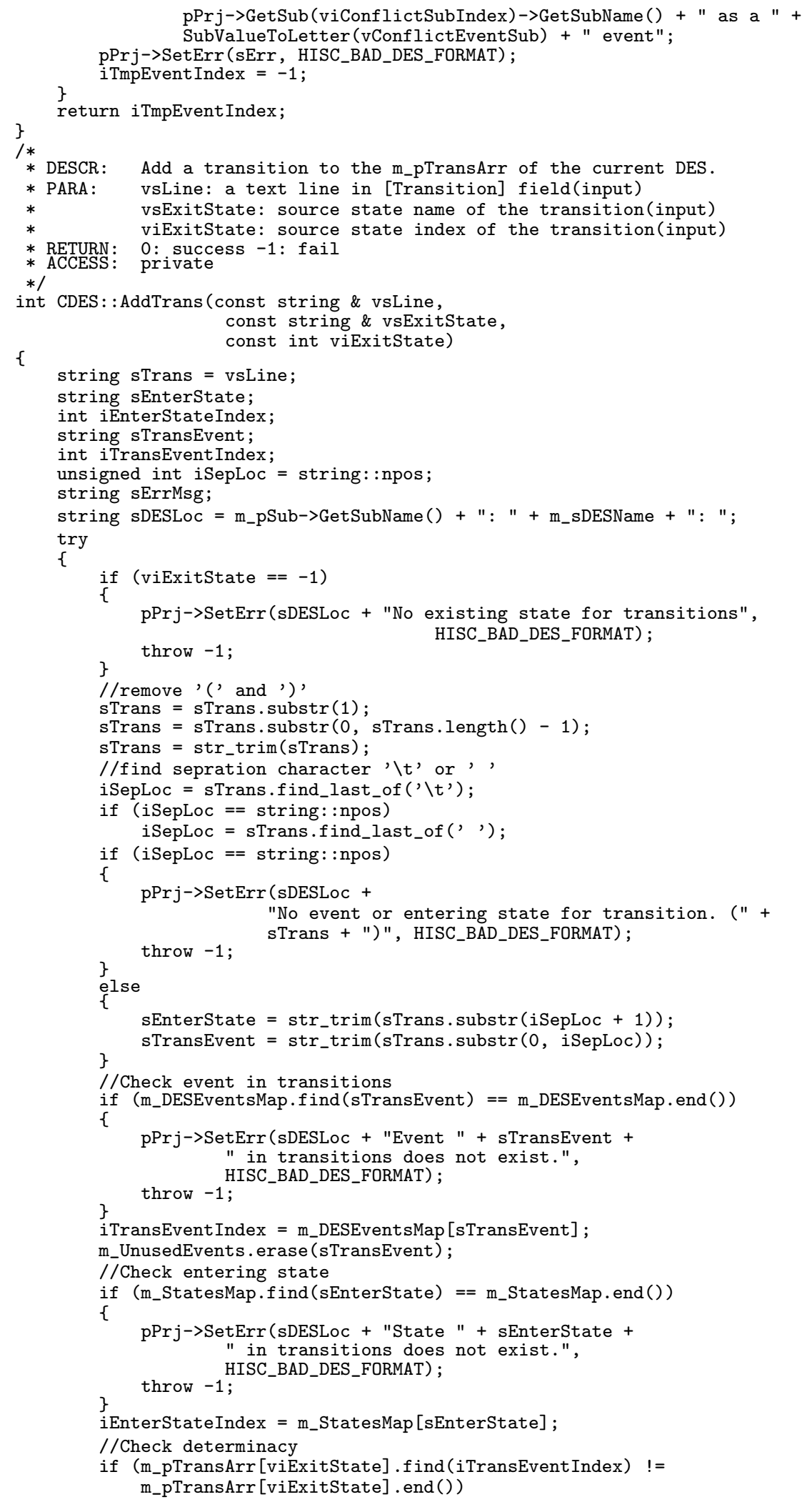




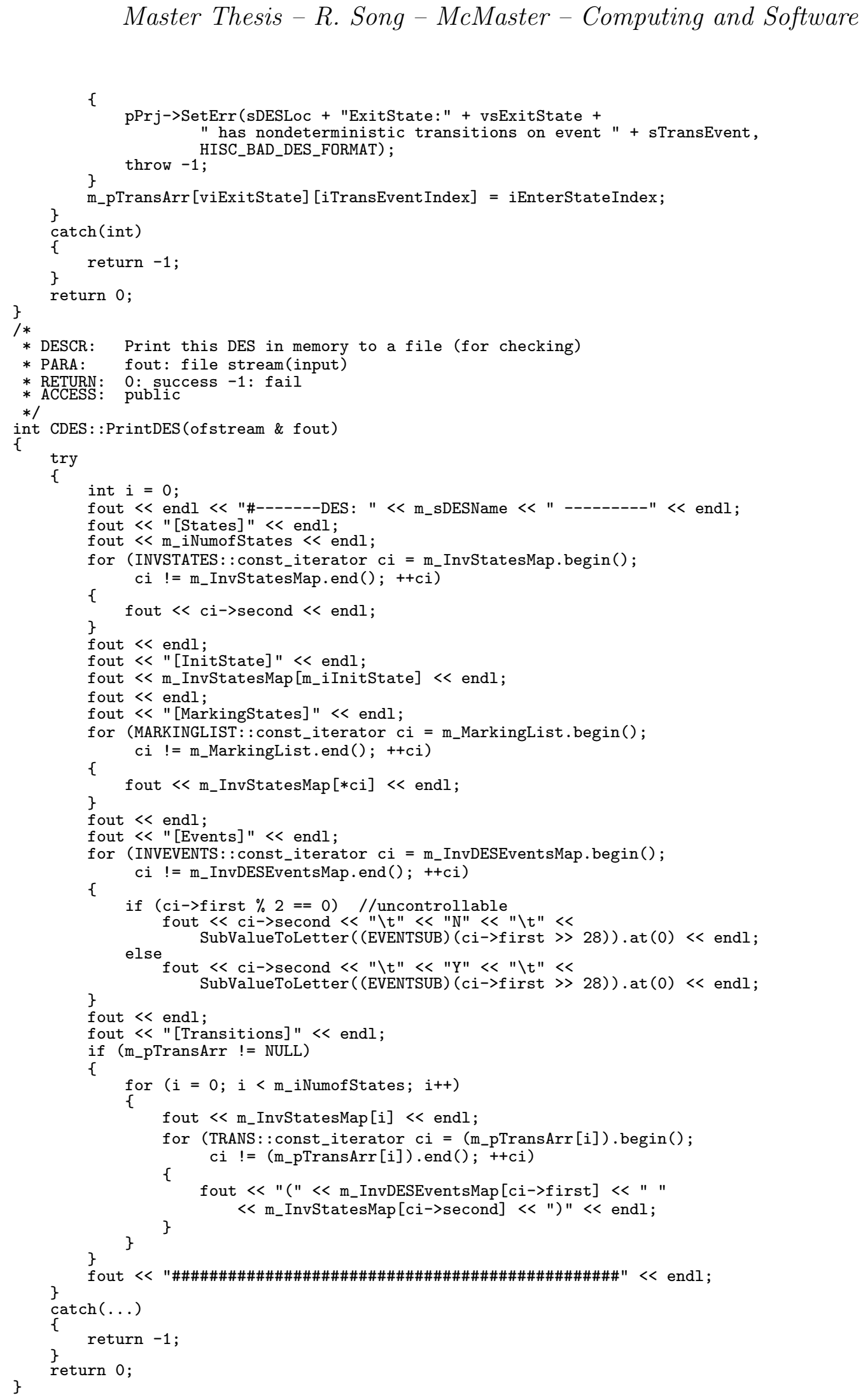




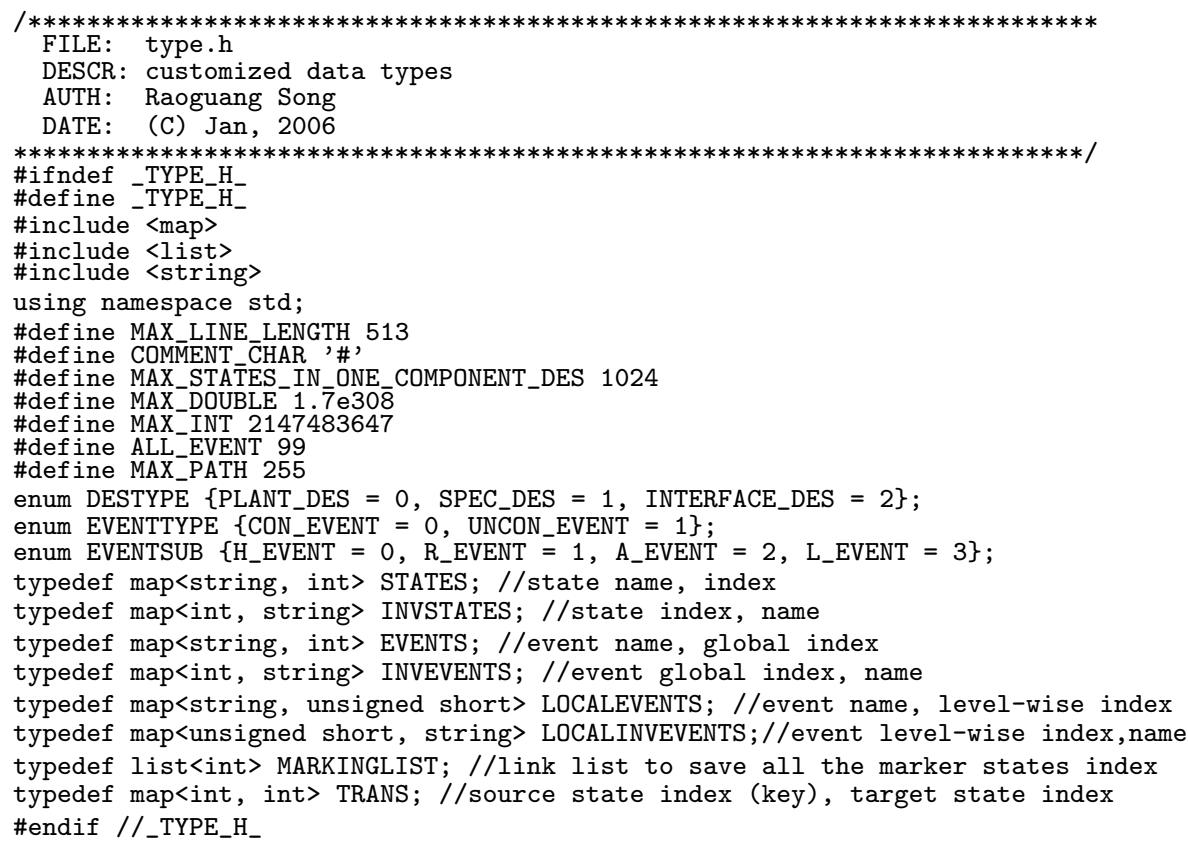




\section{Master Thesis - R. Song - McMaster - Computing and Software}

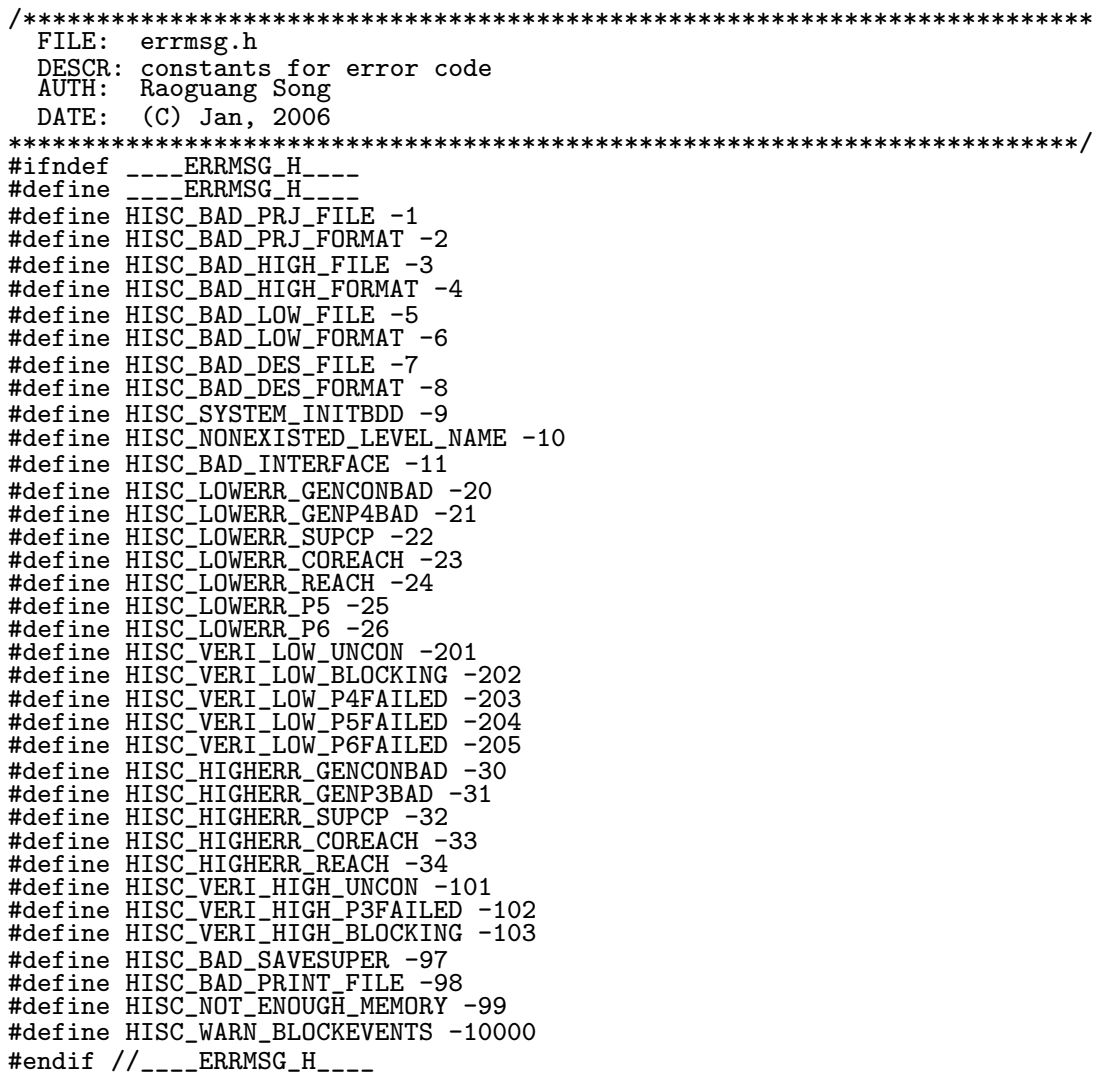




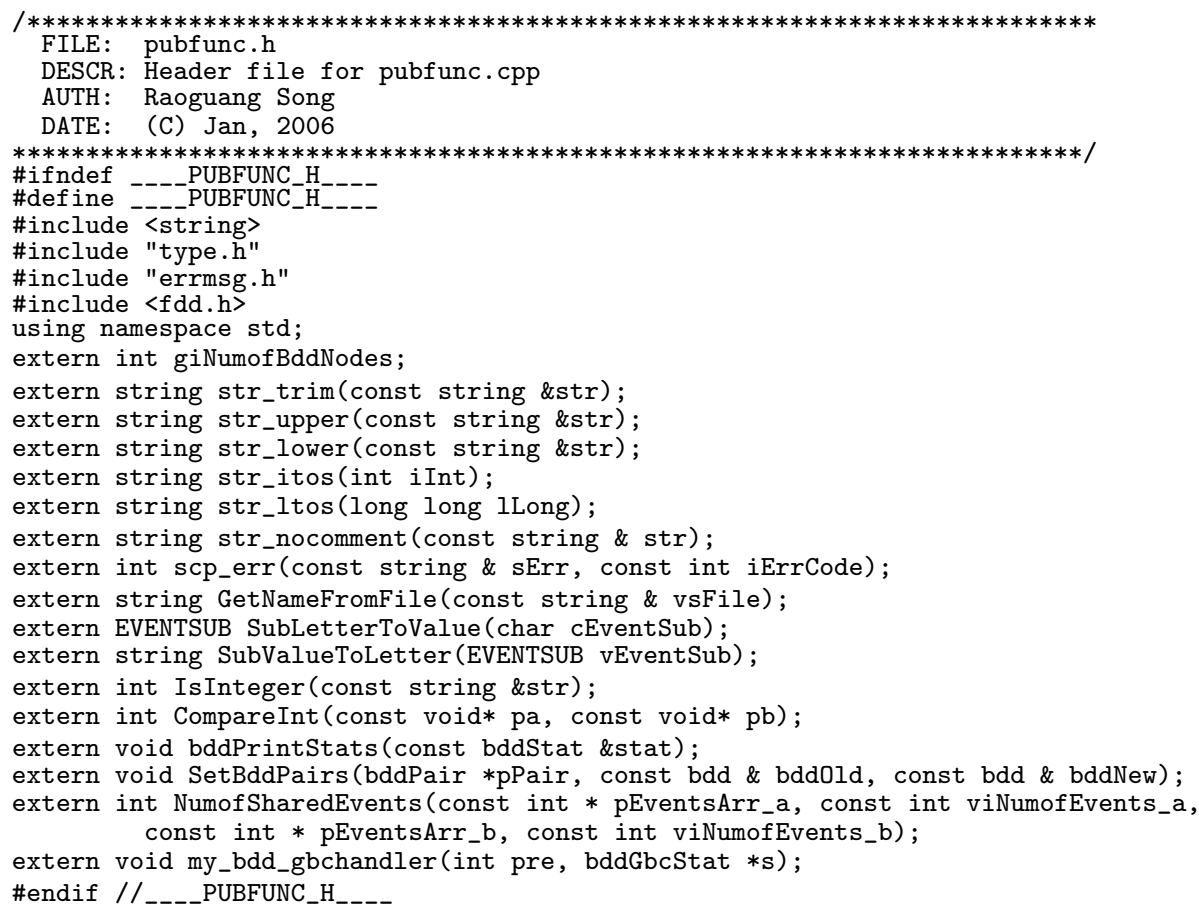




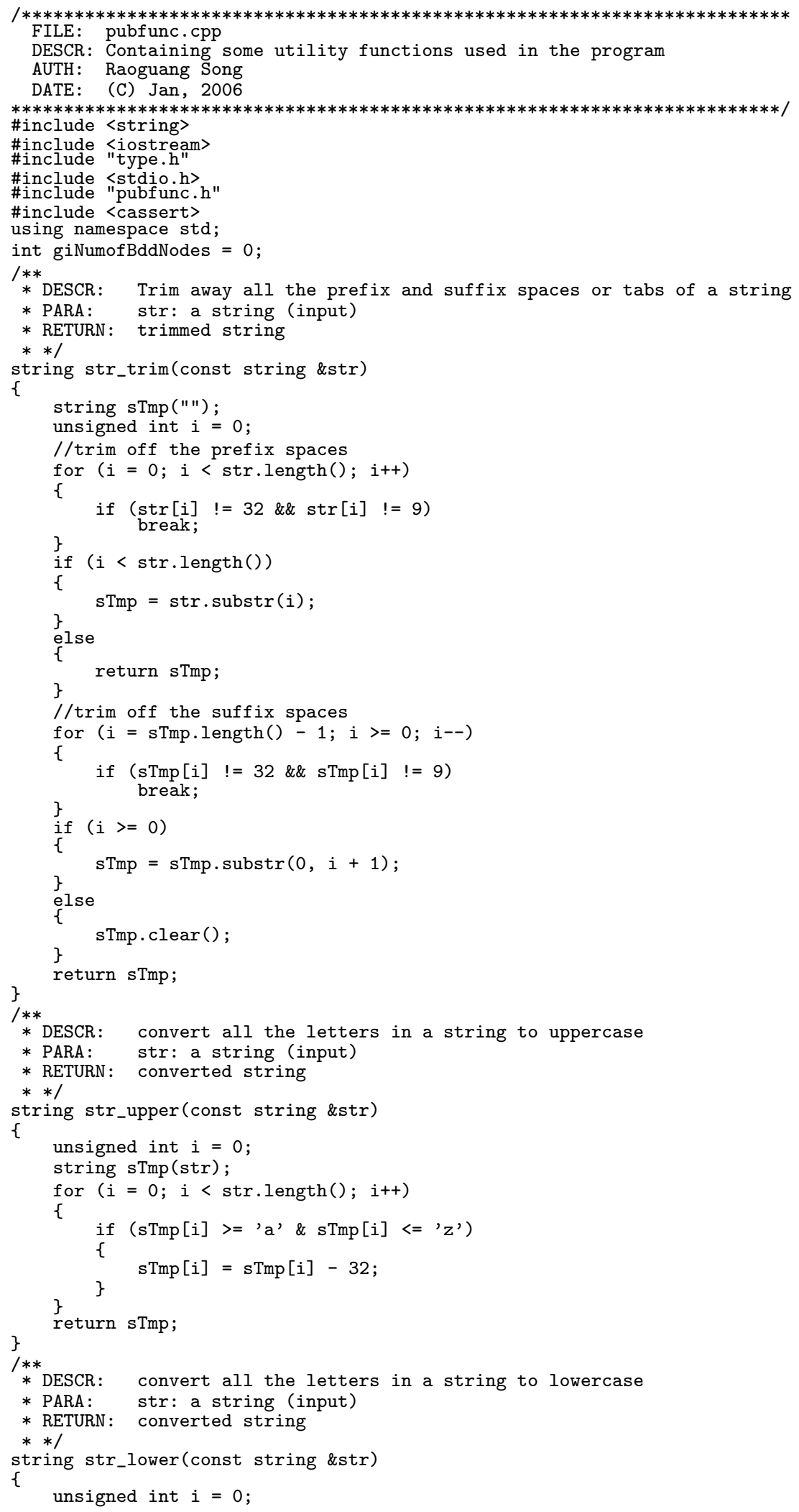




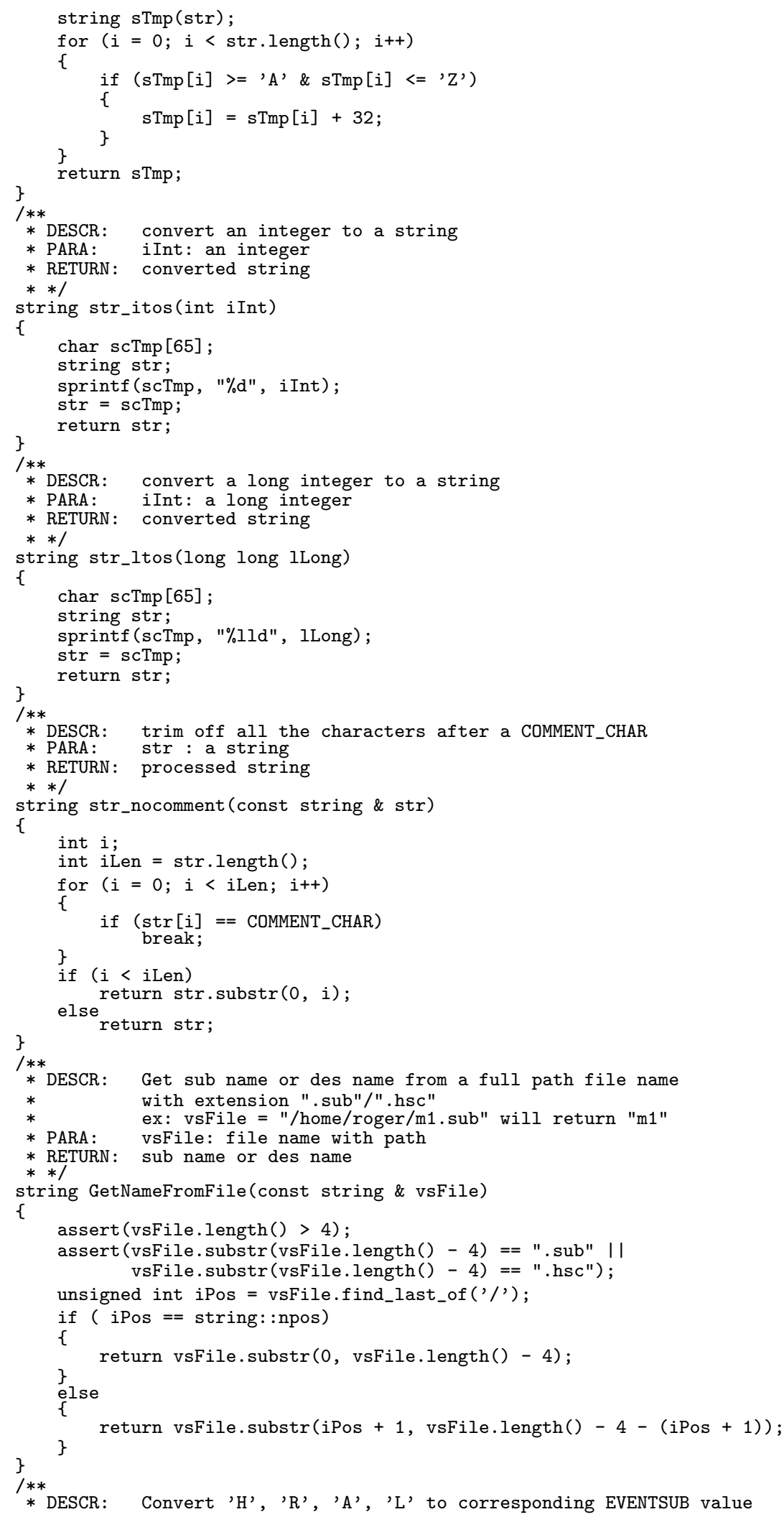




\section{Master Thesis - R. Song-McMaster - Computing and Software}

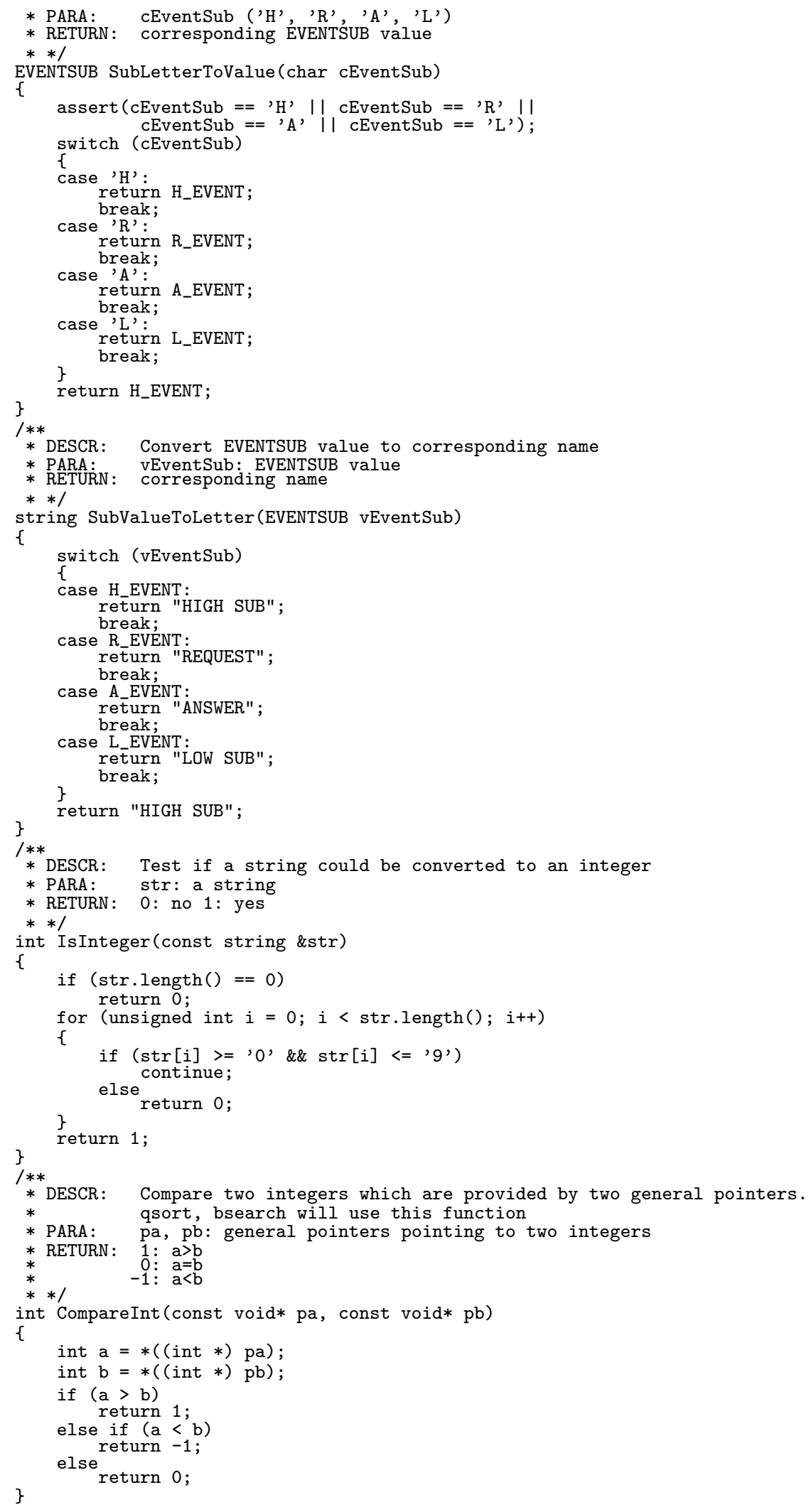




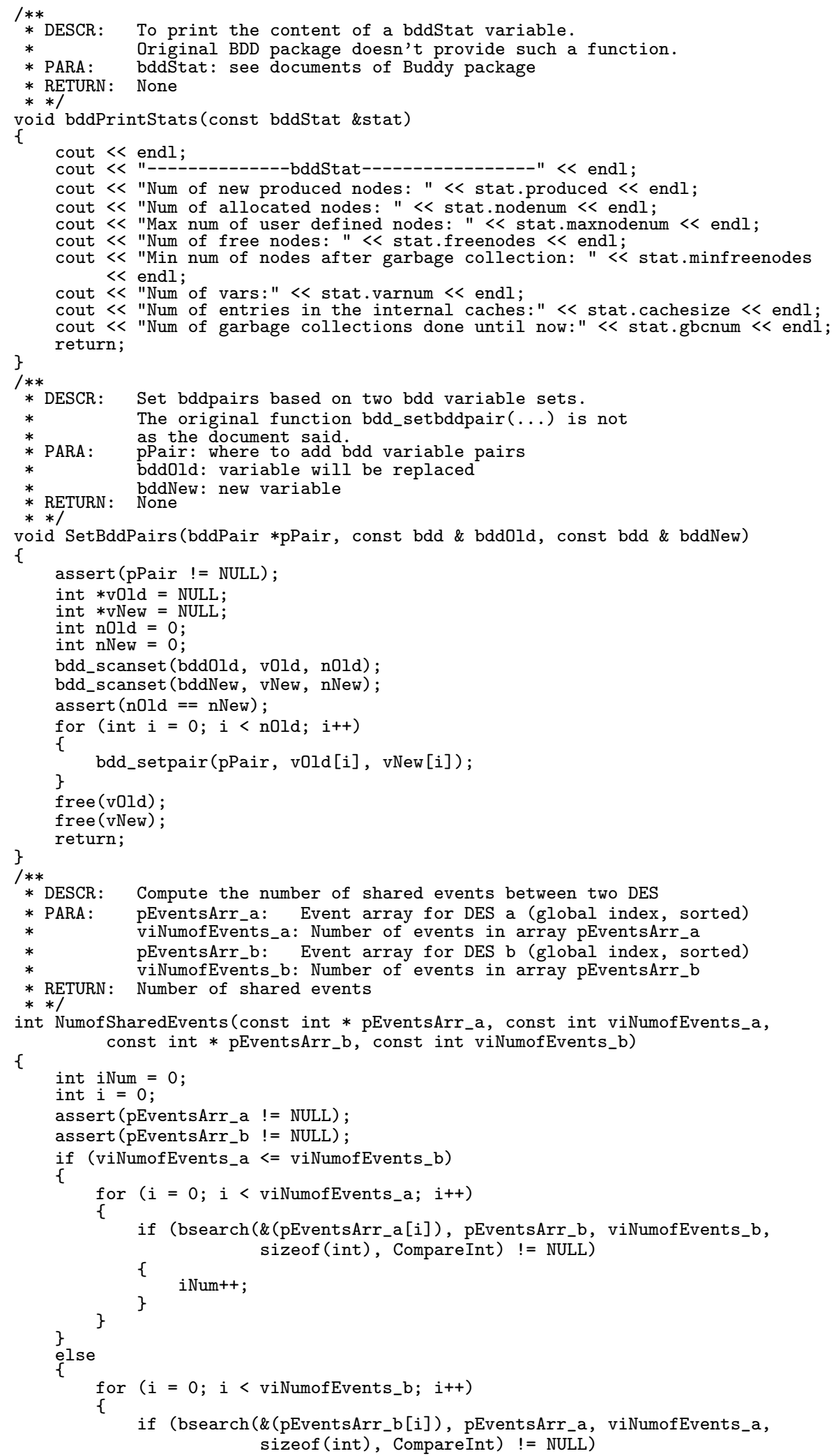




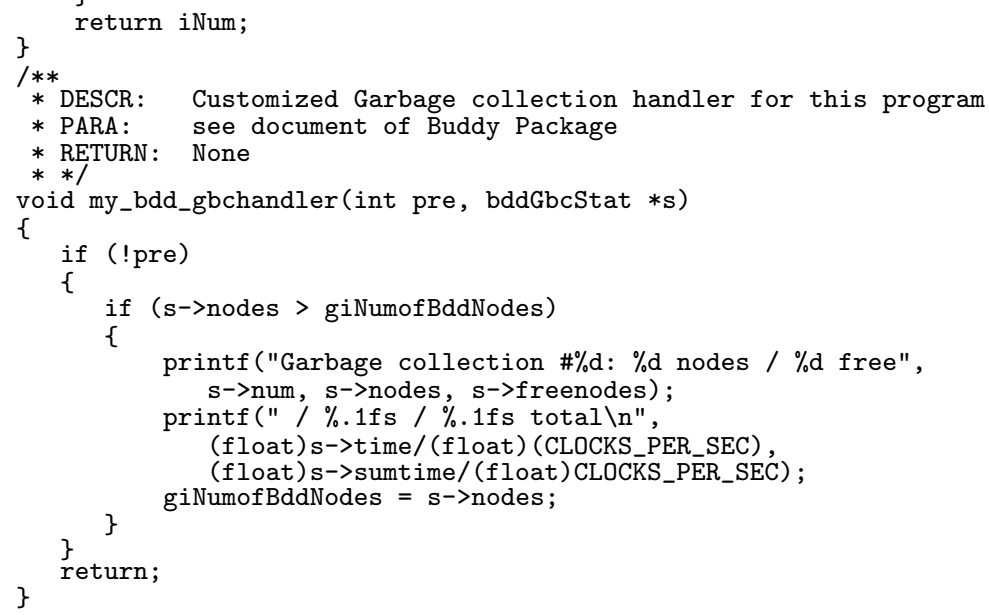


Master Thesis - R. Song - McMaster - Computing and Software

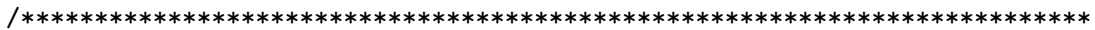 \\ FILE: main.h \\ AUTH : Raoguang Song \\ DATE: (C) Jan, 2006 \\ $* * * * * * * * * * * * * * * * * * * * * * * * * * * * * * * * * * * * * * * * * * * * * * * * * * * * * * * * * * * * * * * * * * * * * * * * * * * *)$ \\ int getchoice(bool bPrjLoaded, const char *scPrjFile); \\ char getkeystroke (char *allowed_choices, int len); \\ int getchoice_savesup(): \\ int getchoice_saveproduct (); \\ int getchoice_tracetype();
}




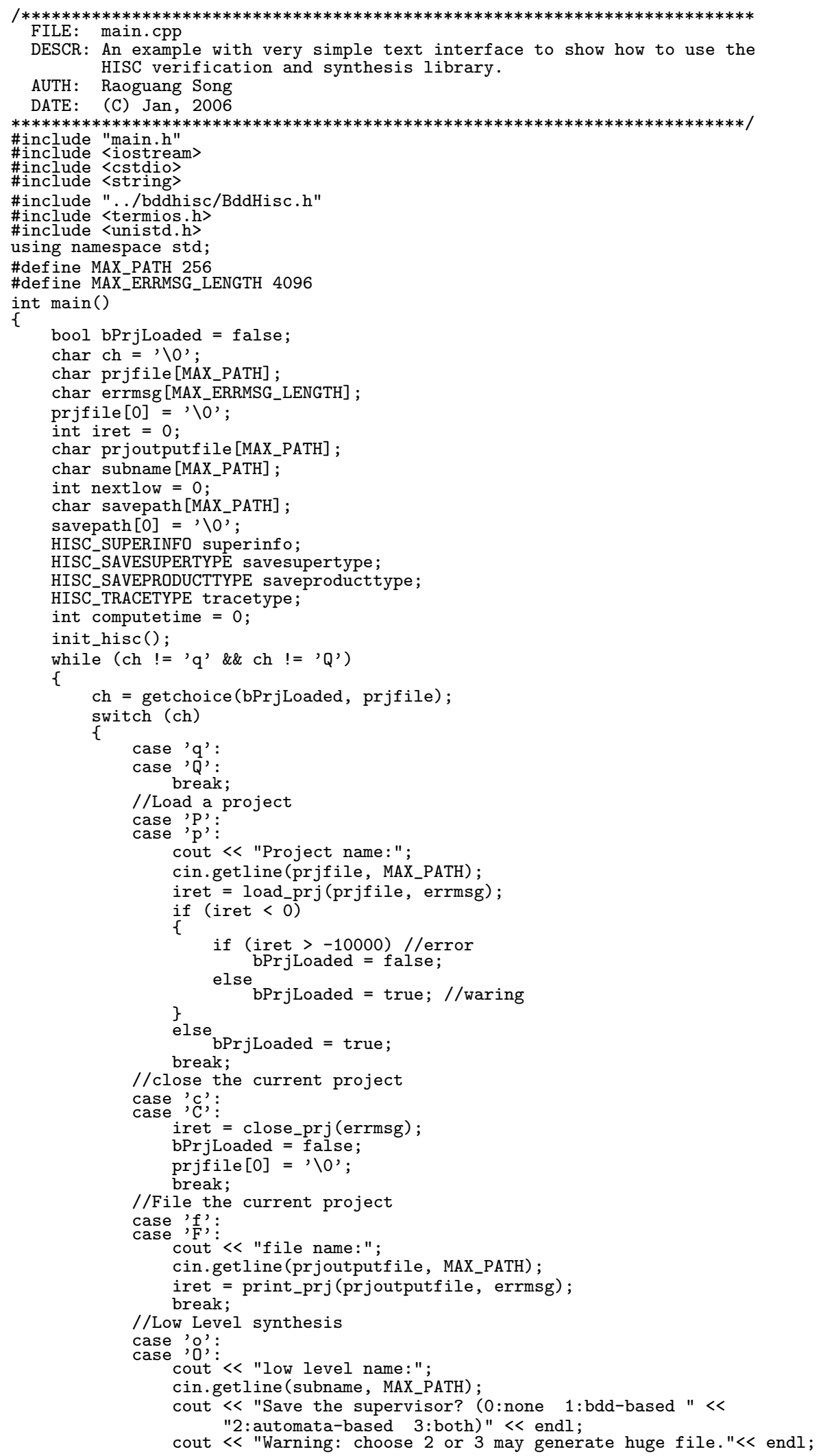




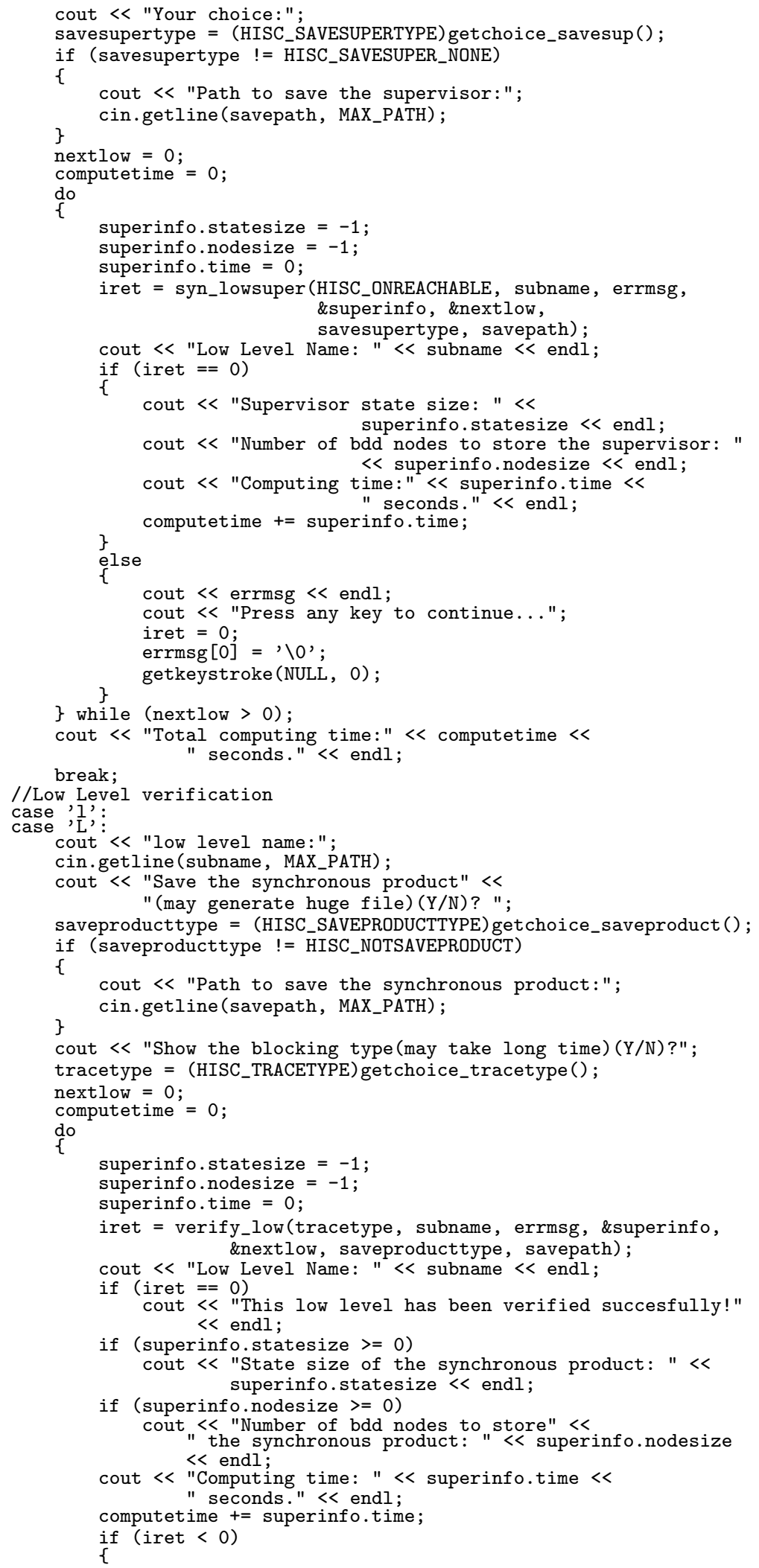


Master Thesis - R. Song - McMaster - Computing and Software

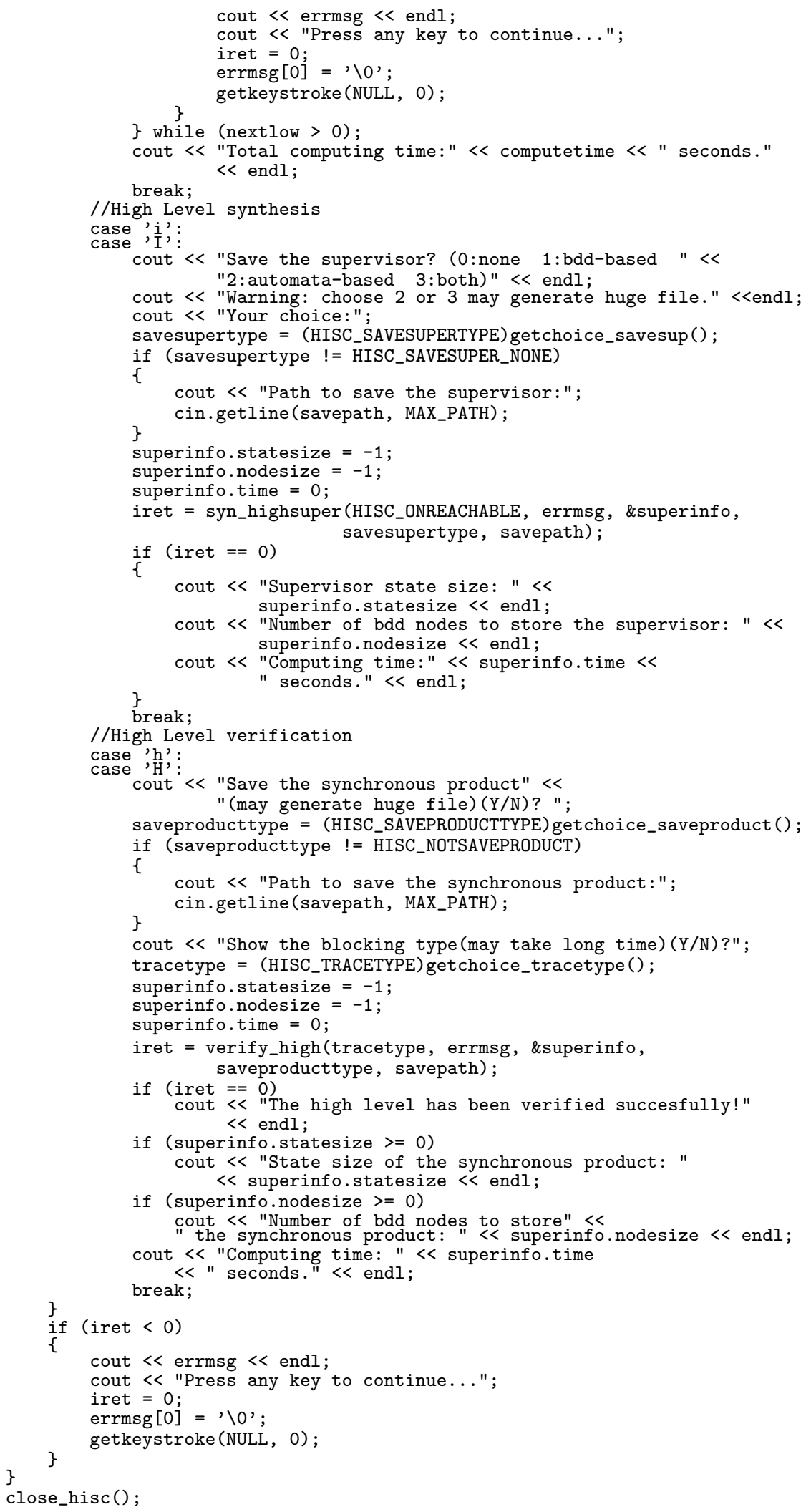


\}

return 0 ;

int getchoice(bool bPrjLoaded, const char *prjfile)

\{

char allowed_choice [50];

int numof choice $=0$;

cout $<<$ endl $<<$ endl $<<$ endl $<<$ endl $<<$ endl;

cout $<<" * * * * * * * * * * * * * * * * * * * * * * * * * * * * * * * * * * * * * * * * * * * * * * * * * * 1)<$ endl;

cout $<$ " Bdd-based HISC Synthesis and Verification Tool " $<$ endl;

cout $<1<* * * * * * * * * * * * * * * * * * * * * * * * * * * * * * * * * * * * * * * * * * * * * * * * * * * "<$ endl;

if (! bPrjLoaded)

\{

allowed_choice $[0]=$ ' $p$ ';

allowed_choice [1] = 'P';

allowed_choice [2] = 'q';

allowed_choice [3] = ' $Q$ ',

numof choice $=4$;

\}

cout $\ll$ " P - Load a HISC project " $<$ endl;

else

allowed_choice $[0]=$ ' $c$ ',

allowed_choice $[1]=$ ' $\mathrm{C}$ ',

allowed_choice [2] = 'q';

allowed_choice [3] = ' $Q$ ',

allowed_choice $[4]=$ ' $h$ ',

allowed_choice [5] = ' $\mathrm{H}$ ',

allowed_choice $[6]=$ 'i',

allowed_choice [7] = 'I';

allowed_choice $[8]=$ ' $\mathrm{F}$ ';

allowed_choice $[9]=$ 'f ';

allowed_choice [10] = ' 0 ';

allowed_choice $[11]=$ 'o',

allowed_choice [12] = 'l';

allowed_choice $[13]=$ ' $L$ ';

numofchoice $=14$

cout $<<"$ H - High level verification $\quad$ " endl;

cout $\ll$ " I - High level synthesis $\quad "<<$ endl;

cout $<<$ " L - Low Level verification $\quad "<<$ endl;

cout $<<" ~ 0$ - Low Level synthesis $\quad "<<$ endl;

cout $<<"$ F - File the current project $"<<$ endl

\}

cout $<$ " C - Close the current project " $\quad<$ endl;

cout $<<"$ Q - Quit " $<<$ endl;

cout $<<" * * * * * * * * * * * * * * * * * * * * * * * * * * * * * * * * * * * * * * * * * * * * * * * * * * * 1<<$ endl;

if ( $\mathrm{bPrj}$ Loaded)

\{

cout $\ll$ "Current Project: " $<$ prjfile $\ll$ endl;

cout $<$ endl;

cout $<<$ "Procedure desired:";

\}

return getkeystroke(allowed_choice, numofchoice);

char getkeystroke(char *allowed_choices, int len)

char choice

struct termios initial_settings, new_settings;

//cin.ignore (255, ' \n');

tcgetattr(fileno(stdin), \&initial_settings);

new_settings = initial_settings;

new_settings.c_lflag \& $=\sim$ ICANON;

$/ /$ new_settings.c_lflag \& = ECHO;

new_settings.c_cc [VMIN] $=1$;

new_settings.c_cc [VTIME] $=0$;

new_settings.c_lflag \& $=\sim$ ISIG;

tcsetattr(fileno(stdin), TCSANOW, \&new_settings);

if $($ len $>0)$

do \{

choice $=$ fgetc $($ stdin $)$;

int $i$;

for $(i=0 ; i<$ len; $i++)$

\{

if (choice == allowed_choices $[i]$ )

\} break;

if $(i==$ len)

choice = ' \n'.

\}

\} while (choice $==$ ' $\backslash \mathrm{n}$ ' || choice $==$ ' $\backslash \mathrm{r}$ ') ; 


\section{Master Thesis - R. Song - McMaster - Computing and Software}

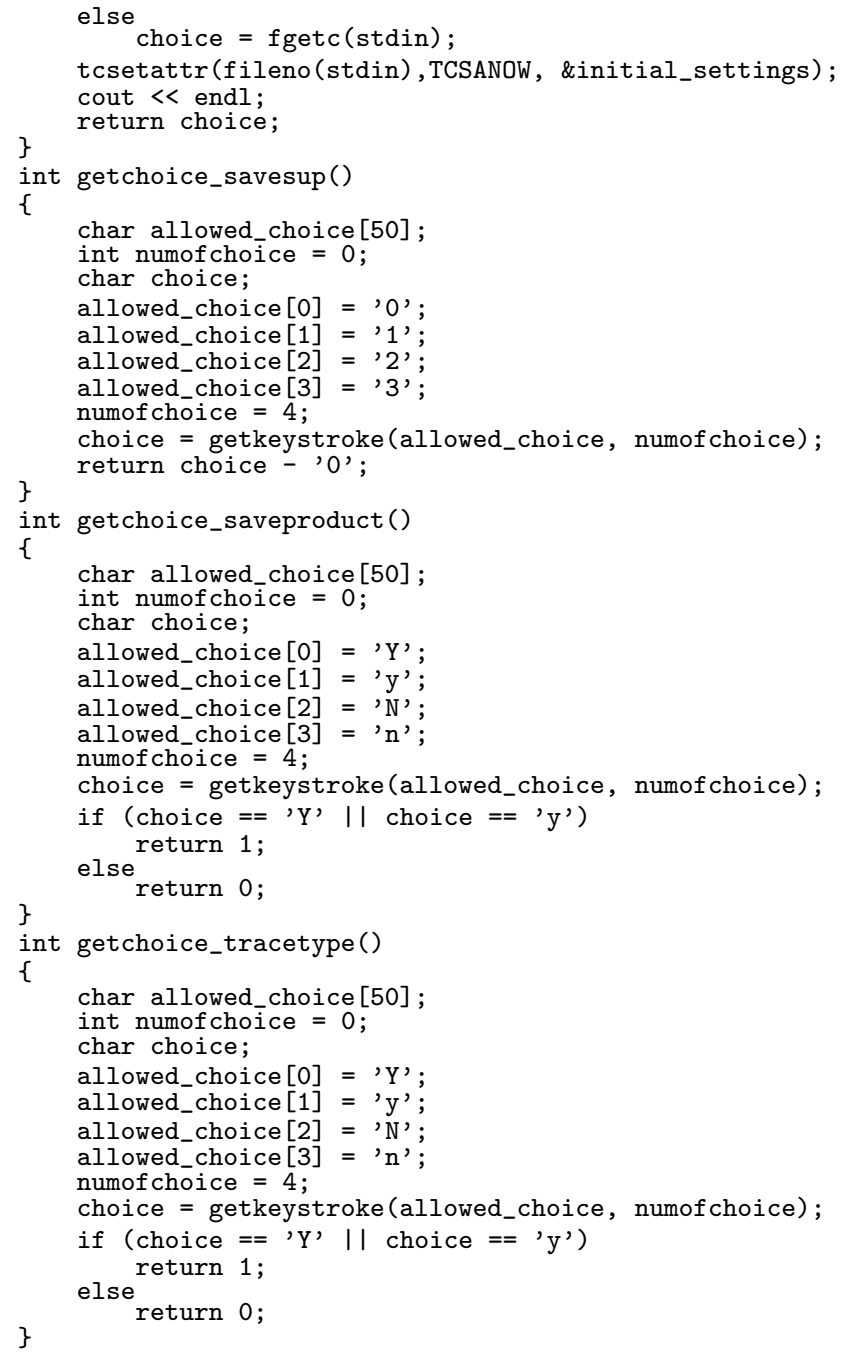

